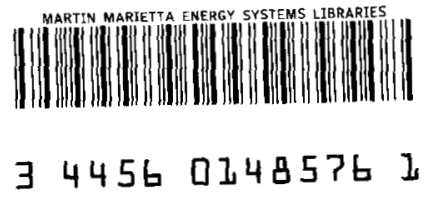

MARTIN MARIETTA

ORNL/CSD/TM-238

\title{
Validation of KENO V.a Comparison with Critical Experiments
}

\author{
W. C. Jordan \\ N. F. Landers \\ L. M. Petrie
}

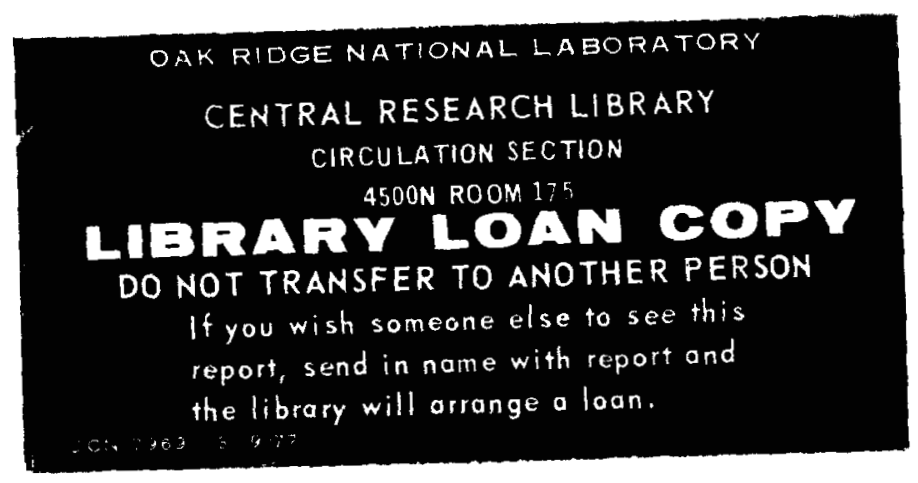

OPERATED BY

MARTIN MARIETTA ENERGY SYSTEMS, INC.

FOR THE UNITED STATES

DEPARTMENT OF ENERGY 

Printed in the United States of America. Available from
National Technical Information Service

U.S. Department of Commerce

5285 Port Royal Road, Springfield, Virginia 22161

NTIS price codes-Printed Copy: A16 Microfiche A0I

This report was prepared as an account of work sponsored by an agency of the United States Government. Neither the United States Government nor any agency thereof, nor any of their employees, makes any warranty, express or implied, or assumes any legal liability or responsibility for the accuracy, completeness, or usefulness of any information, apparatus, product, or process disclosed, or represents that its use would not infringe privately owned rights. Reference herein to any specific commercial product, process, or service by trade name, trademark, manufacturer, or otherwise, does not necessarily constitute or imply its endorsement, recommendation, or favoring by the United States Government or any agency thereof. The views and opinions of authors expressed herein do not necessarily state or reflect those of the United States Government or any agency thereof. 
ORNL/CSD/TM-238

\title{
VALIDATION OF KENO V.a COMPARISON WITH CRITICAL EXPERIMENTS
}

\author{
W. C. Jordan \\ N. F. Landers \\ L. M. Petrie
}

Date Published: December 1986

\author{
Computing and Telecommunications Division \\ at \\ Oak Ridge National Laboratory \\ Post Office Box X \\ Oak Ridge, Tennessee 37831
}

\section{MARTIN MARIETTA ENERGY SYSTEMS, INC., operating the}

Oak Ridge Gaseous Diffusion Plant Oak Ridge National Laboratory

Oak Ridge Y-12 Plant

Paducah Gaseous Diffusion Plant

under Contract No. DE-AC05-84OR21400

for the

U.S. DEPARTMENT OF ENERGY

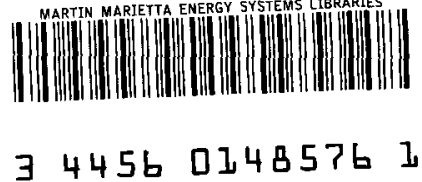





\section{CONTENTS}

$\begin{array}{ll}\text { SECTION } & \text { PAGE }\end{array}$

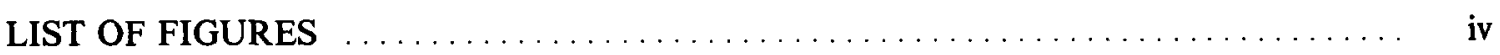

LIST OF TABLES $\ldots \ldots \ldots \ldots \ldots \ldots \ldots \ldots \ldots \ldots \ldots \ldots \ldots \ldots \ldots \ldots \ldots$

ACKNOWLEDGMENTS $\ldots \ldots \ldots \ldots \ldots \ldots \ldots \ldots \ldots \ldots \ldots \ldots \ldots \ldots \ldots$ vii

ABSTRACT $\ldots \ldots \ldots \ldots \ldots \ldots \ldots \ldots \ldots \ldots \ldots \ldots \ldots \ldots \ldots \ldots \ldots \ldots \ldots \ldots$

1. VALIDATION OF KENO V.a IN Y12CSG $\ldots \ldots \ldots \ldots \ldots \ldots \ldots \ldots \ldots \ldots \ldots \ldots \ldots \ldots \ldots$

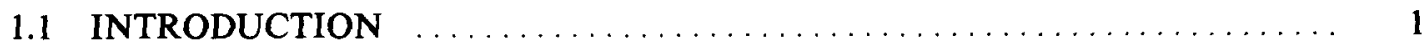

1.2 DESCRIPTION OF THE CODE PACKAGE $\ldots \ldots \ldots \ldots \ldots \ldots \ldots \ldots \ldots \ldots \ldots \ldots \ldots \ldots$

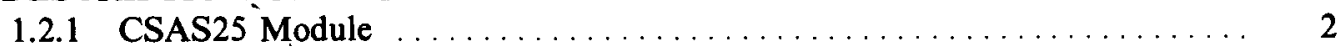

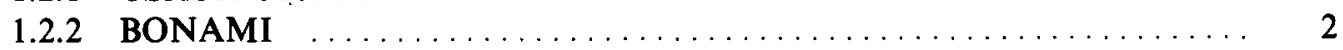

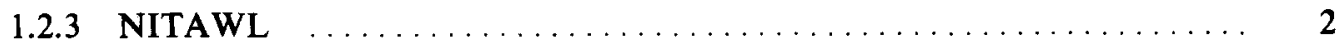

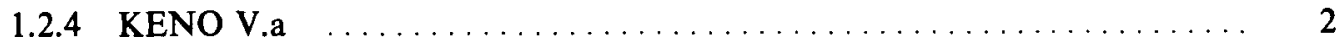

1.2.5 The 27-Group Neutron Cross-Section Library $\ldots \ldots \ldots \ldots \ldots \ldots \ldots$

1.3 CALCULATIONAL PROCEDURE $\ldots \ldots \ldots \ldots \ldots \ldots \ldots \ldots \ldots \ldots \ldots \ldots \ldots \ldots \ldots \ldots$

1.4 PROBLEM DESCRIPTION AND RESULTS $\ldots \ldots \ldots \ldots \ldots \ldots \ldots \ldots \ldots \ldots, 4$

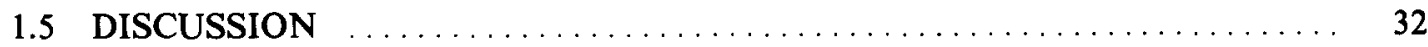

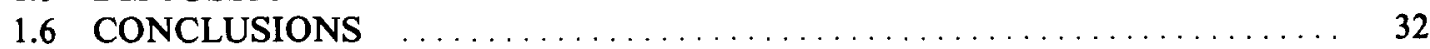

2. EVALUATION OF BIAS IN THE KENO V.a VALIDATION $\ldots \ldots \ldots \ldots \ldots \ldots \ldots 49$

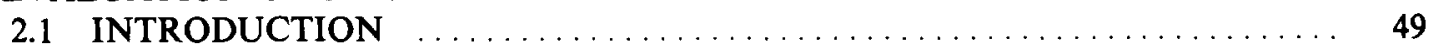

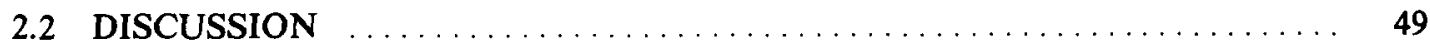

2.2.1 Bias in the 3\% Enriched Green Block Calculations from Ref. $18 \ldots \ldots .49$

2.2.2 Cross-Section Irregularities in the 27- and 218-Group

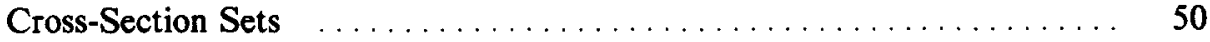

2.2.3 Code Errors Encountered During Validation $\ldots \ldots \ldots \ldots \ldots \ldots \ldots \ldots$

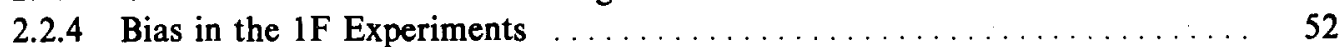

2.2.5 Bias in the Slab Experiments from Ref. $43 \ldots \ldots \ldots \ldots \ldots \ldots$

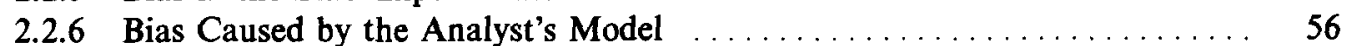

3. GENERAL COMMENTS ON THE EXPERIMENTS REVIEWED $\ldots \ldots \ldots \ldots \ldots \ldots$

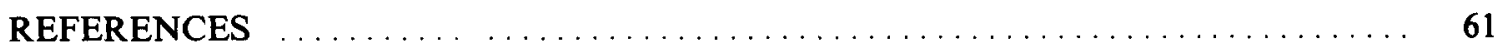

Appendix A Y12CSG INPUT DATA USED FOR VALIDATION CASES $\ldots \ldots \ldots \ldots \ldots$ A-1

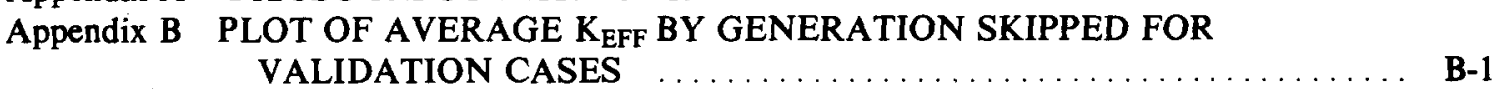

Appendix C CALCULATED RESULTS AND EXPERIMENTAL PARAMETERS $\ldots$ C-1 


\section{LIST OF FIGURES}

FIGURE

PAGE

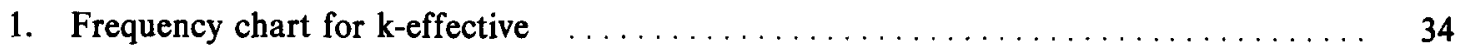

2. Frequency chart for average energy group of neutron causing fission $\ldots \ldots \ldots \ldots \ldots$

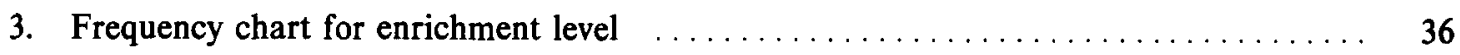

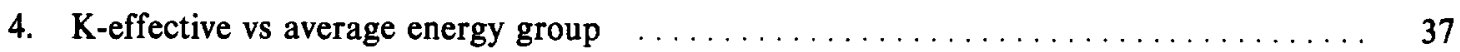

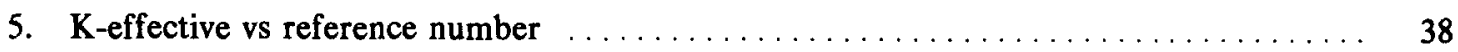

6. K-effective vs enrichment level for low enrichment experiments $\ldots \ldots \ldots \ldots \ldots \ldots \quad 39$

7. K-effective vs enrichment level for high enrichment experiments $\ldots \ldots \ldots \ldots \ldots \ldots \quad 40$

8. K-effective vs average energy group for Table 1 experiments $\ldots \ldots \ldots \ldots \ldots \ldots, 41$

9. K-effective vs average energy group for Table 2 experiments $\ldots \ldots \ldots \ldots \ldots \ldots, 42$

10. K-effective vs average energy group for Table 3 experiments $\ldots \ldots \ldots \ldots \ldots \ldots, 43$

11. K-effective vs average energy group for Table 4 experiments $\ldots \ldots \ldots \ldots \ldots \ldots, 44$

12. K-effective vs average energy group for Table 5 experiments $\ldots \ldots \ldots \ldots \ldots \ldots$

12a. K-effective vs average energy group for Table 5 experiments,

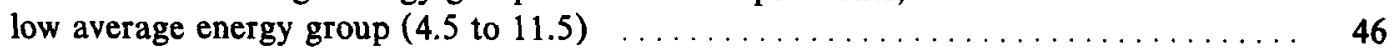

12b. K-effective vs average energy group for Table 5 experiments, high average energy group ( 20 to 25 )

13. K-effective vs average energy group for Table 6 experiments $\ldots \ldots \ldots \ldots \ldots \ldots, 48$

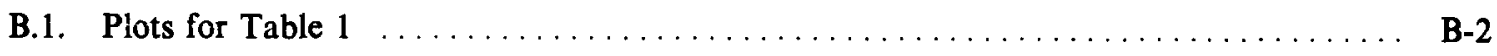

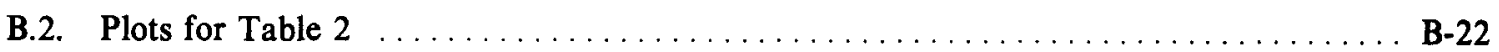

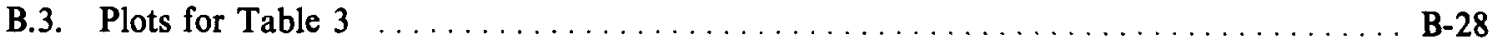

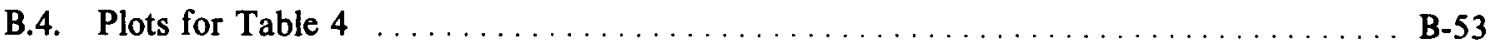

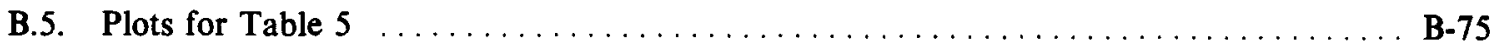

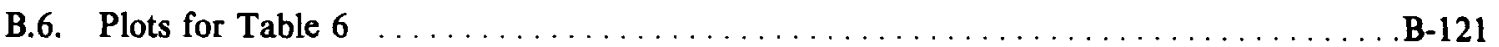




\section{LIST OF TABLES}

TABLE

PAGE

1. KENO V.a calculated $k_{\text {eff }}$ for experimentally critical low enriched uranium systems (last modeled in ref. 8)

2. KENO V.a calculated $k_{\text {eff }}$ for experimentally critical low enriched uranium systems (last modeled in ref. 11)

3. KENO V.a calculated $k_{\text {eff }}$ for experimentally critical low enriched uranium systems (last modeled in ref. 7)

4. KENO V.a calculated $k_{\text {eff }}$ for experimentally critical highly enriched uranium systems (last modeled in ref. 8)

5. KENO V.a calculated $k_{\text {eff }}$ for experimentally critical highly enriched uranium systems (last modeled in ref. 6)

6. KENO V.a calculated $k_{\text {eff }}$ for experimentally critical highly enriched uranium systems

7. Average $\mathrm{k}_{\text {eff }}$ by table

8. Comparison of standard NITAWL treatment vs treating all moderators $\ldots \ldots \ldots \ldots .51$

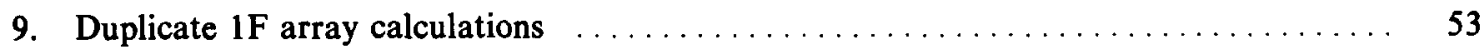

10. Comparison of calculated $k$-eff for the $1 F$ arrays $\ldots \ldots \ldots \ldots \ldots \ldots \ldots \ldots \ldots \ldots \ldots \ldots$

11. Comparison of calculated k-eff for the slab experiments $\ldots \ldots \ldots \ldots \ldots \ldots \ldots \ldots$

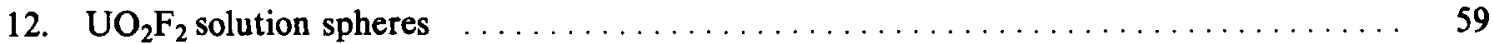

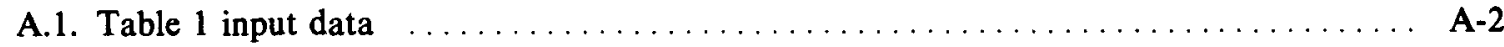

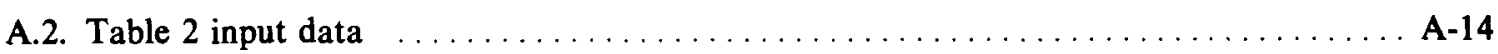

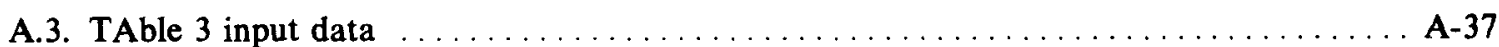

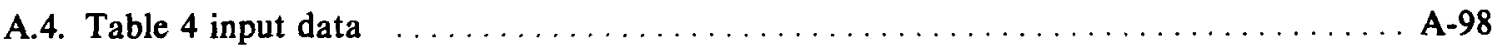

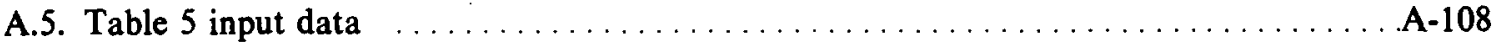

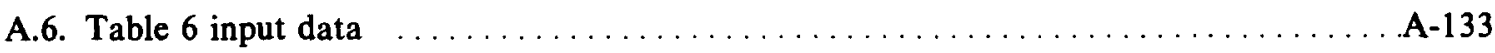

C.1. Calculational results and experimental parameters $\ldots \ldots \ldots \ldots \ldots \ldots \ldots \ldots, C$ 



\section{ACKNOWLEDGMENTS}

This work was supported by the Nuclear Criticality Safety Analytical Methods Resource Center, funded by the Office of Nuclear Safety, U.S. Department of Energy.

The authors would like to acknowledge the helpful assistance provided by M. E. Easter, J. R. Knight, and J. C. Turner in modeling and checking many of the cases run in this validation. A special acknowledgment is extended to Lindy Norris for her patient assistance in preparing this report. 



\begin{abstract}
Section 1 of this report documents the validation of KENO V.a against 258 critical experiments. Experiments considered were primarily high or low enriched uranium systems. The results indicate that the KENO V.a Monte Carlo Criticality Program accurately calculates a broad range of critical experiments. A substantial number of the calculations showed a positive or negative bias in excess of $1 \frac{112 \%}{2}$ $k$-effective $\left(k_{\text {eff }}\right)$. Classes of criticals which show a bias include $3 \%$ enriched green blocks, highly enriched uranyl fluoride slab arrays, and highly enriched uranyl nitrate arrays. If these biases are properly taken into account, the KENO V.a code can be used with confidence for the design and criticality safety analysis of uranium-containing systems.

Section 2 of this report documents the results of investigation into the cause of the bias observed in Sect. 1. The results of this study indicate that the bias seen in Sect. 1 is caused by code bias, crosssection bias, reporting bias, and modeling bias. There is evidence that many of the experiments used in this validation and in previous validations are not adequately documented. The uncertainty in the experimental parameters overshadows bias caused by the code and cross sections and prohibits code validation to better than about $1 \%$ in $\mathrm{k}_{\text {eff. }}$
\end{abstract}





\section{VALIDATION OF KENO V.a IN Y12CSG}

\subsection{INTRODUCTION}

The KENO V.a Monte Carlo Criticality Program is currently being validated in several different ways. Each of these validations will be reported separately. The intent of this study is to validate KENO V. $a^{1}$ in the Y12CSG package against critical experiments. Y12CSG is a frozen version of a system of nuclear criticality safety computer codes similar to the nuclear criticality safety codes available through the $\mathrm{SCALE}^{2}$ package. The sequence which is being validated is the CSAS25 sequence documented in CSAS4 of the SCALE manual. The sequence uses control module CSAS25 created on 85.162 and program modules Q\#\$008 (BONAMI-2) created on 85.011, Q\#\$002 (NITAWL) created on 85.011, and Q\#\$009 (KENO V.a) created on 85.162. The 27-group ENDF/B-IV cross-section master library created on 84.066 and stored in data set Y.LMP12852.Y12CSG.X27 was used for all calculations.

The purpose of a code validation is manyfold. ANSI Standard ANS- $8.1^{3}$ requires that calculational methods used for criticality safety be validated and any bias be determined by correlating the results of critical experiments with calculations. In the past, the primary source of criticality safety information applicable to the handling of fissile material outside of nuclear reactors was obtained from critical experiments or from nuclear safety guides and handbooks based upon critical experiments. These experiments were costly and often took a substantial amount of time before the results were available. With the closing of many of the critical experiment facilities, using critical experiments for operational criticality safety approval has become virtually impossible. Increasing reliance is being placed upon calculational methods to fill in and extend existing experimental data. Extension of data has been accomplished by making use of trends in the bias established in code validation. It is essential that the computational methods used for nuclear criticality safety purposes be sufficiently accurate that one can be confident of subcriticality when adequate safety margins are applied. It is also important that the applied safety margins not be unduly conservative. To accomplish both of these goals simultaneously requires the ability to accurately predict the neutron multiplication factor of a system.

This portion of the KENO V.a validation is based primarily on uranium experiments which have been used in the past for KENO validation. ${ }^{4-8}$ The validation is being done independent of any particular nuclear criticality safety organization, with close cooperation with the authors of the code and personnel familiar both with the codes and many of the critical experiments which were used in the earlier validations. In this manner, trends in the bias can be identified and areas of applicability can be established. In addition, calculational deficiencies may be identified and possibly eliminated.

A total of 258 low enriched ( $<5 \%$ U-235) and highly enriched ( $~ 90 \%$ U-235) experiments have been modeled. These include various solution systems, intermediate density systems, and uranium oxide and metal systems. Four primary sources of bias were identified during the course of the validation. These were (1) bias caused by approximations and the manner in which the theory was implemented in portions of the code (code bias), (2) bias caused by the group structure of the cross sections (cross-section bias), (3) bias caused by the manner in which the experiments were reported versus what was actually done (reporting bias), and (4) bias caused because of the manner in which the analyst chose to model the critical experiment (modeling bias). Mitigation of the bias for the first two cases is fairly straightforward since they are systematic and are introduced by the KENO program and control modules. If trends can be established, it may be possible to eliminate bias in future updates to the codes. The third source of bias, reporting bias, is not well defined and is much more difficult to resolve. Reporting bias is very difficult to distinguish from code and cross-section bias without additional information beyond what was originally reported. The necessary details which have been omitted are different from experiment to experiment and are influenced by the date the experiments were performed along with the prevailing 
belief of the experimenters of what was important and what could be ignored. The fourth source, modeling bias, is bias which stems from the analyst's model and is also difficult to distinguish from other forms of bias, especially when the analyst chooses to ignore (or include) specific portions of a model based on past experience with similar calculations.

The results of this validation are reported in Sect. 1. The discussion of the cause-and-effect of the identified bias for the experiments are presented in Sect. 2. In Sect. 2, many of the calculations were performed with modified versions of the code and with adjusted parameters. The results in Sect. 2 should not be considered part of the validation of the code. They are presented in order to aid in establishing the source of the bias observed in the validation.

\subsection{DESCRIPTION OF THE CODE PACKAGE}

\subsubsection{CSAS25 Module}

The CSAS25 control module allows simplified data input to the functional modules such as BONAMI, NITAWL, and KENO V.a. The module will calculate atomic densities for both mixtures and standard solutions. The module also generates input data for NITAWL, allowing various options for treatment of the cross sections in the resonance region for both homogeneous and heterogeneous systems. Since the earlier validations reported in refs. 6 and 7 , several corrections have been made to the CSAS25 control module.

\subsubsection{BONAMI}

For the 27-group master cross-section library used in this validation, the primary purpose of the BONAMI functional module is to select the required material cross sections and to create a smaller master cross-section library to be processed by NITAWL. There is no data processing done in BONAMI for the 27-group cross-section library.

\subsubsection{NITAWL}

The NITAWL functional module treats the resonance region cross sections for resonance absorbers. The treatment is based on the Nordheim Integral Transform method. The analyst has the option of treating the resonance parameters in the manner most appropriate for the problem detail. The options include (1) an homogeneous medium treatment which treats the resonance region of the fissile mixture as if it were an infinite homogeneous media, and (2) a finite lump treatment which treats the resonance region as if the fissile mixture were in a discrete lump with a $1 / E$ return flux at the boundary for problems where the system is substantially heterogeneous or where reflector effects are important. These options are automatically invoked in the CSAS25 module according to the keywords INFHOMMEDIUM, LATTICECELL, or MULTIREGION. The INFHOMMEDIUM option treats the fissile mixture as an infinite homogeneous medium. The LATTICECELL option utilizes a multiple repeated cell for the resonance self-shielding correction. With the MULTIREGION option, a single-cell resonance self-shielding calculation is made.

\subsubsection{KENO V.a}

KENO V.a is a substantial revision of KENO IV, ${ }^{9}$ and includes an enhanced geometry package which allows modeling of a wide variety of complex three-dimensional geometries. The geometry package allows nested arrays and "holes" to be placed in the geometry model. The code allows the use of reflector options, including mirror reflection, differential albedo reflection, and an automatic reflector; the latter has the capability of using reflector region weighting functions which are based on onedimensional adjoint calculations. The reflector options simplify geometry data input and/or reduce the 
running time of a specific problem. Cross-section input and atom density input are handled by the CSAS25 module. Most of the major KENO parameters have defaults which work for a wide variety of problems, but which can be overridden if the problem or analyst dictates. The major revision to KENO V.a since the validations reported in refs. 6 and 7 is that the program module ICE has been deleted, and KENO V.a mixes its own cross sections. In addition, there have been a few changes in the program logic involving the treatment of computer round-off error during tracking.

\subsubsection{The 27-Group Neutron Cross-Section Library}

The 27-group cross-section library was created in August 1981 from the ENDF/B-IV 218-group master library. ${ }^{10}$ (It should be noted that ref. 10 does not include a discussion of all the nuclides which have been placed in the 218-group library and that several of the nuclides have been regenerated since the document was issued.) Prior to 1981 , the primary cross-section set in use at ORNL was the Knight-Modified Hansen-Roach, 16-group cross-section library. The 27-group set is considered to be an improvement because more thermal neutron groups are available, upscatter is included in the set, and the origin of the base data used to create the data set is better documented. The 27-group set was collapsed with the weighting spectra which was generated with the 218-group library and which was based on the weighting spectra used to prepare the 218-group library from ENDF/B-IV data.

\subsection{CALCULATIONAL PROCEDURE}

In the KENO V.a validation process, many of the available options in CSAS25 and KENO V.a were exercised. These options include the automatic atomic density generation features, the various treatments in NITAWL, and the geometry and reflector options in KENO V.a. This was done in order to validate the mechanics and capabilities of the code for a broad spectrum of problems. The majority of the calculations repeat calculations which were modeled by different people at different times. Usually there are multiple ways that a problem may be specified, all of which are equally correct. The original input data were left substantially unchanged in order to allow testing for discrepancies or bias which might have been caused by the differences in analysts' problem specifications. A complete listing of the input data has been included in Appendix A.

The resonance treatment used was the one which appeared to most accurately fit the problem specifications. All problems were run for 103 generations of 600 neutrons per generation. Some problems were run using the adjoint reflector weighting (bias ID), whereas others were run using full neutron tracking in the reflector in order to test for bias which might be caused by one or the other treatment.

With KENO V.a, there is no single $k_{\text {eff }}$ which is "the answer" for the problem. The code starts with an initial neutron distribution and calculates the effective multiplication for a generation of neutrons. The next generation uses a starting distribution based on the fission points of the previous generation and calculates the effective multiplication for the current generation. Since 103 generations were run, there are 103 different answers. In the ideal situation, once the source has converged and the original (and usually arbitrary) starting distribution has decayed, the difference in the batch-to-batch $\mathrm{k}_{\text {eff }}$ may be attributed to the statistical nature of the Monte Carlo calculation, and the "best" answer should be the average of the remaining calculations. The common practice in KENO V.a is to tabulate the average $\mathrm{k}_{\text {eff }}$ as a function of the number of generations skipped. Considerable judgment is then required in determining the answer. Many calculations do not approach the ideal situation. For example, undersampling may exist where the number of neutrons per generation is not sufficient to sustain a proper source distribution, or the number of generations is insufficient to converge the source distribution. In order to remove the element of judgment from the interpretation of the results and to mechanize the process of validating and comparing results to previous validation, the average $k_{\text {eff }}$ by generation skipped with the lowest calculated standard deviation is reported here. This number appears as the "final value" 
in the plot of average $k_{\text {eff }}$ by generation skipped in the KENO V.a output. The analyst is encouraged to review the tabulated results against the plots in Appendix B when using the validation for establishing safety margins for the different types of criticals which were run.

In each calculation, the optional parameter NUB was set to "yes" in KENO V.a so the program would calculate and print the average energy group of the neutron-causing fission. This parameter has been frequently used in past validations because of trends which have been observed and appear to be correlated.

\subsection{PROBLEM DESCRIPTION AND RESULTS}

A brief problem description and results are presented in Tables 1-6. The experimental models used to generate Table 1 are from low enriched uranium experiments obtained from ref. 8. These include experiments for homogeneous single-unit criticals, both reflected and unreflected, as well as heterogeneous uranium metal rods in water. The fissile compounds considered were $\mathrm{UO}_{2} \mathrm{~F}_{2}$, $\mathrm{U}$ metal, and $\mathrm{U}_{3} \mathrm{O}_{8}$, all at $4.89 \% \mathrm{U}-235$ enrichment. The moderating materials were water, sterotex, and $\mathrm{UO}_{2} \mathrm{~F}_{2}$ solution.

The experimental models used to generate Table 2 are from low enriched uranium experiments obtained from ref. 11. These experiments are formally undocumented experiments for $3.85 \%$ enriched uranium metal rods in water. The heterogeneous systems consist of large diameter rods which are water moderated. In some cases the rods have an internal hole which is also water moderated. The critical experiments were modeled in detail from descriptions in the experiment logbook. Extensive detail was included in these calculations in order to thoroughly test the KENO V.a geometry package.

Table 3 includes models from ref. 7 which were used for the validation of Y12CSG for the Oak Ridge Gaseous Diffusion Plant. The validation considered single-unit criticals, both reflected and unreflected, at several enrichments ranging from 1.4 to $4.98 \%$. The first 30 experiments are $\mathrm{UF}_{4} /$ paraffin-moderated systems and $\mathrm{UO}_{2} \mathrm{~F}_{2}$ solution systems at various moderation levels. The last 20 experiments are $4.46 \%$ enriched damp oxide experiments performed at Rocky Flats. These later experiments test the code's ability to handle both intermixed and interstitial moderation. Several of the damp oxide experiments, which were driven by highly enriched uranium systems, were added to the series.

Highly enriched uranium experiments from ref. 8 were used to generate Table 4 . These are $\mathrm{UO}_{2} \mathrm{~F}_{2}$ and $\mathrm{UO}_{2}\left(\mathrm{NO}_{3}\right)_{2}$ solution systems. Both single-unit and array systems were analyzed under a variety of reflection conditions including unreflected, fully water reflected, concrete reflected, and Plexiglas reflected.

Table 5 includes experiments from ref. 6 which were used for validation of Y12CSG for the Y-12 Plant. Single-unit reflected and unreflected systems for uranium metal, uranium alloy, $\mathrm{UO}_{2} \mathrm{~F}_{2}$ solution and $\mathrm{UO}_{2}\left(\mathrm{NO}_{3}\right)_{2}$ solutions were considered. Extensive calculations were performed on arrays, including (1) arrays of metal units with and without interstitial moderation, (2) arrays of $\mathrm{UO}_{2} \mathrm{~F}_{2}$ slab systems, and (3) arrays of 5-liter containers of $\mathrm{UO}_{2}\left(\mathrm{NO}_{3}\right)_{2}$ having varying reflector thicknesses of paraffin and/or Plexiglas.

Table 6 contains the experimental models and results from the eta experiments performed at Y-12. ${ }^{12,13}$ These experiments are $\mathrm{UO}_{2}\left(\mathrm{NO}_{3}\right)_{2}$ solution experiments in simple unreflected geometry. The systems are typically very dilute. The experiments included boron poisoned solutions and U-233 systems. 
Table 1. KENO V.a calculated $\mathrm{k}_{\text {eff }}$ for experimentally critical low enriched uranium systems (last modeled in ref. 8)

\begin{tabular}{cl}
\hline Case & \multicolumn{1}{c}{ Experimental description } \\
\hline CAA01 & $\begin{array}{l}\text { Experiment 1A. 401-4.89\% U rods, 0.762-cm diam, 30-cm } \\
\text { long, 1.3-cm pitch, } \mathrm{H}_{2} \mathrm{O} \text { moderated and reflected }\end{array}$ \\
CAA02 & $\begin{array}{l}\text { Experiment 4B. 400-4.89\% U rods, 0.762-cm diam, 30-cm } \\
\text { long, 1.3-cm pitch, } \mathrm{H}_{2} \mathrm{O} \text { moderated and reflected }\end{array}$ \\
CAA03 & $\begin{array}{l}\text { Experiment 11A. 240-4.89\% U rods, 0.762-cm diam, 30-cm } \\
\text { long, 1.3-cm pitch, } \mathrm{H}_{2} \mathrm{O} \text { moderated and reflected }\end{array}$ \\
CAA04 & $\begin{array}{l}\text { Experiment 6A. 203-4.89\% U rods, 0.762-cm diam, 30-cm } \\
\text { long, 2.05-cm pitch, } \mathrm{H}_{2} \mathrm{O} \text { moderated and reflected }\end{array}$ \\
CAA05 & $\begin{array}{l}\text { Experiment 8C. 400-4.89\% U rods, 0.762-cm diam, 30-cm } \\
\text { long, 2.05-cm pitch, } \mathrm{H}_{2} \mathrm{O} \text { moderated and reflected }\end{array}$ \\
CAA06 & $\begin{array}{l}\text { Experiment 14A. 398-4.89\% U rods, 0.762-cm diam, 30-cm } \\
\text { long, 1.3-cm pitch, } \mathrm{H}_{2} \mathrm{O} \text { moderated, } \mathrm{H}_{2} \mathrm{O} \text { top reflector, } \\
\text { Plexiglas bottom reflector, Pb reflected 1 face }\end{array}$
\end{tabular}

CAA07 Experiment 23B. 400-4.89\% U rods, 0.762-cm diam, 30-cm long, 1.3-cm pitch, $\mathrm{H}_{2} \mathrm{O}$ moderated, $\mathrm{H}_{2} \mathrm{O}$ top reflector, Plexiglas bottom reflector, $\mathrm{Pb}$ reflected 4 faces

CAA08 Experiment 26A. 215-4.89\% U rods, 0.762-cm diam, 30-cm long, 1.3-cm pitch, $\mathrm{H}_{2} \mathrm{O}$ moderated, $\mathrm{H}_{2} \mathrm{O}$ top reflector, Plexiglas bottom reflector, $\mathrm{Pb}$ reflected 4 faces

CAA09 Experiment 28A. 255-4.89\% U rods, $0.762-\mathrm{cm}$ diam, $30-\mathrm{cm}$ long, 1.3-cm pitch, $\mathrm{H}_{2} \mathrm{O}$ moderated, $\mathrm{H}_{2} \mathrm{O}$ top reflector, Plexiglas bottom reflector, $\mathrm{Pb}$ reflected 4 faces, $\mathrm{SS}$ in center

CAA10 Experiment 30C. 494-4.89\% U rods, 0.762-cm diam, 30-cm long, 1.3-cm pitch, $\mathrm{H}_{2} \mathrm{O}$ moderated, $\mathrm{H}_{2} \mathrm{O}$ to reflector, Plexiglas bottom reflector, $\mathrm{Pb}$ reflected 4 faces, boral in row 6

CAA11 Experiment 31C. 494-4.89\% U rods, 0.762-cm diam, 30-cm long, 1.3-cm pitch, $\mathrm{H}_{2} \mathrm{O}$ moderated, $\mathrm{H}_{2} \mathrm{O}$ top reflector, Plexiglas bottom reflector, $\mathrm{Pb}$ reflected 4 faces, $\mathrm{Cd}$ in row 6

CAA12 Experiment $32 \mathrm{~S} .11 \times 12$ array of $4.89 \% \mathrm{U}$ rods in $-300 \mathrm{~g} \mathrm{U} / 1 \mathrm{UO}_{2} \mathrm{~F}_{2}$ solution, $0.762-\mathrm{cm}$ diam, $2.05-\mathrm{cm}$ pitch, in 95.88-cm-diam tank

$14 \quad 0.9921 \pm 0.0033$

$14 \quad 0.9885 \pm 0.0026$

Reference $\quad \mathrm{k}_{\text {eff }} \pm \sigma$

14

$0.9945 \pm 0.0036$

14

$0.9912 \pm 0.0030$

14

$0.9924 \pm 0.0031$

14

$0.9942 \pm 0.0032$

14

$0.9839 \pm 0.0034$

$14 \quad 0.9957 \pm 0.0036$

14

$0.9778 \pm 0.0036$

14

$0.9966 \pm 0.0030$

14

$0.9950 \pm 0.0029$

14

$1.0010 \pm 0.0032$ 
Table 1 (continued)

\begin{tabular}{|c|c|}
\hline Case & Experimental description \\
\hline CAA13 & $\begin{array}{l}\text { Experiment } 33 \mathrm{~S} .9 \times 10 \text { array of } 4.89 \% \mathrm{U} \text { rods in } \\
-300 \mathrm{~g} \mathrm{U} / 1 \mathrm{UO}_{2} \mathrm{~F}_{2} \text { solution, } 0.762-\mathrm{cm} \text { diam, } 2.05-\mathrm{cm} \\
\text { pitch, in } 95.88-\mathrm{cm} \text {-diam tank }\end{array}$ \\
\hline
\end{tabular}

CAA14 Experiment 36S. $9 \times 10$ array of $4.89 \% \mathrm{U}$ rods in $\sim 300 \mathrm{~g} \mathrm{U} / 1 \mathrm{UO}_{2} \mathrm{~F}_{2}$ solution, $0.762-\mathrm{cm}$ diam, $2.453-\mathrm{cm}$ pitch, in $95.88-\mathrm{cm}$-diam tank

CAA15 Experiment 40S. $6 \times 7$ array of $4.89 \% \mathrm{U}$ rods in $-300 \mathrm{~g} \mathrm{U} / 1 \mathrm{UO}_{2} \mathrm{~F}_{2}$ solution, $1.31-\mathrm{cm}$ diam, $2.99-\mathrm{cm}$ pitch, in $95.88-\mathrm{cm}$-diam tank

CAAl6 Experiment $42 \mathrm{~S} .6 \times 7$ array of $4.89 \% \mathrm{U}$ rods in $-300 \mathrm{~g} \mathrm{U} / 1 \mathrm{UO}_{2} \mathrm{~F}_{2}$ solution, $1.31-\mathrm{cm}$ diam, $3.40-\mathrm{cm}$ pitch, in $95.88-\mathrm{cm}$-diam tank

CAA17 Experiment $44 \mathrm{~S} .6 \times 7$ array of $4.89 \% \mathrm{U}$ rods in $300 \mathrm{~g} \mathrm{U} / 1 \mathrm{UO}_{2} \mathrm{~F}_{2}$ solution, $1.31-\mathrm{cm}$ diam, 3.94-cm pitch, in $95.88-\mathrm{cm}$-diam tank

CAA18 Experiment 45S. $6 \times 7$ array of $4.89 \% \mathrm{U}$ rods in $-300 \mathrm{~g} \mathrm{U} / 1 \mathrm{UO}_{2} \mathrm{~F}_{2}$ solution, $1.31-\mathrm{cm}$ diam, $4.40-\mathrm{cm}$ pitch, in $95.88-\mathrm{cm}$-diam tank

CAA19 $4.89 \% \mathrm{U}_{3} \mathrm{O}_{8}$ - Stereotex blocks $55.5 \mathrm{~g} \mathrm{U}-235 / 1$, unreflected

$14 \quad 0.9921 \pm 0.0025$

$14 \quad 0.9908 \pm 0.0020$

$14 \quad 0.9940 \pm 0.0025$

$14 \quad 0.9885 \pm 0.0025$

CAA20 $4.89 \% \mathrm{U}_{3} \mathrm{O}_{8}$ - Stereotex blocks $40.6 \mathrm{~g} \mathrm{U}-235 / 1$ $(\mathrm{H} / \mathrm{X}=395.0)$, unreflected

$14 \quad 0.9884 \pm 0.0029$

CAA21 4.89\% $\mathrm{U}_{3} \mathrm{O}_{8}$ - Stereotex blocks, $22.1 \mathrm{~g} \mathrm{U}-235 / 1$

$(\mathrm{H} / \mathrm{X}=757.0)$, unreflected

CAA22 $4.89 \% \mathrm{U}_{3} \mathrm{O}_{8}-$ Stereotex blocks $33.3 \mathrm{~g} \mathrm{U}-235 / 1$ $(\mathrm{H} / \mathrm{X}=503.6)$, unreflected

$15 \quad 0.9875 \pm 0.0035$

$15 \quad 1.0030 \pm 0.0031$

$15 \quad 1.0055 \pm 0.0029$

$15 \quad 1.0042 \pm 0.0034$

CAA23 $4.89 \% \mathrm{U}_{3} \mathrm{O}_{8}$ - Stereotex blocks $64.91 \mathrm{~g} \mathrm{U}-235 / 1$

$15 \quad 0.9791 \pm 0.0036$ $(\mathrm{H} / \mathrm{X}=199.3)$, paraffin top reflector, $\mathrm{H}_{2} \mathrm{O}$ reflected bottom and sides

CAA24 $4.89 \% \mathrm{U}_{3} \mathrm{O}_{8}$ - Stereotex blocks $56.2 \mathrm{~g} \mathrm{U}-235 / 1$ $(\mathrm{H} / \mathrm{X}=244.8)$, paraffin top reflector, $\mathrm{H}_{2} \mathrm{O}$ reflected bottom and sides

CAA25 $4.89 \% \mathrm{U}_{3} \mathrm{O}_{8}$ - Stereotex blocks $40.6 \mathrm{~g} \mathrm{U}-235 / 1$ $(\mathrm{H} / \mathrm{X}=396.7)$, paraffin top reflector, $\mathrm{H}_{2} \mathrm{O}$ reflected bottom and sides

$15 \quad 1.0015 \pm 0.0035$ 
Table 1 (continued)

\begin{tabular}{|c|c|c|c|}
\hline Case & Experimental description & Reference & $\mathrm{k}_{\mathrm{eff}} \pm \sigma$ \\
\hline CAA26 & $\begin{array}{l}4.89 \% \mathrm{U}_{3} \mathrm{O}_{8}-\text { Stereotex blocks } 22.2 \mathrm{~g} \mathrm{U}-235 / 1 \\
(\mathrm{H} / \mathrm{X}=756.5) \text {, paraffin top reflector, } \mathrm{H}_{2} \mathrm{O} \text { reflected } \\
\text { bottom and sides }\end{array}$ & 15 & $0.9991 \pm 0.0025$ \\
\hline CAA27 & $\begin{array}{l}4.89 \% \mathrm{U}_{3} \mathrm{O}_{8}-\text { Stereotex blocks } 81.1 \mathrm{~g} \mathrm{U}-235 / 1 \\
(\mathrm{H} / \mathrm{X}=146.8) \text {, paraffin top reflector, } \mathrm{H}_{2} \mathrm{O} \text { reflected } \\
\text { bottom and sides }\end{array}$ & 15 & $0.9991 \pm 0.0040$ \\
\hline CAA28 & $\begin{array}{l}4.89 \% \mathrm{U}_{3} \mathrm{O}_{8}-\text { Stereotex blocks } 107.0 \mathrm{~g} \mathrm{U}-235 / 1 \\
(\mathrm{H} / \mathrm{X}=82.7) \text {, paraffin top reflector, } \mathrm{H}_{2} \mathrm{O} \text { reflected } \\
\text { bottom and sides }\end{array}$ & 15 & $0.9739 \pm 0.0035$ \\
\hline CAA29 & $\begin{array}{l}4.89 \% \mathrm{U}_{3} \mathrm{O}_{8}-\text { Stereotex blocks } 33.3 \mathrm{~g} \mathrm{U}-235 / 1 \\
(\mathrm{H} / \mathrm{X}=504.1) \text {, paraffin top reflector, } \mathrm{H}_{2} \mathrm{O} \text { reflected } \\
\text { bottom and sides }\end{array}$ & 15 & $0.9938 \pm 0.0031$ \\
\hline CAA30 & $\begin{array}{l}4.89 \% \mathrm{UO}_{2} \mathrm{~F}_{2} \text { solution } 42.54 \mathrm{~g} \mathrm{U}-235 / 1 \\
(\mathrm{H} / \mathrm{X}=524) \text { in a } 20 \text {-in.-diam SS cylinder, unreflected }\end{array}$ & 15 & $0.9861 \pm 0.0032$ \\
\hline CAA31 & $\begin{array}{l}4.89 \% \mathrm{UO}_{2} \mathrm{~F}_{2} \text { solution } 42.54 \mathrm{~g} \mathrm{U}-235 / 1 \\
(\mathrm{H} / \mathrm{X}=524) \text { in a } 20 \times 20 \mathrm{in} . \text { aluminum box, unreflected }\end{array}$ & 15 & $0.9936 \pm 0.0032$ \\
\hline CAA32 & $\begin{array}{l}4.89 \% \mathrm{UO}_{2} \mathrm{~F}_{2} \text { solution } 31.79 \mathrm{~g} \mathrm{U}-235 / 1 \\
(\mathrm{H} / \mathrm{X}=735) \text { in a } 20 \text {-in.-diam SS cylinder, unreflected }\end{array}$ & 15 & $0.9936 \pm 0.0028$ \\
\hline CAA33 & $\begin{array}{l}4.89 \% \mathrm{UO}_{2} \mathrm{~F}_{2} \text { solution } 24.04 \mathrm{~g} \mathrm{U}-235 / 1 \\
(\mathrm{H} / \mathrm{X}=1002) \text { in a } 27.3 \text {-in.-diam aluminum sphere, } \\
\text { unreflected }\end{array}$ & 15 & $0.9949 \pm 0.0027$ \\
\hline CAA34 & $\begin{array}{l}4.89 \% \mathrm{UO}_{2} \mathrm{~F}_{2} \text { solution } 24.28 \mathrm{~g} \mathrm{U}-235 / 1 \\
(\mathrm{H} / \mathrm{X}=991) \text { in a } 30 \text {-in.-diam aluminum cylinder, } \\
\text { unreflected }\end{array}$ & 15 & $0.9959 \pm 0.0022$ \\
\hline CAA35 & $\begin{array}{l}4.89 \% \mathrm{UO}_{2} \mathrm{~F}_{2} \text { solution } 42.54 \mathrm{~g} \mathrm{U}-235 / 1 \\
(\mathrm{H} / \mathrm{X}=524) \text { in a } 15 \text {-in.-diam SS cylinder, } \mathrm{H}_{2} \mathrm{O} \text { reflected }\end{array}$ & 15 & $1.0029 \pm 0.0030$ \\
\hline CAA36 & $\begin{array}{l}4.89 \% \mathrm{UO}_{2} \mathrm{~F}_{2} \text { solution } 42.54 \mathrm{~g} \mathrm{U}-235 / 1 \\
(\mathrm{H} / \mathrm{X}=524) \text { in a } 20 \times 20 \text { in. aluminum box, } \mathrm{H}_{2} \mathrm{O} \text { reflected }\end{array}$ & 15 & $1.0106 \pm 0.0026$ \\
\hline CAA37 & $\begin{array}{l}4.89 \% \mathrm{UO}_{2} \mathrm{~F}_{2} \text { solution } 31.79 \mathrm{~g} \mathrm{U}-235 / 1 \\
(\mathrm{H} / \mathrm{X}=735) \text { in a } 15 \text {-in.-diam SS cylinder, } \mathrm{H}_{2} \mathrm{O} \text { reflected }\end{array}$ & 15 & $0.9931 \pm 0.0027$ \\
\hline CAA38 & $\begin{array}{l}4.89 \% \mathrm{UO}_{2} \mathrm{~F}_{2} \text { solution } 22.11 \mathrm{~g} \mathrm{U}-235 / 1 \\
(\mathrm{H} / \mathrm{X}=991) \text { in a } 27.3 \text {-in.-diam aluminum sphere, } \\
\mathrm{H}_{2} \mathrm{O} \text { reflected }\end{array}$ & 15 & $0.9927 \pm 0.0022$ \\
\hline CAA39 & $\begin{array}{l}4.89 \% \mathrm{UO}_{2} \mathrm{~F}_{2} \text { solution } 24.22 \mathrm{~g} \mathrm{U}-235 / 1 \\
(\mathrm{H} / \mathrm{X}=994) \text { in a } 20 \text {-in.-diam SS cylinder, } \mathrm{H}_{2} \mathrm{O} \text { reflected }\end{array}$ & 15 & $1.0001 \pm 0.0025$ \\
\hline
\end{tabular}


Table 2. KENO V.a calculated $k_{\text {eff }}$ for experimentally critical low enriched uranium systems (last modeled in ref. 11)

\begin{tabular}{ll}
\hline Case & \multicolumn{1}{c}{ Experimental description } \\
\hline CAB01 & (EBJ.1) lattice 2. 15-3.85\% U rods, \\
& 7.2 in. diam $\times 30$ in. long in square lattice, \\
& 7.2 in. center to center, $77.8 \mathrm{~cm}$ water height \\
CAB02 & (EBJ.2X) lattice $5.11-3.85 \% \mathrm{U}$ rods, \\
& $\begin{array}{l}7.2 \text { in. diam } \times 30 \text { in. long in square lattice, } \\
7.95 \text { in. center to center, } 72.4 \mathrm{~cm} \text { water height }\end{array}$
\end{tabular}

CAB03 (EBJ.3X) lattice 8. 24-3.85\% U rods,

$16 \quad 0.9927 \pm 0.0025$

7.2 in. diam $\times 30$ in.long in square lattice,

8.70 in. center to center, $75.0 \mathrm{~cm}$ water height

CAB07 (EBJ.4) lattice 3. 16-3.85\% U rods,

$16 \quad 0.9897 \pm 0.0025$

7.2 in. diam $\times 30$ in. long in triangular

16

$1.0025 \pm 0.0029$

lattice, 7.45 in. center to center, $79.3 \mathrm{~cm}$

water height

CAB08 (EBJ.5X) lattice 9. 7-3.85\% U rods,

7.2 in. diam $x 30$ in. long in triangular lattice, 8.20 in. center to center, $53.1 \mathrm{~cm}$ water height

CAB09 (EBJ.6X) lattice 2. 11-3.85\% U rods, 7.2 in. OD x 2.6 in. ID $\times 30$ in. long in triangular lattice, 7.20 center to center, $77.2 \mathrm{~cm}$ water height

CAB10 (EBJ.8) lattice 5. 6-3.85\% U rods,

7.2 in. OD $\times 2.6$ in. ID $\times 30$ in. long in square lattice, 7.95 in. center to center, $91.4 \mathrm{~cm}$ water height ( 2 subcritical)

CAB11 (EBJ.9) lattice 6. 16-3.85\% U rods, 7.2 in. OD x 2.6 in. ID $\times 30$ in. long in square lattice, 8.70 in. center to center, $49.2 \mathrm{~cm}$ water height

CAB12 (EBJ.10) lattice 14. 20-3.85\% U rods, 7.2 in. OD x 2.6 in. ID x 30 in. long in square lattice, $9.07 \mathrm{in}$. center to center, $79 \mathrm{~cm}$ water height 
Table 2 (continued)

\begin{tabular}{|c|c|c|c|}
\hline Case & Experimental description & Reference & $k_{\text {eff }} \pm \sigma$ \\
\hline CAB13 & $\begin{array}{l}\text { (EBJ.11) lattice } 19.8-3.85 \% \mathrm{U} \text { rods, } \\
7.2 \text { in. OD } \times 2.6 \mathrm{in.} \text { ID x } 30 \text { in. long in } \\
\text { square lattice, } 7.2 \mathrm{in.} \text { center to center, } \\
72.5 \mathrm{~cm} \text { water height }\end{array}$ & 16 & $0.9960 \pm 0.0028$ \\
\hline $\mathrm{CAB} 14$ & $\begin{array}{l}\text { (EBJ.12) lattice } 3.22-3.85 \% \mathrm{U} \text { rods, } \\
2.5 \text { in. diam } \times 30 \text { in. long in square lattice, } \\
2.85 \text { in. center to center, } 72.3 \mathrm{~cm} \text { water height }\end{array}$ & 16 & $1.0093 \pm 0.0026$ \\
\hline $\mathrm{CAB} 15$ & $\begin{array}{l}\text { (EBJ.13) lattice } 9.15-3.85 \% \mathrm{U} \text { rods, } \\
2.5 \text { in. diam } \times 30 \mathrm{in} \text {. long in square lattice, } \\
3.25 \text { in. center to center, } 64.8 \mathrm{~cm} \text { water height }\end{array}$ & 16 & $0.9992 \pm 0.0030$ \\
\hline $\mathrm{CAB} 16$ & $\begin{array}{l}\text { (EBJ.14) lattice } 2.23-3.85 \% \mathrm{U} \text { rods, } \\
2.5 \text { in. diam } \times 30 \text { in. long in square lattice } \\
4.00 \text { in. center to center, } 68.9 \mathrm{~cm} \text { water height }\end{array}$ & 16 & $0.9877 \pm 0.0029$ \\
\hline
\end{tabular}

* Job failed in execution. 
Table 3. KENO V.a calculated $\mathrm{k}_{\text {eff }}$ for experimentally critical low enriched uranium systems (last modeled in ref. 7)

Case Experimental description $\quad$ Reference $\quad \mathrm{k}_{\text {eff }} \pm \sigma$

CAS04 An unreflected rectangular parallelepiped of

CAS05 An unreflected rectangular parallelepiped of homogeneous $\mathrm{U}(1.4) \mathrm{F}_{4}$ and paraffin with an $\mathrm{H} / \mathrm{U}-235$ atomic ratio of $421.8 ; 100.0^{\circ} \mathrm{cm} \times 99.9 \mathrm{~cm} \times 103.1 \mathrm{~cm}$

CAS06 An unreflected rectangular parallelepiped of homogeneous $\mathrm{U}(1.4) \mathrm{F}_{4}$ and paraffin with an $\mathrm{H} / \mathrm{U}-235$ atomic ratio of $421.8 ; 130.7 \mathrm{~cm} \mathrm{x} 130.6 \mathrm{~cm} \mathrm{x} 74.2 \mathrm{~cm}$

CAS11 A reflected rectangular parallelepiped of homogeneous $\mathrm{U}(2) \mathrm{F}_{4}$ and paraffin with an $\mathrm{H} / \mathrm{U}-235$ atomic ratio of $195.2 ; 56.22 \mathrm{~cm} \times 56.22 \mathrm{~cm} \times 112.88 \mathrm{~cm}$, reflected with $15.2 \mathrm{~cm}$ of paraffin on top and sides and $15.2 \mathrm{~cm}$ of Plexiglas on the bottom

CAS12 An unreflected rectangular parallelepiped of homogeneous $\mathrm{U}(2) \mathrm{F}_{4}$ and paraffin with an $\mathrm{H} / \mathrm{U}-235$ atomic ratio of $195.2 ; 71.47 \mathrm{~cm} \times 71.47 \mathrm{~cm} \mathrm{x} 94.14 \mathrm{~cm}$

CAS13 A reflected rectangular parallelepiped of homogeneous $\mathrm{U}(2) \mathrm{F}_{4}$ and paraffin with an $\mathrm{H} / \mathrm{U}-235$ atomic ratio of $293.9 ; 51.11 \mathrm{~cm} \times 51.11 \mathrm{~cm} \times 73.87 \mathrm{~cm}$, reflected with $15.2 \mathrm{~cm}$ of paraffin on top

CAS14 An unreflected rectangular parallelepiped of homogeneous $\mathrm{U}(2) \mathrm{F}_{4}$ and paraffin with an $\mathrm{H} / \mathrm{U}-235$ atomic ratio of $293.9 ; 56.22 \mathrm{~cm} \mathrm{x} 56.22 \mathrm{~cm} \mathrm{x} 122.47 \mathrm{~cm}$

CAS15 A reflected rectangular parallelepiped of homogeneous $\mathrm{U}(2) \mathrm{F}_{4}$ and paraffin with an $\mathrm{H} / \mathrm{U}-235$ atomic ratio of $406.3 ; 53.67 \mathrm{~cm} \mathrm{x} 53.67 \mathrm{~cm} \mathrm{x} 54.29 \mathrm{~cm}$, reflected with $15.2 \mathrm{~cm}$ of paraffin on top and sides and $15.2 \mathrm{~cm}$ of Plexiglas on the bottom

CAS16 A reflected rectangular parallelepiped of homogeneous $\mathrm{U}(2) \mathrm{F}_{4}$ and paraffin with an $\mathrm{H} / \mathrm{U}-235$ atomic ratio of $495.9 ; 46.00 \mathrm{~cm} \times 46.00 \mathrm{~cm} \times 96.57 \mathrm{~cm}$, reflected with $15.2 \mathrm{~cm}$ of paraffin on top and sides and $15.2 \mathrm{~cm}$ of Plexiglas on the bottom

CAS17 A reflected rectangular parallelepiped of homogeneous $\mathrm{U}(2) \mathrm{F}_{4}$ and paraffin with an $\mathrm{H} / \mathrm{U}-235$ atomic ratio of $613.6 ; 56.32 \mathrm{~cm} \mathrm{x} 61.29 \mathrm{~cm} \mathrm{x} 54.08 \mathrm{~cm}$, reflected with $15.2 \mathrm{~cm}$ of polyethylene on top and sides and $15.2 \mathrm{~cm}$ of Plexiglas on the bottom 
Table 3 (continued)

\begin{tabular}{ll}
\hline Case & \multicolumn{1}{c}{ Experimental description } \\
\hline CAS18 & $\begin{array}{l}\text { An unreflected rectangular parallelepiped of } \\
\text { homogeneous } \mathrm{U}(2) \mathrm{F}_{4} \text { and paraffin with an } \mathrm{H} / \mathrm{U}-235 \\
\text { atomic ratio of } 613.6 ; 61.3 \mathrm{~cm} \times 66.54 \mathrm{~cm} \times 66.52 \mathrm{~cm}\end{array}$ \\
CAS19 & $\begin{array}{l}\text { A reflected rectangular parallelepiped of } \\
\text { homogeneous } \mathrm{U}(2) \mathrm{F}_{4} \text { and paraffin with an } \mathrm{H} / \mathrm{U}-235 \\
\text { atomic ratio of } 971.7 ; 76.51 \mathrm{~cm} \times 76.44 \mathrm{~cm} \times 82.42 \mathrm{~cm} \\
\text { reflected with } 5.2 \mathrm{~cm} \text { of polyethylene on top and } \\
\text { sides and } 15.2 \mathrm{~cm} \text { of Plexiglas on the bottom }\end{array}$
\end{tabular}

CAS20 An unreflected rectangular parallelepiped of homogeneous $\mathrm{U}(2) \mathrm{F}_{4}$ and paraffin with an $\mathrm{H} / \mathrm{U}-235$ atomic ratio of $971.7 ; 81.45 \mathrm{~cm} \times 86.70 \mathrm{~cm} \times 88.22 \mathrm{~cm}$

CAS21 A reflected rectangular parallelepiped of homogeneous $\mathrm{U}(3) \mathrm{F}_{4}$ and paraffin with an $\mathrm{H} / \mathrm{U}-235$ atomic ratio of $133.4 ; 51.14 \mathrm{~cm} \times 51.14 \mathrm{~cm} \times 51.27 \mathrm{~cm}$, reflected with $15.2 \mathrm{~cm}$ of paraffin on top and sides and $15.2 \mathrm{~cm}$ of Plexiglas on the bottom

CAS22 A reflected rectangular parallelepiped of homogeneous $\mathrm{U}(3) \mathrm{F}_{4}$ and paraffin with an $\mathrm{H} / \mathrm{U}-235$ atomic ratio of $133.4 ; 43.47 \mathrm{~cm} \mathrm{x} 43.47 \mathrm{~cm} \mathrm{x} 86.39 \mathrm{~cm}$, reflected with $15.2 \mathrm{~cm}$ of paraffin on top and sides and $15.2 \mathrm{~cm}$ of Plexiglas on the bottom

CAS23 A reflected rectangular parallelepiped of homogeneous $\mathrm{U}(3) \mathrm{F}_{4}$ and paraffin with an $\mathrm{H} / \mathrm{U}-235$ atomic ratio of $133.4 ; 46.02 \mathrm{~cm} \times 46.02 \mathrm{~cm} \times 67.57 \mathrm{~cm}$, reflected with $15.2 \mathrm{~cm}$ of paraffin on top and sides and $15.2 \mathrm{~cm}$ of Plexiglas on the bottom

CAS24 A reflected rectangular parallelepiped of homogeneous $\mathrm{U}(3) \mathrm{F}_{4}$ and paraffin with an $\mathrm{H} / \mathrm{U}-235$ atomic ratio of $133.4 ; 56.25 \mathrm{~cm} \times 56.25 \mathrm{~cm} \times 43.41 \mathrm{~cm}$, reflected with $15.2 \mathrm{~cm}$ of paraffin on top and sides and $15.2 \mathrm{~cm}$ of Plexiglas on the bottom

CAS25 A reflected rectangular parallelepiped of homogeneous $\mathrm{U}(3) \mathrm{F}_{4}$ and paraffin with an $\mathrm{H} / \mathrm{U}-235$ atomic ratio of $133.4 ; 61.36 \mathrm{~cm} \times 61.36 \mathrm{~cm} \times 38.67 \mathrm{~cm}$, reflected with $15.2 \mathrm{~cm}$ of paraffin on top and sides and $15.2 \mathrm{~cm}$ of Plexiglas on the bottom

CAS26 An unreflected rectangular parallelepiped of homogeneous $\mathrm{U}(3) \mathrm{F}_{4}$ and paraffin with an $\mathrm{H} / \mathrm{U}-235$ atomic ratio of $133.4 ; 56.47 \mathrm{~cm} \times 56.47 \mathrm{~cm} \times 86.64 \mathrm{~cm}$ 
Table 3 (continued)

\begin{tabular}{ll}
\hline Case & \multicolumn{1}{c}{ Experimental description } \\
\hline CAS27 & $\begin{array}{l}\text { An unreflected rectangular parallelepiped of } \\
\text { homogeneous } U(3) \mathrm{F}_{4} \text { and paraffin with an } \mathrm{H} / \mathrm{U}-235 \\
\text { atomic ratio of } 133.4 ; 56.25 \mathrm{~cm} \times 61.36 \mathrm{~cm} \times 74.38 \mathrm{~cm}\end{array}$ \\
CAS28 & $\begin{array}{l}\text { An unreflected rectangular parallelepiped of } \\
\text { homogeneous } \mathrm{U}(3) \mathrm{F}_{4} \text { and paraffin with an } \mathrm{H} / \mathrm{U}-235 \\
\text { atomic ratio of } 133.4 ; 61.4 \mathrm{~cm} \times 61.4 \mathrm{~cm} \times 66.0 \mathrm{~cm}\end{array}$ \\
CAS29 & $\begin{array}{l}\text { A reflected rectangular parallelepiped of } \\
\text { homogeneous } \mathrm{U}(3) \mathrm{F}_{4} \text { and paraffin with an } \mathrm{H} / \mathrm{U}-235 \\
\text { atomic ratio of } 276.9 ; 40.81 \text { cm } \times 40.80 \mathrm{~cm} \times 39.49 \mathrm{~cm}, \\
\text { reflected with } 15.2 \mathrm{~cm} \text { of polyethylene on top and sides } \\
\text { and } 15.2 \mathrm{~cm} \text { of Plexiglas on the bottom }\end{array}$
\end{tabular}

CAS30 An unreflected rectangular parallelepiped of homogeneous $\mathrm{U}(3) \mathrm{F}_{4}$ and paraffin with an $\mathrm{H} / \mathrm{U}-235$ atomic ratio of $276.9 ; 40.90 \mathrm{~cm} \times 40.93 \mathrm{~cm} \times 116.80 \mathrm{~cm}$

CAS31 An unreflected rectangular parallelepiped of homogeneous $\mathrm{U}(3) \mathrm{F}_{4}$ and paraffin with an $\mathrm{H} / \mathrm{U}-235$ atomic ratio of $276.9 ; 48.59 \mathrm{~cm} \times 51.14 \mathrm{~cm} \mathrm{x} 48.53 \mathrm{~cm}$

CAS32 An unreflected rectangular parallelepiped of homogeneous $\mathrm{U}(3) \mathrm{F}_{4}$ and paraffin with an $\mathrm{H} / \mathrm{U}-235$ atomic ratio of $276.9 ; 81.71 \mathrm{~cm} \times 81.66 \mathrm{~cm} \times 31.34 \mathrm{~cm}$

CAS33 A composite cadmium/steel/water side reflected stainless steel cylinder of $0.079 \mathrm{~cm}$ wall thickness and $19.545 \mathrm{~cm}$ IR filled to a height of $54.45 \mathrm{~cm}$ with $\mathrm{U}(4.98) \mathrm{O}_{2} \mathrm{~F}_{2}$ solution at an $\mathrm{H} / \mathrm{U}-235$ atomic ratio of 488

CAS34 A composite 1-in. steel/water side reflected steel cylinder of $0.079 \mathrm{~cm}$ wall thickness and $16.51 \mathrm{~cm}$ IR filled to a height of $143 \mathrm{~cm}$ with $\mathrm{U}(4.98) \mathrm{O}_{2} \mathrm{~F}_{2}$ solution at an $\mathrm{H} / \mathrm{U}-235$ atomic ratio of 488

CAS35 An unreflected sphere of $\mathrm{U}(4.98) \mathrm{O}_{2} \mathrm{~F}_{2}$ with an $\mathrm{H} / \mathrm{U}-235$ atomic ratio of 490 . Solution radius of $25.3873 \mathrm{~cm}$ and stainless steel container wall thickness of $0.0508 \mathrm{~cm}$

CAS36 An unreflected stainless steel cylinder of $0.07874 \mathrm{~cm}$ wall thickness and a $19.55 \mathrm{~cm}$ IR filled to a height of $101.7 \mathrm{~cm}$ with $\mathrm{U}(4.98) \mathrm{O}_{2} \mathrm{~F}_{2}$ solution at an H/U-235 atomic ratio of 496 
Table 3 (continued)

\begin{tabular}{|c|c|c|c|}
\hline Case & Experimental description & Reference & $\mathbf{k}_{\text {eff }} \pm \sigma$ \\
\hline CAR01 & $\begin{array}{l}\text { Experiment } 1.4 .46 \% \text { enriched } \mathrm{U}_{3} \mathrm{O}_{8}, \mathrm{H} / \mathrm{U}=0.77 \text {, } \\
42 \text { fuel cans with } 2.44 \mathrm{~cm} \text { interstitial moderation, } \\
\text { plastic reflected }\end{array}$ & 22 & $1.0081 \pm 0.0034$ \\
\hline CAR02 & $\begin{array}{l}\text { Experiment } 2.4 .46 \% \text { enriched } \mathrm{U}_{3} \mathrm{O}_{8}, \mathrm{H} / \mathrm{U}=0.77 \text {, } \\
43 \text { fuel cans with } 2.44 \mathrm{~cm} \text { interstitial moderation, } \\
\text { plastic reflected }\end{array}$ & 22 & $1.0052 \pm 0.0031$ \\
\hline CAR03 & $\begin{array}{l}\text { Experiment 3. } 4.46 \% \text { enriched } \mathrm{U}_{3} \mathrm{O}_{8}, \mathrm{H} / \mathrm{U}=0.77 \text {, } \\
100 \text { fuel cans with } 0.929 \mathrm{~cm} \text { interstitial moderation, } \\
\text { plastic reflected }\end{array}$ & 22 & $0.9876 \pm 0.0030$ \\
\hline CAR04 & $\begin{array}{l}\text { Experiment } 13.4 .46 \% \text { enriched } \mathrm{U}_{3} \mathrm{O}_{8}, \mathrm{H} / \mathrm{U}=0.77 \text {, } \\
40 \text { fuel cans with } 2.44 \mathrm{~cm} \text { interstitial moderation, } \\
\text { concrete reflected }\end{array}$ & 22 & $1.0089 \pm 0.0029$ \\
\hline CAR05 & $\begin{array}{l}\text { Experiment } 15.4 .46 \% \text { enriched } \mathrm{U}_{3} \mathrm{O}_{8}, \mathrm{H} / \mathrm{U}=0.77 \text {, } \\
98 \text { fuel cans with } 0.929 \mathrm{~cm} \text { interstitial moderation, } \\
\text { concrete reflected }\end{array}$ & 22 & $0.9998 \pm 0.0027$ \\
\hline CAR06 & $\begin{array}{l}4.46 \% \text { enriched } \mathrm{U}_{3} \mathrm{O}_{8}, \mathrm{H} / \mathrm{U}=0.77 \text {, driven by } \\
93.12 \% \text { enriched uranium metal sphere }(29.870 \mathrm{~kg}) \\
120+4 \mathrm{~S} \text { fuel cans, plastic reflected }\end{array}$ & 23 & $0.9951 \pm 0.0031$ \\
\hline CAR07 & $\begin{array}{l}4.46 \% \text { enriched } \mathrm{U}_{3} \mathrm{O}_{8}, \mathrm{H} / \mathrm{U}=0.77 \text {, driven by } \\
\text { high concentration }(14.844 \mathrm{~kg} 351.18 \mathrm{~g} / \mathrm{l}) \text { fuel cans, } \\
\text { plastic reflected }\end{array}$ & 23 & $0.9998 \pm 0.0032$ \\
\hline CAR08 & $\begin{array}{l}4.46 \% \text { enriched } \mathrm{U}_{3} \mathrm{O}_{8}, \mathrm{H} / \mathrm{U}=0.77 \text {, driven by } \\
\text { low concentration }(12.871 \mathrm{~kg} 86.42 \mathrm{~g} / 1) 93.17 \% \\
\text { enriched } \mathrm{UO}_{2}\left(\mathrm{NO}_{3}\right)_{2}, 119+2 \mathrm{~S} \\
\text { fuel cans, plastic reflected }\end{array}$ & 23 & $0.9980 \pm 0.0036$ \\
\hline CAR09 & $\begin{array}{l}4.46 \% \text { enriched } \mathrm{U}_{3} \mathrm{O}_{8}, \mathrm{H} / \mathrm{U}=0.77 \text {, driven by } \\
\text { low concentration }(13.001 \mathrm{~kg} 86.42 \mathrm{~g} / \mathrm{l}) 93.17 \% \\
\text { enriched } \mathrm{UO}_{2}\left(\mathrm{NO}_{3}\right)_{2}, 119+2 \mathrm{~S} \\
\text { fuel cans, plastic reflected }\end{array}$ & 23 & $0.9983 \pm 0.0030$ \\
\hline CAR 10 & $\begin{array}{l}4.46 \% \text { enriched } \mathrm{U}_{3} \mathrm{O}_{8}, \mathrm{H} / \mathrm{U}=0.77 \text {, driven by } \\
\text { low concentration }(12.446 \mathrm{~kg} 86.42 \mathrm{~g} / 1) 93.17 \% \\
\text { enriched } \mathrm{UO}_{2}\left(\mathrm{NO}_{3}\right)_{2}, 119+2 \mathrm{~S} \\
\text { fuel cans, concrete reflected }\end{array}$ & 23 & $1.0008 \pm 0.0034$ \\
\hline CAR11 & $\begin{array}{l}\text { Experiment } A .4 .46 \% \text { enriched } \mathrm{U}_{3} \mathrm{O}_{8} \\
\mathrm{H} / \mathrm{U}=1.25,38 \text { fuel cans with } 2.44 \mathrm{~cm} \text { interstitial } \\
\text { moderation, plastic reflected }\end{array}$ & 24 & $1.0073 \pm 0.0032$ \\
\hline CAR12 & $\begin{array}{l}\text { Experiment B. } 4.46 \% \text { enriched } \mathrm{U}_{3} \mathrm{O}_{8} \\
\mathrm{H} / \mathrm{U}=1.25,78 \text { fuel cans with } 0.929 \mathrm{~cm} \text { interstitial } \\
\text { moderation, plastic reflected }\end{array}$ & 24 & $1.0109 \pm 0.0031$ \\
\hline
\end{tabular}


Table 3 (continued)

\begin{tabular}{|c|c|c|c|}
\hline Case & Experimental description & Reference & $k_{\text {eff }} \pm \sigma$ \\
\hline CAR13 & $\begin{array}{l}\text { Experiment } \mathrm{C} .4 .46 \% \text { enriched } \mathrm{U}_{3} \mathrm{O}_{8} \\
\mathrm{H} / \mathrm{U}=1.25,80 \text { fuel cans with } 0.929 \mathrm{~cm} \text { interstitial } \\
\text { moderation, plastic reflected }\end{array}$ & 24 & $1.0108 \pm 0.0032$ \\
\hline CAR14 & $\begin{array}{l}4.46 \% \text { enriched } \mathrm{U}_{3} \mathrm{O}_{8} \mathrm{H} / \mathrm{U}=1.25 \text {, driven } \\
\text { by high concentration }(12.268 \mathrm{~kg} 351.64 \mathrm{gU} / 1) \text {, } \\
93.17 \% \text { enriched } \mathrm{UO}_{2}\left(\mathrm{NO}_{3}\right)_{2}, 119+2 \mathrm{~S} \text { fuel } \\
\text { cans, plastic reflected }\end{array}$ & 24 & $0.9907 \pm 0.0034$ \\
\hline CAR15 & $\begin{array}{l}4.46 \% \text { enriched } \mathrm{U}_{3} \mathrm{O}_{8} \mathrm{H} / \mathrm{U}=1.25 \text {, driven } \\
\text { by high concentration }(12.400 \mathrm{~kg} 351.65 \mathrm{gU} / 1) \text {, } \\
93.17 \% \text { enriched } \mathrm{UO}_{2}\left(\mathrm{NO}_{3}\right)_{2}, 119+2 \mathrm{~S} \text { fuel } \\
\text { cans, plastic reflected }\end{array}$ & 24 & $1.0106 \pm 0.0033$ \\
\hline CAR16 & $\begin{array}{l}4.46 \% \text { enriched } \mathrm{U}_{3} \mathrm{O}_{8} \mathrm{H} / \mathrm{U}=1.25 \text {, driven } \\
\text { by low concentration }(10.836 \mathrm{~kg} \mathrm{p} 86.60 \mathrm{gU} / \mathrm{l}) \text {, } \\
93.17 \% \text { enriched } \mathrm{UO}_{2}\left(\mathrm{NO}_{3}\right)_{2}, 119+2 \mathrm{~S} \text { fuel } \\
\text { cans, plastic reflected }\end{array}$ & 24 & $0.9978 \pm 0.0036$ \\
\hline CAR17 & $\begin{array}{l}\text { Experiment } \mathrm{F} .4 .46 \% \text { enriched } \mathrm{U}_{3} \mathrm{O}_{8} \\
\mathrm{H} / \mathrm{U}=2.03,48 \text { fuel cans with } 0.92 \mathrm{~cm} \text { interstitial } \\
\text { moderation, plastic reflected }\end{array}$ & 25 & $1.0069 \pm 0.0033$ \\
\hline CAR18 & $\begin{array}{l}\text { Experiment } \mathrm{G} .4 .46 \% \text { enriched } \mathrm{U}_{3} \mathrm{O}_{8} \\
\mathrm{H} / \mathrm{U}=2.03,30 \text { fuel cans with } 2.44 \mathrm{~cm} \text { interstitial } \\
\text { moderation, plastic reflected }\end{array}$ & 25 & $1.0005 \pm 0.0030$ \\
\hline CAR19 & $\begin{array}{l}\text { Experiment } \mathrm{D} .4 .46 \% \text { enriched } \mathrm{U}_{3} \mathrm{O}_{8} \\
\mathrm{H} / \mathrm{U}=2.03 \text {, driven by } 93.12 \% \text { enriched uranium metal } \\
\text { sphere }(13.73 \mathrm{~kg}), 120+4 \mathrm{~S} \text { fuel cans, plastic reflected }\end{array}$ & 25 & $1.0097 \pm 0.0032$ \\
\hline CAR20 & $\begin{array}{l}\text { Experiment } \mathrm{E} .4 .46 \% \text { enriched } \mathrm{U}_{3} \mathrm{O}_{8} \\
\mathrm{H} / \mathrm{U}=2.03 \text {, driven by } 93.12 \% \text { enriched hollow uranium } \\
\text { metal sphere }(12.786 \mathrm{~kg}), 120+4 \mathrm{~S} \text { fuel cans, } \\
\text { plastic reflected }\end{array}$ & 25 & $0.9911 \pm 0.0031$ \\
\hline
\end{tabular}


Table 4. KENO V.a calculated $k_{\text {eff }}$ for experimentally critical highly enriched uranium systems (last modeled in ref. 8)

\begin{tabular}{|c|c|c|c|}
\hline Case & Experimental description & Reference & $\mathrm{k}_{\mathrm{eff}} \pm \sigma$ \\
\hline CAA01 & $\begin{array}{l}\mathrm{U}(93.2) \mathrm{O}_{2} \mathrm{~F}_{2} \text { solution sphere } \mathrm{H} / \mathrm{X}=1112 \text {, } \\
\text { unreflected }\end{array}$ & 26 & $1.0096 \pm 0.0026$ \\
\hline CAA02 & $\begin{array}{l}\mathrm{U}(93.2) \mathrm{O}_{2} \mathrm{~F}_{2} \text { solution sphere } \mathrm{H} / \mathrm{X}=1393 \\
\text { unreflected }\end{array}$ & 26 & $1.0036 \pm 0.0023$ \\
\hline CAA03 & $\begin{array}{l}\mathrm{U}(93.2) \mathrm{O}_{2}\left(\mathrm{NO}_{3}\right)_{2} \text { solution sphere } \\
\mathrm{H} / \mathrm{X}=1379 \text {, unreflected }\end{array}$ & 26 & $0.9998 \pm 0.0023$ \\
\hline CAA04 & $\begin{array}{l}\mathrm{U}(93.2) \mathrm{O}_{2} \mathrm{~F}_{2} \text { solution sphere } \\
\mathrm{H} / \mathrm{X}=76.1, \mathrm{H}_{2} \mathrm{O} \text { reflected }\end{array}$ & 26 & $1.0071 \pm 0.0039$ \\
\hline CAA05 & $\begin{array}{l}\mathrm{U}(93.2) \mathrm{O}_{2} \mathrm{~F}_{2} \text { solution sphere } \\
\mathrm{H} / \mathrm{X}=126.5, \mathrm{H}_{2} \mathrm{O} \text { reflected }\end{array}$ & 26 & $0.9971 \pm 0.0046$ \\
\hline CAA06 & $\begin{array}{l}\mathrm{U}(93.2) \mathrm{O}_{2} \mathrm{~F}_{2} \text { solution sphere } \\
\mathrm{H} / \mathrm{X}=1270, \mathrm{H}_{2} \mathrm{O} \text { reflected }\end{array}$ & 26 & $0.9964 \pm 0.0023$ \\
\hline CAA07 & $\begin{array}{l}\mathrm{U}(93.2) \mathrm{O}_{2} \mathrm{~F}_{2} \text { solution sphere } \\
\mathrm{H} / \mathrm{X}=268.8, \mathrm{H}_{2} \mathrm{O} \text { reflected }\end{array}$ & 27 & $1.0097 \pm 0.0043$ \\
\hline CAA08 & $\begin{array}{l}\mathrm{U}(93.2) \mathrm{O}_{2} \mathrm{~F}_{2} \text { solution sphere } \\
\mathrm{H} / \mathrm{X}=515.1, \mathrm{H}_{2} \mathrm{O} \text { reflected }\end{array}$ & 27 & $1.0212 \pm 0.0033$ \\
\hline CAA09 & $\begin{array}{l}\mathrm{U}(93.2) \mathrm{O}_{2} \mathrm{~F}_{2} \text { solution sphere } \\
\mathrm{H} / \mathrm{X}=203.5 \text { unreflected }\end{array}$ & 27 & $0.9991 \pm 0.0039$ \\
\hline CAA10 & $\begin{array}{l}\mathrm{U}(93.2) \mathrm{O}_{2} \mathrm{~F}_{2} \text { solution sphere } \\
\mathrm{H} / \mathrm{X}=239.3, \mathrm{H}_{2} \mathrm{O} \text { reflected }\end{array}$ & 27 & $1.0164 \pm 0.0042$ \\
\hline CAAl1 & $\begin{array}{l}\mathrm{U}(93.2) \mathrm{O}_{2} \mathrm{~F}_{2} \text { solution sphere } \\
\mathrm{H} / \mathrm{X}=468.2, \mathrm{H}_{2} \mathrm{O} \text { reflected }\end{array}$ & 27 & $1.0351 \pm 0.0039$ \\
\hline CAA12 & $\begin{array}{l}\mathrm{U}(93.2) \mathrm{O}_{2}\left(\mathrm{NO}_{3}\right)_{2} \text { solution } \\
142.92 \mathrm{~g} \mathrm{U} / 1,28.01 \mathrm{~cm} \text { diam } \\
\text { cylinder, unreflected }\end{array}$ & 28 & $1.0090 \pm 0.0039$ \\
\hline CAA13 & $\begin{array}{l}\mathrm{U}(93.2) \mathrm{O}_{2}\left(\mathrm{NO}_{3}\right)_{2} \text { solution } \\
357.71 \mathrm{~g} \mathrm{U} / 1,28.01 \mathrm{~cm} \text { diam } \\
\text { cylinder, unreflected }\end{array}$ & 28 & $1.0060 \pm 0.0035$ \\
\hline CAA14 & $\begin{array}{l}\mathrm{U}(93.2) \mathrm{O}_{2}\left(\mathrm{NO}_{3}\right)_{2} \text { solution } \\
54.89 \mathrm{~g} \mathrm{U} / 1,33.01 \mathrm{~cm} \text { diam } \\
\text { cylinder, unreflected }\end{array}$ & 28 & $1.0119 \pm 0.0042$ \\
\hline CAA15 & $\begin{array}{l}\mathrm{U}(93.2) \mathrm{O}_{2}\left(\mathrm{NO}_{3}\right)_{2} \text { solution } \\
137.4 \mathrm{~g} \mathrm{U} / 1,33.01 \mathrm{~cm} \text { diam } \\
\text { cylinder, unreflected }\end{array}$ & 28 & $1.0027 \pm 0.0046$ \\
\hline
\end{tabular}


Table 4 (continued)

\begin{tabular}{|c|c|c|c|}
\hline Case & Experimental description & Reference & $k_{\text {eff }} \pm \sigma$ \\
\hline CAA16 & $\begin{array}{l}\mathrm{U}(93.2) \mathrm{O}_{2}\left(\mathrm{NO}_{3}\right)_{2} \text { solution } \\
357.71 \mathrm{~g} \mathrm{U} / 1,33.01 \mathrm{~cm} \text { diam } \\
\text { cylinder, unreflected }\end{array}$ & 28 & $1.0024 \pm 0.0045$ \\
\hline CAA17 & $\begin{array}{l}\mathrm{U}(93.2) \mathrm{O}_{2}\left(\mathrm{NO}_{3}\right)_{2} \text { solution } \\
144.38 \mathrm{~g} \mathrm{U} / 1,28.01 \mathrm{~cm} \text { diam cylinder, } \\
\text { concrete reflected }\end{array}$ & 28 & $1.0106 \pm 0.0041$ \\
\hline CAA18 & $\begin{array}{l}\mathrm{U}(93.2) \mathrm{O}_{2}\left(\mathrm{NO}_{3}\right)_{2} \text { solution } \\
334.77 \mathrm{~g} \mathrm{U} / 1,28.01 \mathrm{~cm} \text { diam cylinder, } \\
\text { concrete reflected }\end{array}$ & 28 & $1.0145 \pm 0.0044$ \\
\hline CAA19 & $\begin{array}{l}\mathrm{U}(93.2) \mathrm{O}_{2}\left(\mathrm{NO}_{3}\right)_{2} \text { solution } \\
144.38 \mathrm{~g} \mathrm{U} / 1,33.01 \mathrm{~cm} \text { diam cylinder, } \\
\text { concrete reflected }\end{array}$ & 28 & $1.0056 \pm 0.0043$ \\
\hline CAA20 & $\begin{array}{l}\mathrm{U}(93.2) \mathrm{O}_{2}\left(\mathrm{NO}_{3}\right)_{2} \text { solution } \\
334.77 \mathrm{~g} \mathrm{U} / 1,33.01 \mathrm{~cm} \text { diam cylinder, } \\
\text { concrete reflected }\end{array}$ & 28 & $1.0037 \pm 0.0044$ \\
\hline CAA21 & $\begin{array}{l}\mathrm{U}(93.2) \mathrm{O}_{2}\left(\mathrm{NO}_{3}\right)_{2} \text { solution } \\
144.38 \mathrm{~g} \mathrm{U} / 1,28.01 \mathrm{~cm} \text { diam cylinder, } \\
\text { concrete reflected }\end{array}$ & 28 & $1.0094 \pm 0.0040$ \\
\hline CAA22 & $\begin{array}{l}\mathrm{U}(93.2) \mathrm{O}_{2}\left(\mathrm{NO}_{3}\right)_{2} \text { solution } \\
334.77 \mathrm{~g} \mathrm{U} / 1,33.01 \mathrm{~cm} \text { diam cylinder, } \\
\text { concrete reflected }\end{array}$ & 28 & $1.0092 \pm 0.0037$ \\
\hline CAA23 & $\begin{array}{l}\mathrm{U}(93.2) \mathrm{O}_{2}\left(\mathrm{NO}_{3}\right)_{2} \text { solution } \\
147.66 \mathrm{~g} \mathrm{U} / 1,28.01 \mathrm{~cm} \text { diam cylinder, } \\
\text { Plexiglas reflected }\end{array}$ & 28 & $1.0085 \pm 0.0040$ \\
\hline CAA24 & $\begin{array}{l}\mathrm{U}(93.2) \text { solution } \\
345.33 \mathrm{~g} \mathrm{U} / 1,28.01 \mathrm{~cm} \text { diam cylinder, } \\
\text { Plexiglas reflected }\end{array}$ & 28 & $1.0113 \pm 0.0047$ \\
\hline CAA25 & $\begin{array}{l}\mathrm{U}(93.2) \mathrm{O}_{2}\left(\mathrm{NO}_{3}\right)_{2} \text { solution } \\
147.66 \mathrm{~g} \mathrm{U} / 1,33.01 \mathrm{~cm} \text { diam cylinder, } \\
\text { Plexiglas reflected }\end{array}$ & 28 & $0.9947 \pm 0.0041$ \\
\hline CAA26 & $\begin{array}{l}\mathrm{U}(93.2) \mathrm{O}_{2}\left(\mathrm{NO}_{3}\right)_{2} \text { solution } \\
345.33 \mathrm{~g} \mathrm{U} / 1,33.01 \mathrm{~cm} \text { diam cylinder, } \\
\text { Plexiglas reflected }\end{array}$ & 28 & $1.0033 \pm 0.0040$ \\
\hline CAA27 & $\begin{array}{l}\mathrm{U}(93.2) \mathrm{O}_{2}\left(\mathrm{NO}_{3}\right)_{2} \text { solution } \\
147.66 \mathrm{~g} \mathrm{U} / 1,28.01 \mathrm{~cm} \text { diam cylinder, } \\
\text { Plexiglas reflected }\end{array}$ & 28 & $1.0157 \pm 0.0034$ \\
\hline
\end{tabular}


Table 4 (continued)

\begin{tabular}{|c|c|c|c|}
\hline Case & Experimental description & Reference & $k_{\text {eff }} \pm \sigma$ \\
\hline CAA28 & $\begin{array}{l}\mathrm{U}(93.22) \mathrm{O}_{2}\left(\mathrm{NO}_{3}\right)_{2} \text { solution } \\
345.33 \mathrm{~g} \mathrm{U} / 1,33.01 \mathrm{~cm} \text { diam cylinder, } \\
\text { Plexiglas reflected }\end{array}$ & 28 & $1.0141 \pm 0.0043$ \\
\hline CAA29 & $\begin{array}{l}\mathrm{U}(93.22) \mathrm{O}_{2}\left(\mathrm{NO}_{3}\right)_{2} \text { solution } \\
67.28 \mathrm{~g} \mathrm{U} / 1,21.12 \mathrm{~cm} \text { diam cylinder in } \\
\text { a } 4 \text { x } 4 \text { array, concrete reflected }\end{array}$ & 28 & $1.0046 \pm 0.0033$ \\
\hline CAA30 & $\begin{array}{l}\mathrm{U}(93.12) \mathrm{O}_{2}\left(\mathrm{NO}_{3}\right)_{2} \text { solution } \\
364.11 \mathrm{~g} \mathrm{U} / 1,21.12 \mathrm{~cm} \text { diam cylinder in } \\
\text { a } 4 \times 4 \text { array, concrete reflected }\end{array}$ & 28 & $1.0061 \pm 0.0041$ \\
\hline CAA31 & $\begin{array}{l}\mathrm{U}(93.2) \mathrm{O}_{2}\left(\mathrm{NO}_{3}\right)_{2} \text { solution } \\
83.49 \mathrm{~g} \mathrm{U} / 1,16.12 \mathrm{~cm} \text { diam cylinder in } \\
\text { a } 4 \text { x } 4 \text { array, concrete reflected }\end{array}$ & 28 & $1.0119 \pm 0.0035$ \\
\hline CAA32 & $\begin{array}{l}\mathrm{U}(93.2) \mathrm{O}_{2}\left(\mathrm{NO}_{3}\right)_{2} \text { solution } \\
359.55 \mathrm{~g} \mathrm{U} / 1,16.12 \mathrm{c} \mathrm{diam} \text { cylinder in } \\
\text { a } 4 \times 4 \text { array, concrete reflected }\end{array}$ & 28 & $1.0056 \pm 0.0041$ \\
\hline CAA33 & $\begin{array}{l}\mathrm{U}(93.2) \mathrm{O}_{2}\left(\mathrm{NO}_{3}\right)_{2} \text { solution } \\
76.09 \mathrm{~g} \mathrm{U} / 1,21.12 \mathrm{~cm} \text { diam cylinder in } \\
\text { a } 2 \times 2 \text { array, concrete reflected }\end{array}$ & 28 & $1.0098 \pm 0.0033$ \\
\hline CAA34 & $\begin{array}{l}\mathrm{U}(93.2) \mathrm{O}_{2}\left(\mathrm{NO}_{3}\right)_{2} \text { solution } \\
364.11 \mathrm{~g} \mathrm{U} / 1,21.2 \mathrm{~cm} \text { diam cylinder in } \\
\text { a } 2 \times 2 \text { array, concrete reflected }\end{array}$ & 28 & $1.0014 \pm 0.0038$ \\
\hline CAA35 & $\begin{array}{l}\mathrm{U}(93.2) \mathrm{O}_{2}\left(\mathrm{NO}_{3}\right)_{2} \text { solution } \\
359.55 \mathrm{~g} \mathrm{U} / 1,16.12 \mathrm{~cm} \text { diam cylinder in } \\
\text { a } 2 \times 2 \text { array, concrete reflected }\end{array}$ & 28 & $1.0129 \pm 0.0045$ \\
\hline CAA36 & $\begin{array}{l}\mathrm{U}(93.2) \mathrm{O}_{2}\left(\mathrm{NO}_{3}\right)_{2} \text { solution } \\
359.55 \mathrm{~g} \mathrm{U} / 1,16.12 \mathrm{~cm} \text { diam cylinder in } \\
\text { a } 2 \times 4 \text { array, concrete reflected }\end{array}$ & 28 & $1.0099 \pm 0.0039$ \\
\hline CAA37 & $\begin{array}{l}\mathrm{U}(93.2) \mathrm{O}_{2}\left(\mathrm{NO}_{3}\right)_{2} \text { solution } \\
60.32 \mathrm{~g} \mathrm{U} / 1,21.12 \mathrm{~cm} \text { diam cylinder in } \\
\text { a } 4 \text { x } 4 \text { array, Plexiglas reflected }\end{array}$ & 28 & $1.0000 \pm 0.0034$ \\
\hline CAA38 & $\begin{array}{l}\mathrm{U}(93.2) \mathrm{O}_{2}\left(\mathrm{NO}_{3}\right)_{2} \text { solution } \\
355.94 \mathrm{~g} \mathrm{U} / 1,21.12 \mathrm{~cm} \text { diam cylinder in } \\
\text { a } 4 \text { x } 4 \text { array, Plexiglas reflected }\end{array}$ & 28 & $0.9961 \pm 0.0038$ \\
\hline CAA39 & $\begin{array}{l}\mathrm{U}(93.2) \mathrm{O}_{2}\left(\mathrm{NO}_{3}\right)_{2} \text { solution } \\
60.32 \mathrm{~g} \mathrm{U} / 1,16.12 \mathrm{~cm} \text { diam cylinder in } \\
\text { a } 4 \text { x } 4 \text { array, Plexiglas reflected }\end{array}$ & 28 & $1.0045 \pm 0.0034$ \\
\hline
\end{tabular}


Table 4 (continued)

\begin{tabular}{|c|c|c|c|}
\hline Case & Experimental description & Reference & $k_{\text {eff }} \pm \sigma$ \\
\hline CAA40 & $\begin{array}{l}\mathrm{U}(93.2) \mathrm{O}_{2}\left(\mathrm{NO}_{3}\right)_{2} \text { solution } \\
355.94 \mathrm{~g} \mathrm{U} / 1,16.12 \mathrm{~cm} \text { diam cylinder in } \\
\text { a } 4 \text { x } 4 \text { array, Plexiglas reflected }\end{array}$ & 28 & $0.9985 \pm 0.0039$ \\
\hline CAA41 & $\begin{array}{l}\mathrm{U}(93.2) \mathrm{O}_{2}\left(\mathrm{NO}_{3}\right)_{2} \text { solution } \\
60.32 \mathrm{~g} \mathrm{U} / 1,21.12 \mathrm{~cm} \text { diam cylinder in } \\
\text { a } 2 \times 2 \text { array, Plexiglas reflected }\end{array}$ & 28 & $0.9985 \pm 0.0040$ \\
\hline CAA42 & $\begin{array}{l}\mathrm{U}(93.2) \mathrm{O}_{2}\left(\mathrm{NO}_{3}\right)_{2} \text { solution } \\
355.94 \mathrm{~g} \mathrm{U} / 1,21.12 \mathrm{~cm} \text { diam cylinder in } \\
\text { a } 2 \times 2 \text { array, Plexiglas reflected }\end{array}$ & 28 & $1.0041 \pm 0.0036$ \\
\hline CAA43 & $\begin{array}{l}\mathrm{U}(93.2) \mathrm{O}_{2}\left(\mathrm{NO}_{3}\right)_{2} \text { solution } \\
355.94 \mathrm{~g} \mathrm{U} / 1,16.12 \mathrm{~cm} \text { diam cylinder in } \\
\text { a } 3 \times 2 \text { array, Plexiglas reflected }\end{array}$ & 28 & $0.9982 \pm 0.0038$ \\
\hline
\end{tabular}


Table 5. KENO V.a calculated $k_{\text {eff }}$ for experimentally critical highly enriched uranium systems (last modeled in ref. 6)

\begin{tabular}{|c|c|c|c|}
\hline Case & Experimental description & Reference & $\mathbf{k}_{\mathrm{eff}} \pm \sigma$ \\
\hline CAS01 & $\begin{array}{l}\text { Y-12 validation case A-1. 93.8\% U metal } \\
\text { sphere, unreflected (GODIVA) }\end{array}$ & 29 & $1.0004 \pm 0.0027$ \\
\hline CAS04 & $\begin{array}{l}\text { Y-12 validation case } \mathrm{A}-2.93 .2 \% \text { U-Mo } \\
\text { alloy cylinder annulus, unreflected }\end{array}$ & 30 & $1.0067 \pm 0.0028$ \\
\hline CAS05 & $\begin{array}{l}\mathrm{Y}-12 \text { validation case } \mathrm{A}-3.93 .2 \% \mathrm{UO}_{2} \mathrm{~F}_{2} \\
\text { solution, } 19.992 \mathrm{~g} \mathrm{U} / \mathrm{l} \text {, in } \mathrm{Al} \text { sphere, unreflected }\end{array}$ & 26 & $1.0023 \pm 0.0027$ \\
\hline CAS06 & $\begin{array}{l}\mathrm{Y}-12 \text { validation case } \mathrm{A}-4.93 .18 \% \mathrm{UO}_{2}\left(\mathrm{NO}_{3}\right)_{2} \\
\text { solution, } 20.12 \mathrm{~g} \mathrm{U} / 1 \text {, in } \mathrm{Al} \text { sphere, unreflected }\end{array}$ & 31 & $1.0012 \pm 0.0022$ \\
\hline CAS07 & $\begin{array}{l}\text { Y-12 validation case } \mathrm{A}-5.93 .5 \% \text { U metal } \\
\text { hemispherical shell, } \mathrm{H}_{2} \mathrm{O} \text { reflected }\end{array}$ & 32 & $1.0042 \pm 0.0030$ \\
\hline CAS08 & $\begin{array}{l}\text { Y-12 validation case A- } 6.93 .2 \% \text { U metal } \\
\text { cylinder annulus, graphite reflected }\end{array}$ & 33 & $1.0124 \pm 0.0031$ \\
\hline CASO9 & $\begin{array}{l}\text { Y-12 validation case A-7. } 94 \% \text { U metal } \\
\text { cuboid, natural U reflected }\end{array}$ & 32 & $1.0027 \pm 0.0025$ \\
\hline CAS10 & $\begin{array}{l}\text { Y-12 validation case A-8. } 93.1 \% \text { U metal } \\
\text { hemispherical shell, oil reflected }\end{array}$ & 34 & $1.0114 \pm 0.0034$ \\
\hline CAS11 & $\begin{array}{l}\text { Y-12 validation case A-9. } 93.1 \% \mathrm{U} \text { metal } \\
\text { hemispherical shell, steel center and } \\
\text { oil reflected }\end{array}$ & 34 & $0.9977 \pm 0.0035$ \\
\hline $\mathrm{CAS} 02$ & $\begin{array}{l}\mathrm{Y}-12 \text { validation case } \mathrm{A}-10.97 .67 \% \mathrm{U} \text { metal } \\
\text { sphere, } \mathrm{H}_{2} \mathrm{O} \text { reflected }\end{array}$ & 35 & $0.9995 \pm 0.0031$ \\
\hline CAS03 & $\begin{array}{l}\mathrm{Y}-12 \text { validation case } \mathrm{A}-11.93 .172 \% \mathrm{UO}_{2}\left(\mathrm{NO}_{3}\right)_{2} \\
\text { solution, } 346.7 \mathrm{~g} \mathrm{U} / 1 \text {, in } \mathrm{SS} \text { cylinder, } \\
\text { unreflected }\end{array}$ & 28 & $1.0029 \pm 0.0044$ \\
\hline CAS12 & $\begin{array}{l}\text { Y-12 validation case } B-1.93 .2 \% \text { U metal } \\
\text { cylinder annulus, unreflected, smaller } U \\
\text { cylinder in hole touching one wall }\end{array}$ & 36 & $1.0004 \pm 0.0031$ \\
\hline CAS22 & $\begin{array}{l}\text { Y-12 validation case B-2. } 93.2 \% \text { U metal } \\
\text { cylinder annulus, unreflected, smaller } \mathrm{U} \\
\text { block in hole touching one wall }\end{array}$ & 36 & $1.0082 \pm 0.0034$ \\
\hline CAS23 & $\begin{array}{l}\text { Y-12 validation case B-3. } 93.2 \% \text { U metal, } \\
\text { unreflected cylinders and cuboids in } \\
\text { approximate circular arrangement, cylinder, } \\
\text { cuboid, and hemisphere stack in center }\end{array}$ & 36 & $0.9959 \pm 0.0030$ \\
\hline
\end{tabular}


Table 5 (continued)

\begin{tabular}{llll}
\hline Case & Experimental description & Reference & $k_{\text {eff }} \pm \sigma$ \\
\hline
\end{tabular}

CAS24 Y-12 validation case B-4. 93.2\% U metal

37

$0.9928 \pm 0.0033$

cylinders, $4 \times 4 \times 4$ array, unreflected

CAS25 Y-12 validation case B-5. 93.2\% U metal cylinders, $2 \times 2 \times 2$ array, each unit in

$37 \backslash 1.0079 \pm 0.0030$ the array is a smaller cylinder capped on each end by a larger cylinder, unreflected

CAS26 Y-12 validation case B-6. 93.2\% U metal cylinders, $2 \times 2 \times 2$ array, paraffin reflected

CAS27 Y-12 validation case B-7. 93.2\% U metal cylinders, $2 \times 2 \times 2$ array, each unit in the array is a smaller cylinder capped on each end by a larger cylinder, paraffin reflected

CAS28 Y-12 validation case B-8. 93.2\% U metal cylinders each in a Plexiglas box, $2 \times 2 \times 2$ array of these units unreflected

CAS29 Y-12 validation case B-9. $92.6 \% \mathrm{UO}_{2}\left(\mathrm{NO}_{3}\right)_{2}$ solution, $415 \mathrm{~g} \mathrm{U} / 1$, in Plexiglas cylinders, $3 \times 3 \times 3$ array, unreflected (see also problem U4B271F)

CAS13 Y-12 validation case B-10. 92.6\% UO $\left(\mathrm{NO}_{3}\right)_{2}$ solution, $63.3 \mathrm{~g} \mathrm{U} / 1$, in Plexiglas cylinders, $3 \times 3 \times 3$ array, unreflected (see also problem U6B271F)

CAS14 Y-12 validation case B-11. 93.2\% UO $\left(\mathrm{NO}_{3}\right)_{2}$ solution, $505 \mathrm{~g} \mathrm{U} / 1$, in SS cylinders, $4 \times 4$ array, standing in a solution slab, Plexiglas reflected

CAS15 Y-12 validation case B-12. 93.2\% U metal cylinders, $2 \times 2 \times 2$ array, graphite moderated and polyethylene reflected 
Table 5 (continued)

\begin{tabular}{|c|c|}
\hline Case & Experimental description \\
\hline AS17 & $\begin{array}{l}\mathrm{Y}-12 \text { validation case } \mathrm{B}-14.92 .6 \% \mathrm{UO}_{2}\left(\mathrm{NO}_{3}\right) \\
\text { solution, } 415 \mathrm{~g} \mathrm{U} / \mathrm{l} \text {, in Plexiglas cylinders, } \\
3 \times 3 \times 3 \text { array, paraffin reflected (see } \\
\text { also problem U4R27I1F) }\end{array}$ \\
\hline
\end{tabular}

CAS18 Y-12 validation case B-15. 92.6\% UO $\mathrm{UO}_{2}\left(\mathrm{NO}_{3}\right)_{2}$ solution, $415 \mathrm{~g} \mathrm{U} / \mathrm{l}$, in Plexiglas cylinders, $3 \times 3 \times 3$ array, Plexiglas reflected (see also problem U4R27G1F)

CAS19 Y-12 validation case B-16. 93.1\% UO $\mathrm{UO}_{2}\left(\mathrm{NO}_{3}\right)_{2}$ solution, $450.8 \mathrm{~g} \mathrm{U} / 1$, in SS containers, square central column with 8 perpendicular cylindrical arms unreflected

CAS20 Y-12 validation case B-17. 93.17\% $\mathrm{UO}_{2}\left(\mathrm{NO}_{3}\right)_{2}$ solution, $355.9 \mathrm{~g} \mathrm{U} / 1$, in Al cylinders, $4 \times 4$ array, Plexiglas reflected

CAS21 Y-12 validation case B-18. 93.17\% UO $\mathrm{UO}_{2}\left(\mathrm{NO}_{3}\right)_{2}$ solution, $364.1 \mathrm{~g} \mathrm{U} / 1$, in Al cylinders, $4 \times 4$ array, concrete reflected

CAS30 Problem S333SPO. 93.2\% $\mathrm{UO}_{2} \mathrm{~F}_{2}$ solution, $81.8 \mathrm{~g} \mathrm{U} / \mathrm{l}$, in Al slabs, $37.62 \mathrm{~cm}$ slabs in $3 \times 1$ array, $0 \mathrm{~cm}$ separation, unreflected, cylindrical tank, floor and walls in experiment room included

CAS32 Problem S333SP1. 93.2\% $\mathrm{UO}_{2} \mathrm{~F}_{2}$ solution, $81.8 \mathrm{~g} \mathrm{U} / 1$, in Al slabs, $37.62 \mathrm{~cm}$ slabs in $3 \times 1$ array, $2.54 \mathrm{~cm}$ separation, unreflected, cylindrical tank, floor, and walls in experiment room included

CAS34 Problem S333SP3. 93.2\% $\mathrm{UO}_{2} \mathrm{~F}_{2}$ solution, $81.8 \mathrm{~g} \mathrm{U} / \mathrm{l}$, in Al slabs, $37.62 \mathrm{~cm}$ slabs in $3 \times 1$ array, $7.62 \mathrm{~cm}$ separation, unreflected, cylindrical tank, floor, and walls in experiment room included

CAS36 Problem S333SP4. 93.2\% $\mathrm{UO}_{2} \mathrm{~F}_{2}$ solution, $81.8 \mathrm{~g} \mathrm{U} / 1$, in Al slabs, $37.62 \mathrm{~cm}$ slabs in $3 \times 1$ array, $11.43 \mathrm{~cm}$ separation, unreflected, cylindrical tank, floor, and walls in experiment room included 
Table 5 (continued)

\begin{tabular}{|c|c|c|c|}
\hline Case & Experimental description & Reference & $k_{\text {eff }} \pm \sigma$ \\
\hline CAS38 & $\begin{array}{l}\text { Problem S333SP5. } 93.2 \% \mathrm{UO}_{2} \mathrm{~F}_{2} \text { solution, } \\
81.8 \mathrm{~g} \mathrm{U} / \mathrm{l} \text {, in } \mathrm{Al} \mathrm{slabs,} 37.62 \mathrm{~cm} \text { slabs in } \\
3 \times 1 \text { array, } 13.97 \mathrm{~cm} \text { separation, unreflected, } \\
\text { cylindrical tank, floor, and walls in } \\
\text { experiment room included }\end{array}$ & 43 & $0.9881 \pm 0.0035$ \\
\hline CAS40 & $\begin{array}{l}\text { Problem S333SP6. } 93.2 \% \mathrm{UO}_{2} \mathrm{~F}_{2} \\
\text { solution, } 81.8 \mathrm{~g} \mathrm{U} / 1 \text {, in Al slabs, } \\
37.62 \mathrm{~cm} \text { slabs in } 3 \times 1 \text { array, } \\
15.24 \mathrm{~cm} \text { separation, unreflected, } \\
\text { cylindrical tank, floor, and walls } \\
\text { in experiment room included }\end{array}$ & 43 & $0.9824 \pm 0.0041$ \\
\hline CAS31 & $\begin{array}{l}\text { Problem } \mathrm{S} 333 \mathrm{SPOR} .93 .2 \% \mathrm{UO}_{2} \mathrm{~F}_{2} \\
\text { solution, } 81.8 \mathrm{~g} \mathrm{U} / \mathrm{l} \text {, in } \mathrm{Al} \text { slabs, } \\
37.62 \mathrm{~cm} \text { slabs in } 3 \times 1 \text { array, } \\
0 \mathrm{~cm} \text { separation, } \mathrm{H}_{2} \mathrm{O} \text { reflected }\end{array}$ & 43 & $0.9949 \pm 0.0035$ \\
\hline CAS33 & $\begin{array}{l}\text { Problem S333SP1R. } 93.2 \% \mathrm{UO}_{2} \mathrm{~F}_{2} \\
\text { solution, } 81.8 \mathrm{~g} \mathrm{U} / 1 \text {, in } \mathrm{Al} \text { slabs, } \\
37.62 \mathrm{~cm} \text { slabs in } 3 \times 1 \text { array, } \\
2.54 \mathrm{~cm} \text { separation, } \mathrm{H}_{2} \mathrm{O} \text { reflected }\end{array}$ & 43 & $0.9995 \pm 0.0039$ \\
\hline CAS35 & $\begin{array}{l}\text { Problem S333SP3R. } 93.2 \% \mathrm{UO}_{2} \mathrm{~F}_{2} \\
\text { solution, } 81.8 \mathrm{~g} \mathrm{U} / 1 \text {, in } \mathrm{Al} \text { slabs, } \\
37.62 \mathrm{~cm} \text { slabs in } 3 \times 1 \text { array, } \\
7.62 \mathrm{~cm} \text { separation, } \mathrm{H}_{2} \mathrm{O} \text { reflected }\end{array}$ & 43 & $0.9906 \pm 0.0036$ \\
\hline CAS37 & $\begin{array}{l}\text { Problem S333SP4R. } 93.2 \% \mathrm{UO}_{2} \mathrm{~F}_{2} \\
\text { solution, } 81.8 \mathrm{~g} \mathrm{U} / 1, \text { in } \mathrm{Al} \text { slabs, } \\
37.62 \mathrm{~cm} \text { slabs in } 3 \times 1 \text { array, } \\
11.43 \mathrm{~cm} \text { separation, } \mathrm{H}_{2} \mathrm{O} \text { reflected }\end{array}$ & 43 & $1.0030 \pm 0.0031$ \\
\hline CAS39 & $\begin{array}{l}\text { Problem } \mathrm{S} 333 \mathrm{SP} 5 \mathrm{R} .93 .2 \% \mathrm{UO}_{2} \mathrm{~F}_{2} \\
\text { solution, } 81.8 \mathrm{~g} \mathrm{U} / 1 \text {, in } \mathrm{Al} \text { slabs, } \\
37.62 \mathrm{~cm} \text { slabs in } 3 \times 1 \text { array, } \\
13.97 \mathrm{~cm} \text { separation, } \mathrm{H}_{2} \mathrm{O} \text { reflected }\end{array}$ & 43 & $0.9952 \pm 0.0035$ \\
\hline CAS42 & $\begin{array}{l}\text { Problem } \mathrm{S} 36 \mathrm{SP} 2.93 .2 \% \mathrm{UO}_{2} \mathrm{~F}_{2} \\
\text { solution, } 81.8 \mathrm{~g} \mathrm{U} / 1 \text { in Al slabs, } \\
7.62 \mathrm{~cm} \text { and } 14.834 \mathrm{~cm} \text { slabs in } \\
2 \times 1 \text { array, } 5.08 \mathrm{~cm} \text { separation, } \\
\text { unreflected }\end{array}$ & 43 & $0.9916 \pm 0.0037$ \\
\hline CAS41 & $\begin{array}{l}\text { Problem } \mathrm{S} 36 \mathrm{SP} 15.93 .2 \% \mathrm{UO}_{2} \mathrm{~F}_{2} \\
\text { solution, } 81.8 \mathrm{~g} \mathrm{U} / 1 \text { in } \mathrm{Al} \text { slabs, } \\
7.62 \mathrm{~cm} \text { and } 14.834 \mathrm{~cm} \text { slabs in } \\
2 \times 1 \text { array, } 38.1 \mathrm{~cm} \text { separation, } \\
\text { unreflected }\end{array}$ & 43 & $0.9767 \pm 0.0038$ \\
\hline
\end{tabular}


Table 5 (continued)

\begin{tabular}{ll}
\hline Case & Experimental description \\
\hline CAS43 & $\begin{array}{l}\text { Problem S36SP30.93.2\% UO } \mathrm{F}_{2} \\
\text { solution, } 81.8 \mathrm{~g} \mathrm{U} / 1 \text { in } \mathrm{Al} \mathrm{slabs,}\end{array}$ \\
& $\begin{array}{l}7.62 \mathrm{~cm} \text { and } 14.834 \mathrm{~cm} \text { slabs in } \\
2 \times 1 \text { array, } 76.2 \mathrm{~cm} \text { separation, } \\
\text { unreflected }\end{array}$
\end{tabular}

CAS44 Problem S36SP48. 93.2\% $\mathrm{UO}_{2} \mathrm{~F}_{2}$ solution, $81.8 \mathrm{~g} \mathrm{U} / 1$ in $\mathrm{Al}$ slabs, $7.62 \mathrm{~cm}$ and $14.834 \mathrm{~cm}$ slabs in $2 \times 1$ array, $121.92 \mathrm{~cm}$ separation, unreflected

CAS45 Problem S363SPO. 93.2\% $\mathrm{UO}_{2} \mathrm{~F}_{2}$ solution, $81.8 \mathrm{~g} \mathrm{U} / 1$, in Al slabs, $7.62 \mathrm{~cm}, 14.834 \mathrm{~cm}$, and $7.62 \mathrm{~cm}$ slabs in $3 \times 1$ array, $0 \mathrm{~cm}$ separation, unreflected

CAS46 Problem S363SP10. 93.2\% UO $\mathrm{UF}_{2}$ solution, $81.8 \mathrm{~g} \mathrm{U} / 1$, in Al slabs, $7.62 \mathrm{~cm}, 14.834 \mathrm{~cm}$, and $7.62 \mathrm{~cm}$ slabs in $3 \times 1$ array, $25.4 \mathrm{~cm}$ separation, unreflected

CAS47 Problem S363SP20. 93.2\% $\mathrm{UO}_{2} \mathrm{~F}_{2}$ solution, $81.8 \mathrm{~g} \mathrm{U} / 1$, in Al slabs, $7.62 \mathrm{~cm}, 14.834 \mathrm{~cm}$, and $7.62 \mathrm{~cm}$ slabs in $3 \times 1$ array, $50.8 \mathrm{~cm}$ separation, unreflected

CAS48 Problem S363SP32. 93.2\% $\mathrm{UO}_{2} \mathrm{~F}_{2}$ solution, $81.8 \mathrm{~g} \mathrm{U} / 1$, in Al slabs, $7.62 \mathrm{~cm}, 14.834 \mathrm{~cm}$, and $7.62 \mathrm{~cm}$ slabs in $3 \times 1$ array, $81.28 \mathrm{~cm}$ separation, unreflected

CAS52 Problem S63SP6. 93.2\% $\mathrm{UO}_{2} \mathrm{~F}_{2}$ solution, $81.8 \mathrm{~g} \mathrm{U} / 1$, in Al slabs. One slab is made up from two $7.62 \mathrm{~cm}$ slabs snugly fit together, the other is $7.62 \mathrm{~cm}, 2 \times 1$ array, $15.24 \mathrm{~cm}$ separation, unreflected

CAS49 Problem S63SP12. 93.2\% $\mathrm{UO}_{2} \mathrm{~F}_{2}$ solution, $81.8 \mathrm{~g} \mathrm{U} / \mathrm{l}$, in Al slabs. One slab is made up from two $7.62 \mathrm{~cm}$ slabs snugly fit together, the other is $7.62 \mathrm{~cm}, 2 \times 1$ array, $30.48 \mathrm{~cm}$ separation, unreflected 
Table 5 (continued)

\begin{tabular}{|c|c|c|c|}
\hline Case & Experimental description & Reference & $\mathrm{k}_{\mathrm{eff}} \pm \sigma$ \\
\hline CAS50 & $\begin{array}{l}\text { Problem S63SP18. } 93.2 \% \mathrm{UO}_{2} \mathrm{~F}_{2} \\
\text { solution, } 81.8 \mathrm{~g} \mathrm{U} / 1 \text {, in } \mathrm{Al} \text { slabs. } \\
\text { One slab is made up from two } 7.62 \mathrm{~cm} \\
\text { slabs snugly fit together, the other } \\
\text { is } 7.62 \mathrm{~cm}, 2 \times 1 \text { array, } 45.72 \mathrm{~cm} \\
\text { separation, unreflected }\end{array}$ & 43 & $0.9814 \pm 0.0038$ \\
\hline CAS51 & $\begin{array}{l}\text { Problem S63SP30. } 93.2 \% \mathrm{UO}_{2} \mathrm{~F}_{2} \\
\text { solution, } 81.8 \mathrm{~g} \mathrm{U} / 1 \text {, in } \mathrm{Al} \text { slabs. } \\
\text { One slab is made up from two } 7.62 \mathrm{~cm} \\
\text { slabs snugly fit together, the other } \\
\text { is } 7.62 \mathrm{~cm}, 2 \times 1 \text { array, } 76.2 \mathrm{~cm} \\
\text { separation, unreflected }\end{array}$ & 43 & $0.9791 \pm 0.0036$ \\
\hline CAS54 & $\begin{array}{l}\text { Problem } \mathrm{S} 66 \mathrm{SP} 2.93 .2 \% \mathrm{UO}_{2} \mathrm{~F}_{2} \\
\text { solution, } 81.8 \mathrm{~g} \mathrm{U} / 1 \text {, in } \mathrm{Al} \text { slabs. } \\
\text { One slab is } 14.834 \mathrm{~cm} \text {, and the other } \\
\text { is made up from two } 7.62 \mathrm{~cm} \text { slabs } \\
\text { snugly fit together, } 2 \times 1 \text { array, } 5.08 \mathrm{~cm} \\
\text { separation, unreflected }\end{array}$ & 43 & $0.9831 \pm 0.0034$ \\
\hline CAS58 & $\begin{array}{l}\text { Problem S66SP2. } 93.2 \% \mathrm{UO}_{2} \mathrm{~F}_{2} \\
\text { solution, } 81.8 \mathrm{~g} \mathrm{U} / 1 \text {, in Al slabs. } \\
\text { One slab is } 14.834 \mathrm{~cm} \text {, and the other } \\
\text { is made up from two } 7.62 \mathrm{~cm} \text { slabs } \\
\text { snugly fit together, } 2 \times 1 \text { array, } 15.24 \mathrm{~cm} \\
\text { separation, unreflected }\end{array}$ & 43 & $0.9824 \pm 0.0037$ \\
\hline CAS53 & $\begin{array}{l}\text { Problem } \mathrm{S} 66 \mathrm{SP} 15.93 .2 \% \mathrm{UO}_{2} \mathrm{~F}_{2} \\
\text { solution, } 81.8 \mathrm{~g} \mathrm{U} / 1 \text { in } \mathrm{Al} \text { slabs. } \\
\text { One slab is } 14.834 \mathrm{~cm} \text {, and the other } \\
\text { is made up from two } 7.62 \mathrm{~cm} \text { slabs } \\
\text { snugly fit together, } 2 \times 1 \text { array, } 38.1 \mathrm{~cm} \\
\text { separation, unreflected }\end{array}$ & 43 & $0.9884 \pm 0.0039$ \\
\hline CAS55 & Problem S66SP20. 93.2\% $\mathrm{UO}_{2} \mathrm{~F}_{2}$ & 43 & $0.9876 \pm 0.0038$ \\
\hline
\end{tabular}

solution, $81.8 \mathrm{~g} \mathrm{U} / \mathrm{l}$, in $\mathrm{Al}$ slabs.

One slab is $14.834 \mathrm{~cm}$, and the other is made up from two $7.62 \mathrm{~cm}$ slabs snugly fit together, $2 \times 1$ array, $50.8 \mathrm{~cm}$ separation, unreflected 
Table 5 (continued)

\begin{tabular}{llll}
\hline Case & Experimental description & Reference & $k_{\text {eff }} \pm \sigma$ \\
\hline
\end{tabular}

CAS56 Problem S66SP30. 93.2\% $\mathrm{UO}_{2} \mathrm{~F}_{2}$

$43 \quad 0.9869 \pm 0.0042$

solution, $81.8 \mathrm{~g} \mathrm{U} / 1$, in Al slabs.

One slab is $14.834 \mathrm{~cm}$, and the other

is made up from two $7.62 \mathrm{~cm}$ slabs

snugly fit together, $2 \times 1$ array, $76.2 \mathrm{~cm}$

separation, unreflected

CAS57 Problem S66SP48. 93.2\% $\mathrm{UO}_{2} \mathrm{~F}_{2}$

solution, $81.8 \mathrm{~g} \mathrm{U} / 1$, in Al slabs.

One slab is $14.834 \mathrm{~cm}$, and the other

is made up from two $7.62 \mathrm{~cm}$ slabs

snugly fit together, $2 \times 1$ array, $121.92 \mathrm{~cm}$

separation, unreflected

CAS59 Problem S66SP66. 93.2\% $\mathrm{UO}_{2} \mathrm{~F}_{2}$

solution, $81.8 \mathrm{~g} \mathrm{U} / 1$, in Al slabs.

One slab is $14.834 \mathrm{~cm}$, and the other is made up from two $7.62 \mathrm{~cm}$ slabs snugly fit together, $2 \times 1$ array, $167.64 \mathrm{~cm}$ separation, unreflected

CAS91 Problem U6B271F. 92.6\% UO $\mathrm{UO}_{2}\left(\mathrm{NO}_{3}\right)_{2}$ solution, $63.3 \mathrm{~g} \mathrm{U} / 1$, in Plexiglas cylinders, 3 × 3 × 3 array, unreflected, walls, floor, and tank in experiment room included

CAS60 Problem U2B271F. $92.6 \% \mathrm{UO}_{2}\left(\mathrm{NO}_{3}\right)_{2}$ solution, $279 \mathrm{~g} \mathrm{U} / 1$, in Plexiglas cylinders, $3 \times 3 \times 3$ array, unreflected, walls, floor, and tank in experiment room included

CAS61 Problem U2B81F. 92.6\% UO $\mathrm{UO}_{2}\left(\mathrm{NO}_{3}\right)_{2}$ solution, $279 \mathrm{~g} \mathrm{U} / 1$, in Plexiglas cylinders, $2 \times 2 \times 2$ array, unreflected, walls, floor, and tank in experiment room included

CAS62 Problem U4B1251F. $92.6 \% \mathrm{UO}_{2}\left(\mathrm{NO}_{3}\right)_{2}$ solution, $415 \mathrm{~g} \mathrm{U} / 1$, in Plexiglas cylinders, 5 × 5 × 5 array, unreflected, walls, floor, and tank in experiment room included

43 $0.9836 \pm 0.0036$ 
Table 5 (continued)

\begin{tabular}{llll}
\hline Case & Experimental description & Reference & $k_{\text {eff }} \pm \sigma$ \\
\hline
\end{tabular}

CAS64 Problem U4B641F. $92.6 \% \mathrm{UO}_{2}\left(\mathrm{NO}_{3}\right)_{2}$ walls, floor, and tank in experiment room included

CAS63 Problem U4B271F. $92.6 \% \mathrm{UO}_{2}\left(\mathrm{NO}_{3}\right)_{2}$ solution, $415 \mathrm{~g} \mathrm{U} / \mathrm{l}$, in Plexiglas cylinders, $3 \times 3 \times 3$ array, unreflected, walls, floor, and tank in experiment room included

CAS65 Problem U4B81F. 92.6\% $\mathrm{UO}_{2}\left(\mathrm{NO}_{3}\right)_{2}$ solution, $415 \mathrm{~g} \mathrm{U} / \mathrm{l}$, in Plexiglas cylinders, $2 \times 2 \times 2$ array, unreflected, walls, floor, and tank in experiment room included

CAS90 Problem U4U2B27. $92.6 \% \mathrm{UO}_{2}\left(\mathrm{NO}_{3}\right)_{2}$ solution, $415 \mathrm{~g} \mathrm{U} / 1$, in Plexiglas cylinders, $3 \times 3 \times 3$ array, unreflected, $279 \mathrm{~g} \mathrm{U} / 1$ in 5 central units, walls, floor, and tank in experiment room included

CAS66 Problem U4R27A1F. 92.6\% UO $\mathrm{UN}_{2}\left(\mathrm{NO}_{3}\right)_{2}$ solution, $415 \mathrm{~g} \mathrm{U} / 1$, in Plexiglas cylinders, $3 \times 3 \times 3$ array, reflected, $15.24 \mathrm{~cm}$ paraffin on bottom, $1.27 \mathrm{~cm}$ Plexiglas on other faces

CAS67 Problem U4R27B1F. 92.6\% UO $\mathrm{UO}_{2}\left(\mathrm{NO}_{3}\right)_{2}$ solution, $415 \mathrm{~g} \mathrm{U} / 1$, in Plexiglas cylinders, $3 \times 3 \times 3$ array, reflected, $15.24 \mathrm{~cm}$ paraffin on bottom, $2.54 \mathrm{~cm}$ Plexiglas on other faces

CAS68 Problem U4R27C1F. $92.6 \% \mathrm{UO}_{2}\left(\mathrm{NO}_{3}\right)_{2}$ solution, $415 \mathrm{~g} \mathrm{U} / 1$, in Plexiglas cylinders, $3 \times 3 \times 3$ array, reflected, $15.24 \mathrm{~cm}$ paraffin on bottom, $1.27 \mathrm{~cm}$ Plexiglas on other faces

CAS69 Problem U4R27D1F. 92.6\% UO $\mathrm{UO}_{2}\left(\mathrm{NO}_{3}\right)_{2}$ solution, $415 \mathrm{~g} \mathrm{U} / 1$, in Plexiglas cylinders, 3 × 3 × 3 array, reflected, $15.24 \mathrm{~cm}$ paraffin on 5 faces, $15.24 \mathrm{~cm}$ Plexiglas on 1 face 
Table 5 (continued)

\begin{tabular}{llll}
\hline Case & Experimental description & Reference & $k_{\text {eff }} \pm \sigma$ \\
\hline
\end{tabular}

CAS70 Problem U4R27E1F. 92.6\% $\mathrm{UO}_{2}\left(\mathrm{NO}_{3}\right)_{2} \quad 38 \quad 1.0205 \pm 0.0039$ solution, $415 \mathrm{~g} \mathrm{U} / 1$, in Plexiglas

cylinders, $3 \times 3 \times 3$ array, reflected, $15.24 \mathrm{~cm}$ paraffin on bottom, $3.81 \mathrm{~cm}$ paraffin on other faces

CAS71 Problem U4R27F1F. 92.6\% $\mathrm{UO}_{2}\left(\mathrm{NO}_{3}\right)_{2}$

solution, $415 \mathrm{~g} \mathrm{U} / 1$, in Plexiglas

cylinders, $3 \times 3 \times 3$ array, reflected, $15.24 \mathrm{~cm}$ paraffin on bottom, $7.62 \mathrm{~cm}$ paraffin on other faces

CAS72 Problem U4R27G1F. $92.6 \% \mathrm{UO}_{2}\left(\mathrm{NO}_{3}\right)_{2}$

cylinders, $3 \times 3 \times 3$ array, reflected, $1.27 \mathrm{~cm}$ Plexiglas all faces

CAS73 Problem U4R27H1F. 92.6\% $\mathrm{UO}_{2}\left(\mathrm{NO}_{3}\right)_{2}$

solution, $415 \mathrm{~g} \mathrm{U} / \mathrm{l}$, in Plexiglas

cylinders, $3 \times 3 \times 3$ array, reflected,

$1.27 \mathrm{~cm}$ paraffin all faces

CAS74 Problem U4R27I1F. 92.6\% UO $\mathrm{UO}_{2}\left(\mathrm{NO}_{3}\right)_{2}$

solution, $415 \mathrm{~g} \mathrm{U} / 1$, in Plexiglas

cylinders, $3 \times 3 \times 3$ array, reflected, $15.24 \mathrm{~cm}$ paraffin all faces

CAS75 Problem U4R27J1F. 92.6\% $\mathrm{UO}_{2}\left(\mathrm{NO}_{3}\right)_{2}$ solution, $415 \mathrm{~g} \mathrm{U} / 1$, in Plexiglas cylinders, $3 \times 3 \times 3$ array, reflected, $3.81 \mathrm{~cm}$ paraffin all faces

CAS76 Problem U4R8A1F. 92.6\% $\mathrm{UO}_{2}\left(\mathrm{NO}_{3}\right)_{2}$ solution, $415 \mathrm{~g} \mathrm{U} / 1$, in Plexiglas cylinders, $2 \times 2 \times 2$ array, reflected, $15.24 \mathrm{~cm}$ paraffin on bottom, $1.27 \mathrm{~cm}$ Plexiglas on other faces

CAS77 Problem U4R8B1F. 92.6\% $\mathrm{UO}_{2}\left(\mathrm{NO}_{3}\right)_{2}$ 
Table 5 (continued)

\begin{tabular}{|c|c|c|c|}
\hline Case & Experimental description & Reference & $k_{\text {eff }} \pm \sigma$ \\
\hline CAS78 & $\begin{array}{l}\text { Problem U4R8C1F. } 92.6 \% \mathrm{UO}_{2}\left(\mathrm{NO}_{3}\right)_{2} \\
\text { solution, } 415 \mathrm{~g} \mathrm{U} / 1 \text {, in Plexiglas } \\
\text { cylinders, } 2 \times 2 \times 2 \text { array, reflected, } \\
15.24 \mathrm{~cm} \text { paraffin on bottom, } \\
15.24 \mathrm{~cm} \text { Plexiglas on other faces }\end{array}$ & 38 & $1.0237 \pm 0.0045$ \\
\hline CAS79 & $\begin{array}{l}\text { Problem U4R8D1F. } 92.6 \% \mathrm{UO}_{2}\left(\mathrm{NO}_{3}\right)_{2} \\
\text { solution, } 415 \mathrm{~g} \mathrm{U} / 1 \text {, in Plexiglas } \\
\text { cylinders, } 2 \times 2 \times 2 \text { array, reflected, } \\
15.24 \mathrm{~cm} \text { paraffin on bottom, } \\
2.54 \mathrm{~cm} \text { Plexiglas on other faces }\end{array}$ & 38 & $1.0137 \pm 0.0036$ \\
\hline CAS80 & $\begin{array}{l}\text { Problem U4R8E1F. } 92.6 \% \mathrm{UO}_{2}\left(\mathrm{NO}_{3}\right)_{2} \\
\text { solution, } 415 \mathrm{~g} \mathrm{U} / 1 \text {, in Plexiglas } \\
\text { cylinders, } 2 \times 2 \times 2 \text { array, reflected, } \\
15.24 \mathrm{~cm} \text { paraffin on bottom, } \\
4.45 \mathrm{~cm} \text { Plexiglas on other faces }\end{array}$ & 38 & $1.0223 \pm 0.0045$ \\
\hline CAS81 & $\begin{array}{l}\text { Problem U4R8F1F. } 92.6 \% \mathrm{UO}_{2}\left(\mathrm{NO}_{3}\right)_{2} \\
\text { solution, } 415 \mathrm{~g} \mathrm{U} / 1 \text {, in Plexiglas } \\
\text { cylinders, } 2 \times 2 \times 2 \text { array, reflected, } \\
15.24 \mathrm{~cm} \text { paraffin on bottom, } \\
6.35 \mathrm{~cm} \text { Plexiglas on other faces }\end{array}$ & 38 & $1.0197 \pm 0.0037$ \\
\hline CAS82 & $\begin{array}{l}\text { Problem U4R8G1F. } 92.6 \% \mathrm{UO}_{2}\left(\mathrm{NO}_{3}\right)_{2} \\
\text { solution, } 415 \mathrm{~g} \mathrm{U} / 1 \text {, in Plexiglas } \\
\text { cylinders, } 2 \times 2 \times 2 \text { array, reflected, } \\
15.24 \mathrm{~cm} \text { paraffin on bottom, } \\
1.27 \mathrm{~cm} \text { Plexiglas on other faces }\end{array}$ & 38 & $1.0195 \pm 0.0038$ \\
\hline CAS83 & $\begin{array}{l}\text { Problem U4R8H1F. } 92.6 \% \mathrm{UO}_{2}\left(\mathrm{NO}_{3}\right)_{2} \\
\text { solution, } 415 \mathrm{~g} \mathrm{U} / 1 \text {, in Plexiglas } \\
\text { cylinders, } 2 \times 2 \times 2 \text { array, reflected, } \\
15.24 \mathrm{~cm} \text { paraffin on bottom, } \\
3.81 \mathrm{~cm} \text { Plexiglas on other faces }\end{array}$ & 38 & $1.0225 \pm 0.0039$ \\
\hline CAS84 & $\begin{array}{l}\text { Problem U4R8I1F. } 92.6 \% \mathrm{UO}_{2}\left(\mathrm{NO}_{3}\right)_{2} \\
\text { solution, } 415 \mathrm{~g} \mathrm{U} / \mathrm{l} \text {, in Plexiglas } \\
\text { cylinders, } 2 \times 2 \times 2 \text { array, reflected, } \\
15.24 \mathrm{~cm} \text { paraffin on bottom, } \\
7.62 \mathrm{~cm} \text { Plexiglas on other faces }\end{array}$ & 38 & $1.0247 \pm 0.0041$ \\
\hline
\end{tabular}


Table 5 (continued)

\begin{tabular}{|c|c|c|c|}
\hline Case & Experimental description & Reference & $k_{\text {eff }} \pm \sigma$ \\
\hline CAS85 & $\begin{array}{l}\text { Problem U4R8J1F. } 92.6 \% \mathrm{UO}_{2}\left(\mathrm{NO}_{3}\right)_{2} \\
\text { solution, } 415 \mathrm{~g} \mathrm{U} / 1 \text {, in Plexiglas } \\
\text { cylinders, } 2 \times 2 \times 2 \text { array, reflected, } \\
1.27 \mathrm{~cm} \text { Plexiglas all faces }\end{array}$ & 38 & $1.0175 \pm 0.0046$ \\
\hline CAS86 & $\begin{array}{l}\text { Problem U4R8K1F. } 92.6 \% \mathrm{UO}_{2}\left(\mathrm{NO}_{3}\right)_{2} \\
\text { solution, } 415 \mathrm{~g} \mathrm{U} / \mathrm{l} \text {, in Plexiglas } \\
\text { cylinders, } 2 \times 2 \times 2 \text { array, reflected, } \\
1.27 \mathrm{~cm} \text { paraffin all faces }\end{array}$ & 38 & $1.01675 \pm 0.0042$ \\
\hline CAS87 & $\begin{array}{l}\text { Problem U4R8LlF. } 92.6 \% \mathrm{UO}_{2}\left(\mathrm{NO}_{3}\right)_{2} \\
\text { solution, } 415 \mathrm{~g} \mathrm{U} / \mathrm{l} \text {, in Plexiglas } \\
\text { cylinders, } 2 \times 2 \times 2 \text { array, reflected, } \\
15.24 \mathrm{~cm} \text { paraffin all faces }\end{array}$ & 38 & $1.0177 \pm 0.0042$ \\
\hline CAS88 & $\begin{array}{l}\text { Problem U4R8M1F. } 92.6 \% \mathrm{UO}_{2}\left(\mathrm{NO}_{3}\right)_{2} \\
\text { solution, } 415 \mathrm{~g} \mathrm{U} / 1 \text {, in Plexiglas } \\
\text { cylinders, } 2 \times 2 \times 2 \text { array, reflected, } \\
3.81 \mathrm{~cm} \text { paraffin all faces }\end{array}$ & 38 & $1.0177 \pm 0.0041$ \\
\hline CAS89 & $\begin{array}{l}\text { Problem U4R8N1F. } 92.6 \% \mathrm{UO}_{2}\left(\mathrm{NO}_{3}\right)_{2} \\
\text { solution, } 415 \mathrm{~g} \mathrm{U} / 1 \text {, in Plexiglas } \\
\text { cylinders, } 2 \times 2 \times 2 \text { array, reflected, } \\
7.62 \mathrm{~cm} \text { paraffin all faces }\end{array}$ & 38 & $1.0169 \pm 0.0046$ \\
\hline
\end{tabular}


Table 6. KENO V.a calculated $k_{\text {eff }}$ for experimentally critical highly enriched uranium systems

\begin{tabular}{|c|c|c|c|}
\hline Case & Experimental description & Reference & $\mathrm{k}_{\mathrm{eff}} \pm \sigma$ \\
\hline CASO1 & $\begin{array}{l}\text { Experiment } 1 . \mathrm{U}(93.2) \mathrm{O}_{2}\left(\mathrm{NO}_{3}\right)_{2} \\
\mathrm{H} / \mathrm{X}=1378 \text { in } 27.24 \text {-in. diam sphere }\end{array}$ & 12,13 & $0.9979 \pm 0.0022$ \\
\hline CAS02 & $\begin{array}{l}\text { Experiment 2. } \mathrm{U}(93.2) \mathrm{O}_{2}\left(\mathrm{NO}_{3}\right)_{2} \\
\mathrm{H} / \mathrm{X}=1177, \mathrm{~B} \text { poisoned, in } 27.24 \text {-in. diam sphere }\end{array}$ & 12,13 & $0.9960 \pm 0.0023$ \\
\hline CASO3 & $\begin{array}{l}\text { Experiment 3. } \mathrm{U}(93.2) \mathrm{O}_{2}\left(\mathrm{NO}_{3}\right)_{2} \\
\mathrm{H} / \mathrm{X}=1033 \text {, B poisoned, in } 27.24 \text {-in. diam sphere }\end{array}$ & 12,13 & $0.9920 \pm 0.0027$ \\
\hline CASO4 & $\begin{array}{l}\text { Experiment } 4 . \mathrm{U}(93.2) \mathrm{O}_{2}\left(\mathrm{NO}_{3}\right)_{2} \\
\mathrm{H} / \mathrm{X}=972, \mathrm{~B} \text { poisoned, in } 27.24 \text {-in. diam sphere }\end{array}$ & 12,13 & $1.0020 \pm 0.0024$ \\
\hline CAS05 & $\begin{array}{l}\text { Experiment 5. U-233 } \mathrm{O}_{2}\left(\mathrm{NO}_{3}\right)_{2} \\
\mathrm{H} / \mathrm{X}=1533 \text {, in } 27.24 \text {-in. diam sphere }\end{array}$ & 12,13 & $1.0086 \pm 0.0022$ \\
\hline CAS06 & $\begin{array}{l}\text { Experiment 6. } \mathrm{U}-233 \mathrm{O}_{2}\left(\mathrm{NO}_{3}\right)_{2} \\
\mathrm{H} / \mathrm{X}=1470 \text {, B poisoned, in } 27.24 \text {-in. diam sphere }\end{array}$ & 12,13 & $1.0079 \pm 0.0025$ \\
\hline CAS07 & $\begin{array}{l}\text { Experiment 7. } \mathrm{U}-233 \mathrm{O}_{2}\left(\mathrm{NO}_{3}\right)_{2} \\
\mathrm{H} / \mathrm{X}=1417 \text {, B poisoned, in } 27.24 \text {-in. diam sphere }\end{array}$ & 12,13 & $1.0045 \pm 0.0023$ \\
\hline CAS08 & $\begin{array}{l}\text { Experiment } 8 . \mathrm{U}-233 \mathrm{O}_{2}\left(\mathrm{NO}_{3}\right)_{2} \\
\mathrm{H} / \mathrm{X}=1368, \mathrm{~B} \text { poisoned, in } 27.24 \text {-in. diam sphere }\end{array}$ & 12,13 & $1.0065 \pm 0.0023$ \\
\hline CAS09 & $\begin{array}{l}\text { Experiment 9. U-233 } \mathrm{O}_{2}\left(\mathrm{NO}_{3}\right)_{2} \\
\mathrm{H} / \mathrm{X}=1835 \text {, in 48.04-in. diam sphere }\end{array}$ & 12,13 & $1.0084 \pm 0.0023$ \\
\hline CAS10 & $\begin{array}{l}\text { Experiment } 10 . \mathrm{U}(93.2) \mathrm{O}_{2}\left(\mathrm{NO}_{3}\right)_{2} \\
\mathrm{H} / \mathrm{X}=1835 \text {, in } 48.04 \text {-in. diam sphere }\end{array}$ & 12,13 & $0.9979 \pm 0.0017$ \\
\hline CAS11 & $\begin{array}{l}\text { Experiment } 11 . \mathrm{U}-233 \mathrm{O}_{2}\left(\mathrm{NO}_{3}\right)_{2} \\
\mathrm{H} / \mathrm{X}=1986 \text {, in } 48.04 \text {-in. diam sphere }\end{array}$ & 12,13 & $0.9959 \pm 0.0017$ \\
\hline CAS12 & $\begin{array}{l}\text { Experiment } 12 . \mathrm{U}(93.2) \mathrm{O}_{2}\left(\mathrm{NO}_{3}\right)_{2} \\
\mathrm{H} / \mathrm{X}=1604 \text {, in } 60.92 \text {-in. diam cylinder }\end{array}$ & 12,13 & $0.9989 \pm 0.0023$ \\
\hline CAS13 & $\begin{array}{l}\text { Experiment 13. } \mathrm{U}(93.2) \mathrm{O}_{2}\left(\mathrm{NO}_{3}\right)_{2} \\
\mathrm{H} / \mathrm{X}=1634 \text {, in } 60.92 \text {-in. diam cylinder }\end{array}$ & 12,13 & $0.9983 \pm 0.0022$ \\
\hline CAS14 & $\begin{array}{l}\text { Experiment } 14 . \mathrm{U}(93.2) \mathrm{O}_{2}\left(\mathrm{NO}_{3}\right)_{2} \\
\mathrm{H} / \mathrm{X}=1821 \text {, in } 60.92 \text {-in. diam cylinder }\end{array}$ & 12,13 & $0.9992 \pm 0.0017$ \\
\hline
\end{tabular}


Table 6 (continued)

\begin{tabular}{|c|c|c|c|}
\hline Case & Experimental description & Reference & $\mathrm{k}_{\mathrm{eff}} \pm \sigma$ \\
\hline CAS15 & $\begin{array}{l}\text { Experiment } 15 . \mathrm{U}(93.2) \mathrm{O}_{2}\left(\mathrm{NO}_{3}\right)_{2} \\
\mathrm{H} / \mathrm{X}=1905 \text {, in } 60.92 \text {-in. diam cylinder }\end{array}$ & 12,13 & $0.9925 \pm 0.0019$ \\
\hline CAS16 & $\begin{array}{l}\text { Experiment 16. } \mathrm{U}(93.2) \mathrm{O}_{2}\left(\mathrm{NO}_{3}\right)_{2} \\
\mathrm{H} / \mathrm{X}=1981 \text {, in } 60.92 \text {-in. diam cylinder }\end{array}$ & 12,13 & $0.9976 \pm 0.0016$ \\
\hline CAS17 & $\begin{array}{l}\text { Experiment } 17 \text {. U-233 } \mathrm{O}_{2}\left(\mathrm{NO}_{3}\right)_{2} \\
\mathrm{H} / \mathrm{X}=1819 \text {, in } 60.92 \text {-in. diam cylinder }\end{array}$ & 12,13 & $0.9952 \pm 0.0020$ \\
\hline CAS18 & $\begin{array}{l}\text { Experiment } 18 . \mathrm{U}-233 \mathrm{O}_{2}\left(\mathrm{NO}_{3}\right)_{2} \\
\mathrm{H} / \mathrm{X}=1900 \text {, in } 60.92 \text {-in. diam cylinder }\end{array}$ & 12,13 & $0.9973 \pm 0.0018$ \\
\hline CAS19 & $\begin{array}{l}\text { Experiment } 19 . \mathrm{U}-233 \mathrm{O}_{2}\left(\mathrm{NO}_{3}\right)_{2} \\
\mathrm{H} / \mathrm{X}=1996 \text {, in } 60.92 \text {-in. diam cylinder }\end{array}$ & $12,13^{\circ}$ & $0.9916 \pm 0.0015$ \\
\hline CAS20 & $\begin{array}{l}\text { Experiment 20. U-233 } \mathrm{O}_{2}\left(\mathrm{NO}_{3}\right)_{2} \\
\mathrm{H} / \mathrm{X}=2106 \text {, i } 60.92 \text {-in. diam cylinder }\end{array}$ & 12,13 & $0.9927 \pm 0.0015$ \\
\hline CAS21 & $\begin{array}{l}\text { Experiment 21. } \mathrm{U}(93.2) \mathrm{O}_{2}\left(\mathrm{NO}_{3}\right)_{2} \\
\mathrm{H} / \mathrm{X}=1955 \text {, in } 107.7 \text {-in. diam cylinder }\end{array}$ & 12,13 & $0.9923 \pm 0.0015$ \\
\hline CAS22 & $\begin{array}{l}\text { Experiment 22. } \mathrm{U}(93.2) \mathrm{O}_{2}\left(\mathrm{NO}_{3}\right)_{2} \\
\mathrm{H} / \mathrm{X}=2004 \text {, in 107.7-in. diam cylinder }\end{array}$ & 12,13 & $0.9915 \pm 0.0014$ \\
\hline CAS23 & $\begin{array}{l}\text { Experiment 23. } \mathrm{U}(93.2) \mathrm{O}_{2}\left(\mathrm{NO}_{3}\right)_{2} \\
\mathrm{H} / \mathrm{X}=2052 \text {, in } 107.7 \text {-in. diam cylinder }\end{array}$ & 12,13 & $0.9991 \pm 0.0014$ \\
\hline
\end{tabular}




\subsection{DISCUSSION}

The experiments modeled were parameterized such that trend analysis could be performed. Table C.1 (Appendix C) is a relisting of the results of the 258 experiments which were calculated, along with the parameters which describe the experiments. Table C.1 is partitioned to correspond to Tables 1-6. Table 7 gives the average value of $k_{\text {eff }}$ for each table of results. The mean values of $k_{\text {eff }}$ are in excellent agreement with the experiments. The spread in data, however, indicates that there may be problems with certain types of calculations. The cause of the spread in data will be discussed more fully in Sect. 2.

Figures 1-3 are histograms showing frequency of $k_{\mathrm{eff}}$, average energy group of the neutrons causing fission (average energy group), and the assay distribution for all 258 experiments. From Fig. 2 it can be seen that the majority of the calculations were for thermal systems. Figure 3 indicates that the modeled experiments did not include enrichments between 5 and $90 \%$.

Figure 4 shows the value of calculated $k_{\text {eff }}$ as a function of the average energy group of the neutroncausing fission.

Figure 5 is a plot of calculated $k_{\text {eff }}$ as a function of the reference from which the experiments were modeled. In some of the cases where a number of experiments were run from the same reference, there seems to be a natural break. If all the cases had been equivalent as far as modeling and code treatment, $\mathrm{k}_{\mathrm{eff}}$ should be normally distributed around a mean value. The natural break may be an indication of possible code and/or cross-section problems.

Figures 6 and 7 show the value of $k_{\text {eff }}$ as a function of the assay for low-enriched and high-enriched experiments, respectively. In Fig. 6 , the calculated $k_{\text {eff }}$ of the $3 \%$ enriched experiments are considerably higher than those of other low-enriched experiments. Reasons for this trend are discussed in Sect. 2.

Figures 8-13 are plots of $k_{\text {eff }}$ versus average energy group for each table of results. Closed-band tolerance limits similar to those discussed in ref. 7 have been plotted. The dashed line on these curves represents the lower tolerance limit for $99.9 \%$ of the population with a $95 \%$ confidence level.

Figure 12 was split into two portions (Figs. 12a and 12b) because of the large gap in average energy group of the neutron-causing fission between groups 12 and 20 . This caused the tolerance band width to be smaller in Figs. 12a and 12b. The slope of the curve fit through the data changed considerably in Fig. 12b. Figure 12b highlights the importance of knowing the cause of bias in calculated results. If the bias were caused by a systematic code or cross-section error, then, according to Fig. 12b, systems similar to those used in the figure and with an average energy group in the range of 21 would have a tolerance limit of 0.97 . However, if the bias were caused because of reporting bias, then Fig. 12b shows a false trend; and the tolerance limit is unknown.

\subsection{CONCLUSIONS}

The KENO V.a code accurately calculates a broad range of critical experiments. In some past validations, the average energy group of the neutron-causing fission has been used to report calculated results. There is no strong evidence that calculated results are highly correlated to the average energy group of the neutron-causing fission. In review, a substantial number of the calculations show a positive or negative bias in excess of $1-1 / 2 \%$ in $k_{\text {eff. }}$. Classes of criticals which show a bias include $3 \%$ enriched green blocks, highly enriched uranyl fluoride slab arrays, and highly enriched uranyl nitrate arrays. If these biases are properly taken into account, the KENO V.a code in the Y12CSG package can be used 
Table 7. Average $k_{\text {eff }}$ by table

\begin{tabular}{|c|c|c|c|c|c|c|}
\hline Variable & Label & $\mathbf{N}$ & Mean & $\begin{array}{l}\text { Standard } \\
\text { Deviation }\end{array}$ & $\begin{array}{l}\text { Minimum } \\
\text { Value }\end{array}$ & $\begin{array}{l}\text { Minimum } \\
\text { Value }\end{array}$ \\
\hline & & & Table $=1$ & & & \\
\hline \multirow[t]{2}{*}{$k_{\text {eff }}$} & k-effective & 39 & 0.99369744 & 0.00771453 & 0.97390000 & 1.01080000 \\
\hline & & & Table $=2$ & & & \\
\hline \multirow[t]{2}{*}{$\mathrm{k}_{\text {eff }}$} & k-effective & 12 & 0.99568333 & 0.00620349 & 0.98770000 & 1.00930000 \\
\hline & & & Table $=3$ & & & \\
\hline \multirow[t]{2}{*}{$\mathrm{k}_{\text {eff }}$} & k-effective & 49 & 1.00434082 & 0.00911375 & 0.98460000 & 1.02280000 \\
\hline & & & Table $=4$ & & & \\
\hline \multirow[t]{2}{*}{$k_{\text {eff }}$} & k-effective & 43 & 1.00673721 & 0.00759245 & 0.99470000 & 1.03510000 \\
\hline & & & Table $=5$ & & & \\
\hline \multirow[t]{2}{*}{$k_{\text {eff }}$} & k-effective & 91 & 1.00278132 & 0.01630369 & 0.97570000 & 1.05850000 \\
\hline & & & Table $=6$ & & & \\
\hline $\mathbf{k}_{\text {eff }}$ & k-effective & 23 & 0.99842609 & 0.00554061 & 0.99150000 & 1.00860000 \\
\hline
\end{tabular}


FREQUENCY CHART FOR K-EFFECTIVE

INCLUDES 258 EXPERIMENTS

FREQUENCY

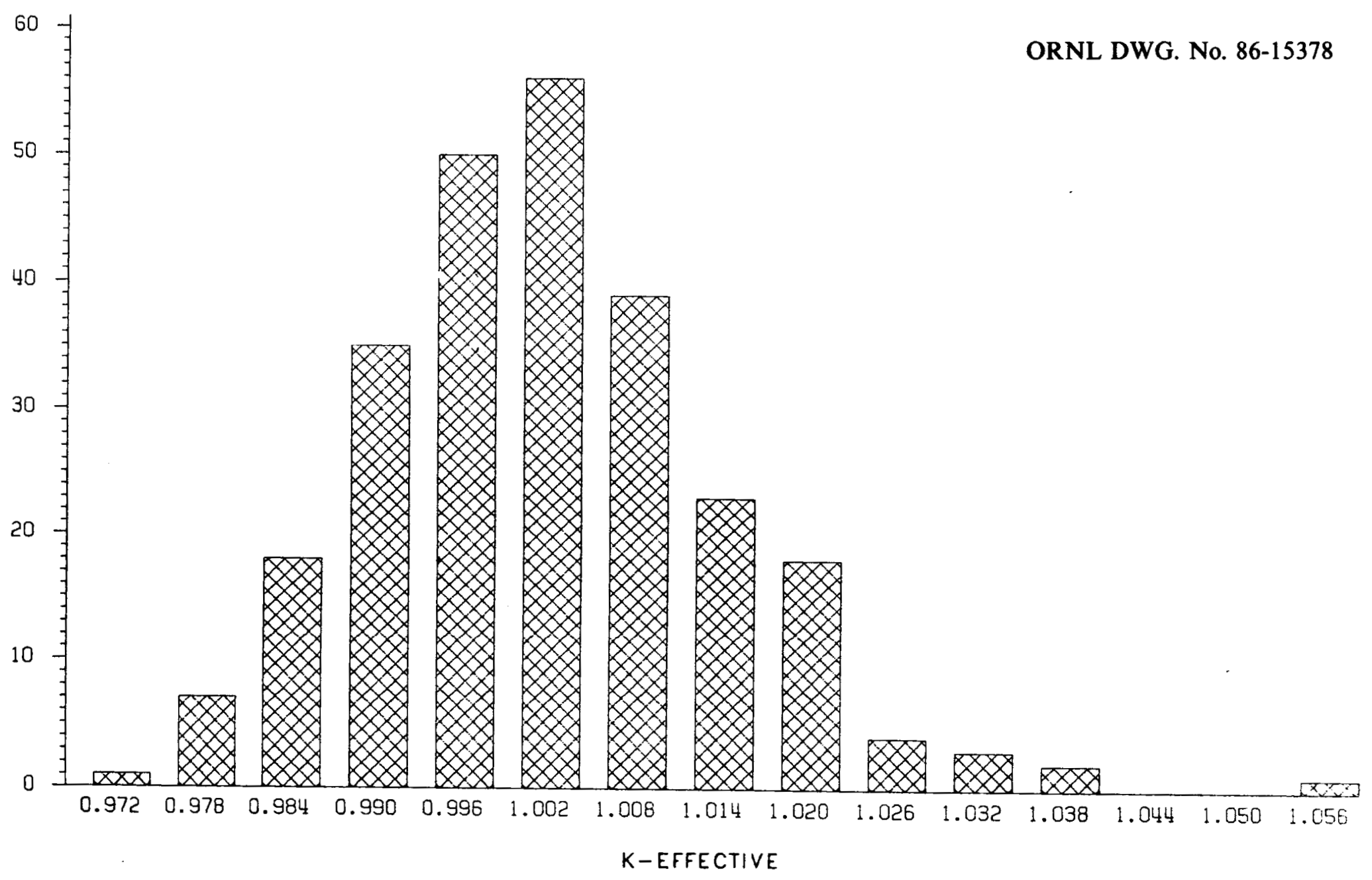

Fig. 1. Frequency chart for k-effective. 
FREQUENCY CHART FOR AVERAGE ENERGY GROUP

INCLUDES 258 EXPERIMENTS

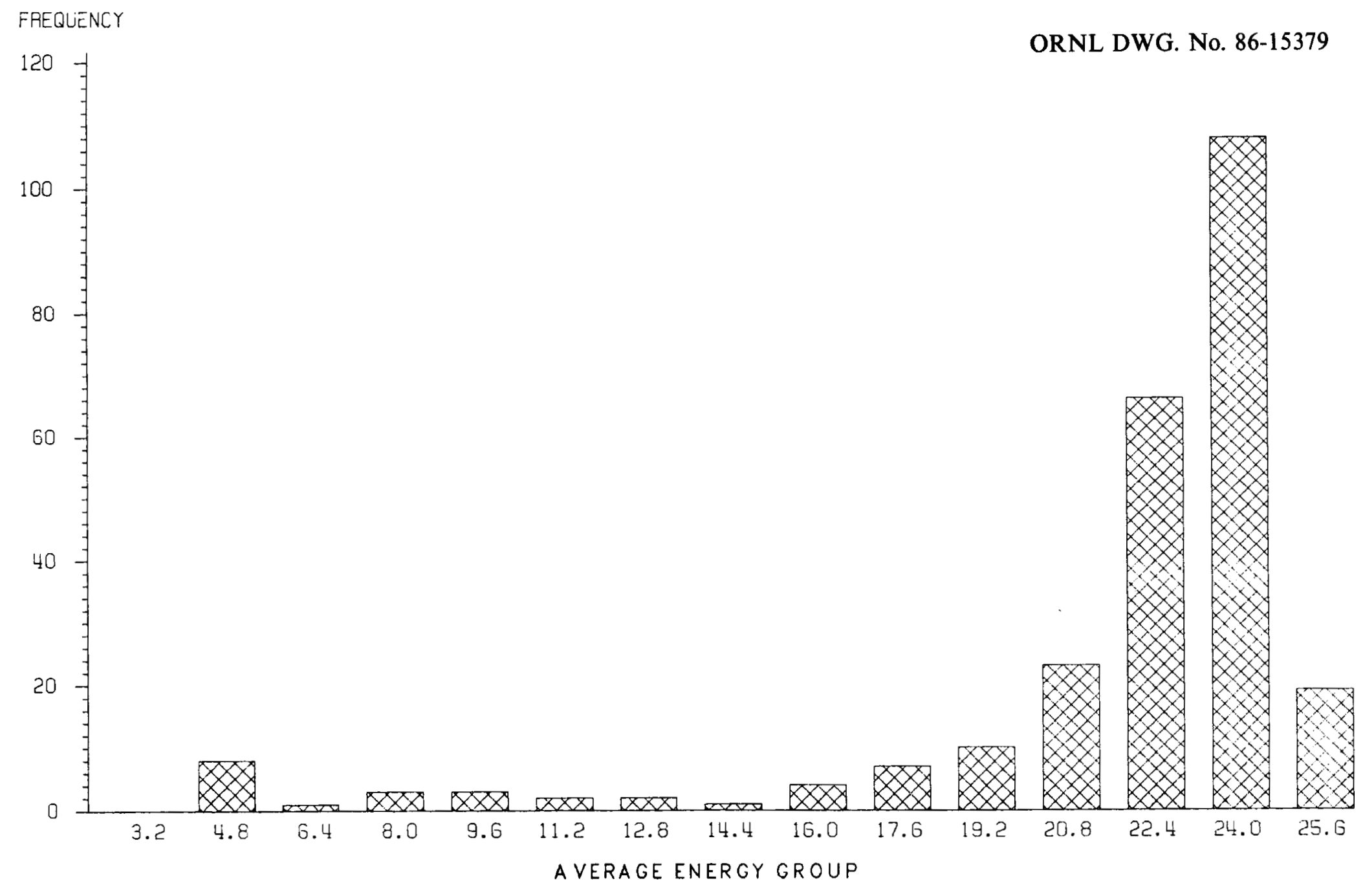


FREQUENCY CHART FOR ENRICHMENT LEVEL INCLUDES 258 EXPERIMENTS

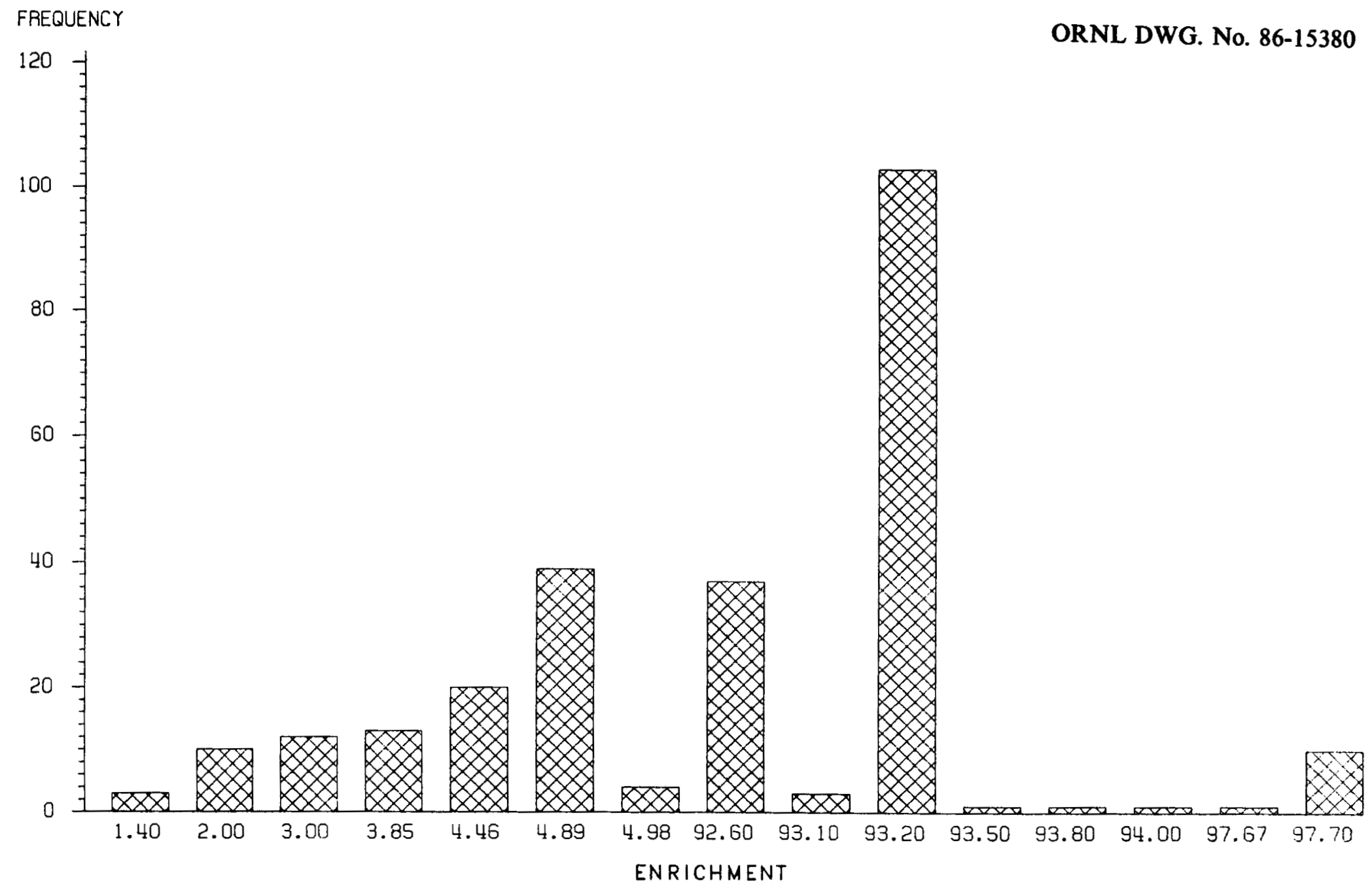

Fig. 3. Frequency chart for enrichment level. 


\section{K-EFFECTIVE VS AVERAGE ENERGY GROUP INCLUDES 258 EXPERIMENTS \\ ORNL DWG. No. 86-15381}

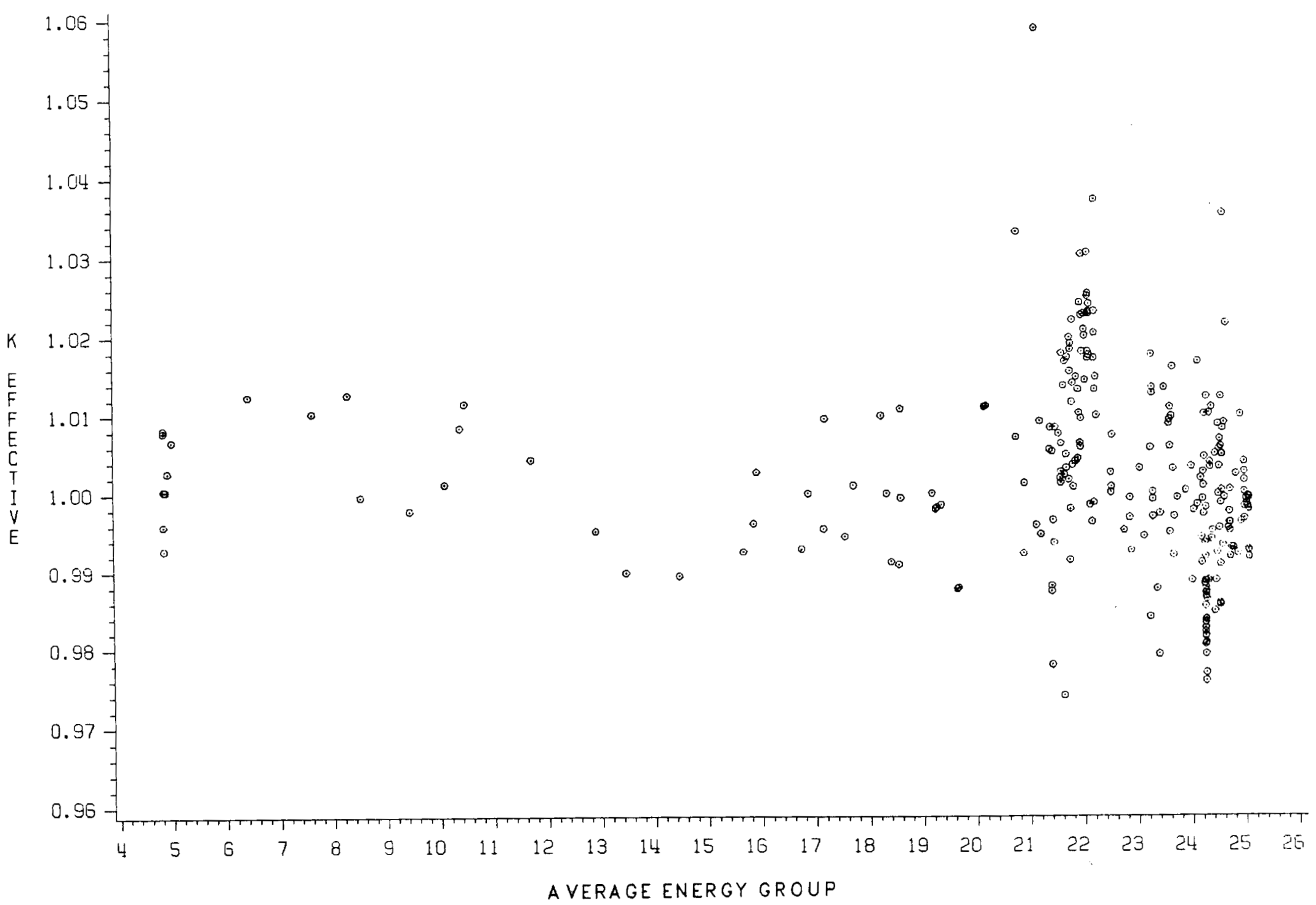

Fig. 4. K-effective vs average energy group. 


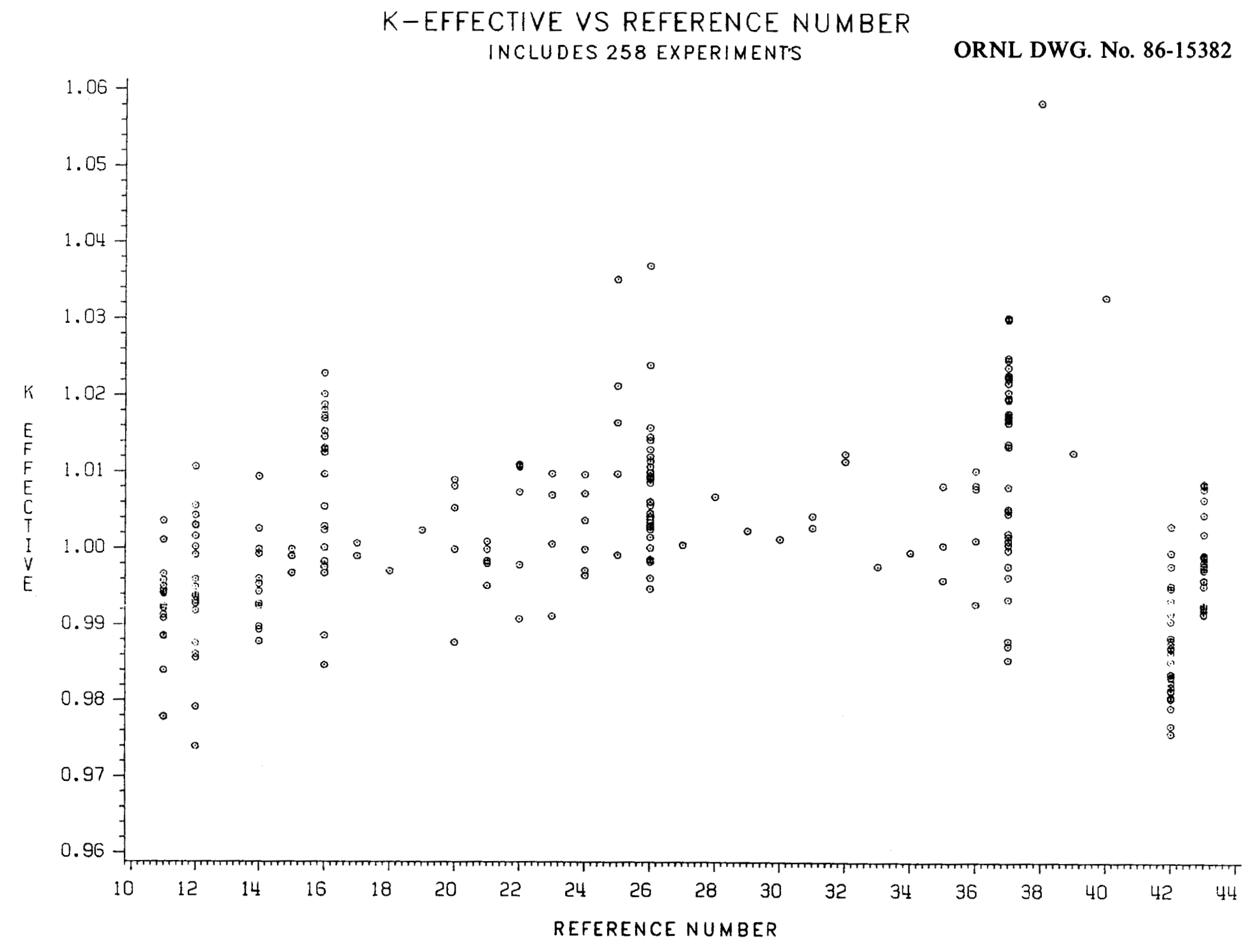

Fig. 5. K-effective vs reference number. 
K-EFFECTIVE VS ENRICHMENT LEVEL

LOW ENRICHMENT EXPERIMENTS

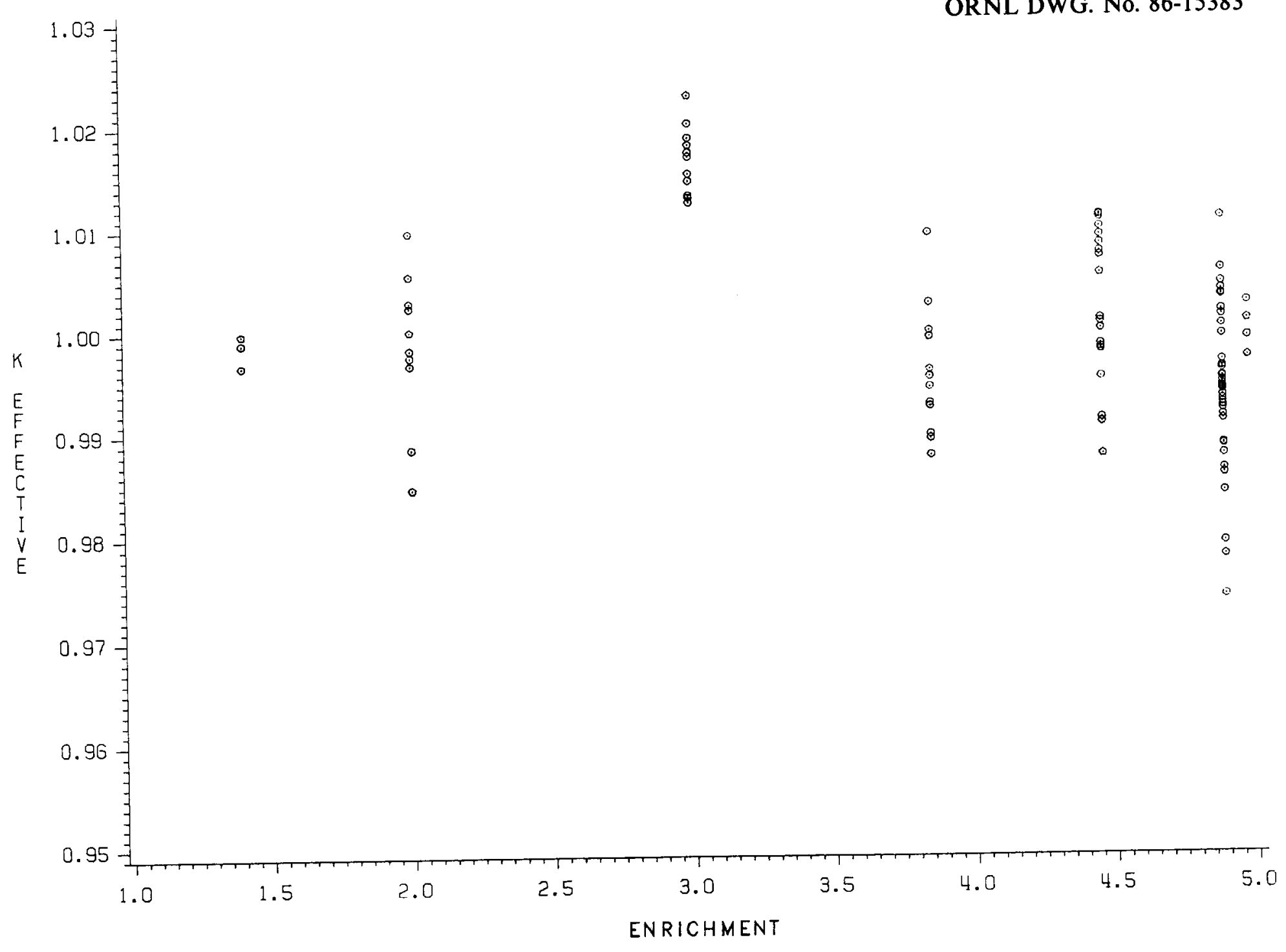

Fig. 6. K-effective vs enrichment level for low enrichment experiments. 


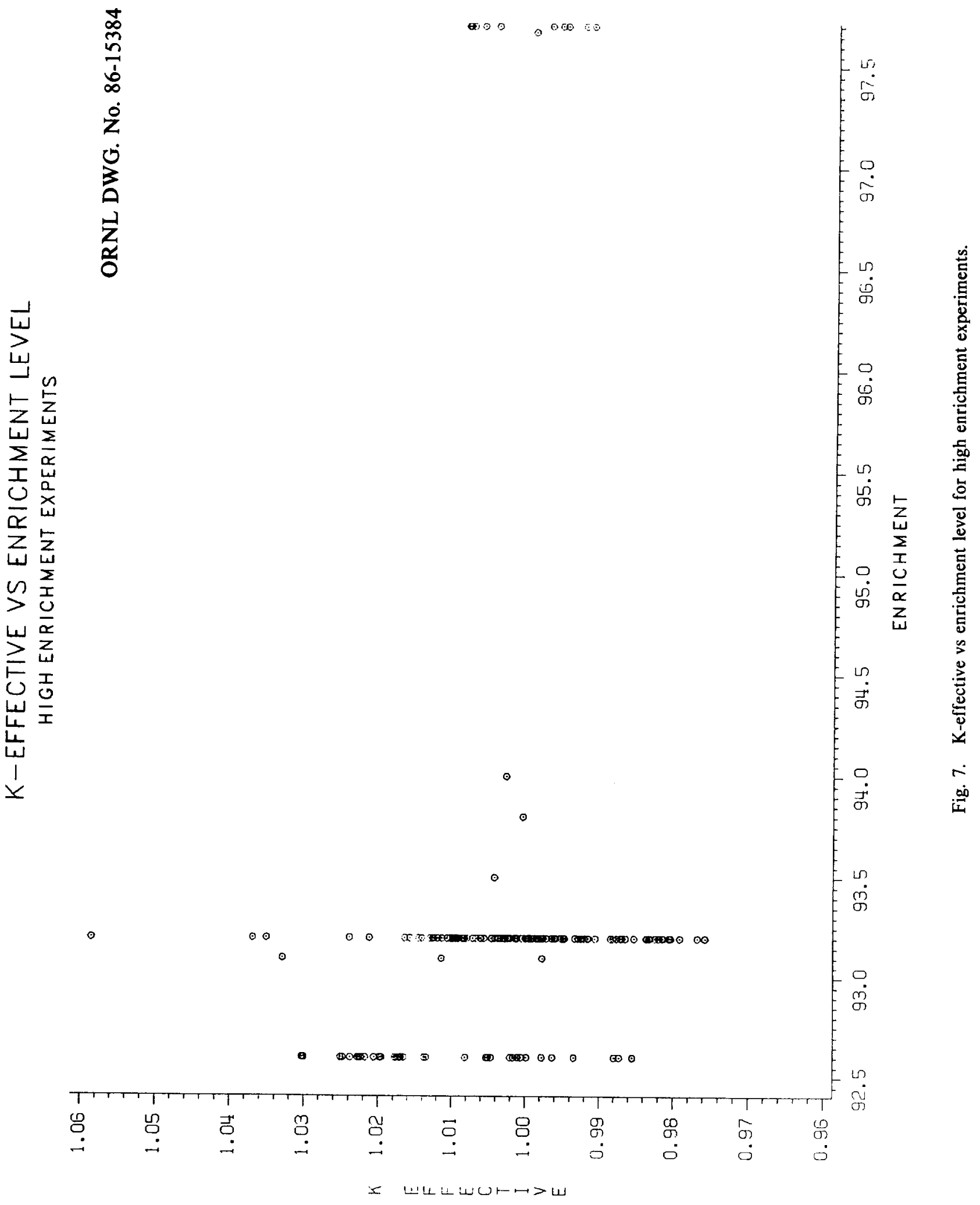




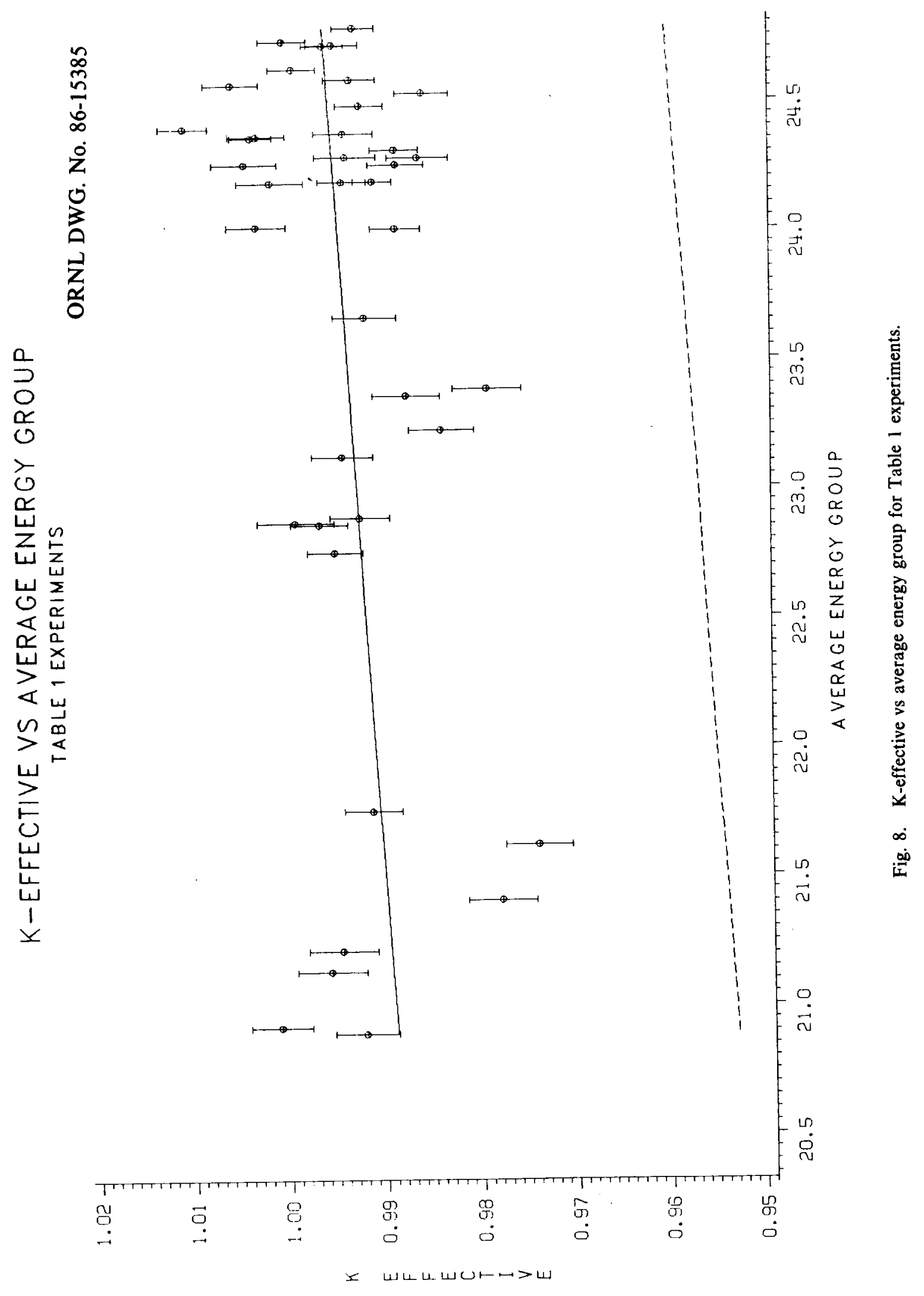




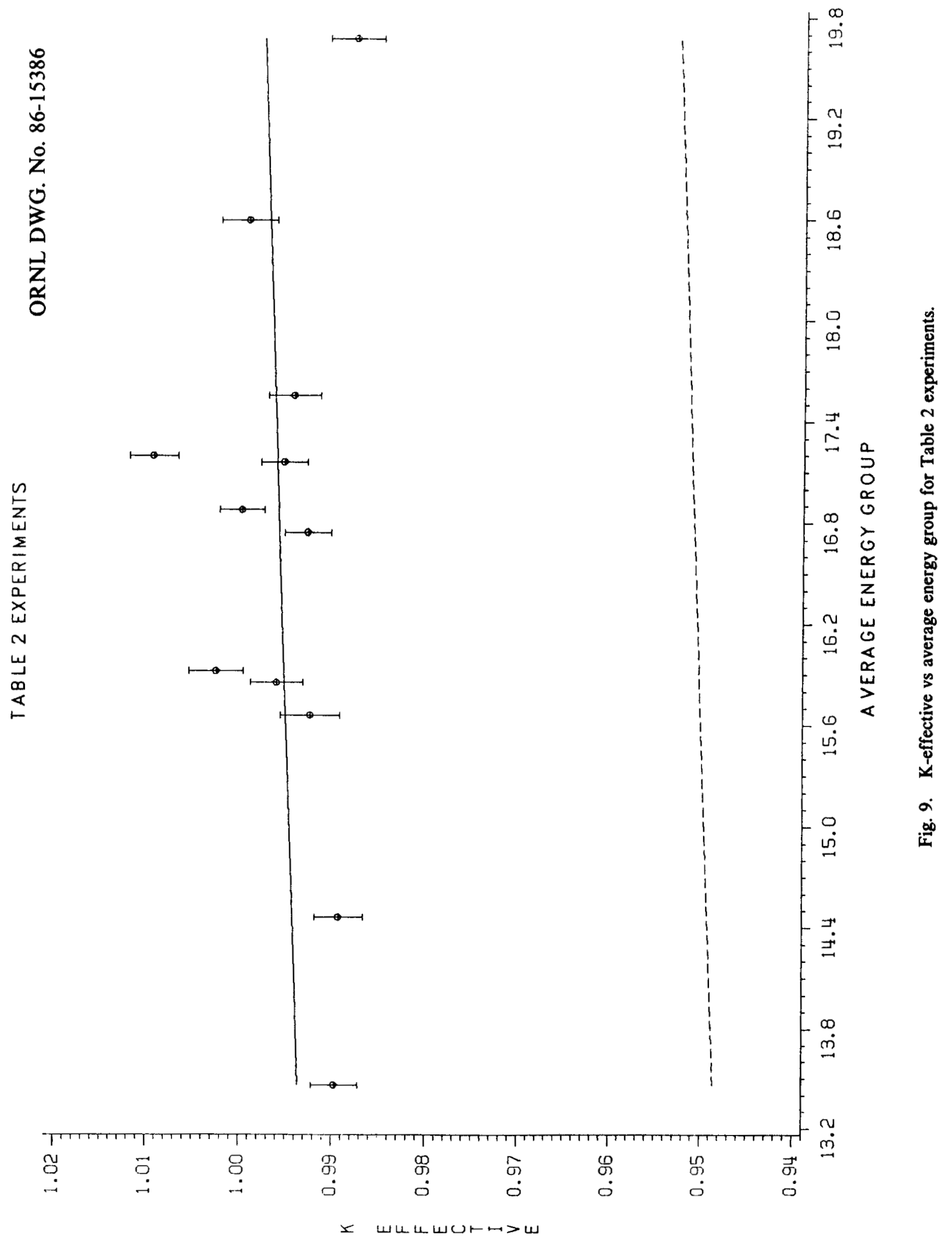




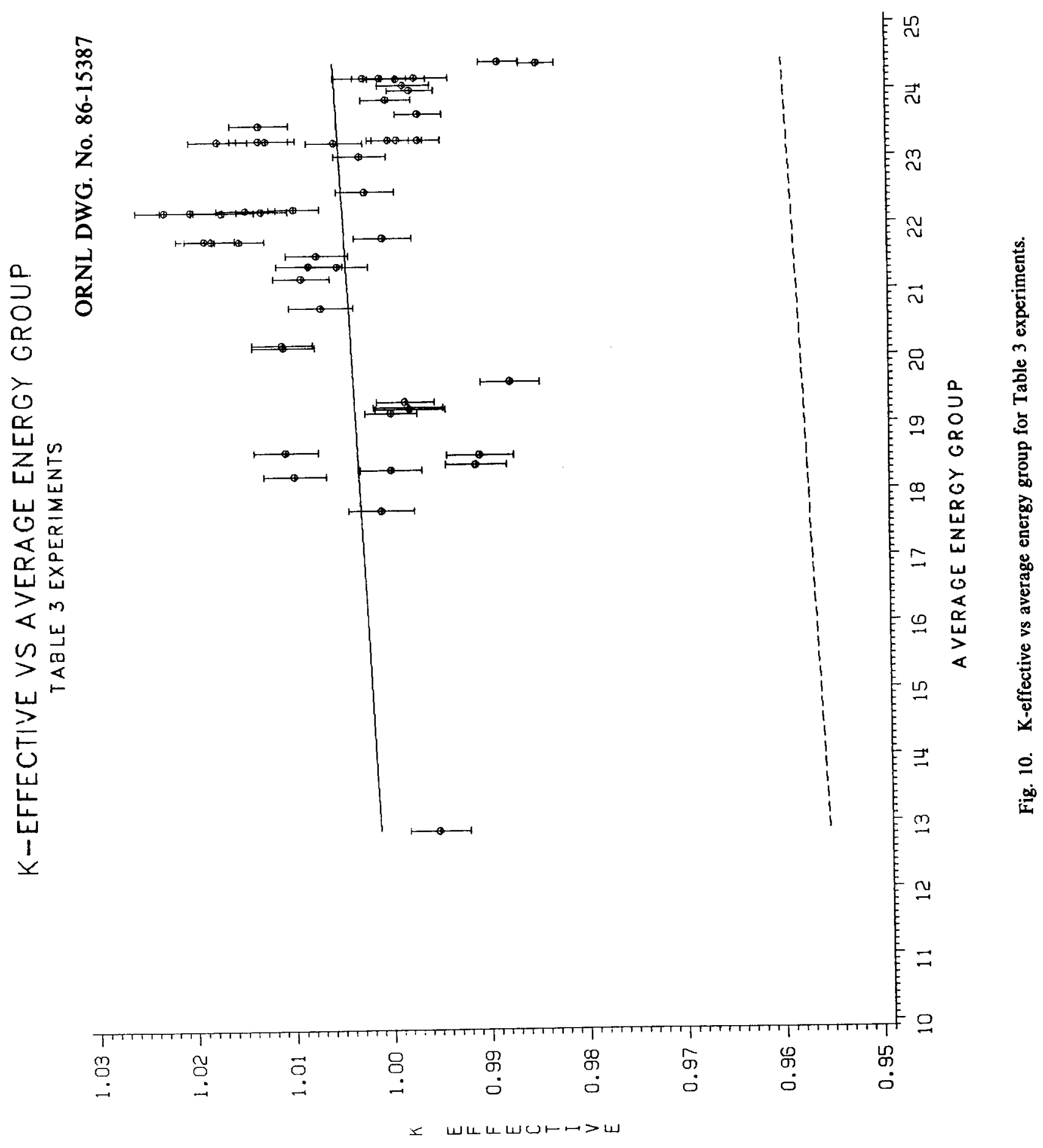




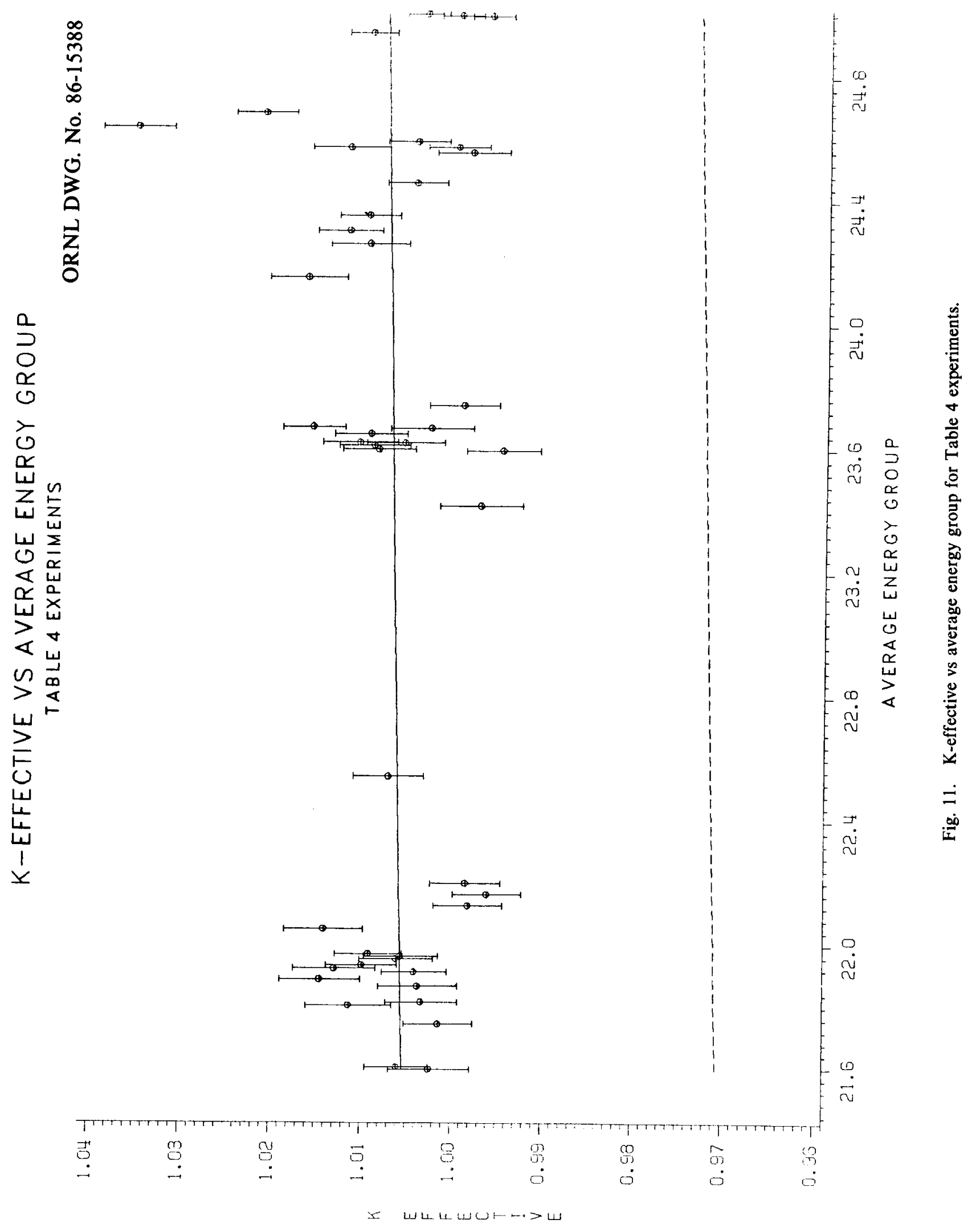




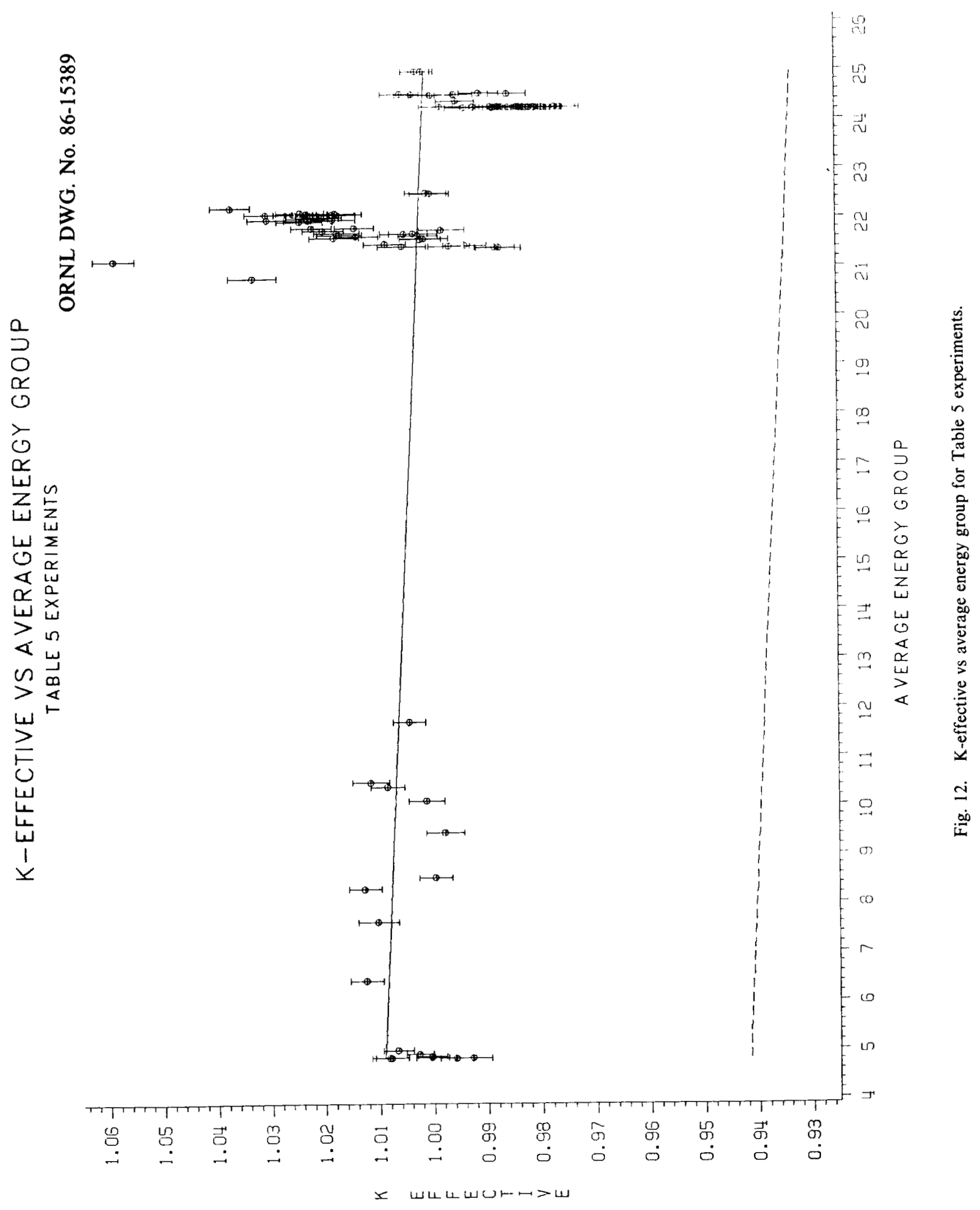


K-EFFECTIVE VS A VERAGE ENERGY GROUP

ORNL DWG. No. $86-15390$

TABLE 5 EXPERIMENTS - LOW AVERAGE ENERGY GROUP (4.5 TO 11.5)

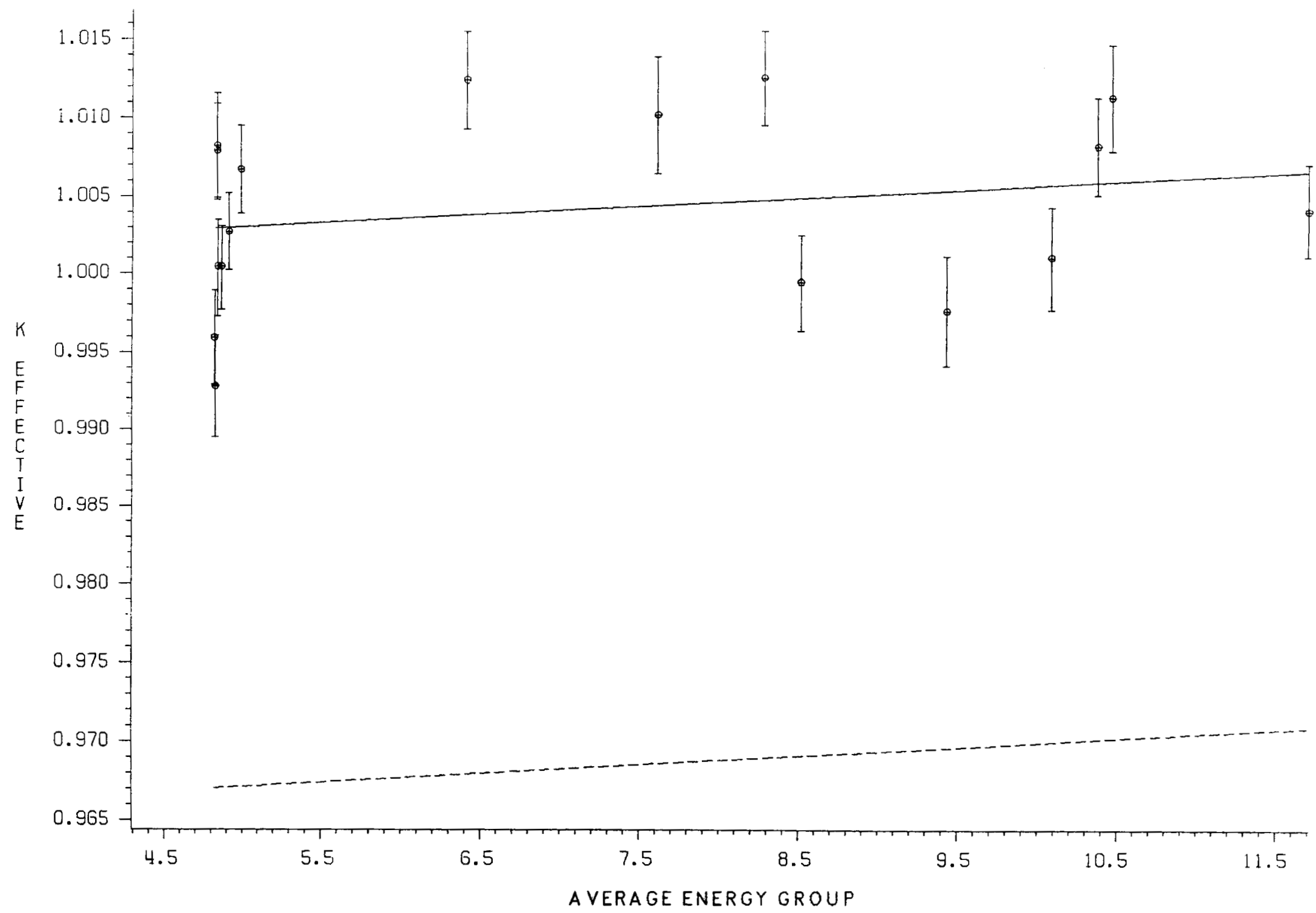

Fig. 12a. K-effective vs average energy group for Table 5 experiments, low average energy group (4.5 to 11.5). 


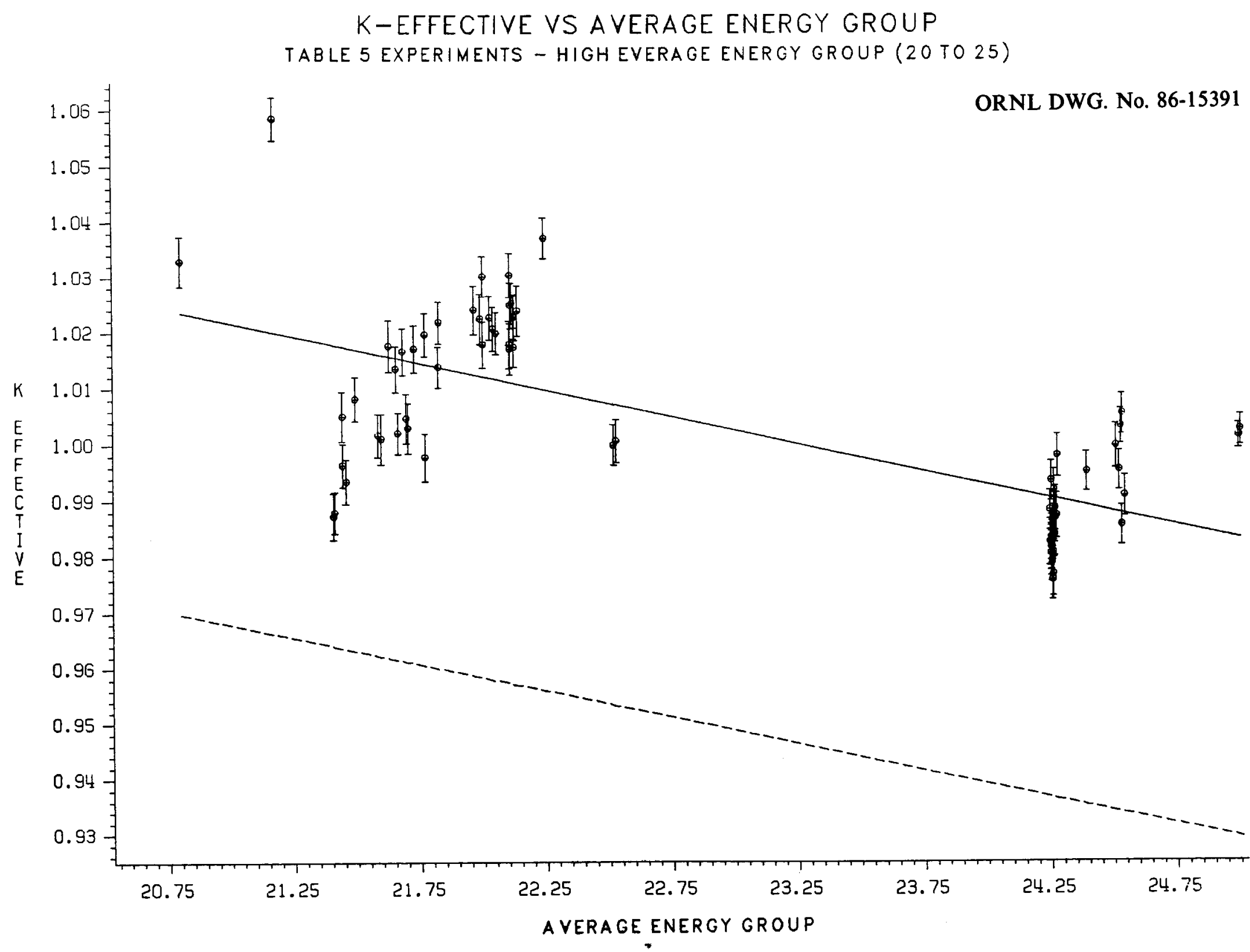

Fig. 12b. K-effective vs average energy group for Table 5 experiments, high average energy group (20 to 25 ). 


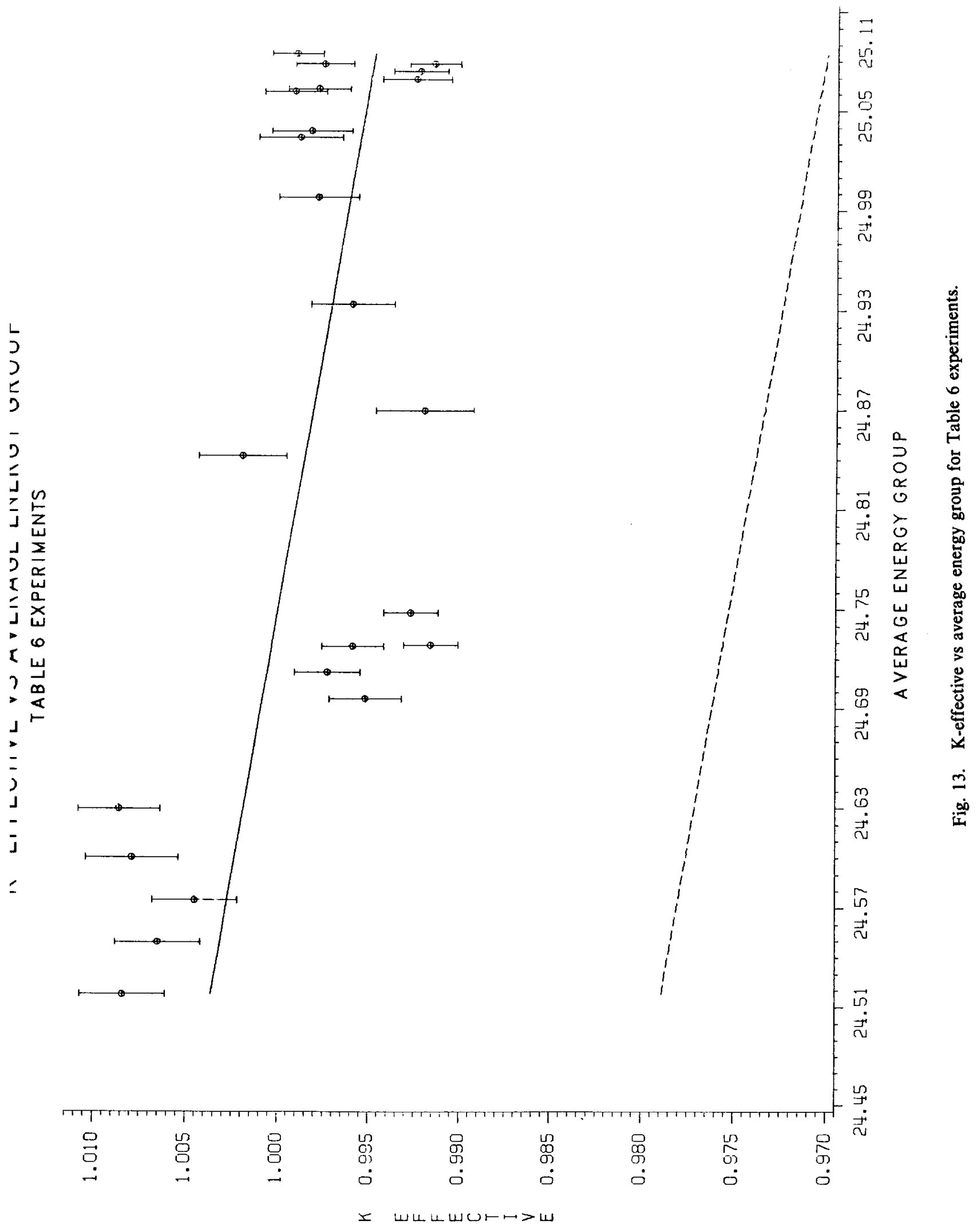


with great confidence for the design and criticality safety analysis of uranium-containing systems. It appears that slight extensions outside the range of validation would be acceptable. It should be noted that only a fraction of the available options and geometry types have been exercised. Proper performance of the options validated here does not imply that all the options in the code perform as well. It must be emphasized that the calculated $k_{\text {eff }}$ from KENO V.a is an estimate of the eigenvalue of the system and has an associated degree of uncertainty due to the statistical nature of the code. Several suggestions are offered to the users of the code.

1. Never base the design or safety of a system on a single calculation. Make at least two calculations in which the random sequence is somehow changed (starting random number, slight change in geometry or materials, etc.) to ensure that the calculated $k_{\text {eff }}$ is a reasonable estimate of the calculational mean for the system.

2. Substantial effort may be required to ensure that the problem has converged to a reasonable estimate of the true $k_{\text {eff }}$ of the system. Undersampling (insufficient neutrons per generation) or problems with convergence of the source distribution (insufficient generations or a very poor initial starting distribution) are not uncommon.

3. Only validated options and geometry types should be used for the criticality safety design of a system.

\section{EVALUATION OF BIAS IN THE KENO V.a VALIDATION}

\subsection{INTRODUCTION}

Several sources of bias were observed during the validation. Most of the validation cases were problems which were already modeled and which had been included in previous validations. A detailed study of all of the experiments included in this validation was not done. Instead, the experimental models were cursorily reviewed against the experimental descriptions in the reference documents. Obvious errors in the experimental models were corrected; otherwise, the models were left unchanged.

The bias, in a series of critical experiments, was studied if (1) the calculated $k_{\text {eff }}$ differed from 1.00 by more than about $1 \%$ for several of the experiments or, (2) the calculated $k_{\text {eff }}$ differed from 1.00 by more than about $2 \%$ for a single experiment. For the majority of the calculations, the standard deviation was on the order of \pm 0.004 . For a single calculation, it could be expected that about $5 \%$ of the time the calculated $k_{\text {eff }}$ would be more than $1 \%$ above or below 1.00 due to the statistics of the calculation. It would be highly unusual for the statistics to give a $2 \%$ bias. For any series of experiments, if more than $5 \%$ of the calculations exceed $1 \%$ deviation from 1.00 , it is an indication that a bias exists.

When bias was suspected, the experimental model was checked against the experimental description. If no discrepancies were observed, then a detailed review of the potential sources of bias was done. The results for the problems reviewed are given in this section. Some recommendations based on the findings of the review are included.

\subsection{DISCUSSION}

\subsubsection{Bias in the 3\% Enriched Green Block Calculations from Ref. 18}

Cases CAS21-CAS32 in Table 3 all show a positive bias of greater than 1.5\%. Review of the reference document did not show major discrepancies between the experimental model and the experimental description. Since the experimental logbook was available for review, a comparison of the logbook description versus the reported description of the experiments was made. It was noted that the reported critical dimensions were not measured dimensions but, rather, were based on nominal block dimensions 
with a correction factor applied for stacking voids. The correction factor was obtained from measurements of a very few stacked assemblies which resulted in a conversion factor for nominal size in inches to reported critical size in centimeters. In most cases, the nominal horizontal dimension was increased by $0.61 \%$, and the nominal vertical dimension was increased by $0.45 \%$, as reported in ORNL-CF-59-4-120. ${ }^{44}$ The effects of this approximation were investigated. The results indicated that the potential error in the experimental size did not account for the $1.5 \%$ bias in the $3 \%$ enriched experiments, and did not explain why the $2 \%$ enriched experiments (CAS11-CAS20 in Table 3) gave good results, whereas the $3 \%$ calculations were biased.

Case CAS21 was arbitrarily chosen for detailed examination. A 218-group calculation was performed with 120,000 neutron histories yielding a $k_{\text {eff }}=1.0012 \pm 0.0020$. The difference between the 27-group and 218-group calculations was examined by running XSDRN and collapsing the cross sections by flux weighting to a 27 -group structure. The infinite media multiplication factors calculated by the 27 - and 218 -group cross-section sets were 1.279 and 1.261 , respectively, or about $1-1 / 2 \%$ difference. Differences in the U-238 resonance region cross sections accounted for $-91 \%$ of the difference between the 27- and 218-group calculations. It was observed that the NITAWL treatment normally accounts for two admixed moderators, whereas, for these experiments, three admixed moderators were present, namely, H, F, and C. The control module was modified to input the second moderator to NITAWL as an effective moderator which approximated the effects of all of the moderating elements. This modification decreased the difference in calculated $k_{\text {eff }}$ between the 27-group and 218-group to about $0.6 \%$ with values of 1.260 and 1.252 , respectively. All of the calculations from ref. 18 were rerun with this modification. A comparison of the results using the standard and modified control

modules are given in Table 8. Based on these results, it is recommended that this modification or a comparable one be incorporated into the Y12CSG cross-section processing routines. This would change the random number sequence and would therefore necessitate a subsequent revalidation.

\subsubsection{Cross-Section Irregularities in the 27- and 218-Group Cross-Section Sets}

During the study of the $3 \%$ enriched green block experiments, a RADE ${ }^{45}$ calculation was performed for several elements in the 27- and 218-group cross-section sets. The thermal cross sections for hydrogen were flagged because the absorption cross section (MT-27) did not equal the sum of the neutron disappearance (MT-101) and total fission (MT-18) as it should. The difference was about $1 / 4 \%$ for the entire thermal range. It was determined that this was caused by a systematic error in XLACS2, which failed to account for the presence of oxygen when the thermal data for $\mathrm{H}_{2} \mathrm{O}$ was used for the generation of the 218 hydrogen cross-section set from ENDF/B-IV data. The exact impact on the hydrogen data has not as yet been determined. There is good agreement between the calculated $k_{\text {eff }}$ for hydrogen-moderated experiments. Therefore, it is not recommended that hydrogen (and all other elements which used thermal kernels based on compounds) be regenerated at this time. However, this should be considered as a potential source of bias in future validations.

The collapsing spectrum used to generate the 27 -group cross-section set from the 218 set was also studied. The carbon collapsing function appeared highly irregular with large peaks in the energy ranges of 5 and $500 \mathrm{eV}$. These peaks should not have occurred considering the Fission$1 / E^{*}$ SIGT-Maxwellian flux spectrum which was used to generate the data, as described in ref. 10. The irregular shape does not affect the total scatter or total absorption cross sections because of carbon's flat cross section, but instead affects the transfer matrix, and, in particular, the within-group scatter. It is not known if the unusual weighting function was caused by a systematic error in XLACS2, or if it was caused by incorrect input data for XLACS2 when the 218 carbon set was generated. A special 218 carbon set was generated using XLACS3 and ENDF/B-IV data, and did not exhibit these irregularities. There is some indication that the existing carbon cross-section set may not be adequate for systems where carbon is the major moderator. 
Table 8. Comparison of standard NITAWL treatment vs treating all moderators

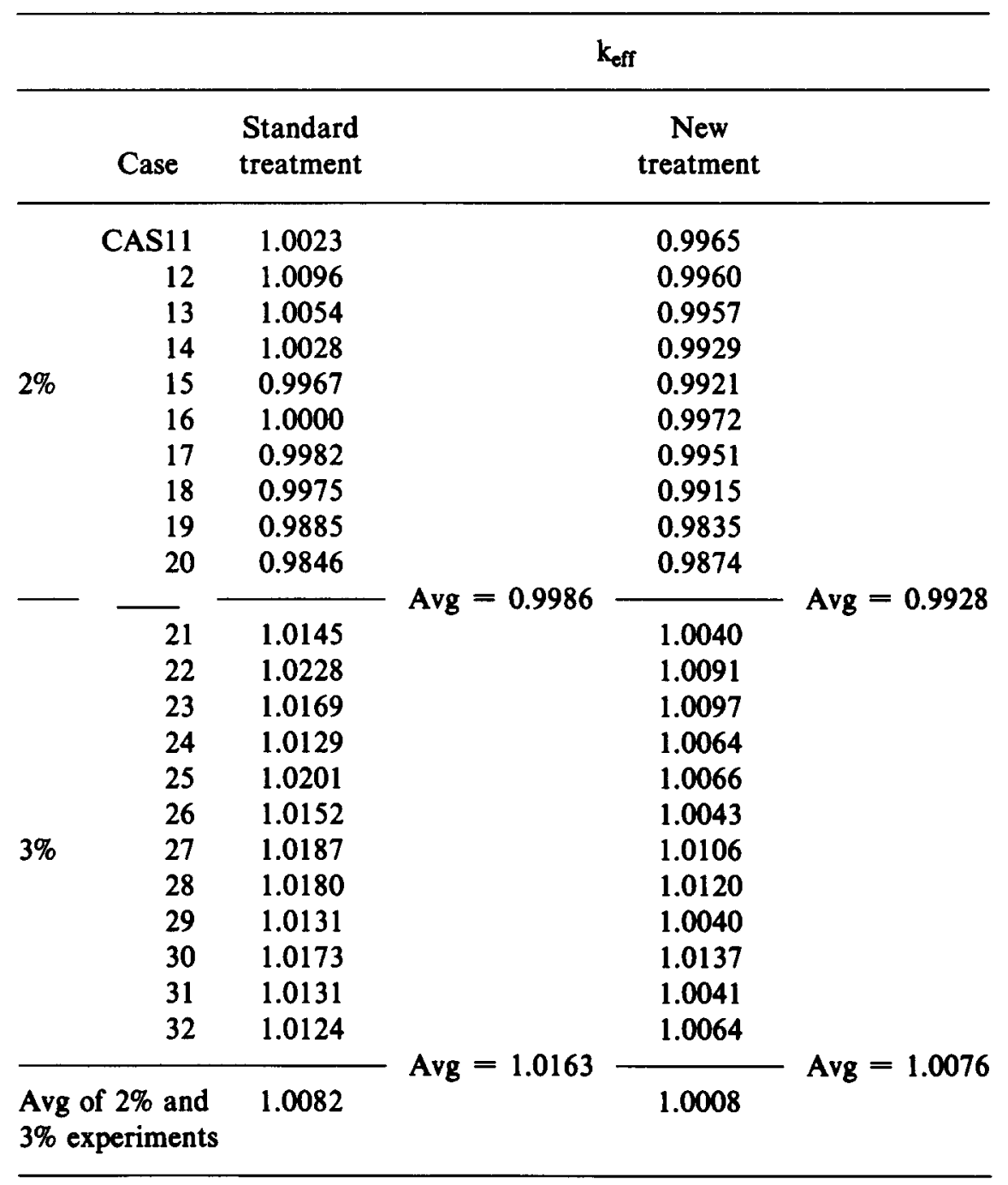


In general, it appears that the collapsing spectrum for generating the 27-group cross-section set from the 218 cross-section set is not adequate for certain classes of problems. This observation is based on the results of infinite media calculations where up to $7 \%$ difference in $k_{\text {eff }}$ was observed between the 27 - and 218-group calculations. The problem appears to occur for models of experiments which have fairly hard spectrums and contain moderators of intermediate mass. This includes intermediate and highly enriched $\mathrm{UF}_{4}-\mathrm{CF}_{2}$ systems and some low enriched $\mathrm{U}_{3} \mathrm{O}_{8}$ - sterotex systems. It should be noted that these observations are based on a study of a very few experiments (which were not included in the validation). The systems studied may not be adequate for inclusion in a validation report. It is recommended that the available experimental information for this class of systems be studied and included in future validations. If these observations are confirmed, there may be a need to incorporate a modification into the Y12CSG sequence to allow a problem-dependent collapse of the fine-group cross sections to a 27 -group set.

\subsubsection{Code Errors Encountered During Validation}

Case CAB09 from Table 2 failed in execution after 42 minutes. It was determined that the cause of failure was due to a divide check in subroutine CROS in KENO V.a. The divide check was traced to a problem with round-off error when a neutron path terminates at the end of a cylinder. This problem is similar to problems which have been previously encountered.

In one study case, differential albedos were used. It was found that turning on the print option PAX in the KENO parameter card caused a change in the random sequence. This problem was traced to an incorrect resetting of a pointer when the print option was triggered.

The correction for both of these problems is straightforward. The proposed corrections should not affect the random sequence on any of the validation runs except for CAB09. It is recommended that these corrections be made to Y12CSG KENO V.a. These corrections would not require a revalidation of the code.

\subsubsection{Bias in the 1F Experiments}

The complete series of experiments at $415 \mathrm{~g} \mathrm{U} / \mathrm{l}$, commonly referred to as the $1 \mathrm{~F}$ arrays, from ref. 38, were included in the validation as cases CAS60-CAS91 in Table 5. Five of these experiments had been included in several previous validations, ${ }^{5,6}$ whereas the complete set of experiments was first included in ref. 6. As a result, two different models of these five cases were included in this validation. Experiments which were included twice are listed in Table 9.

The primary modeling difference is that cases CAS13, CAS19, and CAS29 did not include the experimental room, whereas cases CAS91, CAS64, and CAS63 did. A best estimate of the room was included in the second modeling based on layout drawings of the experiment and descriptions of the experiments from personnel involved in the experiment. When room reflection was included in the model, a $1-1 / 2 \%$ bias for the unreflected cases was eliminated. The two reflected cases are models of the same experiment, except they were prepared by different analysts in a slightly different manner. It is recommended that cases CAS13, CAS16, CAS17, CAS18, and CAS29 be dropped from future validations since they are duplicates and are less accurately modeled.

The balance of the 1F array experiments shows a strong 1-1/2\% bias for the reflected cases. These experiments were studied in detail. ${ }^{46}$ The experimental logbook was available, and a comparison of the reported experiments versus the logbook description was done on a case-by-case basis. A number of details had been omitted from the experimental description in ref. 38. The most important of the details seem to be as follows. 
Table 9. Duplicate 1F array calculations

\begin{tabular}{ccccc}
\hline Case & $\mathrm{k}$ & $\begin{array}{c}\text { Corresponding } \\
\text { Case }\end{array}$ & $\mathrm{k}$ & Reflection \\
\hline CAS13 & 0.9854 & CAS91 & 1.0052 & $\mathrm{U}^{\mathrm{a}}$ \\
CAS16 & 0.9879 & CAS64 & 0.9963 & $\mathrm{U}$ \\
CAS17 & 1.0250 & CAS74 & 1.0172 & $\mathbf{R}^{\mathrm{b}}$ \\
CAS18 & 1.0010 & CAS72 & 1.0016 & $\mathrm{R}$ \\
CAS29 & 0.9872 & CAS63 & 1.0050 & $\mathrm{U}$ \\
\hline
\end{tabular}

${ }^{a} U$ - unreflected

${ }^{\mathbf{b}} \mathrm{R}$ - reflected 
1. The reported critical arrays are interpolations or extrapolations of the actual experiments. In some cases, a single experiment was used and then extrapolated to an ideal array (an array of identical units equally spaced in three dimensions).

2. Details of the reflector were omitted and only nominal compositions and thicknesses were reported for the experimental materials. In many instances the reflector contained streaming paths (holes) for which no description was given.

Based on the best information available, the experiments were remodeled. Only documentable information was incorporated into these refined models. The results of the calculations for the original and refined models are given in Table 10.

The average of the calculated $k_{\text {eff }}$ of the 28 experiments which were remodeled was $1 / 2 \%$ lower for the refined models when compared against the original models. This is considered significant with respect to the statistics of the calculations. The remaining bias is still unexplained.

In the logbook review, it was found that the experiment described in CAS74 was not run. For this experiment, five faces of the array were paraffin-reflected and one was Plexiglas-reflected. (There was insufficient paraffin available to fully reflect the $3 \times 3 \times 3$ array with a $15.24-\mathrm{cm}$ reflector.) It is recommended that CAS74 be dropped from future validations, since it is not a model of a critical experiment.

During this sequence of calculations several observations were made.

1. The automatic solution generator in the Y12CSG package caused a small (but noticeable) change in the uranium density and $\mathrm{H} / \mathrm{U}$ ratio in the nitrate solution. For some cases this caused as much as $1 / 2 \%$ change in the infinite media multiplication factor from XSDRN calculations when compared to results using hand-calculated atomic number densities. It is recommended that the automatic solution generation option not be used if precise detail is available.

2. A comparison was made of the calculated $k_{\text {eff }}$ for models in which a default weighting function of 0.5 was used in the reflector versus the use of reflector biasing. For these problems it was found that reflector biasing saved an average of $38 \%$ on the CPU time. No irregularities were observed between the full tracking versus "reflector biased" calculations except when paraffin weighting functions were used for thick Plexiglas reflectors. Based on an inspection of adjoint calculations for Plexiglas (as a reflector), it was found that the weighting function for Plexiglas is more like water than paraffin. It is recommended that the paraffin reflector bias not be routinely used for Plexiglas. Plexiglas reflector bias weights may need to be incorporated into the Y12CSG package.

3. It appears that these arrays may be susceptible to undersampling because a different starting random number seed changed the results of several calculations by more than three standard deviations. There was no other apparent indication of undersampling. A neutron batch size of 600 may not be sufficient to hold a good neutron starting distribution for these arrays.

4. A definite trend in $\mathrm{k}_{\text {eff }}$ in the refined models was observed as the reflector thickness increased. This may be an indication that some important parameter was omitted from the experimental description and/or model. The possibility of poor cross sections or a problem in code physics exists also. At present, the calculated $k_{\text {eff }}$ for this series of experiments is considered biased an average of 1 to $1-1 / 2 \%$ high.

\subsubsection{Bias in the Slab Experiments from Ref. 43}

Cases CAS39-CAS50 of Table 5 are experiments involving 3- and 6-in.-thick slabs of $\mathrm{UO}_{2} \mathrm{~F}_{2}$ solution at $81.8 \mathrm{~g} \mathrm{U} / \mathrm{l}$. Six of these experiments appeared in Table 5 of ref. 6 and showed no bias. The balance appeared in Table 6 of ref. 6 and showed a strong 2\% negative bias but were flagged as "neglecting external scatter that may be significant." A model of a large tank (Big Sid) had been 
Table 10. Comparison of calculated $k_{\text {eff }}$ for the $1 F$ arrays

\begin{tabular}{|c|c|c|c|}
\hline $\begin{array}{l}\text { Reflection } \\
\text { conditions }\end{array}$ & $\begin{array}{l}\text { Number } \\
\text { of units }\end{array}$ & $\begin{array}{c}\text { Original } \\
\text { model }\end{array}$ & $\begin{array}{l}\text { Refined } \\
\text { model }\end{array}$ \\
\hline \multirow[t]{5}{*}{ Unreflected } & (8) & 1.0081 & 1.0039 \\
\hline & (27) & 1.0050 & 0.9940 \\
\hline & (64) & 0.9963 & 0.9969 \\
\hline & $(125)$ & 0.9934 & 0.9983 \\
\hline & & 1.0007 & 0.9983 \\
\hline \multirow[t]{3}{*}{ 1.27 paraffin reflected } & (27) & 1.0134 & 1.0000 \\
\hline & $(8)$ & 1.0165 & 1.0084 \\
\hline & & 1.0149 & 1.0042 \\
\hline \multirow[t]{3}{*}{1.27 plex reflected } & (27) & 1.0016 & 1.0048 \\
\hline & (8) & 1.0175 & 1.0048 \\
\hline & & 1.0095 & 1.0048 \\
\hline \multirow[t]{3}{*}{3.81 paraffin reflected } & (27) & 1.0300 & 1.0249 \\
\hline & (8) & 1.0177 & 1.0187 \\
\hline & & 1.0239 & 1.0218 \\
\hline \multirow[t]{3}{*}{7.62 paraffin reflected } & (27) & 1.0302 & 1.0311 \\
\hline & $(8)$ & 1.0169 & 1.0186 \\
\hline & & 1.0236 & 1.0249 \\
\hline \multirow[t]{3}{*}{ 15.24 paraffin reflected } & $(8)$ & 1.0177 & 1.0110 \\
\hline & (27) & 1.0117 & 1.0160 \\
\hline & & 1.0202 & 1.0135 \\
\hline
\end{tabular}

15.24-cm bottom reflector, 5 remaining sides uniform reflector

\begin{tabular}{lrrr}
1.27 paraffin reflected & $(27)$ & 1.0170 & 1.0020 \\
& $(8)$ & 1.0195 & 1.0024 \\
\cline { 2 - 3 } 1.28 plex reflected & $(8)$ & 1.0183 & 1.0022 \\
& $(27)$ & 1.0046 & 1.0023 \\
\cline { 2 - 3 } & & 1.0033 & 1.0091 \\
2.54 plex reflected & $(8)$ & 1.0137 & 1.0030 \\
& $(27)$ & 1.0217 & 1.0166 \\
\hline 3.81 paraffin reflected & $(27)$ & 1.0205 & 1.0202 \\
& $(8)$ & 1.0225 & 1.0178 \\
\hline & & 1.0215 & 1.019 \\
4.45 plex reflected & $(8)$ & 1.0223 & 1.0146 \\
6.35 plex reflected & $(8)$ & 1.0197 & 1.0167 \\
7.62 paraffin reflected & $(8)$ & 1.0247 & 1.0147 \\
& $(27)$ & 1.0302 & 1.0286 \\
\hline & & 1.0275 & 1.0217 \\
11.43 plex reflected & $(8)$ & 1.0225 & 1.0179 \\
15.26 plex reflected & $(8)$ & 1.0237 & 1.0220
\end{tabular}


included in the experimental model for the cases reported in Table 5 of ref. 6. Reference 43 was carefully reviewed, and a model of the experimental room and tank were incorporated as appropriate into all of the models. The room was based on a layout drawing of the experimental facility with a "best estimate" of the actual location of the slabs in the room.

The new models substantially improved the calculations for the experiments done inside Big Sid; however, the experiments done outside of Big Sid still showed a $1-1 / 2 \%$ bias. The experimental logbooks were available and reviewed against the reported experiments. Two items of potential significance were observed:

1. There was no detailed description of the tank support structures including how far from the floor the tanks were positioned during the experiments. There was also no description of a control blade structure which was suspended above at least one of the tanks during all of the experiments.

2. There were fragmented notes made at the time of the experiment, and on two later dates, expressing a concern with the accuracy of the uranium concentration analysis and the possibility that evaporation may have increased the concentration. The experiments were reported as being run at an $H / X=337$; notes in the logbook indicated that the solution concentration was between $H / X=333$ and $\mathrm{H} / \mathrm{X}=335$ at the end of the experiments.

Table 11 shows a comparison of the original model versus the refined model for these calculations. In the refined models the tanks were 12 in. above the floor (a best estimate based on discussion with personnel involved in the experiments). All calculations included a room model and were at an $H / X=337$. The experiments are reported in the order in which they were performed. All of the experiments done inside Big Sid calculate well. However, all of the experiments done outside of Big Sid still show a bias. Based on the results in Table 11, it appears that the bias in the calculations was either a function of the time at which the experiments were performed or the location at which they were done. The first case could indicate a potential problem with solution concentration; the second would indicate inadequacies in the experimental model. One of the personnel involved in these experiments was questioned as to the possibility of evaporation and an incorrectly reported $\mathrm{H} / \mathrm{X}$ ratio. It was commented that at the time the experiments were performed, the accuracy of a concentration analysis was usually no better than $1 \%$ and that the experiments were reported as "preliminary" because the experimentalists had problems with some of the results. It was suggested that the eta experiments performed some years later be modeled and calculated.

Several infinite media calculations were done using the XSDRN code to study the impact of an incorrect $\mathrm{H} / \mathrm{X}$ ratio for this solution. The results indicate that the $1-1 / 2 \%$ to $2 \%$ bias observed in the experiments done outside Big Sid could have been caused because of an incorrect concentration used in the calculations; however, insufficient evidence is available to discount the possibility of modeling bias.

\subsubsection{Bias Caused by the Analyst's Model}

When modeling critical experiments, the analyst must decide how much detail to incorporate into his/her model, and, in some cases, how to approximate the various details of the experiment which cannot be modeled explicitly. The amount of detail which is modeled is usually a function of the cost, either time or money, required to include the detail. Many times an analyst calls on past experience in determining what is important and what may be omitted from a model without significantly affecting the results. It was found that cases CAS20 and CAS21 from Table 5 were repeats of Cases CAA30 and CAA38 from Table 4 . The calculated $k_{\text {eff }}$ for these cases was $1.03654,1.0239,0.9961$, and 1.0061 , respectively. The discrepancies were briefly reviewed. It was found that the cases had been set up by two different analysts at different times. Each experimental model was imprecise in that certain details of the experiment were omitted. It was observed that the major difference between CAS20 and 
Table 11. Comparison of calculated $k_{\text {eff }}$ for the slab experiments

\begin{tabular}{|c|c|c|c|}
\hline \multicolumn{4}{|c|}{$\mathrm{k}_{\mathrm{eff}}$} \\
\hline Case & Comments & $\begin{array}{c}\text { Original } \\
\text { model }\end{array}$ & $\begin{array}{c}\text { Refined } \\
\text { model }\end{array}$ \\
\hline CAS30 & The first 15 experiments & 0.9978 & 0.9950 \\
\hline CAS52 & were performed inside & 0.9852 & 0.9901 \\
\hline CAS49 & Big Sid and took ten & 0.9831 & 0.9980 \\
\hline CAS50 & days from start to & 0.9814 & 0.9941 \\
\hline CAS51 & completion & 0.9791 & 0.9919 \\
\hline CAS40 & & 0.9824 & 0.9868 \\
\hline CAS34 & & 0.9803 & 0.9878 \\
\hline CAS35 & & 0.9906 & 0.9906 \\
\hline CAS36 & & 0.9933 & 0.9918 \\
\hline CAS37 & & 1.0030 & 1.0030 \\
\hline CAS38 & & 0.9881 & 0.9913 \\
\hline CAS39 & & 0.9952 & 0.9952 \\
\hline CAS32 & & 0.9805 & 0.9921 \\
\hline CAS33 & & 0.9995 & 0.9995 \\
\hline CAS31 & & 0.9949 & 0.9949 \\
\hline & \multicolumn{3}{|c|}{21 days of related experimenting with same solution } \\
\hline$\overline{\text { CAS53 }}$ & The last 15 experiments & 0.9884 & 0.9834 \\
\hline CAS41 & were performed outside of & 0.9767 & 0.9821 \\
\hline CAS55 & Big Sid and took ten days & 0.9876 & 0.9923 \\
\hline CAS56 & from start to completion & 0.9869 & 0.9847 \\
\hline CAS43 & & 0.9835 & 0.9803 \\
\hline CAS57 & & 0.9864 & 0.9954 \\
\hline CAS44 & & 0.9757 & 0.9803 \\
\hline CAS58 & & 0.9824 & 0.9793 \\
\hline CAS42 & & 0.9916 & 0.9825 \\
\hline CAS54 & & 0.9831 & 0.9859 \\
\hline CAS59 & & 0.9836 & 0.9852 \\
\hline CAS47 & & 0.9819 & 0.9875 \\
\hline CAS46 & & 0.9832 & 0.9729 \\
\hline CAS45 & & 0.9871 & 0.9946 \\
\hline CAS48 & & 0.9813 & 0.9858 \\
\hline
\end{tabular}


CAA30 was that the analyst who set up CAA30 included fire-retardent Plexiglas in the modeling of the upper and lower reflector, whereas the analyst who set up CAS20 used regular Plexiglas for the entire reflector. The result was a $3-1 / 2 \%$ positive bias for CAS20. In CAS21, the analyst chose to ignore a $1.25-\mathrm{cm}$ gap at the junction of the upper and lower reflectors. This omission accounted for approximately $70 \%$ of the difference between cases CAS21 and CAA30. Based on these observations, it is recommended that CAS20 and CAS21 be dropped from future validations, and that cases CAA12-CAA43 be reviewed to ensure that sufficient detail has been incorporated into the models. These two cases highlight the importance of accurately modeling reflector compositons and streaming paths in the reflector.

Case CAS14 from Table 5 calculated a $k_{\text {efr }}$ of 1.0585 . Reference 39 was reviewed, and it was found that several details of the experiment had been omitted from the model. These included the omission of two steel lateral support plates and a detailed model of the stainless-steel slab tank. The model was refined, and the calculated $\mathbf{k}_{\text {eff }}$ dropped to 1.03 . The $3 \%$ positive bias has not been accounted for. Reference 39 documents more than 100 critical experiments. Many of the experiments are similar to the $1 \mathrm{~F}$ array experiments which show a positive bias. Based on this, it is recommended that several more experiments from ref. 39 be included in future validations in order to better establish the bias for these experiments. If possible, additional information beyond what was reported in ref. 39 (such as the experimental logbook and experimentalists' notes) should be reviewed. There is an indication from this experiment (and several others which contain Plexiglas) that the chemical and/or impurity composition of Plexiglas has not been adequately reported. Trace impurities in paraffin or Plexiglas seem to have a strong effect on the calculated $k_{\text {eff. }}$ (There is some evidence that high leakage systems, such as arrays, which are reflected by thin reflectors, are biased. This may indicate code or cross-section problems.)

Evidence of analyst bias was also observed in cases CAA08, CAA10, and CAA11 from Table 4. The analyst used a fissile isotope density which was tabulated at $25^{\circ} \mathrm{C}$ in ref. 27 , whereas the experiments were actually done at different temperatures and had different fissile isotope densities. Instead of simply remodeling only the four incorrect experiments, all of the experiments documented in ref. 27 were modeled and run. For these cases, the radius of the "effective" sphere was calculated based on the reported critical volume (since there appeared to be only slight variation due to temperature). The fissile isotope density was based on the reported critical mass and volume. (It was noted that all of the reported masses for experiments performed in the 32-cm-diam tank were flagged as being $2 \%$ high; however, the reported masses used.) The concentration and masses were high because a layer of plastic paint used to protect the inside of the tank was not taken into account in the volume measurements. This plastic was not modeled in these calculations. The calculations were done using the modified control module which treats all of the admixed moderators. The results are listed in Table 12.

The results indicate that the U-233 experiments are biased $-2-1 / 2 \%$ high. The U-235 experiments in the 26.4-cm-diam sphere appear to be unbiased, but the experiments in the $32-\mathrm{cm}$-diam sphere appear to be biased $1-1 / 2$ to $2 \%$ high.

Based on these results, it is recommended that cases CAA08-CAA11 be refined or be omitted from future validations. The cause of the bias in the results in Table 12 is not known at this time. If the plastic paint used on the interior surface contained impurities such as chlorine or boron, then omission from the model could explain the results.

\subsubsection{Bias in CAS19, Table 5}

Case CAS19 from Table 5 showed a 3.29\% positive bias. The experimental model for the case was compared against the reference document. It was found that the model contained more information than was reported in ref. 41. These arrays have been calculated many times with earlier versions of KENO and the 16-group cross-section set. ${ }^{47,48}$ The input for the earlier calculations was compared. It was found that the automatic solution generator option had been used for the Y12CSG validation, whereas the atomic number densities had been hand calculated and input for previous validations. The 
Table $12 . \quad \mathrm{UO}_{2} \mathrm{~F}_{2}$ solution spheres

\begin{tabular}{|c|c|c|c|c|}
\hline $\mathrm{H} / \mathrm{X}$ & $\begin{array}{c}\text { Temp. } \\
{ }^{\circ} \mathrm{C}\end{array}$ & $\operatorname{gm~X/1}$ & Calculated k & $\begin{array}{c}\text { Original } \\
\text { calculation }\end{array}$ \\
\hline \multicolumn{5}{|c|}{$\mathrm{U}-233 / 26.4-\mathrm{cm}$ diam $/ \mathrm{H}_{2} \mathrm{O}$ reflected } \\
\hline 418.3 & 36.0 & 61.14 & 1.0149 & \\
\hline 414.6 & 39.5 & 61.63 & 1.0287 & \\
\hline 400.5 & 65.5 & 62.96 & 1.0250 & \\
\hline 389.6 & 83.2 & 64.07 & 1.0088 & \\
\hline 378.1 & 96.5 & 65.52 & 1.0231 & \\
\hline \multicolumn{5}{|c|}{$\mathrm{U}-233 / 32.0-\mathrm{cm} \operatorname{diam} / \mathrm{H}_{2} \mathrm{O}$ reflected } \\
\hline 663.1 & 26.3 & 38.72 & 1.0268 & \\
\hline 643.1 & 56.0 & 39.49 & 1.0261 & \\
\hline 602.8 & 99.5 & 41.17 & 1.0411 & \\
\hline \multicolumn{5}{|c|}{ U-233/32-cm diam/unreflected } \\
\hline 381.0 & 27.0 & 67.33 & 1.0251 & \\
\hline \multicolumn{5}{|c|}{$\mathrm{U}-235 / 26.4-\mathrm{cm} \operatorname{diam} / \mathrm{H}_{2} \mathrm{O}$ reflected } \\
\hline 268.8 & 27.5 & 95.05 & 1.0028 & \\
\hline 253.3 & 39.5 & 96.67 & 0.9999 & \\
\hline 245.4 & 74.0 & 101.90 & 1.0191 & \\
\hline 239.3 & 85.5 & 103.86 & 1.0055 & CAA10, $\mathrm{k}=1.0164$ \\
\hline \multicolumn{5}{|c|}{$\mathrm{U}-235 / 32.0-\mathrm{cm}$ diam $/ \mathrm{H}_{2} \mathrm{O}$ reflected } \\
\hline 515.1 & 27.0 & 50.28 & 1.0045 & $\mathrm{CAA} 08, \mathrm{k}=1.0212$ \\
\hline 502.6 & 43.0 & 51.20 & 1.0173 & \\
\hline 503.4 & 43.0 & 51.13 & 1.0094 & \\
\hline 496.5 & 54.0 & 51.59 & 1.0131 & \\
\hline 487.6 & 64.5 & 52.22 & 1.0218 & \\
\hline 476.8 & 87.5 & 52.72 & 1.0190 & (averaged) CAA11, $\mathrm{k}=1.0351$ \\
\hline 459.6 & 87.5 & 54.61 & 1.0220 & \\
\hline \multicolumn{5}{|c|}{$\mathrm{U}-235 / 32.0-\mathrm{cm}$ diam/unreflected } \\
\hline 203.5 & 27.0 & 125.08 & 1.0078 & CAA09, $k=0.9991$ \\
\hline
\end{tabular}


difference was an $0.6 \%$ increase in the uranium density and an $8 \%$ increase in the $\mathrm{H} / \mathrm{X}$ ratio for the number densities calculated by the automatic solution generator. It was also found that the composition for stainless steel 304 differed from what the code uses in standard composition SS304.

The KENO output indicated a potential source convergence and/or undersampling problem in that the average value of $\mathrm{k}_{\text {eff }}$ was 1.0198 , where the calculated $\mathrm{k}$ with the lowest standard deviation was 1.0329. The plot of $k_{\text {eff }}$ by generation skipped showed an unusual trend. The plot of average $k_{\text {eff }}$ indicated that the source distribution had not converged after more than 40 generations, and convergence was from low $k_{\text {eff }}$ toward the final value. It appears that calculations which approach the final value from below tend to have more difficulty converging the source than those which approach the final value from above. The calculation was rerun using the atomic number densities used in previous validations. In addition, a cosine starting distribution was used over a section at the central portion of the column. The calculated $\mathrm{k}$ was 1.0201 . Several of the pipe intersection experiments should be included in future validations in order to establish the bias for this type of system.

\section{GENERAL COMMENTS ON THE EXPERIMENTS REVIEWED}

Based on the study of the experiments which showed some evidence of bias, several observations may be made. The most significant is that a large proportion of the experiments which have been included in this validation and in previous validations should be scrutinized to determine their adequacy for use in validating codes. There is a surprising lack of detail in the descriptions of the experiments, including estimates of the accuracy of concentration in the fissile mixture, the actual dimensions and composition of the materials involved in the experiment and the experimental layout itself. Details such as the description of the experimental room and the location of the experiment in the room have been omitted from most reports. For some systems, these details are extremely important; and their inclusion or omission may bias the calculated results. In some of the experiments studied, the uncertainty in the experimental parameters could easily bias calculated results $2 \%$ high or low, but the uncertainties were not reported. This deficiency indicates a need for a set of experiments which has been carefully evaluated specifically for validation purposes such that reporting bias is eliminated (or at least is well known).

In some instances, the modeling of the critical experiment was found to be poor and biased the results by more than $2 \%$. This again shows a lack of attention to detail. In other cases, the models which were set up were incorrect. These deficiencies indicate a need to verify the accuracy of the models used for validation. Reporting bias and/or modeling bias tend to mask potential problems with the code or cross sections being used. Since these forms of bias are not systematic, the inclusion of these results in any trend analysis should be avoided in order to eliminate the possibility of artificial trends. Extrapolation and/or interpolation of critical experiment information based on artificial trends may not be correct.

In order for the codes and cross sections used for criticality safety to be improved, validation efforts should continue. The cause of the bias in the calculations should be determined and eliminated if possible. 


\section{REFERENCES}

1. L. M. Petrie and N. F. Landers, "KENO V.a An Improved Monte Carlo Criticality Program With Supergrouping," SCALE: A Modular Code System for Performing Standardized Computer Analyses for Licensing Evaluation, Section F11, NUREG/CR-0200, Vol. 2, Rev. 2, U.S. Nuclear Regulatory Commission, June 1983.

2. Staff of Technical Applications, Computing and Telecommunications Division at ORNL SCALE: A Modular Code System for Performing Standardized Computer Analyses for Licensing Evaluation, Vols. 1-3, NUREG/CR-0200, U.S. Nuclear Regulatory Commission (originally issued July 1980, reissued January 1982, Revision 1 issued July 1982, Revision 2 issued June 1983, Revision 3 issued December 1984).

3. Nuclear Criticality Safety in Operations with Fissionable Material Outside Reactors, ANSI/ANS-8.1, American Natl. Standards Institute, New York, 1983.

4. G. R. Handley and C. M. Hopper, Validation of the KENO Code for Nuclear Criticality Safety Calculations of Moderated Low-Enriched Uranium Systems, Y-1948, Union Carbide Corporation, Nuclear Division, Oak Ridge Y-12 Plant, 1974.

5. G. R. Handley et al., Validation of the Monte Carlo Criticality Program KENO IV and the Hansen-Roach Sixteen-Energy-Group Cross Sections for High Assay Uranium Systems, Y-2234, Union Carbide Corporation, Nuclear Division, Oak Ridge Y-12 Plant, 1981.

6. J. R. Knight, Validation of the Monte Carlo Criticality Program KENO V.a for Highly Enriched Uranium Systems, ORNL/CSD/TM-221, Martin Marietta Energy Systems, Inc., Oak Ridge National Laboratory, 1984.

7. M. E. Easter, Validation of KENO V.a and Two Cross-Section Libraries for Criticality Calculations of Low-Enriched Uranium Systems, ORNL/CSD/T-223, K/HS-74, Martin Marietta Energy Systems, Inc., Oak Ridge National Laboratory, 1985.

8. L. M. Petrie and J. T. Thomas, Assessment of Computational Performance in Nuclear Criticality, ORNL/CSD/TM-224, Martin Marietta Energy Systems, Inc., Oak Ridge National Laboratory, 1985.

9. L. M. Petrie and N. F. Cross, KENO IV An Improved Monte Carlo Criticality Program, ORNL4938, Union Carbide Corporation, Nuclear Division, Oak Ridge National Laboratory, 1975.

10. CSRL - A 218-Group Neutron Cross-Section Reference Library in the AMPX Master Interface Format for Criticality Safety Studies, DLC-49, Radiation Shielding Information Center Data Library Collection, Union Carbide Corporation, Nuclear Division, Oak Ridge National Laboratory, 1978.

11. Davis Reed, Validation of KENO V.a for Critical Lattices of Low Enrichment (3.85\% U-235) Uranium Metal Cylinders and Annuli in Water, to be published at Oak Ridge National Laboratory.

12. R. Gwin and D. W. Magnuson, Critical Experiments for Reactor Physics Studies, ORNL/CF-60-4-12, Union Carbide Corporation, Nuclear Division, Oak Ridge National Laboratory, 1960.

13. R. Gwin and D. W. Magnuson, "The Measurement of Eta and Other Nuclear Properties of U-233 and U-235 in Critical Aqueous Solutions, Nucl. Sci. Eng. 12, 364-380 (1962).

14. E. B. Johnson, The Criticality of Large Uranium Metal Units of Low Enrichment, to be published at Oak Ridge National Laboratory. 
15. D. F. Cronin, Critical Mass Studies, Part $X$, ORNL-2968, Union Carbide Nuclear Corporation, Oak Ridge National Laboratory, 1960.

16. E. B. Johnson, The Criticality of Large Uranium Metal Units of Low Enrichment in U-235, to be published at Oak Ridge National Laboratory.

17. F. Abbey, comp, Handbook of Experimental Criticality Data, AHSB(s) Handbook 5, 1968.

18. S. J. Raffety and J. T. Mihalczo, Homogenized Critical Assemblies of 2 and 3\% Enriched Uranium in Paraffin, Y-DR-14, Union Carbide Corporation, Nuclear Division, Oak Ridge Y-12 Plant, 1969.

19. E. B. Johnson, "Effect of Steel-Water Reflectors on the Criticality of Low Enriched Uranyl Fluoride Solution," p. 47 in Neutron Physics Division Annual Progress Report for Period Ending May 31, 1968, ORNL-4280, Union Carbide Corporation, Nuclear Division, Oak Ridge National Laboratory, 1968.

20. E. B. Johnson, Criticality of a Sphere of $U(4.98) 0_{2} F_{2}$ Solution, ORNL-3973, Union Carbide Corporation, Nuclear Division, Oak Ridge National Laboratory, 1966, p. 14.

21. J. W. Webster and E. B. Johnson, Criticality of a Single Unit of Aqueous Uranyl Fluoride Solution Enriched to 5\% in U-235, ORNL-TM-1195, Union Carbide Corporation, Nuclear Division, Oak Ridge National Laboratory, 1965.

22. R. E. Rothe et al., Critical Experiments with Interstitially Moderated Arrays of Low Enriched Uranium Oxide, NUREG/CR-1071, RFP-3008, U.S. Nuclear Regulatory Commission, 1980.

23. G. Tuck and I. Oh, Benchmark Critical Experiments on Low-Enriched Uranium Oxide Systems With $H / U=0.77$, NUREG/CR-0674, U.S. Nuclear Regulatory Commission, 1979.

24. G. R. Goebel et al., Critical Experiments on Low-Enriched Uranium Oxide Systems With $H / U=$ 1.25, NUREG/CR-1653, RFP-3129, U.S. Nuclear Regulatory Commission, 1980.

25. R. E. Rothe and G. R. Goebel, Critical Experiments on Low-Enriched Uranium Oxide Systems With $H / U=2.03$, NUREG/CR-2500, RFP-3277, U.S. Nuclear Regulatory Commission, 1982.

26. J. K. Fox, "Critical Parameters of Uranium Solutions in Simple Geometry," p. 42 in Neutron Physics Division Annual Progress Report for Period Ending September 1, 1958, ORNL-2609, Union Carbide Corporation, Nuclear Division, Oak Ridge National Laboratory, 1958.

27. J. T. Thomas et al., "A Direct Comparison of Some Nuclear Properties of U-233 and U-235," Nucl. Sci. Eng. 1, 20 and 32 (1956).

28. R. E. Rothe, Benchmark Critical Experiments on High Enriched Uranyl Nitrate Solution Systems, NUREG/CR-0041, RFP-2710, U.S. Nuclear Regulatory Commission, 1978.

29. G. E. Hansen, "Properties of Elementary Fast Neutron Critical Assemblies," p. 84 in Proceedings of the Second United Nations International Conference on Peaceful Uses of Atomic Energy, Vol. 12, Geneva, 1958.

30. J. T. Mihalczo and W. E. Kinney, Trans. Am. Nucl. Soc. 4, 302 (1961).

31. R. Gwin and D. W. Magnuson, Nucl. Sci. Eng. 12, 364 (1962).

32. H. C. Paxton, Los Alamos Critical Mass Data, LAMS-3067, Los Alamos National Laboratory, Los Alamos, New Mexico, 1964.

33. J. T. Mihalczo, Trans. Am. Nucl. Soc. 8, 442 (1965).

34. G. Tuck, "Critical Masses of Spherical and Hemispherical Enriched Uranium Assemblies," J. Nucl. Energy 23, 663 (1969). 
35. C. C. Byers, J. J. Koelling, G. E. Hansen, D. R. Smith, and H. R. Dyer, "Critical Measurements of a Water-Reflected Enriched Uranium Sphere," Trans. Am. Nucl. Soc. 27, 412 (1977).

36. D. C. Irving and J. T. Mihalczo, Trans. Am. Nucl. Soc. 7, 287 (1964).

37. J. T. Thomas, Critical Three-Dimensional Arrays of Neutron-Interacting Units, Part II - U(93.2) Metal, ORNL-TM-868, Union Carbide Corporation,Nuclear Division, Oak Ridge National Laboratory, 1964.

38. J. T. Thomas, Critical Three-Dimensional Arrays of Neutron-Interacting Units, ORNL-TM-719, Union Carbide Corporation, Nuclear Division, Oak Ridge National Laboratory, 1963.

39. G. Tuck and H. E. Clark, "Critical Parameters of a Uranium Solution Slab-Cylinder System," Nucl. Sci. Eng. 40, 407-413 (1970).

40. E. C. Crume and J. T. Thomas, Critical and Near Critical Graphite Moderated Arrays of U(93.2) Cylinders, Y-DD-32, Union Carbide Corporation, Nuclear Division, Oak Ridge Y-12 Plant, 1969.

41. B. B. Ernst and C. L. Schuske, Empirical Method for Calculating Pipe Intersections Containing Fissile Solution, RFP-1197, Rockwell International, Rocky Flats, Golden, Colorado, 1968.

42. D. Dickinson and C. L. Schuske, "An Empirical Model for Safe Pipe Interactions Containing Fissile Solution," Nucl. Tech. 10, 179-187 (1971).

43. J. K. Fox and L. W. Gilley, Preliminary Report of Critical Experiments in Slab Geometry, ORNL/CF-56-7-148, Union Carbide Corporation, Nuclear Division, Oak Ridge National Laboratory, 1956.

44. J. T. Mihalczo et al., Preliminary Report on $2 \%$ U-235 Enriched $\mathrm{UF}_{4}-\mathrm{C}_{25} \mathrm{H}_{52}$ Critical Assemblies, ORNL/CF-59-4-120, Union Carbide Corporation, Nuclear Division, Oak Ridge National Laboratory, 1959.

45. AMPX II: Modular Code System for Generating Coupled Multigroup Neutron-Gamma-Ray Cross-Section Libraries from Data in ENDF Format, PSR-63, Union Carbide Corporation, Nuclear Division, Oak Ridge National Laboratory, 1978.

46. W. C. Jordan, Logbook Calculations of the $415 \mathrm{gm} \mathrm{U/l} \mathrm{UO}_{2}\left(\mathrm{NO}_{3}\right)_{2}$ sl Arrays, unpublished data, July $17,1985$.

47. D. Dickinson, Nominally Reflected Pipe Intersections Containing Fissile Solution, unpublished data, July 1986.

48. N. F. Landers et al., "Monte Carlo Analysis of Experimentally Critical Pipe Intersections," Trans. Am. Nucl. Soc. 17, 268 (1973). 


\section{-}




\section{Appendix A}

\section{Y12CSG INPUT DATA USED FOR VALIDATION CASES}

Input data for the validation appears in the same order as listed in Tables 1-6 of Sect. 1 of this report.

Table A.1. Table 1 input data A-2

Table A.2. Table 2 input data

A-14

Table A.3. Table 3 input data

A-37

Table A.4. Table 4 input data

A-98

Table A.5. Table 5 input data

A-108

Table A.6. Table 6 input data

A-133 
Table A.1. Table 1 input data
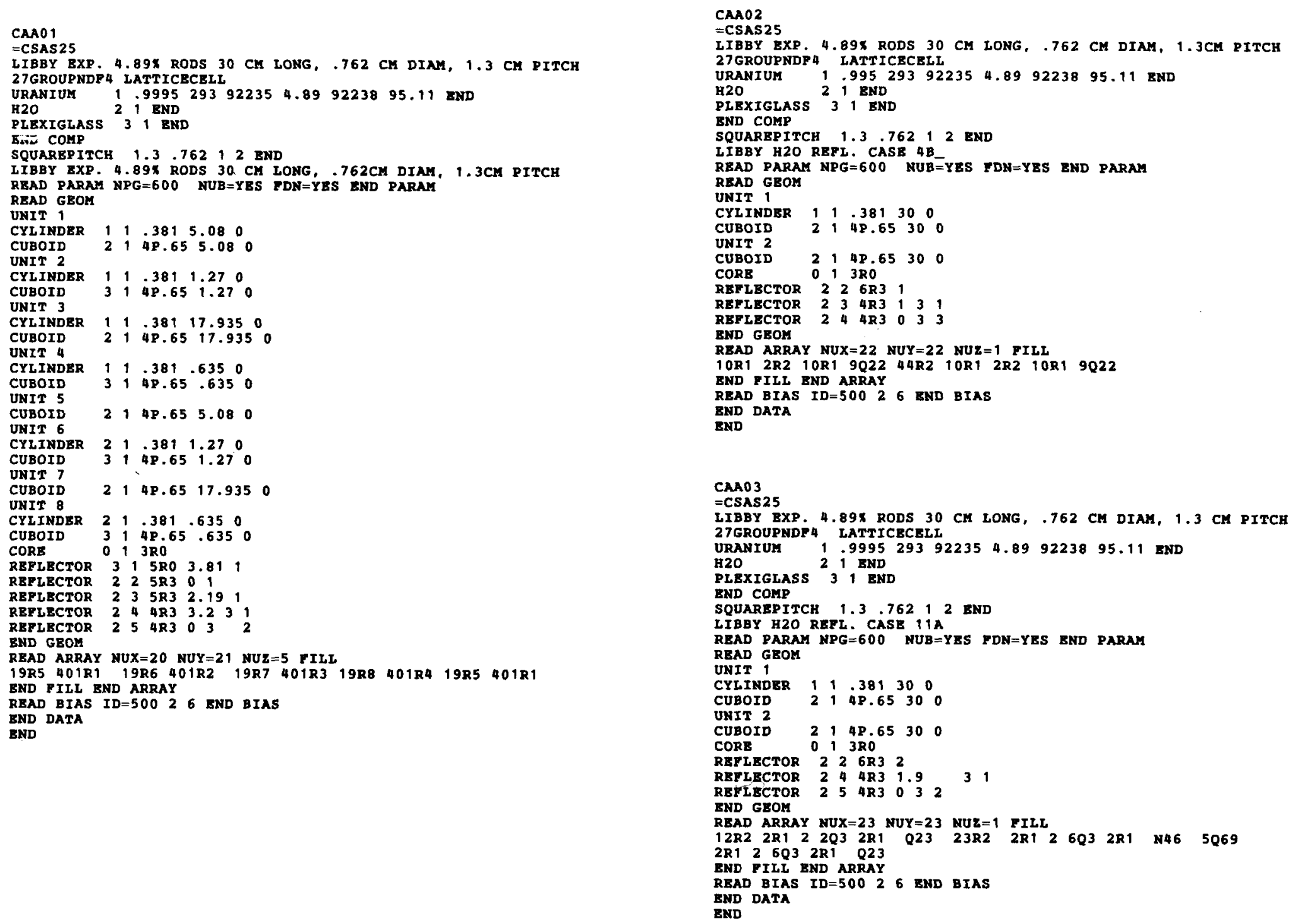
Table A.1 (continued)

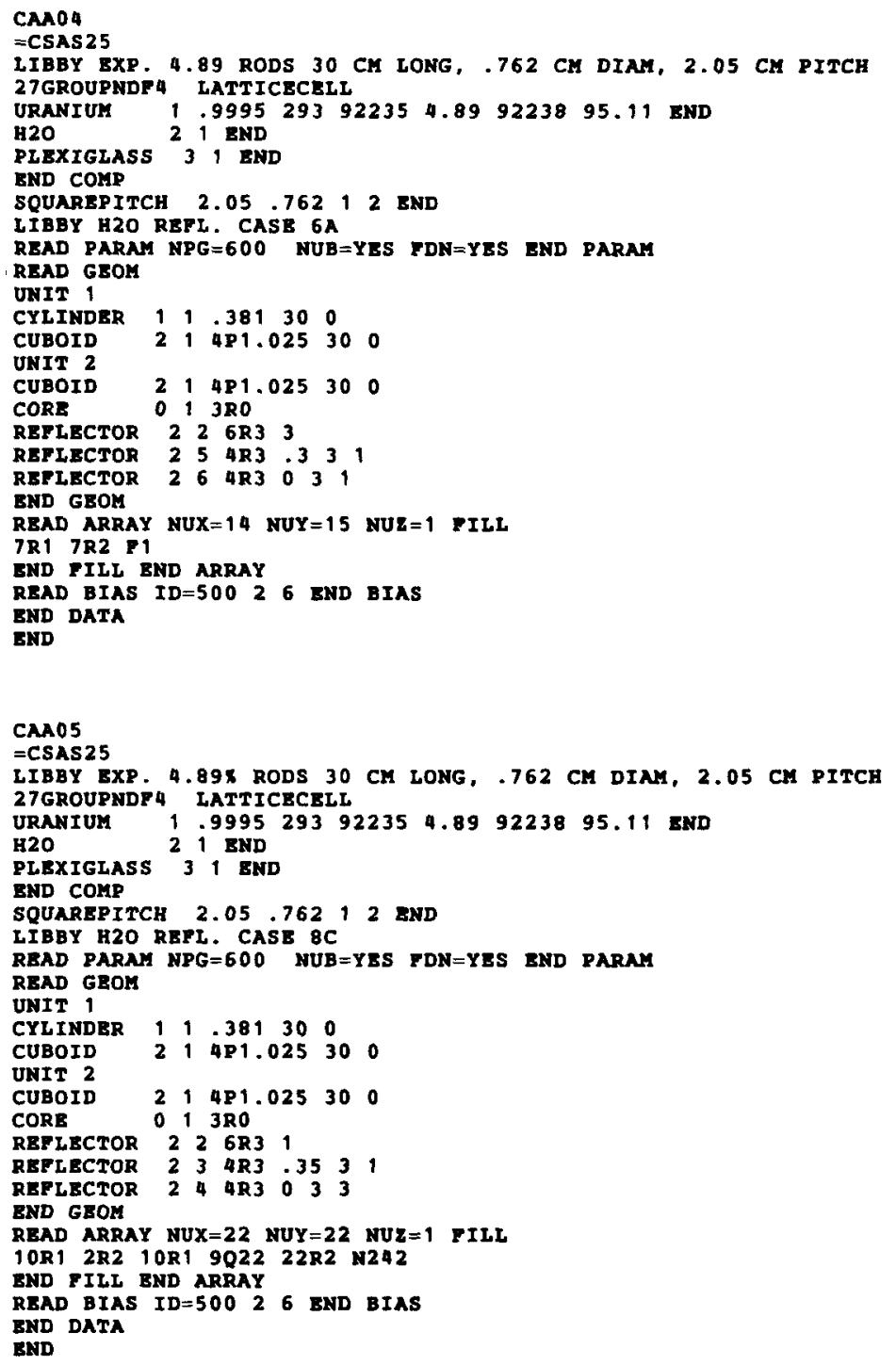

CAAOS

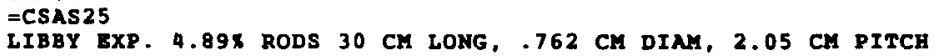

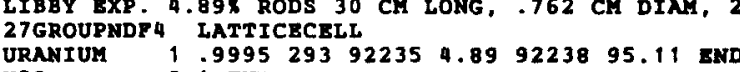

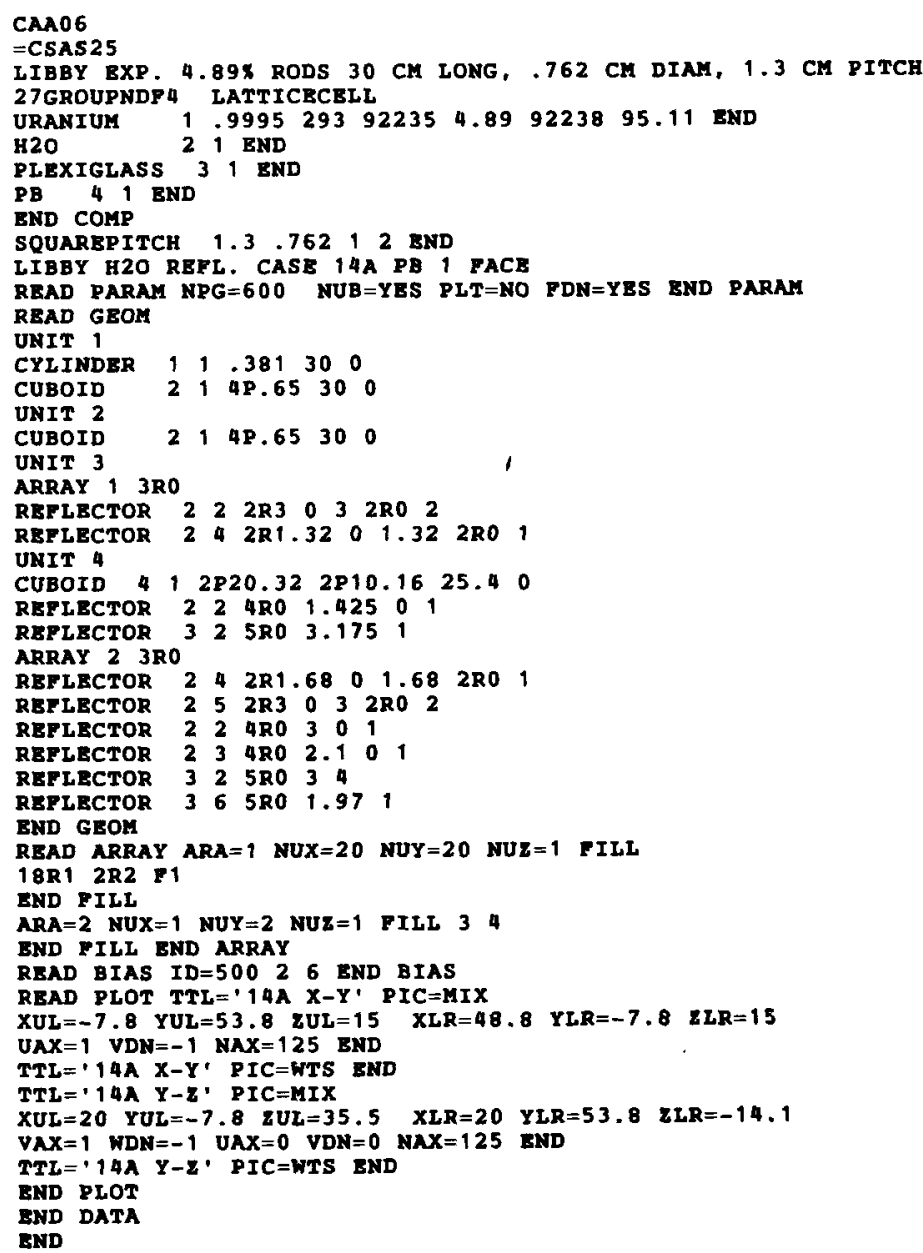


Table A.1 (continued)

CAMOT

IIBAY EXP. 4.89\% RODS $30 \mathrm{CM}$ LOMG, $.762 \mathrm{CM}$ DIAK, $1.3 \mathrm{CM}$ PITCH

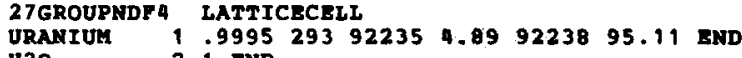
2 i 1995

PLEXIGLASS 31

END COMP

SQUAREPITCH $1.3 \quad 762,2$ END

READ PARAM NPG $=600$ NUB $=$ YES PLT=NO TDN=YES END PARAM

READ GEOM

$\begin{array}{llllll}\text { CYLINDER } & 1 & 1 & \mathbf{3 8 1} 27^{\circ} \\ \text { CUBOID } & 2 & 1 & 48.65 & 27 & 0\end{array}$

UnIT

CYLINDER $1,1.38130$

CUBOID O, 48.6530

UNIT

0148.6530

ARRAY $13 R 0$

REFIECTOR $2,483.221,280$

UNIT 9

RRRAY 4 TRO 3 RETTOR

REFLECTOR O 1 4R3.221 2RO

UNIT 10

URRAT 4

$12810.16 \quad 2 P 16.87125 .40$

REPLECTOR 014 4RO 1.4250

REPLECTOR 322 5RO 3.1751

UNIT 5

3RO

UNIT 6

4 $12 P 37.1912 p 10.1625 .40$

REPLECTOR 001 4RO $1.425 \quad 01$

RRPAY 3 3RO

REFLECTOR 3 2 5 5RO 3 A

REFLECTOR

READ ARRAY ARA $=1$ NUX $=21$ NUY $=21$ NUE $=1$ IILL

IOR1 2 10R1 9Q21 21 R2 10R1 2 10R1 9Q21

END FILI
ARA $=4$ NUX $=21$ NUY $=21 \quad$ NUZ $=1 \quad$ FILI

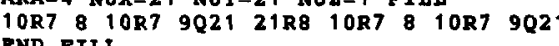

BND FILL

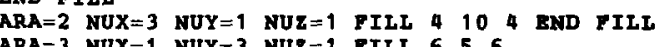

END FILI

$A R A=5$ NUX $=1$ NUY=1 NUZ=2 FILI 39

END PILL END ARRAY

READ BIAS ID $=50026$ END BIAS

$X U L=0 \quad Y U Z=75 \quad$ ZUL $=15 \quad$ XLR $=75 \quad Y L R=0 \quad$ ZLR $=15$

$\mathrm{UAX}=1 \quad \mathrm{VDN}=-1 \quad \mathrm{NAX}=125$ END

TTL $=1^{\prime} X-Y^{\prime}$ PIC $=$ WTS BND

$X U L=33$ YUL $=0$ I $=M I X$

YAX $=1 \quad Y U L=0 \quad Z U L=31 \quad X L R=33 \quad$ YLR $=75 \quad$ \&LR $=-14,1$

$\begin{array}{ll}\text { VAX } & =1 \\ \text { TTL } & =1 \\ Y-z & Y, \quad \text { PIC }=\text { WTS END }\end{array}$
END PLOT
END DATA
END

\section{CMA08}

IIBBY EXP. 4.89X RODS $30 \mathrm{CM}$ LONG, $.762 \mathrm{CM}$ DIAY, $1.3 \mathrm{CM}$ PITCH

27 GROUPNDP A LATTICECELI

1 END

PLEXIGLASS 31 END

PB 411 END

SQUAREPITCH $1.3,762,12$,

RBAY H2O REFI CASE $26 A$ PB A TACES

READ PARAM NPG $=600$ NUB=YES PLT=NO FDN=YES END PARAM

READ GEOF

CYLINDER 111.381300

CUBOID $214 P .65300$

UNIT 3

ARRAY 1 3RO

$\begin{array}{lllllllllll}\text { REPLECTOR } & 2 & 1 & .62 & .62 & .62 & 4.52 & 2 \mathrm{RO} & 1\end{array}$

CUBOID 12 P10.16 2P15.57 25.4

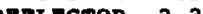

$\begin{array}{llllll}\text { REFLECTOR } & 3 & 2 & 4 R 0 & 3.475 & 1\end{array}$

UNIT 5

ARRAY 2 3RO

UNIT 6

a $12 P 35.892810 .16 \quad 25.40$

$\begin{array}{lllllll} & & \\ \text { CABPLCTOR } & 2 & 2 & 4 R 0 & 1.425 & 0 & 1\end{array}$

ARRAY 3 3R

RETLECTOR 22 ARO 302

REFLECTOR 2 4 ARO 1.950

REFLECTOR 3 3 2 5RO 334

END GEOM

READ APPAY ARA $=1$ NUX $=23$ NUY $=20$ NUE $=1$ IIU

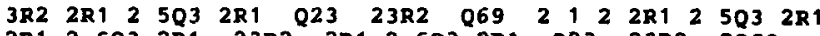

$\begin{array}{lllll}2 R 1 & 2 & 6 Q 3 & 2 R 1 & 23 R 2 \\ 2 R 1 & 2 & 6 Q 3 & 2 R 1 & Q 23\end{array}$

ARA $=2$ NUX=3 NUY=1 NUZ=1 FILI 4 34 END FILL

$A R A=3 \quad N U X=1 \quad N U Y=3 \quad N U Z=1$ PILI 65

BND FIIL END ARRAY

READ BIAS ID $=50026$ END BIAS

$\begin{array}{lll}\text { XUL }=0 & \text { YUL }=72 \quad \text { \&UL }=15 & \text { XLR }=72 \quad \text { YLR }=0 \quad \text { ZLR }=15\end{array}$

UAX $=1$ VDN $=-1 \quad$ NAX $=125$ END

TTL=' $X-Y, \quad$ PIC $=$ WTS END

$40 \quad X L P=33 \quad Y L R=72 \quad \geq L R=-14,1$

$\checkmark A X=1$ WDN $=-1 \quad$ UNX $=0$ VDN=0 NAX $=125$ END

TTI $=1, Y-Z, \quad$ PIC $=$ WTS END

END DATA

END 
Table A.1 (continued)

CNA09
$=$ CSAS 25

IIBBY EXP. $4.89 \times$ RODS $30 \mathrm{CM}$ LONG, $.762 \mathrm{CM}$ DIAM, $1.3 \mathrm{CM}$ PITCH

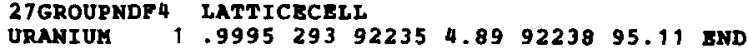

$\mathrm{K} 2 \mathrm{O}$ $2 i$ END

PB 41 END

SS304 51 BND

SND COMP

SQUAREPITCH $1.3 \quad 76212$ END

FACES, SS PLATE IN CENTER

READ GBOM

UNIT 1

$\begin{array}{lllll}\text { CYLINDER } & 1 & 1 & 381300 \\ \text { CUBOID } & 2 & 1 & 48.6530 \%\end{array}$

UNIT 2

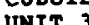

148.65300

ARRAY $13 R 0$

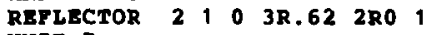

UNIT 7

ARRAY 3 3RO

$\begin{array}{lllllllll} & & & \end{array}$

CUBOID

REPLEC

2R.3325 2RO 1.4253 .175

CUBOID 12 2P10.16 2P15.5725.4O

REFLECTOR 222 4RO 1.425 O 1

UNIT 5 R

ARRAY 2

UNIT 6

CUBOID 4 2P35.89 2P10.16 25.40

$\begin{array}{llllll}\text { REFIECTOR } & 2 & 2 & 4 R 0 & 1.425 & 0 \\ \text { REFLECTOR } & 3 & 2 & 5 R 0 & 3.175 & 1\end{array}$

REPLECTOR

REFLECTOR 22 ARO 305

REPTECTOR 226 ARO 10

$\begin{array}{llllll}\text { REPLECTOR } & 3 & 2 & 5 R 0 & 3 & 4 \\ \text { REFLECTOR } & 3 & 6 & 580 & 1.97\end{array}$

REFLECTOR

END GEOM

AD ARRAY ARA $=1$ NUX=11 NUY $=23$ NUZ $=1$ IRI

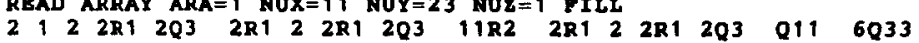

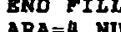

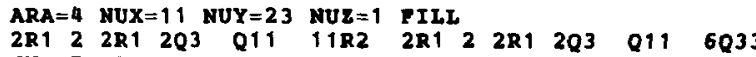

END PILI

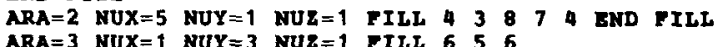

NUX $=1$ NUY $=3$ NUZ $=1$ FILL 6556

BND FTLL $=1$ MOY ARRAY

READ PLOT TTL =1 2 - 6 DND BIAS

$X U L=0 \quad Y U L=72 \quad$ ZUL $=15 \quad X L R=72 \quad$ YLR=0 $\quad$ \&LR=15

$\mathrm{UNX}=1$ VDN=-1 NAX $=125$ SNO

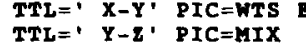

$X U L=33 \quad$ YUL $=0 \quad$ QUL $=40 \quad$ XLR $=33 \quad$ YLR $=72 \quad$ \&LR $=-14.1$

VAX $=1$ WDN $=-1$ UAX $=0$ VDN=0 NAX $=125$ SND

$T T L==Y-Z, P I C=$ WTS END

BND PLOT

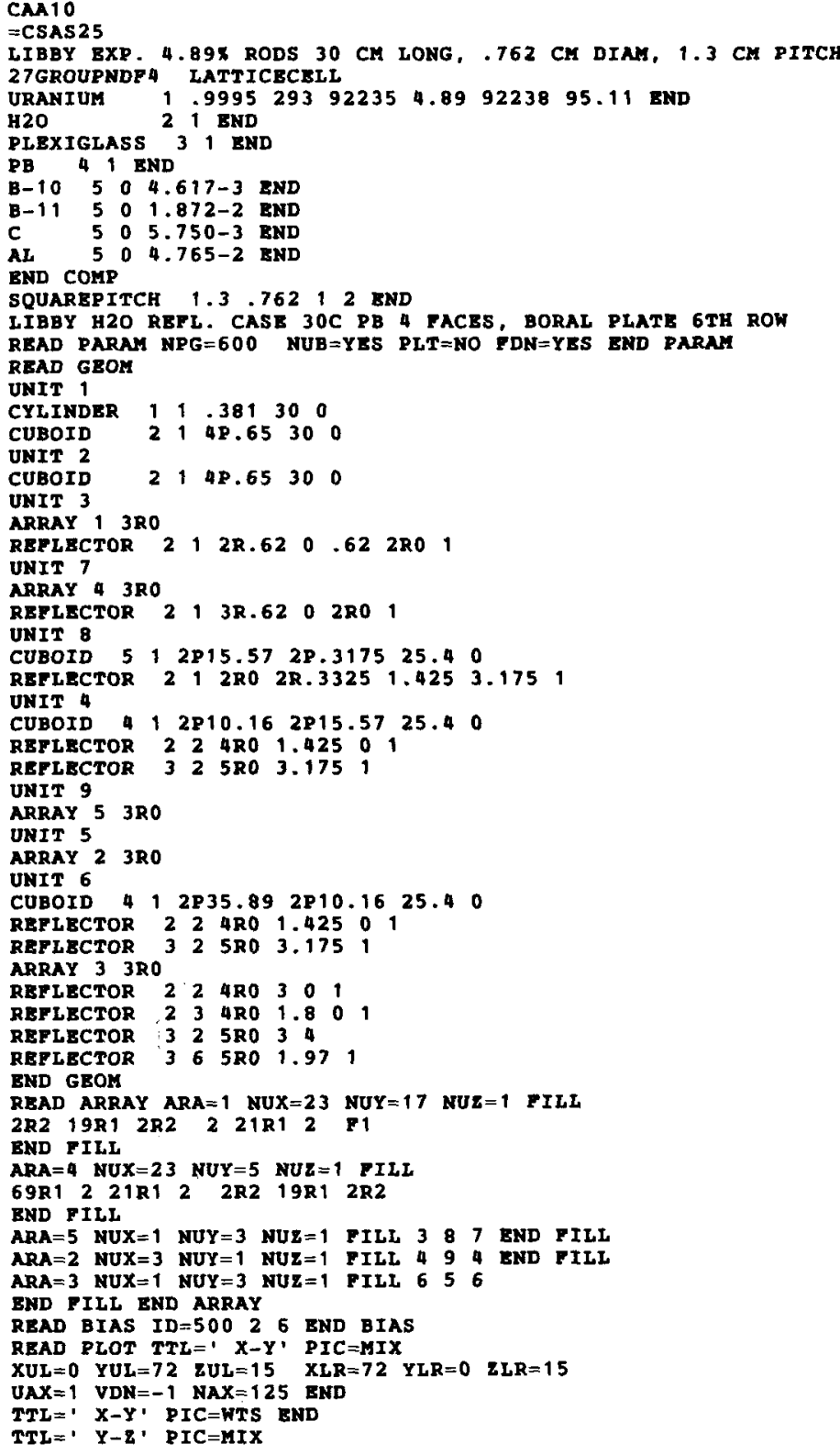


Table A.l (continued)

XUL $=33 \quad$ YUL $=0 \quad 2 U L=40 \quad X L R=33 \quad$ YLR $=72 \quad$ 2LR $=-14.1$
TTL $==9 \quad Y-Z, \quad$ PIC $=$ WTS

BND PLOT

END

\section{CAA 11
$=$ CSAS 25}

$=$ CSAS 25
LIBBY EXP. $4.89 \times$ RODS $30 \mathrm{CM}$ LONG, $.762 \mathrm{CM}$ DIAM, $1.3 \mathrm{CM}$ PITCH

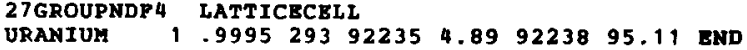

H2O 2 i 2 END

PLEXIGLASS 3 1 END

PB 5 C 51 END

END COMP

SQUAREPITCH $1.3 \quad 762 \quad 1 \quad 2$ END

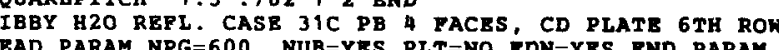
READ GEOM

UNIT 1

$\begin{array}{llllll}\text { CYLINDER } & 1 & 1 & 381300 \\ \text { CUBOID } & 2 & 1 & 48.65 & 30 \%\end{array}$

$214 P .6530$

214 P. 65300

$\begin{array}{lllllllll}\text { RERAYCTOR } & \text { 3RO } & \text { 2R.62 } & 0 & .62 & \text { 2RO } & 1\end{array}$

UNTT 7

TRRAY 430

24 3R.62 O 2RO

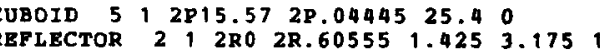

UNIT 4

COID 4 2P10.16 2P15.5725.40

$\begin{array}{lllllll} & \\ \text { REPLECTOR } & 2 & 2 & 4 R 0 & 1.425 & 0 & 1\end{array}$

UNIT 9

ARRAY 5 3RO

UNIT 5 3RR 2 3RO

ARRAY 2 3R

CABOID 4 2P35.89 2P10.16 25.4O

REPLECTOR 222 4RO 1.425 0 1

REPLECTOR $\begin{array}{lllll}3 & 2 & 5 R O & 3.175 & 1\end{array}$

ARRAY 3 3RO

$\begin{array}{llllll} & & & \\ \text { REPLECTOR } & 2 & 2 & 4 R 0 & .5 & 0\end{array}$

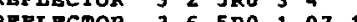

BND GBOM

READ ARRAY ARA $=1$ NUX=23 NUY=11 NUZ=1 FIII

$11 R 112 R 2 \quad P 1$

END FILL

ARA $=4$ NUX=23 NUY=5 NUZ=1 PILL

END FILL

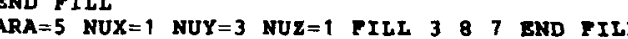

ARA $=2$ NUX $=3$ NUY $=1$ NUZ $=1$ FILI 4 g

$A R A=3$ NUX $=1$ NUY $=3$ NUZ=1 FILL 656

END FILL END ARRAY
READ BIAS ID $=500226$ END BIAS

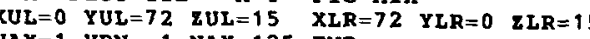

TTL $=1, x-Y$, PIC $=$ WTS

TTL $=$, $Y-2, \quad$ PIC $=$ MIX

$X U L=33 \quad Y U L=0 \quad Z U L=40 \quad X L R=33 \quad Y L R=72 \quad \geq I R=-14.1$

VAX $=1$ WDN $=-1$ UAX $=0$ VDN $=0$ NAX $=125$ END

TTL $=1$ Y $Y-2$ PIC $=$ WTS END

END PLOT
BND DATA

END

CAA 12

$=$ CSAS 25

I.IBBY BXP. $4.89 \times$ RODS $30 \mathrm{CM}$ LONG, $.762 \mathrm{CM}$ DIAM, $2.05 \mathrm{CM}$ PITCH IN UO2F2 SOLN URANIUY 90995293 9 $4.899223895 .11 \mathrm{BND}$

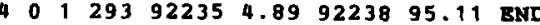
PLEXIGLASS 31 END

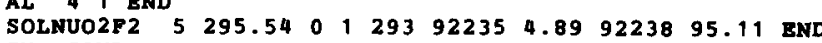

SQUAREPITCH 2.05 .76212 END

READ PARAM NPG $=600$ NUB $=$ YES FDN=YES END PARAM

READ GBOM

UNIT 1

CYLINDER $91,3815.080$

UNIT 2

CYLINDER $11,1,381 \quad 1.27 \quad 0$

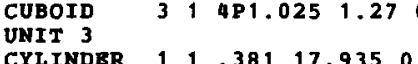

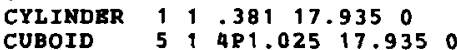

UNIT 4

CYLINDER $1: 381.6350$

CUBOID $314 P 1.025 .6350$

$\begin{array}{llll}\text { UNIT } 5 & \\ \text { CUBOID } & 2 & 1.025 & 5.080\end{array}$

UNIT

$\begin{array}{lllllll}\text { CYLINDER } & 2 & 1 & 381 & 1.27 & 0 \\ \text { CUBOID } & 3 & 1 & 4 P 1 & 025 & 1.27\end{array}$

CUBOID $214 P 1.025 \quad 17.9350$

UNIT 8

$\begin{array}{lllllll}\text { CYLINDER } & 2 & 1 & .381 & .635 & 0\end{array}$

$\begin{array}{llllll}\text { CUBOID } & 3 & 1 & 481.025 & .635 & 0 \\ \text { CORE } & 0 & 1 & -11.275 & -12.30\end{array}$

REPLBCTOR 31 SR0 $3.81^{-12}$

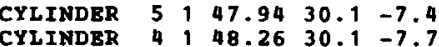

$\begin{array}{llllll}\text { CYLINDER } & 4 & 48.26 & 30.1 & -7.72\end{array}$

SND GEON

READ ARRAY NUX $=11$ NUY $=12$ NUZ $=5$ FILL

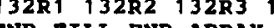

END DATA 
Table A.1 (continued)

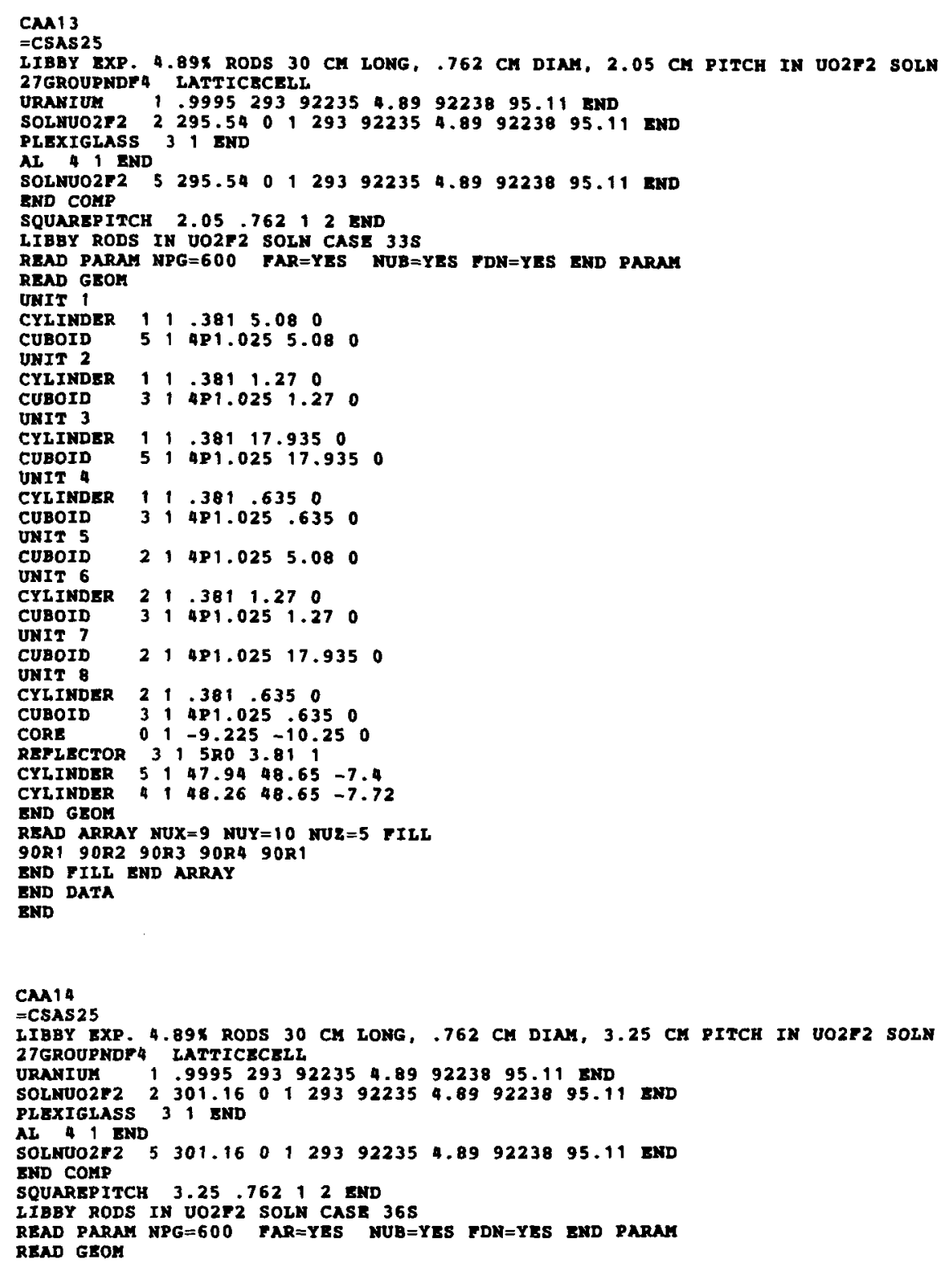

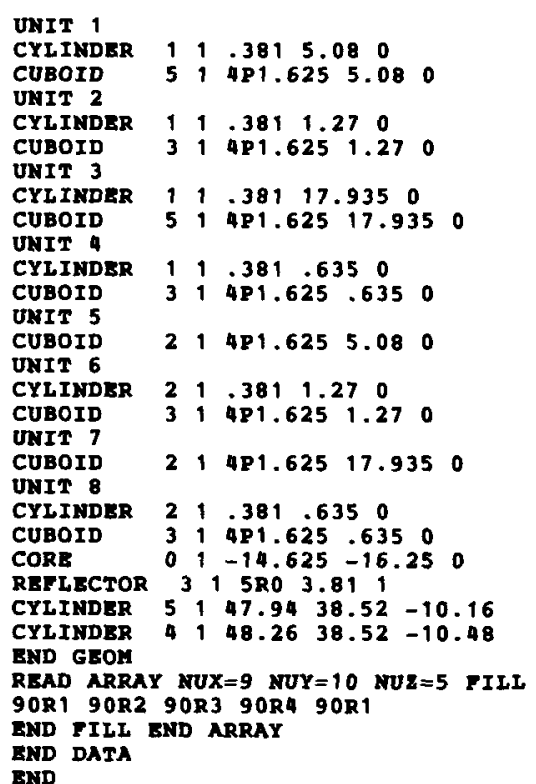

CAA15

LIBBY EXP. $4.89 \times$ RODS $30 \mathrm{CM}$ LONG, $1.31 \mathrm{CM}$ DINM, 2.99 CK PITCH IN UO2F2 SOLN 27GROUPNDF 4 LATTICBCELI

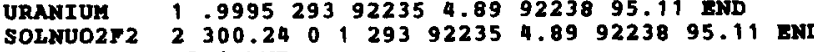
PLDXIG

SOLNUO2F2 $5300.24 \quad 0129392235 \quad 4.899223895 .11$ END END COMP

SQUAREPITCH 2.991 .3112 END

LIBAY RODS IN UO2F2 SOLN CASZ $40 \mathrm{O}$

READ PARAM
READ GEOM
UNIT

CYZINDER 11.6555 .08 .0

CUBOID 5 1 4 PP1.4955.08 5.08

UNIT 2 (2) 1.6551 .270

$\begin{array}{llllll}\text { CYLINDSR } & 1 & 1 & 655 & 1.27 & 0 \\ \text { CUBOID } & 3 & 1 & 41.495 & 1.27\end{array}$

CYLINDER 11.65517 .9350

UNIT

$\begin{array}{lllll}\text { CYL IXDER } & 1 & 1 & .655 .635 & 0 \\ \text { CUBOID } & 3 & 1 & \text { AP1.495 } & .6350\end{array}$

CUBOID $214 P 1.4955 .080$ 
Table A.1 (continued)

UNIT 6

$\begin{array}{lllllll}\text { CYLINDER } & 2 & 1 & \mathbf{6 5} 5 & 1.27 & 0 \\ \text { CUBOID } & 3 & 1 & 4 \mathrm{P} 9 & 495 & 1.27 & 0\end{array}$

CUBOID 214 P1.495 17.935

COBOID

CYLINDER 221.655 .6350

CUBOID 3 , 4 P 1.495 .6350

RBPLECTOR 3 1 1 5R0 3.81 1

$\begin{array}{llllll}\text { CYLINDER } & 5 & 1 & 47.94 & 43.95 & -10.16 \\ \text { CYLINDER } & 4 & 1 & 48.26 & 43.95 & -10.48\end{array}$

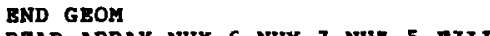

42R1 42R2 $42 R 3$ 42R4 $42 R 1$

BND FILL END ARRAY

END DATA

CAA16

$=\operatorname{CSAS} 25$

LIBBY EXP. $4.89 \times$ RODS $30 \mathrm{CM}$ LONG, $1.31 \mathrm{CM}$ DIAK, 3.40 CK PITCH IN UO2T2 SOL 27GROURNDS LATTICBCEL

293922354.89922389511 sND SOLNUO2F2 $2309.240129392235 \quad 4.899223895 .11$ END

AL 41 END

$\begin{array}{lllllllllll}\text { SOLNUO2F2 } & 5 & 300.24 & 0 & 1 & 293 & 92235 & 4.89 & 92238 & 95.11 & \text { EKD }\end{array}$ END COMP

UAREPITCH 3.401 .3112 END

READ PARAM NPG $=600$ FAR $=Y$ SS NUB $=Y$ YS $Y D Y=Y$ ES READ GEOM

UNIT 1

CYLINDER $111.655 \quad 5.080$

CUBOID

CULOID

UNIT 3

CYUBDER $11 . .65517 .9350$

CNist

$\begin{array}{llllll}\text { CYLINDER } & 1 & 1 & 655 & .635 & 0 \\ \text { CUBOID } & 3 & 1 & 9 P 1.70^{\circ} .635 & 0\end{array}$

UNIT

CUBOID

21491.705 .080

crituse

$\begin{array}{lllllll}\text { CYLINDER } & 2 & 1 & 655 & 1.27 & 0 \\ \text { CUBOID } & 3 & 1 & 4 P 1.70 & 1.27 & 0\end{array}$

CUBOID

CNIT 8

$\begin{array}{llllll}\text { CYLINDER } & 2 & 1 & 655 & .6350 \\ \text { CUBOID } & 3 & 1 & 4 P 1.70^{\circ} & 035 & 0\end{array}$

REFLECTOR 3 1 1 5RO 3 - 11.9

$\begin{array}{lllllll}\text { CYLINDER } & 5 & 1 & 47.94 & 39.1 & -10.16 \\ \text { CYLINDER } & 4 & 1 & 48.26 & 39.1 & -10.48\end{array}$

END GEOM

READ ARRAY NUX $=6$ NUY $=7$ NUZ $=5$ FILI

T2R $42 R 2$

BND DATA
$\operatorname{caA} 17$

LIBBY EXP. $4.89 \times$ RODS $30 \mathrm{CM}$ LONG, $1.31 \mathrm{CM}$ DIAM, $3.940 \mathrm{CM}$ PITCH IN UO2F2 SOLN 27GROUPNDF 4 LATTICBCELL

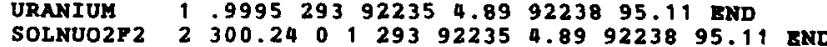
PLEXIGLASS 34 BND

AL 4,1 END

$\begin{array}{lllllllll} & & & \end{array}$ END CONP

SQUAREPITCH $3.94 \quad 1.3112$ END

IIBBY RODS IN UO2P2
READ PARAM NPG $=600$ YAR CASE $44 \mathrm{Y}$
NUB $=$ YES $P D N=Y$ YSS END PARAM READ GBOM

$\begin{array}{lllll}\text { CYLINDER } & 1 & 1 & 6555.080 \\ \text { CUBOID } & 5 & 1 & 4 P 1.975 .08 & 0\end{array}$

UNIT2 2 CYINDER 111.6551 .270

UNIT 3

CYLINDER $\quad 1 \quad 1 \quad .655 \quad 17.935 \quad 0$

CUBOID 51481.9717 .9350

UNIT 4

CYLINDER $1, .655 .6350$

UNIT 5

CUBOID

UNIT 6

$\begin{array}{llllllll}\text { CUBOID } & 3 & 1 & 6655 & 1.27 & 0 \\ \text { CP1.97 } & 1.27 & 0\end{array}$

CUBOID $21481.97 \quad 17.9350$

UNIT

$\begin{array}{llllll}\text { CYLINDER } & 2 & 1 & .655 & .6350 \\ \text { CUBOID } & 3 & 1 & 4 P 1.97^{6} & .635\end{array}$

CORE O $1,-11.82-13.790$

CYLINDER 5 , $147.9439 .25-10.16$

$\begin{array}{llllll}\text { CYLINDER } & 5 & 1 & 47.94 & 39.25 & -10.16 \\ \text { CYLINDER } & 4 & 1 & 48.26 & 39.25 & -10.49\end{array}$

NUX $=6$ NUY $=7$ NUE $=5$ FIL

READ ARRAY NUX $=6$ NOY
$42 R 1$ 42R2 $42 R 3$ 42R4 42R1

BND FILL END ARRA

END 
Table A.1 (continued)

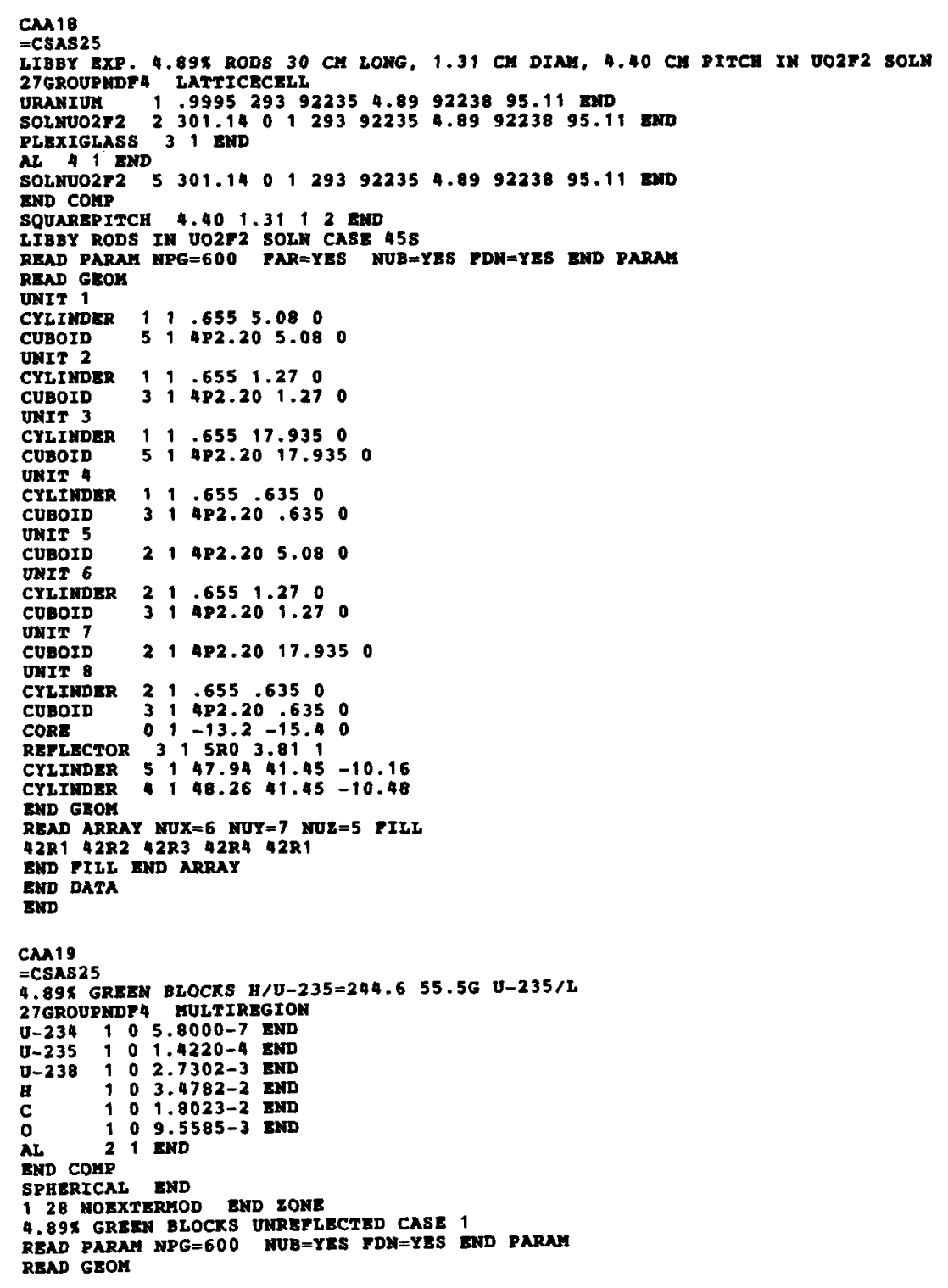


Table A.1 (continued)

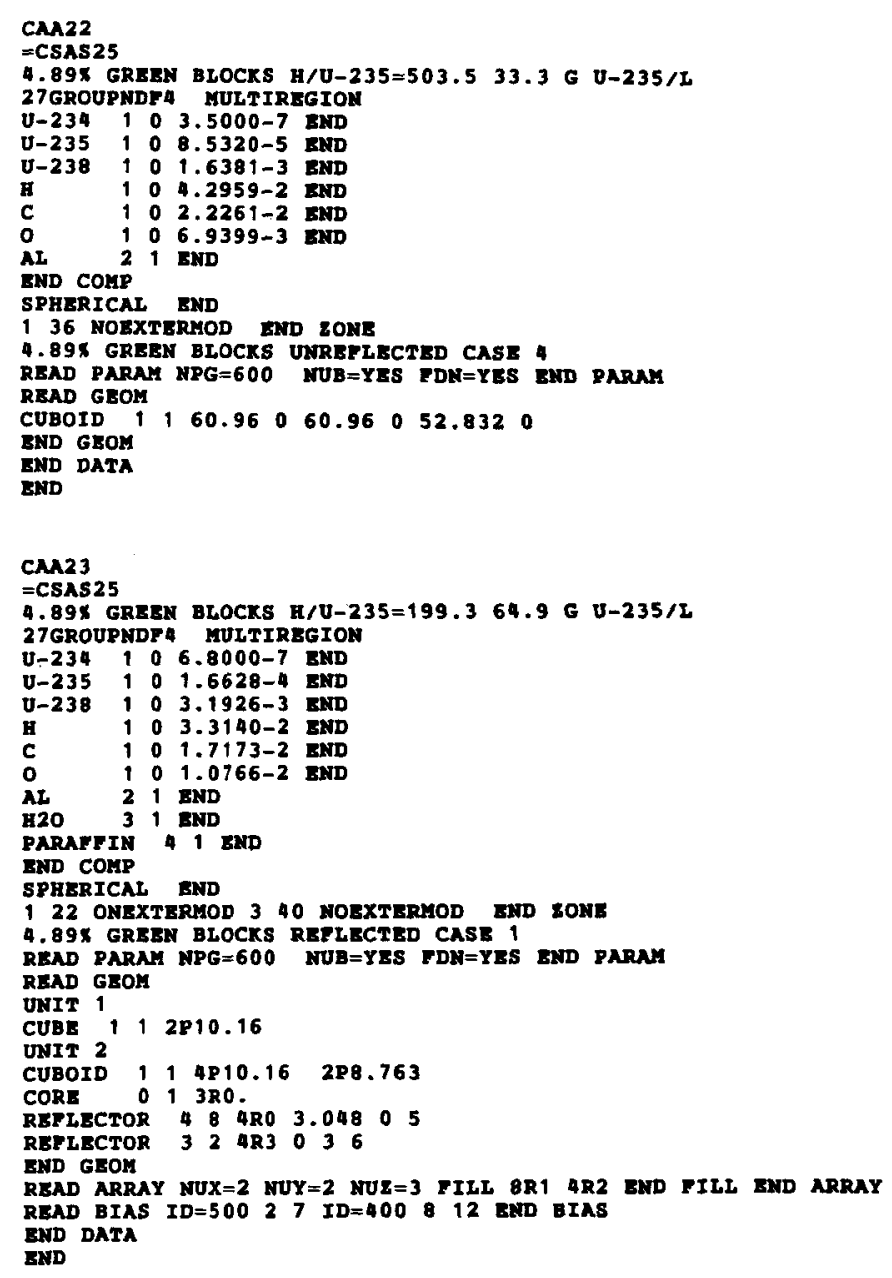

$\operatorname{CAN} 24$
$=\operatorname{CSAS} 25$

$4.89 \times$ GREEN BLOCXS H/U-235=244.8 56.2 G $\mathrm{v}-235 / \mathrm{L}$

27GROUPNDF 4 KULTIREGION

$\begin{array}{llllll}U-235 & 1 & 0 & 1.4399-4 & \text { END } \\ U-238 & 1 & 0 & 2.7647-3 & \text { END }\end{array}$

$\begin{array}{lllll}U-238 & 1 & 0 & 2.7647-3 & \text { END } \\ H & 1 & 0 & 3.5249-2 & \text { END }\end{array}$

c $\quad 100 \begin{array}{lll}1.8265-2 \\ 0\end{array}$

$\begin{array}{lllll}\text { AL } & 2 & 1 & \text { END } \\ \text { H2O } & 3 & 1 & \text { END }\end{array}$

PARAFYIN 41 END

END COMP

122 ONEXTERMOD 340 NOEXTERMOD END ZONE

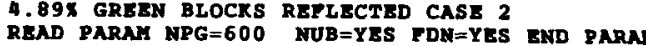

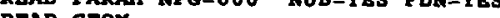

CUBOID $1+40.64040 .64057 .9120$

REFLECTOR 48 4R0 3.048 O 5

REPLECTOR 322 AR 3036

READ BIAS ID $=50027$ ID $=400 \quad 12$ END BIAS

END DATA

$\operatorname{CMA25}$
$=\operatorname{CSAS} 25$

4.89 GREEN BLOCKS H/U-235=396.7 40.6 G U-235/L

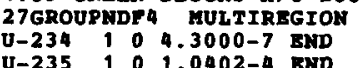

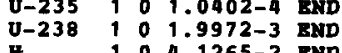

H $1004.1265-2$ END

$\begin{array}{llll}c & 0 & 2.1383-2 \\ 0 & 1 & 0 & 7.855 \\ 0 N D & & \end{array}$

$\begin{array}{llll}\text { AL } & 2 & 1 & \text { END } \\ \text { H2O } & 3 & 1 & \text { END }\end{array}$

PARATEIN 1 END

END COMP

SPHERICAL END

TEXTERMOD 3 38 NOEXTERMOD END ZONE

4. 89 9 GREEN BLOCRS REFLECTED CASE 3 LONE

CUBOID 1140.64040 .64057 .6580

REFHECTOR 48 4RO 3.048 O

READ BIAS ID=500 27 ID=400 812 END BIAS

END DATA 
Table A.1 (continued)

$\operatorname{cm} 27$

4.89x GREEN BLOCKS H/U-235=146.8 81.1 G U-235/L 27 GROUPNDP4 RULTIREGION

$\mathrm{U}-234$ 1 $08.5000-7$ END

U-235 $1002.0779-4 \mathrm{END}$

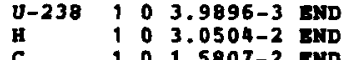

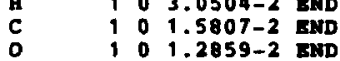

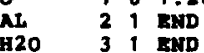

PARATFIN 31 END 1 END

EMD COHP

122 ONEXTEDDOD 3 40 NOEXTERMOD END ZONE

220 GREEN BLOCKS REFLECTED CASE 5 DONE

REND PARAM NPG $=600$ RUB $=$ YES TDN=YES END PARAM

CUBOID 1150.8050 .8042 .1640

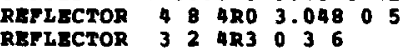

REPLECTOR 32 पR 30

READ BIAS ID $=500 \quad 27$ ID $=400$ \& 12 END BIAS

FMD DACA

$\operatorname{CAN} 28$
$=\operatorname{CsAS} 25$

4.891 GRAEN BLOCXS H/U-235=82.7 107. G $U-235 / L$

27GROUPNDFY MULTIREGIO

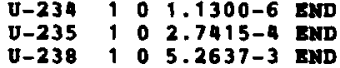

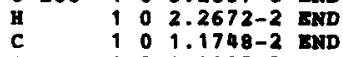

$0 \quad 1001.6007-2$ END

$\begin{array}{llll}A L & 2 & \text { END } \\ \mathrm{H} 2 \mathrm{O} & 3 & 1 & \mathrm{END}\end{array}$

PARAFTIN A 1 END

IND COMP

126 ONEXTERMOD 3 44 NOEXTERMOD END EONE

4. 898 GPEEN BLOCXS REPLECTED CASE 6

READ PARAM NPG $=600$ NUB $=$ YES PDN=YES END PARAY

READ GEOM

QBDID 150.8060 .96056 .1340

REPTECTOR 32 4R3 035

RED GEOM

READ BIA
END DATA
EHD
CMA26

89X GRERN BLOCKS H/U-235=756.6 22.2 G U-235/L

27GROUPNDFA MULTIREGION

U.235 1 0 $5.3080-7$ END

प-238, 0 , $1.0921-3$ ExD

H $\quad \begin{array}{llll}1 & 0 & 4.3035-2 & \text { END } \\ 1 & 0 & 2.2300-2 & \text { END }\end{array}$

\begin{tabular}{llll}
1 & 0 & $2.2300-2$ & END \\
0 & 1 & 0 & $5.4119-3$ \\
\hline & 1 & 1 &
\end{tabular}

AI $\quad 2$ 1 3 END

PARATTIN 1 END

IND COMP

SPHERICAL END

20 46 NOEXTERMOD END ZONE

896 GREEN BLOCKS REFLECTED CASE 4 FND PARAY

READ GEOM

COBOID 1971.12060 .96051 .562

REFLECTOR

READ BIAS ID $=50027$ ID=400 812 END BIAS

END DATA

\section{CAN29}

893 GREEM BLOCKS H/U-235=504,133,3 G U-235/L

27GROUPNDP
U-234 100 HULTIREGION
U-2355 $1000-7$ END

$\begin{array}{lllll}\mathrm{U}-235 & 1 & 0 & 8.5320-5 & \text { END } \\ \mathrm{U}-238 & 1 & 0 & 1.6381-3 & \mathrm{END}\end{array}$

H 10 \%.3010-2 END

$\begin{array}{lllll}\mathrm{C} & 1 & 0 & 2.2287-2 & \mathrm{END} \\ 0 & 1 & 0 & 6.9427-3 & \mathrm{END}\end{array}$

AZ 2 I 1 END

PARA

END COMP

129 ONEXTERUYOD 347 MOEXTERMOD END ZONE

4.89X GREEN BLOCKS REFLBCTED CASE 7

CUBOID 1150.8050 .8041 .148

RETLECTOR 8 4RO 3.048 O 5

32483 O 36

READ BIAS ID $=50027$ ID $=400 \quad 8 \quad 12$ END BIAS

END DATA 
Table A.1 (continued)

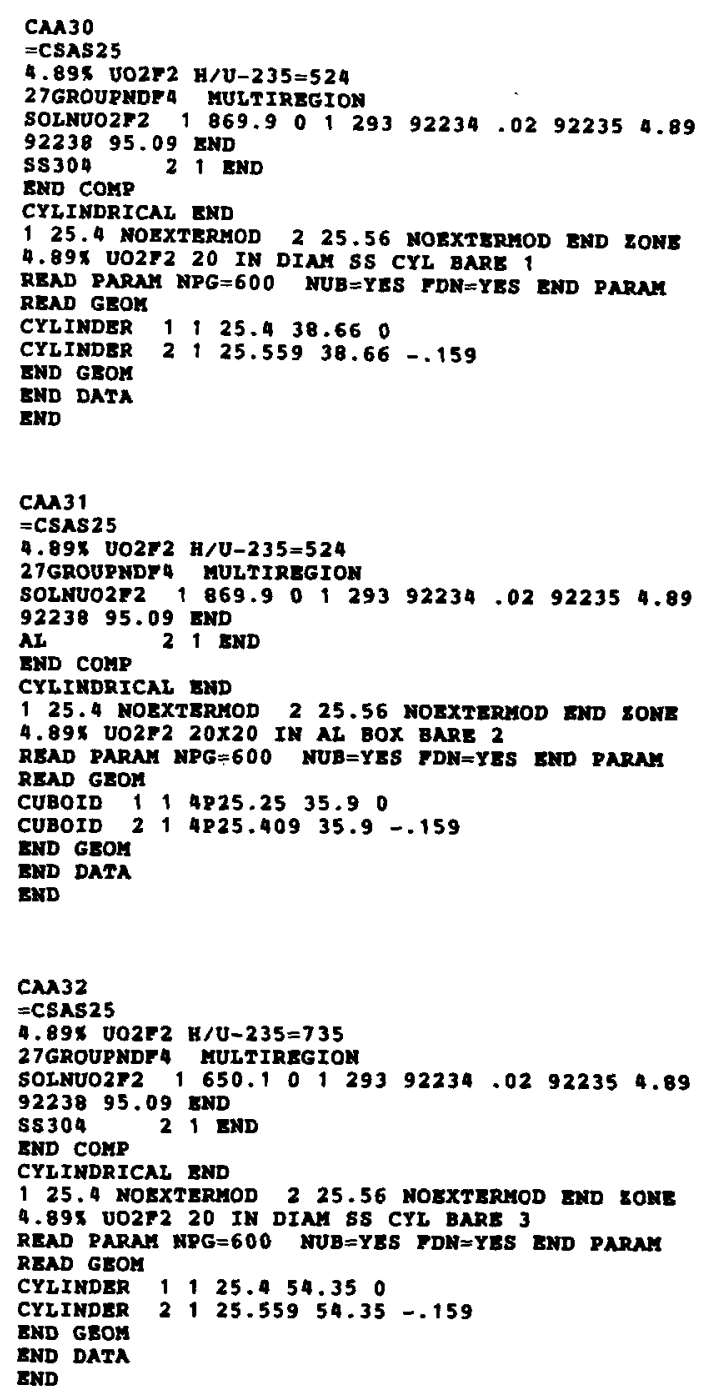

CAN32
$=$ CSAS25
C.

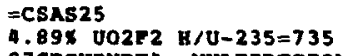

SOLNUO2P2 1650.10129392234 .02922354 .89 9238
SS304 95.09
END COMP

END COMP

(2)

25.4 NOEXTERMOD 225.56 MOEXTERMOD 4.898 V0272 20 IN DIAM SS CYL BARE

Ge

CYA

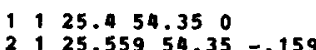

END GEOS
END DATA

$\underset{\text { END }}{\text { END }}$

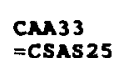

4.898 บ02ק2 H/O-235=1002

BGION 923895.09 END

ALD COMP 21

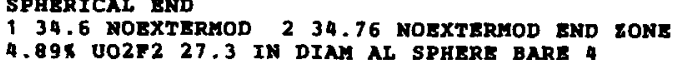
READ PARAM NPG $=600$ NUB $=$ YES PDN=YES END PARAM

$\begin{array}{llllll}\text { READ GEOM } & \\ \text { HEMISPHE- } & 1 & 1 & 34.6 & \text { CHORD } 30 .\end{array}$

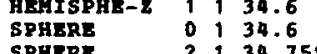

RND DATA

CAN34

C. $89 \times$ บ0272 $\mathrm{H} / \mathrm{U}-235=991$

4.89x

SOLNUO2F2 $1496.50129392234 \quad 0292235 \quad 4.89$

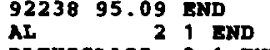

AL
PLEXIGLASS

END COMP

CYLINDRICAL END

138.1 NOEXTERMOD 238.26 NOEXT TRMOD END ZONE

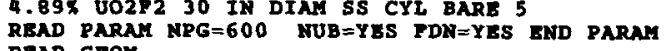

CYYINDER 11138.194 .750

CXLTNDER 2 1 $38.25994 .75 \quad-.159$

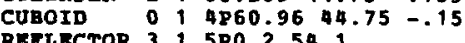

END GEOM

END DATA 
Table A.1 (continued)

$\operatorname{cAN} 35$
$=\operatorname{CSAS} 25$

$4.89 \times$ UO2P2 $\mathrm{H} / \mathrm{U}-235=524$

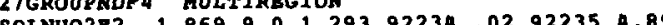

9223895.09 RND

\begin{tabular}{llll}
$\$ 5304$ & 2 & 1 & END \\
420 & 3 & 1 & \\
\hline
\end{tabular}

H2O 31 END

CYL INDRICAL END 219.2 NOEXTERMOD 337.2 NOEXTERMOD END EONE $4.89 \times 10022^{2} 15$ IN DIAT SS CYL REFL

READ PARAM NPG $=600$ NUB $=$ YES FDN=YES END PARA

READ GEOM

CYLINDER 1 i 19.0550 .370

REFLECTOR 3253 B 36

END GEOM

READ BIAS ID $=50027$ END BIAS

CAA36

$=$ CSAS25
4.898 UOPY 2 H/U-235=524

SOLNUO2F2 $1869.90129392234 \quad 0292235 \quad 4.89$ $\begin{array}{lrlll}92238 & 95.09 & \text { END } \\ \text { AL } & 2 & 1 & \text { END } \\ \text { H2O } & 3 & 1 & \text { END }\end{array}$

CYD COMP

125.4 ONEXTERMOD 225.56 NOEXTERMOD 343.56 NOEXTERMOD END ZONE 1..89K 00252 $20 \times 20$ IN AL BOX RENL 2

YES END PARAM

READ GBOM

CUBOID 214 4P25.409 $25.559-.15$

REPLECTOR 326 SR 6

END GEOM

ATAS ID=500 27 END BIAS

BND DATA

CAA37
$=$ CSAS25

4.89X บ02F2 H $/ U-235=735$

(902010252 $1650.10129392234 \quad 02922354.89$ 9223895.09 END

H2O 31 END

END COMP

and

19 ONEXPRMOD 219.2 NOEXTERMOD 337.2 NOEXTERMOD END ZONE

4.89\% UO2F2 15 IN DIAS SS CYL REPL 3 3 .

READ PARAM

READ GEOM

$\begin{array}{lllllll}\text { CYLINDER } & 1 & 1 & 19.05 & 153.0100 & \\ \text { CYLINDER } & 2 & 1 & 19.209 & 153.169 & -.159\end{array}$

REFLECTOR $3223 R 36$

END GEOM
READ BIAS ID $=50027$ END BIAS

END DATA

CAA38

$=$ CSAS25

27GROUPNDY 4 MULTIREGION

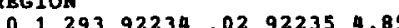

9223895.09 END

$420 \quad 3 \quad 1$ BND

BND COMP

SPHERICAL BND
14.6 ONEXTERMOD

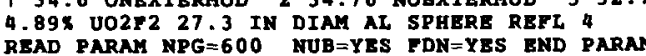

READ GEOM
HEMISSPHE-2

$\begin{array}{llll}\text { HEMISPHE-2 } & 1 & 1 & 34.6 \\ \text { SPHERE } & 0 & 1 & 34.6 \\ \text { SPHERE } & 2 & 14.759\end{array}$

REFLECTOR 323.6

END GEOM $D=50027$ END BIAS

READ BIAS
END DATA
END

CAA39

$4.89 \times$ บ02F2 H $/ \mathrm{U}-235=994$

SOLNUO222, $495.30129392234 \quad 02922354.89$

9223895.09 END

$\begin{array}{lllll}55304 & 2 & 1 & \text { END } \\ \text { H20 } & 3 & 1 & \text { END }\end{array}$

END COMP

125.4 ONBXTEPMOD 225.56 NORXTBRMOD 343.56 NOEXTERMOD END ZONE

READ PARAM NPG $=600$ NUB $=$ YES PDN $\approx$ YES END PARAM

CYLINDER 11 1 25.485 .7200

REPLBCTOR $32 \begin{array}{llll}3 R 3 & 6\end{array}$

END GEOM

READ BIAS ID $=5002$, END BIAS 
Table A.2. Table 2 input data

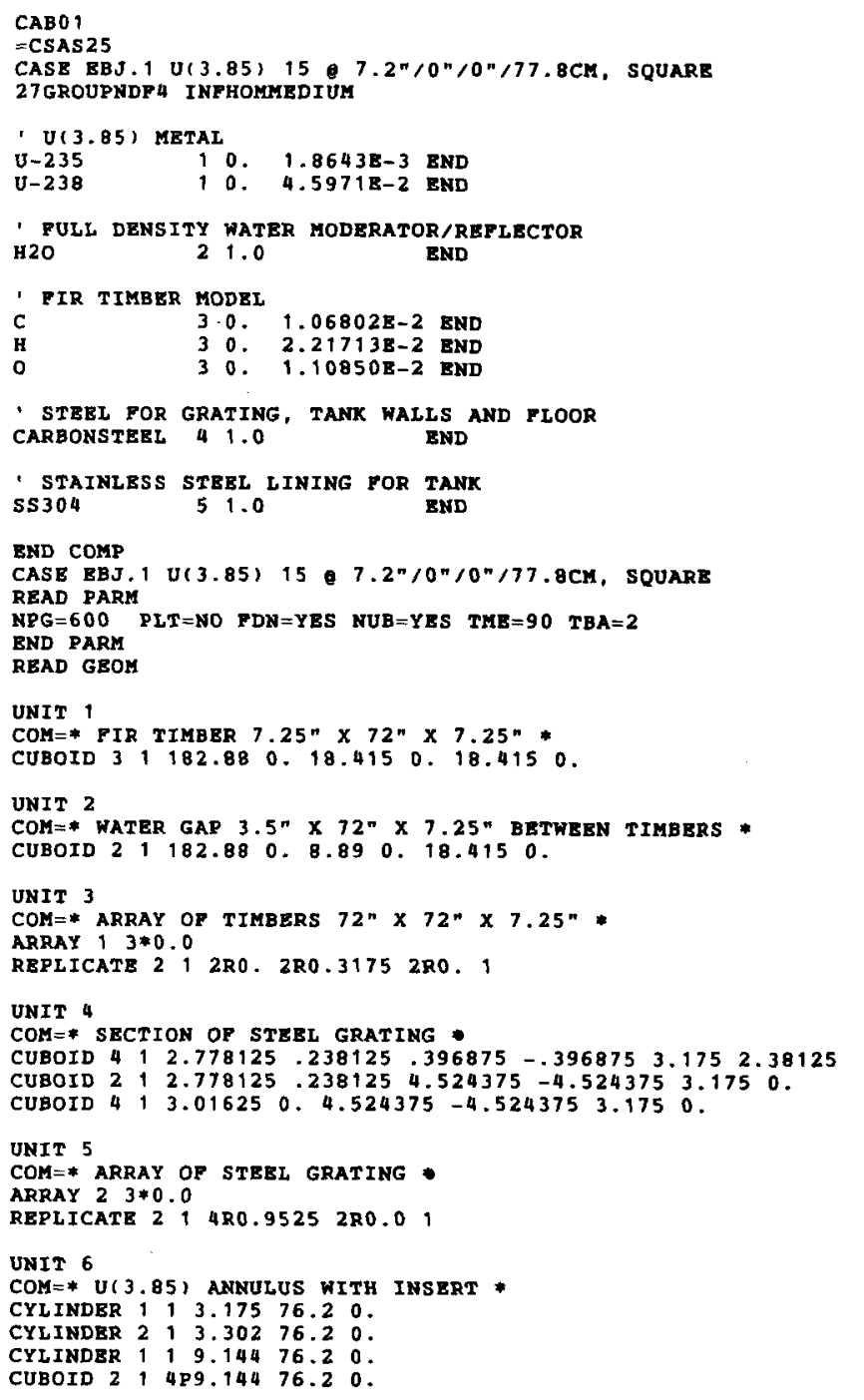

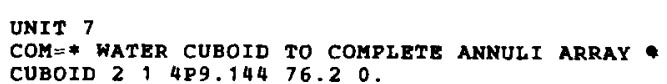

TTL="PLAN VIEH $1 / 6$ SCALE OF CORB, Z=MTDLEVBL OP ANNULI/INSERTS"

UAX $=1$. VDN $=-1, \quad D L X=1.524$ NCH=* U. WGSC* BND

TTL="PLAN VIEW $1 / 6$ SCALE OF CORE, $z=$ MIDLEVEL OF GRATING" $X I R=91.44$ YLR $=-91.44$ \&UL $=20.0025$

UAX $=1$. $V D N=-1$. DLX $=1.524 \quad$ NCH $=*$ U. WGSC* END

TTL="PLAN VIEW $1 / 6$ SCALE OF CORE, $2=$ MIDLEVEL OF PIR TIMBERS" $X U L=-91.44 \quad Y U L=91.44 \quad Z U L=9.2075$

$U A X=1$. $V D N=-1$. DLX $=1.524$ NCH $=*$ U. WGSC* END

TTL $=$ "PLAN VIEW $1 / 10$ SCALE OF MOCKUP, $Z=$ MIDLEVEL OF U UNITS" $X U L=-121.92 \quad Y U L=121.92 \quad 2 U L=59.69$

UAX $=1$. VDN $=-1$. DLX $=2.54 \quad \mathrm{NCH}=*$ U. WGSC* BND

TTL="PLAN VIEW $2 X$ SCALE OF SINGLE U UNIT, $2=$ MIDLEVEL OF UNIT" $X U L=0$
$X L R=18.288 \quad Y L R=18.288 \quad Z U L=59.69$

$U A X=1$. VDN $=-1$. DIX $=0.127$ NCH=* U. WGSC* END

TTL $=$ "CROSS SECTION OP CORE $1 / 6$ SCALE, $Y=9.144$ "

$X U L=-91, A 4$ YUL $=9$, $144 \quad$ ZUL $=102$

$U A X=1$. WDN $=-1$. DLX $=1,524$ NCH=* U. WGSC* BND 
Table A.2 (continued)

TTL="CROSS SECTION OF MOCRUP $1 / 10$ SCALE, $Y=9.144$ " $X U L=-121.92 \quad Y U L=9.144 \quad Z U L=105$
$X L R=121.92 \quad Y L R=9.144$

$X L R=121,92$ YLR $=9.144 \quad 2 L R=-65$.
$U A X=1$. WDN $=-1$, DLX $=2.54$ NCH= U. WGSC* END

TIL="CROSS SECTION OF GRATING/FIR TIMBERS, FULE SCALE"

$X U L=0, Y U L=0 . \quad Z U L=24,13$

$U A X=1$. WDN $=-1$. DLX $=.254 \quad N C H=*$ U. WGSC * END

TTL="PLAN VIEH OF GRATING THRU TIBBARS, FULE SCALE"

$X U L=0 . \quad Y U L=25.4 \quad Z U L=21.193$
$X L R=25.4 \quad Y L R=0 . \quad Z L R=21.193$

UAX $=1$. VDN $=-1$. DLX $=.254$ NCH $=*$ U. WGSC* END

TTL="PLAN VIEW OF GRATING O GRATING MIDLEVEI, TULL SCAEE"

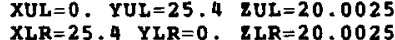

UAX $=1$, VDN $=-1$, DEX $=.254$ NCH $=*$ U. WGSC* END

TTL $=$ "PLAN VIRW OF TIMBERS TIMBRR MIDLEVEL, FULL SCALE" $X U L=0 . \quad Y U L=25.4 \quad 2 U L=9.2075$

UAX $=1$. VDN $=-1$. DLX $X=254$ NCH $=*$ U. WGSC* END

END PLOT

END DaxA

\section{CABO2}

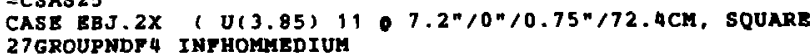

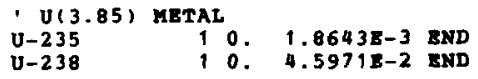

C PULL DENSITY WATER MODERATOR/REKLECTOR

TIR TIMBER MODEL

C
H
0

.0 BND

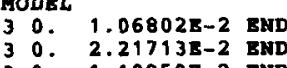

CARBEL FOR GRATING, TANK WALLS AND FLOOR

STATNLESS STEEL LINING FOR TANK
SS 304

END COMP READ PARM $T$ MPG $=600$ MUE $=$ YES $P D Y=Y B S$ TME $=90 \quad T B A=2$ RND PARM
UNIT 1

COM=* PIR TIMBER $7.25^{n} \times 72^{\prime \prime} \times 7.25 " *$

UNIT 2

COM=* WATBR GAP $3,5^{\prime \prime} \times 72^{\prime \prime} \times 7.25^{\prime \prime}$ BETWEEN TIMBERS *

CUBOID 21182.880 .8 .890 .18 .4150 .

UNIT 3

年 ARRAY $13 \neq 0.0$

REPLICATE 2 2 1 2RO. 2RO.3175

UNIT 4

COM=* SECTION OF STERL GRATING *

CUBOID $4 \quad 12.778125 .238125 \quad 396875 \quad-.396875 \quad 3.175 \quad 2.38125$

CUBOID $212.778125 .238125 \quad 4.524375-4.524375 \quad 3.1750$

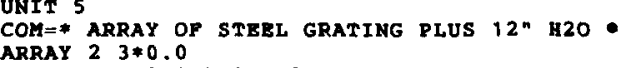

REPLICATE 2 1 4 R0.9525 2R0.0

UNIT 6 U

CYLINDER $1.13 .175 \quad 72.4 \quad 0$

CYLINDER 199.14472 .40

CUBOID 2,4 P 10.096572 .40

UNIT 7

COM=* WATER CUBOID TO COMPLETE ANWULY ARRAY $(72.4 \mathrm{~cm} \mathrm{H})$ -

UNIT 8

COM=* ARRAY OF 11 ANNULI WITH U(3.85) INSERTS $(72.4 \mathrm{CM}$ H) *

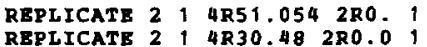

UNIT 9

COK=* U(3.85) ANNULUS WITH INSERT $(3.8 \mathrm{cM} \mathrm{H})$.

CYLINDER 1113.1753 .80$.

CYLINDER 013.3023 .80$.

(P10.0965.3.8.

UNIT 10

COM=* VOID CUBOID TO COMPLETE ANNULI ARRAY $(3.8 \mathrm{cM} \mathrm{H})$ -

CUBOID 014 P 10.09653 .80 .

UNIT 11

COM= ARRAY OF 11 ANNULI WITH U(3.85) INSERTS $(3.8 \mathrm{cM} \mathrm{H})$

REPLICATE 0014 4R51.054 2R0.0 
Table A.2 (continued)

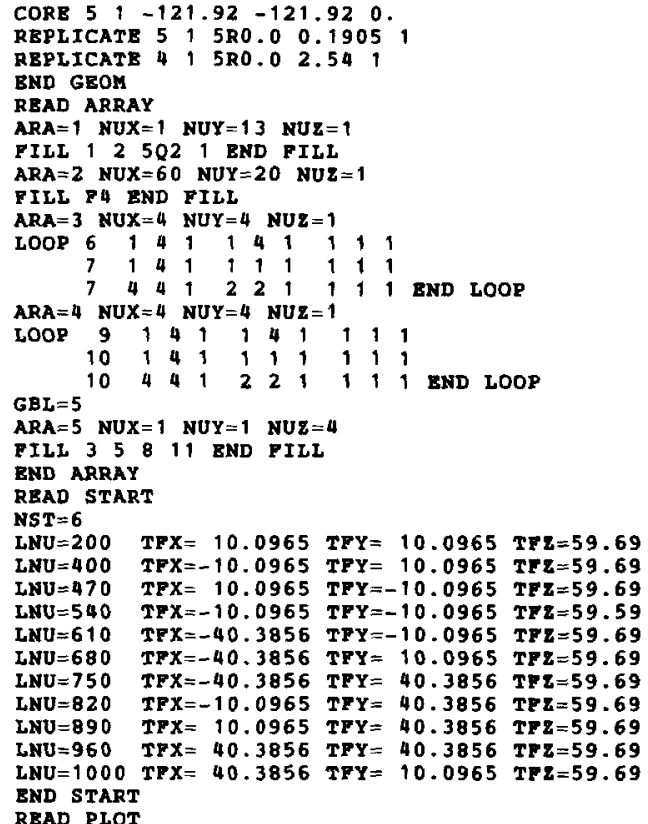

TTL="PLAN VIEW $1 / 6$ SCALE OP PALIET, $Z=$ =MIDLEVEL OP ANNULI/INSERTS" $X U L=-91.44 \quad Y U L=91.44 \quad Z U L=59.69$

$U A X=1$. VDN $=-1$. DLX $=1.524$ NCH=* U.WGSC* END

TTL="PLAN VIBW $1 / 6$ SCALB OF PALIET, $2=$ MIDLEVEL OF GRATING" $X U L=-91.44 \quad Y U L=91.44 \quad Z U L=20.0025$

$U A X=1$. $V D N=-1$, DLX $=1.524 \quad N C H=*$ U. WGSC $*$ END

TTL="PLAN VIEW 1/6 SCALE OF PALLET, $Z=$ MIDLEVEL OF PIR TIMBERS $X U L=-91,44 \quad Y U L=91.44 \quad 2 U L=9.2075$
$X L R=91.44$ Y YLR $=-91.44$ Q

UAX $=1$. VDN $=-1$. DLX $X=9.524$ NCH=* U. WGSC* BND

TTL="PLAN VIEW $1 / 10$ SCALE OF MOCKUP, $z=$ MIDLEVEL OP $U$ UNITS" $X U L=-137 . Y Y U L=137 . \quad 2 U L=59.69$
$X L R=137 . Y Y L R=-137 . \quad 2 L R=59.69$

UAX $=1$. YDN $=-1$, DLX $=2.54 \quad \mathrm{NCH}=*$ U. WGSC* END

TTL="PLAN VIEW $2 X$ SCALE OP SINGLE U UNIT, $Z=M$ MDLEVEL OF UNIT" $X L R=20.193=2 Y R=0, \quad L L=59.69$

UAX $=1$. VDN $=-1$. DLX $=0.127$ NCH $=*$ U. WGSC* END
TTL $=$ "CROSS SECTION OF PALLET 116 SCALE, $Y=10.0965$ " $X L R=91.44 \quad Y L R=10.0965$ aLR=-10. UAX $=1$. WDN $=-1$. DLX $=1.524$ NCH $=$ U. WGSC* END

TTL ="CROSS SBCTION OF MOCKUP $1 / 10$ SCALE, $Y=10.0965$ " $X L Q=137$. $Y Z R=10.0965$ ZUL $=113$.

UAX $=1$. WDN $=-1$. DIX $X=2.54 \quad \mathrm{NCH}=$ * U. WGSC* END

TTL = "CROSS SBCTION OF FLOOR/GRATING/PIR TIMBERS, PULL SCALE" $X U R=25$ Y $Y L R=0.2 L R=5.4$

$U A X=1$. WDN $=-1$. DLX $=.254$ NCH $=* U$ U. WGSC* END

TTL=" PLAN VIBW OP GRATING THRU TIEBARS, PULL SCALB $X L R=25,4 \quad Y L R=0, \quad L R=21,193$

UAX $=1$. VDN $=-1$. DLX $=.254$ NCH=* U. WGSC* END

TTL $="$ PLAN VIEW OF GRATING GRATING MIDLEVEL, PULL SCALB $X I R=25$, 4 YLR=0. $\quad \mathrm{Z} L \mathrm{~L}=20.0025$

UAX $=1$. VDN $=-1$. DLX $=.254$ NCH $=$ U. WGSC* END

TTL="PLAN VIBW OP TIMBERS O TIMBRR MIDLEVEL, FULL SCALE" $X I R=25,4 \quad Y L R=-25,4 \quad Z T R=9,2075$

UAX $=1$. VDN $=-1$. DLX $=.254 \quad \mathrm{NCH}=*$ U. WGSC $*$ END

TTL $=$ "CROSS SBCTION AT TOP OF UNITS, $2 X$ SCALE"

UAX $=1$. WDN $=-1$. DLX $=.127$ NCH=* U. WGSC* END END PLOT

END 
Table A.2 (continued)

CABO3

CASE EBJ.3X ( U(3.85) 24 7.2"/0"/1.5"/75, OCM, SQUARE)

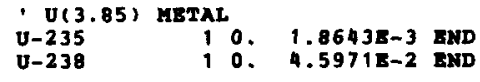

GULL DENSTTY WATER MODERATOR/REFLECTOR

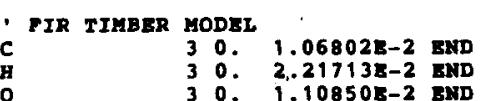

CARBEL FOR GRATING, TANK WALLS AND YLOOR

STAINLESS STEET LINING FOR TANR
SS 304

CASE $\mathbf{E B J} .3 \times$

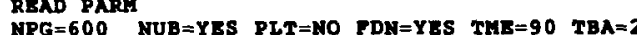

END PARM
READ GEOM

UNIT 1

COM=* PIR TIMBER $7.25 " \times 72 " \times 7.25 n " * 0$

UNIT 2

COM=*

2182.880 .8 .890 .18 .4150

UNIT ${ }^{3}$ COMERAY OF TIMBERS 72" $\times 72^{\prime \prime} \times 7.25{ }^{\prime \prime}$ PLUS 12" H2O *

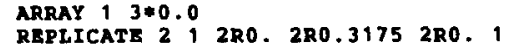

REPLICATE 2 2R0. 2R0.3175 2R0.

\section{UNIT 4}

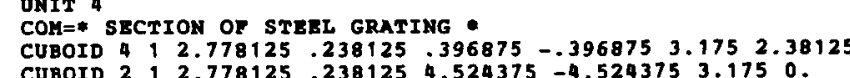

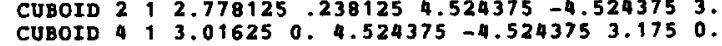

\section{UNIT 5}

COM=* ARRAY OF STEEI GRATING PLUS 12" H20 * REPLICATE 2 , 4 RO. 9525250.001

\section{UNIT 6}

COM=* U(3.85) ANNULUS YITH INS ERT $(75.0 \mathrm{cM} \mathrm{H})$ * CYLINDER 1 1 3.17575 .00

CYLINDER 213.30275 .00

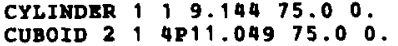

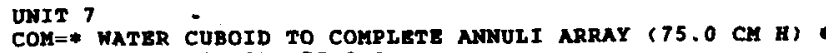
CUBOID 2 , 411.04975 .00

UNIT 8

COM= ARRAY OF 24 ANNULI WITH U(3.85) INSERTS $(75.0 \mathrm{cM}$ B) ARRAY $33 * 0.0$ REPLICATE 2 4R36.195 2RO.

UNIT 9

COM=* U(3.85) ANNULUS WTTH INSERT $(1.2 \mathrm{CM}$ 月)

CYYIINDER i 13.1751 .20 .

CYLINDER 0193.302 1.2

CUBOID 1 AP11.049 120 .

UNIT 10

COM=* VOID CUBOID TO COMPLETE ANNULI ARRAY $(1.2 \mathrm{cM} \mathrm{H})$ * CUBDID 01 AP11.049 1.20 .

UNIT 11

COM=* ARRAY OF 24 ANNULI MITH U(3.85) INSERTS $(1.2 \mathrm{cM} \mathrm{H})$ *

ARRAY $43 * 0.0$
REPLICATE 0
REPLICATE
O

CORE $51-121.92-121.920$.

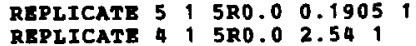

REPLICATE

ARA $=1$ NUX $=1$ NUY $=13 \quad N U E=1$

$\begin{array}{llllll}\text { PILLL } & 1 & 2 & 502 & 1 & \text { END } \\ \text { ARA ILI } & \text { PIUX }\end{array}$

FILL 74 BND PILL

ARA $=3 \quad$ NUX $=5 \quad$ NUY $=5 \quad$ NUZ $=9$

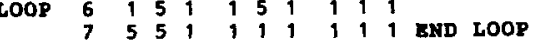

ARA $=4$
LOOP

LOOP $\begin{array}{rrrrrrrrrrr}9 & 1 & 5 & 1 & 1 & 5 & 1 & 1 & 1 & 1 & \\ 10 & 5 & 5 & 1 & 1 & 1 & 1 & 1 & 1 & 1 & \text { END LOOP }\end{array}$

$\begin{aligned} & \text { GBL }=5 \\ & A R A\end{aligned}=5$ NUX $=1 \quad$ NUY $=1 \quad$ NUZ $=4$

FILI $3 \quad 5 \quad 8 \quad 11$ END FILL

END ARRAY

READ STA

$X S M=-55.245 \quad X S P=55.245 \quad$ YSM=-55.245 YSP=55.245 \&SH=21.59 ZSP=97.79 RED START

TTL="PLAN VIEW $1 / 6$ SCALE OF PALLET, $8=$ MIDLEVEL OF ANNULI/INSERTS" $X U L=-91.44 \quad Y U L=91.44 \quad 2 U L=59.69$

XIR $=91.44$ YLR $=-91.44 \quad$ ZLR=59.69
UAX $=1$. VDN $=-1$. DLX $=1.524$ NCH $=*$ U. WGSC* END

TTL="PLAN VIEW 1/6 SCALE OF PALLET, $z=$ MIDLEVEL OF GRATING" $\mathrm{XUL}=-91.44 \quad \mathrm{YUL}=91.44 \mathrm{ZUZ}=20.0025$

$X L R=91.44$ YLR $=-91.44 \quad 2 L R=20.0025$
$U N X=1$. VDN $=-1$. DLX $X=1.524$ NCH $=*$ U. WGSC* END 
Table A.2 (continued)

TTL="PLAN VIEW 1/6 SCALE OF PALIET, $Z=$ MIDLEVEL OF PIR TIMBERS" $X U L=-91.44$ YUL $=91.44 \quad Z U L=9.2075$

XLR $=91.44$ YLR $=-91.44 \quad$ ZLR $=9.2075$
UAX $=1$. VDN $=-1$. DLX $=1.524 \quad$ NCH $=*$ U. WGSC* END

TTL="PLAN VIEW $1 / 10$ SCALE OF MOCKUP, $Z=$ MIDLEVEL OF U UNITS" $X U L=-137 . \quad Y U L=137 . \quad Z U L=59.69$

XLR=137. YLR $=-137.2 L R=59.69$
UAX $=1$. VDN $=-1$. DLX $=2.54$ NCH=* U. WGSC* END

TTL="PLAN VIBW $2 X$ SCALE OF SINGLE U UNIT, $Z=$ MIDLEVEL OF UNIT"

$X U L=-11.049$ YUL $=11.049 \quad \mathrm{ZUL}=59.69$

$X I R=11.049$ YLR $=-11.049$ ZLR $=59.69$
UAX $=1$. VDN $=-1$. DLX $=0.127$ NCH $=0$ U. WGSC* END

TTL $=$ "CROSS SBCTION OF PALLET $1 / 6$ SCALE, $Y=0.0$ "

$X U L=-91.44 \quad Y U L=0.0 \quad Z U L=113$.

$X L R=91.44$ YLR $=0.0$ PLR $=-18$.
$U A X=1$. WDN $=-1$. DLX $=1.524$ NCH=* U. WGSC* END

TTL $=$ "CROSS SBCTION OF MOCKUP $1 / 10$ SCALE, $Y=0.0$ "

$\mathrm{XUL}=-137 . \quad \mathrm{YUL}=0.0 \quad \mathrm{ZUL}=113$

$X L R=137$. YLR=0.0 $2 L R=-18$.
$U A X=1$. WDN $=-1$. DLX $=2.54$ NCH=* U. WGSC* END

TTL $=$ "CROSS SBCTION OF PLOOR/GRATING/FIR TIMBERS, FULL SCALE"

$X U L=0 . \quad Y U L=0, \quad 2 U L=24,13$

$X L R=25.4$ YLR $=0 . \quad$ ZLR $=-5.4$
$U A X=1$. WDN $=-1$. DLX $=.254^{4} \mathrm{NCH}=*$ U. WGSC* END

TTL $="$ PLAN VIBW OF GRATING THRU TIEBARS, FULI SCALE

$X U L=0 . \quad Y U L=25.4 \quad 2 U L=21.193$

$X L R=25.4 \quad Y L R=0 . \quad 2 L R=21.193$
$U A X=1 . \quad$ VDN $=-1 . \quad$ DLX $=.254$ NCH=* U. WGSC $\quad$ END

TTL="PLAN VIBW OF GRATING O GRATING MIDLEVEL, FULL SCALE"

$X U L=0, Y U Z=25,4 \quad Z U L=20.0025$

$X I R=25.4$ YLR $=0, \quad Z L R=20.0025$
UAX $=1$. VDN $=-1$. DLX $=.254$ NCH=*U. UGSC* END

TTL $=$ "PLAN VIRW OF TIMBERS TIMBER MIDLEVEL, FULL SCALE"

UAX $=1$. VDN $=-1$. DLX $=.254$ NCH $=*$ U. WGSC* END

TTL $=$ "CROSS SECTION AT TOP OP UNITS, $2 X$ SCALE"

$X U L=-11.049$ YUL $=0 . \quad 2 U L=105$.
$X L R=11.049$ Y

UAX $=1$. WDN $=-1$. DLX $=.127$ NCH $=*$ U. WGSC $*$ END

END PLOT
END DATA
CAB07

CASB EBJ.4 U(3.85) 16 e $7.2 n / 0 " / 0.25 n / 79.3 \mathrm{CM}$, TRIANGULAR

27GROUPNDF 4 INFHOMMEDIUM

U(3.85) METAL

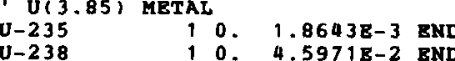

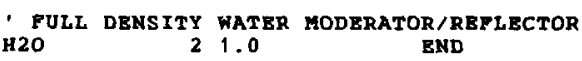

PIR TIMBE MODEL

$\begin{array}{lllll}C & \text { PIR TIMBER MODEL } & & \\ \text { C } & 30 . & 1.06802 \mathrm{E}-2 & \mathrm{END} \\ \mathrm{H} & 3 & 0 . & 2.21713 \mathrm{E}-2 & \mathrm{END} \\ 0 & 3 & 0 . & 1.10850 \mathrm{E}-2 & \mathrm{END}\end{array}$

C STEEL FOR GRATING, TANK WALLS AND FLOOR

SS STAMINLESS STEEL LINING FOR TANK

$$
\begin{aligned}
& 51.0 \\
& 5 \text { TEED LINING FOR TANK }
\end{aligned}
$$

CASE BBJ.4 U(3.85) 16 ?.2n/0"/0.25"/79.3CM, TRIANGULAR

RBA

READ GBOM

UNIT 1

COM=* FIR TIMBER 7.25" $\times 72^{\prime \prime} \times 7.25^{n} *$

UNIT 2

COM=* WATER GAP $3.5^{n} \times 72^{n} \times 7.25^{n}$ BETHEEN TIMBERS *
CUBOID 21182.880 .88 .890 .18 .4150 .

UNIT 3

COM=* ARRAY OF TIMBERS 72" $\times 72^{*} \times 7.25 " *$

$\begin{array}{llll}\text { ARRAY } 13 * 0.0 \\ \text { REPLICATE } 2 & 1 & 2 R 0.2 R 0.3175 \text { 2RO. } 1\end{array}$

UNIT "

CUBOID $412.778125 \cdot 238125,396875-396875 \quad 3.1752 .38125$

CUBOID $212.778125 .238125 .396075-.39687503 .1752$

UNIT 5

COM=* ARRAY OF STEEL GRATING *

ARRAY $23 * 0.0$

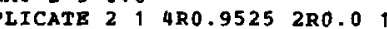

UNIT 6

COM=* U(3.85) ANNULUS WTTH INSERT *

CYLINDER $11113.175 \quad 76.20$.

CYLINDER $213.302 \quad 76.20$. 
Table A.2 (continued)

UNIT 7

COM= ARRAY OF 16 ANNULI WITH U(3.85) INSERTS *

CUBOID 214 4P91.44 79.3 O.

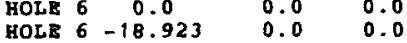

$\begin{array}{lll}-9.4615 & 16.3878 & 0.0\end{array}$

HOLE $6 \quad 9.4615 \quad 16.3878 \quad 0.0$

HOLE $618.923 \quad 0.0 \quad 0.0$

HOLE $6 \quad-9.4615-16.38780 .0$

HOL8 $6-28.3845 \quad 16.3878 \quad 0.0$

$\begin{array}{llll}\text { HOLE } 6 & 0.0 & 32.7756 & 0.0\end{array}$

$\begin{array}{lllll}\text { HOLE } & 6 & 28.3845 & 16.3878 & 0.0 \\ \text { HOLE } & 6 & 37.846 & 0.0 & 0.0\end{array}$

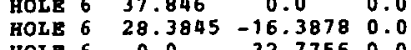

$\begin{array}{lllll}\text { HOLE } & 6 & 0.0 & -32.7756 & 0.0 \\ \text { HOLE } & 6 & -18.923 & -32.7756 & 0.0 \\ \text { HOLE } & 6 & -28.3845 & -16.3878 & 0.0\end{array}$

CORE $31-91.44-91.440$.

REPLCATE 5 TRO.0 0.19051

REPLICATE 411580.02 .541

END GEOM

READ ARRAY

$A R A=1 \quad$ NUX $=1 \quad$ NUY $=13 \quad$ NUZ $=1$

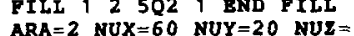

FILL F4 END FILL

$G B L=3$

$A R A=3 \quad$ NUX $=1 \quad$ NUY $=1 \quad$ NUZ $=3$

ILL 335 ENA FILI

READ START

RST $=1$
$X S H=-37.5285 \quad X S P=46.99$

YSM=-41.9196 YSP $=41.9196$

END START

TTL="PLAN VIEW 1/6 SCALE OF CORE, Z=MIDLEVBL, OP ANNULI/INSERTS" $\mathrm{XUL}=-91.44 \quad \mathrm{YUL}=91.44 \quad 2 \mathrm{UL}=59.69$

$X L R=91.44$ YLR $=-91.44 \quad 2 L R=59.69$
$U A X=1$. VDN $=-1$. DLX $X=1.524$ NCH $=*$ U.WGSC* END

TTI=" PLAN VIEW $1 / 6$ SCALE OF CORE, $Z=$ MIDLEVEL OF GRATING"

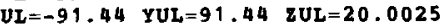

$X L R=91.44$ YLR $=-91.44 \quad 2 L R=20.0025$
$U N X=1$. VDN $=-1$. DL $X=1.524$ NCH $=*$ U. WGSC* END

TTI="PLAN VIBW $1 / 6$ SCAIE OP CORE, $Q=$ MIDLBVEL OF FIR TIMBERS" $X U L=-91.44 \quad Y U L=91.44 \quad 2 U L=9.2075$

$X L R=91.44$ YLR $=-91.44 \quad 2 L R=9.2075$
$U A X=1$. VDN $=-1$. DLX $=1.524$ NCH=* U. WGSC* BND

TTI="PLAN VIEW $1 / 10$ SCALE OF MOCKUP, $\&=$ MIDLEVEL OF U UNITS" $X U L=-121.92$ YUL $=121.92 \quad 2 U L=59.69$

UAX $=1$. VDN $=-1$. DLX $=2.54$ NCH=* U. WGSC* END
TTL=" PLAN VIEW FULL SCALE OF SINGLE U UNIT, $Z=M I D L B V E L$ OF UNIT" $X I R=16.75$ YLR $=-16.75$. $21 \mathrm{R}=59.69$

UAX $=1$. VDN $=-1$. DL $X=0.254$ NCH=* U. WGSC* END

TTL $=$ "CROSS SECTION OF CORE $1 / 6$ SCALE, $Y=0.0$ $X U L=-91.44 \quad$ YUL $=0.0 \quad$ ZUL $=102$

$U A X=1$. WDN $=-1$. DLX $=1.524$ NCH=* U. WGSC* END

TTL $=$ "CROSS SECTION OF MOCKUP $1 / 10$ SCALE, $Y=0.0$ " $X U L=-121.92$ YUL $=0.0 \quad 2 U L=105$.

$U A X=1$. WDN $=-1$. DLX $=2.54 \quad \mathrm{NCH}=*$ U. WGSC* END

TTL="CROSS SECTION OF GRATING/FIR TIMBERS, FULL SCALE"

$X U L=0 . \quad Y U L=0 . \quad 2 U L=24,13$

$U A X=1$. WDN $=-1$, DLX $=.254$ NCH=* U. WGSC* END

TTL="PLAN VIEW OF GRATING THRU TIEBARS, FULI SCALE"

$X U L=0 . \quad Y U L=25,4 \quad Z U L=21.193$

UAX $=1$. VDN $=-1$. DLX $=.254$ NCH $=*$ U. WGSC* END

TTL="PLAN VIEW OP GRATING G GRATING MIDLEVEL, FULL SCALP $X U L=0$. $Y U L=25.4$ ZOL $=20.0025$

UAX $=1$. VDN $=-1$, DLX $=.254 \quad$ NCH $=$ U. WGSC* BND

TTL="PLAN VIEW OP TIMBERS A TIMBER MIDLEVEL, TULI SCALE" $X U L=0$. $Y U L=25.4 \quad Z U L=9.2075$

$\mathrm{VDN}=-1 . \quad \mathrm{DLX}=.254$ NCH=* U. HGSC* END

END PLOT 
Table A.2 (continued)

CABOB

CASE EBJ.5X (U(3.85) $7.2 * / 0 n / 1.0 \% / 53.1 \mathrm{CM}$, TRIANGULAR)

- U(3.85) META

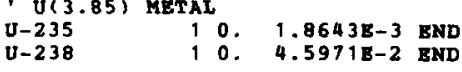

HOLL DENSITY YATER MODERATOR/RETLECTOR

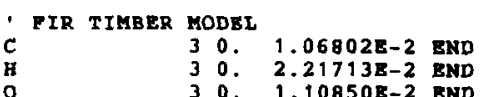

STEEL FOR GRATING, TANK WAILS AND FLOOR
CARBONSTEEL 4 BND

STAINLESS STEEL IINING FOR TANK
SS 304

BND COMP

CASE EBJ. $5 X$

TBA=3 NPG $=600$ NUB $=$ YBS PLT=NO PDN $=$ YBS TME $=90$

BND PARM

UNIT 1

COM=* PIR TIMBER $7.25 n \times 72^{n} \times 7.25 n *$

UNIT 2 WATER GAP 3.5" $\times 72 " \times 7.25 "$ BETWEEN TIMBERS -

8.890 .18 .4150

UNIT 3

COM=* ARRAY OF TIMBERS 72" $\times 72^{\prime \prime} \times 7.25$ "

REPLICATE 2 2 2R0. 2RO 3175 2RO.

REPLICATE $2,4 R 30.48280 .0$

UNIT 4

COM=* SBCTION OF STEBL GRATING *

$\begin{array}{lllllll} & \end{array}$ CUBOID $212.778125 \quad .238125 \quad 4.524375 \quad-4.524375 \quad 3.1750$.

CUBOID $13.016250 .4 .524375-4.5243753 .1750$

UNIT 5

ARRAY OP STEEL GRATING *

ARRAY 2 3*0.0

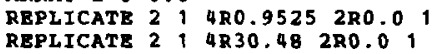

UNIT 6

COM=* U(3.85) UNIT, RLOODED, H=1.905, LEVELS 1 AND $3 *$

CYLINDER $119.175,1.9050$

$\begin{array}{lllll}\text { CYLINDER } & 1 & 3.302 & 1.905 & 0 \\ \text { CYLINDER } & 1 & 9.144 & 1.905 & 0\end{array}$
UNIT 7

COM=* ARRAY OF ? UNITS, PLOODED, H=1,905, LEVELS 1 AND 3 *

CUBOID 314 4P91.44 1.9050 .

HOLE 60.000 .00 .0

$\begin{array}{llrrr}\text { HOLB } & 6 & -10.414 & 18.037577 & 0.0 \\ \text { HOLE } & 6 & 10.414 & 18.037577 & 0.0\end{array}$

HOLE $6 \quad 20.828 \quad 0.0 \quad 0.0$

HOLE $6-10.414-18.0375770 .0$

REPLICATE 214 AR30.48 2R0.0

UNIT 8

COM=* U(3.85) UNIT, FLOODED, H=35.2425, LEVEI 2 .

年

$\begin{array}{llllll}\text { CYLINDRR } & 2 & 1 & 3.302 & 35.2425 & 0 . \\ \text { CYLINDER } & 1 & 1 & 9.144 & 35.2425 & 0 .\end{array}$

UNIT 9

COM=* ARRAY OF 7 UNITS, FLOODED, H=35.2425, LEVBL 2 ,

CUBOID 214 P91.44 35.24250 .

$\begin{array}{llll}\text { HOLE } 8 & 0.0 & 0.0 & 0.0\end{array}$

HOLE $8-20.828 \quad 0.0$

HOLE $8 \quad 10.414 \quad 18.037577 \quad 0.0$

HOLE $820.828 \quad 0.0 \quad 1870.0$

HOLE $8 \quad 10.414-18.037577 \quad 0.0$

HOLE $8-10.414-18.0375770 .0$

UNIT 10

COM=* U(3.85) UNIT, FLOODED, H=14.0475, LEVEL 4 *

CYLINDER 2 2 $13.302 \quad 14.04750$.

UNIT 11

COM=* ARRAY OF 7 UNITS, FLOODED, H=14.0475, LEVEI 4 *

HOLE 10210.01 .4414 .04750$.

HOLE $10-20.828 \quad 0.0$

HOLE $10-10.414 \quad 18.0375770 .0$

HOLE $10 \quad 10.414$ 18.037577 0.0

HOLE 10 10.414 -18.0375770 .0$

HOLE $10-10,414-18.0375770 .0$

REPLICATE 2 1 4R30.48 280.0 ?

UNIT 12

COM=* U( 3.85 ) UNIT, DRY, H=11.9875, LEVEL 5 *

OER 13.17511 .98750$.

$\begin{array}{lllllll}\text { CYLINDER } & 0 & 1 & 3.302 & 11.9875 & 0 . \\ \text { CYLINDER } & 1 & 1 & 9.144 & 11.9875 & 0 .\end{array}$ 
Table A.2 (continued)

UNIT 13 CUBOID O 1 4P91.44 11.98750

HOLE $12 \quad 0.000 .0 \quad 0.0$

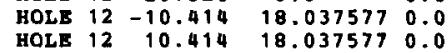

$\begin{array}{lllll}\text { HOLE } & 12 & 10.414 & 18.03 & 12 \\ \text { HOLE } & 12 & 20.828 & 0.0 & 0.0\end{array}$

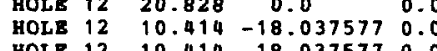

REPLICATE $0,4830.48$ 2RO. $0^{\circ} i^{\circ}$

UNIT 14

COM=* U(3.85) UNIT, DRY, H=1.905, LEVEL 6 * CYLINDBR $0,3.302,9050$.

CYLINDER $119.144 \quad 1.9050$.

\section{UNIT 15}

COM=* ARRAY OF 7 UNITS, DRY, H=1.905, LEVEL 6 *

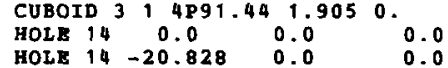

$\begin{array}{lllll}\text { HOLE } & 14 & -20.828 & 0.0 & 0.0 \\ \text { HOLB } & 14 & -10.414 & 18.037577 & 0.0 \\ & & \end{array}$

HOLE $14 \quad 10.414$ 18.037577 0.0

HOLE $14 \quad 10.414-18.0375770 .0$

HOLB $14-10.414-18.037577$
REPLICATB $0.14830 .48 \quad 280.0$

UNIT 16
COM=* U( 3.85$)$ UNIT, DRY, H=9.2075, LEVEL,

CYLINDER I $19.175,9.20750$.

$\begin{array}{lllllll}\text { CYLINDER } & 0 & 1 & 3.302 & 9.2075 & 0 . \\ \text { CYL INDER } & 1 & 1 & 9.144 & 9.2075 & 0 .\end{array}$

\section{UNIT 17}

COM=* ARRAY OF 7 UNITS, DRY, H=9.2075, IEVEL 7 *

CUBOID 014 P 91.449 .2075

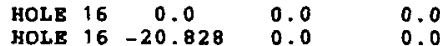

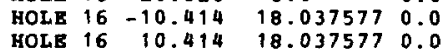

$\begin{array}{lcccc}\text { KOLE } & 16 & 10.414 & 18.037577 & 0.0 \\ \text { HOLE } & 16 & 20.828 & 0.0 & 0.0\end{array}$

HOLE $16-10.414-18.0375770 .0$

HOLE $16-10.414-18.037577$
REPLICATE $0.14 R 30.48 \quad 280.0$

CORE $3 \quad 1-121.92-121.920$.

REPLICATE 5 S 1980.00 .1905

END GEOM

READ ARRAY

$A R A=1 \quad N U X=1 \quad N U Y=13 \quad N U Z=1$

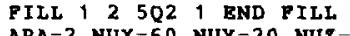

NUY $=20$ NUZ $=$

54 END FILI

$A R A=3$ NUX $=1 \quad$ NUY $=1 \quad$ NUZ $=9$

ARA $=3$ NUX-1 NUY $=1 \quad$ NUZ $=9$
FILL $35797 \quad 11 \quad 13 \quad 15 \quad 17$ RND FILL

\section{READ START \\ $X S M=-20.828 \times S P=20.828$ \\ $\begin{array}{ll}X S M=-20.828 & \text { YSP }=20.828 \\ Z S M=21.59 & \mathrm{ZSP}=74.69\end{array}$ \\ END START}

TTL="PLAN VIEW $1 / 6$ SCALE OP CORE, $Z=M$ MDLEVEL OP ANNULI/INSERTS $X U L=-100 . \quad Y U L=100 . \quad 2 U L=59.69$

$X L R=100$. YLR $=-100 . \quad 2 L R=59.69$

TTL ="PLAN VIEW $1 / 6$ SCALE OP CORB, $z=$ MIDLEVEL OF GRATING"

$X U L=-100, Y U L=100 . \quad 2 U L=20.0025$

$X L R=100, Y L R=-100.2 L R=20.0025$
UAX $=1$. VDN $=-1$. DLX $=1.524$ NCH $=*$ U. WGSC* BND

TTI="PLAN VIEN $1 / 6$ SCALE OF CORE, $\mathrm{z}=$ MIDLEVEL OF PIR TIMBERS" XUL $=-100 . \quad Y U L=100 . \quad 2 U L=9.2075$

XLR $=100$, YLR $=-100,2 L R=9.2075$

TTL="PLAN VIEW $1 / 10$ SCALE OF MOCXUP, $z=$ MIDLEVEL OF U UNITS $X U L=-130 . \quad Y U L=130 . \quad 2 U L=59.69$

$X L R=130, Y L R=-130 . \quad 2 L R=59.69, \quad U$ WGSC * END

TTL="PLAY VIBH, PULT SCALE AT CENTER OF ARRAY, $Z=M I D L E V B L$

$X U L=-16.75$ YUL $=16.75 \quad 2 U L=59.69$

$X L R=16.75$ YLR $=-16.75$ \&LR=59.69

TTL="CROSS SECTION OF CORE $1 / 6$ SCALE, $Y=0.0^{\circ}$

$X U \mathrm{~L}=-100 . \quad Y U \mathrm{~L}=0.0 \quad \mathrm{zUL}=102$

$X L R=100, \quad Y L R=0.0 \quad 2 L R=0$.

TTL $=$ "CROSS SECTION OF MOCKUP $1 / 10$ SCALE, $Y=0,0$ "

$X O L=-130 . \quad Y U L=0.0 \quad Z U L=105$.

$X L R=130$. YLR $=0.0 \quad \geq L R=-65$
WAX $=1$, WDN $=-1$. DLX $=2.54$ NCH $=*$ U. WGSC* END

TTL ="CROSS SECTION OF GRATING/FIR TIMBERS, FULL SCAIE"

$X U L=0 . \quad Y U L=0 . \quad 2 U L=24,13$

$X L R=25.4 \quad$ YLR $=0 . \quad 2 L R=-5.0$
UAX $=1$. WDN $=-1$. DIXX $=.254^{\circ}$ NCH $=*$ U. WGSC* END

TTL="PIAN VIBW OP GRATING THRU TIBBARS, FULL SCALE"

$X U L=0 . \quad Y U L=25.4 \quad 2 U L=21.193$

$X L R=25.4 \quad$ YLR =0. $\quad$ ZLR $=21.193$
$U A X=1$. VDN $=-1 . \quad$ DLX $=254 \quad$ NCH=* U. WGSC* END

TTL="PLAN VIEW OF GRATING O GRATING MIDLEVEL, PULL SCALE"

TTL $=" P L A N$
$X U L=0 . \quad$ YUL $=25.4 \quad$ ZUL $=20.0025$

XLR $=25.4 \quad$ YLR $=0 . \quad$ ZLR $=20.0025$
UAX $=1$. VDN $=-1 . \quad$ DLX $=.254 \quad$ NCH $=*$ U. WGSC* END

TTL="PLAN VIEW OF TIMBERS T TIMBER MIDLEVEL, FULL SCALE"

$X U L=0$. YUL $=25.4 \quad 2 U L=9.2075$

$X I R=25.4$ YLR $=-25.4 \quad 2 L R=9.2075$
UAX $=1$. VDN $=-1$. DLX $=.254$ NCH $=*$ U. WGSC* END 
Table A.2 (continued)

TTL $=$ "CROSS SBCTION OF CBNTRAL UNIT, $2 X$ SCALE" $X U L=0.0 \quad Y U L=0.0 \quad$ QUL $=102$
$X L R=15.24 \quad$ YLR $=0,0 \quad$ ZLR

UAX $=1$. WDN $=-1$. DLX $=0.127$ NCH $=*$ U. WGSC * END

\section{END PLOT
END DATA}

END

\section{CABO9}

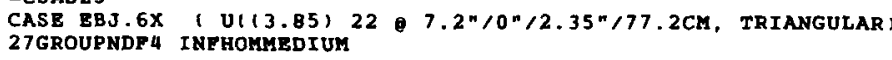

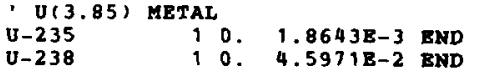

H2O PULL DENSITY WATER MODERATOR/REFLECTOR
HEN
1.0

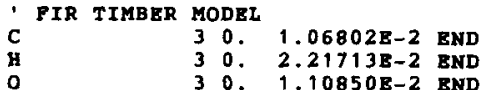

STBEL POR GRATING, TANK WALLS AND FLOOR
CARBONSTEEL 4.0

STAINLESS STBEL LINING FOR TANK
SS304
51.0

END COMP

CASB EBJ. $6 x$

TBA $=3 \quad \mathrm{NPG}=600 \quad \mathrm{NUB}=\mathrm{YES} \quad \mathrm{PLT}=\mathrm{NO} \quad \mathrm{FDN}=$ YBS $\quad \mathrm{TME}=90$

RND PARM

UNIT 1

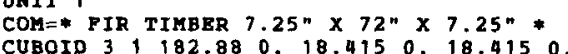

UNIT 2

COM=* WATER GAP $3.5 " \times 72 " \times 7.25$ " BETHEEN TIMBERS -

UNIT
COM $=*$ ARRAY OF TIMEERS $72 " \times 72 " \times 7.25 " *$

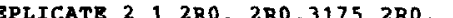

REPLICATE 214 4R30.48 2RO.0

UNIT 4

SECTION OF STEEI GRATING

CUBOID $4 \quad 1 \quad 2.778125 \quad .238125 \quad .396875 \quad-.396875 \quad 3.175 \quad 2.38125$

CUBOID $2142.778125 .238125 \quad 4.524375 \quad-4.524375 \quad 3.1750$.

CUBOID $413.016250 .4 .524375-4.5243753 .1750$.
UNIT 5

ARPAY 2 3*0 OP STEEL GRATING

REPLICATE 2
REPLICATE $2,4 R 0.95252 R 0.0$
$4 R 30.48280 .0$

UNIT 6

COM=* U(3.85) UNIT, PLOODED, $H=1.905$, LEVELS 1,3 , AND 5 . CYLINDER +1 T $3.1759 .905 \%$

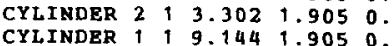

UNIT $?$

COM=* ARRAY OF 22 UNITS, PLOODED, H=1.905, LEVELS 1, 3, AND 5 . CUBOID 31 4 P91.44 1.9050 .

$\begin{array}{llll}\text { HOLE } 6 & 0.0 & 0.0 & 0.0\end{array}$

HOLE $6-12.1285 \quad 21.0071780 .0$

HOLB $6 \quad 12.1285 \quad 21.007178 \quad 0.0$

HOLE 6 24.257 $0.0 \quad 0.0$

HOLE $6-12.1285-21.0071780 .0$

HOLE $6-36.3855-21.0071780 .0$

HOLE $6-48.514 \quad 0.0 \quad 0.0$

HOLE $6-36.3855 \quad 21.0071780 .0$

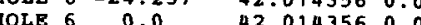

HOLE $6 \quad 24.257 \quad 42.0143560 .0$

HOLE 6 $36.3855 \quad 21.0071780 .0$

(1) 0.0

HOLE $6 \quad 24.257 \quad-42.0143560 .0$

HOLE $60.0 \quad-42.0143560$

HOLE $6-24.257-42.014356 \quad 0.0$

HOLE $6-48.514-42.014356 \quad 0.0$

HOLE 6 60.6425 -21.0071780 .0
REPLICATE 214 R $30.48280 .00^{\circ}$

UNIT 8

COM $=*$ U( 3.85 ) UNYT, PLOODRD, H=35.2425, LEVEL $2 *$ CYIINDER $2,3.30235 .24250$.

CYLINDER 119.14435 .24250$.

UNIT 9

COM=* ARRAY OF 22 UNITS, FLOODED, H=35.2425, IEVEI 2 "

CUBOID 214 P91.44 35.24250.

$\begin{array}{llll}\text { HOLE } 8 & 0.0 & 0.0 & 0.0\end{array}$

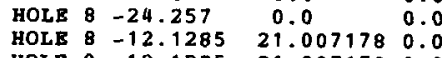

$\begin{array}{llll}\text { HOLE } & 12.1285 & 21.007178 & 0.0 \\ \text { HOLE } & 24.257 & 0.07178 & 0.0\end{array}$

HOLE $8 \quad 12.1285-21.0071780 .0$

HOLE $8-12.1285-21.007178 \quad 0.0$

HOLE $8-48.514 \quad-21.0071780 .0$

$\begin{array}{lllll}H O L E & 8 & -36.3855 & 21.007178 & 0.0\end{array}$

$\begin{array}{llll}\text { HOLE } 8 & -24.257 \quad 42.014356 & 0.0\end{array}$

$\begin{array}{ccccc}\text { HOLE } 8 & 0.0 & 42.014356 & 0.0 \\ \text { HOLE B } & 24.257 & 42.014356 & 0.0\end{array}$

$\begin{array}{llll}\text { HOLE } 8 & 24.257 & 42.014356 & 0.0\end{array}$ 
Table A.2 (continued)

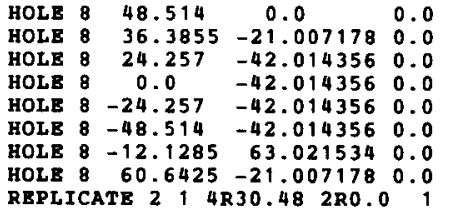

UNIT 10

COM=* U(3.85) UNIT, FLOODED, H=26.035, LEVEL 4 $\begin{array}{lllllll}\text { CYLINDER } & 1 & 1 & 3.175 & 26.035 & 0 . \\ \text { CYLINDER } & 2 & 1 & 3.302 & 26.035 & 0 .\end{array}$

$\begin{array}{llllll}\text { CYLINDER } & 2 & 1 & 3.302 & 26.035 & 0 \\ \text { CYLINDER } & 1 & 1 & 9.144 & 26.035 & 0\end{array}$

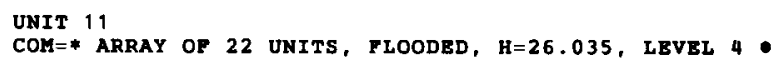

CUBOID 214 P91.44 26.035 0 .

HOLE 100.0 0 $0.0 \quad 0.0$

$\begin{array}{lllll}\text { HOLB } & 10 & -24.257 & 0.0 & 0.0 \\ \text { HOLB } & 10 & -12.1285 & 21.007178 & 0.0\end{array}$

HOLE $10 \quad 12.1285 \quad 21.0071780 .0$

$\begin{array}{lllll}\text { HOLE } & 10 & 24.257 & 0.0 & 0.0\end{array}$

HOLE $100 \quad 12.1285-21.0071780 .0$

HOLE $10-12.1285-21.0071780 .0$

HOLE $10-46.514-21.0071780 .0$

HOLE $10-36.3855 \quad 21.007178 \quad 0.0$

HOLE $10-24.257 \quad 42.014356 \quad 0.0$

HOLE $10 \quad 0.0 \quad 42.014356 \quad 0.0$

HOLE 10 24.257 42.0143560 .0

HOLE 1036.385521 .007780 .0

HOLE $10 \quad 36.3855 \quad-21.007178 \quad 0.0$

HOLE $10 \quad 24.257 \quad-42.014356 \quad 0.0$

HOLE $1000.07-42.0143560 .0$

HOLE $10-24.257 \quad-42.0143560 .0$

HOLE $10-12.1285 \quad 63.0215340 .0$

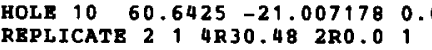

UNIT 12

U(3.85) UNIT, FLOODED, H=9.2075, LEVEL 6 *

CYLINDER 113.175 , 9.207500

CYLINDER 2 2 13.302 3.20750 .

CYLINDER 119.1449 .20750

UNIT 13

COM=* ARRAY OF 22 UNITS, FLOODED, $H=9.2075$, LEVEI 6 * CUBOID 214 P91.44 9.20750 .

HOLE $12 \begin{array}{llll}12 & 0.0 & 0.0 & 0.0\end{array}$

HOLE $12-24.257 \quad 0.0 \quad 0.0$

HOLE $12-12.285521 .007178 \quad 0.0$

HOLE $12 \quad 24.257 \quad 0.071780 .0$

HOLE $12 \quad 12.1285 \quad-21.007178 \quad 0.0$

HOLE $12-12.1285-21.007178 \quad 0.0$

HOLE $12-36.3855-21.0077780 .0$

$\begin{array}{lllll}\text { HOLE } & 12 & -48.514 & 0.0 & 0.0 \\ \text { HOLE } & 12 & -36.385 & 21.007178 & 0.0\end{array}$

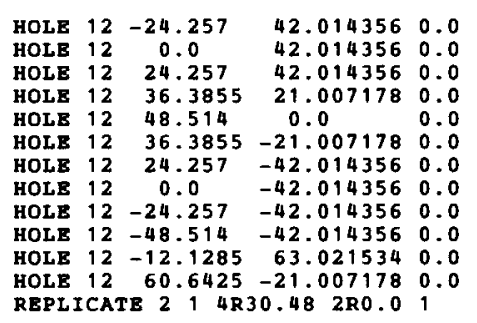

CORE $31-121.92-121.920$.

REPLICATE 2 1 4 RO. 0 1.0 0.0

REPLICATE 5 1 5 RO.0 0.1905

REPLICATE

ARA $=1$ NUX $=1 \quad$ NUY $=13 \quad$ NUZ $=1$

$A R A=2 \quad N U X=60 \quad$ NUY $=20 \quad$ NUZ $=1$

PILL F4 END FILL

$\begin{aligned} & G B L=3 \\ & A R A=3\end{aligned} \quad \mathrm{NUX}=1 \quad \mathrm{NUY}=1 \quad \mathrm{NUZ}=8$

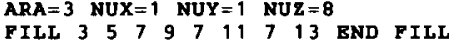

END ARRAY

READ STARI

NST $=1$

$X S M=-57.658 \quad X S P=57.658$

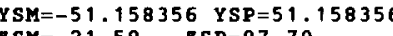

END START

$T T L=" P L A N$ VIEW $1 / 6$ SCALE OF CORE, $Z=M$ IDLEVEL OP ANNULI/INSBRTS" $X U L=-100 . \quad Y U L=100 . \quad 2 U L=59.69$

UAX $=1$. VDN=-1. DLX $=1.524$ NCH=* U. WGSC* END

TTL="PLAN VIEW $1 / 6$ SCALE OF CORE, $Z=M I D L B V B L$ OF GRATING"

$X U L=-100 . Y U L=100 . \quad Z U L=20.0025$

UAX $=1$. VDN=-1. DLX $X=1.524$ NCH $=*$ U. WGSC* END

TTL="PLAN VIEW 1/6 SCALE OF CORE, Z=MIDLBVEL OF FIR TIMBERS

$X U L=-100$. $Y U L=100 . \quad Z U L=9.2075$

$X L R=100$ Y YLR $=-100$
UAX $=1$. VDN $=-1$. DLX $=1.524 \quad$ NCH=* U. WGSC* END

TTL="PLAN VIBW 1/10 SCALE OF MOCKUP, \&=MIDLEVEL, OF U UNITS" $X U L=-130$. YUL $=130.2 U L=59.69$

$X L R=130, Y L R=-130, \quad$ ZLR $=59.69$
$\mathrm{UAX}=1$, VDN $=-1$, DLX $=2.54 \quad \mathrm{NCH}=*$ U. WGSC* END

TTL=" PLAN VIBW, PULL SCALE AT CENTER OF ARRAY, $Z=$ MIDLEVEL * $X U L=-16.75 \quad Y U L=16.75 \quad 2 U L=59.69$

$X L R=16.75$ YLR $=-16.75 \quad$ \&LR $=59.69$. 
Table A.2 (continued)

TTL $=$ "CROSS SECTION OF CORE $1 / 6$ SCALE, $Y=0.0$ " $X U L=-100$. YUL $=0.0 \quad 2 U L=102$

XLR $=100$. YLR=0.0 $2 L R=0$
UAX $=1$. WDN $=-1$. DLX $=1.524$ NCH=* U. WGSC* END

TTL $=$ "CROSS SECTION OF MOCKUP $1 / 10$ SCALE, $Y=0.0$ $X U L=-130 . \quad Y U L=0.0 \quad 2 U L=105$.

$X L R=130$. YLR $=0.0 \quad 2 L R=-65$
$U A X=1$. WDN $=-1$. DLX $=2.54$ NCH=* U. WGSC* END

TTL="CROSS SBCTION OF GRATING/FIR TIMBERS, PULL SCALB"

$X U L=0 . \quad Y U L=0 . \quad 2 U L=24.13$

UAX $=1$. WDN $=-1$. DLX $=.254$ NCH $=$ U. WGSC* END

TTL=" PLAN VIEH OF GRATING THRU TIBBARS, FULL SCALE"

$X U L=0 . \quad Y U L=25.4 \quad 2 U L=21.193$

$X L R=25.4$ YLR $=0$. ZLR $=21.193$
UAX $=1$ VDN $=-1$. DLX $=.254$ NCH=* U. WGSC* END

TTL="PLAN VIEW OF GRATING G GRATING MIDLEVEL, FULL SCALE" $X U L=0 . \quad Y U L=25.4 \quad 2 U L=20.0025$
$X L R=25.4 \quad Y L R=0 . \quad 2 L R=20.0025$

$X L R=25.4 Y L R=0.2 L R=20.0025$. $U$.

TTI="PLAN VIEH OF TIMBERS A TIMBER MIDLEVEL, PULI SCALE" $X U L=0 . \quad Y U L=25.4 \quad 2 U L=9.2075$

END

TTL = "CROSS SECTION OF CENTRAL UNIT, $2 X$ SCALE" $X U L=0.0 \quad$ YUL $=0.0 \quad 2 U L=102$.

$X L R=15.24 \quad$ YLR $=0.0 \quad 2 L R=-5$.
$U A X=1$. WDN $=-1$, DLX $=0.127$ NCH $*$ U. WGSC* END

END PLOT
BND DATA

END DAT
CAB 10
$=$ CSAS 25 CASE EBJ.8 U( 3.85$) 6$ e $7.2 \% / 2.6 " / 0.75 \% / 91.4 \mathrm{CM}$, SQUARE
27GROUPNDF4 INFHOMMEDIUM

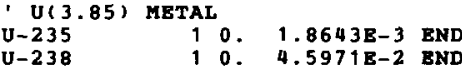

PULL DENSITY WATER MODERATOR/RBFLECTOR

FIR TIMBER MODBL

$\begin{array}{lll} & \\ 30 D E L & 1.06802 \mathrm{E}-2 & \mathrm{END} \\ 30 . & 2.21713 \mathrm{E}-2 & \mathrm{END}\end{array}$

CARTERE FOR GRATING, TANK WALIS AND FLOOR

STAINLESS STEEL LINING FOR TANK
SS 304

END COMP 8 U(3.85) 6 ?.2n/2.6n/0.75"/91.4CM, SQUARE

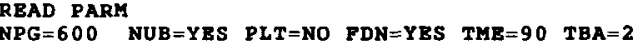

END PARM

READ GBOM

UNIT 1

COM=* PIR TIMBER $7.25 n \times 72^{n} \times 7.25^{n *} *$

UNIT 2

UNIT 2 COM=* WATER GAP $3.5^{n} \times 72^{n} \times 7.25 "$ "BETWEEN TIMBERS *

UNIT 3

COM=* ARRAY OF TIMBERS 72" $\times 72^{n} \times 7.25^{\prime \prime}$

ARRAY $13 * 0.002$ REPLATE 2 2RO 3175 2RO.

REPLICATE 2 2RO. 2RO.3175 $2 \mathrm{R}$

UNIT 4

COM=* SECTION OF STEEL GRATING -

CUBOID 4 1 $2.778125 .238125 .396875 \quad-.396875 \quad 3.175 \quad 2.38125$ CUBOID $212.778125, .238125,5.524375-4.5243753 .17$

UNIT 5

COM=* ARRAY OF STEEL GRATING *

ARRAY $2 \quad 3 * 0.0$

RBPLICATE 214 LRO 9525 2RO.0 
Table A.2 (continued)

UNIT 6

COM-* U(3.85) ANNULUS, H=76.2, SUBMERGED, ON CORNER

CYLINDER 213.30276 .20$.

CYLINDER 114.61413976 .20

CYIINDER 1195.62826476 .20$.

CYLINDER 117.24234176 .20

CYLINDER 1177.92707976 .20

CYLINDER 1119.14400076 .20$.

COM=* U(3.85) ANNULUS, $\mathrm{H}=76.2$, SUBMERGED, ON SIDE

CYLINDER 213.30276 .20 .

$\begin{array}{llllll}\text { CYLINDER } & 1 & 4.614139 & 76.2 & 0 \\ \text { CYLINDER } & 1 & 1 & 5.628264 & 76.2 & 0\end{array}$

CYLINDER 1116.48571076 .20

CYLINDER 117.92707976 .20

CYLINDER $1118.55719976 .2 \quad 0$

CYLINDER I 19.14400076 .20 .

UNIT 8

COM=* ARRAY OF 6 ANNULI, H=76.2, SUBMERGED -

ARRAY $33 * 0.0$

REPLICATE 2 2 $12 R 61.1505 \quad 2 R 71.247 \quad 2 R 0$.

CORE $41-121.92-121.920$.

REPLICATE 5 S 11580.00 .1905

REPD GBOM

NUY 13 NUZ 1

FILE 12502 NOY $=13$ NUZ

$A R A=2$ NUX $=60$ NUY $=20$ NUZ $=1$

FILL P4 END PILI

ARA $=3$ NUXX $=3$ NUY $=2 \quad$ NUZ $=1$

FILL=4 $\begin{array}{llllllll}6 & 6 & 6 & 7 & \text { END PI }\end{array}$

ARA $=4$ NUX $=1 \quad$ NUY $=1$ NUZ

PILL 358 BND PILI

END ARRAY

READ STAR

$X S M=-30.2895 \quad X S P=30.2895$

YSM $=-20.193 \quad$ YSP $=20.193$

$2 S M=21.59$ ZSP $=97.79$

BND START

TTL="PLAN VIEW $1 / 6$ SCALE OF CORB, $Z=$ MIDLEVEL OF ANNULI/INSERTS" $X U L=-91.44$ YUL $=91.44 \quad 2 U L=59.69$

$X L R=91.44$ YLR $=-91.44$ ZLR $=59.69$

69 U WS*

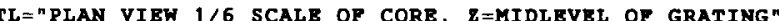

$X U L=-91.44 \quad Y U L=91.44 \quad 2 \mathrm{UL}=20.0025$

XLR $=91.44$ YLR $=-91.44$
UAX $=1$. VDN $=-1$. DLX $=1.524$ NCH $=20$ U. WGS $*$ BND
TTL="PLAN VIEW 1/6 SCALE OF CORE, Z $=$ MIDLEVEL OF FIR TIMBERS" $X U L=-91.44 \quad Y U L=91.44 \quad \mathrm{ZUL}=9.2075$

$X L R=91.44$ YLR $=-91.44 \quad$ ZLR $=9.2075$
UAX $=1$. VDN $=-1$. DLX $=1.524 \quad$ NCH=* U. WGS $*$ END

TTL="PLAN VIEW $1 / 10$ SCALE OF MOCKUP, $Z=$ MIDLBVEL OF U UNITS" $X U L=-135$. YUL $=135 . \quad Z U L=59.69$

XIR $=135$. YLR $=-135, \mathbf{Z L R}=59.69$
UAX $=1$. VDN $=-1$. DLX $=2.54 \quad$ NCH=* U. WGS* END

TTL="PLAN VIEW $2 X$ SCALE OF SINGLE U UNIT, $2=$ MIDLEVEL OF UNIT" $X U L=0.0 \quad Y U L=20.193 \quad \mathrm{ZUL}=59.69$

$X L R=20.193$ YLR $=0.00212=59.69$
UAX $=1$. VDN $=-1$. DLX $=0.127$ NCH $=$ U. WGS* END

TTL $=$ "CROSS SECTION OF CORE $1 / 6$ SCAIE, $Y=10.0965$ "

$X U L=-91,44 \quad Y U L=10.0965 \quad$ ZUL $=125$

UAX $=1$. WDN $=-1$. DLX $=1.524$ NCH=* U. WGS* END

TTL $=$ "CROSS SECTION OF MOCKUP $1 / 10$ SCALE, $Y=10.0965$

$X U L=-135 . Y U L=10.0965 \quad Z U L=125$

UAX $=1$, WDN $=-1$, DIX $=2.54$ NCH $=$ * U, WGS* END

TTL="CROSS SECTION OF GRATING/FIR TIMBERS, FULL SCALE"

$X U L=0 . \quad Y U L=0$. $Z U L=24.13$

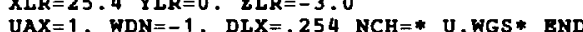

TTL="PIAN VIE OF GRATING THRU TIEBARS, FULI SCALB"

$X U L=0 . \quad Y U L=25.4 \quad 2 U L=21.193$

XAR $=1$. YDN $=-1$. $\mathrm{DLX}=254$ NCH $=$ * U.WGS* END

TTL="PLAN VIEN OF GRATING G GRATING MIDLEVEL, FULL SCAIE" $X U L=0 . \quad Y U L=25.4 \quad Z U L=20.0025$

UAX $=1$. VDN $=-1$. DLX $=254$ NCH $=$ * U. WGS * END

TTL="PLAN VIEW OF TIMBERS A TIMBER MIDLEVEL, FULL SCALE" $X U L=0 . \quad Y U L=25.4 \quad Z U L=9,2075$

$X I R=25.4 \quad$ YLR $=-25.4 \quad$ ZLR $=9.2075$
UAX $=1$ VDN $=-1$. DLX $=254 \quad \mathrm{NCH}=*$ U. WGS* END

TTL="CROSS SECTION OF CENTRAL UNIT, FULL SCALE, $Y=10.0965^{\circ}$ $\mathrm{XUL}=-10.0965 \quad \mathrm{YUL}=10.0965 \quad \mathrm{ZUL}=125$

XLR $=10.0965$ YLR=10.0965 $\mathrm{ZLR}=-10$.
UAX $=1$. WDN $=-1$. DLX $=.254$ NCH $=*$ U. WGS $*$ END

END PLOT

END DA 
Table A.2 (continued)

CAB 11
$=$ CSAS25 CASE EBJ.9 U(3.85) 160 (6.2" $72.6 " / 1.50 " / 49.2 \mathrm{CM}$, SQUARE
27GROUPNDF4 INFHOMMEDIUM

$\begin{array}{lcccc}\mathbf{U}(3.85) & \text { METAL } & & \\ \mathbf{U}-235 & 10 . & 1.8643 \mathrm{~B}-3 & \text { END } \\ \mathbf{U}-238 & 1 & 0 . & 4.5971 \mathrm{E}-2 & \text { END }\end{array}$

$\begin{array}{ccc}\text { FULL DENSITY WATER MODERATOR/REFLECTOR } \\ \text { H20 } & 2 \text { 1.0 } & \text { BND }\end{array}$

FIR TIMBER MODEL $30.06802 \mathrm{E}-2$ END

O 30 . $1.10850 \mathrm{~B}-2$ END

C STEEL FOR GRATING, TANK WALIS AND FLOOR

STAINLESS STERL LINING FOR TANK
SS 304

END COMP 9 (3.85) 16 7.2"/2.6n/1.50"/49.2CM, SQUARE RPG $=600$ NUB $=$ YES PLT=NO FDN=YBS THE=90 TBA=2

END PARM

UNIT 1

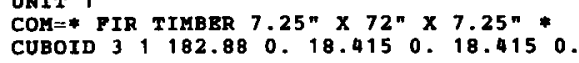

UNIT 2

COM=* WATRR GAP $3.5^{n} \times 72^{n} \times 7.25^{n}$ BETWEEN TIMBERS CUBOID 29182.880 .8 .890 . 18.4150 .

UNIT 3

COM=* ARRAY OF TIMBERS $72 n \times 72^{n} \times 7.25 n$

REPLICATE 212 2RO. 2R0 3175 2RO. 1

REPLICATE 214 R 30.48 2RO. 1

UNIT 4

COM=* SECTION OF STRBI GRATING -

CUBOID $412.778125 \quad .238125 \quad .396875 \quad-.396875 \quad 3.1752 .38125$ CUBOID $2112.778125 \quad 238125 \quad 4.524375 \quad-4.524375 \quad 3.1750$.

$3.016250 .4 .524375-4.5243753 .1750$.

UNIT 5

COM=* ARRAY OF STBEL GRATING *

$\begin{array}{lllll}\text { REPLICATE } & 2 & 1 & 4 R 0.9525 \quad 2 R 0.0 & 1 \\ \text { REPLICATE } & 2 & 1 & 4 R 30.48 & 2 R 0.1\end{array}$
UNIT 6

COM=* U (3.85) ANNULUS, H=49.2, SUBMERGED, ON CORNER * CYLINDER 2 1 3.30249 .20 .

CYLINDER , 15.62826449 .20 .

CYLINDER 1116.48571049 .20

CYLINDER 1177.24234149 .20

CYLINDER 117.92707949 .20$.

CYLINDER 1199.14400049 .20

COMT ' U( 3.85, ANNULUS, H=49.2, SUBMERGED, ON SIDE *

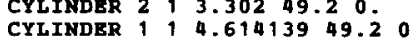

CYLINDER $1,5.62826449 .20$.

CYLINDER 1116.48571049 .20

CYIINDER 117.24234149 .20

CYLINDER 117.92707949 .20$.

CYIINDER $1,9.14400049 .20$.

CUBOID 214 P 11.04949 .20 .

UNIT 8

(3.85) ANNULUS, H=49.2, SUBMERGED, IN MIDDLE -

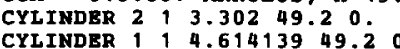

CYLINDER $115.628264 \quad 49.20$

$\begin{array}{lllllll}\text { CYLINDER } & 1 & 1 & 6.485710 & 49.2 & 0 . \\ \text { CYLINDER } & 1 & 1 & 7.242341 & 49.2 & 0 .\end{array}$

$\begin{array}{llllll}\text { CYLINDER } & 1 & 1 & 7.242341 & 49.2 & 0 \\ \text { CYLINDER } & 1 & 7.927079 & 49.2 & 0 \\ \text { CYINDER } & 7 & \end{array}$

CYLINDER 117.92707949 .200

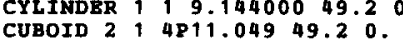

UNIT 9

U( 3.85 ) ANNULUS, H=27, 0 , DRY, ON CORNER *

CYLINDER O $13.302 \quad 27.00$.

$\begin{array}{lllllll}\text { CYLINDER } & 1 & 1 & 4.614139 & 27.0 & 0 \\ \text { CYLINDER } & 1 & 1 & 5.628264 & 27.0 & 0\end{array}$

CYLINDER 116.48571027 .00

CYLTDER 19.24234127 .00 .

CYLINDER 1118.55719927 .00$.

$\begin{array}{lllll}\text { CYLINDER } & 1 & 1 & 9.14400027 .0 & 0 \\ \text { CUBOID } 0 & 1 & 4 P 11.049 & 27.0 & 0\end{array}$

UNIT 10
COM $=*$ U (3.85) ANNULUS, $H=27.0$, DRY, ON SIDE *

CYLINDER 0 1 3.30227 .00

CYLINDER 114.61413927 .00

CYLINDER 115.62826427 .00 .

CYLINDER 19.48571027 .00

CYLINDER 117.92707927 .00 .

CYLINDER 1118.55719927 .00 .

CYLINDER 119.14400027 .0 
Table A.2 (continued)

UNIT 11

COM=* U( 3.85) ANNULUS, H=27.0, DRY, IN MIDDLE -

$\begin{array}{llllll}\text { CYLINDER } & 0 & 1 & 3.302 & 27.0 & 0.0 \\ \text { CYLINDER } & 1 & 1 & 4.614139 & 27.0 & 0 .\end{array}$

$\begin{array}{llllll}\text { CYLINDER } & 1 & 1 & 4.614139 & 27.0 & 0 . \\ \text { CYLINDER } & 1 & 1 & 5.628264 & 27.0 & 0 \\ \text { CYLINDER } & 1 & 0\end{array}$

CYLINDER 1196.48571027 .00 .

$\begin{array}{llllll}\text { CYLINDER } & 1 & 1 & 7.242341 & 27.0 & 0 \\ \text { CYLINDER } & 1 & 1 & 7.927079 & 27.0 & 0\end{array}$

CYLINDER 118.55719927 .00

CYLINDER 11199.14400027 .0

UNIT 12
COM=* ARRAY OP 16 ANNULI, H=49.2, SUBMERGED -

REPLICATE 2 1 4 R47.244 2RO. 1

UNIT 13
COM=* ARRAY OP 16 ANNULI, H=27.0, DRY *

REPLICATE 0014 R47.244 2R0. 1

CORB 5 - $1-121.92-121.920$.

REPLICATE 5 S 1 5RO.0 0.1905

BND GBOM

ARA $=1 \quad$ NUX $=1 \quad$ NUY $=13 \quad N U Z=1$

PILL 12 2 5 Q2 1 END FIII

ARA $=2$ NUX $=60$ NUY $=20 \quad$ NUZ $=1$
FILL F4 END PILI

PILI 67767888778876776 END FILL

$A R A=4$ NUX $=4$ NUY $=4$ NUE $=1$

PIII $9 \begin{array}{lllllllllllllll}10 & 10 & 9 & 10 & 11 & 11 & 10 & 10 & 11 & 11 & 10 & 9 & 10 & 10 & 9 \\ \text { END } & \text { PILI }\end{array}$

$G B L=5$

ARA $=5$ NUXX $=1 \quad$ NUY $=1 \quad$ NUZ $=4$

ILI 351213 END FILL

READ START

NST $=1$
$X S Y=-44.196 \quad X S P=44.196$

$Y S M=-44.196 \quad Y S P=44,196$

BND START
READ PLOT

TTL="PLAN VIEW $1 / 6$ SCALE OF CORE, Z=MIDLEVEL OP ANNULI/INSERTS"

TTL $=" P L A N$ VIEW $1 / 6$ SCALE OF CORE,
$X U L=-91.44 \quad$ YUL $=91.44 \quad 8 U L=59.69$

$X L R=91.44$ YLR $=-91.44 \quad 2 L R=59.69$

UAX $=1$. VDN $=-1$. DLX $=1.524 \mathrm{NCH}=*$ U. WGS * BND

TTL="PLAN VIEW $1 / 6$ SCALE OF CORE, $Z=$ MIDLEVEL OF GRATING"

$X U L=-91.44$ YUL $=91.44 \quad 2 U L=20.0025$

$\mathrm{UAX}=1$. $\mathrm{VDN}=-1 . \quad \mathrm{DLX}=1.524 \quad \mathrm{NCH}=*$ U. WGS* BND

TTL="PLAN VIEW $1 / 6$ SCALE OF CORE, $Z=M$ MDLBVEL OF PIR TIMBERS"

$X U L=-91.44 \quad Y U L=91.44 \quad 2 U L=9.2075$
$X L R=91.44 \quad Y L R=-91.44 \quad Z L R=9.2075$

$U A X=1$. VDN $=-1$. DLX $=1.524 \quad \mathrm{NCH}=*$ U. WGS $*$ END
TTL="PLAN VIBW $1 / 10$ SCALB OF MOCKUP, $\mathrm{Z}=$ MIDLEVBL OF U UNITS" $X U L=-135 . \quad Y U L=135 . \quad 2 U L=59.69$

UAX $=1$, VDN $=-1$, DIX $=2.54$ NCH=* U. WGS* END

TTL="PLAN VIEW $2 X$ SCALB OF SINGLE U UNIT, $Z=$ MIDLEVEL OF UNIT" XUL $=0.0 \quad$ YUL $=22.098 \quad$ $\quad$ UL $=59.69$

XIR $=22.098$ YLR $=0 . \quad 2 L R=59.69$
UAX $=1$ VDN $=-1$ DLX $=0.127 \quad$ NCH $=*$ U. WGS* END

TTL $=$ "CROSS SECTION OF CORE $1 / 6$ SCALE, $Y=11.049 "$

$X U L=-91.44 \quad Y U L=11.049 \quad 2 U L=110$.

$X I R=91.44$ YLR $=11.049 \quad 2 L R=-10$.
UAX $=1$. WDN $=-1$. DLX $=1.524$ NCH=* U. WGS* BND

TTL="CROSS SECTION OF MOCKUP 1/10 SCALE, Y=11.049" $X U L=-135$. YUL $=11.049$ \&UL $=125$

XLR $=135$. YLR $=11.049$ 2LR $=-10$.
UAX $=1$. WDN $=-1$. DLX $=2.54$ NCH $=*$ U. WGS $*$ END

TTL="CROSS SECTION OF GRATING/PIR TIMBERS, FULL SCALE"

$X U L=0, \quad Y U L=0, \quad Z U L=24.13$

$X L R=25.4$ YLR $=0$. $\mathrm{ZLR}=-3.0$
$\mathrm{UAX}=1$. WDN $=-1$. DLX $=.254^{\circ}$ NCH=* U. WGS* END

TTL="PLAN VIEK OF GRATING THRU TIEBARS, PULL SCALE"

$X U L=0, \quad Y U L=25.4 \quad Z U L=21.193$

UAX $=1$. VDN $=-1$. DLX $=.254$ NCH $=*$ U. WGS* END

TTI="PLAN VIEN OF GRATING O GRATING MIDLEVEL, FULL SCALE" $X U L=0, Y U L=25.4 \quad Z U L=20.002$

$\mathrm{UAX}=1$. $\mathrm{VDN}=-1$. $D L X=.254 \quad \mathrm{NCH}=* \mathrm{U}$.WGS $\mathrm{END}$

TTL="PLAN VIEW OF TIMBERS TIMBER MIDLEVEL, FULI SCALE $X L R=25$. $Y$ Y $=-25,4=25 R=9.2075$

UAX $=1 . \quad \mathrm{VDN}=-1$. DLX $=.254 \quad \mathrm{NCH}=*$ U. WGS $*$ END

TTL $=$ "CROSS SBCTION OF CENTRAI UNIT, FULI SCALE, $Y=11.049$ $X U L=0.0 \quad Y U L=22.098 \quad 2 U L=110$.

UAX $=1$, WDN $=-1$. DLX $=.254$ NCH $=*$ U. WGS * END

BND PLOT
END DATA 
Table A.2 (continued)

CAB 12
$=$ CSAS25

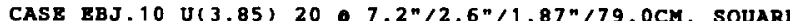
27GROUPNDP 4 INFHOMMEDIUM

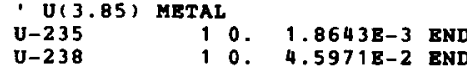

FULL DENSITY WATER MODERATOR/REFLECTOR
H2O
BND

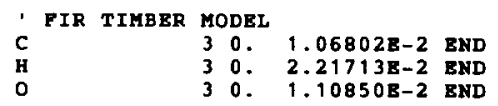

C STEEL POR GRATING, TANK WaLLS AND FLOOR

CARBOnSTEEL G

SS 304

END

\section{END COMP} 51.0

CASB BBJ. 10 U(3.85) $20 \bullet 7.2^{n / 2.6^{n / 1}}$ (1.87"/79.0CK, SQUARE NPG $=600$ NUB $=$ YES PLT=NO $\quad$ PDN=YES TME $=90 \quad$ TBA $=2$ BND PARM

UNIT 1

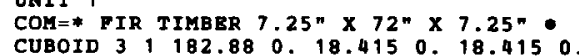

UNIT 2

COM=* WATER GAP $3.5^{n} \times 7^{n} \times 7.25 "$ BETWEEN TIMBERS

182.880 .8 .890 .18 .4150

UNIT 3

COM=* ARRAY OF TIMBERS $72^{\prime \prime} \times 72^{n} \times 7.25^{n}$.

REPLICATE 2 1 2RO. 2RO.3175 2R0. 1

REPLICATE $2114 \mathrm{R} 30.48$ 2RO .

UNIT 4

COM=* SECTION OF STEEL GRATING

$\begin{array}{lllllll} & \end{array}$

CUBOID $413.016250 .4 .524375-4.5243753 .1750$.

UNIT 5

COM=* ARRAY OF STEEL GRATING

$\begin{array}{llllll}\text { REPLICATE } & 1 & 4 R 0.9525 & 2 \mathrm{RO} 0.0 & 1 \\ \text { REPLICATE } & 2 & 1 & 4 \mathrm{R} 30.48 \quad 2 \mathrm{RO} 0.1\end{array}$

UNIT 6

(3.85) ANNULUS, $\mathrm{H}=76.2$, SUBMERGED

CYLINDER 2 1 $3.302 \quad 76.20$.

CUBOID 2 2 14 4P11.5189 99.00.
UNIT 7

COM=* U ( 3.85 ) ANNULUS, H=76.2, SUBMERGED

CYLINDER $213.302 \quad 76.20$.

CYLINDER 1 1 199.14476 .20 .0

UNIT 8 COM $=$ ( 3.85 ) ANNULUS, $H=76.2$, SUBMERGED * $\begin{array}{llllll}\text { CYLINDER } & 2 & 1 & 3.302 & 76.2 & 0 . \\ \text { CYLINDER } & 1 & 1 & 9.144 & 76.2 & 0 .\end{array}$

CUBOID $214 P 11.518979 .00$.

COMIT 9 U $(3.85$ ) ANNULUS, $H=76.2$, SUBMERGED * CYLINDER $1,9.14476 .20$.

CUBOID 214 P $1 \uparrow .518979 .00$

UNIT 10

CYLINDER $2 \quad 1 \quad 3.30276 .20$

CUBOID 214 P 11.518979 .0

UNIT 11

CYIINDER 2 2 $3.302 \quad 76.20$.

CYLINDER $199.14476 .20^{\circ}$.

UNIT 12

COM=* U( 3.85 ) ANNULUS, $H=76.2$, SUBMERGED

$\begin{array}{llllll}\text { CYLINDER } & 2 & 1 & 3.302 & 76.2 & 0 . \\ \text { CYLINDER } & 1 & 1 & 9.144 & 76.2 & 0 .\end{array}$

CUBOID 214 P 11.518979 .00

UNIT 13 COM=* U(3.85) ANNULUS, $H=76.2$, SUBMERGED $\begin{array}{llllll}\text { CYLINDER } & 2 & 1 & 3.302 & 76.2 & 0 . \\ \text { CYLINDER } & 1 & 1 & 9.144 & 76.2 & 0 .\end{array}$

CUBOID 214 P11.5189 79.00 .

UNIT 14

COM $=$ U ( 3.85 ) ANNULUS, H=76.2, SUBMERGED *

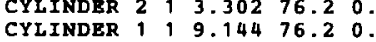

CUBOID 214 P11.518979.0 0 .

UNIT 15

COM=* U( 3.85 ) ANNULUS, $H=76.2$, SUBMERGED * CYLINDER 2173.30276 .20$.

CUBOID 214 P 11.518979 .00 .

UNIT 16

COM=* U(3.85) ANNULUS, $y=76.2$, SUBMERGED * CYLINDER 213.30276 .20$.

CUBOID 214 P 11.518979 .00 .

UNIT 17

* U ( 3.85) ANNULUS, H=76.2, SUBMERGED *

CYLINDER 293.30276 .20 .

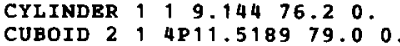



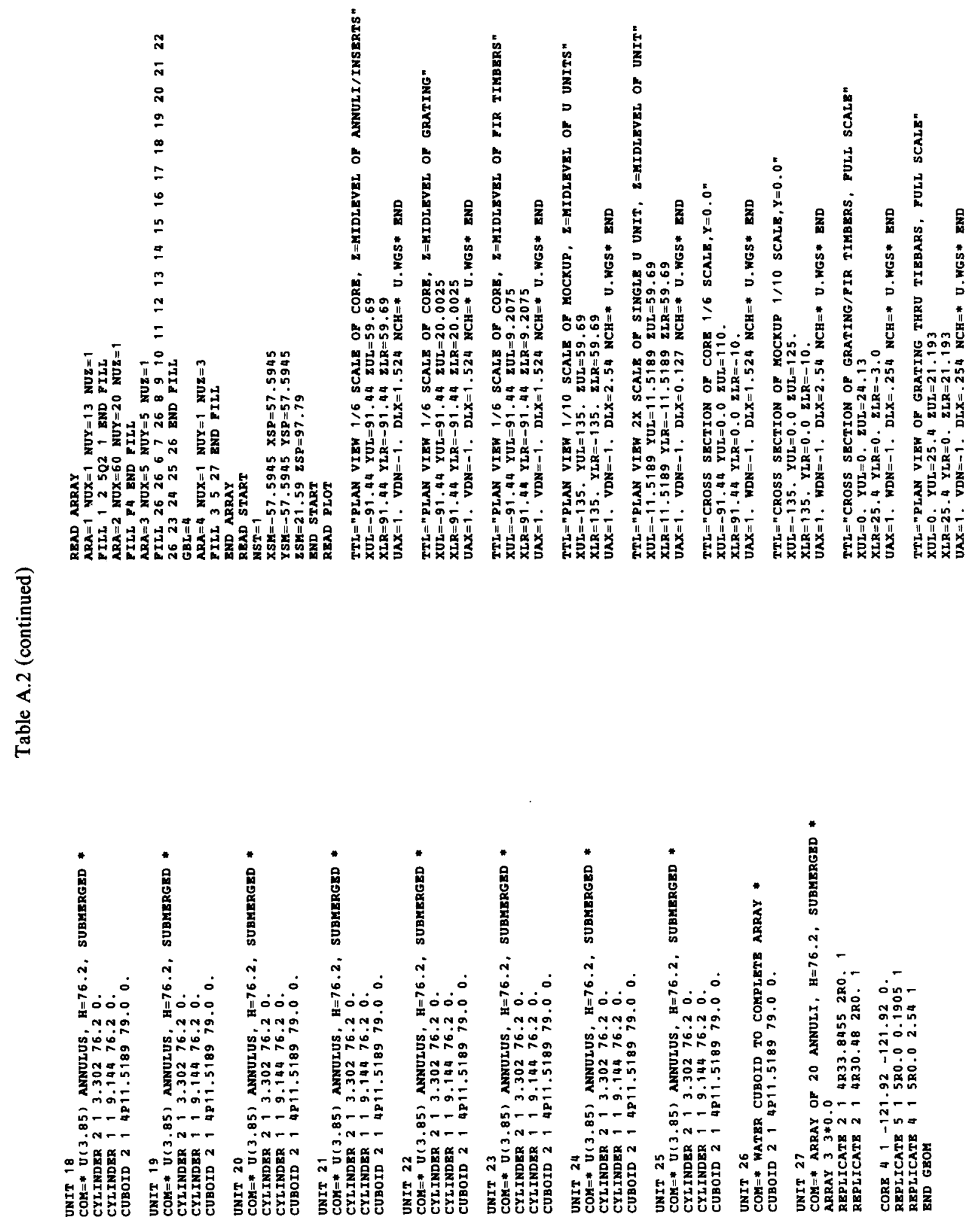
Table A.2 (continued)

TTL="PLAN VIEW OF GRATING O GRATING MIDLEVEL, TULL SCALE" XUL $=0$. YUL $=25.4 \quad \mathrm{ZUL}=20.0025$

$X I R=25.4$ YLR=0. $2 L R=20.0025$
$U A X=1$. VDN $=-1$. DLX $=.254$ NCH $=*$ U. WGS* END

TTL="PLAN VIEW OF TIMBERS Q TIMBER MIDLEVEI, FULL SCALE" $X U L=0 . \quad Y U L=25.4 \quad Z U L=9.2075$

XLRR $=25.4$ YLR=-25.4 $\quad$ YLR=9.2075
UAX $=1$. VDN $=-1$. DLX $=.254$ NCH $=*$ U. WGS* END

TTL="CROSS SBCTION OF CENTRAL UNIT, FULL SCALE, $Y=0$, 0 " $X U L=-11.5189$ YUL $=0.0 \quad$ \&UL $=110$

XIR $=11.5189$ YLRR=0.0 ZLR $=-10$.
UAX $=1$. WDN $=-1$. DLX $=.254$ NCH=* U . WGS* END

END PLOT
END DATA

CAB13

CASE EBJ. 11 U(3.85) 8 o $7.2 n / 2.6 n / 0.0 " / 72.5 \mathrm{CM}$, SQUARE
27GROUPNDF4 INFHOMMEDIUM

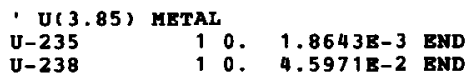

FULL DENSITY WATER MODERATOR/REFLECTOR
H20

FIR TIMBER MODEL

$\mathrm{C}$
$\mathrm{H}$
$\mathrm{O}$

3 $0.1 .10850 \mathrm{~B}-2$ END

STERL FOR GRATING, TANK WALLS AND FLOOR
CARBONSTEEL 4.0

STAINLESS STEEL LINING POR TANK
SS 304

END COMP

CASE EBJ.11 U(3.85) 8 ?.2"/2.6"/0.0"/72.5CM, SQUARE READ PARM

BNG PARM

UNIT

COM=* YIR TIMBER 7.25n $\times 72^{n} \times 7.25^{n} *$

COBOT

" 7.25 " BETHEEN TIMBERS

COM=* WATER GAP $3.5^{n} \times 7^{n \prime} \times 7.25^{n}$ BET
CUBOID $21182.880 .8 .890 .18 .415^{\circ} 0$.
UNIT 3

CONi=* ARRAY OF TIMEERS $72^{n} \times 72^{n} \times 7.25^{n}$ *

ARRAY $13 * 0.0$

REPLICATE 2 2RO. 2R0.3175 2RO.

UNIT 4

COM=* SBCTION OF STEEI GRATING *

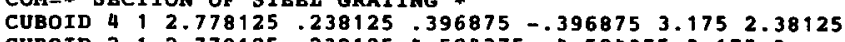

CUBOID $212.778125 .2381254 .524375-4.52437533 .17$

UNIT 5

COM=* ARRAY OF STEEL GRATING *

ARRAZ $23 * 0.0$

REPLICATR 2 I 4 RO $0.9525 \quad$ 2R0.0

UNIT 6

COM=* U(3.85) ANNULUS, H=72.5, SUBMERGED, ON CORNER

CYLINDER 213.30272 .50 .

CYLINDER 1175.62826472 .50 :

CYLINDER 116.48571072 .50$.

CYLINDER 117.24234172 .50 .

CYLINDRR 177.92707972 .50 .

CYLINDER 1199.14400072 .50

CYLINDBR 1199.14400072 .5

UNIT 7

COM=* U( 3.85 ) ANNULUS, H=72.5, SUBMERGED, ON SIDE C

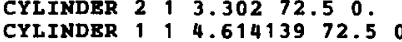

CYLINDER 115.62826472 .50 .

CYLINDER 1116.48571072 .50 .

CYLINDER 1117.24234172 .50 .

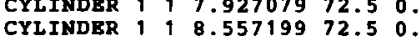

CYLINDER 1119.14400072 .50

CUBOID 214 P9.144 72.5 0 .

UNIT 8

COM=* U( 3.85 ) ANNULUS, $H=3.7$, DRY, ON CORNER *

CYLINDRR 0113.3023 .70

CYLINDER 115.6282643 .70 .

CYLINDER 1115.4857103 .70 .

CYLINDER 197.2423413 .70 .

CYLINDER 118.5571993 .70 .

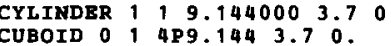

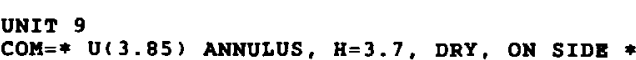

CYLINDER O 13.3023 .70

CYLINDER 114.6141393 .70

CYLINDER $1145.628264 \quad 3.70$

CYLINDER 1196.4857103 .70

CY.

$\begin{array}{llllll}\text { CYLINDER } & 1 & 1 & 8.557199 & 3.7 & 0 \\ \text { CYLINDER } & 1 & 1 & 9.144000 & 3.7 & 0\end{array}$ 
Table A.2 (continued)

UNIT 10

ARRAY OF 8 ANNULI, $H=72.5$, SUBMERGED * 2R54.864 2R73.152 2RO. 1

REPLICATE $214 R 30.48$ 2RO. 1

UNIT 11

COM=* ARRAY OF 8 ANNULI, H=3.7, DRY

ARRAY $4 \quad 3 * 0$.

2RO. 1

RBPLICATE 01 4R30.48 2R0. 1

CORE $51-121.92-121.920$.

REPLICATE 4 1 5 RO.0 2.541

BND GEOM

READ ARRAY

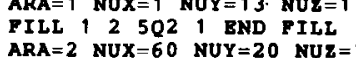

FILL F4 END FILL

ARA $=3$ NUX $=4 \quad$ NUY $=2 \quad$ NUZ $=1$

FILL 677766776 END $F I L$

FILL 89988998 BND PILL

ARA $=5 \quad$ NUX $=1 \quad$ NUY $=1 \quad$ NUZ $=4$

FILL $3 \quad 5 \quad 1011$ END FILI

READ START

NST $=1$

$X S M=-36.576 \quad X S P=36.576$

$Y S M=-18.288 \quad Y S P=18.288$

ZSM $=21.59 \quad 2 S P=94.09$

END START

TTL="PLAN VIEW 1/6 SCALB OF CORE, Z=MIDLEVEL OF ANNULI/INSERTS" $X U L=-91.44 \quad Y U L=91.44 \quad 20 L=59.69$

$X L R=91.44$ YLR $=-91.44 \quad$ ZLR $=59.69$
UAX $=1$. VDN $=-1$. DLX $=1.524$ NCH=* U. WGS $*$ END

TTL="PLAN VIBW 1/6 SCALB OF CORE, Z=MIDLEVEL OF GRATING"

$X U L=-91.44 \quad Y U L=91.44 \quad 2 U L=20.0025$

$X L R=91.44$ YLR $=-91.44 \quad$ ZLR $=20.0025$
UAX $=1$. VDN $=-1 . \quad$ DLX $=1.524$ NCH $=*$ U. WGS * END

TTL=" PLAN VIEW 1/6 SCALB OP CORE, $z=$ MIDLEVEL OF FIR TIMBERS" $X U L=-91.44 \quad Y U L=91.44 \quad 2 U L=9.2075$

$X L R=91.44$ YLR $=-91.44 \quad 2 L R=9.2075$
UAX $=1$. VDN $=-1$. DL $X=1.524 \quad$ NCH=* U. WGS* END

TTL="PLAN VIEW $1 / 10$ SCALE OF MOCKUP, $Z=$ MIDLEVEL OF $U$ UNITS" $X U Z=-135 . \quad Y U L=135 . \quad 2 U L=59.69$

XLR $=135$. YLR $=-135 . \quad 2 L R=59.69$
UAX $=1$. VDN $=-1$. DLX $=2.54 \quad \mathrm{NCH}=*$ U. WGS * END

TTL="PLAN VIEW $2 X$ SCALE OF SINGLE U UNIT, $Z=$ MIDLEVEL OF UNIT"

$X L R=18.288$ YLR $=0.02 L R=59.69$
$U A X=1$. VDN $=-1$. DLX $=0.127 \quad \mathrm{NCH}=*$ U. WGS $*$ END
TTL=" CROSS SECTION OF CORE $1 / 6$ SCALE, $Y=9.144^{n}$ $X U L=-91,44$ YUL $=9,144 \quad 2 U L=110$

UAX $=1$. WDN $=-1$. DLX $=1.524$ NCH $=*$ U. WGS* END

TTL $=$ "CROSS SBCTION OP MOCKUP $1 / 10$ SCALE, $Y=9.144^{\circ}$

$X U L=-135$. YUL $=9.144 \quad 2 U L=125$.

$X I R=135$. YIR $=9.144$ ZLR $=-10$.
UAX $=1$. WDN $=-1$. DLX $=2.54$ NCH $=*$ U.WGS* END

TTL="CROSS SECTION OP GRATING/FIR TIMBERS, FULI SCALE

$X U L=0 . \quad Y U L=0 . \quad 2 U L=24.13$

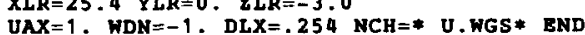

TTL="PLAN VIBW OP GRATING THRU TIBBARS, FULL SCALE"

$X U L=0 . \quad Y U L=25.4 \quad Z U L=21.193$

$X L R=25.4 \quad$ YLR=0. $\quad 2 L R=21.193$

TTL="PLAN VIEW OF GRATING O GRATING MIDLEVEL, PULL SCALB" $X U Z=0 . \quad Y U L=25.4 \quad 2 U L=20.0025$

XAR $=1$.

TTL $=$ "PLAN $\mathrm{YIEW}$ OF TIMBERS

$X U L=0 . \quad Y U L=25.4 \quad 2 U L=9.2075$

$X L R=25.4$ YLR $=-25.4 \quad 2 L R=9.2075$
$U A X=1$. VDN $=-1$. DLX $=.254$ NCH=* U. WGS* END

TTL $=$ "CROSS SECTION OF CENTRAL UNIT, FULL SCALB, Y=9.144"

$X U L=0.0 \quad Y U L=9.144 \quad$ ZUL $=110$.

$X L R=18.288$ YLR $=9.144 \quad 2 L R=-10$.
$U A X=1$. WDH $=-1$. DLX $=.254$ NCH=* U. WGS* END

END PLOT
BND DATA

CAB14

CASE EBJ.12 U(3.85) 22 2.5"/0.0"/0.35"/72.3CM, SQUARE 27GROUPNDF 4 INFHOMMEDIUM

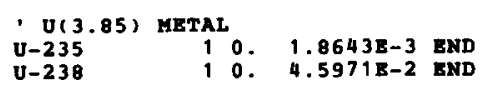

1 PULI DENSITY WATER MODERATOR/REFLECTOR
H2O

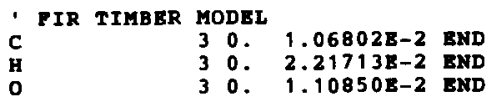

- STEEI FOR GRATING, TANK WALLS AND FLOOR

STAINLESS STERL LINING POR TANK
SS 304 
Table A.2 (continued)

END COMP

CASE EBJ .12 U (3.85) 22 2.5"/0.0"/0.35"/72.3CK, SQUARE

TPG $=600$ NUB $=$ YES PLT $=$ NO FDN=YES TME $=90 \quad$ TBA=2

RND PARM

UNIT 1

COM=* FIR TIMBER $7.25 n \times 72 " \times 7.25 n *$

UNIT 2

COM=* HATER GAP 3.5" $\times 72^{n} \times 7.25$ " BETHEEN TIMBERS *

CUBoID 21182.880 .8 .890 .18 .4150 .

UNIT 3

COM=* ARRAY OF TIMBERS 72" $\times 72 " \times 7.25 \%$ "

COMAT ARAY OR

REPLICATE 2 2 1 2R0. 2R0.3175 2RO. 1

UNIT "

CUBOID 4 $12.778125 \quad .238125 \quad .396875 \quad-.396875 \quad 3.175 \quad 2.38125$

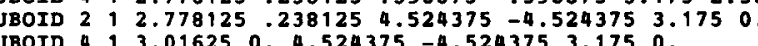

UNIT 5

COM=* ARRAY OP STEEL GRATING -

REPLICATE 2 1 1 4R0.9525 2R0.0

UNIT 6

U(3.85) ROD, H=72,3, SUBMERGED *

CYLINDER 1111.41990372 .30

CYLINDER 1112.45934472 .30

CYLINDER $1112.839806 \quad 72.30$.

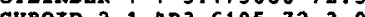

UNIT 7

COM=* WATER CUBOID TO COMPLETE ARRAY 3 -

CUBOID 214 P3.6195 72.30 .

UNIT 8

COM $=*$ U(3.85) ROD, H=3.9, DRY *

CYLINDER $i$ i 1.4199033 .90 .

CYIINDER 11112.0080463 .90$.

CYLINDER 113.0080463 .90$.

$\begin{array}{lllllll}\text { CYLINDER } & 1 & 1 & 2.839806 & 3.9 & 0 \\ \text { CYLINDBR } & 1 & 1 & 3.175000 & 3.9 & 0\end{array}$

CUBOID 014 P3.6195 3.90.

UNIT 9

COM=* VOID CUBOID TO COMPLETE ARRAY 4 *

UNIT 10

COM=* U( 3.85 ) ROD ARRAY, $H=72.3$, SUBMERGED *

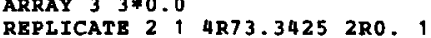

$\begin{array}{llll}\text { REPLICATE } & 2 & 1 & 4 R 73.3425 \quad 2 R 0 \\ \text { REPLICATE } & 2 & 1 & 4 R 30.48 \quad 2 R 0 \text {. }\end{array}$

UNIT 11

COM=* ARRAY OF 22 U(3.85) RODS, H=3.9, DRY

REPLICATE $0.14 R 73.3425$ 2R0;

CORE $51-121.92-121.920$.

REPLICATE 5 S 1 5R0.0 0.1905

END GEOH

READ ARRAY

ARA $=1$ NUX=1 NUY=13 NUZ $=1$

FILL 125021 END FILL

ARA $=2$ NOX $=60$ NUY $=20$

FILI F4 END FII

ARA=3 NOX $3 R 7$ NUY $=5$ NUZ $=1$

NRA $=4$ NUX $=5 \quad$ NUY $=5 \quad$ NUZ $=1$

ILL 2R8 3R9 $20 R 8$ END FIL

ARA $=5 \quad$ NUX $=1$
NILI

END ARRAY

$X S M=-18.0975$ XSP=18.0975

YSR=-18.0975 XSP $=18.0975$

ZSH=21.59 ZSP=93.89

RND START

TTL="PLAN VIEW $1 / 6$ SCALE OF CORE, $Z=M I D L E V E I$ OF ANNULI/INSERTS"

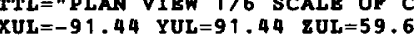

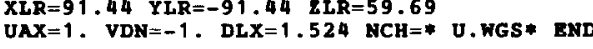

TTL="PLAN VIBW OF ARRAY, FULL SCALE"

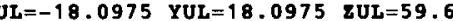

XIR $=18.0975$ YLR $=-18.0975 \quad$ ZLR $=59.69$
UAX $=1$. VDN $=-1$. DLX $=.254$ NCH $=*$ U. WGS $*$ END

$T T L=" P L A N$ VIEW $1 / 6$ SCALE OF CORE, $z=M I D L E V E L$ OF GRATING"

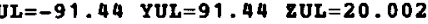

$X L R=91.44$ YLR $=-91.44 \quad 2 L R=20.0025$
$U A X=1$. VDN $=-1$. DLX $=1.524$ NCH $=*$ U. WGS* END

TTL="PLAN VIEW $1 / 6$ SCALE OF CORE, $Q=$ MIDLEVEL OF FIR TIMBERS" XUI $=-91.44 \quad$ YUL $=91.44 \quad 2 U L=9.2075$

$U A X=1$. VDN $=-1$. DLX $=1.524$ NCH=* U. WGS* BND

TTL="PLAN VIEW $1 / 10$ SCALE OP MOCKUP, $Z=$ MIDLEVEI, OF U UNITS" $X U L=-135 . Y U L=135 . \quad 2 U L=59.69$

UAX $=1$. VDN $=-1$. $D L X=2.54$ NCH=* U. WGS* END

TTI ="PLAN VIEW $2 X$ SCALE OF SINGLE U UNIT, $2=$ MIDLEVEL OF UNIT $X U L=-3.6195$ YUL $=3.6195 \quad \mathrm{ZUL}=59.69$

UAX $=1$. VDN $=-1$. DLX $=0.127$ NCH=* U. WGS* END 
Table A.2 (continued)

TTL ="CROSS SECTION OF CORE $1 / 6$ SCALE, $Y=0.0$ " $X U L=-91.44$
$X L R=91.44$ YUL $=0.0 \quad 2 U L=110$

UNX $=1$. WDN=-1. DLX $=1.524$ NCH=* U. WGS* END

TTL $=$ "CROSS SECTION OF MOCXUP $1 / 10$ SCALE, $Y=0.0$ " $X U L=-135 . \quad Y U L=0.0 \quad 2 U L=125$.

$U A X=1$. WDN $=-1$. DLX $=2.54$ NCH $=$ U. WGS $*$ END

TTL="CROSS SBCTION OF GRATING/FIR TIMBERS, PULI SCALE"

$X U L=0$. YUL $=0$. $Z U L=24.13$

XLR $=25.4$ YLR $=0$. XLR $=-3.0$
UAX $=1$. WDN $=-1$. DLX $=.254$ NCH $=*$ U. WGS* END

TTL="PLAN VIEW OF GRATING THRU TIEBARS, FULL SCALE"

$X U L=0 . \quad Y U L=25.4 \quad$ ZUL $=21.193$

$X I R=25.4 Y$ Y Y

TTL="PLAN VIEW OF GRATING GRATING MIDLEVEL, FULI SCALE" $X U L=0 . \quad Y U L=25.4 \quad 2 U L=20.0025$
$X L R=25.4 \quad Y L R=0 . \quad Z L R=20.0025$

$X L R=25.4$ YLR $=0 . \quad Z L R=20.0025$
$U A X=1$.

TTL="PLAN VIEW OP TIMBERS TIMBER MIDLEVEL, FULE SCALE" $X U L=0 . \quad Y U L=25.4 \quad 2 U L=9.2075$

XLR $=25.4 \quad Y L R=-25.4 \quad$ \&LR=9. 2075 U. WG * END

TTL="CROSS SBCTION OF CENTRAL UNIT, $2 \times 1$ SCALE, $Y=0.0$ " $X U L=-3.6195$ YUL $=0.0 \quad$ IUL $=110$

XLR $=3.6195$ YLR $=0.0 \quad$ OLR $=-10$.
UAX $=1$. WDN $=-1$. DLX $=.127$ NCH=* U. WGS * END

END PLOT
BND DATA
CAB15

CASB EBJ.13 U(3.85) 15 2.5"/0.0"/0.75"/64.8CM, SQUARE 27GROUPNDY INFHOMMEDIUH

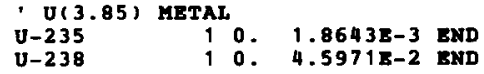

HULL DENSITY WATER MODERATOR/REFLECTOR

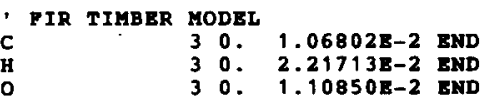

STEEL FOR GRATING, TANK WALLS AND PLOOR

CARBONSTEEL 41.0

' STAINLESS STEEL LINING FOR TANK
SS 304

BND COMP 13 U(3.85) $1502.5 \% / 0.0 \% / 0.75 \% / 64.8 \mathrm{CM}$, SQUARE

NPG $=600$ NUB $=$ YES PLT $=$ NO FDN=YES TME $=90 \quad$ TBA $=2$

END PARM

READ GEOM

UNIT 1

182.880 .18 .4150 .18 .4150

UNIT 2

WATER GAP $3.5^{\prime \prime} \times 72^{\prime \prime} \times 7.25^{n}$ BETWEEN TIMBERS *

CUBOID $2,182.880,8.890,18.4150$

UNIT 3

COM=* ARRAY OF TIMBERS $72^{n} \times 72^{n} \times 7.25^{n}$ *

ARRAY $13 * 0.0$

1 2R0. 2R0 3175 2RO

REPLICATE 2 1 1 4R30.48 2R0.

UNIT 4

COM=* SECTION OF STEEL GRATING

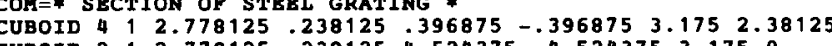

CUBOID $4,3.016250 .4 .524375-4.5243753 .1750$

UNIT 5

COM=* ARRAY OF STEEL GRATING *

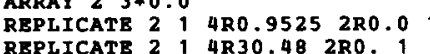

COMIT 6 U(3.85) ROD, $H=64.8$, SUBMERGED *

CYLINDER 111.41990364 .80$.

CYITDDER 112.45934464 .80$.

CYLINDER $1112.839806 \quad 64.80$

CYLINDER 113.17500064 .80

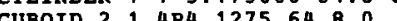


Table A.2 (continued)

UNIT 7

COM=* WATER CUBOID TO COMPLBTE ARRAY 3 -

CUBOID 214 P4.1275 64.80

UNIT 8

85) ROD, $H=11,4$, DRY

CYLINDER 1 1 $1.419903 \quad 11.40$.

CYLINDER 112.45934411 .40

CYLINDER $1112.839806 \quad 11.40$

CUBOID $014 \mathrm{P} 4.1275,11.40$.

\section{UNIT 9}

COM=* VOID CUBOID TO COMPLETE ARRAY 4 -

UNIT 10

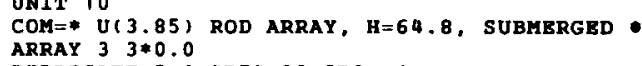

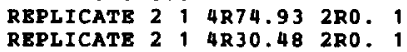

UNIT 11

COM=* ARRAY OF 15 RODS, H=11.4, DRY *

ARRAY $43 * 0.0$

REPLICATE 0 . 1 4R74.93 2RO. 1

CORB $51-121.92-121.920$

REPLICATE 51 5R0.0 0.1905

END GEOM

ARA $=1 \quad N U X=1 \quad N U Y=13 \quad$ NUZ $=1$

FILL 125021 BND FILL

ARA $=2$ NUX $=60$ NUY $=20$ NUZ $=$

PILL P4 END FIL

ARA $=3$ NUX $=4$ NUY $=4$ NUZ $=1$

ARA $=4 \quad N U X=4 \quad$ NUY $=4 \quad$ NUZ $=1$

FILL 3R8 9 12R8 END PILL

GBL $=5$
$A R A=5$ NUX $=1 \quad$ NUY $=1 \quad$ NUZ $=4$

PILI 35

READ START

NST $=1$

$X S M=-16.51 \quad X S P=16.5$

$Y S M=-16.51 \quad Y S P=16.51$

RND START

TTL="PLAN $V I E W 1 / 6$ SCALB OP CORE, $Z=M I D L E V E L$ OF ANNULI/INSERTS" $X U L=-91.44 \quad Y U L=91.44 \quad \mathrm{ZUL}=59.69$
$X L R=91.44 \quad Y L R=-91.44 \quad \mathrm{ZLR}=59.69$

UAX $=1$. VDN $=-1$. DLX $=1.524$ NCH=* U. WGS* END

TTL=" PLAN VIEW PULI SCALE OF ARRAY, $Z=$ MIDLEVEL, OF UNITS"

$\begin{array}{lll}X U L=-16.51 & Y U L=16.51 & Z U L=59.69 \\ X I R=16.51 & Y L R=-16.51 & \end{array}$

UAX $=1$. VDN $=-1$. DLX $=.254$ NCH $=*$ U. WGS $*$ END
TTL="PLAN VIEW $1 / 6$ SCALE OF CORE, $z=$ MIDLEVEL OF GRATING" XUL $=-91.44 \quad$ YUL $=91.44 \quad \mathrm{zUL}=20.0025$

XLR=91. 44 YLR $=-91.44 \quad 2 L R=20.0025$
UAX $=1$. VDN $=-1$. DLX $=1.524$ NCH=* U. WGS* END

TTL="PLAN VIBW $1 / 6$ SCALE OF CORE, $Z=M$ IDLEVEL OF FIR TIMBERS" $X U L=-91.44 \quad$ YUL $=91.44 \quad 2 U L=9.2075$

XLR=91. 44 YLR $=-91.44$ ZLR $=9.2075$
UAX $=1$. VDN $=-1$. DLX $=1.524$ NCH= U. WGS $*$ END

TTL=" PLAN VIEW 1/10 SCALE OF MOCKUP, $z=M I D L E V E L$ OF $U$ UNITS" $X U L=-135 . \quad Y U L=135 . \quad Z U L=59.69$

$U A X=1$. VDN=-1. DLX $X=2.54$ NCH=* U. WGS* END

TTL="PLAN VIEW $2 X$ SCALE OF SINGLE U UNIT, $Z=$ MIDLEVEL OF UNIT" $X U L=0.0 \quad Y U L=8.255 \quad Z U L=59.69$

UAX $=1$. VDN $=-1$. DLX $=0.127$ NCH=* U. WGS* BND

TTL $=$ "CROSS SECTION OF CORE $1 / 6$ SCALE, $Y=4.1275^{n}$

$X U L=-91,44 \quad Y U L=4,1275 \quad Z U L=110$.

$\begin{aligned} & X L R=91.44 \\ & U A X\end{aligned}=1$. WDN $=-1$. DLX $=1.524$ NCH $=$ U. WGS* END

TTI $=$ "CROSS SECTION OP MOCKUP $1 / 10$ SCALB , $Y=4.1275$

$X U L=-135 . \quad Y U L=4.1275 \quad Z U L=125$

UAX $=1$. WDN $=-1$. DLX $=2.54$ NCH $=*$ U. WGS $*$ END

TTL="CROSS SECTION OF GRATING/FIR TIMBERS, FULL SCALE"

$X U L=0$. $Y U L=0$. $\quad$ ZUL $=24.13$

$X L R=25.4$ YLR $=0 . \quad 2 L R=-3.0$ MCH

TTL="PLAN VIEW OF GRATING THRU TIEBARS, PULL SCALE"

$X U L=0 . \quad Y U L=25.4 \quad$ ZUL $=21.193$
$X I, R=25.4 \quad Y L R=0$.

$X L R=25.4 \quad$ YLR=0. $\quad$ ZLR=21.193
UAX $=1$. VDN=-1. DLX $=.254 \quad$ NCH=* U.WGS* END

TTL=" PLAN VIEW OF GRATING O GRATING MIDLEVEL, PULL SCALE" $X U L=0 . \quad Y U L=25.4 \quad Z U L=20.0025$

$X L R=25.4$ YLR $=0 . \quad$ ZLR $=20.0025$
UAX $=1$. VDN $=-1$. DLX $=.254$ NCH $=*$ U. WGS $*$ END

TTL=" PLAN VIEW OF TIMBERS TIMBER MIDLEVEL, PULL SCALE" $X U L=0 . \quad Y U L=25.4 \quad Z U L=9.2075$

$X L R=25.4 \quad Y L R=-25.4 \quad$ ZLR $=9.2075$

UAX $=1$. VDN $=-1$. DLX $=.254$ NCH=* U. WGS* END

TTL $=$ "CROSS SECTION OF CENTRAL UNIT, $2 X$ SCALE, $Y=4.1275^{\circ}$

$X U L=0.0 \quad$ YUL $=4.1275 \quad \mathrm{zUL}=110$.

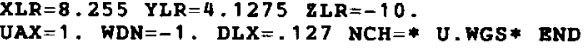

END PLOT

END DATA 
Table A.2 (continued)

CAB 16

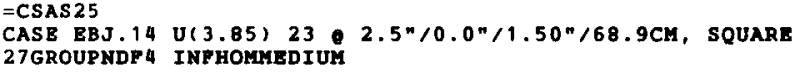

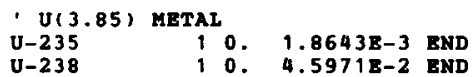

GULL DENSITY WATER MODERATOR/RETLECTOR

- FIR TIMBER MODE

\%. $2.21713 \mathrm{~B}-2$ BND

C STEEL FOR GRATING, TANK WALLS AND TLOOR STAINLESS STEEL LINING FOR TANK
SS 304

END COMP

CASE RBJ.14U(3.85) 23 2.5"/0.0"/1.50"/68.9cm, SQUARE

READ PARM

NPG $=6000$

UNIT

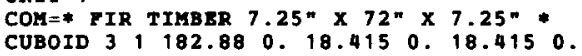

UNIT

TIMBRS

CUBOID 21182.880 .8 .890 .18 .4150 .

UNIT 3

COM=* ARRAY OF TIMBERS 72" $\times 72^{n} \times 7.25 n *$

3*0.0

REPLICATE 2 1 2RO. 280,3175 2RO. 1

UNIT 4

COM=* SECTION OF STERI GRATTMG

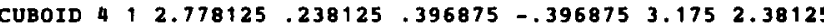
CUBOID $24 \begin{aligned} & 2.778125 \\ & \text { CUBOID }\end{aligned}$ CUBOID $4,3.016250 .4 .524375-4.5243753 .1750$.

UNIT 5

COH=* ARRAY OF STEEI GRATING *

ARRAY 2 3*0.0

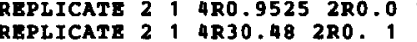

UNIT 6

COM=* U(3.85) ROD, $H=68.9$, SUBMERGED *

CYLINDER $; 11.41990368 .90$.

CYLINDER 1112.00804668 .90 .

CYLINDER 112.45934468 .90$.

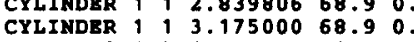

CUBOID 214 P5.08 68.90 .
UNIT 7

COM=* WATER CUBOID TO COMPLETE ARRAY 3 *
CUBOID 214 P5.08 68.90 .

UNIT 8

CYLINDER 1191.4199037 .30$.

CYIINDER 11 1 2.4593447 .30 .

CYLINDER $1192.839806 \quad 7.30$

CUBOID 0 \% 4 P5.08 7.30.

UNIT 9

COM=* VOID CUBOID TO COMPIETE ARRAY 4 "

CUBOID 014 P5.08 7.30

UNIT 10

COM=* U (3.85) ROD ARRAY, H=68.9, SUBMERGED *

ARRAY $33 * 0.0$

REPLICATE 2 1 4R66.04 2RO.

UNIT 11

COM=* ARRAY OF 22 U(3.85) RODS, H=7.3, DRY -

ARRAY $4 \quad 3 * 0.0$

REPLICATE 014 4R66.04 2RO.

CORE $5 \quad 1 \quad-121.92 \quad-121.92 \quad 0$.

REPLICATE 5 $11580.0 \quad 0.1905$

END GEOM

$A R A=1 \quad N U X=1 \quad N U Y=13 \quad$ NUZ $=1$

FILL 125021 END PILI

ARA $=2$ NUX $=60$ NUY $=20 \quad$ NUZ $=1$

FILL 74 END FILI

PRA $=3$ NUX $=5 \quad$ PUY $=5 \quad N U Z=1$

$A R A=4$ NUX $=5$ NUY

FILL 3RB 2R9 20R8 END FILI

$G B L=5$

ARA $=5$ NUX $=1$ NUY $=1 \quad$ NUZ $=4$

ILL $3 \quad 5 \quad 1011$ END PILL

RND ARRAY

NST $=1$

$X S M=-25: 4 \quad X S P=25,4$

$Y S M=-25$.4 $Y S P=25.4$

BND START

TTL="PLAN VIEW $1 / 6$ SCALE OF CORE, $Z=$ MIDLEVEL OF ANNULI/INSERTS" $X U L=-91.44 \quad Y U L=91.44 \quad Z U L=59.69$
$X L R=91.44 \quad Y L R=-91.44 \quad 2 L R=59.69$

UAX $=1$. VDN $=-1$, DLX $=1.524 \quad$ NCH $=*$ U.WGS* END

TTL="PLAN VIEW OF ARRAY, FULI SCALE"

$X U L=-25.4 \quad$ YUL $=25.4 \quad$ ZUL $=59.69$

VAX $=1$. VDN $=-1$, DIX $=254$ NCH=* U. WGS* END 
Table A.2 (continued)

TTL=" PLAN VIEW $1 / 6$ SCALE OF CORE, $Z=$ MIDLBVEL OF GRATING" $X U L=-91.44 \quad Y U L=91.44 \quad$ ZUL $=20.0025$
$X L R=91.44$ YLR $=-91.44$ ZL $=20.0025$

UAX $=1$. VDN $=-1$. DLX $=1.524$ NCH $=*$ U. WGS* END

TYL=" PLAN VIEH $1 / 6$ SCALE OF CORE, $Z=$ MIDLEVEL OF FIR TIMBERS XUL $=-91.44$ YUL $=91.44 \quad 8 U L=9.2075$

XLR $=91.44$ YLR $=-91.44 \quad$ ZLR $=9.2075$
UAX $=1$. VDN $=-1$. DLX $=1.524$ NCH=* U.WGS* END

TTL="PLAN VIEW $1 / 10$ SCALE OF MOCXUP, $z=$ MIDLEVEL OF $U$ UNITS" $X U L=-135 . Y U L=135, \quad 2 U L=59.69$

$X L R=135$. YLR $=-135.2 L R=59.69$
$U A X=1$. VDN $=-1$. DLX $=2.54$ NCH=* U. WGS* END

TTL=" PLAN VIEW $2 X$ SCALE OF SINGLE U UNIT, $Z=$ MIDLEVEL OF UNIT $X U L=-5.08 \quad$ YUL $=5.08 \quad Z U L=59.69$
$X L R=5.08 \quad$ YLR $=-5.08 \quad 2 L R=59.69$

XLR $=5.08$ YLR $=-5.08$ ZLR $=59.69$
$U A X=1$. VDN $=-1$. DLX $=0.127$ NCH

TTL $=$ "CROSS SECTION OF CORE $1 / 6$ SCALE, $Y=0.0$ "

$X U L=-91.44 \quad$ YUL $=0.0 \quad Z U L=110$.

$X L R=91.44 \quad Y L R=0.0 \quad 2 L R=-10$.

TTL $=$ "CROSS SECTION OF MOCKUP $1 / 10$ SCALE, $Y=0.0 "$

$X U L=-135 . \quad Y U L=0.0 \quad 20 L=125$.

$X L R=135$. YLR $=0.0 \quad 2 L R=-10$.

$\mathrm{NX}=1$. WDN=-1, DEX 2.54 NCH=* U.WGS* END

TTL="CROSS SECTION OF GRATING/FIR TIMBERS, FULL SCALE"

$X U L=0 . \quad Y U L=0 . \quad 2 U L=24.13$

XLR=25. 4 YLR $=0 . \quad 2 L R=-3.0$
UAX $=1$. WDN $=-1$. DLX $=.254^{\circ}$ NCH=* U.WGS* END

TTL=" PLAN VIEW OF GRATING THRU TIEBARS, FULL SCALE"

$X U L=0 . \quad Y U L=25.4 \quad 2 U L=21.193$

$X L R=25.4$ YLR=0. $2 L R=21.193$
UAX $=1$. VDN $=-1$. DLX $=.254$ NCH=* U.WGS* END

TTL="PLAN VIEW OF GRATING O GRATING MIDLEVEL, FULL SCALE" $X U L=0 . \quad$ YUL $=25.4 \quad$ QUL $=20.0025$

UAX $=1$. VDN $=-1$. DLX $=.254$ NCH=* U.WGS* END

TTL $=$ "PLAN VIEW OF TIMBERS - TIMBER MIDLEVEL, FULL SCALE"

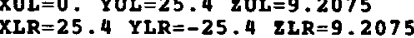

UAX $=1$. VDN $=-1$. DLX $=.254$ NCH $=$ U.WGS END

TTL $=$ "CROSS SECTION OF CENTRAL UNIT, $2 X$ SCALE, $Y=0.0$ "

XL $=-5.08$ YUL $=0.0 \quad 2 U L=110$.

UAX $=1$. WDN $=-1$. DLX $=.127$ NCH=* U. WGS * END

END PLOT
END DATA 
Table A.3. Table 3 input data

CASO4

BRITISH HANDBOOK OF CRITICALITY SAFETY U(1.42)F4 ( PARAFTIN (CASE 04) 27GROUPNDF4 INFHOMMEDIUM $1.40239223898 .5977 \mathrm{END}$

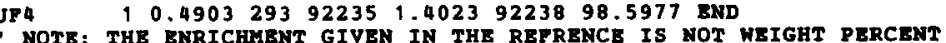
PARATE : THE BNRICHEENT 10.4572 BND

- PARATYIN (CASE O4 READ PARM NPG $=600$ NUB=YES FDN=YES END PARM

CUBOID $112846.552 P 46.502 P 61.9$

END GEOM

END DATA

CAS05
$=$ CSAS2

BRITISH HANDBOOK OF CRITICALITY SAFETY U(1.42)P4 E PARAFTIN (CASE 05) 27GROUPNDP4 TNPHOMMEDIUM 14023923899.5977 END

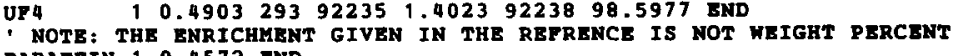
PARAFPIN 10.4572 END

BRITISH HANDBOOK OP CRITICALITY SAFETY U(1.42)T4 S PARAFFIN (CASE 05 READ PARM NPG $=600$ NUB $=$ YES PDN=YES END PARM

CUBOID $112950.002949 .95 \quad 2851.55$

END GEOM

BND DATA

CAS 06
$=$ CSAS 25

BRITISH HANDBOOK OF CRITICALITY SAFETY U(1.42)/4 \& PARAFTIN (CASE 06, 27GROUPNDF 4 INFHOMMEDIUM

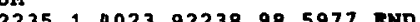

NOTE: THE ENRICHMENT

BND COMP

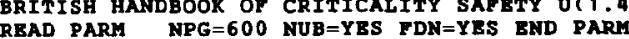

READ GEOY

READ GEOM

BND GEOM
END DATA
BND

END

=CSAS25 HANDBOOX OF CRITICALITY SAFETY U(1.42)F4 E PARAFTIN (CASE 06) HANSEN-ROACH INFHOMMEDIU

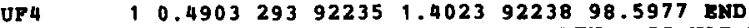

NOTB: THE BNRICHMENT GIVEN IN THE RETRENCE IS NOT WEIGHT PERCENT PARAFFIN 10.4572 END

GRITISH HANDBOOR OF CRITICALITY SATETY U(1.42) FA : PARAFPIM (CASE O6) READ PARA NPG $=600$ NUB $=$ YES FDN=YES END PARM

READ GBOM
CUBOID $1+2865.35 \quad 2865.3 \quad 2837.1$

RND GEOM

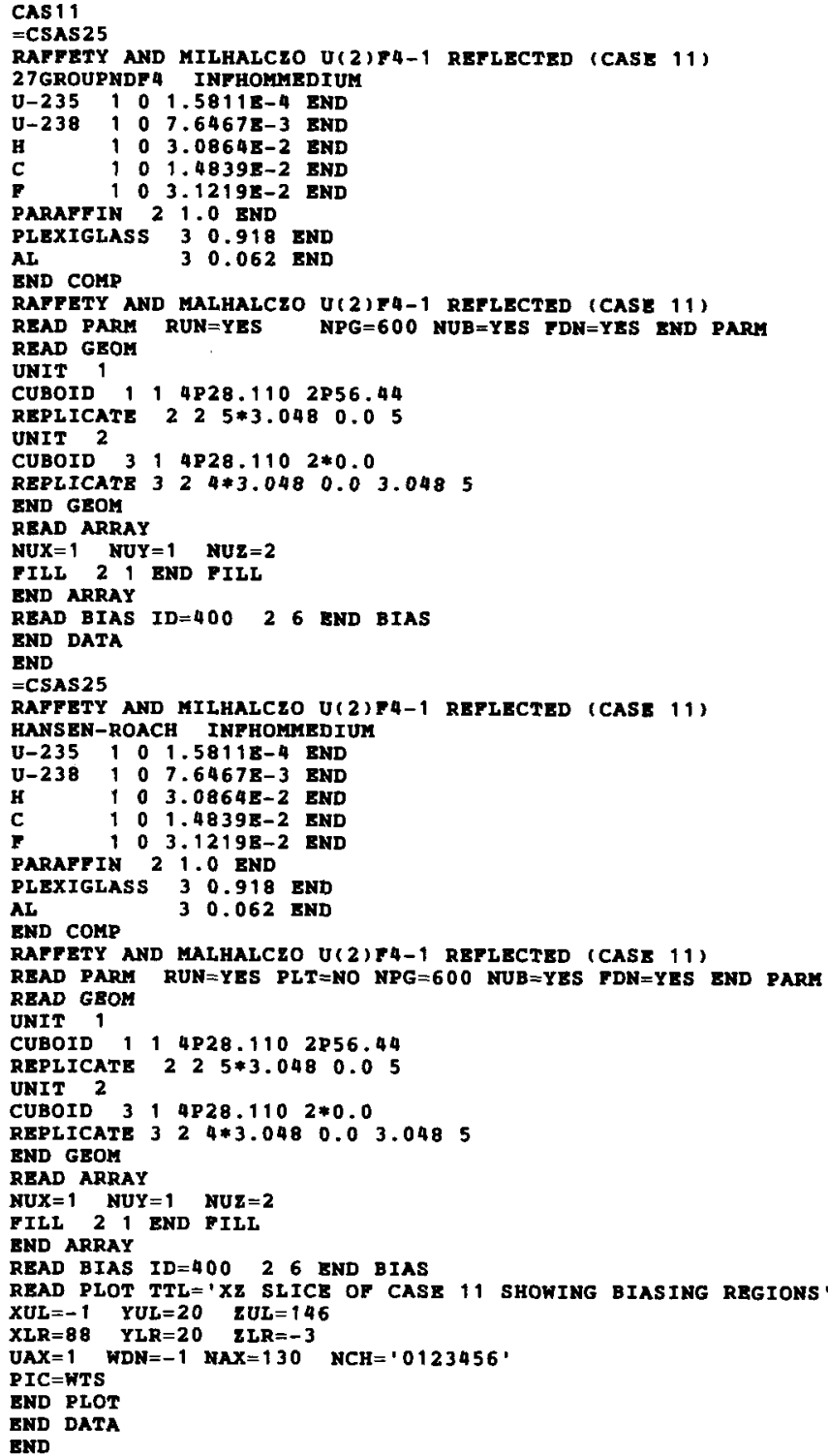


Table A.3 (continued)

CAS 12

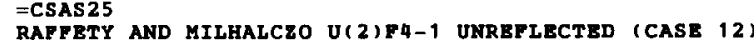

RAFTETY AND MILHALCZO ULC

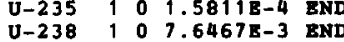

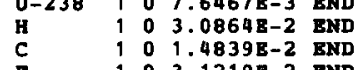

END COMP

RAFTETY AND MALHALCZO U(2)

READ PARM NPG $=600$ NUB=YES FDN=YES END PARM

$\begin{array}{llllllllll}\text { READ GEOM } & & & & & \end{array}$

CUBOID

END GEOM

END $=$ CSAS25

RAFTETY AND MILHALCZO U(2) 4 -1 UNREFLECTED (CASE 12 )

HAFEST-ROACH INFHOMMEDTUM

$\mathrm{U}-235 ; 001.5811 \mathrm{~B}-4$ END

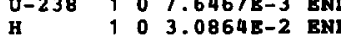

$\begin{array}{lllll}C & 1 & 0 & 1.48398-2 & \text { END } \\ F & 1 & 0 & 3.1219 \mathrm{E}-2 & \text { END }\end{array}$

BND COMP OND MALHALCZO U(2) T4-1 UMRETLECTED (CASE 12)

READ PARM NPG $=600$ NUB=YBS FDN=YES END PARM

READ GEOM $135.735-35.73535 .735-35.73597 .07-47.07$

CUBOID $1135.735 \quad-35.735 \quad 35.735 \quad-35.735 \quad 47.07 \quad-47.07$

BND GBOM

END

CAS 13

RAFPETY AND MILHALCZO U(2)P4-2 REPLECTED (CASE 13) NO BIASING 27GROUPNDF 4 INFHOMMEDIUH

$\begin{array}{lllll}\mathbf{U}-235 & 1 & 0 & 1.3303 \mathrm{E}-4 & \mathrm{END} \\ \mathbf{U}-238 & 1 & 0 & 6.4370 \mathrm{E}-3 & \mathrm{END}\end{array}$

$\begin{array}{llllll}\mathbf{U}-238 & 1 & 0 & 6.4370 \mathrm{E}-3 & \mathrm{END} \\ \mathrm{H} & 1 & 0 & 3.9097 \mathrm{E-2} & \mathrm{END} \\ \mathrm{C} & 1 & 0 & 1.9797 \mathrm{E}-2 & \mathrm{END}\end{array}$

$\begin{array}{lllll}\text { H } & 1 & 0 & 3.9097 \mathrm{E}-2 & \text { END } \\ \mathrm{C} & 1 & 0 & 1.8797 \mathrm{E}-2 & \mathrm{END} \\ \mathrm{r} & 1 & 0 & 2.6280 \mathrm{E}-2 & \mathrm{END}\end{array}$

PARAFTIN 21.0 END

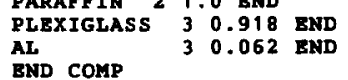

A READ PARM RUN=YES NPG $=600$ NUB=YBS FDN=YES END PARM

READ GEOM

UNIT 1

CUBOID 114 4P25.555 2P36.935

CUBOTD 210 4P25.555 200.0

REPLICATE 3 4P25.555 $2 * 0.0$

END GBOM

NUX=1 $\quad$ NUY $=1 \quad$ NUZ $=2$

FILL 21 END FILL

BND ARRAY

READ BIAS ID $=40026$ END BIAS

BND DATA
$=\operatorname{CSAS} 25$

AND MTLHALCZO U(2)F4-2 REPLECTED (CASE 13) NO BIASING HANSEN-ROACH INFHOMMEDIUI

$\begin{array}{llllll}0-235 & 1 & 0 & 1.3303 E-4 & \text { END } \\ -238 & 1 & 0 & 6.4370 \mathrm{E}-3 & \mathrm{END}\end{array}$

H $\quad 1.03 .9097 \mathrm{E}-2$ END

$102.6280 \mathrm{E}-2 \mathrm{BND}$

PARAFTIN 2 1.0 END

$\begin{array}{lllll}\text { PLEXIGLASS } & 3 & 0.918 & \text { END } \\ \text { AL } & 3 & 0.062 & \text { END }\end{array}$

DND COMP READ PARM RUN $=$ YES PLT $=$ NO $N P G=600$ NUB $=Y$ Y TDY $=$ YES NOP PARY READ GBOM

UNIT 1

CUBOID 11 4P25.555 2P36.935

UNIT 2

REPLICATE $3 \quad 2 \quad 4 * 3.048 \quad 0.0^{\circ} 3.0485$

END GEOM

NUX $=1 \quad$ NUY $=1 \quad$ NUZ $=2$

PILL 21

READ BIAS ID $=400 \quad 26$ END BIAS

13 SHOWING BIASING REGIONS $\begin{array}{lll}X U L=-1 & Y U L=20 & Z U L=106 \\ X L R=83 & Y L R=20 & Z L R=-3\end{array}$

UAX $=1$ WDN $=-1 \quad$ NAXX $=130 \quad$ NCH $=10123456$

PIC $=$ WTS

END PLOT

END D 
Table A.3 (continued)

CAs14

RAFFETY AND MILHALCZO U(2) PA-2 UNRETLECTED (CASE 14) RAFPETY AND MILHALCEO OTO
$27 G R O U P M D Y 4$
INFHOMDIEDIUH

$\mathrm{U}-235 \quad 1 \quad 0 \quad 1.33038-4$ BHD

$\mathrm{U}-238$; 0 6.43708-3 END

$\begin{array}{lllll}\text { H } & 1 & 0 & 3.90978-2 & \text { END } \\ \text { C } & 1 & 0 & 1.8797 \mathrm{E}-2 & \text { END }\end{array}$

END cons

RATPETY AND MALHALCZO U(2)F4-2 UNREFLECTED (CASE 14 ) READ PARM NPG $=600$ NUB=YES FDN=YES END PARM

READ GSOA $120.11-28.1128 .11-28.1161 .235-61.235$

END GEOH

END
$=$ CSAS25

RAFFETY AND MILHALCZO U(2) P4-2 UNRETLECTED (CASE 14) RAFFTY AND

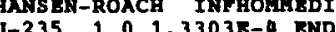

$\mathrm{U}-238 \quad 1006.4370 \mathrm{E}-3$ END

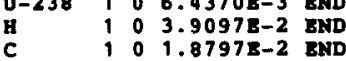

- $\quad 0 \quad 2.6280 \mathrm{~B}-2$ END

DND COMP PARM NPG $=600$ NUB=YES YDN=YSS END PARA

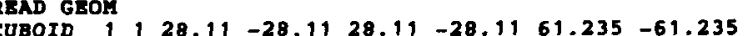
END GEOR

END

CAS15

RAFPTY AND MALHALC2O U(2)F4-3 REPLECTED (CASE 15,

27GROUPNDF 4 INFHOKHEDIUM

U-235 $1001.11915-4$ END

$\begin{array}{llllll}\mathrm{U}-238 & 1 & 0 & 5.4152 \mathrm{E}-3 & \text { END } \\ \mathrm{H} & 1 & 0 & 4.5472 \mathrm{E}-2 & \mathrm{END} \\ \mathrm{C} & 1 & 0 & 2.18515-2 & \end{array}$

$102.21098-2$ END

PARATTIN 21.0 END

PLEXIGLASS 30.918 END

END COMP

30.062 END

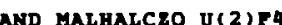

READ PAPM RUY =YES NPG $=600$ NUB $=$ YES FDN $=$ YES END $P A B M$

READ GBOH

CUBOID 1 AP26.835 2P27.145

U⿻15 2

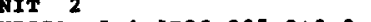

CUBOID 311 AP26.835 $2 * 0.0$

END GEOH

MUXX=1 2 MUY $=1$ MUZ $=2$

PILD ARRAY 21

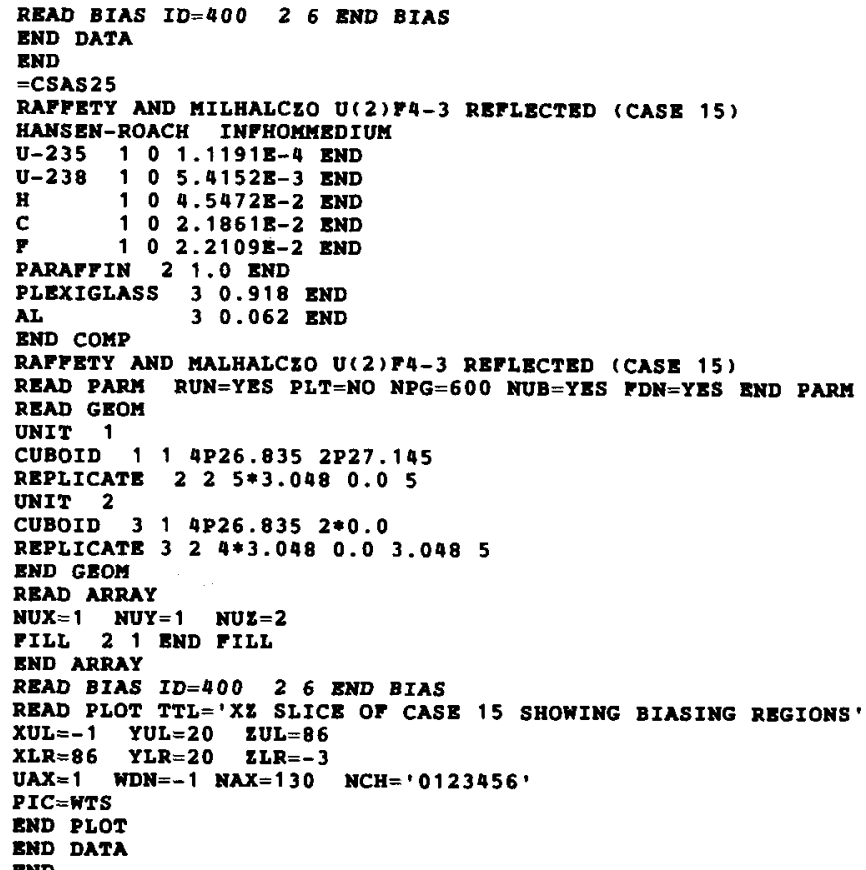

RND COKP$$
30.062 \text { END }
$$

REND PARH RUN=YES PLT $=$ NO NPG $=600 \quad$ NUB $=Y$ YSS $\quad$ DNN=YBS END PARM GAD GROM

UNIT T

R7Pा 4 P26.835 2P27.145

UNIT 2 CUE

REPLICATE $320 \% 3.048 \quad 0.0 \quad 3.048$

END GEOM

NUX $=1 \quad$ NUY $=1 \quad$ NUZ $=2$

NUX=1 $\quad$ NUY $=1 \quad$ NUZ $=2$

END ARRAY

READ PLOT TTL $=$ ' $X Z$ SLICE OF CASE 15 SHOWING BIASING REGIONS.

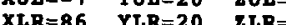

UAX $=1$ WDN $=-1$ NAX $=130$ NCH $=\cdot 0123456^{\prime}$

PIC $=$ WTS
END PLOT

END DATA 
Table A.3 (continued)

CAS16

RAFPETY AND MILHALCEO U(2)PA-4 REFLECTED (CASE 16)

27GROUPNDF 4 INFHOMAEDIU

U-238 $1000.99248-4$ END

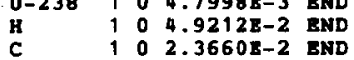

$F \quad 1001.95968-2$ END

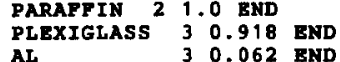

30.062 END

RAFTETY AND MALHAICZO U(2)

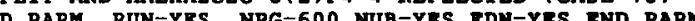
READ GROM

CUBOID 11 1 4 P23.000 2848.285

REPLICATE $225 \% 3.0480 .05$

UNIT 2

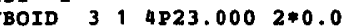

REPLICATE

READ ARRAY

NUX $=1 \quad$ NUY $=1 \quad$ NUZ $=2$

FILL 21 END FILI

READ BIAA

ID $=40026$ END BIAS

BND DATA

END
$=$ CSAS25

PAFPETY AND MILHAICZO U(2)F4-4 REFLECTED (CASE 16 )

HANSEN-ROACH INFHOMMEDIUH

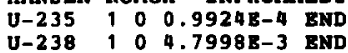

$\begin{array}{lllll}0-238 & 1 & 0 & 4.7998 B-3 & \text { END } \\ \mathrm{H} & 1 & 0 & 4.9212 \mathrm{~B}-2 & \mathrm{BND}\end{array}$

F $\quad 1$ o $12.3660 \mathrm{E}-2 \mathrm{BND}$

PARAFFIN 2 i.0 END

$\begin{array}{llll}\text { PLEXIGLASS } & 3 & 0.918 & \text { END } \\ \text { AL } & 3 & 0.062 & \text { END }\end{array}$

ALN COMP

AND MALHALCZO U(2)FA-4 REFLECTED (CASE 16)

UNIT 1

CUBOID 114 4P23.000 2P48.28

REPLICATE $2225 * 3.048 \quad 0.05$

CUBOID 314 P23.000 2*0.0

REPLICATB 32 4*3.048 $0.0 \quad 3.048$

END GEOM

READ ARRAY

NUX $=1 \quad$ NUY $=1 \quad$ NUZ $=2$

III 21 END FILI

READ BIAS ID $=40026$ END BIAS

READ PLOT TTL='XE SLICE OP CASB 16 SHOWING BIASING REGIONS

$X U L=-1$ YUL $=20 \quad 20 L=129$

UAX $=1$ WDN $=-1$ NAX $=130$ NCH $=\cdot 0123456$,

PIC=WTS

PIC
END PLOT
END DATA
CAS 17
$=$ CSAS2

RAFFETY AND MILHALC2O U(2)FA-5 REFLECTED (CASE 17 )

27GROUPNDF 4 INFHOMMEDIUM

U-238 $100.19418-3$ END

H $\quad 105.3187 \mathrm{E}-2$ BND

C $\quad 1: 02.5570 \mathrm{E}-2$ END

POLYETHYLENE 2 1.00 END

$\begin{array}{llll}3 & 0.918 \text { END } \\ \text { AL } & 3 & 0.062 \text { BND }\end{array}$

END COMP

MALHALCZO U( 2) F4-5 REFLECTED (CASE 17)

READ PAPY RUN $Y$ TH

READ GEOH

CUBOID $112 \mathrm{P28.1602P30.645 \quad 2P27.040}$

REPLICATE $22 \quad 5 * 3.0480 .05$

UNTT 202828.1602030 .045200 .0

CUBOID 312 2P28.160 2P30.645 $2 * 0.0$

END GEOM

READ ARRAY

NUX=1 NUY=1 NUE $=2$

READ BIAS

END DATA

$=$ CSAS 25

(CASE 17)

HANSEM-ROACH INFHOMAREIUI

U-238 $1000.8667 \mathrm{E}-4$ END

H $1005.3187 \mathrm{E}-2$ END

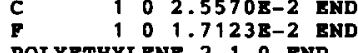

AL $\quad 30.062$ END

END COMP

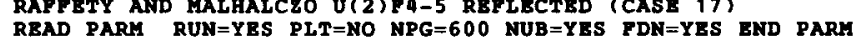
READ GEOY

UNIT 1

CUBOID 1 1 2P28.160 2P30.645 2P27.040

REPLICATE $222 \quad 5 \% 3.048 \quad 0.0$

CUBOID 312828.1602 2P30.645 2*0.0

REPLICATE $324 \% 3.048 \quad 0.03 .0485$

END GEOM

RUX $=1$ ARRYY NUY $=1$ NUZ $=2$

FND ARRAY

READ BIAS ID $=40026$ END BIAS

READ PLOT TTL='XZ SLICE OF CASE 17 SHOWING BIASING REgIONS

$X U L=-1 \quad Y U L=20 \quad 20 L=86$

UAX $=1$ WDN $=-1$ NAX $=130$ NCH $=10123456$

PIC $=$ WTS

END PLOT 


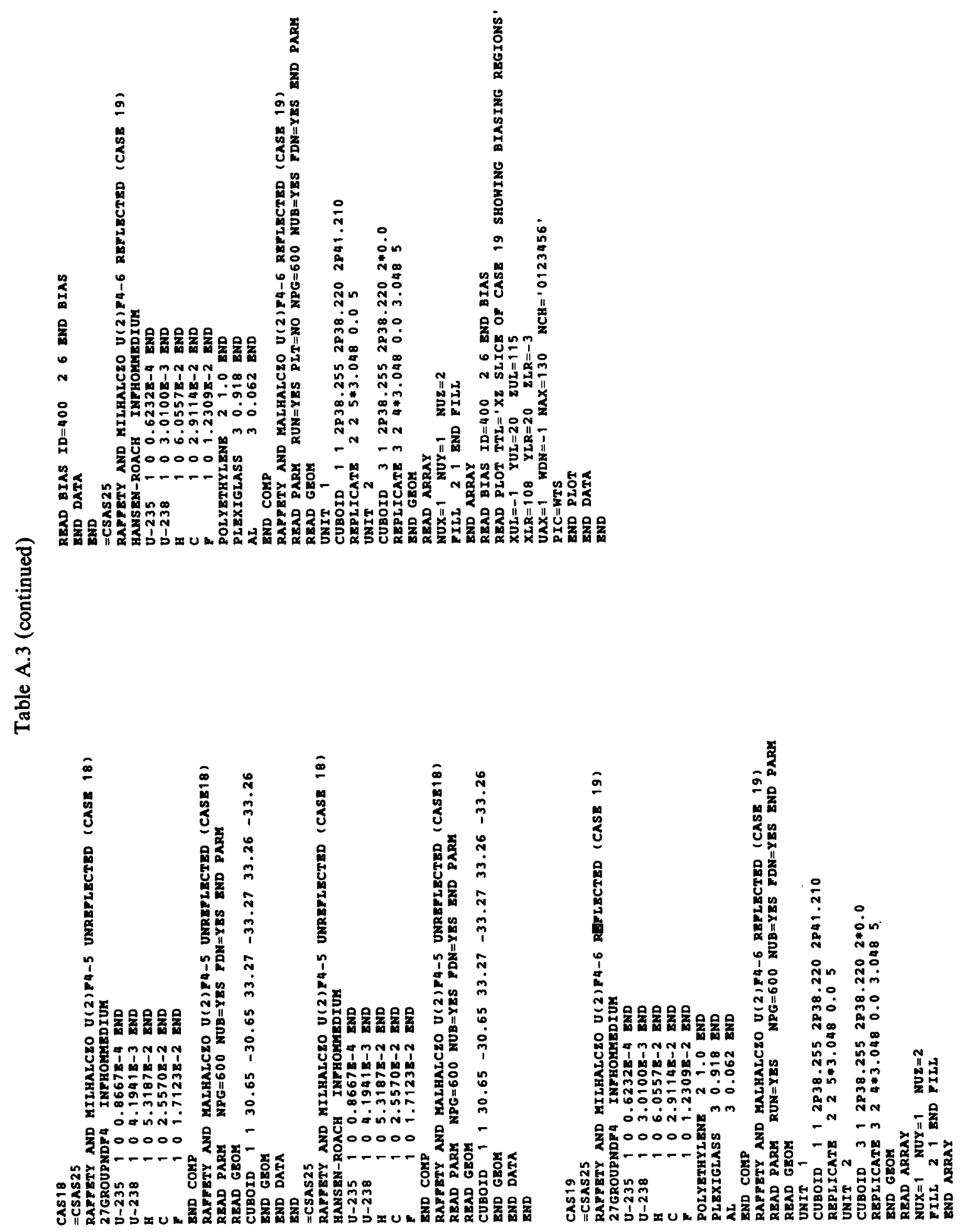


Table A.3 (continued)

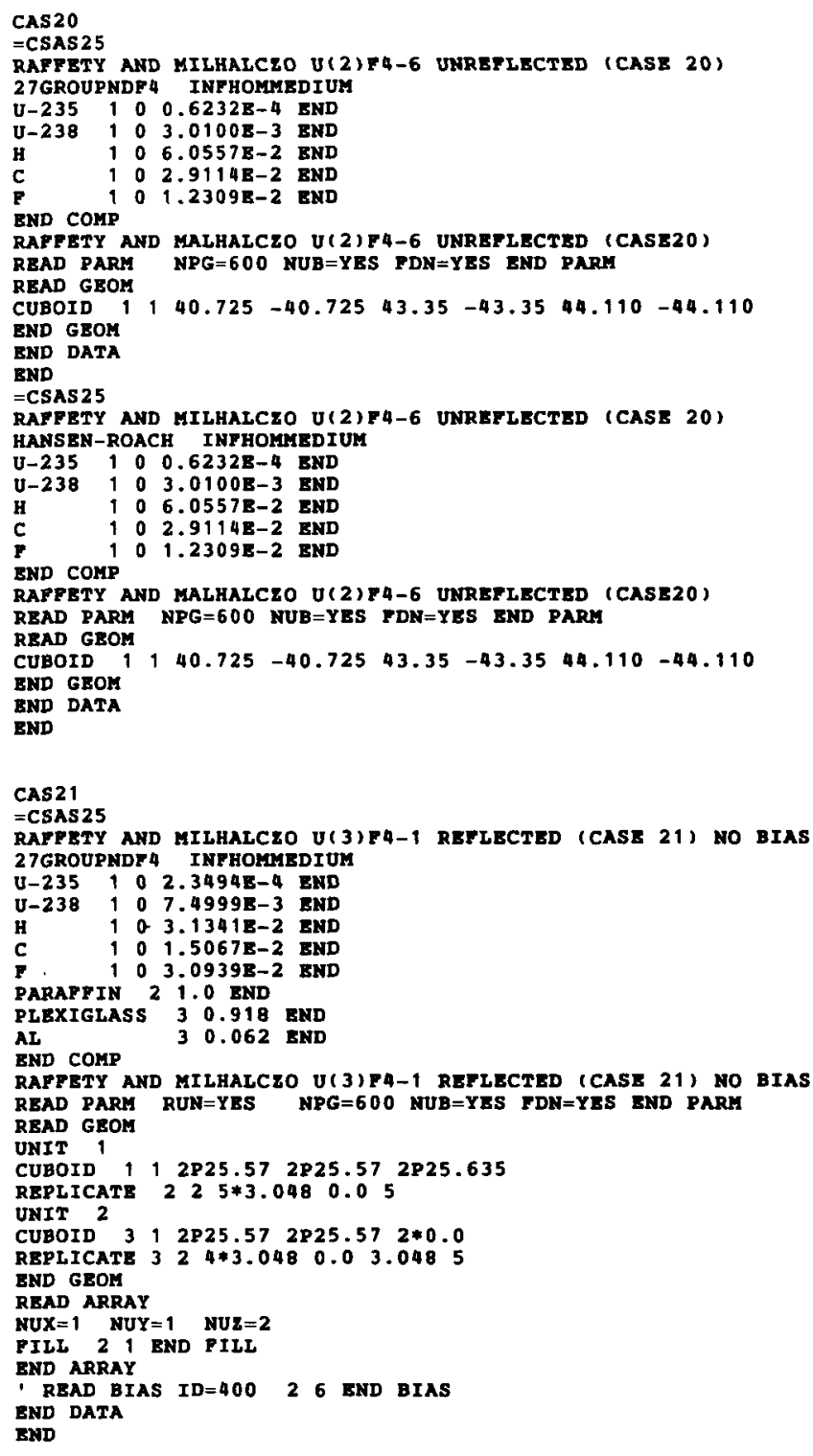


Table A.3 (continued)

CAS23

RAFPETY AND MILHALCEO U(3)P4-1 RETLECTED (CASE 23)

27GROUPNDF 4 INFHOMMEDIUM

\begin{tabular}{lllll}
$\mathrm{U}-235$ & 1 & 0 & $2.3494 \mathrm{E}-4$ \\
$\mathrm{U}-238$ & 1 & 0 & $7.4999 \mathrm{EN}-3$ \\
$\mathrm{H}$ & & 1 & 0 & 3.1341 \\
\hline
\end{tabular}

$\begin{array}{llllll}0-238 & 1 & 0 & 7.4999 \mathrm{E}-3 & \text { END } \\ \mathrm{H} & 1 & 0 & 3.1341 \mathrm{E}-2 & \mathrm{END} \\ \mathrm{C} & 1 & 0 & 1.5067 \mathrm{E}-2 & \mathrm{END}\end{array}$

F 100 3.0939E-2 END

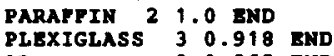

AI 3 O 0.062 END

RND COMP

READ PARM RUN=YES NPG $=600$ NUB $=$ YES FDN=YES END PARA

READ GEOM

UNIT ${ }^{1}{ }^{\prime}$ CUBOID $12 P 23.0102 P 23.0102 P 33.785$

CUBOID $1122 P 23.0102 P 23.010$

UNIT 2

REPLICATE 32 2P13.048 $0.0 \quad 3.0485$

END GEOH

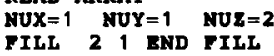

END ARRAY

READ BIAS ID $=400 \quad 26$ END BIAS

END

RAFPSTY AND MILHALCZO U(3) F4-1 REFLECTED (CASE 23)

HANSEN-ROACH INFHOMMEDIUH

$\mathrm{U}-235$ 1 $02.3494 \mathrm{E}-4$ END

$\begin{array}{llll}0-238 & 1 & 0 & 7.4999 \mathrm{E}-3 \\ \mathrm{H} & 1 & 0 & 3.1341 \mathrm{END} \\ \mathrm{C} & 1 & 0 & 1.5067 \mathrm{END}\end{array}$

$1003.09398-2$ END

PARAFTIN 21.0 END

PLEXIGLASS 330.918 END

AND COMP

30.062 END

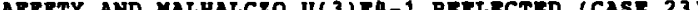

READ PARM RUN=YES PLT $=$ NO NPG $=600$ NUB $=Y$ YS $\quad$ FDN=YES END PARA READ GEOH

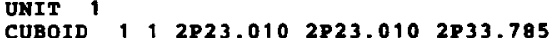

REPLICATE $225 \% 3.0480 .05$

ONIT 2

COBOID $312823.0102823 .0102 * 0.0$

REPLICATE $324 * 3.0480 .03 .0485$

END GEOM

MUX $=1 \quad$ NUY $=1 \quad$ NUZ $=2$

END ARRAY

READ BIAS ID $=400 \quad 26$ END BIAS

作

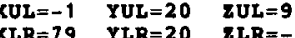

UNX $=1$ NDN $=-1 \quad$ NAX $=130^{\circ}$ NCH $=10123456^{\circ}$

PIC = WTS

END PLOT

END DATA

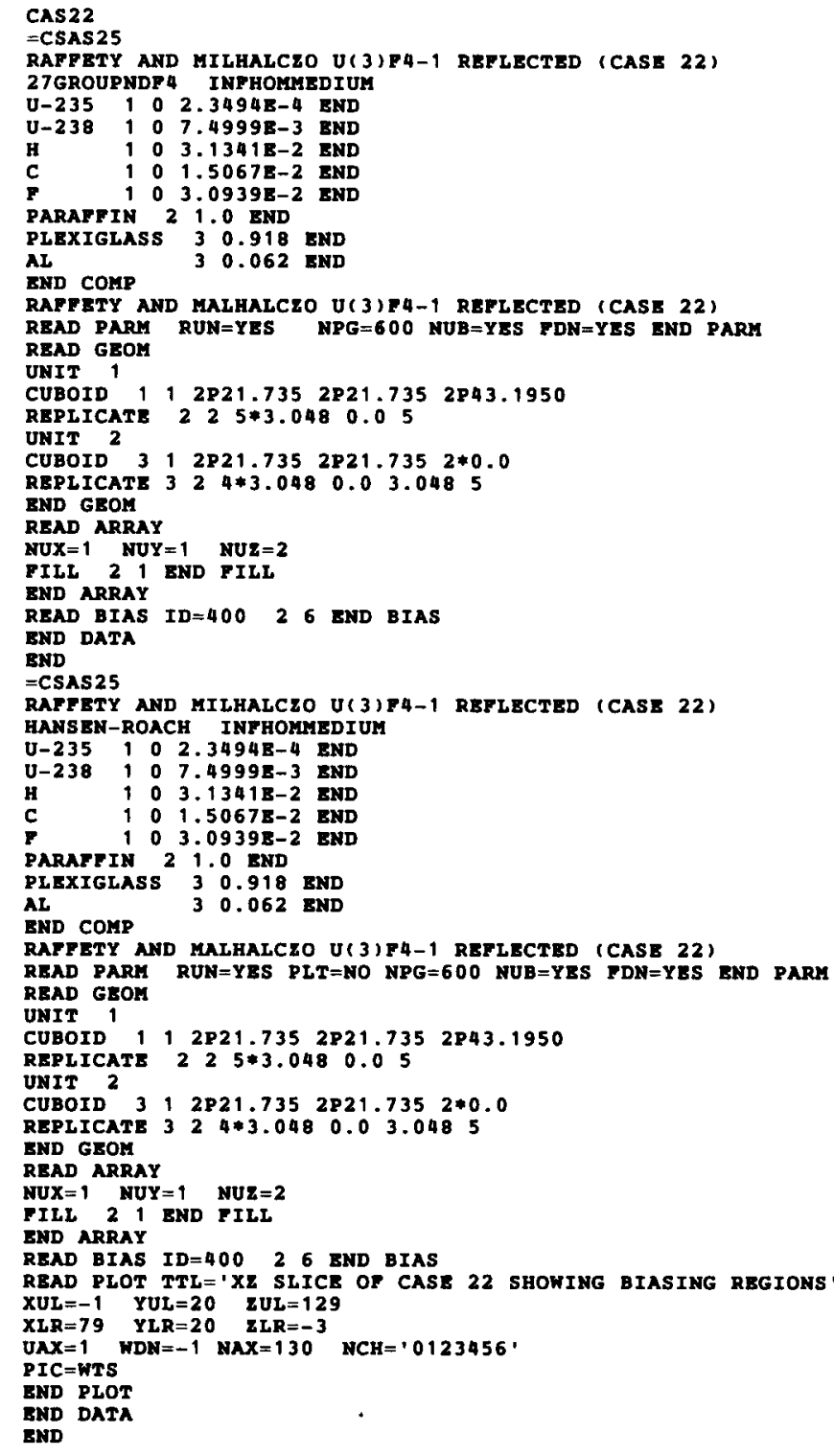




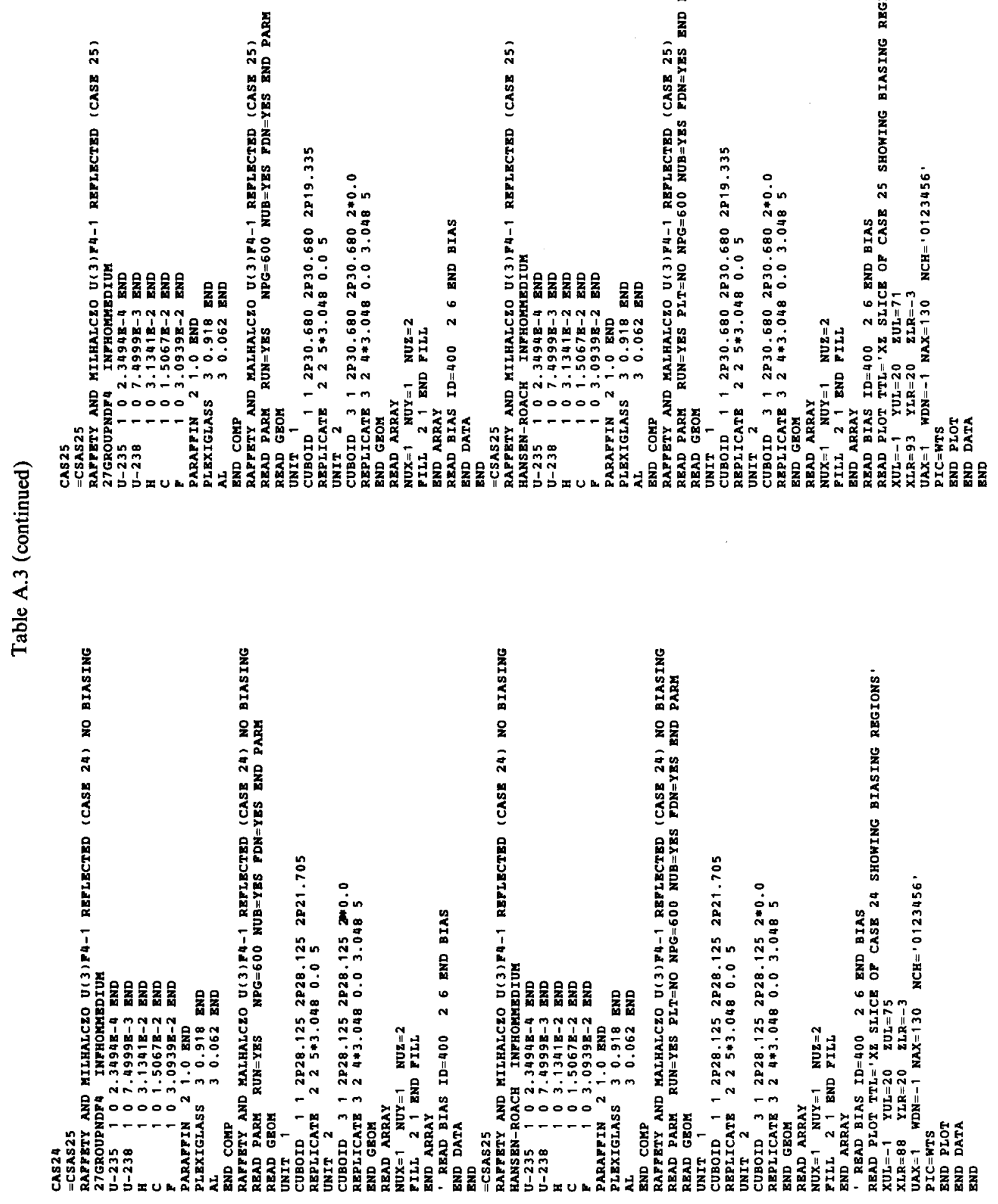


Table A.3 (continued)

$\operatorname{CAs} 26$
$=$ CSAS2

RAFFETY AND MILHALCZO U(3)P4-1 UNREFLECTED (CASE 26) 27GROUPNDF 4 INPHOMMEDIUM

$0-235102.34948-4$ END

$\mathrm{U}-238 \quad 1007.49998-3 \mathrm{END}$

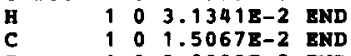

END COKP

SCTED (CASE 26 )

READ PARM

READ GEOM

(a)

END GEOM
BND DATA

BND

RAFFBTY AND MILHAICZO U(3)F4-1 UNREFLECTED (CASE 26)

AANSEN-ROACH INFHOMHEDIU

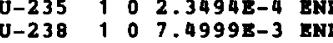

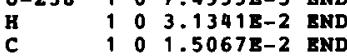

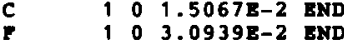

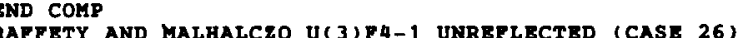
RRAD PARM NPG $=600$ NUB=YES FDN=YES END PARM

$\begin{array}{llllllll}\text { READ GEOM } & & & \end{array}$ CUBOID

END GEOM
END DATA
END

CAS27

RAFPETY AND MILHALCZO U(3)F4-1 UNREFIECTED (CASE 27) 27GROUPNDF 4 INFHOKMEDIUM

U-235 $1002.3494 E-4$ END

\begin{tabular}{lllll}
$\mathrm{U}-238$ & 1 & 0 & $7.4999 \mathrm{E}-3$ & $\mathrm{END}$ \\
$\mathrm{H}$ & 1 & 0 & $3.1341 \mathrm{E}-2$ & $\mathrm{END}$ \\
\hline & 1 & 0 & $1.5057 \mathrm{E}-2$ &
\end{tabular}

C $\quad 1001.5067 \mathrm{E-2} \mathrm{END}$

END COMP

RAFFBTY AND MALHALCZO U(3) 74-1 UNREFIECTED (CASE 27) READ PARM NPG $=600$ NUB=YES PDN=YES END PARY

$\begin{array}{llllllllll}\text { READ GEOM } & & & & & \end{array}$

BND GBOM

BND

$=$ CSAS 25

RAFPETY AND MILHALCZO U(3)F4-1 UNREFLECTED (CASE 27) RANBN

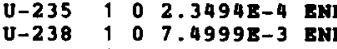

$\begin{array}{llll}\mathrm{H} & 1 & 0 & 3.1341 \mathrm{E}-2 \\ \mathrm{C} & 1 & 0 & 1.5067 \mathrm{END} \\ \mathrm{C} & 1 & \mathrm{END}\end{array}$

F $1003.0939 \mathrm{~B}-2 \mathrm{END}$
RAFFETY AND MALHALCZO U( 3 )F4-1 UNREFLECTED (CASE 27)

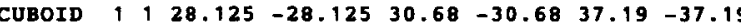

END GBOM

END DATA

\section{CAS28}

RAFPBTY AND MILHALCZO U(3)F4-1 UNREFLECTED (CASE 28 ,

RAFFETY AND MILHALCEO U(3)

27GROUPNDPA TNPHOMMEDIUM

$\begin{array}{lllll}\mathrm{U}-235 & 1 & 0 & 2.3494 \mathrm{E}-4 & \mathrm{END} \\ \mathrm{U}-238 & 1 & 0 & 7.4999 \mathrm{~B}-3 & \mathrm{END}\end{array}$

$\begin{array}{lllll}\mathrm{H} & 1 & 0 & 3.13418-2 & \mathrm{END} \\ \mathrm{C} & 1 & 0 & 1.5067 \mathrm{E}-2 & \mathrm{ENDD}\end{array}$

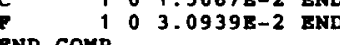

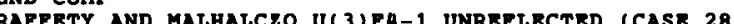
READ PARM NPG $=600$ NUB $=$ YES YDN=YES END PARM

$\begin{array}{llllllllll}\text { READ GEOM } & & & & & & \end{array}$

END GEOM

END

RAPPETY AND MILHALCEO U(3)P4-1 UNREFLECTED (CASE 28 ) HANSEN-ROACH INPHOMMEDIUM

U-235 $1002.3494 E-4$ END

10 3.43418-2 END

C $\quad 1001.50678-2$ END

END COMP 0 3.0939:-2 BND

RAPPETY AND MALHALCZO U(3) P4- 1 UNREFLECTED (CASE 28) READ PARM NPG $=600$ NUB $=$ YES PDN=YES END PAPA

$\begin{array}{llllllllll}\text { CUBOID } & 1 & 1 & 30.7 & -30.7 & 30.7 & -30.7 & 33.00 & -33.00\end{array}$

END GEOM

END DATA 
Table A.3 (continued)
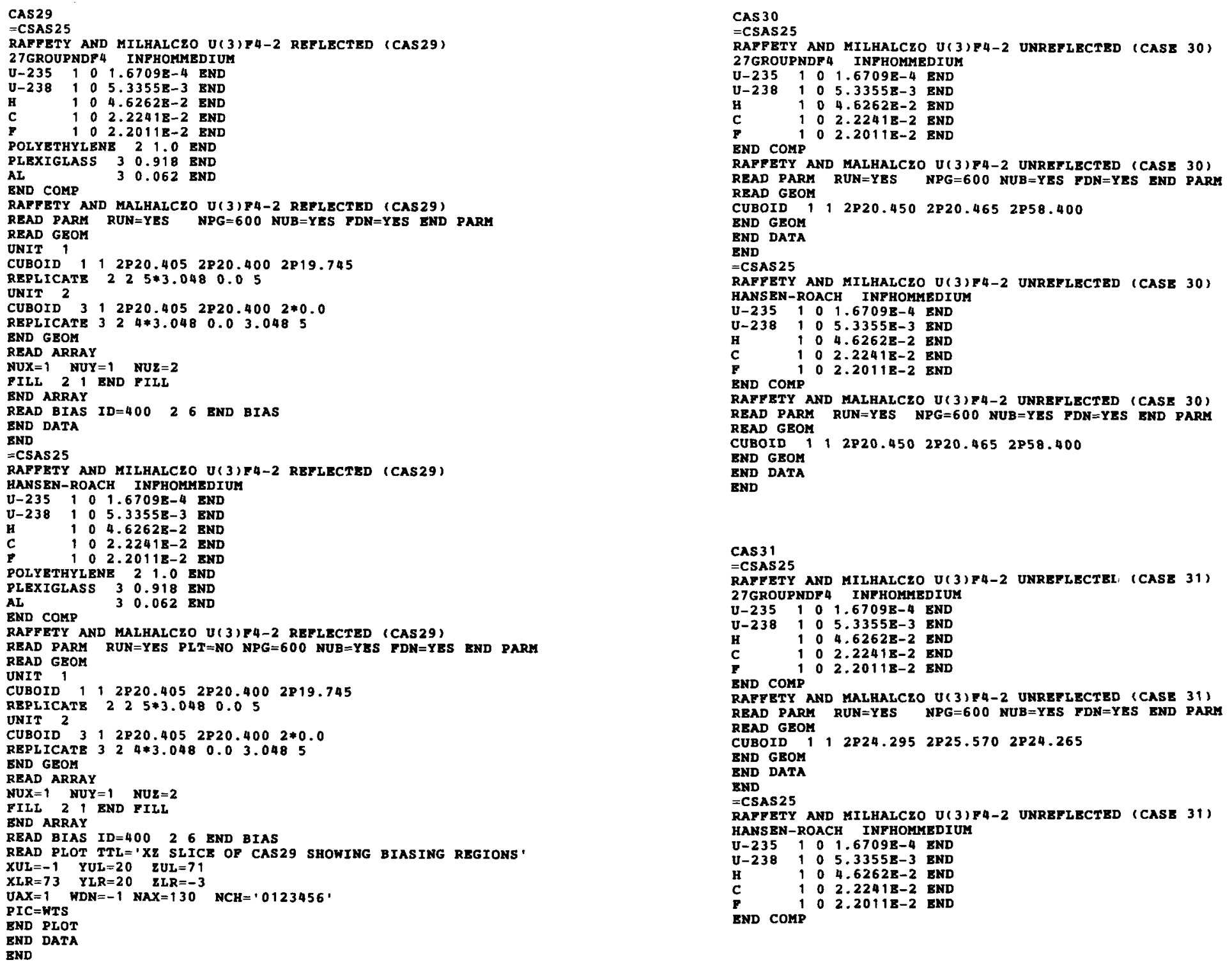
Table A.3 (continued)

RAFTETY AND MALHALCZO U(3)F4-2 UNREPLECTED (CASE 31 ) CUBOID $1112824.295 \quad 2825.5702824 .265$

END DATA

CAS 32
$=$ CSAS25

RAFTETY AND MILHALCZO U(3) P4-2 UNREFLECTED (CASE 32) 27GROUPWDPA INFHOHHEDIUI

$\begin{array}{lllll}\mathrm{U}-235 & 1 & 0 & 1.6709 \mathrm{E}-4 & \mathrm{END} \\ \mathrm{U}-238 & 1 & 0 & 5.3355 \mathrm{~B}-3 & \mathrm{END}\end{array}$

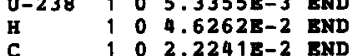

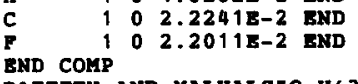

END COMP

RAFTETY AND MALHALCEO U ( 3 ) P4-2 UNREFLECTED (CASE 32 ) PQD CUBOID 112 P40.855 2P40.830 2P15.670

END GEOM

END DA

$=$ END

RAFTETY AND MILHALCZO U( 3 ) F4-2 UNREFLECTED (CASE 32) HANSEN-ROACH INFHOMMEDIUM

U-235 $1001.67095-4$ END

H $104.6262 \mathrm{E}-2 \mathrm{END}$

C $\quad 102.2241 \mathrm{E}-2 \mathrm{BND}$

END COMP

RAFFETY AND MALHALCZO U( 3) FA-2 UNREYLECTED (CASE 32) READ PARM RUN $=$ YES NPG $=600$ NUB $=$ YES $F D=Y$ YES END PARM

READ GEOM

CUBOID 112 P40.855 2P40.830 2P15.670

END GEOM

EYD

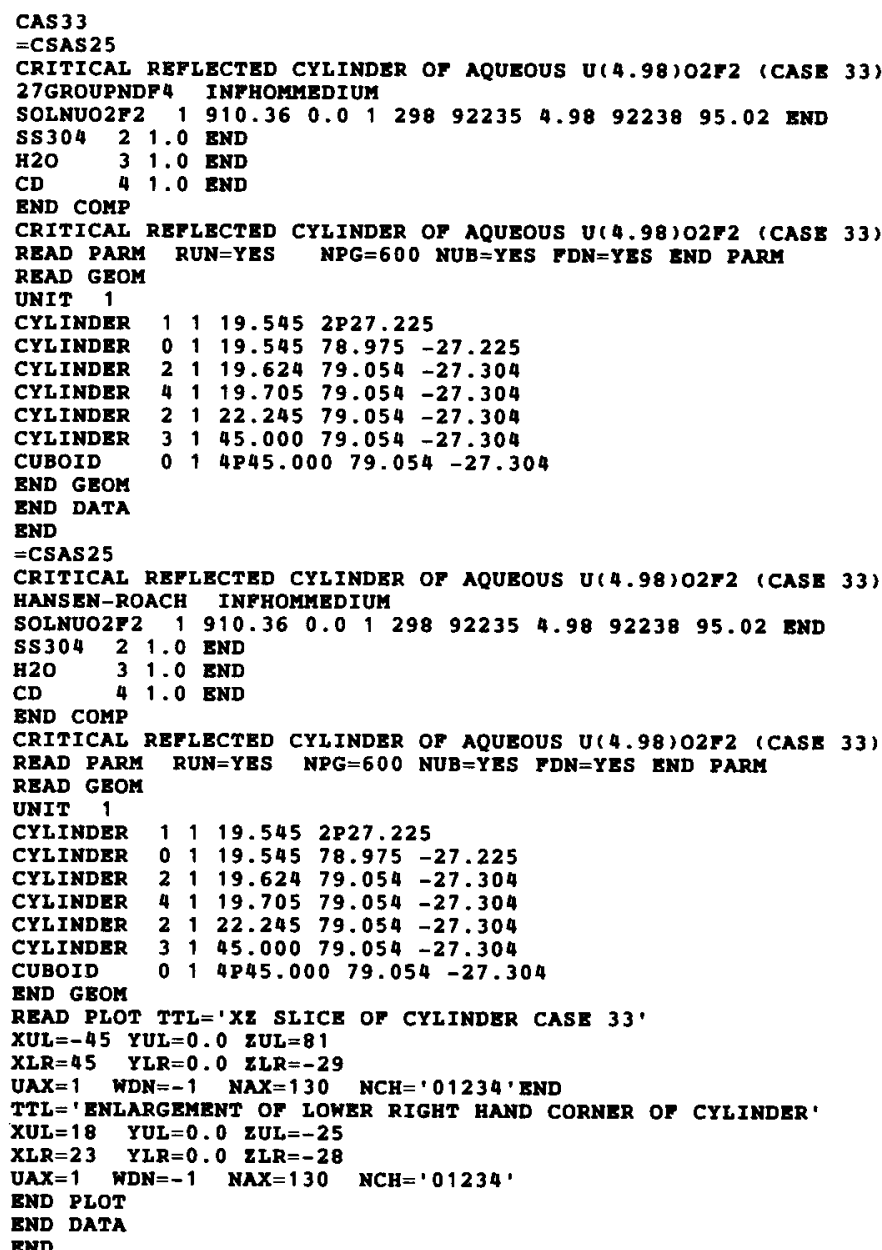


Table A.3 (continued)

CAS34

CRITICAL REPLECTED CYLINDER OP AQUEOUS U(4.98)O2P2 (CASE 34) 27GROUPNDF4 INFHOMMEDIUM SOLNUO2F2 1910.360 .0 $\begin{array}{llllll}U-235 & 1 & 0 & 1.15998-4 & \text { END } \\ \mathrm{U}-238 & 1 & 0 & 2.1852 \mathrm{E}-3 & \mathrm{END}\end{array}$

$\begin{array}{lllll}P & 1 & 0 & 4.6024 \mathrm{E}-3 & \text { END } \\ \mathrm{H} & 1 & 0 & 5.6605 \mathrm{E}-2 & \text { END } \\ 0 & 1 & 0 & 3.2904 \mathrm{E}-2 & \text { END }\end{array}$

SS304 201.0 END

BND COMP 1.0 END

CRITICAI REPLECTED CYIINDER OF AQUEOUS U(4.98)02F2 (CASE 34)

READ GEOM

RNIT 1

CYTy 116.5102871 .500

CYINDER $00116.510 \quad 172.400-71.500$

CYLINDER $\quad 2 \quad 1 \quad 19.129 \quad 172.479-71.579$

CYLINDER $33145.000 \quad 172.479-71.579$

CUBOID

GND GEOM

BND

CSAS 25

CRITICAL REPLECTED CYLINDER OF AQUEOUS U(4.98)02F2 (CASE 34)

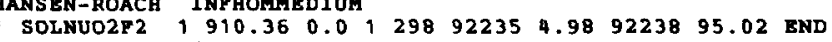

$-2351001.1599 \mathrm{E}-4$ END

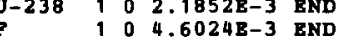

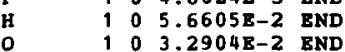

5530421.0 END

END COMP

CRITICAL REFLECTED CYLINDER OF AOUBOUS U( 4.98 ) O2F2 (CASE 34) READ PARM RUN $=$ YES NPG $=600 \quad$ NUB $=Y B S$ PDN $=Y E S$ END $P A R M$

READ GEOM

CYLINDER $1116.510 \quad 2$ P71.500

CYLINDER $001 \quad 16.510 \quad 172.400-71.500$

$\begin{array}{lllllll}\text { CYLINDER } & 2 & 1 & 16.589 & 172.479 & -71.579 \\ \text { CYLINDER } & 2 & 1 & 19.129 & 172.479 & -71.579\end{array}$

$\begin{array}{llllll}\text { CYLINDER } & 2 & 1 & 19.129 & 172.479 & -71.579 \\ \text { CYLINDER } & 3 & 1 & 45.000 & 172.479 & -71.579\end{array}$

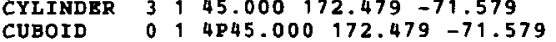

SLICB OP CYLINDER CASB $34^{\prime}$

$X U L=-45 \quad Y U L=0.0 \quad 2 U L=17$

$X L R=45 \quad$ YLR $=0.0 \quad \sum L R=-73$

TTL=' BNLARGEMENT OF LOWER RIGHT CORNER OF CYLINDER

$X U L=15 \quad Y U L=0.0 \quad$ ZUL $=-69$

XLR $=20 \quad$ YLR $=0.0 \quad$ ZLR $=-73$
UAX $=1$ WDN $=-1 \quad$ NAX $=130 \quad$ NCH $=.0123$

BND PLOT

BND DATA
CAS 35

CRITICAL SPHERE OF AQUEOUS U(4.98)02F2 (CASE 35)

27GROUPNDF4 INFHOMMEDIUM $129292235 \quad 4.98 \quad 92238 \quad 95.02 \quad$ END U-235 $1001.1613 E-4$ END

$\mathrm{U}-238 \quad 1002.1878 \mathrm{~B}-3 \mathrm{END}$

$\begin{array}{lllll}\mathrm{H} & 1 & 0 & 5.6947 \mathrm{E}-2 & \mathrm{END} \\ \mathrm{O} & 1 & 0 & 3.3081 \mathrm{E}-2 & \mathrm{END}\end{array}$

SS $30421.0 \mathrm{END}$

READ PARM RUN-YBS AQUEOGS READ GROM

SPHERE 11125.387

$\begin{array}{llll}\text { SPHBRE } & 2 & 1 & 25.4127 \\ 0 & 1 & 6 \text { P25.4127 }\end{array}$

BND GBOM

END DATA

$=\operatorname{CSAS} 25$

CRITICAL SPHERE OF AQUEOUS U (4.98)02F2 (CASE 35

HANSEN-ROACH
SOLNUO2F2 SOLNO2F2,1910.18

$\mathrm{U}-238 \quad 1 \quad 0 \quad 2.1878 \mathrm{E}-3$ END

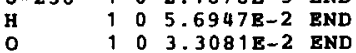

$\begin{array}{llll}1 & 0 & 3.3081 \mathrm{E}-2 & \mathrm{END} \\ \mathrm{F} & 1 & 0 & 4.6080 \mathrm{E}-3 \\ \mathrm{END}\end{array}$

533042

$4.6080 \mathrm{E}-3 \mathrm{END}$
$1.0 \mathrm{END}$

CRITICAL SPHERE OF ADUEOUS U(4.98)O2F2 (CASE 35$)$

READ PARM RUN $=Y$ YBS NPG $=600$ NUB $=$ YES $P D N=Y E S$ END PARM

READ GEOM

SPHERE 19925.387

$\begin{array}{lrrrr}\text { SPHERE } & 2 & 1 & 25.4127 \\ \text { CUBOID } & 0 & 1 & 62525.412\end{array}$

END GEOM

作

$X U L=-26 \quad Y U L=0.0 \quad 2 U L=26$

$\mathrm{UAX}=1$ WDN $=-1 \quad \mathrm{NAX}=130 \quad \mathrm{NCH}$

TTL $=$ ' ENLARGEMENT OF SPHERE WALL

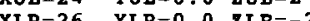

UAX $=1$ WDH $=-1$ NAX $=130 \quad \mathrm{NCH}=012$

END PIOT

END DATA 
Table A.3 (continued)

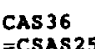

CRITICAL CYLINDER OF AOUEOUS U(4.98) 02F2 (CASE 36)

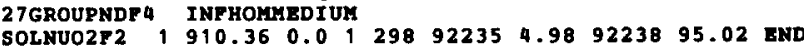
SS 304221.0 END BND COMP
CRITICAL CYLINDER OF AQUEOUS U(4.98) $02 F 2$ (CASE 36)
READ PARM RUN=YES NPG $=600$ NUB=YES PDN=YES END PARM READ GEOM

CYLINDER $1119.5500 \quad 2 P 50.85$

CYIINDER $0011919.5500 \quad 74.16 \quad \mathbf{5 0 . 8 5}$

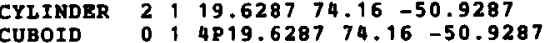

END GEOM

END DATA

END

CRITICA CYLINDBR OF AQUROUS U(4.98)02P2 (CASE 36) HANSEN-ROACH INTHOMMEDTUM $\begin{array}{llll} & \end{array}$ SS304 21.0 END

CRITICAL CYLINDER OF AQUEOUS U(4.98) 02F2 (CASE 36)

READ PARM

READ GEOM

CYLINDER $1119.5500 \quad 2 P 50.85$

CYLINDER $00119.5500 \quad 74.16 \quad-50.85$

CYLINDER $20119.6287 \quad 74.16-50.9287$

BND GBOM 1 4P

READ PLOT TTL $=1 X Z$ SLICE
XUL $=-21$ YUL $=0.0$ \&UL $=76$

$X L R=21 \quad Y L R=0.0 \quad 2 L R=-52$

TTL= ' ENLARGEMENT OF LOWER RIGHT CORNER OF CYLINDER'

$X U L=18 \quad Y U L=0.0 \quad 2 U L=-49$

$X I R=21 \quad Y L R=0.0 \quad \mathrm{ZLR}=-52$

MCH $=1012$

END PLOT
CARO 1
$=$ CSAS 25

ROCKY PLATS CRITICALS NUREG/CR-1071 EXPERIMENT NUMBER 1 (27 GROUP) 48 PUEL CANS $2.44 \mathrm{CM}$ MODERATOR GEE. HU77. DATA (OPT1)

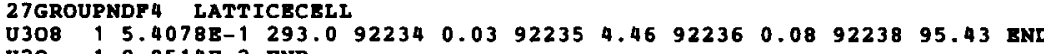
ARBM-BAGGIE 1.03 O $000100114.01601284 .980161 .2011 .9134 E-2$ BND $1100 \quad 1.030011302799 .18260000 .5290000 .229 .53908-1$ ARBM-TAPB(VINYL) 1.0700010015 .92601245 .91801610 .8217000

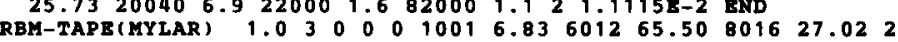
$1.7491 \mathrm{~B}-2 \mathrm{END}$

ARBM-MODERATOR $1.185330001001 \quad 7.83 \quad 601259.49801632 .483$ END ARBM-PLEX (REG)

1.1773 END

(1.0 3000 10016.48601242 .17801649 .54

$3.7534 \mathrm{~B}-3 \mathrm{BND}$

$1.1648 \mathrm{~B}-3$ (1.0 30000100111.67601286 .2980161 .204

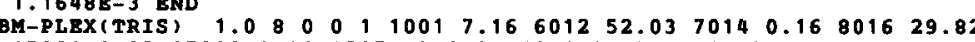

$150311.02170001 .81350794 .260350812 .8405 \quad 1.2757$ END

MLAX(GLUE) 1.030000100111 .67601286 .2980161 .205

$1.1648 \mathrm{E}-3$ END

ARBM-FILLER $1.1853000010017 .83601259 .49801632 .486 \quad 88$ END

THIS MATERIAL IS ACTUALLY MODRRATOR ADJUSTED POR THE VO

SPHTRIANGP $19.9462 \quad 18.5857 \quad 1 \quad 3 \quad 18.95792$ END

ROCKY FLATS CRITICALS NUREG/CR-1071 EXPERIMENT NURBER 1 ( 27 GROUP)

48 PUEL CANS 2.44 CM MODERATOR GEE.HU77.DATA(OPT1)

YES FDN=YES $\quad P L T=N O$

BND PARM

UNIT 1

COM=' PUEL BOX $15.28 \mathrm{cM}$ ON A SIDE WITH $.15 \mathrm{cK}$ WALIS .05CM STACKING VOID

CUBOID 11 1 6 6P7.49

CUBOID $0116 P 7.6650$

UNIT 2

COM='X-PACR INTRRSTITIAL MODERATOR'

CUBOID 31281.2200487 .66

COY=IY-FACE INTERSTITIAI MODERATOR.

CUBOID $312 P 7.665 \quad 281.2200 \quad 287.665$

UNIT 4

COM='Z-FACE INTERSTITIAL MODERATOR'

CUBOID 5

COM=' MORE X-FACE MODERATOR'

CUBOID 31 4P1.2200 2 P7.665

UNIT 6

COM=' MORE Y-PACB MODERATOR'

CUBOID, 3 T 2 PT.665 4 W

COM=' MORE Z-FACE MODERATOR

CUBOID 3 , 2P1.2200 2P7.665 2P1.2200 
Table A.3 (continued)

UNIT
COM=LAST OF INTERSTITIAL MODERATOR:

CUBOID 316 G1.2200

UNIT,

COME' NORTH SPLIT TABLE CORE'

ARRAY $13 * 0$

COM='SOUTH SPLIT TABLE CORE'

ARRAY $2 \quad 3 * 0.0$

UNIT 11

COM='PLEXIGLASS REPLECTOR SHEET WITHOUT TRIS, NORTH BOTTOM REFLECTOR CUBOID

COM='PLEXIGLASS RBPLECTOR SHEBT WITH TRIS, NORTH BOTTOM REPLECTOR'

CUBOID 512 2P16.5500 2P38.7500 2P0.6150

COM=' UPPER PORTION NORTH BOTTOM REPLECTOR WITH TRIS

COM=' UPPER PORTION NORTK BOTTOM REPL
CUBOID 5 1 2 P16.550 2838.75 2P8.24

UNIT 14

COM='LOWER PORTION NORTH BOTTOM REFLECTOR WITH TRIS'

CUBOID 512 P16.550 2838.75 2P3.69

UNIT 15 COM= NORTH BOTTOM RBPLECTOR INCLUDES REGULAR AND TRIS' ARRAY $3 \quad 3 * 0.0$

UNIT 16

COM='PLBXIGLAS SHEET BOTTOM SOUTH REFLECTOR WITHOUT TRIS

CUBOID $012 \mathrm{P} 5.12 \mathrm{P2} .552 \mathrm{PO} .615$

CUBOID $4144.3-5.12 P 38.75$ 2P0.615

COY= PLEXIGLAS SHEET BOTTOY SOUTH REPLECTOR WITH TRIS,

CUBOID 012 2P5.1 2P2.55 $2 \mathrm{P0} 0.615$

CUBOID $5144.3-5.12838 .75280 .6150$

UNIT 18
COM=L LOWER PORTION SOUTH BOTTOM REFLECTOR WITH TRIS '
CUBOID O 2 2P5.1 2 P2.55 2 P8.855

CUBOID 5 1 $44.3-5.12$ P38.75 2P8.855

UNIT 19

COM=' SOUTH BOTTOM REFLECTOR WITH REGULAR AND TRIS.

ARRAY $43 * 0.0$

UNIT 20 COM=' BAST AND WEST REPLECTORS POR NORTH REPLBCTOR WITH TRIS CUBOID 512816.5502812 .652854 .2825

COMT = ARRAY FOR EAST AND WEST REFLECTORS FOR NORTH REFLECTOR' ARRAY $53 * 0,0$

COM=' 'BAST AND WEST REPIECTORS TOR SOUTH REFLECTOR WITH TRIS' CUBOID 5 T 2 P24.700 2P12.65 2 P54.2825

UNIT 23

'ARRAY POR BAST AND WBST REFLECTORS FOR SOUTH REFLECTOR' ARRAY $63 * 0.0$ COM=' NORTH TOP REPLECTOR WITH TRIS'
CUBOID 5,2 2P29.100 2P64.05 2P12.1 UNIT COM=' ARRAY TOR NORTH TOP REFLECTOR' ARRAY $7 \quad 3 * 0.0$

COM=. SOUTH TOP REPLECTOR WTRH TRTS'

CUBOID 0 1 2 P5.1 2 P2.55 2 P12 15

CUDID 5 1 $69.4-5.12564 .05$ 2P12.15
COMT 27 ARRAY POR SOUTH TOP REFLECTOR

ARRAY $83 * 0.0$

COM=' NORTH END REFLECTOR $9.8 \mathrm{CM}$ PORTION WITHOUT TRIS CUBOID $4,2 \mathrm{P} 4.9000 \quad 2 \mathrm{P} 64.0500 \quad 2 \mathrm{P} 54.2825$

UNIT 29

COM=' NORTH END RELPECTOR 5.2 CM PORTION

CUBOID 512 2P2.6 2P64.0500 2P54.2825 WITH TRIS

UNIT 300 COM= NORTH END RELTECTOR $10.1 \mathrm{CM}$ PORTION YITHOUT TRIS CUBOID 412 2P5.05 2P64.05 2P54.2825

UNIT 31 COM='ARRAY POR NORTH END REPLECTOR.

COM=' ARRAY POR

ARRAY 932

COM=.

CUBOTD 5 1 2P12.55 2P64.05 2P54.2825

UNIT 33

COM=' ARRAY TO

ARRAY 100

COM='BOTTOM MODERATING PLASTIC NORTH CORE '

CUBOID 612 2P16.550 2P38.7500 2P13.0500

UNIT 35

COM=' TOP MODERATING PLASTIC NORTH CORE:

UNIT 36 , 300.0

UNIT 37

ARRAY $12 \quad 3 * 0.0$

COM=' NORTH CORE

COM= NORTH 13 , $3 * 0.0$

COM= ' NORTH CORE

ARRAY $14 \quad 3 * 0.0$

BAST AND WEST REPLECTOR'

COM= ' NORTH COR

COM=' NORTH SPLIT TABLE FACEPLATE'

CUBOID ${ }^{3} 1$ 2P0.6150 2 EAC.0500 2P66.4325

COM='NORTH CORE WITH TOP REPLECTOR'

ARRAY 16 3*0.0

UNIT 43

COM = ' NORTH CORE WITH FACEPLATE'

ARRAY $173 * 0.0$

COM $=\cdot 12.95 \mathrm{CM}$ THICK MODERATOR SOUTH COPE

CUBOID 6 1 2 P23.924 2P6.475 2P40.183

UNIT 45 THICK MODERATOR SOUTH CORE.

COM='2.95 THICK MODERATOR SOUTH CORE'
CUBOID 6 1 2P23.924 2P38.5285 2P1.475

UNIT 46

COM='COMBINATION OF CORE WITH 12.95 THICK MODERATOR' ARRAY $18 \quad 3 * 0.0$

COM=' COMBINATION OF CORE WITH 2.95 THICK MODERATOR'

ARRAY 19
UNIT 48

COM='SOUTH CORE WITH EAST WEST REPLECTORS
ARRAY $20 \quad 3 * 0.0$ 
Table A.3 (continued)

UNIT 49
COM=. SOUTH CORE WITH EAST WEST REFLECTORS ' ARRAY $213 * 0$.

COM=' SOUTH CORE END REPLECTOR'

ARRAY $22 \quad 3 * 0.0$

UNIT 5

COM=' FACEPLATE POR SOUTH SPLIT TABLE'

CUBOID 3 UNIT 280.4620 2864.0500 2566.43

COM='SOUTH CORE HITH TOP RETIECTOR'

ARRAY $23 \quad 3 * 0.0$
UNIT 53

COM='SOUTH CORE WITH FACEPLATE

ARRAY 24
UNIT 54

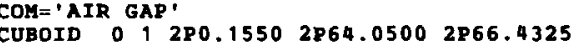

GLOBAI

UNIT 55

ARRAY $25 \quad 3 * 0.0$

UNIT 56

COM= IMMPTY FUEI LOCATION

CUBOID 01687.6650

COM=' SIDE MODERATOR'

CUBOID $6 \quad 1 \quad 2 P 16.550 \quad 2 P 4.4300 \quad 2 P 25.4350$

UNIT 58 12

COM=' END MODERATOR' COM=. SOUTH CORE BOT:OM MODEPATING PIASTIC CUBOTD 6,2 2P24.7000 2P38.7500 2P13.0500 UNIT 60 COM=' SOUTH CORE TOP MODERATING PLASTICC CUBOID 61

CUBOID $012 P 7.665 \quad 2 P 1.2200 \quad 2 P 7.665$

END GEOMETRY

$A R A=1 \quad$ NUX $=3$ NUY $=7$ YUZ $=5$

COM= ' NORTH SPLIT TABLE COR

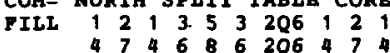

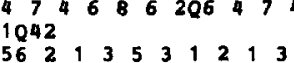

ARA $=2$ NUXX 3 NUYY 7 NUE $=5$

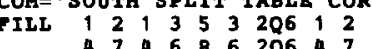

47468
1042

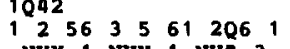

ARA $=3$ NUX $=1$ NUY=1 NUE $=3$

COM=' NORTH BOTTOM REFLECT

$A R A=4 \quad N U X=1$ NUY $=1$ NUZ $=7$

COM=' SOUTH BOTTOM REFLECTOR'

ARA $=5$ NUX $=1$ NUY $=1$ NUZ $=1$
COM=' RAST AND WEST WALLS OF NORTH REPLECTOR'

PILL 20 END PILI NUR

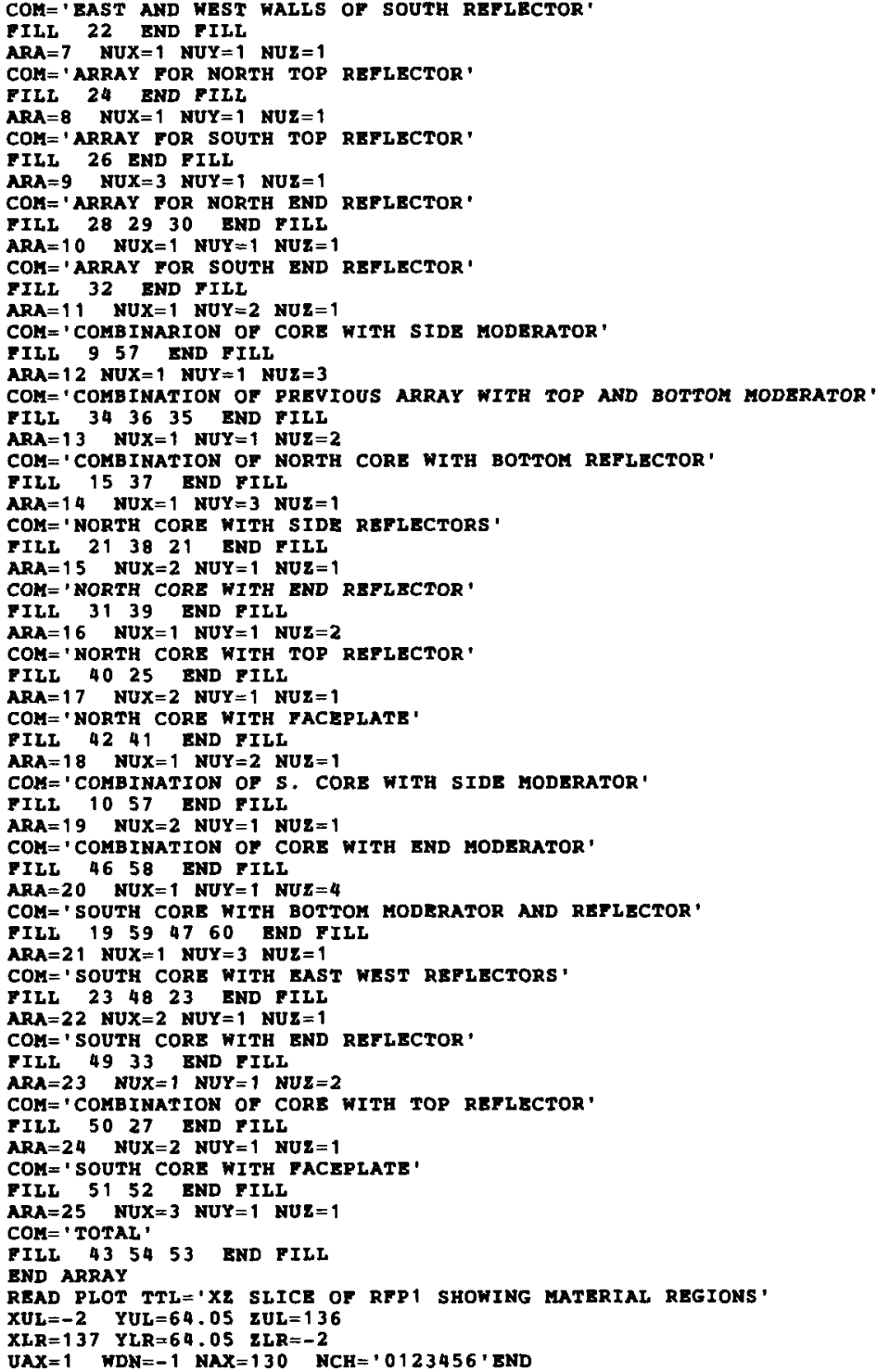


Table A.3 (continued)

UNIT 24

TOP REPLECTOR WITH TRIS' CUBOID $512 \mathrm{2P29.100} \mathrm{2P64.05 \quad 2P12.15}$ COM= ARRAY FOR NORTH TOP REFLECTOR' ARRAY 7 3*0.0

COM= ' SOUTH TOP REFLECTOR WITH TRIS CUBOID 5 1 $69.4-5.12 \mathrm{P} 64.05 \quad 2 \mathrm{P} 12.15$ UNIT 27

COH=' ARRAY FOR SOUTH TOP REFLECTOR'

ARRAY $83 * 0.0$

COM=' NORTH END REPLECTOR $9.8 \mathrm{CM}$ PORTION WITHOUT TRIS' CUBOID $412 \mathrm{P} 4.90002 \mathrm{P} 64.05002 \mathrm{P} 54.2825$

UNIT 29 MT 12 MRT

CUBODD $5,282.62864 .0500^{\circ} 2854.2825$

COM= ' NORTH END RELPECTOR 10.1 CM PORTION WITHOUT TRIS CUBOID 4 1 2P5.05 2P64.05 2 P54.2825

ONIT 31 COM=' ARRAY POR NORTH END REFLECTOR'

ARRAT $323 * 0.0$

COM='SOUTH END REFTECTOR'

CUBOID 51 2P12.55 2P64.05 2P54.2825

COA = ARRAY FOR SOUTH END REFLECTOR'

ARRAY $10 \quad 3 * 0.0$

UNIT 34

COM='BOTTOM MODERATING PLASTIC NORTH CORE

CUBOID 612 2P16.550 2P38.7500 2P13.0500

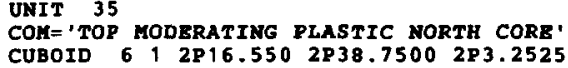

$\begin{array}{lll}\text { UNIT } & 36 & 1 \\ \text { ARRAY } & 11 & 3 * 0.0\end{array}$

UNIT 37

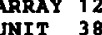

COM = ' NORTH CORS

ARRAY 13 3* 0.0

COMIT ' 39 ORTH CORE WITH EAST AND WEST REFLECTOR'

COM=' NORTH CORE

UNIT 40

COM=' NORTH CORE WITH END REFLECTOR'

ARRAY $15 \quad 3 * 0.0$

ANIT

CUBOID 312 2P0.6150 2P64.0500 2P66.4325

UNIT 42 COM

COME ' NORTH CONE
ARRAY $16 \quad 3 * 0.0$

TAcrotate

ARRAY $17 \quad 3 * 0.0$

UNIT 44

COM=112.95 CM THICK MODERATOR SOUTH CO

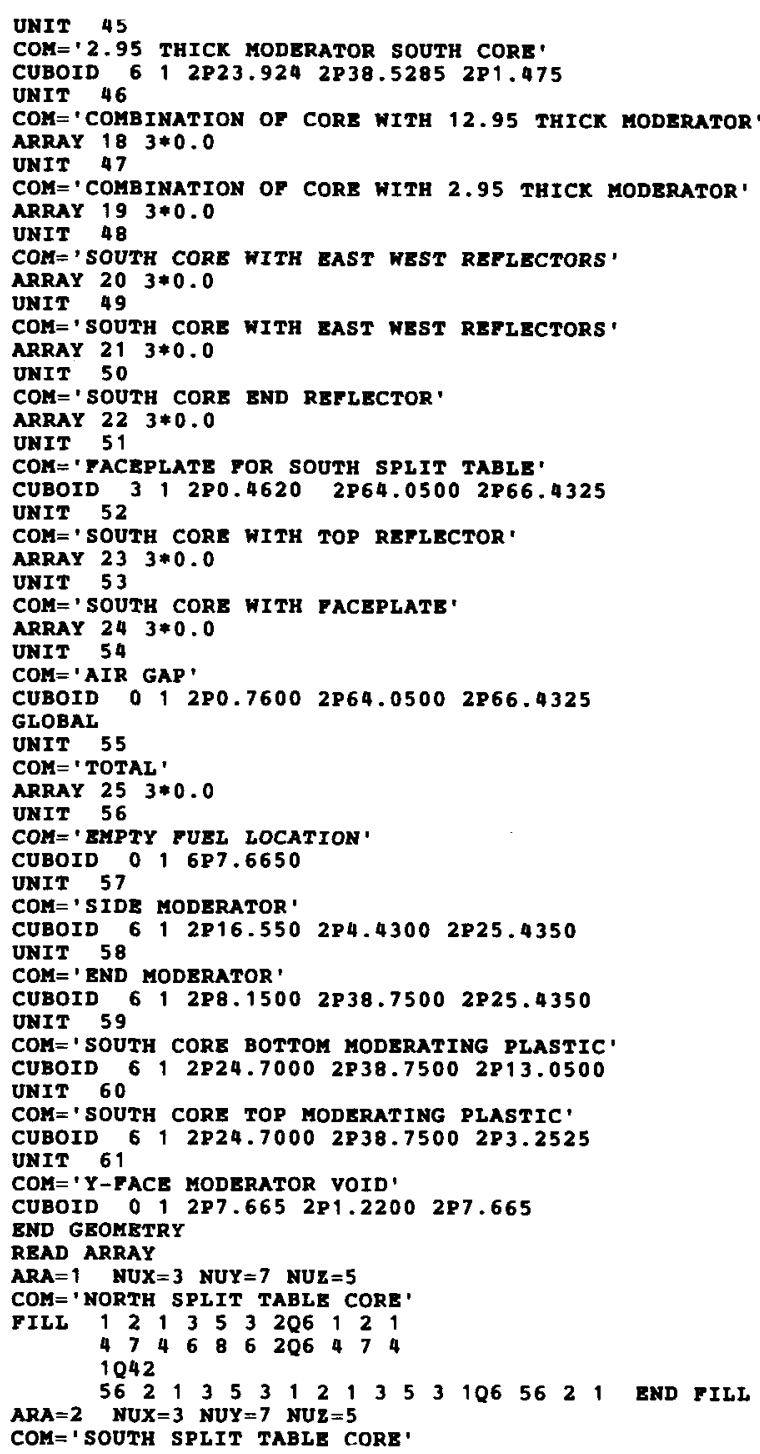


Table A.3 (continued)

FILI $\begin{array}{llllllllll}1 & 2 & 1 & 3 & 5 & 3 & 206 & 1 & 2 & 1 \\ 4 & 7 & 4 & 6 & 8 & 6 & 206 & 4 & 7 & 4\end{array}$

$11^{42} 56353121353125635611256$ END PILL

ARA $=3 \quad \mathrm{NUX}=1 \quad \mathrm{NUY}=1 \quad \mathrm{NUZ}=3$

COM=' NORTH BOTTOM REPLECTOR

FILL $14 \quad 11 \quad 13$ BND FILI

COM=' SOUTH BOTTOM REPLECTOR'

FILL $1816 \quad 17 \quad 16 \quad 1617 \quad 17$ BND PILL

COM=' BAST AND WEST WALLS OR

ARA $=6$. 20 BNX PILY

FILL 22 BND FILI

WOUTH REPLECTOR'

COM= 'ARPAY FOR NORTH TOP RETIECTOR'

FILL 24 END PILI

$A R A=8$ NUX $=1$ NUY $=1$ NUZ $=1$
COM= ARRAY

FTL2 26 END FTIL

NieA=9 2 NUX $=3$ FULE

COM= 'ARRAY FOR NORTH END REFLECTOR'

FILI 282930 BND FILL

COM= 'ARRAY FOR SOUTH END REPLECTOR

FILI 32 END PILI

$A R A=11$ NUX $=1$ NUY $=2$ NUZ $=1$

COM= ' COMBINARION OF CORE WITH SIDE MODERATOR'

TILL 957 BND PILL

COM=' COMBINATION OF PREVIOUS ARRAY WITH TOP AND BOTTOM MODERATOR'

FILL 343635 MST FILL

$A R A=13$ NUX $=1$ NUY $=1$ NUZ $=2$

COM =' COMBINATION OF NORTH CORB WITH BOTTOM REFLECTOR

FILI $15 \quad 37$ END PILL

COA= 'NORTH CORE WITH SIDE REFLECTORS

COR= NORTH CORE WITH SID

ARA $=15$ NUX $=2$ NUY $=1$ NUZ=1

COM=' NORTH CORE WITH BND REFLECTOR'

FILI 3139 BND FILL

ARA $=16$ NUX $=1$ NUY $=1$ NUZ $=2$
COM= ' NORTH CORE WITH TOP REFLECTOR'

COM $=$ ' NORTH CORE WITH

ARA $=17$ NUX $=2$ NUY $=1$ NUZ $=1$

COM= ' NORTH CORE WITH FACEPLATE'

FILI 4241 END FILL

$A R A=18$ NUX $=1$ NUY=2 NUZ $=1$

COM=' COMBINATION OP $S$. CORE WITH SIDE MODERATOR'

FILI 1057 BND FILL

COM=' COMBINATION OF CORE WITH END MODERATOR'

PILL 4658 END PIL

ARA $=20 \quad$ NUX $=1 \quad$ NUY $=1$ NUZ $=4$

COM='SOUTH CORE WITH BOTTOM MODERATOR AND REFIECTOR

FILL 19594760 END FIL

COM=' SOUTH CORE WITH EAST WEST REFLECTORS '

PILL 234823 BND FILI

$A R A=22$ NUX $=2$ NUY $=1$ NUZ $=1$

COM='SOUTH CORE WITH END REPLECTOR'

PILI 4933 BND FILI

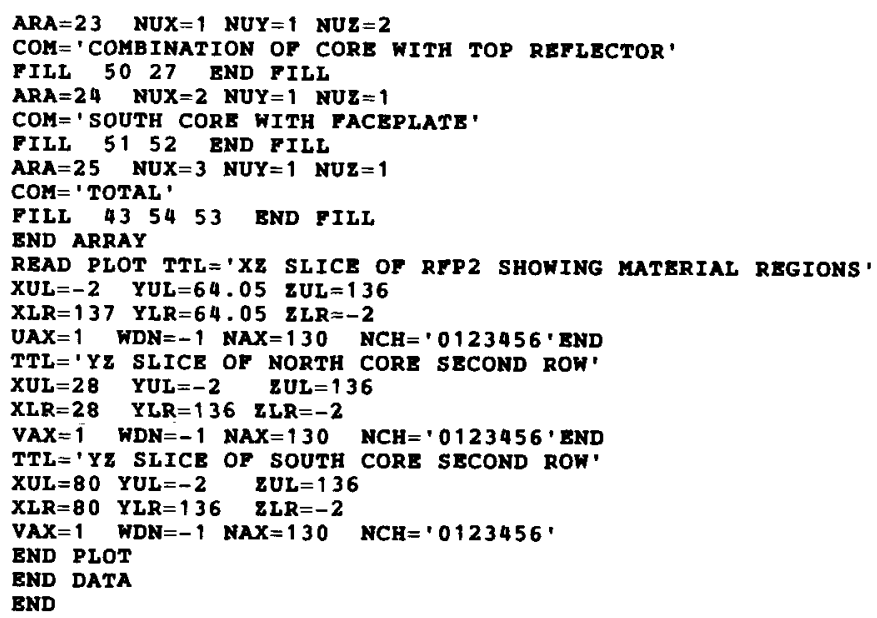

$A R A=23 \quad N U X=1 \quad N U Y=1 \quad N U Z=2$

CORE WITH TOP REFIECTOR

ARA $=24$ NUX $=2$ NUY $=1$ NUZ $=1$

COM='SOUTH CORE WITH FACEPLATE'

PILL $51 \quad 52$ BND FILL
$A R A=25 \quad$ NUX $=3$ NUY $=1$ NUZ $=1$

COYA ' ' TOTAL'

FILL 435453 END FILI

BND ARRAY

READ PLOT TTL='XZ SLICE OF RTP2 SHONING MATERIAL REGIONS'

$X U L=-2 \quad Y U L=64.05 \quad 2 U L=136$

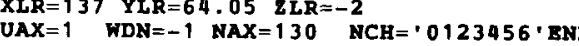

TTL= ' YZ SLICE OF NORTH CORE SECOND ROW

$X U L=28 \quad Y U L=-2 \quad Z U L=13$

.

TTL $=$ ' YZ SLICB OF SOUTH CORE SECOND ROW'

$X U L=80 \quad Y U L=-2 \quad Z U L=136$

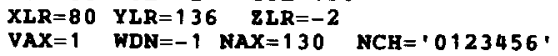

BND PLOT

END

CAR03

$=\operatorname{CSAS} 25$

ROCKY PLATS CRITICALS NUREG/CR-1071 EXPERIMENT NUMBER 3 (27 GROUP) 100 FUEL CANS 0.929 CM MODERATOR GEE. HU77. DATA(UND)

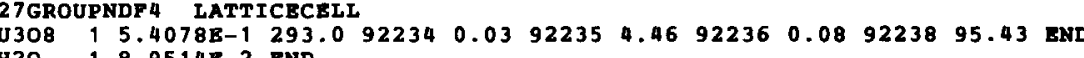

ARBM-BAGGIE $1.030000100114 .01601284 .980161 .2011 .9134 E-2$

$\begin{array}{lllllllllllllll}\text { END } & & & \end{array}$

BND

ARBM-TAPE(VINYL) $\quad 1.07000010015 .92601245 .91801610 .8217000$

$25.73200406 .9220001 .6820001 .121 .11158-2$ END

ARBM-TAPE (KYLAR) 1.0300010016 .83601265 .50801627 .02

$1.7491 \mathrm{E}-2 \mathrm{END}$

ARBM-MODERATOR 1.18530000010017 .83601259 .49801632 .48

AREM-PLEX(REG) 1.03000010017 .84601259 .59801632 .234

$\begin{array}{lllllllll} & & \end{array}$

ARBM-PIEX(GLUE)

000010011.67601286 .2980161 .204

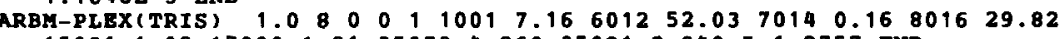

$150311.0217000 \quad 1.81350794 .260350812 .840 \quad 5 \quad 1.2757$ END

$\begin{array}{lllllllllllll}\text { ARBM-PLEX(PAPER) } & 1.0 & 3 & 0 & 0 & 0 & 1001 & 6.48 & 6012 & 42.17 & 8016 & 49.5 & 5\end{array}$

ARBM-PLEX(GLUE) $1.0300001001 \quad 11.67601286 .2980161 .205$

ARBM-FILLER $1.18530000010017 .83601259 .498016 \quad 32.486 \quad 0.7$ END

; THIS MATERIAL IS ACTUALLY MODERATOR ADJUSTED FOR THE VOLUME

- FRACTION OF THE VOID FILLED BETWEEN CORE AND REFLECTOR.

END COMP

STANGP 19.0018 .58571318 .95792 END

(27 GROUP) - 100 FUEL CANS $0.929 \mathrm{CM}$ MODERATOR GEE.HU77.DATA (UND)

READ PARM RUN $=$ YES NPG $=600$

NUB=YBS PDN=YES PLT $=$ NO
END PARH 
Table A.3 (continued)

READ GROM

COM=' PUEI BOX $15.28 \mathrm{cM}$ ON A SIDE WITH $.15 \mathrm{CM}$ WALLS .05CM STACKING VOID' CUBOID 1116 6P7.49

CUBOID $2216 \mathrm{~F} 7.64$

CUBOID

UNIT

COM='X-PACE INTERSTITIAL MODERATOR'

CUBOID 312 P0.4645 4P7.665

COME='Y-PACR INTERSTITIAL MODERATOR'

UNIT

COM='Z-FACE INTERSTITIAL MODERATOR

CUBOID 31 4P7.665 280.4645

UNIT

COM='MORE X-PACE MODBRATOR'
CUBOID 314 P0.4645 2 P 7.665

UNIT 6

COM=' MORE Y-FACE MODERATOR'

CUBOID $312 P 7.665$ 4P0.4645

UNIT 7

COM=' MORE Z-PACB MODERATOR

$6652 P 0.4645$

COM='LAST OF INTERSTITIAL MODERATOR'

CUBOID 316 PO 0.4645

UNIT 9

COM=' NORTH SPLIT TABLE CORE'

ARRAY $13 * 0$.

COTI 1000.0

ARRAY $2 \quad 3 * 0.0$

COM='PLEXIGLASS REFLECTOR SHEET WITHOUT TRIS, NORTH BOTTOM REFLECTOR' CUBOID $412 \mathrm{P} 15.80002 \mathrm{P38.7500} \mathrm{2P0.6150}$

UNIT 12

COM='PLEXIGLASS REFLECTOR SHEBT WITH TRIS, NORTH BOTTOM REFLECTOR

CUBOID $512 P 15.80002 \mathrm{P} 38.7500 \quad 2 \mathrm{PO} .6150$

COM = UPPER PORTION NORTH BOTTOM REFLECTOR WITH TRIS

CUBOID 512 2P15.8 2 P38.75 2P8.29

UNIT 14

COM=' LOWER PORTION NORTH BOTTOM REPLECTOR WITH TRIS

CUBOID 5 1 2 P15.8 2 P38.75 2 P3.69

UNIT 15

TERT REGUAR AND TRIS

ARRAY $33 * 0.0$

COM='PLEXIGLAS SHEET BOTTOM SOUTH RETLECTOP WITHOUT TRIS

CUBOID 0 1 2 2P5.1 2 P2.5 2 P0.6150

CUBOID 4 1 $44.3-5.12$ P38.75 280.615

UNIT 17

COM='PLEXIGLAS SHEET BOTTOM SOUTH REFIECTOR WITH TRIS

CUBOID 0 1 2 2P5.1 2P2.5 2P0.6150

CUBOTD 5 1 $44.3-5.12 \mathrm{P} 38.75$ 2P0.6150

COM, LOWER PORTION SOUTH BOTTOM REPIECTOR WITH TRIS',

CUBOID 0 1 2 P5.12P2.5 2 PQ.85

CUBOID 5 1 $44.3-5.12 \mathrm{P38} .75288 .855$

COM=' SOUTH BOTTOM REFLBCTOR WITH REGULAR AND TRIS '

ARRAY $43 * 0.0$

COM= ' EAST AND WEST REPLECTORS FOR NORTH REFIECTOR WITH TRIS '
UNIT 21

TER ARRAY 5 3*0.0

BCTOR WITH TRIS

COM='BAST AND WEST REFLECTORS POR SOUT
CUBOID 512 2P24.700 2 P12.65 2P54.2825

NIT 23

COM= ARRAY FOR EAST AND WEST REFLECTORS FOR SOUTH REFLECTOR' ARRAY 6 3*0.0

COM=' NORTH TOP REFLECTOR WITH TRIS.

CUBOID 5 1 12 P28.35 2 P64.05 2 P 12.15

UNIT 25

COM= ' ARRAY POR NORTH TOP REFLECTOR

ARRAY $73 * 0.0$

UNIT 26 COM='SOUTH TOP REFLECTOR WITH TRIS '

CUBOID 012 P5.1 2P2.5 2P12.15

CUBOID 5 1 69.4 -5.1 2P64.05 2P12.15

UNIT 27

COM=' ARRAY FOR SOUTH TOP REFLECTOR'

ARRAY $83 * 0.0$

UNIT 28

TEFLCTOR $9.8 \mathrm{CM}$ PORTION WITHOUT TRIS

CUBOID $4,12 \mathrm{P4} .90002 \mathrm{P} 64.0500 \quad 2 \mathrm{P} 54.2825$

UNIT 29 .

CUBOID $51282.62 P 64.05002 P 54.2825$

UNIT 30

COM= NORTH END RELFECTOR $10.1 \mathrm{CK}$ PORTION WITHOUT TRIS

CUBOID 4,2 P5.05 2P64.05 2P54.2825

UNIT 31

'ARRAY FOR NORTH END RBFLECTOR'

ARRAY $93 * 0.0$

UNIT 32

COM='SOUTH END REPLECTOR'
CUBOID 5,2 P12.55 2P64.05 2 P54.2825

UNIT 33

COM='ARRAY FOR SOUTH END REFLECTOR'

ARRAY $10 \quad 3 * 0.0$

COM=112.95 THICK MODERATING PLASTIC NORTH CORE CUBOID $612 \mathrm{P} 15.79452 \mathrm{P} 6.47502 \mathrm{P} 40.1830$

UNIT 35 THICK MODERATING PLASTIC NORTH CORE'

CUBOID 612 2P15.7945 2P38.5285 2P1.475

UNIT 36

ARRAY $113 * 0.0$

NIT 37

$\begin{array}{lllllll}0.0 & 0.011 & 0.443 & 0.0 & 0.1590 & 0.0\end{array}$

UNIT 38

COR= NORTH CORE

ARRAY $13 \quad 3 * 0.0$

COM=

ARRAY $14 \quad 3 * 0.0$

UNIT 40

COM=' NORTH CORB WITH END REPLECTOR'

ARRAY $15 \quad 3 * 0.0$

UNIT 41

CUBOTD 12 PO.6150 2P64.0500 2P66.4325 
Table A.3 (continued)

UNIT 42

COM= 'NORTH CORE WITH TOP REFLECTOR

ARRAY $16 \quad 3 * 0.0$

TACEPLATE

UNIT 44

COM $=12.95 \mathrm{CM}$ THICK MODERATOR SOUTH CORE

CUBOID 6 1 2 P23.924 2P6.475 2P40.183

UNIT 45

COM='2.95 THICK MODERATOR SOUTH CORE'

UNIT 46

COM='COMBINATION OF CORE WITH 12.95 THICK MODERATOR' ARRAY $18 \quad 3 * 0.0$

UNIT 47

OF CORE WITH 2.95 THICK MODERATOR' ARRAY $19 \quad 3 * 0.0$ UNIT 48

COM='SOUTH CORE WITH EAST WEST REFLECTORS'

ARRAY $20 \quad 3 * 0.0$

UNIT

COY-'SOUTH CORE WITH BAST WEST REFLECTORS'

ARRAY $213 * 0.0$

COM='SOUTH CORE END REFLECTOR

ARRAY $22 \quad 3 * 0.0$

UNIT 51

$\begin{array}{llll}\text { CUBOID } 312 \mathrm{PO} .4620 & 2 \mathrm{P} 64.0500 & 2 \mathrm{P66} .432\end{array}$

UNIT 52 12

ARRAY $23 \quad 3 * 0.0$

UNIT 53

COM=' SOUTH CORE WITH PACBPLATE'

ARRAY $24 \quad 3 * 0.0$

UNIT 54

CUBOID 0 1 2PO.5250 2P64.0500 2P66.4325

UNIT 55

ARRAY $25 \quad 3 * 0.0$

END GEOMETRY

READ ARRAY

ARA $=1$ NUX $=3 \quad$ NUY $=7 \quad$ NUZ $=9$

SPLIT TABLE CORE

$\begin{array}{lllllllll}2 & 1 & 3 & 5 & 3 & 206 & 1 & 2 & 1 \\ 7 & 4 & 6 & 8 & 6 & 206 & 4 & 7 & 4\end{array}$

$\begin{array}{ccccccccc}3 Q 42 & 1 & 3 & 5 & 3 & 206 & 1 & 2 & 1\end{array}$ END $\mathrm{FIL}$

$A R A=2 \quad N U X=5 \quad N U Y=7 \quad N U Z=9$

FILL $\begin{array}{lllllllllllllllll} & 2 & 1 & 2 & 1 & 3 & 5 & 5 & 3 & 2 Q & 10 & 1 & 2 & 1 & 2 & 1\end{array}$

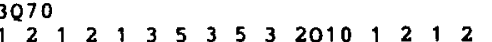

ARA $=3 \quad$ NUX $=1$ NUY $=1 \quad$ NUZ $=3$

COM $=$ 'NORTH BOTTOM REPLECTOR'

$A R A=4 \quad N U X=1 \quad$ NUY $=1$ NUZ $Z=7$

COM $=$ 'SOUTH BOTTOM REFLECTOR'

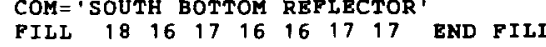

ARA $=5 \quad$ NUX $=1 \quad$ NUY $=1 \quad$ NUZ $=1$

COM='BAST AND WEST WALLS OF NORTH RBPLBCTOR'

FILL 20 END FILI

COM $=1$ NUX $=1$ NUY $=1 \quad$ NUZ $=1$

PILI 22 END FILI

$A R A=7$ NUX $=1$ NUY $=1$ MUR $=1$

COM='ARRAY FOR NORTH TOP REPLECTOR'

FILL 24 END FIL

COMA $=8$ NUX $=1 \quad$ NUY $=1 \quad$ NUZ $=1$

PILL 26 BND PILI

ARA $=9 \quad$ NUX $=3$ NUY $=1$ NUE

= 'ARRAY POR NORTH END REFLECTOR'

FILL $28 \quad 29 \quad 30$ END FILL

COM='ARRAY FOR SOUTH END REPLECTOR'

PILL 32 BND FILL

ARA $=11$ NUX $=1$ NUY $=2 \quad \mathrm{NUZ}=1$

COM=' COMBINARION OF CORE WITH $12.95 \mathrm{CM}$ THICK MODERATOR'

PILL 9 34 END PILL

COM=' COMBINATION OF PREVIOUS ARRAY WITH $2.95 \mathrm{CM}$ THICK MODERATOR'

FILI $36 \quad 35$ BND PILI

ARA $=13$ NUX $=1$ NUY $=1 \quad \mathrm{NUZ}=2$

COM=' COMBINATION OF NORTH CORE WITH BOTTOM REPLECTOR'

FILI $15 \quad 37$ END FILL

COM='NORTH CORE WITH SIDE REFLECTORS .

COM=' NORTH CORE WITH SIDE

ARA $=15$ NUX $=2$ NUY $=1$ NUZ $=1$

COM=' NORTH CORE WITH E

FILL 3139 END FILL
ARA $=16$
NUX $=1$ NUY $=1$ NUZ $=2$

ARA $=16$ NUX $=1$ NUY $=1$ NUZ $=2$
COM= $=$ NORTH CORE WITH TOP REPLECTOR

COM $=$
PIL NORTH CORE WITH TO 25 END PILI

ARA $=17$

COM $=$ ' NORTH CORE WITH FACEPLATE

FILI 4241 END FILI

COM=' COMBINATION OF $S$. CORE WITH $12.95 \mathrm{cM}$ THICK MODERATOR'

PILL 10 GA BND FILI

COM=' COMBINATION OF CORE WITH $2.95 \mathrm{CM}$ THICK MODEPATOR'

FILI $46 \quad 45$ BND FILI

$A R A=20 \quad$ NUX $=1$ NUY $=1$ NUZ $=2$

COM=' SOUTH CORE WITH BOTTOM REFLECTOR'

FILI 1947 END FILL

ARA $=21$ NUX $=1$ NUY $=3$ NUZ $=1$
COM $=$ ' SOUTH CORE WITH EAST WEST REPLECTORS

FILL 234823 END FILL

COM=' SOUTH CORE WITH END RRPLECTOR'

FILL 4933 BND FILI

$A R A=23$ NUX $=1 \quad$ NUY $=1 \quad$ NUZ $=2$

ARA $=$ COMBINATION OF CORE WITH TOP REFLECTOR.

FILL $50 \quad 27$ END FILL

COM = ' SOUTH CORB WITH FACEPLATE'

PILL 5152 END FILI

$A R A=25 \quad N U X=3$ NUY $=1$ NUZ $=1$

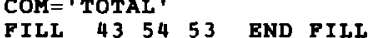


Table A.3 (continued)

END ARRAY

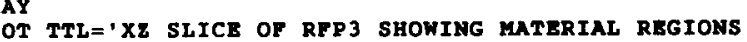

$X U L=-1 \quad$ YUL $=64.05 \quad$ ZUL $=135$

$\mathrm{UAX}=1$ WDN $=-1$ NAX $=130$ NCH $=10123456$, END

TTI = 1 Y SLICE OF NORTH CORE SECOND ROW'

$X U L=28 \quad Y U L=-2 \quad Z U L=136$

$X L R=28 \quad$ YLR $=136 \quad$ ZLR $=-2$
VAX=1 WDN=-1 NAX=130 NCH $=10123456 \cdot$ END

TTI $=$ 'YZ SLICE OF SOUTH

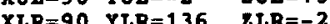

VAX $=1$ WDN $=-1$ NAX $=130 \quad \mathrm{NCH}=\cdot 0123456$ '

BND PLOT

END

\section{CAR04}

=CSAS25
ROCKY FLATS CRITICALS NUREG/CR-1071 EXPERIMENT NUMBER 13 (27 GROUP)

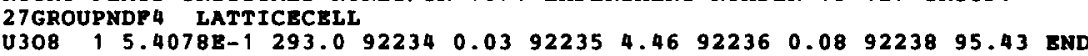

ARBM-BAGGIB $1.030001001 \quad 14.01601284 .98016 \quad 1.2011 .9134 \mathrm{E}-2$ ARBM-AL1100 $1.0300011302799 .1826000 \quad 0.529000 \quad 0.229 .53908-1$ ARBM-TAPE(VINYL) $\quad 1.0700010015 .92601245 .918016 \quad 10.8217000$ $25.73200406 .9220001 .6820001 .121 .1115 \mathrm{~s}-2$ END

ARM-TAPE (MYLAR) 1.030000016 .83601265 .50801627 .022

$1.7491 \mathrm{~B}-2$ BND

ARBM-MODERATOR 1.1853

END COMP

ROCKY FLATS CRITICALS NUREG/CR-1071 EXPERIMENT NUMBER $13(27$ GROUP) READ PARM RUN $=$ YBS NPG $=600$

RND PARM

UNIT 1

COM=' FUEL BOX $15.28 \mathrm{CM}$ ON A SIDE WITH .15 CM WALLS .05CM STACKING VOID' CUBOID 1 1 1 687.49

CUBOID 221687.64

CUBOID

UNIT

COM='X-FACE INTERSTITIAL MODERATOR'

UNIT

COM $=$ ' $Y$ - PACB INTER

CUBOID 31287.665 2P1.2200 2P7.665

UNIT

INACE INTERSTITIAL MODERATOR

CUBOID

COM= $'$ MORE X-PACE MODEPATOR'

CUBOID 314 P1.2200 2 P7.66

UNIT 6

COM='MORE Y-FACE MODERATOR'

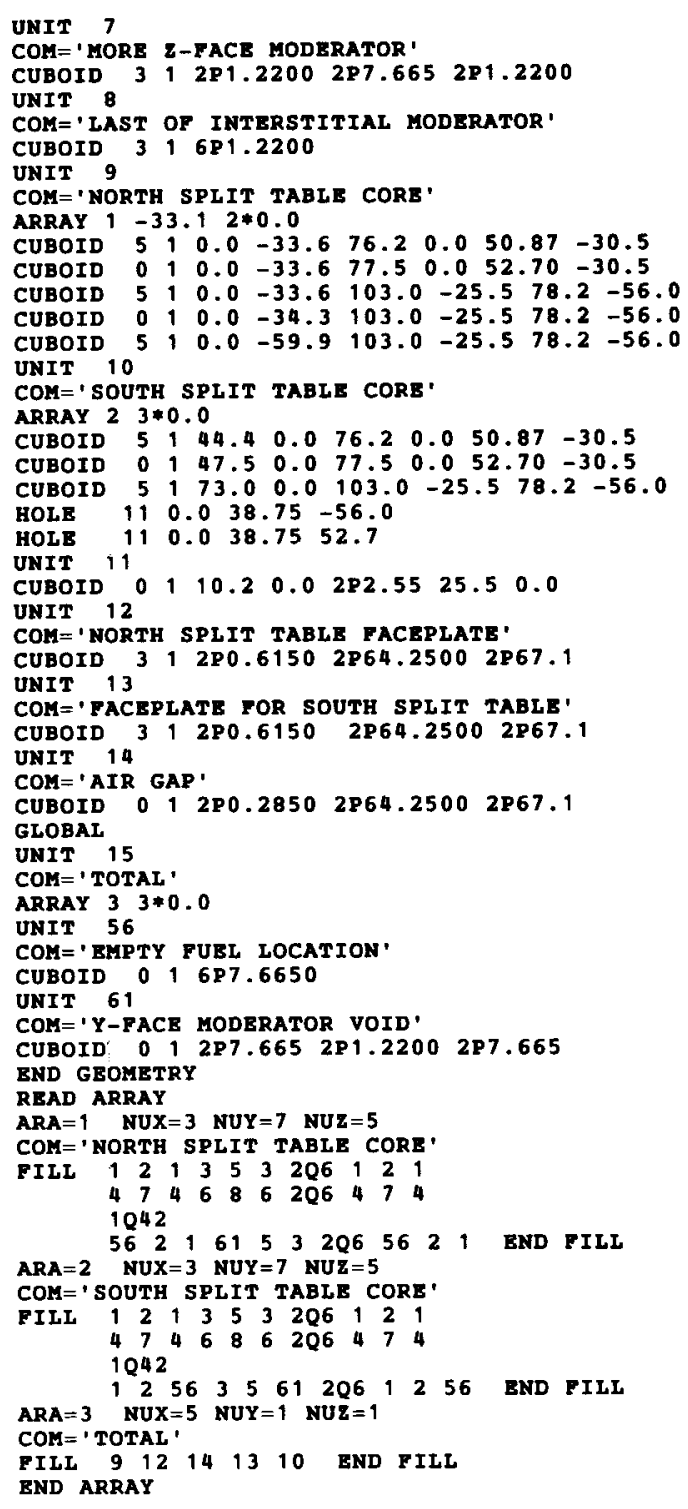


Table A.3 (continued)

READ PLOT TTL='XZ SLICE OF RPP 1 SHOWING MATERIAL REGIONS' $X U L=-2 \quad Y U L=64.05 \quad 2 U L=136$

UAX $=1$ WDN $=-1 \quad$ NAX $=130 \quad$ NCH $=\cdot 0123456 \cdot \mathbf{E N D}$

TTL='YZ SLICE OF NORTH CORE SECOND ROW

$X U L=28$ YUL $=-2 \quad 2 U L=136$

VAX $=1$ WDN $=-1$ MAX $=130 \quad \mathrm{NCH}=0123456^{\circ} \cdot \mathrm{END}$

TTL $=$ YY SLICE OP SOUTH CORE SECOND ROW'

$X U L=80 \quad Y U L=-2 \quad Z U L=136$

$X L R=80 \quad$ YLR $=136 \quad 2 L R=-2$
VAX $=1$
WDN $=-1 \quad$ NAX $=130 \quad \mathrm{NCH}=\cdot 0123456$.

END PLOT

STAT $=1$

$X S M=15$ XSP

BND

\section{CAR05}

ROCKY FLATS CRITICALS NUREG/CR-1071 EXPERIMENT NUMBER 15 (27 GROUP) 27GROUPNDF 4 LATTICECBL

U308 $15.4078 \mathrm{E}-1293.0922340 .0392235 \quad 4.4692236 \quad 0.089223895 .43$ END

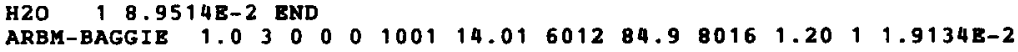$$
1.03001000130279 .18260000 .5290000 .220 .53908-1
$$

MAL1100 $1.0300011302799 .18260000 .529000 \quad 0.2229 .53908-1$

B-TAPE(VINYL) 1.0700010015 .92601245 .91801610 .8217000

$25.73200406 .922000 \quad 1.682000 \quad 1.1221 .11158-2$ END

I.TAPE (MYLAR)

1.0300010016 .83601265 .50801627 .022

$\begin{array}{lll}\text { ARBM-MODERATOR } & 1.185 & 3 \\ \text { RFCONCRETB } & 5 & 1.0 \\ \text { BND }\end{array}$

RND COMP

SPHTRIANGP $19.000018 .5857 \quad 1 \quad 3 \quad 18.95792$ END

ROCKY FLATS CRITICALS NUREG/CR-1071 EXPERIMENT NUMBER 15 (27 GROUP)

READ PARM RUN $=$ YES NPG $=600 \quad$ PLT $=$ NO

FDN $=$ YBS NUB $=$ YBS

BND PARM

READ GEOM

COM=' FUEL BOX $15.28 \mathrm{CM}$ ON A SIDE "ATITH $.15 \mathrm{CM}$ WALLS .05CM STACKING VOID' CUBOID 111087.49

CUBOID 0 1 6 6P7.665

UNIT ${ }^{2}$

X-FACE INTERSTITIAL MODERATOR

CUBOID $312 P 0.4645487 .665$

COMI $=$ Y ${ }^{3}$ - PACE INTERSTITIAL MODERATOR'

CUBOID $312 \mathrm{P7} .665 \quad 2 \mathrm{PO} 0.4645 \quad 2 \mathrm{P7} .665$

UNIT 4

COM='Z-FACE INTERSTITIAL MODERATOR

CUBOID $314 P 7.665 \quad 280.4645$

COM= $\operatorname{mos}$

COM= MORE X-PACE MODERATOR'
CUBOID 314 PO.4645 2P7.665

UNIT 6

COM $=$ MORE $Y$ - FACE MODERATOR

CUBOID 31287.665490 .4645

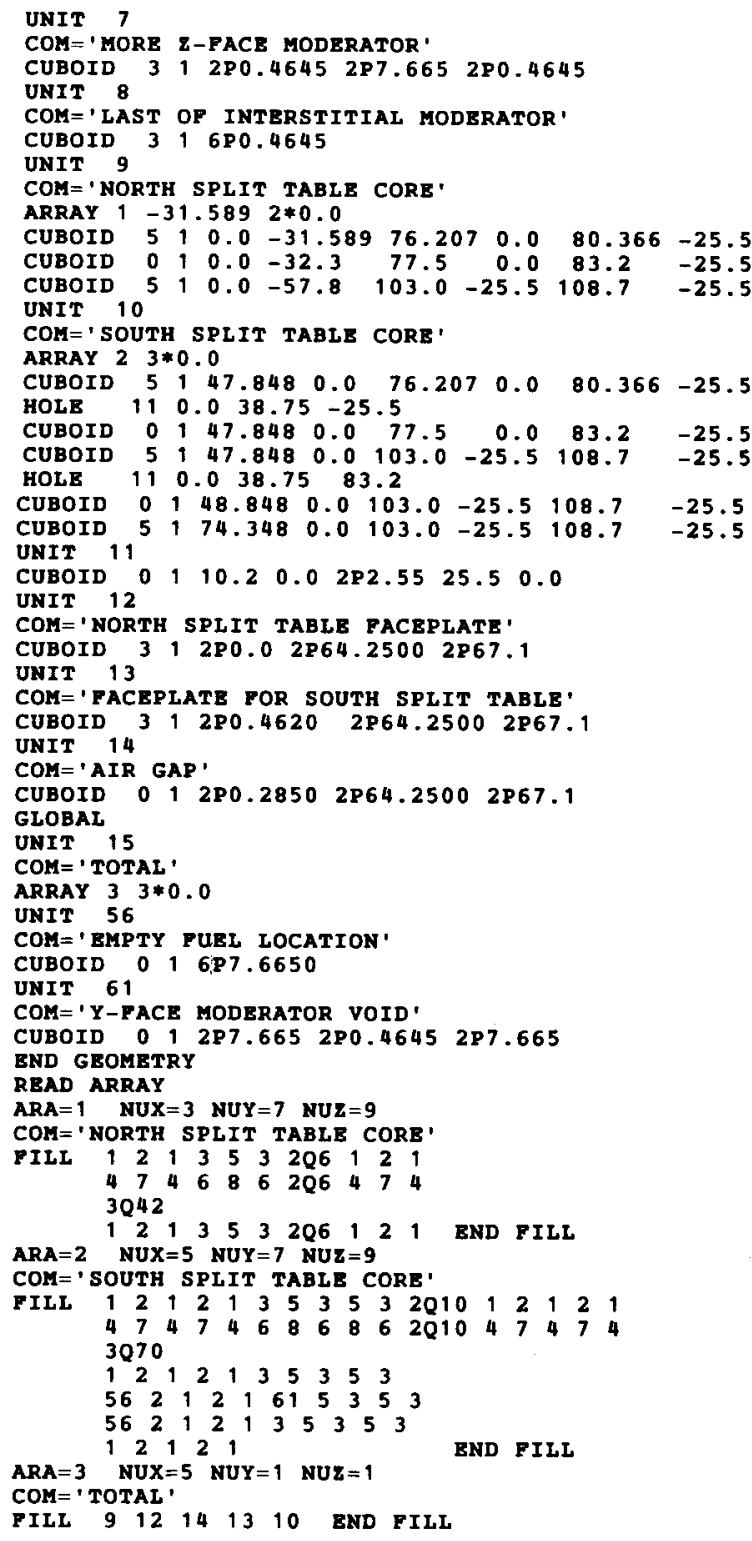


Table A.3 (continued)

RND ARRAY

READ PLOT TTI='XZ SLICE OF RPP1 SHOWING MATERIAI REGIONS'

$X U L=-2 \quad Y U L=64.05 \quad Z U L=136$

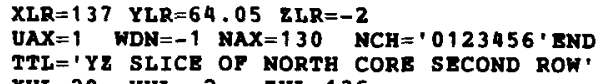

$\begin{array}{lll}X U L=28 & \text { YUL }=-2 & \text { ZUL }=136 \\ X L R=28 & \text { YLR }=136 & \text { yLR }\end{array}$

VAX $=1$ WDN $=-1$ NAX $=130$ NCH $=0123456 \cdot$ END

TTL $=$ YZ SLICE OF SOUTH CORE SECOND ROW

$\begin{array}{lll}X U L=80 & Y U L=-2 & Z U L=136 \\ X L R=80 & Y L R=136 & \text { ZLR }=-2\end{array}$

$X L R=80$ YLR $=136 \quad$ ZLR $=-2$
VAX $=1$

BND PLOT

READ START NST $=1$

$X S M=15 \quad x S P=122 \quad$ YSM=15 YSP=122 \&SM=30 zSP=122 END START END DATA

CAR06

=CSAS25 ROCKY PLATS CRITICALS NUREG/CR-0674 EXPERIMENT NUMBER? (27 GROUP MODEI)
FUEL CUBE $15.28 \mathrm{CH}, \mathrm{U}(4.46) 308$ H/U=0.77, HI ENRICHED SPHERE DRIVEN 27GROUPNDF 4 INFHOMMEDIUM U308 $15.4078 \mathrm{E}-1 \quad 293.0922340 .03922354 .4692236 \quad 0.089223895 .43$ END

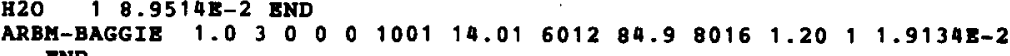

$\begin{array}{llllllllllllll}\text { END } & \\ \text { ARBY-AL1100 } & 1.0 & 3 & 0 & 0 & 1 & 13027 & 99.18 & 26000 & 0.5 & 29000 & 0.2 & 2 & 9.53908-1\end{array}$

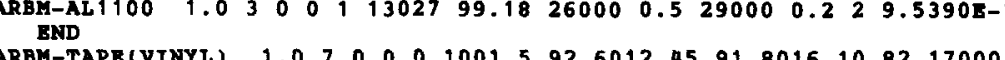

ARBM-TAPB (VINYL) 1.0700010015 .92601245 .918016

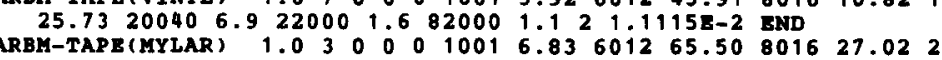

$1.7491 \mathrm{E}-2 \mathrm{END}$

ARBM-MODERATOR $1.185300001001 \quad 7.83601259 .498016 \quad 32.483$ END

ARBM-PLEX (REG)

ARBM-PLEX (PAPER)

$3.7534 \mathrm{E}-3$ END

1.1648 E -3 BND

ARBM-PLEX(TRIS) 1.08 \& 0 O $110017.16 \quad 601252.03 \quad 70140.16801629 .82$ 150311.02170001 .81350794 .260350812 .8405
1.2757

AREM-PLEX PAPER

MRM-PLEX (GLUE)

ARBM-FILLER $1.18530000010017 .83601259 .498016 \quad 32.4860 .7$ END

; THIS MATERIAL IS ACTUALLY MODERATOR ADJUSTED FOR THE VOLUME

FRACTION OF THE VOID FILLED BETHEEN CORE AND REFLECTOR.

URANTUS 0.951629300223410023593 .9290000 .272 .715 END ARBM-PJ 0.8162000100185 .1601214 .980 .00410 .4

SS304 91.0 END

BND COM

ROCKY PLATS CRITICALS NUREG/CR-0674 EXPERIMENT NUMBER ? (27 GROUP MODEI) ' PUEL CUBE $15.28 \mathrm{CM}, \mathrm{U}(4.46) 308$ H $\mathrm{H} / \mathrm{U}=0.77$, HI ENRICHED SPHERE DRIVEN READ PARM

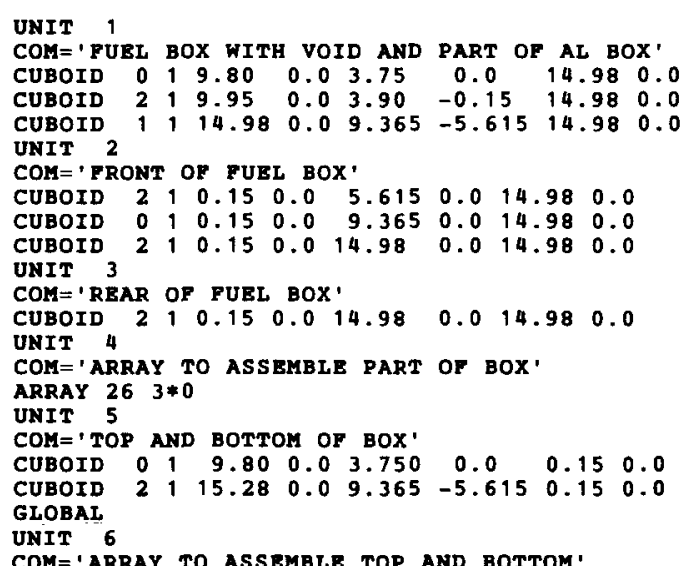

UNIT 1

COM=' PUBL BOX WITH VOID AND PART OF AL BOX'

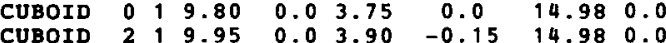

CUBOID $1114.98 \quad 0.0 \quad 9.365-5.615 \quad 14.98 \quad 0.0$

UNIT 2

COM=' FRONT OF FUEL BOX

CUBOID 2 1 $10.15 \quad 0.0 \quad 5.615 \quad 0.0 \quad 14.98 \quad 0.0$

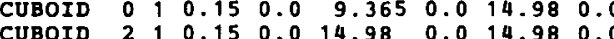

UNIT 3

COM=' REAR OF FUEL BOX

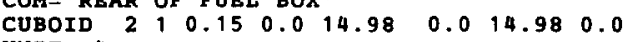

UNIT 4

COM=' ARRAY TO ASSBMBLE PART OF BOX

ARRAY $26 \quad 3 * 0$

COM= ' TOP AND BOTTOM OF BOX.

$\begin{array}{lllllllll}\text { CUBOID } & 0 & 1 & 9.80 & 0.0 & 3.750 & 0.0 & 0.15 & 0.0 \\ \text { CUBOID } & 2 & 1 & 15.28 & 0.0 & 9.365 & -5.615 & 0.15 & 0.0\end{array}$

GLOBAL

UNIT 6

COM=' ARRAY TO ASSBMBLE TOP AND BOTTOM

ARRAY $273 * 0$

$\begin{array}{llllllllll}\text { CUBOID } & 2 & 1 & 15.28 & 0.0 & 15.13 & -0.15 & 15.28 & 0.0 \\ \text { CUBOID } & 0 & 1 & 15.305 & -0.025 & 15.155 & -0.175 & 15.305 & -0.025\end{array}$

UNIT 7

COM=' FUEL BOX $15.28 \mathrm{CM}$ ON A SIDE WITH $.15 \mathrm{CM}$ WALLS .05CM STACKING VOID

CUBOID 11967.49

$\begin{array}{lllll}\text { CUBOID } & 2 & 1 & 6 P 7.64 \\ \text { CUBOID } & 0 & 1 & 6 P 7.6650\end{array}$

CUBOID

COM $=$ 'BOX FOR DRIVBR

CYIIINDER $9,0.3175 \quad 2 P 7.32$

HEMISPHE $+X \quad 8 \quad 17.334$ CHORD 6.818

CUBOID $018.512-6.818 \quad 287.665 \quad 287.665$

$\begin{array}{llllll}\text { HOLE } 37 & 0.0 & 0.0 & -7.66\end{array}$

COY='ALUMINUM WEIGHT DISTRIBUTION PLATE FOR SPECIAL BOX'

CUBOID $0199.80 \quad 0.0 \quad 3.750 \quad 0.000 .16 \quad 0.0$

UNIT 35

COM=-ALUMINUM WEIGHT DISTRIBUTION PLATE POR BOXES

$\begin{array}{llll}\text { CUBOID } 71 & 4 \text { P7.665 } & 0.16 \quad 0.0\end{array}$

COMIT ' DRIVER MOUNT'

$\begin{array}{llllll}C Y I I N D E R & 9 & 1 & 1.56 & 0.331 & 0.0\end{array}$

UNIT 9

COM=' NORTH SPLIT TABLE CORB'

$\begin{array}{lllllllll}\text { ARRAY } 1 & 3 * 0.0 \\ \text { CUBOID } 0 & 0 & 30.66 & -0.94 & 77.5 & 0.0 & 83.475 & 0.0\end{array}$

UNIT 10

COM=' SOUTH SPLIT TABLB CORE

CUBOID 2010

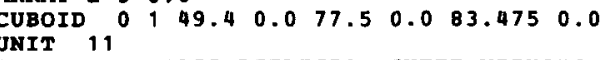

COM= PLEXIGLASS REPLECTOR SHEET WITHOUT

CUBOID 412 2P15.8000 2P38.7500 2P0.6150

CUBOID 512 2P15.8000 2P38.7500 2P0.6150

COM=' UPPER PORTION NORTH BOTTOM REPLECTOR WITH TRIS

COM= UPPER PORTION NORTH BOTTOM RE
CUBOID 5,2 P15.8 2 2838.75 2P8.24 
Table A.3 (continued)

UNIT 14 CUBOID 5 1 2P15.8 2838.752 P3.69

UNIT 15

COM=' NORTH BOTTOM REFLBCTOR INCLUDES REGULAR AND TRIS ARRAY 3300 .

UNIT 16 COM='PLEXIGLAS SHEET BOTTOM SOUTH REPLECTOR WITHOUT TRIS CUBOID 0 T 2P5.1 2P2.5 2P0.6150

CUBOID $4+44.3-5.12$ P 38.75 2P0.615

UNIT 17

COM= PLEXIGLAS SHEET BOTTOM SOUTH REFLECTOR WITH TRIS'

CUBOID 0 1 2 P5. $12 \mathrm{PP} 2.5 \quad 2 \mathrm{PO} .6150$

CUBOID 5 1 $44.3-5.12838 .75280 .6150$

COM=' LOKER PORTION SOUTH BOTTOM

CUBOID 5 1 $44.3-5.12$ P38.75 288.855

UNIT 19

COM='SOUTH BOTTOM REFLECTOR WITH REGULAR AND TRIS'

ARRAY $43 * 0$.

COM='BAST AND WEST REPLECTORS FOR NORTH REFLECTOR WITH TRIS' CUBOID $512815.82812 .652 P 54.2825$

COM='ARRAY FOR GAST AND WBST RBPLECTORS FOR NORTH REFLECTOR'

ARRAY 5 3*0.0

UNIT 22 20.0 AND COM='EAST AND WEST REFLECTORS FOR SOUTH
CUBOID $512824.700 \quad 2812.65 \quad 2854.2825$

UNIT 23

COM=' APRAY POR BAST AND WEST REPLBCTORS POR SOUTH REFLECTOR'

ARRAY $6 \quad 3 * 0.0$

UNIT 24

COM=' NORTH TOP REFLECTOR WITH TRIS

CUBOID $5 \quad 12$ 2P28.35 2P64.05 2P12.15

COM. 25 TYY 1 POR NORT TOP REFLECTOR

ARRAY $73 * 0.0$

UNI T 26

COM='SOUTH TOP REPLBCTOR WITH TRIS'

CUBOID $0,2 \mathrm{P} 5.12 \mathrm{P2} .52 \mathrm{P} 12.15$

CUBOID $5169.4-5.12 \mathrm{P} 64.05$ 2P12.15

UNIT 27

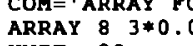

UNIT

CUBOID 412 2P4.9000 2P64.0500 2P54.2825

UNIT 29

CUBOID $5,282.6$ 2P64.0500 2P54.2825

CUBOID $30^{5}$

COM='NORTH END RELPECTOR 10.1 CM PORTION WITHOUT TRIS'

CUBOID 412 P5.05 2P64.05 2P54.2825

UNIT 31 COM= ARRAY FOR NORTH END REFLECTOR

COM $=1$ ARRAY FOR
ARRAY 9 \% $3 * 0.0$

COM=1 SOUTH

CUBOID 512 P12.55 2P64.05 2P54.2825

UNIT 33
COM $=$ ARRAY FOR SOUTH END REFLECTOR

ARRAY 10 3*0.
UNIT 38
COM='NORTH CORE WITH BOTTOM REFLECTOR.

COME' NORTH CORE
ARRAY $133 * 0.0$
UNIT 39

COM='NORTH CORE WITH EAST AND WEST REFLECTOR'

ARRAY $14 \quad 3 * 0.0$

UNIT 40

COM='NORTH CORE WITH END REFLECTOR'

ARRAY $15 \quad 3 * 0$ - 0

COM = ' NORTH CORE WITH TOP REFLECTOR'

ARRAY $16 \quad 3 * 0.0$

UNIT 48

COM='SOUTH CORE WITH BOTTOM REFLECTORS

ARRAY $20 \quad 3 * 0.0$

COM=, SOUTH CORE WITH EAST WEST REPLECTORS

ARRAY $213 * 0.0$

UNIT 50

COM='SOUTH CORE END REPLECTOP'

ARRAY $22 \quad 3 * 0.0$

UNIT 52

COM='SOUTH CORE WITH TOP REFLECTOR'

ARRAY $23 \quad 3 * 0.0$

COM = ' AIR GAP'

$\begin{array}{llllllllll}\text { HEMISPHE } X & 8 & 7 & 7.334 & \text { CHORD } & -6.818 & & & \\ \text { CUBOID } 0 & 1 & -6.818 & -7.619 & 64.475 & -63.625 & 69.13 & -63.735\end{array}$

GLOBAL

UNIT 55

COME ' TOTAL'

ARRAY $25 \quad 3 * 0.0$

READ ARRAY

ARA $=1 \quad$ NUX=2 NUY $=5 \quad N U Z=9$

COM='NORTH SPLIT TABLE CORE'

$10 R 35$
$10 R 7$
$10 R 35$
$10 R 7$

$10 \mathrm{R} 35$

$10 \mathrm{R} 7$

$10 \mathrm{R} 35$

$A R A=2$ END FILL

COM=' SOUTH SRIT TABLE CORE'

FILL 6R7 6 8R7

6R35 $34 \quad 8 R 35$

6R7 $6 \quad 8 R 7$

$6 R 35348 R 3$

6R7 88 8R7

6R $75348 R 35$

6R35 $348 R 35$
$6 R 7$ 6 $8 R 7$ END FILL

$A R A=3 \quad N U X=1 \quad N U Y=1 \quad N U Z=3$

COM= ' NORTH BOTTOM RBFLBCTOR

FILL 4 T1 3 END FILL

COM= ' SOUTH BOTTOM RERL

$\begin{array}{lllllllllll}\text { PILL } 18 & 16 & 17 & 16 & 16 & 17 & 17 & \text { END FILL }\end{array}$

ARA $=5$ NUX $=1$ NUY $=1$ NUE $=1$
COM='BAST AND WEST WALLS OF NORTH REPLECTOR'

FILL 20 END FILI 
Table A.3 (continued)

ARA $=6$ NUX=1 NUY $=1 \quad \mathrm{NUZ}=1$

COM= ' BAST AND WEST WALLS OF SOUTH REPLBCTOR'

FILL 22 SND FILL

COM=' $A R R A Y$ YOR NORTH TOP REFLECTOR'

CILL 24 END VILL

ARA $=8$ NUX $=1$ NUY $=1$ NUZ $=1$

COM=' ARRAY FOR SOUTH TOP REFLECTOR'

FILL 26 END PILL

ARA $=9$ NUX $=3$ NUY $=1 \quad$ NUZ $=1$

COH=' ARRAY FOR NORTH END

ILL $28 \quad 2930 \quad$ END FILI

COM=1APRAY FOR SOUTH END RETLECTOR.

PILL 32 END FILL

ARA $=13$ NUX $=1$ NUY $=1$ NUZ $=2$

COM=' COMBINATION OF NORTH CORE WITH BOTTOM RETLECTOR

FILL 15 9 END FILL

COM=' NORTH CORE WITH SIDE REFLECTORS

COM=' NORTH CORE WLTH SID

$A R A=15$ NUX $=2$ NUY $=1$ NUZ $=1$

COM=' 'NORTH CORE WITH END REFLECTOR'

PILL 3139 END FILL

ARA $=16$ NOX $=1$ NOY $=1$ NUZ $=2$

COH = ' NORTH CORE WITH TOP

PILL $40 \quad 25$ END FILI

COM $=$ ' SOUTH CORE WITH BOTTOM REPLECTOR

FILL 1910 BND FILL

ARA $=21$ NUX $=1 \quad$ NUY $=3 \quad$ NUZ $=1$

COM='SOUTH CORE WITH EAST WEST RETLECTORS

FILL 234823 END FILL

COM=' SOUTH CORE WITH END REFLCTOR'

COM= 4933 S 30 TIS

ARA $=23$ NUX $=1 \quad$ NUY $=1$ NUZ $=2$

COM $=$ ' COMBINATION OF CORE WITH TOP REPLECTOR'

FILL 5027 END FILL

$A R A=25 \quad N U X=3$ NUY $=1$ NUZ $=1$

FILL 425452 END FIII

COM='ASSEMBLY OF TUEL BOX TRONT AND REAR'

FILL $2 \begin{array}{llll}1 & 3 & \text { END FILI }\end{array}$

$A R A=27$ NUX $=1$ NUY $=1$ NUZ $=3$

COM=' ASSBMBLY OF FUEL BOX TOP AND BOTTO

FILI 5 4 5 END FILI

READ PLOT TTL='YZ SLICZ OF SOUTH CORE FIRST ROW

$X U L=64.319$ YUL $=55.625 \quad 2 U L=71.735$

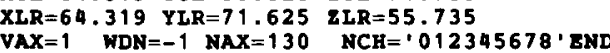

TTL $=$ ' $X 2$ SLICE OF SPHERE CUBOID

$X U L=56.0 \quad Y U L=63.625 \quad \mathrm{ZUL}=71.735$

作

END PLOT

READ START NST $=6$ TFX=65 TPY=63.625 TPZ=63.735 LNU $=300$ END START READ START NST $=1$ XSM $=56.981 \quad$ XSP $=71.649$ YSH $=56.219 \quad$ YSP $=70.959$

ZSM $=56.401$ ZSP $=71.069$ END START

BND DATA
CAR07

ROCKY FLATS CRITICALS NUREG/CR-0674 EXPERIMENT NUMBER ? (27 GROUP MODEL) FUEI CUBE $15.28 \mathrm{CM}, \mathrm{U}(4.46) 308 \mathrm{H} / \mathrm{U}=0.77$, HI CONC. SOLUTION DRIVEN

J308 1 5.4078E-1 293.0 $922340.0392235 \quad 4.46922360 .089223895 .43$ END H2O $18.9514 \mathrm{E}-2 \mathrm{END}$

$1.03000100114 .01601284 .980161 .2011 .91348-2$

END

DND $1.030011302799 .18260000 .5290000 .229 .53908-1$

BND

10.8217000

$25.73200406 .9220001 .6820001 .121 .1115 \mathrm{~B}-2$ END

ARB-TAPE(NYLAR) 1.0300010016 .83601265 .50801627 .022

$\begin{array}{llllllllllll}.7491 E-2 & & \end{array}$

ARBM-PLEX(REG) 1.0300010017 .84601259 .59801632 .234

$1.1773 \mathrm{BND}$

ARBM-PLEX(PAPER) 1.03000010016 .48601242 .17801649 .5

$3.75342-3$ END

(1.0

$1.1648 \mathrm{E}-3$ END

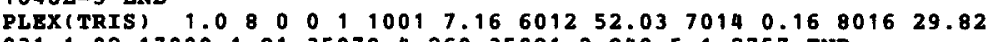

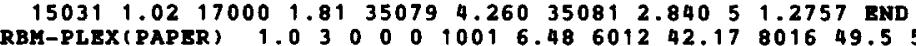

3.7534E-3END
1.030

$1.1648 \mathrm{~B}-3$ END

ARBM-FILLER $11.185300001001 \quad 7.836012 \quad 59.498016 \quad 32.48 \quad 6 \quad 0.7 \quad$ END

THIS MATERIAL IS ACTUALLY MODERATOR ADJUSTED FOR THE VOLUAE

ARBM-AI1100 1.0 30011302799.18260000 .5290000 .272 .715 END SOLNUO2(NO3) 2 \& $351.18 \quad 0.549 \quad 1.0293 .0922341 .0229223593 .172$

922360.434922385 .372 END

$5 S 304 \quad 91.0$ END

ROCKY FLATS CRITICALS NUREG/CR-0674 EXPERIMENT NUMBER ? (27 GROUP MODEL)

PUEL CUBE 15.28 CM U (4. 46)308 READ PARY

READ GEOH

COM=' TUEL BOX WITH VOID AND PART OF AL BOX'

CUBOID $0119.80 \quad 0.0 \quad 3.75 \quad 0.0 \quad 14.980 .0$

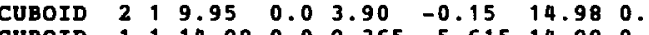

$\begin{array}{lllll} & \end{array}$

FRONT OF FUEL BOX

$\begin{array}{lllllllll}\text { CUBOID } 2 & 1 & 0.15 & 0.0 & 5.615 & 0.0 & 14.98 & 0.0\end{array}$

$\begin{array}{lllllllll}\text { CUBOID } & 0 & 0.15 & 0.0 & 9.365 & 0.0 & 14.98 & 0.0\end{array}$

CUBOID

COME.

$\begin{array}{lllllllll} & & & \end{array}$

UNIT

ARRAY $26 \quad 3 * 0$

UNIT 5

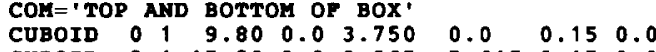

cUomat 
Table A.3 (continued)

UNIT 6

COMA' ARRAY TO ASSEMBLE TOP AND BOTTOM.

$\begin{array}{lllllllll}\text { CUBOID } & 2 & 1 & 15.28 & 0.0 & 15.13 & -0.15 & 15.28 & 0.0 \\ \text { CUBOID } & 0 & 1 & 15.305 & -0.025 & 15.155 & -0.175 & 15.305 & -0.025\end{array}$

CoBoro 0

COM= ' FUBL BOX $15.28 \mathrm{CM}$ ON A SIDE WITH $.15 \mathrm{CR}$ WALLS .05CM STACKING VOID

CUBOID 119 . 17.49 $\begin{array}{lll}2 & 1 & 6 P 7.64 \\ 0 & 1 & 6 P 7.6650\end{array}$

UNIT 34

ECIAI BOX

$\begin{array}{llllllll}\text { CUBOID } & 9 & 9.80 & 0.0 & 3.750 & 0.0 & 0.16 & 0.0 \\ \text { CUBOID } 7 & 1 & 15.33 & 0.0 & 9.54 & -5.79 & 0.16 & 0.0\end{array}$

COM=' ALUMINUM WEIGHT DISTRIBUTION PLATE FOR BOXES

CUBOID 714 P7.665 0.160 .0

UNIT 9

COM=' NORTH SPLIT TABLE CORE'

ARRAY $13 * 0.0$

$\begin{array}{lllllll}30.66 & -0.94 & 77.5 & 0.0 & 83.475 & 0.0\end{array}$

COM='SOUTH SPLIT TABLE CORE

ARRAY $23 * 0$.

CUBOID $01949.4 \quad 0.0 \quad 77.5 \quad 0.0 \quad 83.475 \quad 0.0$

COM= ' PLEXIGLASS RETLECTOR SHEET WITHOUT TRIS, NORTH BOTTOM REFLBCTOR COM= PLEXIGLASS REFLECTOR SHEET WITHOUT
CUBOID $412 \mathrm{P} 15.80002 \mathrm{P} 38.75002 \mathrm{PO} .6150$

COM=' PLEXIGLASS REFLECTOR SHEET WITH TRIS, NORTH BOTTOM REFLECTOR' CUBOID $512 P 15.80002838 .75002 P 0.6150$

UNIT 13

COM=' UPPER PORTION NORTH BOTTOM REPLECTOR WITH TRIS

CUBOID 5 , 2 P15.8 2 P38.75 2PB.24

UNIT 14

CUBOID 5,2 P15.8 2838.75 2P3.69

COM=' NORTH BOTTOM REPLECTOR INCLUDES RBGULAR AND TRIS

ARRAY $33 * 0.0$

UNIT 16

= ' PLEXIGLAS SHEET BOTTOM SOUTH REFLECTOR WITHOUT TRIS'

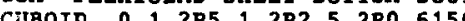

CUBOID 17

COM=' PLEXIGTAS SHERT BOTTOY SOUTH REPLECTOR WTTH TRIS

CUBOID 0 1 $285.12 \mathrm{P2} .5 \quad 2 \mathrm{PO} 0.6150$

CUBOID $5144.3 \quad-5.12838 .75$ 2P0.615

UNIT 18

COM='LOWRR PORTION SOUTH BOTTOM REFLECTOR WITH TRIS'

CUBOID 0 1 12 2P5.1 2 P2.5 288.855

CUBOID 5 1 $44.3-5.12 \mathrm{P} 38.75$. 288.85

COMT 19 SOUTH BOTTOM REFLECTOR WITH REGULAR AND TRIS

ARRAY 4 3*0.0

UNIT 20 COM=1 20 ANT AND KEST REFLECTORS TOR NORTH REFLECTOR WITH TRIS COM=' RAST AND WEST REPLECTORS FOR NORTH
CUBOID 5 1 2815.8 2812.65 2854.2825

UNIT 21

POR BAST AND WEST REFLECTORS FOR NORTH RETLECTOR ARRAY $5 \quad 3 * 0.0$

UNIT 22

COM= EAST AND HEST REFTECTORS POR SOUTH REFLECTOR WITH TRTS

CUBOID 5 AND24.700 2P12.65 2 P54.2825
UNIT 23

POR RAST AND WEST REPLBCTORS POR SOUTH REFLECTOR

UNIT 24 TOO

COM=' NORTH TOP REFLECTOR WITH TRIS
CUBOID 5 , 2 P28.35 2 P64.05 2 P 12.15

UNIT 25

ARPARAY FOR NORTH TOP REFLECTOR

$73 * 0.0$

COM=' SOUTH TOP REFLECTOR WITH TRIS

CUBOID $01285.12 P 2.5 \quad 2 P 12.15$

CUBOID $5169.4-5.12 P 64.05 \quad 2 P 12.15$

UNIT 27

ARRAY FOR SOUTH TOP REFLECTOR

WITHOUT TRIS

CUBOID 412 P4.9000 2P64.0500 2P54.2825

UNIT 29

CUBOID $5,282.6 \quad 2864.0500 \quad 2854.2825$

COM=' NORTH END RELPECTOR $10.1 \mathrm{CM}$ PORTION WITHOUT TRIS'

CUBOID 412 P5.05 2P64.05 2P54.2825

UNIT 31 COM= ARRAY FOR NORTH END REPLECTOR

COM= ARRAY FOR
ARRAY $9 \quad 3 * 0.0$

.

CUBOID $512 P 12.552 P 64.052 P 54.2825$

UNTT 33

SOUTH END REFLECTOR

ARRAY $10 \quad 3 * 0.0$

UNIT 38

DEPLECTOR.

UNIT 39 TH CORE WITH EAST AND WEST REPLECTOR.

ARRAY $14 \quad 3 * 0.0$

UNIT 40

NORTH CORE WITH END REPLECTOR

ARRAY $15 \quad 3 * 0.0$

COM='NORTH CORE WITH TOP REPLECTOR'

ARRAY $16 \quad 3 * 0.0$

UNIT 48

COM='SOUTH CORE WITH BOTTOM REPLECTORS'

ARRAY $203 * 0.0$

UNIT ' SOUTH CORE WITH EAST WEST REFLECTORS'

ARRAY $213 * 0.0$

UNIT 50

COM='SOUTH CORE BND REPLECTOR'

ARRAY $22 \quad 3 * 0.0$

UNIT 52 SOMT CORE WITH TOP REPLECTOR

ARRAY $23 \quad 3 * 0.0$

$\begin{array}{lllllll}\text { COM=1AIR GAP } & & & & & & \end{array}$

GLOBAL

UNIT 55

CORT- $25,3 \div 0.0$ 
Table A.3 (continued)

UNIT 56

OF TWO REgULAR FUEI BOXES

ARRAY 28
UNIT 57

COM=' COMBINATION OF REgULAR FUEL BOX AND SPECIAL FUEL BOX' ARRAY $293 * 0.0$

UNIT 5

COM= COMBIMATION OF TWO REGULAR WETGHT DISTRIBUTION PLATES ARRAY $30 \quad 3 * 0.0$

COM= ' COMBINATIOH

UNIT 60

COM= ' NORTH CORE SOLUTION DRIVER'

CUBOID $811287.5 \quad 2 P 14.93 .710-7.5$

CUBOID 012 PP7.5 2814.9287 .5

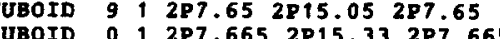

UNIT 61

COR= ' MORTH CORE SOLUTIOH DRIVER'

CUBOID $81287.5 \quad 2 P 14.93 .630-7.5$

CUBOID $011287.52 P 14.9287 .5$

$\begin{array}{llllll}\text { CUBOID } & 9 & 1 & 2 \mathrm{P} 7.65 & 2 \mathrm{P} 15.05 & 2 \mathrm{P} 7.65 \\ \text { CUBOID } & 0 & 1 & 2 \mathrm{P} 7.665 & 2 \mathrm{P} 15.33 & 2 \mathrm{P} 7.665\end{array}$

CUBOID 01

RNDAD GROMET

ARA $=1 \quad$ NUX=2 NUY $=4 \quad$ NUZ $=9$

COM $=$ ' HORTH SPLIT TABLE CORE'

FILL

$\begin{array}{lll}2 R 7 & 2856 & 4 R 7\end{array}$

2R7 2856 4R7

2R35 2R58 4R35

$\begin{array}{llll}2 R 7 & 56 & 60 & 4 R 7\end{array}$

$2 R 7 \quad 2 R 56 \quad 4 R 7$

$\begin{array}{lll}2 R 35 & \text { 2R58 } & 4 R 35 \\ 2 R 7 & 2 R 56 & 4 R 7\end{array}$

END IIL

COHA = SOUTH SPLIT

$\begin{array}{lll}3 R 7 & 3 R 56 & 6 R 7 \\ 3 R 35 & 3 R 58 & 6 R 35\end{array}$

$3 R 7 \quad 3 R 56 \quad 6 R 7$

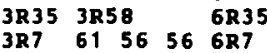

$3 R 35 \quad 3 R 58 \quad 6 R 35$

$3 R 355958586835$

END PIIL
$A R A=3$ NUX $=1$ NUY $=1 \quad$ NUZ $=3$

$\begin{array}{lllll}3 R 35 & 59 & 58 & 58 & 6 R 3 \\ 3 R 7 & 57 & 56 & 56 & 6 R 7\end{array}$

COH= 'NORTH BOTTOK REFLECTOR'

FILL $14 \quad 1113$ END FILL

COM=' SOUTH BOTTOM REFTECTOR'

IIL $\begin{array}{llllllll}18 & 16 & 17 & 16 & 16 & 17 & 17 & \text { END FIL }\end{array}$

ARA $=5$ NUX $=1$ NUY $=1$ NUZ $=1$
COM=' BAST AND WEST WALLS OF NORTH REFLECTOR'

FILL 20 END FILL

ARA $=6$ NUX $=1$ NUY $=1$ NUZ $=1$
COM= 'BAST AND WEST WALLS OF SOUTH REFLECTOR'

COM= BAST AND WEST

$A R A=7 \quad$ NUX $=1 \quad$ NUY $=1 \quad$ NU $z=1$

COM=' ARRAY FOR NORTH TOP REFLECTOR'

ILI 24 END FILL
ARA $=8 \quad$ NUX $=1 \quad$ NUY $=1 \quad$ NUZ $=1$

REFLECTOR.

ILL 26 END PIL

COM $=9$ NUX $=3$ NUY $=1$ NUZ $=1$ REPLECTOR

COM= $A$ ARRAY POR NORTH END

TRA 282930 END FILL

COM $=$ ' ARRAY FOR SOUTH END REFLECTOR

FILI 32 TND

$A R A=13$ NUX $=1$ NUY $=1$ NUY $=2$

COM $=$ ' COMBINATION OF NORTH

PILI 159 FND PILL

ARA $=14$ NUX $X=1$ NUY $=3$ NUZ $=1$

ARA 15 3821 BND

WITH END REFLECTOR

FILL 3139 BND PILL

$A R A=16 \quad N U X=1 \quad N U Y=1 \quad$ NUZ $=2$

COM='NORTH CORE WITH TOP REFLECTOR'

FILI $40 \quad 25$ BND FILL

$A R A=20 \quad$ NOX $=1 \quad$ NUY $=1$ NUZ $=2$

COHL $=$ SOUTH 19 CORE

$A R A=21$ NUX $=1$ NUY $=3$ NUZ $=1$

COM=' SOUTH CORE WTTH BAST WEST REFLECTORS'

FILI $23 \quad 4823$ END FILL

ARA $=22$ NUX $=2$ NUY $=1$ NUZ $=1$

COH= SOUTA CORE WITH END REFLECTOR'

PILL 4933 SND FILL

COM-'COMBINATION OF CORE WITH TOP REFLECTOR'

FILI 5027 BYD FILI

ARA $=25 \quad$ NUX $=3 \quad$ NUY $=1 \quad$ NUZ $=1$

COM= TOTAL

FILL 425452 END FILL

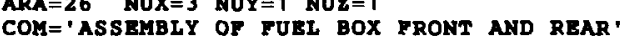

FILI 213 BND PILL

ARA $=27$ NUX $=1$ NUY $=1$ NUZ $=3$

COM='ASSEMBLY OF FUEL BOX TOP AND BOTTOM

FILL 5 4 5 EHD FILL

ARA $=28 \quad N U X=1 \quad N U Y=2 \quad N U Z=1$

COM $=$ ' COMBINATION OF TWO FUBL BOXES

ILL 77 END FIL

COM=' COMBINATION OF SPECIAL AND REGULAR FUEL BOXES '

FILL 76 BND FIL

MRA $=30$ NUX=1 NUY=2 NUZ $=1$

COM=' COMBINATION OF TWO WBIGHT DISTRIBUTION PLATES

PILL 3535 BND FILL

COH=' COMBINATION OF SPBCIAL AND REGULAR WEIGHT DISTRIBUTION PLATES' FILI 35 34 END FILL

END ARRAY

READ PLOT TTL='YZ SLICE OF SOUTH CORB FIRST ROW

$X U L=64.319$ YUL $=40.000 \quad 2 U L=72.000$

$X L R=64.319$ YLR $=72.000$
VAX $=1$ WDN $=-1$ NAX $=130 \quad$ NCH $=1012345678 \cdot$ END

VAX $=1$ NDN=-1 NAX $=130$ NCH='01234

$X U L=56.0$ YUL $=63.625 \quad 2 U L=71.735$

$X L R=72.0 \quad Y L R=63.625 \quad z L R=55.735$

$\mathrm{UAX}=1$ WDN $=-1$ NAX $=130 \quad \mathrm{NCH}=.012345678^{\circ}$

END PLOT 
Table A.3 (continued)

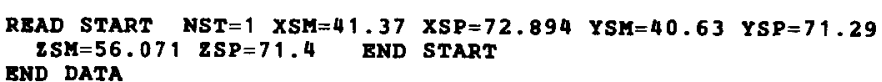
END

CAROB

$=\operatorname{csas} 25$

ROCKY PLATS CRITICALS NUREG/CR-0674 EXPERIMENT NUMBER ? ( 27 GROUP MODEL PUEL CUBE $15.28 \mathrm{CM}, \mathrm{U}(4.46) 308$ H/U=0.77, LOW CONC. SOLUTION DRIVEN U308 $15.4078 \mathrm{8}-1 \quad 293.0922340 .03922354 .4692236 \quad 0.089223895 .43$ END H2O $18.9514 \mathrm{E}-2$ END

AREM-BAGGIE $1.0300001001 \quad 14.01601284 .980161 .2011 .91345-2$

M-AL1100 $1.030011302799 .18250000 .5290000 .229 .53908-1$

EMATAPE(VINYL) 1.0700010015 .92601245 .91801610 .8217000

$25.73200406 .9220001 .6820001 .1211115 E_{-2}$ E4D

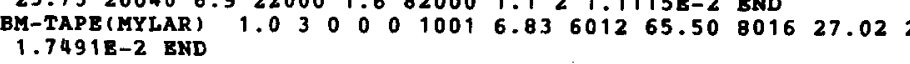

ARBM-MODERATOR $1.18530000010017 .83601259 .498016 \quad 32.483$ END

ARBM-PLEX REG

ARBM-PLEX (PAPER

$3.75348-3$ BND

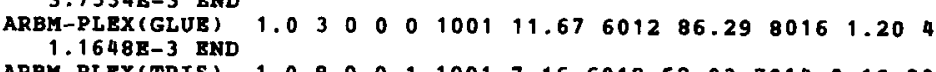

M-PLEX(TRIS) $1.08000110017 .16 \quad 601252.03 \quad 7014 \quad 0.16 \quad 8016 \quad 29.82$

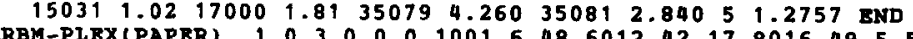

$3.7534 \mathrm{E}-3$ BND

M-PLEX(GLUB) 1.030000100111 .67601286 .2980161 .205

$1.1648 \mathrm{~B}-3$ END

ARBK-FILLER $1.185 \quad 3 \quad 0 \quad 0 \quad 0 \quad 1001 \quad 7.83 \quad 601259.498016 \quad 32.48 \quad 6 \quad 0.7$ END

THIS MATERIAL IS ACTUALLY MODERATOR ADJUSTED FOR THE VOLUME

ARBM-AL $1100,1.0$ OO 0 1 13027 .

A

922360.434922385 .372 END

5530491.0 BND

END COMP

ROCKY FLATS CRITICALS NUREG/CR-0674 EXPERIMENT NUMBER ? (27 GROUP MODEL) PUEL CUBE $15.28 \mathrm{CM}, U(4.46) 308$ \& $H / U=0.77$, LOW CONC. SOLUTION DRIVEN READ PARM

UNIT 1

\begin{tabular}{l} 
COM='PUEL BOX WITH VOID AND PART OF AL BOX' \\
CUBOID 0 \\
\hline
\end{tabular}

$15 \quad 14.98 \quad 0.0$

UNIT 2

COM=' TRONT OP FUEL BOX'

$\begin{array}{lllllllll} & & & & & & & \\ \text { CUBOID } 2 & 1 & 0.15 & 0.0 & 5.615 & 0.0 & 14.98 & 0.0\end{array}$

$\begin{array}{llllllllll}\text { CUBOID } & 0 & 1 & 0.15 & 0.0 & 9.365 & 0.0 & 14.98 & 0.0\end{array}$

$\begin{array}{llllllll} & & & & \end{array}$

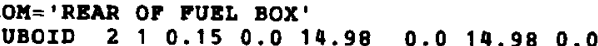

UNIT 4

ASSEMBLE PART OF BOX

ARRAY $26 \quad 3 * 0$

Thris 26

TOP AND BOTTOM OF BOX

$\begin{array}{llllllll}\text { CUBOID } & 0 & 9.80 & 0.0 & 3.750 & 0.0 & 0.15 & 0.0\end{array}$

GLOBAI

UNIT 6

COM=' ARRAY TO ASSEMBLE TOP AND BOTTOM.

$\begin{array}{lllllllllll}\text { CUBOID } & 2 & 1 & 15.28 & 0.0 & 15.13 & \mathbf{- 0 . 1 5} & 15.28 & 0.0 \\ \text { CUBOID } & 0 & 1 & 15.305 & -0.025 & 15.155 & -0.175 & 15.305 & -0.025\end{array}$

UNIT 7

COM='FUEL BOX 15.28 CM ON A SIDE WITH .15 CM WALLS .05CM STACKING VOID'

CUBOID 1 1 16 6P7.49

$\begin{array}{lllll}\text { CUBOID } & 2 & 1 & \text { 6P7.64 } \\ \text { CUBOID } & 0 & 1 & 6 P 7.6650\end{array}$

UNIT $_{34}$

COM='ALUMINUM WEIGHT DISTRIBUTION PLATE FOR SPECIAL BOX'

CUBOID $0199.800 .0 \quad 3.750 \quad 0.0 \quad 0.160 .0$

CUBOID $7115.33 \quad 0.0 \quad 9.54 \quad-5.790 .16 \quad 0.0$

COM $=$ ' ALUMINUM WEIGHT DISTRIBUTION PLATE FOR BOXES.

CUBOID 714 4P7.665 0.160 .0

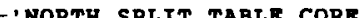

ARRAY $13 * 0.0$

CUBOID $0130.66-0.94 \quad 77.5 \quad 0.083 .475 \quad 0.0$

COM=' SOUTH SPLIT TABIE CORE.

ARRAY 2 3*0.0

$149.40 .077 .5 \quad 0.083 .4750 .0$

COM='PLEXIGLASS REFLECTOR SHEET WITHOUT TRIS, NORTH BOTTOM REFLECTOR' CUBOID 412 P15.8000 2P38.7500 2P0.6150

UNIT 12 COM=.

COM='PLEXIGLASS REFLECTOR SHEET WITH TRIS, NORTH BOTTOM REPLECTOR' CUBOID $512 \mathrm{P} 15.80002 \mathrm{P} 38.7500 \quad 2 \mathrm{PO} .6150$

COM= ' UPPER PORTION NORTH BOTTOM REPLECTOR WITH TRIS'

CUBOID 5 1 2P15.8 2P38.75 2P8.24

UNIT 14

COM= ' LOWER PORTION NORTH BOTTOM REFLECTOR WITH TRIS

CUBOID 5 1 2P15.8 2 P38.75 2P3.69

UNIT 15 COM, NORTH BOTTOM REFLECTOR INCLUDES REGULAR AND

ARRAY $3 * 0.0$

UNIT 16

COM='PLEXIGLAS SHEET BOTYOM SOUTH REPLECTOR WITHOUT TRIS

CUBOID 0 1 2 P5.1 2 P2.5 2 P0.6150

CUBOID 4 1 $44.3 \quad-5.12 \mathrm{~PB} 3.75 \quad 2 \mathrm{PO} 0.615$

COM=-PLEXIGLAS SHEET BOTTOM SOUTH

RELETOR WITH TRIS.

CUBOID 5 , $44.3^{-5}-1.52838 .75280 .6150$

UNIT 18

COM=' LOWER PORTION SOUTH BOTTOM REFLECTOR WITH TRIS'

CUBOID 00112 P 5.12 2P2.5 2 PP8.85

CUBOID $5 \quad 1 \quad 44.3 \quad-5.1 \quad 2$ P $38.75 \quad 2$ P8.855

COM=' SOUTH BOT 
Table A.3 (continued)

UNIT 20

COM='EAST AND WEST REPLECTORS FOR NORTH RETLECTOR WITH TRIS CUBOID 512 2P15.8 2P12.65 2P54.2825

COME ARRAY TOR EAST AHD ARRAY $5 \quad 3 * 0.0$

UNIT 22

RETLECTOR WITH TRIS CUBOID $512 \mathrm{P24} .700$ 2P12.65 2854.2825

UNIT 23 TOY ARRAY 6 \% $3 \neq 0,0$

UNRAT 24

COY = 'NORTH TOP RETLECTOR WITH TRIS

CUBOID 5,2 2P28.35 2P64.05 2P12.15

UNIT 25 COMRAY YOR NORTH TOP REFLECTOR'

ARRAY $73 * 0.0$

COM=. 26 TOUTH TOP REPLECTOR WITH TRIS

CUBOID $012 \mathrm{P} 5,12 \mathrm{P2} .52 \mathrm{P} 12.15$

CUBOID 5 1 69.4 -5.1 2 P 64.05 2P12.1

UNIT 27

COM= ' ARRAY FOR SOUTH TOP RETLECTOR'

ARRAY 8 B*0.0

COM=1 28 CUBOID 4 2P4.9000 2P64.0500 2P54.2825

UNIT 29

(1)

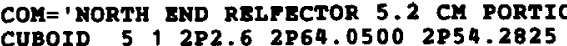

CUBOID 30 2P2.6 2P64.0500 2P54.2825

COM=' NORTH END RELFECTOR $10.1 \mathrm{CM}$ PORTIO

CUBOID 4 1 2P5.05 2P64.05 2P54.2825

COM = ARRAY TOR NORTH END REFLECTOR'

ARRAY 9 3*0.0

UNIT 32

OM=' SOUTH END REPLECTOR

CUBOID 5,2 P12.55 2P64.05 2854.2825

UNIT 33 .

ARRAY $10 \quad 3=0.0$

UNIT 38

COM= ' NORTH CORE WTTH BOTTOM RETLECTOR'

ARRAY $13,3 * 0.0$

UNIT 39

WEST REPLCTOR

ARRAY $14 \quad 3 * 0.0$

UNIT 40 TO CORE WITH END REFLECTOR.

ARRAY $15 \quad 3 * 0$.

UNIT 42

ARAY 15 300.0

ARRAY $16 \quad 3 * 0.0$

COM= I SOUTH CORE WITH BOTTOM REPLECTORS'

ARRAY $203 * 0$.

UNIT 49

EAST WEST REPLECTORS

ARRAY $21 \quad 3 * 0.0$

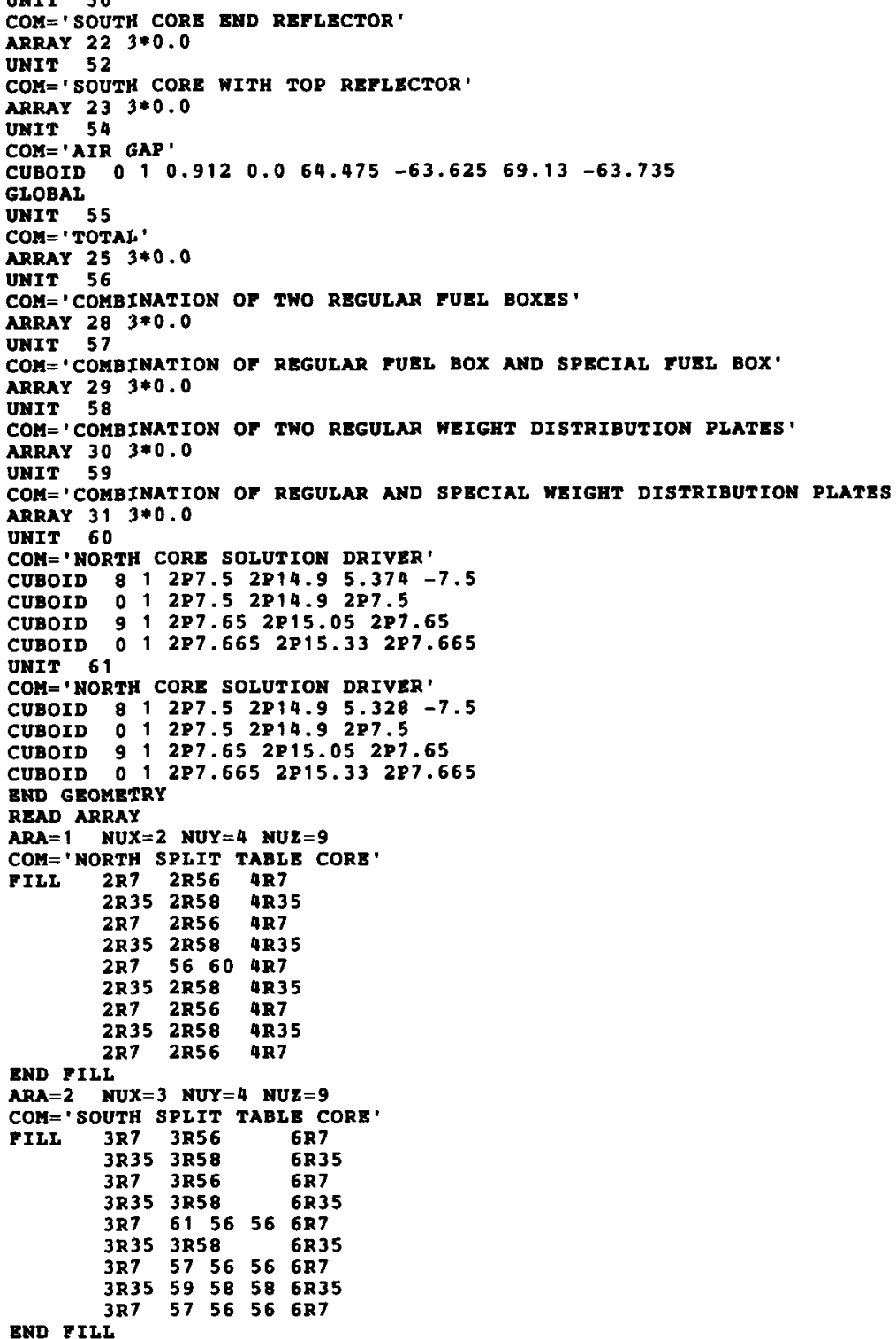




\section{Table A.3 (continued)}

ARA $=3 \quad$ NUX $=1 \quad$ NUY $=1 \quad$ NUZ $=3$

COE= NORTH BOTTOM REFLECTOR

$\begin{array}{lllll}\text { FILL } 14 & 11 & 13 & \text { END FILI } \\ \text { ARA }=4 & \text { NUX }=1 & \text { NUY }=1 & \text { NUZ }=7\end{array}$

COM=' SOUTH BOTTOM REFLECTOR'

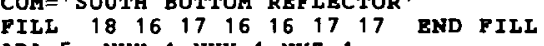

COM ' BAST AND WEST WALLS OF NORTH REPLECTOR.

ARA $=6$ NUX $=1$ NUY $=1$ NUZ $=1$ SOUTH REPLECTOR

COM= BAST AND WEST

ARA $=7$
NUX $=1$ NUY $=1$ NUZ $=1$

COM $=$ ' ARRAY FOR NORTH TOP REFLECTOR'

FILL 24 END FILL

COM=' ARRAY FOR SOUTH TOP REFLECTOR

FILL 26 END FILL

ARA $=9 \quad \mathrm{NUX}=3 \quad \mathrm{NUY}=1 \quad \mathrm{NUZ}=1$

COM=' ARRAY POR NORTH END REFLECTOR

FILL $28 \quad 29 \quad 30$ BND FILI

ARA =

FILL 32 END FILL

$A R A=13 \quad$ NUX $=1 \quad$ NUY $=1$ NUZ $=2$

COH= COMBINATION OF NORTH CORE WITH BOTTOM REFLECTOR

FILI 159 END FILL

ARA $=14$ NUX $=1$ NUY $=3$ NUZ $=1$
COM $=$ ' NORTH CORE WITH SIDE REPLECTORS '

FILI 213821 TND IILI

ARA $=15$ NUX $=2$ NUY $=1$ NUZ $=1$

COM= ' NORTH CORE WITH END REPLECTOR'

FILL $3139 \quad$ END PILL

COM $=$ ' NORTH CORE WITH TOP REFLECTOR'

COM $=$ 'NORT CORE WITH TO

ARA $=20 \quad$ NUX $=1$ NUY $=1$ NUZ $=2$

COM = ' SOUTH CORE WITH BOTTOM REFLECTOR'

FILL 1910 END PILL

ARA $=21$ NUX $=1$ NUY $=3$ NUZ $=1$

COK=' SOUTH CORE WITH EAST WEST REPLECTORS.

PILI $23 \quad 48 \quad 23$ END PILI
$A R A=22$ NUX $=2$ NUY $=1$ NUZ $=1$

COM='SOUTH CORE WITH RND REFLECTOR'

PILL 4933 BND PILL

ARA $=23$ NUX $=1 \quad$ NUY $=1 \quad$ NUZ $=2$

COM=' COMBINATION OF CORE WITH TOP REFLECTOR'

FILI 5027 END FILL

$A R A=25$ NOX

PILL 425452 END FILL

$A R A=26 \quad N U X=3 \quad N U Y=1 \quad N U Z=1$

COM='ASSEMBLY OF FUEL BOX PRONT AND REAR'

FILI 213 BND FILL

ARA $=27$ NUX $=1$ NUY $=1$ NUZ $=3$
COM='ASSEMBLY OF PUEL BOX TOP AND BOTTON'

COM= ASSEMBLY OF PUEL

FRA $=28$ NUX $=1$ NUY $=2$ NUI $=1$

COM=' COMBINATION OF TWO FUEL BOXES'

FILL 77 END FIL

$A R A=29 \quad N U X=1 \quad N U Y=2 \quad N U Z=1$

PBCIAL AND REgULAR FUBL BOXES

CIII COMBINATION OF
$A R A=30 \quad N U X=1 \quad N U Y=2 \quad N U Z=1$

COM=' COMBINATION OP TWO WEIGHT DISTRIBUTION PLATES'

FILL 3535 BND FILL

COM=' COMBINATION OF SPECIAL AND REGULAR WEIGHT DISTRIBUTION PLATES PILL $35 \quad 34$ END FILL

READ PLOT TTL='YZ SLICE OF SOUTH CORE FIRST ROF

$X U L=64.319$ YUL $=40.000 \quad 2 U L=72.000$

VAX $=1$ WDN $=-1$ NAX $=130 \quad$ NCH $=012345678$, RND

TTL $=$ ' $X Z$ SLICE OF SPHERE CUBOID

$X U L=56.0 \quad Y U L=63.625 \quad \mathrm{ZUL}=71.735$

UAX $=1$ WDN $=-1$ NAX $=130$ NCH $=1012345678^{\circ}$

END PLOT

作

ZSM $=56.071 \quad$ ZSP $=71.4$ END START

BND DATA

CAROS

CAROY

ROCKY FLATS CRITICALS NURBG/CR-0674 EXPERIMENT NUMBER 2 (27 GROUP MODEI PUEL CUBE $15.28 \mathrm{CM}, \mathrm{U}(4.46) 308$ \& $\mathrm{H}=0.77$, LOW CONC. SOLUTION DRIVEN

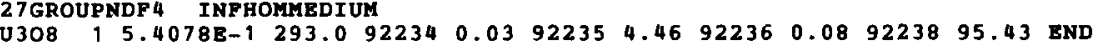

$1.9514 E-2$
H2O END

END 1.0300213027 99.18 $260000.5290000 .229 .5390 \mathrm{~B}-1$

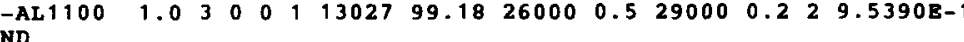

ARBM-TAPE (VINYL) $\quad 1.07000010015 .92601245 .918016 \quad 10.8217000$

$25.73200406 .9220001 .6820001 .12211115 \mathrm{E}-2$ END 27.02

ARBE-TAPEIMYLARI

ARBM-MODERATOR $1.18530000010017 .83 \quad 601259.49 \quad 8016 \quad 32.48 \quad 3$ END

ARBM-PLEX(REG) $\quad 1.03000010017 .84601259 .598016 \quad 32.234$

1.1773 BND

00010016.48601242 .17801649 .54 $3.7534 \mathrm{E}-3$ END

1.03000100111 .67601285 .2980161 .204

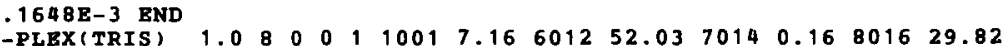

$5031 \quad 1.0217000^{\circ} 1.81350794 .260350812 .84051 .2757$ END

ARBM-PLEX(PAPER) $1.03000010016 .486012 \quad 42.17801649 .55$ $3.7534 \mathrm{E}-3$ END

ARBM-PLEX(GLUE) $1.0300001001 \quad 11.67601286 .2980161 .205$

$\begin{array}{lllllllllllllll}\text { ARBM-FILLER } & 1.185 & 3 & 0 & 0 & 0 & 1001 & 7.83 & 6012 & 59.49 & 8016 & 32.48 & 6 & 0.7 & \mathrm{END}\end{array}$

I THIS MATERIAL IS ACTUALLY MODERATOR ADJUSTED POR THE VOLUME

FRACTION OF THE VOID PILLED BETWEEN CORE AND REFLECTOR.

ARBM-AL $1100 \quad 1.0 \quad 300011302799.1826000 \quad 0.5 \quad 290000.272 .715$ END

SOLNUO2(NO3) $2 \quad 8 \quad 86.42 \quad 0.149 \quad 1.0293 .092234 \quad 1.02292235 \quad 93.172$

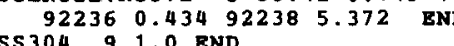

ROCKY FLATS CRITICALS NUREG/CR-0674 EXPERIMENT NUMBER 2 (27 GROUP MODEL) - FUEL CUBE $15.28 \mathrm{CM}, \mathrm{U}(4.46) 308 \mathrm{H} / \mathrm{U}=0.77$, LOW CONC. SOLUTION DRIVEN READ PARM 
Table A.3 (continued)

UNIT 1

COM='FUEL BOX WITH VOID AND PART OF AL BOX'

$\begin{array}{lllllllll}\text { CUBOID } & 0 & 1 & 9.80 & 0.0 & 3.75 & 0.0 & 14.98 & 0.0 \\ \text { CUBOID } & 2 & 1 & 9.95 & 0.0 & 3.90 & -0.15 & 14.98 & 0.0\end{array}$ $\begin{array}{lllllllll}\text { CUBOID } 1 & 1 & 14.98 & 0.0 & 9.365 & -5.615 & 14.98 & 0.0\end{array}$

UNIT 2

COM='PRONT OF PUEL BOX

$\begin{array}{lllllllllll}\text { CUBOID } 2 & 1 & 0.15 & 0.0 & 5.615 & 0.0 & 14.98 & 0.0\end{array}$

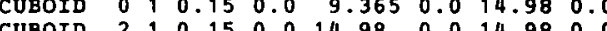

UNIT 3

COM='REAR OF PUEL BOX

$\begin{array}{llllllllll}\text { CUBOID } & 2 & 1 & 0.15 & 0.0 & 14.98 & 0.0 & 14.98 & 0.0\end{array}$

UNIT 4

COM=' ARRAY TO ASSEMBLE PART OF BOX

ARRAY 26 3*0

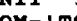

COM= TOP AND BOTTOM OF BOX'

$\begin{array}{lllrllllll}\text { CUBOID } 0 & 1 & 9.80 & 0.0 & 3.750 & 0.0 & 0.15 & 0.0\end{array}$

GLOBAI

UNIT 6
COM $=$ ARRAY TO ASSEMBLE TOP AND BOTTOM.

ARRAY $27 \quad 3 * 0$

$\begin{array}{llllllllll}\text { CUBOID } 2 & 1 & 15.28 & 0.0 & 15.13 & -0.15 & 15.28 & 0.0\end{array}$

UNIT 7

COM=' PUEL BOX $15.28 \mathrm{CM}$ ON A SIDE WITH $.15 \mathrm{CM}$ WALLS .05CM STACKING VOID

CUBOID 119687.49

$\begin{array}{lllll}\text { CUBOID } & 2 & 1 & 6 P 7.64 \\ \text { CUBOID } & 0 & 1 & 6 P 7.6650\end{array}$

CUBOID

IT 34

COM='ALUMINUM WEIGHT DISTRIBUTION PLATE POR SPECIAL BOX

CUBOID $00199.80 \quad 0.0 \quad 3.750 \quad 0.0 \quad 0.160 .0$

CUBIT 35

COM=' ALUMINUM WEIGHT DISTRIBUTION PLATE FOR BOXES

CUBOID 71 4P7.665 0.160 .0

UNIT 9

COM=' NORTH SPLIT TABLE CORE'

CUBOID $0130.66-0.94 \quad 77.5 \quad 0.0833475 \quad 0.0$

COM=' SOUTH SPLIT TABLE CORE'

ARRAY $23 * 0.0$

CUBOID $0149.4 \quad 0.0 \quad 77.5 \quad 0.0 \quad 83.475 \quad 0.0$

COM='PLEXIGLASS REFLECTOR SHEET WITHOUT TRIS, NORTH BOTTOM REPLBCTOR CUBOID 412 P15.8000 2P38.7500 2P0.6150

CUBOID 12

作

CUBOID 512 P15.8000 2P38.7500 2P0.6150

UNIT 13 COM = UPPER PORTION NORTH BOTTOM REFIECTOR WITH TRIS CUBOID 512 P15.8 2 P38.75 2P8.24

(

CUBOID 5 T2P15.8 2P38.75 2P3.69

UNIT 15 COM='NORTH BOTTOM REFLECTOR INCLUDES REGULAR AND TRIS ARRAY $3 \quad 3 * 0.0$
UNIT 16

COM-' PLEXIGLAS SHEET BOTTOM SOUTH REPLECTOR WITHOUT TRIS

CUBOID 0 1 2 2P5.1 2 P2.5 2 P0.6150

CUBOID $4144.3-5.12 \mathrm{P} 38.75 \quad 2 \mathrm{PO} 0.615$

UNIT 17 7 TOM='PLBXIGLAS SHEET BOTTOM SOUTH REFLECTOR WITH TRIS

CUBOID 0 1 2 2P5.1 2 P2.5 2 P0.6150

UNIT 18 .

CUBOID 0112 P5.1 2 P2.5 2 P8.855

CUBOID 5 1 $44.3 \quad-5.12$ 2P38.75 2 P8.85

UNIT 19

COM=' SOUTH BOTTOM REFLECTOR WITH REGULAR AND TRIS'

ARRAY 4 U*0.

COM = BAST AND WEST REFLECTORS POR NORTH REPLECTOR WITH TRIS CUBOID 512 P15.8 2P12.65 2P54.2825

UNIT 21
COM='ARRAY FOR EAST AND WEST REFLECTORS FOR NORTH REPLECTOR ARRAY 5 , $3 * 0.0$

COM='BAST AND WEST REPLBCTORS POR SOUTH REFLECTOR WITH TRIS' CUBOID 512 P24.700 2P12.65 2P54.2825

CONT 23

COM='ARRAY FOR EAST AND WEST REFLECTORS FOR SOUTH REFLECTOR ARRAY $6 \quad 3 * 0.0$

UNIT 24

COM=' NORTH TOP REFLECTOR WITH TRIS'

$\begin{array}{lllll}\text { CUBOID } 5 & 1 & 2 \mathrm{P} 28.35 & 2 \mathrm{P} 64.05 & 2 \mathrm{P} 12.1\end{array}$

COMI ' ARRAY FOR NORTH TOP REPLECTOR'

ARRAY $73 * 0.0$

UNIT 26

COM=' SOUTH TOP REFLECTOR WITH TRIS

CUBOID 0 1 2P5.1 2P2.5 2 P12.15

CUBOID $5169.4-5.12864 .05$ 2P12.15

UNIT 27

COM='ARRAY FOR SOUTH TOP REPLBCTOR'

ARRAY $83 * 0.0$

9.8CM PORTION WITHOUT TRIS

CUBOID 412 2P4.9000 2P64.0500 2P54.2825

UNIT 29 . 19 RN

COM=' NORTH END RELPBCTOR $52^{2}$ CM PORTION
CUBOID 5,2 2.6 2 P64.0500 2 P54.2825

UNIT 30

COM=' NORTH BND RELFECTOR $10.1 \mathrm{CM}$ PORTION WITHOUT TRIS

CUBOID 4 1 2 P5.05 2P64.05 2P54.2825

UNIT 31 COM=1 ARRAY FOR NORTH BND REPLECTOR'

COM= ARRAY FON
ARRAY $9 \quad 3 * 0.0$

ARRAY 9
UNIT 32

COM=' SOUTH END REPLECTOR'

$\begin{array}{llll}\text { CUBOID } 5 & 12 \mathrm{P} 12.55 & 2 \mathrm{P} 64.05 & 2 \mathrm{P} 54.2825\end{array}$

COM='ARRAY FOR SOUTH END REFLECTOR'

ARRAY $10 \quad 3 * 0.0$

UNIT 38

' NORTH CORE WITH BOTTOM REFLECTOR'

ARRAY 13
UNIT 39

COM='NORTH CORE WITH BAST AND WEST REFLECTOR'

ARRAY $14 \quad 3 * 0.0$ 
Table A.3 (continued)

UNIT 40
COM= ' NORTH CORE WITH END REFLBCTOR.

ARRAY $15 \quad 3 * 0.0$

COM=' NORTH CORE

UNIT 48

SOM = SOUTH CORE WITH BOTTOM REPLECTORS.

ARRAY $20 \quad 3 * 0.0 \quad 3$

COM=' SOUTH CORE

ARRAY 21

COM = 'SOUTH CORE BND REPLECTOR'

ARRAY $22 \quad 3 * 0.0$

UNIT 52

ART TOP REFLECTOR'

ARRAY $23 \quad 3 * 0.0$

COMIT 54 G

$\begin{array}{lllllll} & \end{array}$

GLOBAL

UNIT 55

COM= TOTAL.

ARRAY 25

COM=' COMBINATION OF TWO REGULAR FUEL BOXES

ARRAY $283 * 0.0$

UNIT 57

COM-. COMBINATION OF REgULAR FURI BOX AND SPBCtAL FUBL BOX ARRAY 29 3*0.0

UNIT 59

ARRAY $30 \quad 3 * 0.0$
COME

ANIT 59

COM=' COMBINATION OF REGULAR AND SPECIAL WEIGHT DISTRIBUTION PLATES ARRAY $31 \quad 3 * 0.0$

UNIT 60

COR=

CUBOID 81 2P7.5 2P14.9 $5.499-7.5$

CUBOID 8 \& 1 2P7.5 2 P 14.95 .49

CUBOID 912 P 7.65 2P15.05 287.65

CUBOID $011227.665 \quad 2 \mathrm{P} 15.33 \quad 2 \mathrm{P} 7.66$

COH= ' NORTH CORB SOLUTION DRIVER'

CUBOID \& $112 \mathrm{PP} 7.52 \mathrm{P} 14.95 .445-7.5$

CUBOID $9287.52 \mathrm{P} 14.92 \mathrm{P} 7.57$

CUBOID $012 \mathrm{PP} .6652 \mathrm{P} 15.332 \mathrm{P} 7.665$

END GEOMETRY

READ ARRAY

$A R A=1 \quad N U X=2 \quad N U Y=4 \quad N U Z=9$

COM=' NORTH SPIIT TABLE CORE'

2R7 $2 \mathrm{R} 56 \quad 4 \mathrm{R} 7$

$2 R 35$ 2R58 $4 R 35$

$2 R 35 \quad 2 R 58 \quad 4 R 35$

$\begin{array}{llll}2 R 7 & 56 & 60 & 4 R 7\end{array}$

2R35 2R58 $\quad 4 R 35$

2R7 $2 R 56 \quad 4 R 7$

BND PILL

ARA $=2 \quad$ NUX $=3 \quad$ NUY $=4 \quad$ NUZ $=9$
COM= ' SOUTH SPLIT TABLE CORE

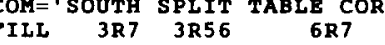

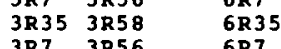

$3 \mathrm{R} 35 \quad 3 \mathrm{R} 58 \quad 6 \mathrm{R} 35$

$\begin{array}{lllll}3 R 7 & 61 & 56 & 56 & 6 R 7\end{array}$

$3 R 35$ 3R58 $6 R 35$

3R7 57 56 $56 \quad 6 R 7$

END FILI $\begin{array}{lllll} & \\ & & 0\end{array}$

ARA $=3$ NUX $=1$ NUY $=1$ NUZ $=3$

COM= NORTH BOTTOM REPLECTOR'

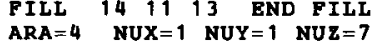

COM=' SOUTH BOTTOM REFLECTOR'

$\begin{array}{llllllllll}\text { FILL } 18 & 16 & 17 & 16 & 16 & 17 & 17 & \text { END FILL }\end{array}$

FILL 20 END FILI WALLS

ARA $=6$ NUX=1 NUY $=1 \quad$ NUZ $=1$

COM= BAST AND WEST

ARA 7 NUX $=1$ NUY $=1$ NUZ $=1$

COM=' ARRAY FOR NORTH TOP REFLECTOR'

FILL 24 END EILI

ARA $=8$ NUX $=1 \quad$ NUY $=1 \quad$ NUZ $=1$

COM=' ARRAY FOR SOUTH TOP REFLECTO '

PILL 26 END FILL

CARA=9 NOX $=3$ NUYT=1 NOZ=1

FILL 2829 R

ARA $=10$, $29 \mathrm{X}=1$ NUY $=1$ NUZ

作

FILL 32 END FILL

$A R A=13$ NUX $=1 \quad$ NUY $=1 \quad$ NUZ $=2$

COM=' COMBINATION OF NORTH CORE WITH BOTTOM REPLECTOR'

FILL 159 END FILI

COM=1 NOPTH

PILL $21 \quad 38 \quad 21$ END FILI

$A R A=15$ NUX $=2$ NUY $=1 \quad$ NUZ $=1$

COM='NORTH CORE WITH END REFLECTOR'

FILL 3139 END FILL

ARA $=16$ NUX $=1$ NOY $=1$ NUZ $=2$

PIII $40 \quad 25$ BND PILT

$A R A=20$ NUX $=1 \quad$ NUY $=1$ NUZ $=2$

COM-'SOUTH CORE WITH BOTTOM REFLECTOR'

FILL 1910 END FILL

ARA $=21 \quad$ NUX $=1 \quad$ NUY $=3 \quad$ NUZ $=1$

COM='SOUTH CORE WITH BAST WEST REPLECTORS'

PILL 234823 BND PILL

COM='SOUTH CORE WITH END REFLECTOR

FILL 4933 END FILL

ARA $=23 \quad$ NUX $=1 \quad$ NUY $=1 \quad$ NUZ $=2$

COM=' COMBINATION OF CORE WITH TOP REPLECTOR'

PILL 5027 END PILL

$\triangle R A=25 \quad N U X$

COML 425452 END FILL 
Table A.3 (continued)

ARA $=26 \quad N U X=3 \quad N U Y=1 \quad N U Z=1$

COM=' ASSEMBLY OF PUEL BOX FRONT AND REAR'

PILL 2 13 END PILL

ARA $=27 \quad$ NUX $=1 \quad$ NUY $=1 \quad$ NUZ $=3$

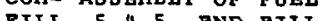

ARA $=28$ NUX $=1$ ENDY $=2$ NUZ

COM= COMBINATION OP TWO PUEL BOXES

FILL 77 END FILI

$A R A=29$ NOX $=1$ NUY $=2$ NUZ $=1$

COM=' COMBINATION OF SPECIAL AND REGULAR FUBL BOXES'

FILL 76 END FILI

$=1$ TRTH DISTRIBUTTOM PLATES

FILL $35 \quad 35$ END FILI

ARA $=31 \quad$ NUX $=1 \quad$ NUY $=2 \quad$ NUZ $=1$

COM=' COMBINATION OF SPECIAL AND REGULAR WEIGHT DISTRIBUTION PLATES'

PILL 3534 BND FILL

READ PLOT TTL='YZ SLICE OF SOUTH CORE FIRST ROW

$X \mathrm{XUL}=64.319 \quad \mathrm{YUL}=40.000 \quad \mathrm{ZUL}=72.000$

VAX $=1$ WDN $=-1 \quad$ NAX $=130 \quad \mathrm{NCH}=1012345678 \cdot \mathrm{BND}$

TTL $=\cdot X Z$ SIICE OF SPHERE CUBOID

$X U L=56.0 \quad Y U L=63.625 \quad Z U L=71.735$

UAX $=1$ WDN $=-1$ NAX $=130$ NCH $=.012345678$.

END PLOT

READ START NST $=1 \quad$ XSM=41.37 XSP $=72.894 \quad$ YSM $=40.63 \quad$ YSP $=71.29$ ZSM $=56.071 \quad$ ZSP $=71.4$ BND START

END DATA

\section{CAR10}

ROCKY FLATS CRITICALS NUREG/CR-0674 CONCRETE REFLECTED (27 GROUP MODEL) - FUEL CUBE $15.28 \mathrm{CM}, \mathrm{U}(4.46) 308 \mathrm{H} / \mathrm{U}=0.77$, HI CONC. SOLUTION DRIVBN

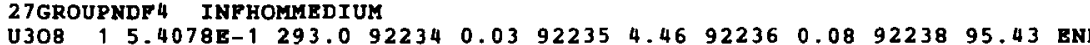

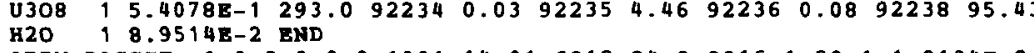
ARBM-BAGGIE $1.0300001001 \quad 14.01601284 .98016 \quad 1.20 \quad 1 \quad 1.9134 \mathrm{E}-2$ END $1100 \quad 1.030001,1302799.18 \quad 26000 \quad 0.5 \quad 29000 \quad 0.2229 .5390 \mathrm{~B}-1$ ARBM-TAPE(VINYL) $\quad 1.070000010015 .92601245 .91801610 .8217000$

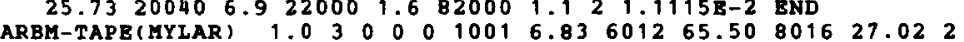
ARBH-TAPB(MYLAR) $\begin{array}{llllllllllllll}1 & & & \end{array}$ RECONCRETE 5 1.0

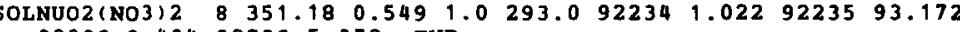
$92236 \quad 0.434922385 .372$ BND

55304991.0 END

ROCKY FLATS CRITICALS NUREG/CR-0674 CONCRETE REFLECTED (27 GROUP MODEL) 1. FUEL CUBE $15.28 \mathrm{CM} U(4,46) 308$ H $/ U=0.77$, HI CONC. SOLUTION DRIVEN READ PARM NPG $=600$ NUB $=$ Y 4 S PDN=YES PLT $=$ NO READ GBOM

UNIT 1

COM=' FUEL BOX WITH VOID AND PART OF AL BOX'

CUBOID $0 \begin{array}{lllllll} & 9.80 & 0.0 & 3.75 & 0.0 & 14.98 & 0.0\end{array}$

$\begin{array}{lllllllll}\text { CUBOID } & 2 & 1 & 9.95 & 0.0 & 3.90 & 0.0 .15 & 14.98 & 0.0 \\ \text { CUBOID } & 1 & 1 & 14.98 & 0.0 & 9.365 & -5.615 & 14.98 & 0.0\end{array}$
UNIT 2

COM=' FRONT OF FUBL BOX

CUBOID $2 \begin{array}{llllllll}2 & 0.15 & 0.0 & 5.615 & 0.0 & 14.98 & 0.0\end{array}$

$\begin{array}{llllllllll}\text { CUBOID } & 1 & 0.15 & 0.0 & 9.365 & 0.0 & 14.98 & 0.0\end{array}$

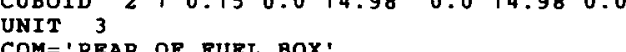

$\begin{array}{llllllllll}\text { CUBOID } & 2 & 1 & 0.15 & 0.0 & 14.98 & 0.0 & 14.98 & 0.0\end{array}$

UNIT 4

COM='ARRAY TO ASSEMBLE PART OF BOX.

ARRAY $26 \quad 3 * 0$.

COM $=$ TOP AND BOTTOM OP BOX

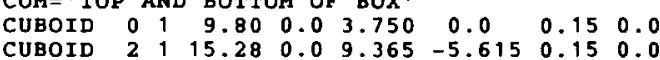

GLOBAL

COM='ARRAY TO ASSEMBLE TOP AND BOTTOM

ARRAY $27 \quad 3 * 0$

$\begin{array}{llllllllll}\text { CUBOID } & 2 & 1 & 15.28 & 0.0 & 15.13 & -0.15 & 15.28 & 0.0 \\ \text { CUBOID } & 0 & 1 & 15.305 & -0.025 & 15.155 & -0.175 & 15.305 & -0.025\end{array}$

UNIT 7

COM='FUEL BOX 15.28 CM ON A SIDE WITH .15 CM WALLS .05CM STACKING VOID'

CUBOID 1 1 16 6P7.49

$\begin{array}{llll}\text { CUBOID } & 2 & 1 & 6 P 7.64 \\ \text { CUBOID } & 0 & 1 & 6 P 7.6650\end{array}$

UNIT 34

COM='ALUMINUM WEIGHT DISTRIBUTION PLATE FOR SPECIAL BOX'

CUBOID $019.80 \quad 0.0 \quad 3.750 \quad 0.0 \quad 0.160 .0$

CUBOID $71115.33 \quad 0.0 \quad 9.54 \quad-5.79 \quad 0.16 \quad 0.0$

UNIT 35

COM=' ALUMINUM WEIGHT DISTRIBUTION PLATE POR BOXES

CUBOID 714 4P7.665 $0.16 \quad 0.0$

COM='NORTH SPLIT TABLE CORE AND REFLECTOR'

ARRAY $1-30.660 .000 .0$

CUBOID 0 1 $0.0-32.3 \quad 77.5 \quad 0.083 .2 \quad 0.0$

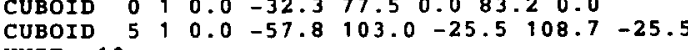

UNIT 10

COM='SOUTH SPLIT TABLE CORE AND REFLECTOR'

ARRAY $23 * 0: 0$

CUBOID $001.47 .5 \quad 0.0 \quad 77.5 \quad 0.083 .20 .0$

HOLE

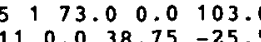

$\begin{array}{llll}11 & 0.0 & 38.75 & 83.2\end{array}$

UNIT 11

CUBOID $0110.2 \quad 0.0282 .5525 .5 \quad 0.0$

UNIT 54 GAP

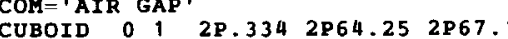

GLOBAL

UNIT 55

COM= ' TOTAL '

ARRAY $25 \quad 3 * 0.0$

UNIT 56

COM=' COMBINATION OF TWO REGULAR FUBL BOXES

ARRAY 28
UNIT 57

COM=' COMBINATION OF REGULAR FUEL BOX AND SPECIAL PUBL BOX ARRAY $29 \quad 3 * 0.0$

UNIT 58 COM= ' COMBINATION OP TWO REGULAR WEIGHT DISTRIBUTION PLATES'

COM= ' COMBINATION 
Table A.3 (continued)

UNIT 59

COM= COMBINATION OF REGULAR AND SPECIAL WEIGHT DISTRIBUTION PLATES ARRAY $313 * 0.0$

UNIT 60

COM=' NORTH CORE SOLUTION DRTVER'

$\begin{array}{llllll}8 & 1 & 2 P 7.5 & 2 P 14.9 & 3.122 & -7.5\end{array}$

CUBOID $01287.52 P 14.9287 .5$

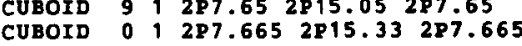

UNIT 61

COM=' SOUTH CORE SOLUTION DRIVER'

CUBOID 811 2P7.5 2P14.9 $3.047-7.5$

CUBOID $1287.552 P 15.05787$

CUBOID O 1 2P7.655 $2815.33 \quad 287.665$

END GEOMETRY

$\underset{A R A=1}{\text { RRAD NUX }=2 \text { NUY }=4 \text { NUY }=9}$

COM=' NORTH SPLIT TABLS COPE'

FILL $2 R 7$ 2R56 $4 R 7$

2R35 2R58 4R3

2R7 2856 4R7

2R7 $56 \quad 60 \quad 4 R 7$

2R35 2R58 4R3

$\begin{array}{lll}2 R 7 & 2 R 56 & 4 R 7 \\ 2 R 35 & 2 R 58 & 4 R 3 \\ 2 R 7 & 2 R 56 & 4 R 7\end{array}$

BND FILI

NRA $=2$ NUX $=3$ NUY $=4$ NUE $=9$

COM='SOUTH SPLIT TABLE CORE'

$3 R 7 \quad 3 R 56 \quad$ 6R7

$\begin{array}{lll}3 R 35 & 3 R 58 & 6 R 35 \\ 3 R 7 & 3 R 56 & \text { 6R7 }\end{array}$

$\begin{array}{lll}3 R 7 & 3 R 56 & 6 R 7 \\ 3 R 35 & 3 R 58 & 6 R 35\end{array}$

$3 R 7 \quad 6156 \quad 56 \quad 6 R 7$

$3 R 353 R 58 \quad 6 R 35$

$\begin{array}{lllll}3 R 7 & 57 & 56 & 56 & 6 R 7 \\ 3 R 35 & 59 & 58 & 58 & 6 R 35\end{array}$

$\begin{array}{lllll}3 R 35 & 59 & 58 & 58 & 6 R 35 \\ 3 R 7 & 57 & 56 & 56 & 6 R 7\end{array}$

\section{BND FILI}

NUXX $=3$ NUY $=1$ nUz $=1$

PILL $954 \quad 10$ END FILL

ARA $=26$
NUX $=3$
COM NUY $=1$ NUZ

COM= ASSEMBLY OF FURI

$A R A=27 \quad$ NUX $=1 \quad$ NUY $=1$ NUZ $=3$

COM= ASSEKBLY OF FUEL

FILL 5 4 5 BND FILL

COM $=$ ' COMBINATION OF TWO FUEL BOXES'

FILI 77 RND FILL

ARA $=29$ NUX $=1$ NUY $=2$ NUE $=1$
COM=. COMBINATION OF SPECIAL AND REGULAR FUEL BOXES

PILL 7 -

ARA $=30^{7}$ NUX $=1$ NUY PILL NUZ $=1$

COM=' COMBINATION OF THO WEIGHT DISTRIBUTION PLATES'

FILI. $35 \quad 35$ END FILL

ARA $=31$ NUX $=1$ NUY $=2$ NUE $=1$
COM=' COMBINATION OF SPECIAL AND REGULAR WEIGHT DISTRIBUTION PLATES FILL $35 \quad 34$ END FILI
READ PLOT TTL='YZ SLICE OF SOUTH CORE FIRST ROW'

$X U L=64.319$ YUL $=40.000 \quad \& U L=72.000$

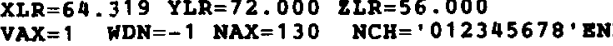

TTL $=$ 'XZ SLICE OF SPHERE CUBOID

$X U L=56.0$ YUL $=63.625$ SUL $=71.735$

UAX $=1$ WDN $=-1$ NAX $=130$ NCH $=.012345678^{\prime}$

END PLOT

START NST $=1 \quad X S M=15.0 \quad X S P=122.0 \quad$ YSM $=15.0 \quad$ YSP $=122.0$

ZSM $=15.0 \quad$ ZSP $=122.0$ END START

END DATA

CAR11

ROCKY FLATS CRITICAIS NUREG/CR-1653 EXPERIMENT A ( 27 GROUP)

48 PUEL CANS 2.44 CM

(1 5 4078E-1 293 H2O $11.5627 \mathrm{~B}-1$ END

ARBM-BAGGIE $1.0 \quad 3 \quad 00001001 \quad 14.01601284 .98016 \quad 1.2011 .91348-2$

$\begin{array}{lllllllllllll}\text { END } & & 13027 & 99.18 & 26000 & 0.5 & 29000 & 0.2 & 2 & 9.53908-1\end{array}$

ARBM-AL 11

BND

$25.73200406 .9220001 .6820001 .121 .1115 \mathrm{~B}-2$ SND

ARBM- $\pi$ :FE(MYLAR) $1.03000010016 .83601265 .50 \quad 8016 \quad 27.02 \quad 2$

$1.74918-2$ END
ARBM-MODERATOR 1.18530

ARBY-PLEX(REG) 1.03000010017 .84601259 .59801632 .23

ARBM-PLEX (PAPER) 1.03000010016 .48601242 .17801649 .54

3.7534E-3 END
ARBM-PLEX (GLUE) $1.0 \quad 3 \quad 0 \quad 0 \quad 0 \quad 1001 \quad 11.67 \quad 601286.298016 \quad 1.204$

$1.1648 E-3$ END
ARBX-PLEX(TRIS) $1.08000010017 .16 \quad 601252.03 \quad 70140.16801629 .82$

$150311.02 \quad 170001.81350794 .260350812 .8405 \quad 1.2757$ END

ARBA-PLSX (PABSR)

ARBY

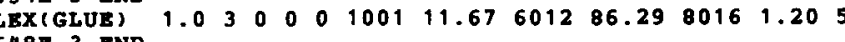

ARBM-FILLER $1.1853000001001 \quad 7.836012 \quad 59.49801632 .486 .888$ END

PRACTION OF THE VOID FILLED BETWEEN CORE AND REFLECTOR.

SND COMP

$19.946218 .5857 \quad 1 \quad 3 \quad 18.95792$ END

(2) GROUP)

- 48 FUEL CANS 2.44 CM MODERATOR GEE. HU125. DATAIOPT

READ PARM RUN=YES NPG $=600 \quad$ PDN=YES NUB $=$ YES PLT=NO END PARM READ GBOM

UNIT 1

'PUEL BOX 15.28 CM ON A SIDE WITH $.15 \mathrm{CM}$ WALLS .05CM STACKING VOID' CUBOID 111677.49

CUBOID 016 T 67.6650

UNIT 2

COM='X-PACB INTERSTITIAI MODERATOR'

CUBOID 3 T 2 P1.2200 487.665 
Table A.3 (continued)

UNIT 3

COM='Y-FACE INTERSTITIAL MODERATOR'

CUBOID 31 2P7.665 2P1.2200 2P7.665

CON= $=2$-FACE INTERSTITIAL, MODERATOR

CUBOID 314 4P7.665 2P1.2200

UNIT 5

CUBOID 3 X-FACE MODERATOR.

UNIT 6

COM=' HORE Y-FACE MODERATOR'

CUBOID 31287.665 4P1.220

UNIT 7

COM= MORE Z-FACE MODERATOR

$6652 P 1.2200$

IT

COY ='LAST OF INTERSTITIAL YODERATOR'

CUBOID 316 P1.2200

UNIT 9

ARRAY $13 * 0.0$

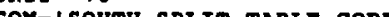

Coray $23 * 0$.

ARRAY 2 UTIT

COM=' PLEXIGLASS REFIECTOR SHEET MITHOUT TRIS, NORTH BOTTOM REFLECTOR'

CUBOID 412816.55002838 .7500 2PO.6150

UNIT 12

COM='PLEXIGLASS REFLECTOR SHEET WITH TRIS, NORTH BOTTOM REFLETOR

CUBOID $512 P 16.55002 P 38.7500 \quad 2 P 0.6150$

COY='UPPER PORTION NORTH BOTTOM REPLECTOR WITH TRIS'

CUBOID $512816.550 \quad 2838.75 \quad 288.24$

UNIT 14 14 PORTION

COM=' LOWER PORTION NORTH BOTTOM REFLECTOR WITH TRIS

CUBOID 512 P16.550 2P38.75 2P3.69

COM='NORTH BOTTOM REFLECTOR INCLUDES REGULAR AND TRIS

ARRAY $3 \quad 3 * 0.0$

UNIT 16

COM='PLEXIGLAS SHEET BOTTOM SOUTH REPLECTOR WITHOUT TRIS'

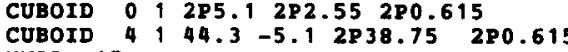

COM= P PEXIGLAS SHEET BOTTOK SOUTH RETLECTOR WTTH TRIS

CUBOID 0 1 2 P5 12 2P2.55 280.615

75280.6150

COM='LOWER PORTION SOUTH BOTTOM REFLECTOR WITH TRIS'

CUBOID 012 P5.1 2P2.55 2P8.855

CUBOID 5 1 $44.3-5.12$ P38.75 2 P8.855

UNIT 19

ARRAY $4 \quad 3 * 0$.

UNIT 20

COM='EAST AND WEST REPLECTORS FOR NORTH RETLECTOR WITH TRIS'

CUBOID

CUPT 2 2P16.550 2P12.65 2P54.2825

COM='ARRAY YOR EAST AND WEST REFLECTORS FOR NORTH REFLECTOR' ARRAY 5 3*0.0

COY = CUBOID 512 P24.700 2P12.65 2P54.2825
UNIT 23 COY A ARRAY TOR EAST AND WEST REFLECTORS POR SOUTH REPLECTOR ARRAY $6 \quad 3 * 0.0$

UNIT 24

COM=' NORTH TOP REFLECTOR WITH TRIS CUBOID 5,2 P29.100 2P64.05 2P12.15 UNIT 25

TOR NORTH TOP REFLECTOR.

ARRAY $73 \% 0.0$

COM='SOUTH TOP REPLECTOR WITH TRIS.

CUBOID 012 2P5.1 2P2.55 2P12.15 CUBOID $5169.4-5.12 P 64.05$ 2P12.15

UNIT 27

COM= ' ARRAY POR SOUTH TOP REPLECTOR'

ARRAY $83 * 0.0$

COM=' NORTH END REFLECTOR $9.8 \mathrm{CM}$ PORTION WITHOUT TRIS

CUBOID 1 2P4.9000 2P64.0500 2P54.2825

UNIT 2 ' NORTH END RELFECTOR 5.2 CM PORTION WITH TRIS'

CUBOID 512 2P2.6 2P64.0500 2P54.2825

COM='NORTH END RELPECTOR 10.1 CM PORTION WITHOUT TRIS'

CUBOID 412 P5.05 2P64.05 2P54.2825

UNIT 31

COR=' ARRAY FOR NORTH END REFIECTOR'

ARRAY $9 \quad 3 * 0.0$

UNIT 32

CUBOID $512812.552864 .05 \quad 2854.2825$

UNIT 33

COM='ARRAY FOR
ARRAY $10 \quad 3 * 0.0$

UNIT 34

COM='BOTTOM MODERATING PLASTIC NORTH CORE'

$\begin{array}{lll} & \end{array}$

COH=' TOP MODERATING PLASTIC NORTH CORE'

CUBOID 612 2P16.550 2P38.7500 2P3.2525

UNIT 36

ARRAY $113 * 0.0$

URRAY 12 (3)

ARRAY $12 \quad 3 * 0.0$

COM ='NORTH CORE WITH BOTTOM REPLECTOR'

ARRAY $13 \quad 3 * 0.0$

UNIT 39

COM=' NORTH CORE WITH EAST AND WEST REFLECTOR'

ARRAY $14 \quad 3 * 0.0$

UNIT 40

COM=' NORTH CORE WITH END REPLECTOR'

ARRAY $15 \quad 3 * 0.0$

ANIT

COM=

CUBOID $3+2 \mathrm{PO} .6150$ 2P64.0500 2P66.4325

UNIT 42

COF=' NORTH CORE WITH TOP REFLECTOR'

ARRAY $16 \quad 3 * 0.0$

COM= ' YORTH CORE WITH FACEPLATE.

ARRAY $97,3 * 0.0$ 
Table A.3 (continued)

UNIT 44

COM='12.95 CM THYCK MODRRATOR SOUTH CORE'

CUBOID 612 2P23.924 2P6.475 2P40.183

UNIT 45

COM $=22.95$ THICK MODERATOR SOUTH CORE'

UNTT 46 2823.924

CON=' COMBINATION

ARRAY 18
UNIT 47

OP CORE WITH 2.95 THICK MODERATOR

ARRAY $19 \quad 3 * 0.0$

COM='SOUTH CORE WITH EAST WEST REFLECTORS'

ARRAY $20 \quad 3 * 0.0$

UNIT 49

COM='SOUTH CORE WITH EAST WBST RETLECTORS'

ARRAY $213 * 0.0$

UNIT 50

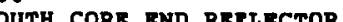

ARRAY $22 \quad 3 * 0.0$

COM= $I$ FACEPLATE FOR SOUTH SPLIT TABLE

CUBOID 312 2P0.4620 2P64.0500 2P66.4325

UNIT 52

COM $=$ - SOUTH CORE WITH TOP REFLECTOR

ARRAY $233 * 0.0$

COM='SOUTH CORE WITH YACEPLATE'

ARRAY $243 * 0.0$

UNIT 54

$\begin{array}{llll} & \\ \text { CUBOID } 01 & 12 P 0.1550 & 2 P 64.0500 & 2 P 66.4325\end{array}$

GLOBAL

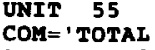

ARRAY $25 \quad 3 * 0.0$

UNIT 56

COM=' BMPTY FUEL LOCATION

CUBOID 01687.6650

COM 2510 MODERATOR,

CUBOID 512 2P16.550 2P4.4300 2P25.4350

UNIT 58

CUBOID 612 2PB.1500 2P38.7500 2P25.4350

UNIT 59

COM='SOUTH CORE BOTTOK MODERATING PLASTIC'

UNIT 60

COM='SOUTH CORE TOP MODERATING PLASTIC

CUBOID $6,12 \mathrm{P24} .7000 \quad 2 \mathrm{P} 38.7500 \quad 2 \mathrm{P} 3.2525$

UNIT 61

COM='Y-FACE MODERATOR VOID'

CUBOID $0 \quad 12 P 7.665 \quad 281.2200 \quad 2 P 7.665$

COM $=$ 'X-FACE MODERATOR VOID'

COBOID 0 1 2P1.2200 4P7.665

BEAD

ARA $=1$ NUX $=3$ NUY

$C O M=1$ NOX $=3$ NUY $=7$ NUZ $=5$
FILL $\quad \begin{array}{llllllllll}1 & 2 & 1 & 3 & 5 & 3 & 206 & 1 & 2 & 1 \\ 4 & 7 & 4 & 6 & 8 & 6 & 206 & 4 & 7 & 4\end{array}$

1042468620647

562,6153562515153106565256 END FIL

$A R A=2 \quad N U X=3 \quad N U Y=7 \quad N U Z=5$

COM=' SOUTH SPLIT TABLE CORB'

$\begin{array}{llllllllll}1 & 2 & 1 & 3 & 5 & 3 & 206 & 1 & 2 & 1 \\ 4 & 7 & 4 & 6 & 8 & 6 & 206 & 4 & 7 & 4\end{array}$

$\begin{array}{lllllll}2 & 56 & 3 & 5 & 61 & 206 & 56\end{array}$

$A R A=3$ NOX

FILL $14 \quad 11 \quad 13$ BND PILI

ARA $=4 \quad$ NUX $=1 \quad$ NUY $=1 \quad$ NUZ $=7$

COM=' SOUTH BOTTOH REFLECTOR

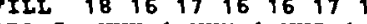

WFIS NORTH REFLECTOR'

ARA $=6$ NUX=1 NUY $=1$ NUZ=1

COM= BAST AND WEST

$A R A=7$,

COM= ' ARRAY FOR NORTH TOP REPLECTOR'

FILL 24 END FILI

COM=' ARRAY FOR SOUTH TOP REFLECTOR'

FILL 26 BND FILL

$A R A=9$ NUX $=3$ NUY $=1 \quad \mathrm{NUZ}=1$

PME

FILL $28 \quad 2930$ END FILI

COM=' ARRAY FOR SOUTH END REFLECTOR'

FILL 32 END FILI

$A R A=11$ NUX $=1$ NUY $=2$ NUZ $=1$

COM=' COMBINARION OF CORB WITH SIDE MODERATOR'

IILL 957 END FILI

COA $=12$ NUX $=1$ NUY $=1$ NUE $=3$
COM=' COMBINATION OF PREVIOUS ARRAY WITH TOP NND BOTTOM MODERATOR'

FILL $34 \quad 36 \quad 35$ END FILL

COM=' COMBINATION OF NORTH CORE WITH BOTTOM REPLECTOR'

FILL 1537 END FILI

COM= ' NORTH CORE WITH SIDE REFLECTORS'

PILL 2138 21

$A R A=15$ NUXX $=2$ NUY $=1$ MUZ $=1$

COM=' NORTH CORE WITH END REFLECTOR'

FILL 3139 END FILL

$A R A=16 \quad N U X=1$ NUY $=1$ NUZ $=2$

COM=' NORTH CORE WITH TOP REFLECTOR'

ARA $=17$, 25 BND $=2$ FILL

COM=' NORTH CORE WITH PACEPLATE'

FILL 4241 END FILL

$A R A=18$ NUX $=1 \quad$ NUY $=2$ NUZ $=1$

COM=' COMBINATION OF $\mathrm{S}$. CORE WITH SIDS MODERATOR'

PILL $10 \quad 57$ BND FILL

COM= ' COMBINATION OF CORE WITH END MODERATOR'

FILL 4658 END FILL

ARA $=20 \quad N U X=1 \quad N U Y=1 \quad N U Z=4$

COM=' SOUTH CORE WITH BOTTOM MODERATOR AND REFLECTOR'
FILL 19594760 BND FILL 
Table A.3 (continued)

$A R A=21 \quad N U X=1 \quad N U Y=3 \quad N U Z=1$

COM=' SOUTH CORE WITH EAST WEST REFLECTORS'

PILL 234823 END FILI
ARA $=22$ NUX 22 NUY $=1$ NUE $=1$

COM='SOUTH CORE WITH END REFLECTOR'

PILL 4933 BND FILL

ARA $=23 \quad$ NUX $=1 \quad$ NUY $=1 \quad$ NUZ $=2$

COM=' COMBINATION OF CORE

FILL $50 \quad 27$ BND FILL
$A R A=24 \quad$ NUX $=2$ NUY $=1$ NUZ $=1$

ARA $=24$ NUX $=2$ NUY $=1$ NUQ $=1$
COM=' SOUTH CORB WITH FACEPLATE '

TILL 5152 END FILL

ARA $=25$ NUX $=3$

COM='TOTAI'

FILL 4354

SND ARRAY 53 END FILE

READ PLOT TTL='XZ SLICE OF RPP1 SHONING MATERIAL REGIONS

$\mathrm{XUL}=-2 \quad \mathrm{YUL}=64.05 \quad \mathrm{ZUL}=136$

UAX $=1$ WDN=-1 NAX $=130$ NCH $=10123456,8 \mathrm{ND}$

TTL $=$ 'YZ SLICE OF NORTH CORE PIRST ROW

$X U L=48 \quad Y U L=-2 \quad Y U L=136$

$X L R=48 \quad Y L R=136 \quad \& L R=-2 \quad 2 \quad$ L

NAX $=1$ WDN=-1 NAX $=130$ NCH= $01234566^{\prime}$ SND

$X I R=28 \quad Y L R=136, Z L R=-2$

$\checkmark N X=1 \quad \mathrm{WDN}=-1 \quad \mathrm{MAX}=130 \quad \mathrm{NCH}=0123456, \mathrm{BND}$

TTL $=$ ' YZ SLICE OF SOUTH CORE PIRST ROH'

$\begin{array}{ll}X U L=62 & \text { YUL }=-2 \\ X L R=62 & \mathrm{YUL}=136\end{array}$

$X L R=62 \quad Y L R=136 \quad 2 L R=-2 \quad 2 R$

NAX $=12$ YLR $=-1$ NAX 130 NCH $=$ '0123456 END

$X U L=80 \quad Y U L=-2 \quad, \quad U L=136$

$X L R=80 \quad Y L R=136 \quad Z L R=-2$

YAX $=1$ WDN $=-1 \quad$ NAX $=130^{\circ}$ NCH $=10123456^{\prime}$ END

END PLOT

ND DATA
CAR 12

ROCKY PLATS CRITICALS NUREG/CR-1653 EXPERIMENT B (27 GROUP )

- 78 PUEL CANS 0.929 CH MODERATOR GEE. HU125. DATA(UND1)

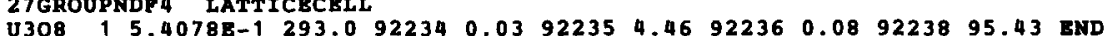

H2O $11.5627 \mathrm{E}-1$ END

ARBM-BAGGIE $1.0 \quad 30001001 \quad 14.01601284 .980161 .2011 .91348-2$

MAL1100 $1.030001302799 .18260000 .5290000 .229 .5390 \mathrm{E}-1$

ARBM-TAPE (VINYL) 1.0700010015 .92601245 .91801610 .8217000

$25.73200406 .9220001 .6820001 .121 .1115 \mathrm{E}-2$ END

1.0300010016 .83601265 .50801627 .022

$1.7491 \mathrm{~B}-2 \mathrm{BND}$

ARBM-MODERATOR 1.1853000010017 .83601259 .49801632 .4833 END

RBM-PLEX (REG)

3.7534E-3 END 1.0300010016 .48601242 .17801649 .54

ALEX(GLUE) 1.030000100111 .67601286 .2980161 .20

ARBM-PLEX(TRIS) $1.08000110017 .16 \quad 601252.03 \quad 7014 \quad 0.16801629 .82$

150311.02170001 .81350794 .260350812 .84051 .2757 END

ARBM-PLEX(PAPER) 1.0300010016 .48601242 .17801649 .55

$3.7534 \mathrm{E}-3$ END

ARBM-PLEX(GLUE) $1.0300001001 \quad 11.67601286 .2980161 .205$

$1.1648 E-3$
END

- THIS MATBRIAL IS ACTUALIY MODERATOR ADJUSTED FOR THE VOLUME FRACTION OP THE VOID FILLED BETMEEN CORE AND REFLECTOR.

SPHTRIANGP $19.00 \quad 18.5857 \quad 1 \quad 3 \quad 18.95792$ END

ROCKY FLATS CRITICALS NUREG/CR-1653 EXPERIMENT B ( 27 GROUP)

18 FUEL CANS 0.929 CM MODERATOR GEE. HU1 25.DATA (UND 1 )

READ GEOM

COM= PUEL BOX 15.28 CM ON A SIDE WITH .15 CM WALLS .05CM STACKING VOID

CUBOID 1 1 6 PP7.49

CUBOID 2 T 16 6

CUBOID 01687.665

COM='X-FACB INTERSTITIAL MODERATOR'

CUBOID

COY $=$ ' $Y$-PACE INTERSTITIAL MODERATOR'

CUBOID 31287.665 2P0.4645 2P7.665

UNIT

COM='Z-PACB INTERSTITIAL MODEPATOR'

CUBOID 314 P7.665 2 PO.4645

COM=' MORE X-PACE MODERATOR

CUBOID 314 PO.4645 287.665

UNIT 6

COM= ' YORE Y-FACE MODERATOR'

CUBOID 312 PP7.665 4 P0.4645

COY $=1$ Mone

' MORE Z-FACE MODERATOR'

CUBOID $312 P 0.46452 P 7.665 \quad 2 P 0.4645$

UNIT

COM= 'LAST OF INTERSTITIAL MODERATOR

CUBOID 316 PO.4645 
Table A.3 (continued)

UNIT 9

GOM='NORTH SPLIT TABLE CORE

ARRAY $13 * 0.0$

COM=.

ARRAY $2 \quad 3 * 0.0$

COM='PLEXIGLASS REFLECTOR SHEET WITHOUT TRIS, NORTH BOTTOM REFLECTOR CUBOID $412 \mathrm{P} 15.80002 \mathrm{P} 38.7500$ 2P0.6150

UNIT 12

COM='PLEXIGLASS REFIBCTOR SHEET WITH TRIS, NORTH BOTTOM REFLECTOR' CUBOID 512 2P15.8000 2P38.7500 2P0.6150

UNIT 13

COM='UPPER PORTION NORTH BOTTOM REFLECTOR WITH TRIS' CUBOID 512815.82838 .752 P8.24

(28)

CUBOID 512 P 15.8 2 P 38.75 2P3.69

UNIT 15

COM=' NORTH BOTTOM REFLECTOR INCLUDES REGULAR AND TRIS ARRAY $33 * 0.0$

COM=' PLRXIGLAS SHEET BOTTOM SOUTH REPIECTOR WITHOUT TRIS CUBOID 0012 P5.1 2 P2.55 2 P0.615

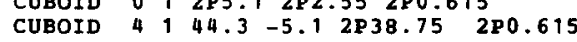

UNIT 17

COM='PLEXIGLAS SHEET BOTTOM SOUTH REPLECTOR WITH TRIS

CUBOID 0012 P $5.1282 .55 \quad 2 P 0.615$

CUBOID 5 1 $44.3-5.12838 .75280 .6150$

COM= LOHER PORTION SOUTK BOTTOM REFLECTOR HITH TRIS

CUBOID 012 TP5.1 2 P2.55 298.855

CUBOID $5144.3-5.12$ 2P38.75 2P8.85

UNIT 19

COM=' SOUTH BOTTOM REFLBCTOR WITH RBGULAR AND TRIS

ARRAY $43 * 0$.

COM=' EAST AND WEST REFLECTORS FOR NORTH REFLECTOR WITH TRIS' CUBOID $512215.8 \quad 2912.652854 .2825$

UNIT 21

COM=' ARRAY FOR BAST AND WEST REPLECTORS FOR NORTH REFLECTOR' ARRAY $5 \quad 3 * 0.0$

UNIT 22 AND REST REFLECTORS FOR SOUTH REFLECTOR WITH TRIS. CUBOID $512824.700 \quad 2812.65 \quad 2854.2825$

COM='ARRAY FOR BAST AND WEST REFLECTORS POR SOUTH REFLECTOR' ARRAY $6 \quad 3 * 0.0$

COM='

CUBOID $5,2828.352864 .052 \mathrm{P} 12.15$

UNIT 25 COM= ARRAY FOR NORTH TOP REPLECTOR'

ARRAY $73 * 0.0$

UNIT 26 (2)

CUBOID $0,2 P 5,12$.

CUBOID 5 1 69.4 - 5 .1 $2 \mathrm{P}_{64} .05 \quad 2 \mathrm{P} 12.15$

UNIT 27

COM=' ARRAY FOR SOUTH TOP REFLECTOR

ARRAY 8 3*0.0

COM = ' NORTH END RETLECTOR $9.8 C M$ PORTION WITHOUT TRIS

COM='NORTH END REFLECTOR 9 .8CM PORTION
CUBOID $4+284.90002864 .0500 \quad 2854.2825$
UNIT 29 COM=' NORTH BND RELPBCTOR $5.2 \mathrm{CM}$ PORTION WITH TRIS'

CUBOID $5,2 \mathrm{P2.6} 2 \mathrm{P64.0500} 2 \mathrm{P5} 4.2825$

UNIT 30
COM=' NORTH END RELPECTOR 10.1 CM PORTION WITHOUT TRIS CUBOID 4,2 P5.05 2P64.05 2P54.2825

COMIT A ARRAY FOR NORTH END REFLECTOR.

ARRAY 9300.0

ARRAY 32

COM='SOUTH END REFLECTOR'

CUBOID 5 , 2P12.55 2P64.05 2P54.2825
CUBI

UNIT 33

COM=' ARRAY FOR SOUTH BND REFLECTOR'

ARRAY $10 \quad 3 * 0.0$

COM='12.95 THICK MODE

CUBOID 512 P15.7945 2P6.4750 2P32.0535

UNIT 35 THICK MODERATING PLASTIC NORTH CORE'

CUBOID 692 P $15.7945 \quad 2$ P38.5285 2 P1.475

UNIT

ARRAY $113 * 0.0$

UNIT 37

REPLICATB $\begin{array}{lllllllllllll}0 & 0.0 & 0.011 & 0.443 & 0.0 & 0.1590 & 0.0\end{array}$

UNIT 38

COM='NORTH CORE WITH BOTTOM REPLECTOR'

ARRAY $133 * 0.0$

COM $=$ ' NORTH CORE WITH EAST AND WEST REFLECTOR

ARRAY $14 \quad 3 * 0.0$

UNIT 40

COM=' NORTH CORE WITH END REPLECTOR'

ARRAY 15
UNIT 41

COM=' NORTH SPLIT TABLE FACEPLATE'

CUBOID $3,2 P 0.61502864 .05002 P 66.4325$

UNIT 42 21

COM= 'NORTH COR

ARRAY $16 \quad 3 * 0.0$

COM=' NORTH CORE WITH FACEPLATE'

ARRAY $173 * 0.0$

UNIT 44

COM='12.95 CM THICK MODERATOR SOUTH CORE

CUBOID 612 P23.924 2P6.475 2P32.0535

COM='2.95 THICK MODERATOR SOUTH CORE :

UNIT 46

COM=' COMBINATION OP CORE WITH 12.95 THICK MODERATOR ARRAY $18 \quad 3 * 0$.

UNIT 47
COM= COMBINATION OF CORE WITH 2.95 THICK MODERATOR.

ARRAY 19

COM='SOUTH CORE WITH EAST HEST REFLCTORS'

ARRAY $20 \quad 3 * 0.0$

UNIT 49

COM=' SOUTH CORB WITH EAST WEST REFLECTORS' 
Table A.3 (continued)

UNIT 50

COA SOUTH CORE END REFLECTOR'

ARRAY $223 * 0.0$

COM=' PACEPLATE FOR SOUTH SPLIT TABLE' CUBOID 312 2PO.4620 $2 \mathrm{P} 64.0500 \quad 2 \mathrm{P} 66.4325$ UNIT 52 COM='SOUTH CORE WITH TOP REFLECTOR' ARRAY $233 * 0.0$

COMTIS 53

WITH FACBPLATB

ARRAY $24 \quad 3 * 0.0$

UNIT 54

CUBOID 012 P0.3990 2P64.0500 2P66.4325

GLOBAI

UNIT 55

UNIT 56

COM=' NORTH BOTTOM MODERATOR'

CUBOID 612 2P15.8000 2P38.7500 2P8.1295

COM = BOTTOM SOUTH MODERATOR

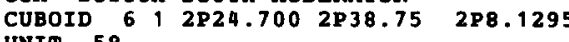

COML LOCATION

END GEOMETRY

READ ARRAY

$A R A=1 \quad N U X=3 \quad N U Y=7 \quad N U Z=7$

COM=' NORTH SPLIT TABLE CORE'

FILL $\quad \begin{array}{lllllllllll}1 & 2 & 1 & 3 & 5 & 3 & 206 & 1 & 2 & 1\end{array}$

$$
2 Q^{4} 2
$$

Q42 2 (2)

$A R A=2$ NUX $=5$ NUY $=7$ NUZ $=7$

COA=' SOUTH SPLIT TABLE CORE'

$\begin{array}{llllllllllllllll}2 & 1 & 2 & 1 & 3 & 5 & 3 & 5 & 3 & 2010 & 1 & 2 & 1 & 2 & 1 \\ 4 & 4 & 7 & 4 & 6 & 8 & 6 & 8 & 6 & 2010 & 4 & 7 & 4 & 7 & 4\end{array}$

$\begin{array}{llllllllllllllll}2070 & 1 & 2 & 1 & 3 & 5 & 3 & 5 & 3 & 2 Q 10 & 1 & 2 & 1 & 2 & 1 & \text { END PIL }\end{array}$

ARA $=3$ NUX $=1$ NUY $=1$ NUZ $=4$ A

COM=' NORTH BOTTOM MODERATOR

ARA $=4$ NUX $=1$ NUY $=1 \quad$ NUZ $=8$

MUTH BOTTOM MODERATOR AND REFLECTOR

FILI $\begin{array}{lllllllll}18 & 16 & 17 & 16 & 16 & 17 & 17 & 57 & \text { END FILI }\end{array}$

COM=' EAST AND WEST WALLS OF NORTH REFLECTOR.

FILL 20 END PILI

$A R A=6 \quad N U X=1 \quad$ NUY $=1$ NUZ $=1$

COM='BAST AND WEST WALLS OF SOUTH REFLECTOR'

FILL 22 END FILI

CON=' ARRAY FOR NORTH TOP REPLECTOR'

FILI, 24 BND PILI

$=1$ NUY $=1 \quad$ NUZ $=1$

FILI 26 BND PILI

ARA $=9$ NUX $=3$ NUY $=1$ NUZ $=$

COM= 'ARRAY POR NORTH END REFLECTOR
$A R A=10 \quad N U X=1 \quad N U Y=1 \quad N U Z=1$

ARA 10 NUX 1 NUY 1 NUZ 1 REPLECTOR

CORA $=112$ BND FILL

COM=' COMBINARTON OF CORE

FILL 934 END FILI

COM=' COMBINATION OP PREVIOUS ARRAY WITH $2.95 \mathrm{CM}$ THICK MODERATOR'

FILL $36 \quad 35$ BND FILI

$A R A=13$ NUX $=1 \quad N U Y=1 \quad$ NUZ $=2$

COM= COMBINATION OF NORTH CORE WITH BOTTOM REFLECTOR

FILL 15 37 END FILL

ARA $=14 \quad$ NUX $=1 \quad$ NUY $=3$ NUZ $=1$

COH $=$ ' NORTH CORE WITH SIDE REPLECTORS'

PILL 213821 END PILL

COM=' NORTH CORE WITH END REPLECTOR'

FILL 3139 END FILI

ARA $=16 \quad \mathrm{NUX}=1 \quad \mathrm{NUY}=1 \quad \mathrm{NUZ}=2$

COM=' NORTH CORE WITH TOP REFLECTOR'

FILL 4025 BND

COM= ' NORTH CORE WITH FACEPLATE'

FILL 4241 END FILI

$A R A=18$ NUX $=1 \quad$ NUY $=2$ NUZ $=1$

COM=' COMBINATION OF $S$. CORE WITH $12.95 \mathrm{CM}$ THICK MODERATOR

PILL 1044 BND FILL

ARA $=19 \quad$ NUX $=1 \quad$ NUY $=1 \quad$ NUZ $=2$

COM=' COMBINATION OF CORB WITH 2.95 CM THICK MODERATOR'

FILL 46 45 S 1 NUY $=1$ LL

COM='SOUTH CORE WITH BOTTOM REFLECTOR'

FILI 1947 END FILI

ARA $=21$ NUX $=1$ NUY $=3 \quad$ NUZ $=1$

COM $=$ ' SOUTH CORE WITH BAST WEST REPLECTORS'

FILL 234823 BND FILL

COM=' SOUTH CORE WITH END REFLECTOR'

FIIL 4933 END FILL

$A R A=23$ NUX $=1 \quad$ NUY $=1$ NUZ $=2$

COM=' COMBINATION OF CORE WITH TOP REFLECTOR

FILL 5027 BND FILL

$A R A=24 \quad N U X=2 \quad N U Y=1 \quad$ NUZ $=1$

COM='SOUTH CORE WITH FACEPLATE'

$A R A=25 \quad$ NUX $=3$ YUY $=1$ NUZ $=1$

COM= 'TOTAI'

FILI 4354

END ARRAY

READ PLOT TTL='XZ SLICE OF RFP 3 SHOWING MATERIAL REGIONS.

$X U L=-1 \quad$ YUL $=64.05 \quad$ \&UL $=135$

UAX $=1$ WDN $=-1$ NAX $=130$ NCH $=0123456 \cdot$ BND

TTL $=$ 'YZ SLICE OF NORTH CORE FIRST ROW'

$X U L=48 \quad Y U L=-2 \quad$ qUL $=136$

$X L R=48 \quad$ YLR $=136 \quad \mathrm{ZLR}=-2$

VAX $=1$ WDN $=-1 \quad$ NAX $=130 \quad \mathrm{NCH}=0123456^{\circ}$ END

TTL $=$ 'YZ SLICE OF NORTH CORE SECOND ROW'

$\begin{array}{lll}X U L=28 & Y U L=-2 & Z U L=13 \\ X L R=28 & Y L R=136 & \end{array}$

VAX $=1$ WDN $=-1$ NAX $=130$ NCH $=10123456$, END
TTL= $=$ YZ S.ICE OF SOUTH CORE SECOND ROW' 
Table A.3 (continued)

$X U L=80 \quad Y U L=-2 \quad Z U L=136$

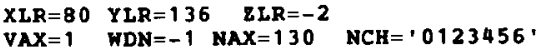

BND PLOT

END

CAR 13

$=$ CSAS 25

ROCKY PLATS CRITICALS NUREG/CR-1653 EXPBRIMENT C (27 GROUP

80 FUEL CANS 0.929 CM MODERATOR GBE. HU125. DATA (UND2)

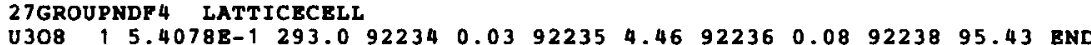

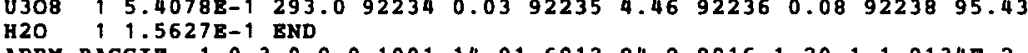

END
AND

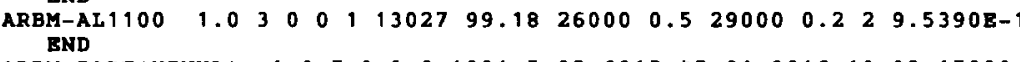

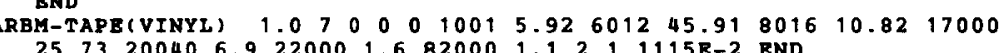

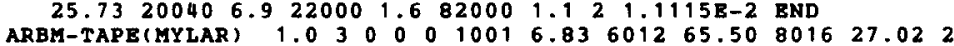

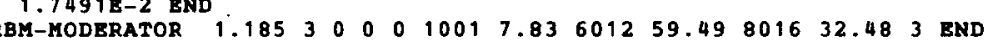

ARBM-PLEX(REG) $\quad 1.0 \quad 3 \quad 0 \quad 0 \quad 0 \quad 10017.84601259 .59801632 .234$

1.1773 END

ARBM-PLEX(PAPER) 1.0300010016 .48601242 .17801649 .54

$3.75348-3$ END

103000100111.67601286 .2980161 .204

ARBM-PLEX(TRIS) $1.08000171001 \quad 7.16 \quad 6012 \quad 52.037014 \quad 0.16801629 .82$

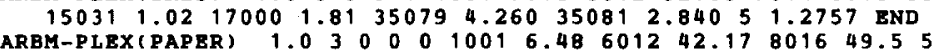

$3.7534 \mathrm{~B}-3$ BND

ARBM-PLBX(GLUB) 1.03000100111 .67601286 .2980161 .205

$\begin{array}{llllllllllllll}1.1648 E-3 & \text { END } & & & & \end{array}$

ARBM-FILLER 1.185 THIS MATERIAL IS ACTUALLY MODERATOR ADJUSTED FOR THE VOLUME

. THIS MATERIAI IS ACTUALLY MODERATOR ADJUSTED FOR THE VO

BND COMP

SPHTRIANGP 19.0018 .58571318 .95792 BND

ROCKY FLATS CRITICALS NUREG/CR-1653 EXPERIMENT C (27 GROUP)

PUEL CANS 0.92 9 CM MODIRATOR GEE. HUI 25 .

END PARM

READ GROM

COM=PPURL BOX $15.28 \mathrm{CM}$ ON A SIDE WITH .15 CM WALLS .05CM STACKING VOID'

CUBOID $116 \mathrm{1} 7.49$

CUBOID 2 I 687.64

UNIT 2

INTERSTITIAL MODERATOR

CUBOID 312 P0.4645 4 P7.665

UNIT 3312 O

COM='Y Y FACE INTERSTITIAL MODERATOR'
CUBOID 312 2P7.665 2 PO. $4645 \quad 287.665$

UNIT 4

COM='Z-PACE INTERSTITIALL MODERATOR'

CUBOID

COM='MORE X-PACE MODERATOR'
CUBOID 314 P0.4645 2P7.665
UNIT 6

COM=' MORE Y-PACE MODBRATOR'

CUBOID 312 P7.665 4 P0.4645

UNIT ${ }^{7}$ COM=I MORE Z-FACE MODERATOR'

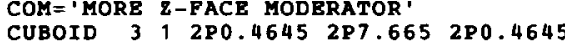

UNIT 8 T 2 TO.4645 2 P7.665 2 PO. 4645

CUBOID 316 PO. 1645

UNIT 9

COM='NORTH SPLIT TABLE CORE'

ARRAY $113 * 0.0$

COM=' SOUTH SPLIT TABLE CORE'

ARRAY $23 * 0.0$

UNIT 11

COM='PLEXIGLASS REFLECTOR SHEET WITHOUT TRIS, NORTH BOTTOM REFLECTOR' CUBOID 41 2P15.8000 2P38.7500 2P0.6150

UNIT 12 COM='PLEXIGLASS REFLECTOR SHEET WITH TRIS, COM='PLEXIGLASS REPLECTOR SHEET WITH TRIS, NORTH BOTTOM REPLECTOR' UNIT 13

COM=' UPPER PORTION NORTH BOTTOM REFLECTOR WITH TRIS'

CUBOID $512 \mathrm{P} 15.82 \mathrm{P} 38.752 \mathrm{~PB} .24$

UNIT 14
COM='LONER PORTION NORTH BOTTOM RBFLECTOR WITH TRIS'

CUBOID $5,2 \mathrm{P} 15.82 \mathrm{P38.75} 2 \mathrm{P3} .69$

COM=' NORTH BOTTOM REFLECTOR INCLUDES REGULAR AND TRIS'

ARRAY $3 \quad 3 * 0.0$

UNIT 16

COM='PLEXIGLAS SHEET BOTTOM SOUTH REFLECTOR WITHOUT TRIS

CUBOID 0 1 2 P5.1 2 P2.55 2 PO.615

CUBOID 4 1 $44.3-5.12 P 38.75$ 2P0.615

COMI'PLEXIGLAS SHEET BOTTOM SOUTH REFLECTOR WITH TRIS '

CUBOID 012 2P5.1 2 P2.55 2 PO.615

CUBOID 5 1 $44.3-5.12$ P 38.75 2P0.6150

UNIT 18

COM=' LOWER PORTION SOUTH BOTTOM REFLECTOR WITH TRIS'

CUBOID $0012 \mathrm{P} 5.12 \mathrm{P2} .55 \quad 2 \mathrm{~PB} .855$

$2 \mathrm{P} 8.855$

UNIT ' 19 SOUTH BOTTOM REFLECTOR WITH REGULAR AND TRIS .

ARRAY 4 3*0.0

UNIT 20

TH REPLECTOR WITH TRIS CUBOID 5 1 2P15.8 2P12.65 2P54.2825

21
UNIT $20 M=$ ARRAY FOR EAST AND WEST REFIECTORS FOR NORTH REFLECTOR. ARRAY $53 * 0.0$

COM=' BAST AND WEST REPLECTORS FOR SOUTH REPLECTOR WITH TRIS CUBOID 512 P24.700 2P12.65 2P54.2825

UNIT 23

COM=' APRAY FOR EAST AND WEST REFLECTORS FOR SOUTH REFLECTOR ARRAY $6 \quad 3 * 0.0$

COM=' NORTH TOP REPLECTOR WITH TRIS' CUBOID 512 2P28.35 2P64.05 2P12.15

COMI - ARRAY FOR NORTH TOP REFLECTOR

ARRAY $7 \quad 3 * 0.0$ 
Table A.3 (continued)

UNIT 26

COM= SOUTH TOP REPLBCTOR WITH TRIS

CUBOID 0 1 12 2P5.1 2P2.55 2P12.15

CUBOID $5169.4 \quad 5.92864 .05$ 2P12.1

UNIT 27

COM= ARRAY FOR SOUTH TOP REFLECTOR

ARRAY 8300

COM=' NORTH END REFLECTOR 9.8CM PORTION YITHOUT TRIS CUBOID 412 2P4.9000 2P64.0500 2P54.2825

COMI' 29 NORTH END RELFECTOR 5.2 CM PORTION WITH TRIS'

CUBOID 512 2P2.6 2P64.0500 2P54.2825

COM=' NORTH END REIFECTOR $10.1 \mathrm{cM}$ PORTION WITHOUT TRIS CUBOID $4,2 \mathrm{P5} .052 \mathrm{P} 64.052 \mathrm{P} 54.2825$

COM= ARRAY POR NORTH END REFLECTOR

ARRAY $93 * 0.0$

UNIT 32

END RETIECTOR

CUBOID 512 2P12.55 2P64.05 2P54.2825

COM=' APRAY FOR SOUTH END REFECTOR

ARRAY $10 \quad 3 * 0.0$

UNIT 34

COM=112.95 THICK MODERATING PLASTIC NORTH CORE

CUBOID 612 P15.7945 2P6.4750 2P32.0535

COM $=2.95$ THICK MODERATING PLASTIC NORTH CORE

CUBOID 612 P15.7945 2P38.5285 2P1.475

UNIT 36

ARRAY $113 * 0.0$

UNIT 37

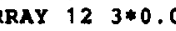

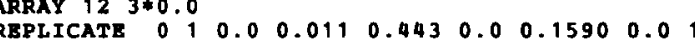

JNIT 38

SOTTOM REFLECTOR.

ARRAY 13 3* 0.0

COM $=$ ' NORTH COR
ARRAY $14 \quad 3 * 0.0$

UNIT 40

STE RELECTOR

ARRAY $15 \quad 3 * 0.0$

COM='NORTH SPLIT TABLE FACEPLATE

CUBOID $312 \mathrm{PO} .6150$ 2P64.0500 2P66.432S

COM=' NORTH CORE WITH TOP REFLECTOR

ARRAY $16 \quad 3 * 0$.

COM=' NOPTH CORE HITH FACEPLATE'

ARRAY $17 \quad 3 * 0.0$

UNIT 44

COM $=12.95$ CM THICK MODERATOR SOUTH CORE

CUBOID 612 P23.924 2P6.475 2P32.0535

UNIT 45

COM= $=2.95$ THICK MODERATOR SOUTH CORE'

UNIT 46

DQ

COM=' COMBINATION OF CORE WITH 12.95 THICK MODERATOR

ARRAY $18 \quad 3 * 0.0$

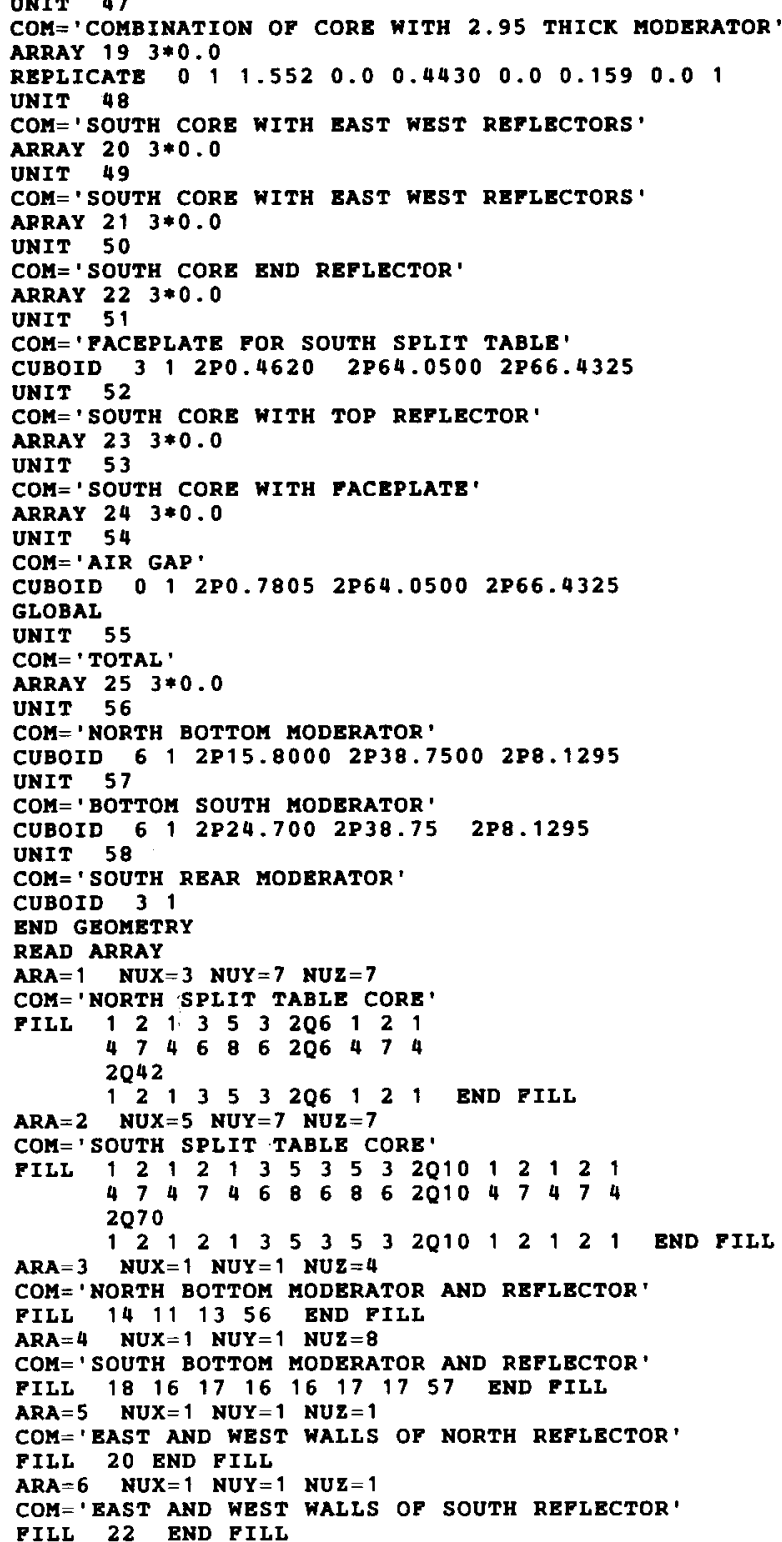


Table A.3 (continued)

MWA=7 NUX=1 NUY=1 NUZ=1

COM=' ARRAY FOR NORTH TOP REPLECTOR'

FILL 24 END PILL

COM=1 NOX $=1$ NOP SOUTH TOP RETLECTOR.

PILL 26 END FILI

ARA $=9$ NUX $=3$ NUY $=1 \quad$ NUZ $=1$

COM= ' ARRAY FOR NORTH END REFLECTOR'

FILL $28 \quad 2930$ BND FILL

ARA $=10$ NUX $=1$ NUY $=1$ NUZ $=1$
COM= ARRAY FOR SOUTH END REFLECTOR

COHE = ARRAY

$A R A=11 \quad$ NUX $X=1$ NUY $=2$ NUZ $=1$

COM= ' COMBINARION OF CORE WITH $12.95 \mathrm{CM}$ THICK MODERATOR'

FILL 934 END FILL

$A R A=12 \quad N U X=1$ NUY $=1$ NUZ $=2$

COM='COMBINATION OF PREVIOUS ARPAY WITH $2.95 \mathrm{CM}$ THICK MODERATOR'

FILL $36 \quad 35$ END FILI

COM=' COMBINATION OF NORTH CORE MITH BOTTOM REFIECTOR'

FILI $15 \quad 37$ END FILL

ARA $=14 \quad$ NUX $=1 \quad$ NUY $=3 \quad$ NUZ $=1$

CON='MORTH CORE WITH SIDE REFLECTORS

FILL 213821 END FILL

COM='

FIL 3139 END FILI

$A R A=16 \quad$ NUX $=1$ NUY $=1 \quad$ NUZ $=2$

COM=' NORTH CORE WITH TOP REFLECTOR'

FILL $40 \quad 25$ END FILL

ARA $=17$ NUX $=2$ NUY $=1$ NUZ $=1$
COM $=$ NORTH CORE WITH FACEPLATE

COM=' NORTH CORE WITH

$A R A=18$ NUX $=1$ NUY $=2$ NUZ $=1$

COM=' COMBINATION OF $\mathrm{S}$. CORE WITH $12.95 \mathrm{CM}$ THICK MODERATOR'

FILL 1044 BND FILI

$A R A=19$ NUX=1 NUY $=1$ NUZ=2

COM=' COMBINATION OF CORE WITH $2.95 \mathrm{CM}$ THICK MODERATOR'

FILL 4645 END FILL

COM=' SOUTH CORE WITH BOTTOM REFLECTOR'

FILL 1947 END PILI

ARA $=21$ NUX $=1$ NUY $=3$ NUZ $=1$

COM=' SOUTH CORE WITH EAST WEST REFLECTORS

FILL 234823 END FILI

ARA $=22$ NUX $=2$ NUY $=1$ NUE $=1$
COM =' SOUTH CORE WITH END RETLECTOR'

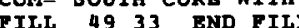

$A R A=23$ NUX $=1 \quad$ NUY $=1$ NUZ $=2$

COM=' COMBINATION OF CORE WITH TOP REFLECTOR.

PILI 5027 END FILL

ARA $=24$ NUX $=2$ NUY $=1$ NUZ $=1$
COM $=$ ' SOUTH CORE WITH FACEPLATE

COM=' SOUTH CORE WITH FAC
FILI 5152 END PILL

ARA $=25$ NUX $=3$ NUY $=1$ MUZ $=1$

$\operatorname{COM}=\cdot$ TOTAL

FILL 435453 END PILL

END ARRAY

TLE='XZ SLICE OP RPP 3 SHOWING MATERIAI REGIONS '

$X U L=-1 \quad$ YUL $=64.05 \quad 2 U L=135$

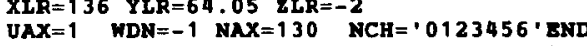

TAX $=1$ WDN=-1 NAX $=130$ NCH $=\cdot 0123456$ SND

XUL $=28 \quad$ YUL $=-2 \quad$ ZUL $=136$

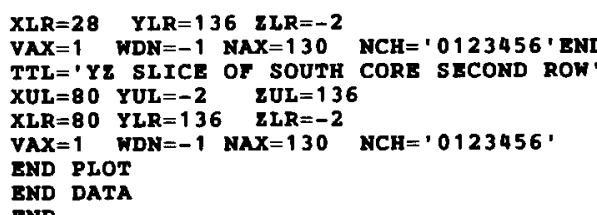

$\begin{array}{lll}X L R=28 & Y L R=136 \quad Z L R=-2\end{array}$

VNX $=1$ WDN $=-1 \quad$ NAX $=130$ NCH $=0123456^{\prime} \mathrm{END}$

XUL $=80$ YUL $=-2$ Q $\quad$ UUL $=136$

$X L R=80 \quad Y L R=136 \quad 2 L R=-2$

$\mathrm{VAX}=1$ WDN $=-1$ NAX $=130$ NCH $=0123456$.

END PLOT

CAR14

ROCKY YLATS CRITICALS NUREG/CR-1653 EXPERIMENT NUMBER ? (27 GROUP MODEL) (1) 27GROUPNDP 4 INFHOMMEDTUM

U308 $95.4078 E-1293.0922340 .03922354 .46922360 .089223895 .43$ END

ARBM-BAGGIE $1.030001001 \quad 14.01601284 .980161 .2011 .91348-2$ BND

$\begin{array}{lllllllllllll} & & \end{array}$ END $25.73200406 .9220001 .6820001 .121 .1115 \mathrm{E}-2$ END

ARBM-TAPE (MYLAR) 1.0300010016 .83601265 .50801627 .02 $1.7491 \mathrm{~B}-2 \mathrm{BND}$

ARBM-MODERATOR 1.1853000010017 .83601259 .49801632 .483 END

1.1773 END

ARM-PLEX (PAPER)

1.03000010016 .48601242 .17801649 .54

$7534 \mathrm{~B}-3$ END

1.03000100111 .67601286 .2980161 .20 4

1648E-3 END

ARBM-PLEX(TRIS) $1.08000110017 .16 \quad 601252.03 \quad 7014 \quad 0.16801629 .82$ 150311.02170001 .81350794 .260350812 .84051 .2757 END

$3.7534 \mathrm{E}-3$ END

PLEC(GLUE) 1.030000100111 .67601286 .2980161 .205

$1.1648 \mathrm{E}-3$ END

ARBM-FILLER 1.18533000010017 .83601259 .49801632 .4860 .7 END

THIS MATERIAL IS ACTUALLY MODERATOR ADJUSTED FOR THE VOLUME

ARAY

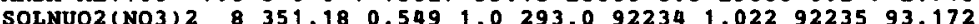
$922360.43492238 \quad 5.372$ END

SS 30491.0 END

ROCKY FLATS CRITICALS NURBG/CR-1653 EXPERIMENT NUMBER ? (27 GROUP MODEI) PUEL CUBE $15.28 \mathrm{CM}, \mathrm{U}(4.46) 308$ H $\mathrm{H} / \mathrm{U}=1.25$, HI CONC. SOLUTION DRIVEN

READ GBOM

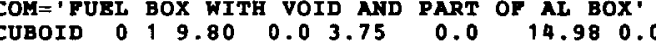

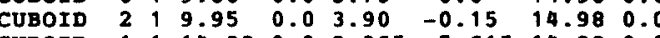

custoris

UNIT 2

COM=' FRONT OF FUEL BOX

$\begin{array}{lllllllll} & & & & & & \\ C U B O I D & 2 & 1 & 0.15 & 0.0 & 5.615 & 0.0 & 14.98 & 0.0\end{array}$

$\begin{array}{lllllllll}\text { CUBOID } & 0 & 1 & 0.15 & 0.0 & 9.365 & 0.0 & 14.98 & 0.0 \\ \text { CUBOID } & 2 & 1 & 0.15 & 0.0 & 14.98 & 0.0 & 14.98 & 0.0\end{array}$ 
Table A.3 (continued)

UNIT 3

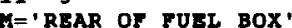

CUBOID $210.15 \quad 0.0 \quad 14.98 \quad 0.0 \quad 14.98 \quad 0.0$

COMT $=$ ARRAY TO ASSEMBLE PART OF BOX

ARRAY $26 \quad 3 \neq 0$

UNIT 5

COM=' TOP AND BOTTOM OF BOX

$\begin{array}{llllllll} & & & & & \end{array}$

$\begin{array}{llll} & \end{array}$

GLOBAL $_{6}$

COM= 'ARRAY TO ASSEMBLE TOP AND BOTTOM

ARRAY 27 3*0

$\begin{array}{llllllllll}\text { CUBOID } & 2 & 1 & 15.28 & 0.0 & 15.13 & -0.15 & 15.28 & 0.0\end{array}$

7

COM=' PUEL BOX $15.28 \mathrm{CM}$ ON A SIDE WITH .15 CK WALLS .05CM STACXING VOID'

CUBOID 11967.49

$\begin{array}{lllll}\text { CUBOID } 2 & 1 & 677.64 \\ \text { CUBOID } 0 & 1 & 687.6650\end{array}$

COMT $=$ ALUMINUM WEIGHT DISTRIBUTION PLATE FOR SPECIAL BOX'

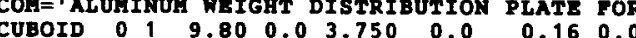

CUBOID 7 1 $15.33 \quad 0.0 \quad 9.54-5.790 .160 .0$

UNIT 35

COK='ALUMINUM WEIGHT DISTRIBUTION PLATE FOR BOXES .

$\begin{array}{llll}\text { CUBOID } 71 & 4 P 7.665 & 0.16 & 0.0\end{array}$

UNIT 9

COM=' NORTH SPLIT TABLE CORE'

$\begin{array}{llllll} & \end{array}$

conit $10^{\circ}$

COM='SOUTH SPLIT TABLE CORE'

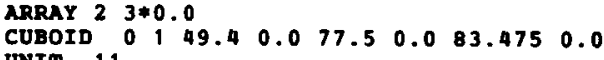

CONT = PLEXIGLASS REPLECTOR SHEET WITHOUT TRIS, NORTH BOTTOM REFLECTOR'

(15.8000 2838.7500 2D0.6150

Coms 12

COM='PIEXIGLASS REFLECTOR SHEET WITH TRIS, NORTH BOTTOM RETLETTOR'

CUBOID 512 2P15.8000 2P38.7500 2P0.6150

UNIT 13

COM='UPPER PORTION NORTH BOTTON REFIECTOR WITH TRIS'

CUBOID 51 2P15.8 2P38.75 2PB.24

(U)

CUBOID 512 2P15.8 2P38.75 2P3.69

UNIT 15

COM=' NORTH BOTTOM RETLECTOR INCLUDES REGULAR AND TRIS

ARRAY $33 * 0.0$

COM-'PIEXIGLAS SHEET BOTTOM SOUTH REFLECTOR WITHOUT TRIS

CUBOID 012 2P5.1 2 P2.5 2 PO 6150

CUBOID $4144.3-5.12838 .75$ 2P0.615

UNIT 17

COM=' PLEXIGLAS SHEET BOTTOM SOUTH REFLECTOR WITH TRIS'

CUBOID $012 \mathrm{PS} .12 \mathrm{2P2} .52 \mathrm{PO} .6150$

CUBOID 5 1 $44.3-5.12 \mathrm{P} 38.75$ 2PO.6150

COH='LOWER PORTION SOUTH BOTTOM RETLECTOR WTTH TRIS'

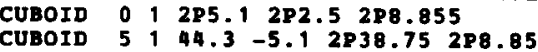

UNIT 19

COM=' SOUTH BOTTOM REPLECTOR WITH REGULAR AND TRIS'

ARRAY 4 \% $3 * 0.0$

COY=' EAST AND WEST REFIECTORS FOR NORTH REFLECTOR WITH TRIS CUBOID 512815.8 2P12.65 2854.2825

UNIT 21

COM=' ARRAY FOR EAST AND WEST REFLECTORS FOR NORTH REFLECTOR ARRAY 5 3*0.0

COM='EAST AND WEST REFLECTORS FOR SOUTH REFIECTOR WITH TRIS' CUBOID 512824.7002812 .652854 .2825

UNIT 23 (23

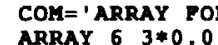

ARRAY $63 * 0.0$

UNIT 24

CM =' NORTH TOP REFLBCTOR WITH TRIS.

BOID 512 2P28.35 2P64.05 2P12.15

25

CON= ' ARRAY FOR NORTH TOP REFLECTOR'

NRRAY $73 * 0.0$

UNIT 26

COM=' SOUTH TOP REFLECTOR WITH TRIS'

CUBOID 0112 2P5. 1 2P2.5 2 P12.15

CUBOID $51269.4-5.12864 .052 P 12.15$

COM $=$ ARRAY FOR SOUTH TOP REFLECTOR

ARRAY $8 \quad 3 * 0.0$

UNIT 28

COM=' NORTH END REPLECTOR $9.8 \mathrm{CM}$ PORTYON WITHOUT TRIS

CUBOID 4 1 2P4.9000 2P64.0500 2P54.2825

COMT 29 . NORTH END RELFECTOR 5.2 CM PORTION WITH TRIS ,

CUBOID $512 \mathrm{P2.6} 2 \mathrm{P64.0500} 2 \mathrm{P5} 4.2825$

COMORTH BND RELPECTOR $10.1 \mathrm{CM}$ PORTION WITHOUT TRIS

CUBOID 412 2P5.05 2P64.05 2P54.2825

UNIT 31

COY='ARRAY FOR NORTH END REFLECTOR'

ARRAY 9 3*0.0

COM=, SOUTH END DETLECTOR.

CUBOID 51 2P12.55 2P64.05 2P54.2825

UNIT 33

COM= ARRAY FOR SOUTH END REPLECTOR'

ARRAY $10 \quad 3 * 0.0$

UNIT 38

WITH BOTTOM RELECTOR

ARRAY 13 3*0.0

CORE WITH EAST AND WEST REFLECTOR

ARRAY $143 * 0.0$

UNIT 40

COY= 'NORTH CORE WITH END REFLECTOR'

ARRAY $15 \quad 3 * 0.0$

COM='NORTH CORE WITH TOP REPLECTOR'

ARRAY $16 \quad 3 * 0.0$

UNIT 48

COM=' SOUTH CORE WITH BOTTON REFLECTORS
ARRAY 20 ? $3 * 0.0$ 
Table A.3 (continued)

\section{UNIT 49}

WITH EAST WEST REFLECTORS

ARRAY 21 3*0.0

UNIT 50

SOUTH CORE END REFLECTOR'

ARRAY 22
UNIT 52

COM=' SOUTH CORE WITH TOP REYLECTOR

ARRAY $233 * 0$.

$\begin{array}{llllllll} & & & \end{array}$

UNIT 55

COM $=$ ' TOTAL'

ARRAY $25 \quad 3 * 0$.

UNIT 56

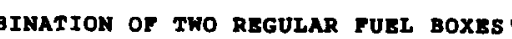

ARRAY 28
UNIT 57

COM= ' COMBINATION OP REGULAR TUEI BOX NND SPECIAI TUET BOX' ARRAY $293 * 0.0$

UNIT 58

COM= ' COMBINATION OF TWO REGULAR WEIGHT DISTRIBUTION PLATES

ARRAY $30 \quad 3 * 0$.

COM= ' COMBINATION OF REGULAR AND SPECIAL METGHT DISTRIBUTION PLATES' ARRAY $313 * 0$.

UNIT 60

COM=' NORTH CORE SOLUTION DRIVER'

CUBOID 89287.5 2P14.9 $1.763-7.5$

CUBOID 12 2P7.5 2P14.9 2P7.5

CUBOID 9 2P7.65 $2 P 15.052 P 7.65$

COM='SOUTH CORE SOLUTION DRIVER'

CUBOID $81287.5 \quad 2 P 14.9 \quad 1.695-7.5$

CUBOID 017287.52814 .9287 .5

CUBOID $912 P 7.652 P 15.05287 .65$

$A R A=1 \quad$ NUX $=2 \quad N U Y=4 \quad$ NUZ $=9$

COM=' NORTH SPLIT TABLE CORE'

$2 R 7$ 2R56 4R7

2R35 $2 R 58$ 4R335

2R7 $2 \mathrm{R} 56$ 4R7

2R7 $56 \quad 60 \quad 4 R 7$

2R35 $2 R 58 \quad 4 R 35$

$\begin{array}{lll}2 R 7 & 2 R 56 & 4 R 7 \\ 2 R 35 & 2 R 58 & 4 R 35\end{array}$

BND FILI $\begin{array}{lll}2 R 7 & 2 R 58 & 4 R 35 \\ 2 R 56 & 4 R 7\end{array}$

NUX $=3$ NUY $=4 \quad \mathrm{NUZ}=9$

FILI SOUTH SPLIT TABLE CORE

$\begin{array}{lll}3 R 7 & 3 R 56 & 6 R 7 \\ 3 R 35 & 3 R 58 & 6 R 35\end{array}$

$\begin{array}{lll}3 R 7 & 3 R 56 & 6 R 7 \\ 3 R 35 & 3 R 58 & 6 R 35\end{array}$

$\begin{array}{llll}3 R 35 & 3 R 58 & 6 R 35 \\ 3 R 7 & 61.56 & 56 & 6 R 7\end{array}$

$3 R 35 \quad 3 R 58 \quad 6 R 35$

$\begin{array}{lllll}3 R 7 & 57 & 56 & 56 & 6 R 7\end{array}$

$\begin{array}{lllll}3 R 35 & 59 & 58 & 58 & 6 R 35 \\ 3 R 7 & 57 & 56 & 56 & 6 R 7\end{array}$
END FILL

$x=1$ NUY $=1$ NUZ $=3$

COY= ' NORTH BOTTOY REFLBCTOR'

$\begin{array}{lllll}\text { FILL } & 14 & 11 & 13 & \text { BND FILI } \\ A R A=4 & \text { NUX }=1 & \text { NUY }=1 \text { NUE }=7\end{array}$

COM=' SOUTH BOTTOM REFLECTOR'

FILL $\begin{array}{lllllll}18 & 16 & 17 & 16 & 16 & 17 & 17\end{array}$ END FILL

COM='BAST AND WEST WALLS OF NORTH REFIECTOR'

FILL 20 BND FILI

ARA $=6$ NUX $=1$ NUY $=1$ MU $E=1$

OUTH REFLCTOR

FILI 22 END FILI

$N R A=7$ NUX=1 NUY $=1$ NUZ $=1$

TOP REPLECTOR

ILL 24 END FILL

COM= ' ARRAY FOR SOUTH TOP RETLECTOR'

PILL 26 END FILI

ARA $=9 \quad$ NUX $=3$ NUY $=1 \quad$ NUZ $=1$

COM= 'ARRAY YOR NORTH END REPLECTOR'

PILL 282930 BND PILL

COM=' ARRAY FOR SOUTH END REPLECTOR'

FILL 32 END FILL

ARA $=13$ NUX $=1$ NUY $=1$ NUZ $=2$
COM $=$ COMBINATION OF NORTH CORE WITH BOTTOM REFLECTOR'

FILL 159 END PILL

ARA $=14$ NUX $=1$ NUY $=3$ NUZ $=1$
COM='NORTH CORE WITH SIDE REFLECTORS '

PILL $2138 \quad 21$ END FILI

$A R A=15$ NUX $=2$ NUY $=1$ NUE $=1$

COH= ' NORTH CORE WITH END REFLECTOR'

PILL 3139 END FILL

ARA $=16$ NUX $=1$ NUY $=1$ NUZ $=2$
COM= ' NORTH CORE WITH TOP REFLECTOR'

FILL $40 \quad 25$ END PILL

$A R A=20$ NUX $=1$ NUY $=1$ NUZ $=2$

COM=' SOUTH CORE WITH BOTTOM REPLECTOR'

FILL 1910 BND FILL

$A R A=21 \quad N U X=1 \quad N U Y=3 \quad N U Z=1$

REPECTORS '

FILI $2348 \quad 23$ END FILL

COM=' SOUTH CORE WITH END REFLECTOR'

FILL 4933 BND PIL

$A R A=23 \quad N U X=1 \quad N U Y=1 \quad N U Z=2$

COM $=$ COMBINATION OF CORE WITH TOP REFLECTOR'

FILI 5027 END FILL

PILL 425452 END PILL

COM='ASSBMBLY OF FUEL BOX FRONT AND REAR'

PILL 2 3 END FILL

$A R A=27$ NUX $=1$ NUY $=1$ NUZ $=3$
COM $=\cdot$ ASS BMBLY OF FUEL BOX TOP AND BOTTON

CIIL 5 4 5 BND FILI

$A R A=28$ NUX $=1$ NUY $=2$ NUZ $=1$

COM=' COMBINATION OP TWO FUEL BOXES'

FILI 77 END FILI

ARA $=29$ NUX $=1$ NUY $=2$ NUZ $=1$.

COM=' COMBINATION OF 
Table A.3 (continued)

$A R A=30 \quad$ NUX $=1 \quad$ NUY $=2 \quad$ NUZ $=1$

mistribution plates.

FIIL 35 35 END FILL

DISTRIBUTION PLATES FILL 35 34 BND PILI

END ARRAY

RAAD PLOT TTI='YZ SLICE OF SOUTH CORE FIRST ROW

$X U I=64.319$ YUL $=40.000 \quad 2 U L=72.000$

YAX $=1$ WDN $=-1$ NAX $=130$ NCH $=.012345678^{\prime}$.

TTL $=$ 'XZ SLICE OF SPHERE CUBOID

$\mathrm{XUL}=56.0$ YUI $=63.625 \quad \mathrm{ZUL}=71.735$

$X L R=72.0 \quad$ YLR $=63.625 \quad$ ZLR $=55.735$
M

UNX=1 HDH

DND PLOT

RSM=56.071 $25 P=71.4$.

END DATA

CAR15
$=$ CSAS25

ROCKY PLATS CRITICALS NUREG/CR-1653 EXPERIMENT MUMBER ? (27 GROUP MODEL FUEL CUBE $15.28 \mathrm{CM}$, U(4.46)308 H/U=1.25, HI CONC. SOLUTION DRIVEN $\begin{array}{llllllll}\text { U } 308 & 5.40788-1 & 293.092234 & 0.0392235 & 4.4692236 & 0.0892238 & 95.43 & \text { END }\end{array}$ H2O $11.5627 E^{-1}=0$

AREM-BAGGIE 1.0 3 000 O $1001 \quad 14.01601284 .98016 \quad 1.2011 .91345-2$

AND $1100 \quad 1.0 \quad 300011302799.1826000 \quad 0.529000 \quad 0.229 .53908-1$

END

NDE (YINY) 1.0700010015 .9260124591801610 .8217000

$25.73200406 .92200016920001 .121111150-201016$

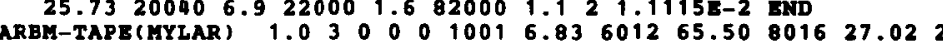

$1.7491 \mathrm{E}-2$ END

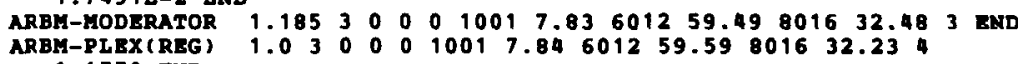

AREA-PLEX(PAPER) $\quad 1.0300010016 .48601242 .17801649 .5$

ARBA-PLEX(PAPER

ARBM-PLEX (GLUE)

$1.16488-3$ END
ARBMLEX(TRS) $1.080000110017 .16601252 .03 \quad 70140.16801629 .8$

1.03000100111 .67601286 .2980161 .20

150311.02170001181350794 .260350812 .8405
S

ARBM-PLEX (PAPER)

$3.7534 E-3$ END
M-PLEX (GLUE)

1.03000100111 .67601206 .2900161 .205

ARBM-PILLER $1.18530000010017 .83601259 .49801632 .486 \quad 0.7$ END

' THIS MATERIAL IS ACTUALLY MODERATOR ADJUSTED FOR THE VOLUME

FRACTION OF THE VOID FILLED BETHEEN CORE AND REFLECTOR.

ARBA-AL $1100 \quad 1.0 \quad 3300191302799.18260000 .529000 \quad 0.272 .715$ END

LNO2 (NO3)2 8351.180 .5491 .0293 .0922341 .0229223593 .172

922360.434922385 .372 Bnd

$5 S 30491.0$ END

DOCXY FLATS CRITICALS NUREG/CR-1653 EXPERIMENT NUMBER ? (27 GROUP MODEL FUEL CUES $15.28 \mathrm{CM}, U(4.46) 308$ H/U $=1.25$, HI CONC. SOLUTION DRIVEN READ PARM
UNIT 1 COM $=$ PUEL BOX WITH VOID AND PART OF AI BOX.

CUBOID $0199.80 \quad 0.0 \quad 3.75 \quad 0.0 \quad 14.98 \quad 0.0$

$\begin{array}{lllllllll}\text { CUBOID } & 2 & 1 & 9.95 & 0.0 & 3.90 & -0.15 & 14.98 & 0.0 \\ \text { CUBOID } & 1 & 1 & 14.98 & 0.0 & 9.365 & -5.615 & 14.98 & 0.0\end{array}$

CONIT ' PRONT OF FUEL BOX '

CUBOID $2,0.15 \quad 0.0 \quad 5.615 \quad 0.014 .98 \quad 0.0$

$\begin{array}{llllllllll}\text { CUBOID } & 2 & 1 & 0.15 & 0.0 & 5.615 & 0.0 & 14.98 & 0.0 \\ \text { CUBOID } & 0 & 1 & 0.15 & 0.0 & 9.365 & 0.0 & 14.98 & 0.0\end{array}$

CUBOID 2 1 $0.15 \quad 0.0 \quad 14.98 \quad 0.0 \quad 14.98 \quad 0.0$

UNIT 3

$\begin{array}{lllllll} & & \end{array}$

COM=' ARRAY TO ASSBMBLE PART OF BOX'

ARRAY $26 \quad 3 * 0$

UNIT 5

COK= TOP AND BOTTOM OF BOX'

$\begin{array}{lllrlllll}\text { CUBOID } & 0 & 9.80 & 0.0 & 3.750 & 0.0 & 0.15 & 0.0\end{array}$

$\begin{array}{llllllll} & & & \end{array}$

GLOBAL 6

COMT= ARRAY TO ASSEMBLS TOP AND BOTTOM

ARRAY $27 \quad 3 * 0$

$\begin{array}{lllllllll}\text { CUBOID } & 2 & 1 & 15.28 & 0.0 & 15.13 & -0.15 & 15.28 & 0.0 \\ \text { CUBOID } & 0 & 1 & 15.305 & -0.025 & 15.155 & -0.175 & 15.305 & -0.025\end{array}$

UNIT 7

COM= PUEL BOX $15.28 \mathrm{CM}$ ON A SIDE WITH $.15 \mathrm{CM}$ WALLS .05CM STACXING VOID

CUBOID 1 1 6 6P7.49

CUBOID 016 67.6650

UNIT 34

COM='ALUMINUK WEIGHT DISTRIBUTION PLATE FOR SPECIAI BOX

CUBOID 0019.800 .03 .7500 .000 .160 .0

CUBOTD 01

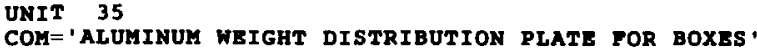

CUBOID $794 \mathrm{P7} .665 \quad 0.160 .0$

UNIT 9

COM='NORTH SPLIT TABLE CORE'

ARRAY $13 * 0.0$

CUBOID $0+30.66 \quad-0.9477 .5 \quad 0.083 .475 \quad 0.0$

COM='SOUTH SPLIT TABLE CORE'

ARRAY 2 3*0.0

CUBOID $0194.4 \quad 0.0 \quad 77.5 \quad 0.0 \quad 83.475 \quad 0.0$

UNIT 11

COM=' PLEXIGLASS REFLECTOR SHEET WITHOUT TRIS, NORTH BOTTOM REPLECTOR CUBOID $412 P 15.80002 P 38.75002 P 0.6150$

UNIT 12 COM=' PLEXIGLASS REFLECTOR SHEET WITH TRIS, NORTH BOTTOM REPLECTOR'

CUBOID 5,2 P15.8000 2838.7500 2P0.6150

COM=' UPPER PORTION NORTH BOTTOM REFLECTOR WITH TRIS'

CUBOID 512 2P15.8 2838.75 2P8.24

UNIT 14

COM='LOWER PORTION NORTH BOTTOM REPLECTOR WITH TRIS '

CUBOID 5 T 2P15.8 2P38.75 2P3.69

COH=' NORTH BOTTOM REFLECTOR INCLUDES REGULAR AND TRIS' 
Table A.3 (continued)

UNIT 16

COM=' PLEXIGLAS SHEET BOTTOM SOUTH REPLECTOR WITHOUT TRIS'

CUBOID 012 P5 12 P2 5 2P0.6150

CUBOID $494.3-5.12 P 38.75$ 2P0.615

UNIT 17

COY=' PLEXIGLAS SHEET BOTTOM SOUTH REYLECTOR WITH TRIS

CUBOID 0 1 2 2P5.1 2 2P2.5 2 PO 615

CUBOID 5 1 $44.3-5.12838 .75 \quad 2 P 0.6150$

COY=. 18 POR PORTON SOUTH BOTTOM REPLETOR MITH TRIS

CUBOID 0 1 2 2P5.1 2P2.5 2P8.855

CUBOID 5 1 $44.3-5.92838 .75$ 2P8.855

UNIT 19

作

ARRAY 20 U $3 * 0$.

COY='EAST AND WEST REFLECTORS TOR NORTH RETLECTOR WITH TRIS CUBOID 512 2P15.8 2P12.65 2P54.2825

UNIT 21

COM= ' ARRAY FOR BAST AND WEST RETLECTORS YOR NORTH REFLECTOR' ARRAY $53 * 0.0$

COM=' CUBOID $5,2824.700 \quad 2 P 12.652 P 54.2825$

UNIT 23

EAST AND WEST REPLECTORS FOR SOUTH REFLECTOR ARRAY $6 \quad 3 * 0.0$

AnTT

- NORTH TOP REFLECTOR WITH TRIS.

CUBOID 512 2828.35 2P64.05 2P12.15

COM= 'ARRAY POR NORTH TOP REFLETTOR'

ARRAY $73 * 0.0$

UNIT 26

COM='SOUTH TOP REFLECTOR WITH TRIS'

$\begin{array}{ll}0 & \\ & \end{array}$

CUBOID $5169.4-5.12 P 64.05$ 2P12.15

COH='ARRAY FOR SOUTH TOP REFLECTOR'

COM=' NORTH END REFIECTOR $9.8 \mathrm{CM}$ PORTION WITHOUT TRIS'

CUBOID 41284.90002 2P64.0500 2P54.2825

COM=' NORTH BND RELPECTOR 5.2 CK PORTION HITH RPIS'

CUBOID $51282.6 \quad 2 \mathrm{P64.0500} 2 \mathrm{P5} 4.2825$

UNIT 30

COM=' NORTH END RELFECTOR 10.1 CM PORTION WITHOUT TRIS

CUBOID 41285.052864 .052854 .2825

COMI ' ARRAY FOR NORTH END REFLECTOR

ARRAY $93 * 0,0$

UNIT 32

COM=' SOUTH END REFLECTOR'

CUBOID $512 \mathrm{P} 12.552864 .05$ 2P54.2825

UNIT 33

TOR soury and pepteron

ARRAY $10 \quad 3 * 0.0$

COME' NORTH CORE
ARRAY $13 \quad 3 * 0.0$
UNIT 39

WITH BAST AND WEST REFLECTOR'

ARRAY $14 \quad 3 * 0.0$

COM='NORTH CORE WITH BND REPLECTOR

ARRAY $15 \quad 3 * 0.0$

UNIT 42

ARRAY $16 \quad 3 * 0.0$

COM=' SOUTH CORE WITH BOTTOM REPLECTORS

ARRAY $20 \quad 3 * 0.0$

ARRAY $213 * 0.0$

UNIT 50

ARTA SOUTH CORE END REFLECTOR'

ARRAY $223 * 0.0$

COM=' SOUTH CORB WITH TOP REFLECTOR'

ARRAY $23 \quad 3 * 0.0$

UNIT 54200.0

COM='AIR GAP

$0.7320 .0-64.475-63.625 \quad 69.13-63.735$

GLOBAI

UNIT 55

COM= 'TOTAL'

ARRAY $253=0$

COM=. COMBINATION OF TWO REGUTAR TUET BOXES

ARRAY $28 \quad 3 * 0.0$ ARAY $29 \quad 3 * 0$.

UNIT 58

COM=' COMBINATION OF TWO REGULAR WEIGHT DISTRIBUTION PLATES'

ARRAY $30,3 * 0.0$

UNIT SO ARRAY $313 * 0.0$

UNIT 60

COM='NORTH CORE SOLUTION DRIVER'

CUBOID $81287.5 \quad 2 P 14.9 \quad 1.863-7.5$

CUBOID $01287.52 P 14.9287 .5$

CUBOID 012 2P7.665 2P15.33 2P7.665

UNIT 61 1 287.6652815 .33287 .665

COM='SOUTH CORE SOLUTION DRIVER'

CUBOID $81287.5 \quad 2 P 14.9 \quad 1.794-7.5$

CUBOID 012 2P7.5 $2 P 14.9287 .5$

CUBOID 912 2P7.65 2 P $15.05 \quad 2$ P7.65

CUB

END GEOMETRY

ARA $=1 \quad$ NUX $=2$ NUY $=4 \quad$ NUZ $=9$

COM=' NORTH SPLIT TABLE CORE'

FILL

2R35 2R58 4R35

2R7 2R56 4R7

2R7 56 60 4R7

$28352258 \quad 4 R 35$

2R7 2R56 4R7

2R35 2R58 4R35

(2) 
Table A.3 (continued)

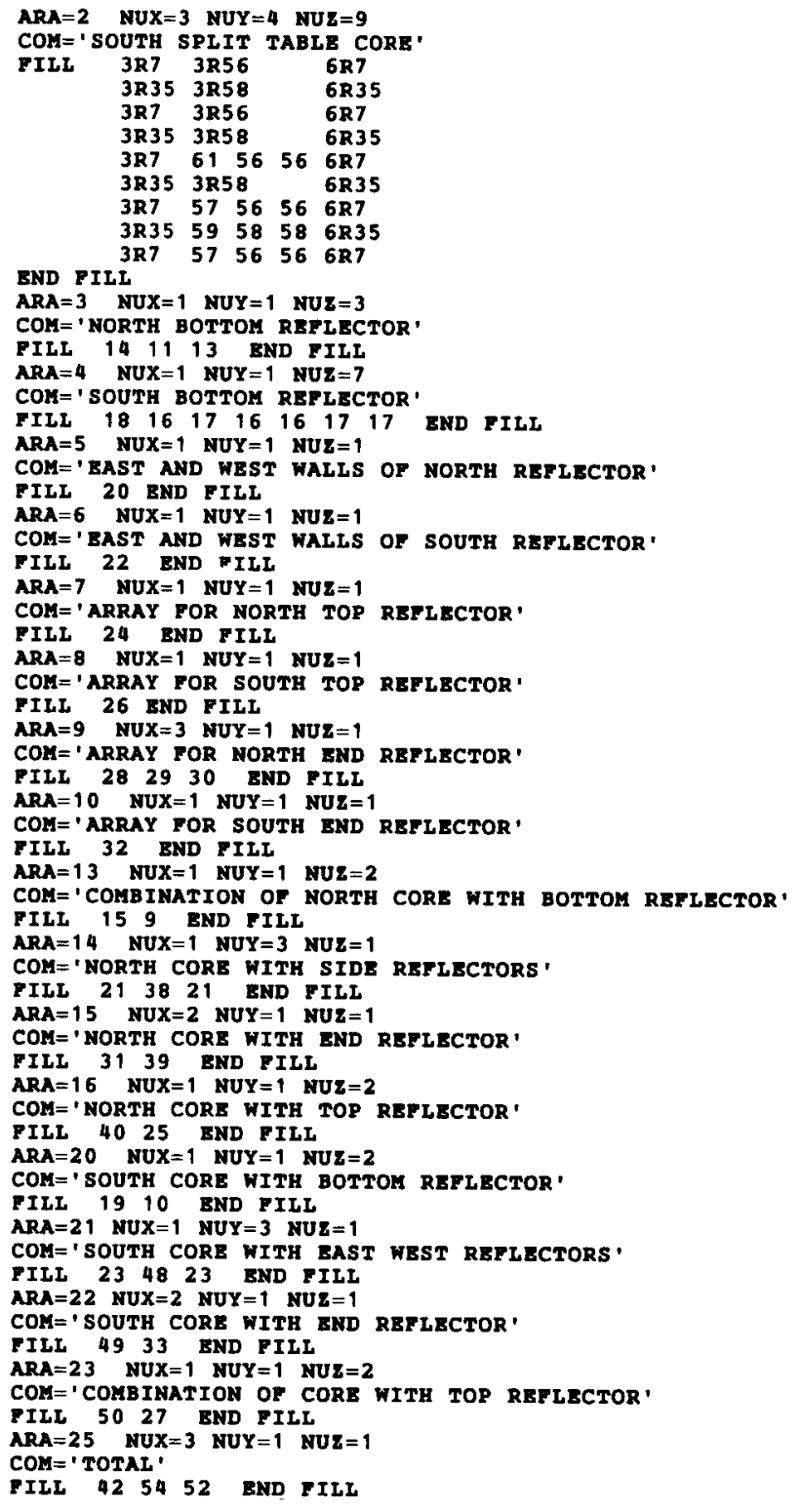

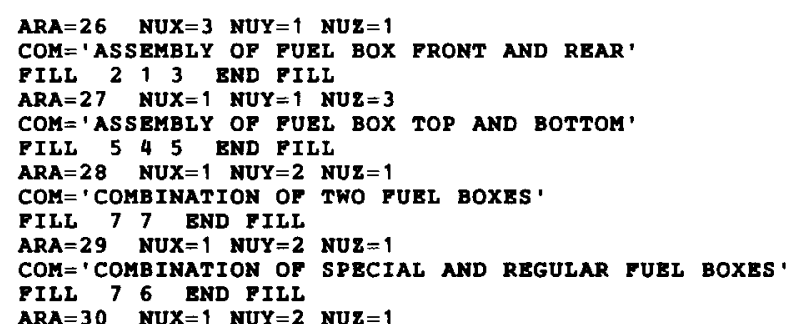

COM $=$ ' COMBINATION

FILL 35 35 END FILI

ARA $=31$ NUX $=1$ NUY $=2$ NUZ $=1$
COM $=$ ' COMBINATION OF SPECIAL AND REGULAR WEIGHT DISTRIBUTION PLATES'

FND ARPAY

READ PLOT TTL='YZ SLICE OF SOUTH CORE FIRST ROW'

$X U L=64.319$ YUL $=40.000 \quad 2 U L=72.000$

XLR $=64.319$ YLR $=72.000 \quad$ 2LR $=56.000$
VAX $=1$ WDN $=-1$ NAX $=130 \quad$ NCH $=\cdot 012345678 \cdot$ END

TTL $=$ 'XZ SLICB OP SPHERE CUBOID

$X \mathrm{~L}=56.0$ YUL $=63.625$ $20 \mathrm{~L}=71.735$

VAX $=1 \quad$ WDN $=-1 \quad \mathrm{NAX}=130 \quad \mathrm{NCH}=012345678$

END PLOT

READ START NST $=1 \quad X S M=41.37 \quad$ XSP $=72.894 \quad$ YSM $=40.63 \quad$ YSP $=71.29$

$Z S M=56.071 \quad 2 S P=71.4$ BND START

RAD START NST=1 END START BND DATA

CAR 16

ROCKY PLATS CRITICALS NUREG/CR-1653 EXPERIMENT NUMBER ? $(27$ GROUP MODEI - FUEI CUBE $15.28 \mathrm{CM}, \mathrm{U}(4.46) 308$ H/U=1.25, LOW CONC. SOLUTION DRIVEN

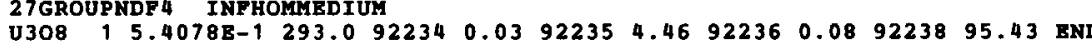

$1.03000100114 .01601284 .980161 .2011 .9134 \mathrm{E}-2$ END $1100 \quad 1.0 \quad 300011302799.1826000 \quad 0.529000 \quad 0.2 \quad 2 \quad 9.53908-1$

M-TAPE(VINYL) 1.07000010015 .92601245 .91801610 .8217000 $25.73200406 .9220001 .6820001 .921 .11158-2$ BND 10.02170

ARBM-TAPB (MYLAR) 1.03000010016 .83$$
1.74918-2 \text { BND } 1.185300010017 .83601259 .49001632 .4830
$$

ARAM

1.1773 END

ARBM-PLEX(PAPER) 1.03000010016 .48601242 .17801649 .5

$3.7534 \mathrm{E}-3 \mathrm{BND}$

ARBM-PLEX(GLUB) 1.03000100111 .67601286 .2980161 .204

$1.1648 \mathrm{~B}-3 \mathrm{BN}$

(1.0 8 00110017.16601252 .0370140 .16801629 .82

ARBM-PIEX(PAPER) 1.0300070016 .48601242 .17801649 .5

$3.75348-3 \mathrm{BND}$ 
Table A.3 (continued)

ARBM-PLEX(GLUE) 1.03000100111 .67601286 .2980161 .205

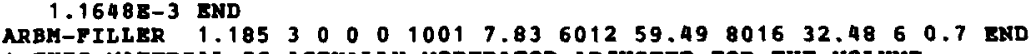
THIS MATERIAL IS ACTUALLY MODERATOR ADJUSTED FOR THE VOLUM

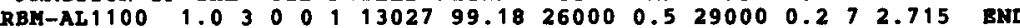
SOLNUO2(NO3)2 $8886.42 \quad 0.1491 .0293 .0922341 .0229223593 .172$ $92236 \quad 0.434922385 .372$ END

$\mathrm{ss} 304$

RND COKP

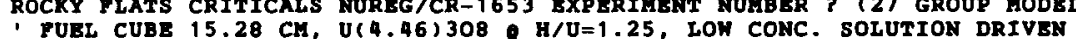
READ PARM NPG $=600$ NUB $=$ YES FDN=YES PLT $=$ YES END PARM READ GEOM

UNIT 1

COA= ' FUBL BOX WITH VOID AND PART OF AL BOX'

CUBOID 0 1 $9.80 \quad 0.0 \quad 3.75 \quad 0.0 \quad 14.980 .0$

CuBOID $119.950 .03 .905-5.61514 .980 .0$

UNIT 2 (1)

COM=' PRONT OF FUEL BOX.

$\begin{array}{lllllllll}\text { CUBOID } 2 & 1 & 0.15 & 0.0 & 5.615 & 0.0 & 14.98 & 0.0\end{array}$

CUBOID $0110.15 \quad 0.0 \quad 9.365 \quad 0.0 \quad 14.98 \quad 0.0$

$\begin{array}{lllllllll}\text { CUBOTD } 2 & 1 & 0.15 & 0.0 & 14.98 & 0.0 & 14.98 & 0.0\end{array}$

UNIT 3

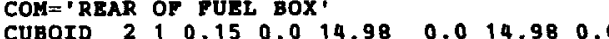

UNIT

CM=' ARRAY TO ASSEMBLE PART OF BOX

ARRAY $26 \quad 3 * 0$

UNIT 5

AOTTOM OF BOX

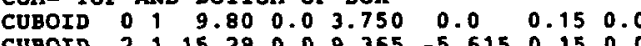

GLOBAL

UNIT

COM=' ARRAY TO ASSEMBLE TOP AND BOTTOM.

ARRAY 27 3*0

$\begin{array}{lllllllll}\text { CUBOID } & 2 & 1 & 15.28 & 0.0 & 15.13 & -0.15 & 15.28 & 0.0\end{array}$

UNIT

COM=' FUEL BOX 15.28 CM ON A SIDE WITH .15 CM WALLS .05CM STACXING VOID

CUBOID 1 1 6 PP7.49

CUBOID 21687.64

CUBOID 01687

COM= 14 ALUMINUM WEIGHT DISTRIBUTION PLATE YOR SPECIAL BOX

CUBOID $019.800 .03 .750 \quad 0.0 \quad 0.160 .0$

CUBOID $7195.33 \quad 0.09 .54 \quad-5.790 .160 .0$

UNIT 35
COA = ALURINUR WEIGHT DISTRIBUTION PLATE FOR BOXES '

CUBOID 714 P $7.665 \quad 0.160 .0$

UNIT 9

COM=' NORTH SPLIT

UNIT 10

COM=' SOUTH SPLIT TABLE CORE'

ARRAY $23 * 0$.
UNIT 11

COM= ' PLEXIGLASS REFLECTOR SHEET WITHOUT TRIS, NORTH BOTTOY RETLECTOR' CUBOID 1 2P15.8000 2P38.7500 2P0.6150

WIT 12

COM=' PLEXIGLASS REFLECTOR SHEET WITH TRIS, NORTH BOTTOM REFLECTOR' CUBOID 512 P15.8000 2838.7500 2P0.6150

COM=IUPPER PORTION NORTH BOTTOM REFLECTOR WITH TRIS'

CUBOID 5 , 2 P15.8 2838.752 P8.24

UNIT 14

COM='LOWER PORTION NORTH BOTTOM REFLECTOR WITH TRIS'

CUBOID 5 T 2 P15.8 2 P38.75 2P3.69

UNIT 15 .

ARRAY $33 \neq 0.0$

UNIT 16

COM='PLEXIGLAS SHEET BOTTOK SOUTH REFLECTOR WITHOUT TRIS

CUBOID $0112 \mathrm{P5} .12 \mathrm{2P2} .52 \mathrm{PO} .6150$

CUBOID $4144.3 \quad-5.12$ P38.75 2P0.615

COH='PLEXIGLAS SHEET BOTTOM SOUTH REFLECTOR WITH TRIS'

CUBOID 012 2P5.1 2P2.5 2 PO.6150

CUBOID

UNIT 18

COM='IOWER PORTION SOUTH BOTTOM REFLECTOR WITH TRIS.

CUBOID 012 P 5.12 2P2.5 2 P8.85

CUBID $51144.3-5.12$ 2P38.75 2P8.855

COM=' SOUTH BOTTOM REFLECTOR WITH REGULAR AND TRTS'

ARRAY $4 * 0.0$

COM='BAST AND WBST REFLECTORS FOR NORTH REFLECTOR WITU TRIS CUBOID 5 1 2P15.8 2P12.65 2P54.2825
CUAT

UNIT 21512 TOMY

ARRAY $53 * 0.0$
UNIT 22

COM=' EAST AND WEST REFLECTORS FOR SOUTH REFLECTOR WITH TRIS

CUBOID 5 I 2P24.700 2P12.65 2P5A 2825

COM=' ARRAY YOR EAST AND WEST REPLECTORS TOR SOUTH RETLECTOR' ARRAY $6 \quad 3 * 0.0$

COM=' NORTH TOP REFLECTOR WITH TRIS'

CUBOID
UNIT 25

COM=.

ARRAY $73 * 0.0$

COYT' 26 SOUTH TOP REPLECTOR WITH TRIS'

CUBOID 0 1 2 P $5.12 \mathrm{P} 2.52 \mathrm{P} 12.15$

CUBOID $5169.4-5.92 P 64.05 \quad 2 P 12.15$

UNIT 27

COM=' ARRAY FOR SOUTH TOP REFLECTOR.

ARRAY $83 * 0.0$

COM=' NORTH END REFLECTOR $9.8 C M$ PORTION WITHOUT TRIS'

CUBOID $1284.90002 P 64.05002854 .2825$

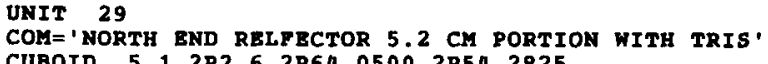

CUBOID 512 P2.6 2P64.0500 2P54.2825

COM=' NORTH END RELFECTOR $10.1 \mathrm{CM}$ PORTION WITHOUT TRIS'

CUBOID 4 1 2P5.05 2P64.05 2 P54.2825 
Table A.3 (continued)

UNIT 31 COH= ARRAY FOR NORTH END REFLECTOR'

ARRAY $93 * 0.0$

COH $=$. SOUTH END RETLECTOR'

CUBOID $5,2 \mathrm{P} 12.552 \mathrm{P64.05} 2 \mathrm{P5} 4.2825$

UNIT 33
COM=' ARRAY FOR SOUTH END REFLECTOR'

ARRAY $10 \quad 3 * 0.0$

COMT 38 . 38 .

ARRAY 13 3* 0.0

UNIT 39

CAST AND WEST RETLECTOR

ARRAY $14 \quad 3 * 0.0$

UNIT 40

COM= NORTH CORE NITH END REFLECTOR'

ARRAY $15 \quad 3 * 0.0$

TOP PEFIECTOR

COM= NORTH CORE

ARRAY $16 \quad 3 * 0.0$

COH = SOUTH CORE WITH BOTTOM REPLETORS

ARRAY $20 \quad 3 * 0.0$

COM='

ARRAY $213 * 0.0$

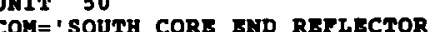

COM $=$ ' SOUTH CORE
ARRAY $22 \quad 3 * 0.0$

UNIT 52 .

ARRAY $233 * 0.0$

GNIT 54 GAP

$\begin{array}{lllllllll} & & \end{array}$

GLOBAL 55

COM= 'TOTAL,

ARRAY 25

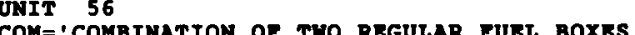

ARRAY $28 \quad 3 * 0.0$

UNIT 57

OF REGUTAR PUEL BOX AND SPECTAI TUEI BOX

ARRAY 29 3*0.0

UNIT 58 COM=' COMBINATION OF TWO REGULAR WEIGHT DISTRIBUTION PLATES ARRAY $30 \quad 3=0.0$

UNIT 59

COM=' COMBINATION OF REGULAR AND SPECIAL MBIGHT DISTRIBUTION PLATES

ARRAY $31 \quad 3 \neq 0.0$

UNIT 60

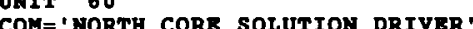

CUBOID $81287.5 \quad 2814.93 .332-7.5$

CUBOID 01287.5 2P14.9 287.5

CUBOID $91287.652815 .052 P 7.65$

CUBOID $01287.665 \quad 2 P 15.33 \quad 2 P 7.665$

UNIT 61

COM=' SOUTH CORE SOLUTIOM DRIVER'

CUBOID 8 1 2P7.5 2P14.9 3.292 -7.5

CUBOID $01287.52 P 14.9287 .5$

CUBOXD $11287.652 \mathrm{P} 15.052 \mathrm{P7} .65$

END GEOKETRY

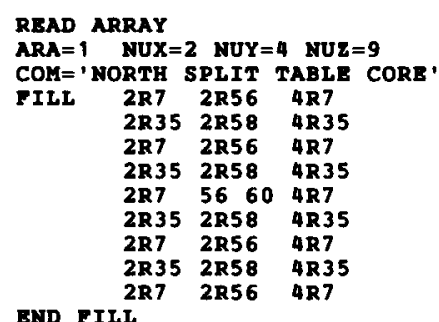

END FIL

$A R A=2$ NUX=3 NUY $=4$ NUZ $=9$

COM=' SOUTH SPLIT TABLE CORE'

FIL $\quad 3 R 7 \quad 3 R 56 \quad 6 R 7$

$\begin{array}{lll}3 R 355 & 3 R 58 & 6 R 35 \\ 3 R 7 & 3 R 56 & 6 R 7\end{array}$

$3 R 353 R 58 \quad 6 R 35$

$\begin{array}{lllll}3 R 7 & 61 & 56 & 56 & 6 R 7 \\ 3 R 35 & 3 R 58 & 6 R 7\end{array}$

$\begin{array}{llll}3 R 35 & 3 R 58 & 6 R 35 \\ 3 R 7 & 5756 & 56 & 6 R 7\end{array}$

$\begin{array}{lllll}3 R 7 & 57 & 56 & 56 & 6 R 7 \\ 3 R 35 & 59 & 58 & 58 & 6 R 35 \\ 3 R 7 & 57 & 56 & 56 & 6 R 7\end{array}$

BND FILI

$A P A=3$ YUX $X=1$ NUY $=1$ NUE $=3$

COM=' NORTH BOTTOM REFLECTOR'

FILL $14 \quad 11,13$ END FILL

ARA $=4$ NOX $=1$ NUY $=1$ NUZ $=7$

COM=' SOUTH BOTTOM REFLECTOR'

CIND FIII WALE

soury

COM= BAST AND WEST

FILA 22 END FILL

COM=' ARRAY FOR NORTH TOP REPLECTOR

FILI 24 END FILL

$X=1$ NUY $=1$ NUY $=1$

COY =' ARRAY FOR SOUTH TOP REFLECTOR'

COMA $=9$ NUX $=3$ NUY $=1$ NUZ $=1$ REPLECTOR

FILI 282930 BND FILL

COY='ARRAY. FOR SOUTH END RETLECTOR'

FILI 32 END

COM $=13$ NUX=1 NUY=1 NUZ=2 CORE WITH BOTTOM REFLECTOR'

FILI 159 END FILI

$A R A=14 \quad$ NUX $=1 \quad$ NUY $=3$ NUZ $=1$

COM=' NORTH CORE WITH SIDE RETLECTORS'

FILI $2138 \quad 21 \quad$ END FILI

COM =' NORTH CORE WITH END REPLECTOR'

FILI 3139 END FILL

$A R A=16 \quad N U X=1 \quad N U Y=1 \quad N U Z=2$

COM='NORTH CORE WITH TOP RETLECTOR'

FILL $40 \quad 25$ END FILL

COM=' SOUTH CORE TITH BOTTOM REPLECTOR'

COM=' SOUTH CORE WITH 
Table A.3 (continued)

ARA $=21 \quad$ NUX=1 NUY=3 NUZ=1

COM=' SOUTH CORE WITH EAST HEST REFLECTORS

FILI $2348 \quad 23$ BND FILL

ARA $=22$ NUX $=2$ NUY $=1$ NUZ $=1$
COM $=$ ' SOUTH CORE WITH END REFLECTOR

COM=' SOUTH CORE WITH

FILI 4933 BND FILL

COM=' COMBINATION OF CORE WITH TOP REPLECTOR'

PILI 5027 END FILL

$A R A=25 \quad N U X=3 \quad$ NUY $=1 \quad$ NUZ $=1$

COM=' TOTAL'

FILL 425452 END FILLL

COM=1 ASSOMBIY OP TUEZ BOX FRONT AND REAR'

FILI 213 END FILL

$A R A=27 \quad N U X=1 \quad N U Y=1 \quad N U Z=3$

COM=' ASSEMBLY OF FUEL BOX TOP AND BOTTOM"

PILL 545 END FILL

ARA $=28$ NUX $=1$ NUY $=2$ NUZ $=1$.

COM= COMB

PILL 77 BND FILI

COM=' COMBINATION OF SPECIAL AND REGULAR FUEL BOXES

PILL 76 BND FILL

ARA $=30 \quad$ NUX $=1$ NUY $=2 \quad$ NUZ $=1$

COM=' COMBINATION OF TWO

FILL 3535 END FILL

COM=' COMBIMATION OF SPBCIAL AND REGUIAR MEIGHT DISTRIBUTION PLATES

PILL 3534 END FILI

BND ARRAY

READ PLOT TTL='YZ SLICE OF SOUTH CORE FIRST ROW'

$X U L=64.319$ YUL $=40.000 \quad$ $\quad$ UUL $=72.000$

$X L R=64.319$ YLR $=72.000 \quad$ ZLR $=56.000$

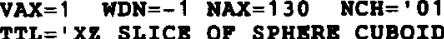

$X U L=56.0$ YUL $=63.625 \quad \mathrm{ZUL}=71.735$

$X L R=72.0 \quad$ YLR $=63.625 \quad$ QLR $=55.73$

UAX $=1 \quad$ WDH $=-1 \quad$ NAX $=130 \quad$ NCH $=.012345678$

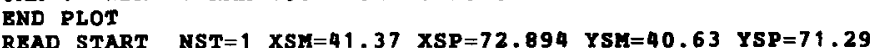

ZSM $=56.071$ \&SP $=71$. 4 END START

BND DATA
CAR17

$=\operatorname{CSAS} 25$

ROCKY FLATS CRITICALS NUREG/CR-2500 EXPERIMENT F (27 GROUP)

48 FUEL CANS 0.929 CM MODERATOR GEE. HU203. DATA (UNDER)

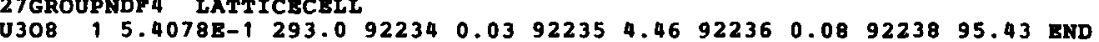

ARBM-BAGGIE $1.03000100114 .01601284 .980161 .2011 .9134 \mathrm{E}-2$

ARBM-AL1100 $1.0 \quad 3 \quad 0 \quad 0 \quad 1 \quad 1302799.1826000 \quad 0.5 \quad 29000 \quad 0.2 \quad 2 \quad 9.53908-1$

END

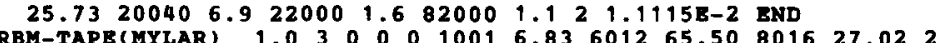

$1.7491 \mathrm{~B}-2$ END

ARBM-MODERATOR 1.18533000010017 .83601259 .498016

ARBM-PLEX (REG) $\quad 1.03000010017 .84601259 .59801632 .234$

1.1773 END

1.03000010016 .48601242 .17801649 .5

3.75348-3 END

$1.1648 \mathrm{E}-3$ END
1.

3000100111.67601286 .2980161 .20

(FLEX(TRIS) $1.08000110017 .16601252 .03 \quad 70140.16801629 .82$

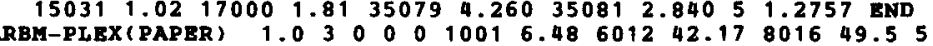

作

1.03000100111 .67601286 .2980161 .205

$1.1648 \mathrm{E}-3$ END

ARBM-FILLER $1.185 \quad 3 \quad 0 \quad 0 \quad 0 \quad 1001 \quad 7.83 \quad 601259.498016 \quad 32.4861 .0$ END

THIS MATERIAL IS ACTUALLY MODERATOR ADJUSTED FOR THE VOLUME

FRACTION OF THE VOTD FILIED BETWEEN CORE AND REPLECTOR.

END COMP

SPHERICAL VACUUM REFLECTED 0.0 END

19.2929 ONEEXTERMOD 3 10.0 NOEXTERMOD END ZONE

ROCXY PLATS CRITICALS NUREG/CR-2500 EXPERIMENT F (27 GROUP)

48 PURL CANS 0.929 CM MODERATOR GEE. HU203.DATA(UNDER)

READ PARM RUN $=$ YES NPG $=600$
PLT $=$ NO NUB $=$ YES FDN $=$ YES

READ GEOV

READ GEO

COM=' PUEL BOX $15.28 \mathrm{cM}$ ON A SIDB WITH $.15 \mathrm{~cm}$ WALLS .05CM STACKING VOID

CUBOID 1116 6P7.49

CUBOID 001687.665

COM='X-FACB INTERSTITIAL MODERATOR'

CUBOID 312 PO.4645 4P7.665

COM='Y-PACE INTERSTITIAI MODERATOR'

$\begin{array}{lll}\text { CUBOID } 31 & \text { 2P7.665 2P0.4645 2P7.665 }\end{array}$

UNIT

COM='Z-FACE INTERSTITIAL MODERATOR'

CUBOID

COM=1 $50 \mathrm{~g}$ -

CUBOID 3 , 4 PO.4645 2P7.665

UNIT 6

COM=' MORB Y-PACE MODERATOR'

CUBOID 3 T 2P7.665 4P0.4645

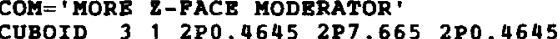


Table A.3 (continued)

UNIT 8

COM = LAST OF INTERSTITIAL MODERATOR

CUBOID 316 PO 0.4645

SPTH TABLE CORE

ARRAY $13 * 0$.

REPLICATE $010.0 \quad 0.0 \quad 0.0 \quad 0.0 \quad 17.8910 \quad 0.0$

UNIT 10

COM='SOUTH SPLIT TABLE CORE'

ARRAY $23 * 0.0$

10.00 .00 .00 .017 .89100 .01

COMT = PLEXIGLASS REFLECTOR SHEET WITHOUT TRIS, NORTH BOTTOM REFLECTOR

CUBOID 412 P15.8000 2P38.7500 2PO.6150

UNIT 12

COM=' PLEXIGLASS REFLECTOR SHEET WITH TRIS, NORTH BOTTOM REFLECTOR'

CUBOTD 512 2P15.8000 2P38.7500 2PO.6150

CNIT 13 UPRE PORTION MORTH BOTTOK REFIECTOR WITH TRIS

CUBOID 512 P15.8 2 P38.75 2PB.24

UNIT 14

COM='LOWBR PORTION NORTH BOTTOK REPLECTOR YITH TRIS'

CUBOID 5 1 2P15.8 2P38.75 2P3.69

UNIT 15

TERLCTOR INCLUDES REGULAR AND TRIS

ARRAY $33 * 0$.

COM - PLEXIGLAS SHEET BOTTOM SOUTH REFLECTOR WITHOUT TRIS

CUBOID $012 \mathrm{P} 5.12 \mathrm{P2} 2.55 \quad 2 \mathrm{PO} 0.615$

CUBOID 4 1 $44.3-5.12$ P38.75 2P0.615

UNIT 17

COM=' PLEXIGLAS SHEET BOTTOK SOUTH REELECTOR WITH TRIS

CUBOID $012 \mathrm{P5}, 1$ 2P2.55 2P0.615

CUBOID

COM='LOWRR PORTION SOUTH BOTTOM REFIECTOR WITH TRIS

CUBOID $0112 \mathrm{2P} 5.12 \mathrm{2P2} .55$ 2P8.85

CUBOID 5 1 $44.3-5.12838 .75$ 2P8.855

UNIT 19

CONT

ARRAY $4 \quad 3 * 0$.

COY=' BAST AND WEST REFLECTORS FOR NORTH RETLECTOR WITH TRIS

CUBOID $512 \mathrm{P} 15.82 \mathrm{P} 12.652 \mathrm{P} 54.2825$

UNIT 21 COM=. ARRAY FOR BAST AND WEST REFLECTORS FOR NORTH REPLECTOR,

ARRAY 5 3*0.0

COMH. CUBOID 512824.7002812 .652854 .2825

UNIT 23

DATT AND WEST REPLECTORS FOR SOUTH REPLECTOR

ARRAY $63 * 0.0$

UNTT $24=0.0$

COK= ' NORTH TOP REFLECTOR WITH TRIS

COMT = 25 ARRAY FOR NORTH TOP REPLECTOR'

ARRAY $73 \neq 0.0$

UNIT 26

COM='SOUTH TOP REFLECTOR WITH TRIS

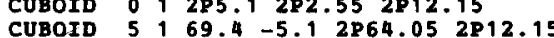

UNIT 27

TOR SOUTH TOP RETLETOR

COM=' NORTH END REFLBCTOR $9.8 \mathrm{CM}$ PORTION

UNIT 29 T2 29.90002 P64.0500 2 P54.2825

CUBOID 512 2P2.6 2P64.0500 2P54.2825

UNIT 30

COM='NORTH END RELFECTOR $10.1 \mathrm{CM}$ PORTION WITHOUT TRIS'

CUBOID 412 2P5.05 2P64.05 2P54.2825

UNIT 31 COM = ARRAY FOR NORTH END REFLECTOR

COM= ARRAY FOR
ARRAY $93 * 0.0$

COH= I SOUTH END PEFLECTOR

CUBOID 512 2P12.55 2P64.05 2P54.2825

UNIT 33

COM='ARRAY FOR SOUTH END REFLECTOR'

ARRAY $10 \quad 3 * 0.0$

COMT 12.95 THICK MODRRATING PLASTIC NORTH CORE

CUBOID 6 1 2P15.7945 2P6.6965 2P32.8695

COM= 2.95 THICK MODERATING PLASTIC NORTH CORE'

CUBOID 0112 P15.7945 2P38.75 2P1.5545

$\begin{array}{llll}\text { UNIT } & 36 & & \\ \text { ARRAY } & 11 & 3 * 0.0\end{array}$

ARRAY 11

ARRAY $12 \quad 3 * 0.0$

UNIT 38

COM=' NORTH CORE WITH BOTTOM REFLECTOR

ARRAY $13 \quad 3 * 0.0$

UNIT 39

COM=' NORTH CORE WITH EAST AND WEST REPLECTOR'

ARRAY $14 \quad 3 * 0$.

COM=' NORTH CORE WITH END REFLECTOR'

ARRAY $15 \quad 3 * 0.0$

UNIT 41

COM=' NORTH SPLIT TABLE FACEPLATE FOR THIS CASE AIR GAP HALP THICX' CUBOID 0,2 P0.1025 2P64.0500 2P66.4325

UNIT 42

.

ARRAY $16 \quad 3 * 0.0$

COM=' NORTH CORE WITH FACEPLATE'

ARRAY $17 \quad 3 * 0.0$

COM=.12.95 CM THICK MODERATOR SOUTH CORE'

CUBOID ${ }^{6} 12 \mathrm{P} 15.79452 \mathrm{P} 6.69652 \mathrm{P} 32.869$

COM= 2.95 THICK MODERATOR SOUTH CORE'

CUBOID $0112815.7945 \quad 2 P 38.752 P 1.5545$

COM=' COMBINATION OF CORE WITH 12.95 THICX MODERATOR'

ARRAY $18 \quad 3 * 0.0$

COMT COMBINATION OF CORE WITH 2.95 THICK MODERATOP

ARRAY $19 \quad 3 * 0.0$

UNIT 48

COM='SOUTH CORE WITH EAST WEST REFLECTORS

ARRAY $20 \quad 3 * 0.0$ 
Table A.3 (continued)

UNIT 49

CON=' SOUTH CORE WITH EAST WEST REPLECTORS

ARRAY $21 \quad 3 * 0.0$

COM $=$ ' SOUTH CORE END REFLECTOR'

ARRAY $22 \quad 3 * 0.0$

COM= ' FACEPLATE FOR SOUTH SPLIT TABLE CUBOID $31280.4645 \quad 2864.0500$ 2P66.4325 UNIT 52 TOM TOP REFLECTOR

COM $=$ S SOUTH CORE
ARRAY $23 \quad 3 * 0.0$

UNIT 53

' SOUTH CORE WITH FACEPLATE

ARRAY $24 \quad 3 * 0.0$

CUBOID 012 P0.1025 2P64.0500 2P66.4325

GLOBAI

UNIT 55

UNIT 56

COM = ' NORTH BOTTOM MODBRATOR

CUBOID 61 2P15.8000 2P38.7500 2P7.3135

UNIT 57 COM $=$ BOTTOM SOUTH MODERATOR'

CUBOID 612 2P24.700 2P38.75 2P7.3135

COM=. REAR FILTER

CUBOID $6 \quad 1$ 2P8.9055 2P38.75 2P34.424

UNIT 59

COM $=$ ' COMBINATION OF SOUTH CORE WITH REAR PILLER

ARRAY $26 \quad 3 * 0.0$

COM= NORTH CORE REAR FIITER

CUBOID $612 P 0.0055 \quad 2 P 38.75 \quad 2 P 34.424$

COM= ' COMBINATION OF NORTH CORE WITH REAR FILLER

ARRAY $273 * 0.0$

END GEOMET:Y

ARA $=1$ NUX $=3$ NUY $=7$ NUE

FIIL $12,3553206,20$

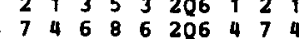

$\begin{array}{lllllllll}2 & 1 & 3 & 5 & 3 & 206 & 1 & 2 & 1 \\ \text { END FILI }\end{array}$

$A R A=2 \quad N U X=3 \quad N U Y=7 \quad N U Z=5$

COM=' SOUTH SPLIT TABLE CORE'

$\begin{array}{llllllllll}4 & 2 & 1 & 3 & 5 & 3 & 206 & 1 & 2 & 1 \\ 4 & 7 & 4 & 6 & 8 & 6 & 206 & 4 & 7 & 4\end{array}$

1042

12135532061221 END FILI

$A R A=3 \quad$ NUX $=1$ NUY $=1$ NUZ $=4$

CMI = 'NORTH BOTTOM MODBRATOR AND REFLECTOR'

PILL 14111356 END FIL

COM =' SOUTH BOTTOM MODBPATOR AND RETLECTOR'

FILL $18 \quad 16 \quad 17 \quad 16 \quad 16 \quad 17 \quad 17 \quad 57 \quad$ END FILI

$A R A=5$ NUX $=1$ NUY $=1$ NUZ $=1$

COM='BAST AND WEST WALLS OF NORTH RBFLECTOR'
ARA $=6 \quad$ NUX $=1 \quad$ NUY $=1 \quad$ NUZ $=1$

NOUTH RETLECTOR

PILL 22 END FILL

COM=' ARRAY TOR YORTH TOP REFLECTOR

ILE 24 END NORTH

ARA $=8$ NUX $=1$ NUY=1 $N U=1$

COM='ARRAY FOR SOUTH TOP REFLECTOR'

PILL 26 END FILI

COM=9 NUX $=3$ NUY $=1$ NUZ $=1$

COM=' ARRAY FOR NORTH END

ARA $=10$ NUX $=1$ NUY $=1$ NUZ $=$ ?

COM=' ARRAY FOR SOUTH END REFLECTOR'

PILL 32 END PILI

COM=' COMBINARION OF CORE WITH $12.95 \mathrm{CM}$ THICK MODERATOR'

FILL 934 END FILL

$A R A=12$ NUX $=1 \quad$ NUY $=1$ NUZ $=2$.

COM=' COMBINATION OF $P$ TEV

PILL 36 BS BND FILL

COM=' COMBINATION OF NORTH CORE WITH BOTTOM REFLECTOR'

FILL $15 \quad 61$ BND FILI

ARA $=14 \quad$ NUX $=1 \quad$ NUY $=3$ NUZ $=1$

COM=' NORTH CORE WITH SIDE REFLECTORS

PILL 213821 BND FILL

DITH BND PECTOR

FILL 3939 END FILI

$A R A=16 \quad N U X=1 \quad N U Y=1 \quad$ NUZ $=2$

COM=' NORTH CORE WITH TOP REFLECTOR'

PILL 4025 BND PILL

COM=' NORTH CORE WITH NAZ $=1$

FILL 42 41 END PILI

CORE WITH $12.95 \mathrm{CM}$ THICK MODERATOR

FILI 1044 END PILL

ARA $=19$ NOX $=1$ NUY $=1$ NUZ $=2$

COM=' COMBINATION OF CORE WITH $2.95 \mathrm{cM}$ THICK MODERATOR'

FILL 4645 END FILL

COM=' SOUTH CORE WITH BOTTOM REFIECTOR'

PILL 1959 END FILL

ARA $=21$ NUX=1 NUY $=3$ NUZ $=1$

COM='SOUTH CORB WITH BAST WEST REFLECTORS

PILL 234823 END FILL

COM=' SOUTH CORE WITH END REFLECTOR'

FILL 4933 BND PILL

$A R A=23$ NUX $=1 \quad N U Y=1 \quad$ NUZ $=2$

COM $=$ ' COMBINATION OF CORE WITH TOP REPLECTOR'

PILL 5027 END FILL

$A R A=24$ NUX $=2$ NUY $=1$ NUZ $=1$

COM=' SOUTH CORE WITH PACEPLATE'

$A R A=25$ NUX $=3$ NUY $=1$ NUZ $=1$

COM= 'TOTAI

FILL 435453 END PIL

IRA $=26$ NUX $=2$ NUY $=1$ NUZ $=1$

COM=' COMBINATION OF SOUTH CORE WITH REAR FILLER' 
Table A.3 (continued)

ARA $=27$ NUX $=2$ NUY $=1$ NUZ $=1$
COM=' COMBINATION OF NORTH CORE WITH REAR FILLER' FILL 3760 END FIL

END ARRAY

READ PLOT TTL $=$ 'X2 SITCE OF RPPF SHOWING MATERIAI REGIONS

$X U L=-1$ YUL $=64.05 \quad 2 U L=135$

$\mathrm{UAX}=1$ WDN $=-1 \quad$ NAX $=130 \quad \mathrm{NCH}=.0123456$, END

TTS $=1$ YZ SHICE OF NORTH CORE

$X U L=48 \quad Y U L=-2 \quad Z U L=136$

XLR=48 YLR=136 \&LR=-2

VAX $=1$ WDN $=-1 \quad$ NAX $=130 \quad \mathrm{NCH}=10123456^{\prime} \cdot \mathrm{END}$

TTL $=$ YZ SLICE OF NORTH CORE SECOND ROW'

$\begin{array}{lll}X U L=28 & Y U L=-2 & Z U L \\ X I R & =13\end{array}$

YAX $=1$ WDY $=-1$ MAX $=130$ NCB $=10123456^{\prime}$ ' BND

TTL $=1 Y 2$ SLICE OF SOUTH CORE PIPST ROW"

$X U L=62 \quad Y U L=-2 \quad Z U L=136$

XLR=62 YLR=136 ZLR=-2

$=130$ NCH $=0123456 \cdot \mathbf{E N D}$

TTL $=$ TOP VIEW OP SOURCE SLOT

$X U L=-2 \quad Y U L=136 \quad$ ZUL $=125$

UNX $=1 \quad$ VDN $=-1 \quad$ MAX $=130 \quad \mathrm{NCH}=.01234566^{\prime}$ END

TTL=' TOP VIEW OF TOP LAYER'

$X U L=-2 \quad Y U L=136 \quad 2 U L=95$

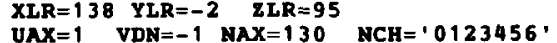

UNX $=1$ VD

READ START NST $=1$

$X S P=108.0$ XSM $=10$.

$Y S P=108.0 \quad Y S M=10.0$

$\mathrm{ZSP}=123.0 \mathrm{ESM}=44.0$

END START

END DATA
CAR 18

ROCKY FLATS CRITICALS NUREG/CR-2500 EXPERIMENT G 127 GROUP

- 30 FUEL CANS 2.44 CH MODERATOR GEE.HU203.DATA (OPTUM)

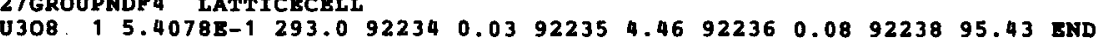
$12.6356 \mathrm{~B}-1$ (1)

ARBM-BAGGIE $1.030001001 \quad 14.01601284 .980161 .2011 .9134 \mathrm{E}-2$

ARBM-AL1100 1.0 3 $0011302799.1826000 \quad 0.529000 \quad 0.229 .5390 \mathrm{E}-1$

END

ARBM-TAPE(VINYL) $\quad 1.07000010015 .92601245 .918016 \quad 10.82 \quad 17000$

$25.73200406 .9220001 .6820001 .121111155-2$ END

627.022

$1.7491 \mathrm{E}-2$

ARBM-RODERATOR 1.1853000010017 .83601259 .49801632 .483 END

ARBM-PIEX (REG)

ARBH-PLEX(PAPER 1.0300010016 .48601242 .17801649

ARBM-PLEX(TRIS) 1.08000110017 .16601252 .0370140 .16801629 .82

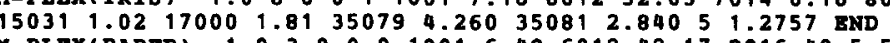

ARBM-PLEX(PAPER) $\quad 1.03000010016 .48601242 .17801649 .55$

3.7534E-3 END
ARBM-PLEX(GLUE) $1.030001001 \quad 11.67601286 .2980161 .205$

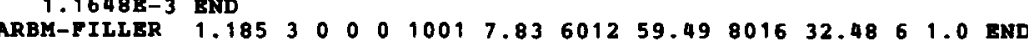

THIS MATERIAL IS ACTUALLY MODERATOR ADJUSTED FOR THE VOLUME

- PRACTION OF THE VOID FILLED BETWEEN CORE AND REFIECTOR.

END COMP

SPHTRIANGP $19.0018 .5857 \quad 1 \quad 3 \quad 18.95792$ END

ROCKY FLATS CRITICALS NUREG/CR-2500 EXPERIMENT G $(27$ GROUP

' 30 FUEI CANS 2.44 CM MODERATOR GEE. HU203.DATA (OPTUM)

END PARM

UNIT 1

COM=' FUEI BOX $15.28 \mathrm{cM}$ ON A SIDE WITH $.15 \mathrm{CM}$ WALLS .05CM STACKING VOID'

CUBOID 1 1 687.49

CUBOID 211687.64

UNIT 2

COM=1X-FACE INTEPSTITIAL MODERATOR.

CUBOID $312 P 1.2200 \quad 4 P 7.665$

UNIT 3

COM='Y-FACE INTERSTITIAL MODERATOR'

CUBOID 31287.665 2P1.2200 2P7.665

UNIT

COM='Z-FACE INTERSTITIAL MODERATOR

UNIT 5

COM=' RORE X-FACE MODERATOR'
CUBOID 31 4P1.2200 2P7.665

UNIT 6

COM='MORE Y-PACE MODERATOR'

CUBOID

COM=' MORE Z-FACE MODERATOR'

CUBOID $312 P 1.2200 \quad 2 P 7.665 \quad 281.2200$

UNIT 8 COM= 'LAST OF INTERSTITIAL MODRRATOR'

CUBOTD 1 (121.2200 
Table A.3 (continued)

UNIT 9

COM= ' NORTH SPLIT TABLE CORE

COM=' SOUTH SPLIT TABLE CORE'

ARRAY $23 * 0.0$

COM='PLEXIGLASS REPLECTOR SHEBT WITHOUT TRIS, NORTH BOTTOM REPLECTOR CUBOID 412 P16.5500 2P38.7500 2P0.6150

, NORTH BOTTOM REFLECTOR'

CUBOID 512 P16.5500 2P38.7500 2P0.6150

UNIT 13
COM=' UPPER PORTION NORTH BOTTOM REPLECTOR WITH TRIS

CUBOID 5 , 2P16.5500 2P38.75 2P8.24

UNIT 14

COM =' LOWER PORTION NORTH BOTTOM REFLECTOR WITH TRIS

CUBOID 5 1 2816.55002838 .75283 .69

UNIT 15

COM=' NORTH BOTTOM REPLBCTOR INCLUDES REGULAR AND TRIS' ARRAY $33 * 0.0$

COY='PIEXIGLAS SHEET BOTTOM SOUTH REFLECTOR WITHOUT TRIS'

CUBOID 0 1 $12 \mathrm{P5} .12 \mathrm{P2} .55 \quad 2 \mathrm{PO} 0.615$

CUBOID 4 1 $144.3 \quad-5.12$ 2P38.75 2 PO.615

UNIT 17

COM=' PLEXIGLAS SHEET BOTTOM SOUTH REPLECTOR WITH TRIS

CUBOID 0 1 2 P5.1 2 P2.55 2P0.615

CUBOID 18

COM='LOWRR PORTION SOUTH BOTTOM REFLECTOR WITH TRIS'

UBOID $O 1928$

CUBOID $5144.3 \quad-5.12838 .75 \quad 288.855$

UNIT 19

ARRAY $4 \quad 3 * 0$.

UNIT 20

PLECTOR WITH TRIS

CUBOID 512 P16.5500 2P12.65 2P52.782

COM=' ARRAY POR EAST AND WEST REPLECTORS FOR NORTH RETIECTOR ARRAY $5 \quad 3 * 0$.

COM=' EAST AND WEST REPLECTOPS FOR SOUTH PEFLECTOR WITH TRIS CUBOID $512 \mathrm{P24.7002P12.652P52.7825}$

UNIT 23 COMRAY YOR EAST AND WEST REFLECTORS FOR SOUTH RETLECTOR' ARRAY $63 * 0.0$

COM= NORTH TOP REPLBCTOR 41 TH TRIS

CUBOID 5 1 2 P29.1 2 P64.05 2 P12.15

COMT 2 ARRAY FOR NORTH TOP REFLECTOR

ARRAY $73 * 0.0$

COMI'SOUTH TOP REFIECTOR WITH TRIS '

COM $=$ ' SOUTH TOP REFLECTOR WITH TRI
CUBOID 0 , 2 P5.1 2P2.55 2P12.15

CUBOID $5169.4-5.12 P 64.05$ 2P12.15

UNIT 27

COM=' ARRAY FOR SOUTH TOP REPLECTOR

ARRAY $83 * 0.0$

COM=' NORTH BND REFLECTOR 9.8CM PORTION WITHOUT TRIS'

COM= NORTH BND REPLECTOR 9.8CM PORTION WITH
CUBOID 4 1 2P4.9000 2P64.0500 2P52.7825
UNIT 29 COM=' NORTH END RELPECTOR 5.2 CM PORTION WITH TRIS'

CUBOID 51282.62864 .05002852 .7825

UNIT 30

COM='NORTH END RELPECTOR $10.1 \mathrm{CM}$ PORTION WITHOUT TRIS

CUBOID 4 1 2P5.05 2P64.05 2P52.7825

UNIT 31

ARRAY $9 \quad 3 * 0.0$

UNIT 32

COM='SOUTH END REPLECTOR'

COM='SOUTH END REPLECTOR'
CUBOID 5 , 2P12.55 2P64.05 2 P52.7825
UNIT 33

COM='ARRAY FOR SOUTH END REPLECTOR'

ARRAY $10 \quad 3 * 0.0$

COM, 12.95 THICK MODEPATING PLASTIC MORTH CORE

CUBOID $012 P 16.5500 \quad 2 P 13.315 \quad 2 P 25.4350$

UNIT 35

COM='2.95 THICK MODERATING PLASTIC NOR

CUBOID 612 P16

UNIT $36 \quad 3 * 0.0$

UNIT 37

ARRAY $123 * 0.0$

UNIT 38

COM='NORTH CORE WITH BOTTOM REFLECTOR'

ARRAY $133 * 0.0$

COM=' MORTH CORE WITH EAST AND UEST DEFHECTOR

ARRAY $143 * 0.0$

UNIT 40

COM=' HORTH CORE WITH END REFLETOR'

ARRAY $15 \quad 3 * 0.0$

COM

CUBOID 31 2P0.61 2P64.0500 2P64. 9325

UNIT 42

COM=' NORTH CORE
ARRAY $16 \quad 3 \neq 0.0$

UNIT 43

' COM=' NORTH CORE WITH PACEPLATE.

ARRAY $17 \quad 3 * 0.0$

UNIT

COM=112.95 CM THICX MODERATOR SOUTH CORE

CUBOID 012 2P16.5500 2P13.315 2P25.4350

COM=-2.95 THICK MODERATOR SOUTH CORE.

CUBOID 612 P16.5500 2P38.75 2P1.7525

COM=' COMBINATION OF CORE WITH 12.95 THICX MODERATOR' ARRAY $18 \quad 3 * 0.0$

UNIT 47

COM=' COMBINATION OF CORE WITH 2.95 THICX MODERATOR ARRAY $19 \quad 3 * 0.0$

COM=' SOUTH CORE WITH EAST WEST REPLECTORS'

ARRAX $20 \quad 3 * 0.0$

UNIT 49

COM=' SOUTH CORE WITH EAST WEST REFLECTORS '

ARRAY $213 * 0.0$

COM='SOUTH CORE END REFIECTOR'

ARRAY $22 \quad 3 * 0.0$ 
Table A.3 (continued)

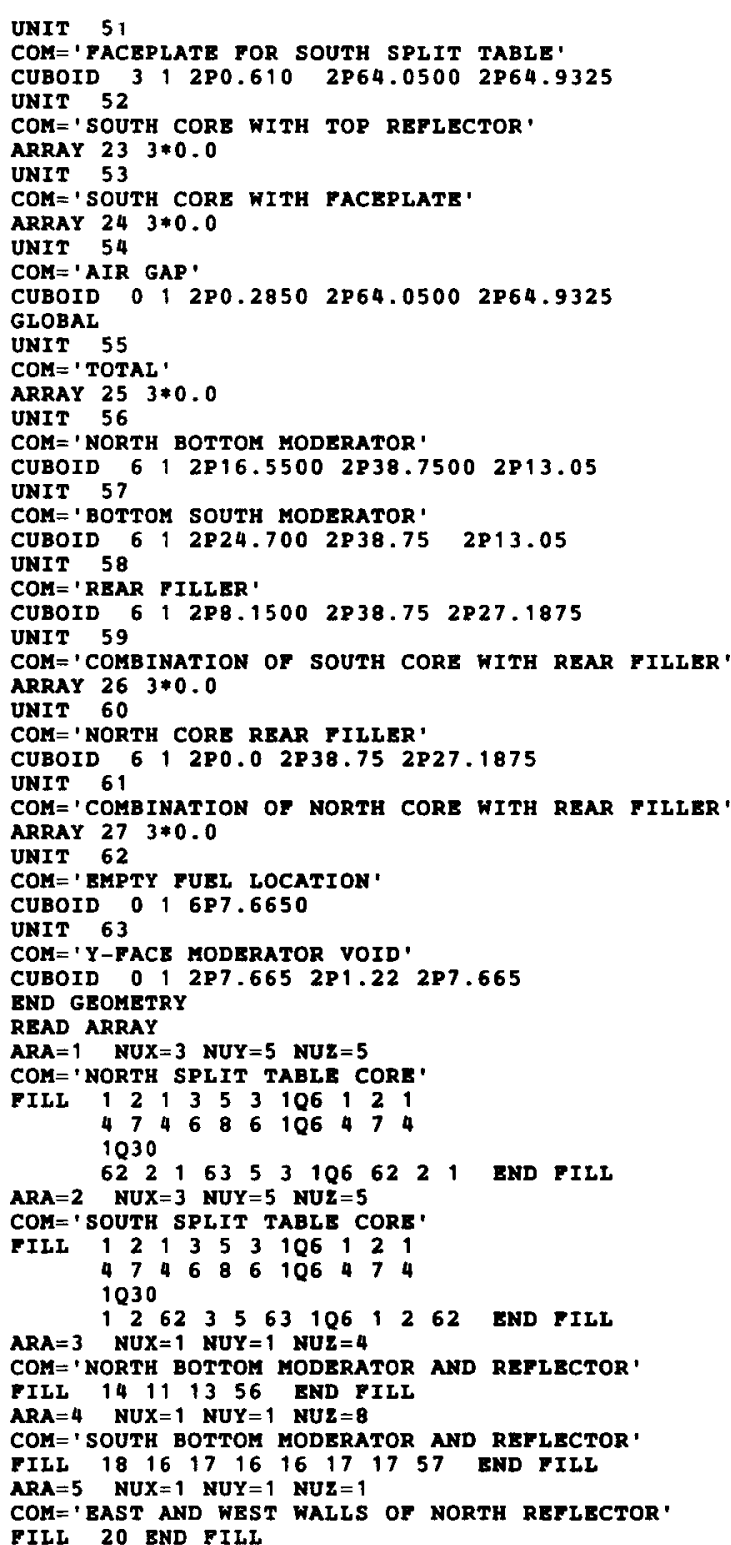

ARA $=6 \quad N U X=1 \quad N U Y=1 \quad N U Z=1$

COM=' EAST AND WEST WALIS OF SOUTH REPLECTOR'

FILL 22 BND PILL

ARA $=7$ NUX=1 NUY $=1 \quad \mathrm{NUZ}=1$

COM= ARRAY POR NORTH TOP REPLECTOR'

ARA $=8$ NUX $=1$ NUY $=1$ NUZ $=1$

COM=1 APRAY FOR SOUTH TOP REFIECTOR'

FILL 25 END RILL

ARA $=9 \quad$ NUX $=3 \quad$ NUY $=1 \quad$ NUZ $=1$

CON= 'ARRAY FOR NORTH END REPLBCTOR'

FILL 282930 BND FILI

COM $=$ 'ARRAY YOR SOUTH END REFLECTOR

FILL 32 BND FILL

COM=' COMBIMARIOY OF CORE WITH $12.95 \mathrm{CM}$ THICK MODERATOR'

FILL 934 END EILI

ARA $=12$ NUX $=1 \quad$ NUY $=1 \quad$ NUZ $=2$

COM=' COMBINATION OP PREVIOUS ARRAY WITH $2.95 \mathrm{CM}$ THICK MODERATOR'

FILI 3635 END FILL

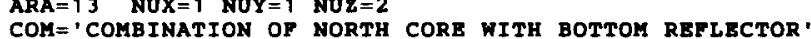

FILL 15 G1

ARA $=14$ NUX 14 BND FIL $=3$ NUZ $=1$

COM $=$ ' NORTH CORE WITH STDE REFLECTORS

FILI 213821 END FILI

$A R A=15$ NOX $=2$ NUY $=1$ NUZ $=1$

COM=' NORTH CORE WITH EMD

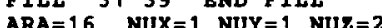

COM='

PILL 4025 END FILI

ARA $=17 \quad$ NUX $=2 \quad$ NUY $=1 \quad$ NUZ $=1$

COM=' NORTH CORE WITH FACEPLATE'

FILL 4241 BND FILL

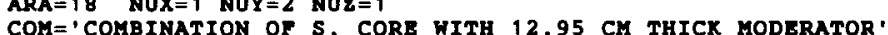

$A R A=19$ NUX $=1$ NUY $=1$ NUZ $=2$

COM=' COMBINATION OF CORE WITH 2.95 CM THICR MODERATOR'

FILL 4645 END FILL

ARA $=20$ NUX $=1$ NUY $=1$ NUZ $=2$
COM $=$ SOUTH CORE WITH BOTTOM REPLECTOR

FILL 1959 BND PILL

COM= SOUTH CORE WITH EAST WEST RETLECTORS '

FILI $234823 \quad$ SKD FILL

$A R A=22 \quad N U X=2 \quad N U Y=1 \quad N U Z=1$

COM=' SOUTH CORE WITH BND REFLBCTOR'

FILL 4933 BND TILL

COM=' COMBINATION OF CORE WITH TOP REYLECTOR'

TII 5027 END

FILI 50 27 END FILL

COM=' SOUTH CORE WITH FACEPLATE'

FILI 5152 END FILL

ARA $=25$ NUX

COM= ' TOTAL'

FILL 435453 END FILL

COM='COMBINATION OF SOUTH CORE WITH RBAR TILLER'

PILL 4758 END PILL 
Table A.3 (continued)

ARA $=27 \quad \mathrm{NUX}=2 \quad \mathrm{NUY}=1 \quad \mathrm{NUZ}=1$

COM= COMBINATION OF NORTH CORE WITH REAR FILLER

PILL 3760 BND FILI

RND ARRAY

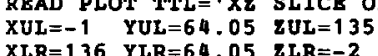

$\mathrm{UAX}=1$ WDN $=-1$ NAX $=130 \quad \mathrm{NCH}=0123456 \cdot \mathrm{END}$

TTL $=$ 'Y2 SLICE OF NORTH CORE FIRST ROW'

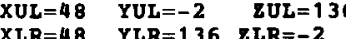

$X L R=48 \quad Y L R=136 \quad \& L R=-2 \quad \mathrm{YCH}=0123456^{\prime} \mathrm{RND}$

TTL='YY SLICE OF NORTH CORE SECOND RON"

$X U L=28 \quad Y U L=-2 \quad z U L=136$

$X L R=28 \quad Y L R=136 \quad$ \&LR=-2

VAX $=1$ WDN=-1 $\mathrm{NAX}=130 \quad \mathrm{NCH}=\cdot 0123456^{\circ} \mathrm{END}$

TTL $=$ 'YZ SLICE OF SOUTH CORE FIRST ROW

$\begin{array}{lll}X U L=62 & Y U L=-2 & Z U L \\ X L R=62 & Y L R=136 & \end{array}$

YAX $=1$ WDN $=-1$ NAX $=130$ NCH $=\cdot 0123456$, END

TTL $=$ ' TOP VIEW OF SOURCE SLOT'

$\begin{array}{lll}X U L=-2 & Y U L=136 & Z U L=125 \\ X L R=138 & Y L R=-2 & Z L R=125\end{array}$

$X I R=138 \quad Y L R=-2 \quad Z L R=125$

OAX $=1 \quad$ VDN $=-1 \quad$ NAX $=130 \quad \mathrm{NCH}=.0123456^{\prime} \mathrm{BN}$

TTL=

$\begin{array}{lll}X L R=138 & Y L R=-2 & 2 U L=9 \\ X L R=95\end{array}$

$\mathrm{UAX}=1 \quad \mathrm{VDN}=-1 \quad \mathrm{NAX}=130 \quad \mathrm{NCH}=.0123456$

BND PLOT

NST $=1$

$X S P=100.0 \quad X S M=10.0$

END DATA

BND

CAR19

ROCKY FLATS CRITICALS NUREG/CR-2500 EXPERIMENT NUMBER? (27 GROUP MODEL) FUEI CUBE 15.28 CH, U(4.46) 308 H/U $=2.03$, HI ENRICHED SPHERE DRIVEM

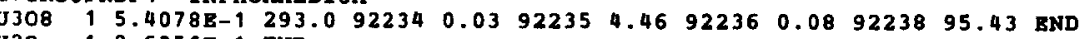
H2O $12.63568-1$

ARBM-BAGGIE $1.030001001 \quad 14.01601284 .98016 \quad 1.2011 .9134 \mathrm{E}-2$ END

AL1100 1.0300, $1302799.18260000 .5290000 .229 .53908-1$ BND

B-TAPE(YINYL) 107000010015.92601245 .91801610 .8217000 $25.73200406 .9220001 .6820001 .12111115 \mathrm{E}-2$ END

BM-TAPE(MYLAR) $1.0300010016 .83601265 .508016 \quad 27.022$

$1.7491 \mathrm{~B}-2$ BND

ARBM-MODERATOR 1.1853000010017 .83601259 .49801632 .483 END

1.1773 END

1.177 END

$3.7534 \mathrm{E}-3$ END

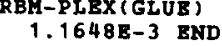

ARBM-PLEX(TRIS) $\quad 1.08000010017 .16 \quad 6012 \quad 52.03 \quad 7014 \quad 0.16801629 .82$ $15031 \quad 1.0217000 \quad 1.81350794 .260 \quad 350812.840551 .2757$ END ARBM-PLEX(PAPER) 1.03000010016 .48601242 .17801649 .55 $3.7534 \mathrm{E}-3$ END

ARBM-PLEX(GLUE) $1.0300001001 \quad 11.67601286 .2980161 .20$

ARBM-FILLER 1.185 300000100177.83601259 .49801632 .4860 .7 END PRACTION OP THE VOIDUALLY MODERATOR ADJUSTED FOR THE VOLU

ARBM-AL1 1 100 $1.0 \quad 3 \quad 0 \quad 0 \quad 1 \quad 1302799.1826000 \quad 0.529000 \quad 0.272 .715$ END URANIUM $80.9483293 .0922341 .09223593 .19922360 .492238 \quad 5.41$ END ARBM-PJ 0.81620 As 304921.0

PLATS CRITICALS NUREG/CR-2500 EXPERIMENT NUMBER \& (27 GROUP MODEL) PUE $15.28 \mathrm{CM}$ U $(4.46) 308$ H $/ U=2.03$, HI BNRICHED SPHERE DRIVEN READ PARM NPG $=600$ NUB=YES PDN=YES PLT=NO END PARM

READ GEOM

UNIT 1

COM=' PUEL BOX WITH VOID AND PART OF AL BOX'

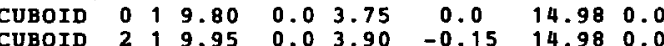

CUBOID $1914.98 \quad 0.0 \quad 9.365-5.61514 .98 \quad 0.0$

UNIT 2

COM= ' FRONT OF FUEL BOX

$\begin{array}{lllllllll} & & & & & & \\ \text { CUBOID } & 2 & 1 & 0.15 & 0.0 & 5.615 & 0.0 & 14.98 & 0.0\end{array}$

$\begin{array}{llllll} & \end{array}$

UNIT 3

COM=' REAR OF FUEL BOX

CUBOID $210.15 \quad 0.0 \quad 14.98 \quad 0.0 \quad 14.98 \quad 0.0$

UNIT 4

COM='ARRAY TO ASSBMBLE PART OF BOX

ARRAY $26 \quad 3 * 0$

A

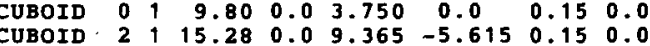

GLOBAL

UNIT

TO ASSEMBLE TOP AND BOTTOM.

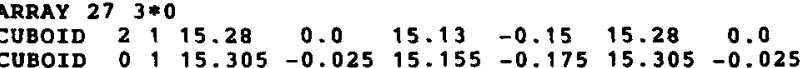

UNIT,

COM=' FUEL BOX 15.28 CM ON A SIDE WITH .15 CM WALLS .05CM STACXING VOID

CUBOID 1 1 16 FP.49

$\begin{array}{lllll}\text { CUBOID } & 2 & 1 & 6 P 7.64 \\ \text { CUBOID } & 0 & 1 & 6 P 7.6650\end{array}$

UNIT 8

COM $=\cdot B O X$ FOR DRIVER'

CYLINDER $9910.3175 \quad 2 P 5.66$

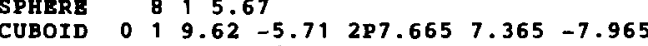

HOLE $37 \quad 0.0 \quad 0.0 \quad-7.965$

COM=' ALUMTNUM WEIGHT DISTRIBUTION PLATE FOR SPECIAI BOX CUBOID $\begin{array}{lllllllll}0 & 9 & 9.80 & 0.0 & 3.750 & 0.0 & 0.16 & 0.0\end{array}$ CUBOID $7115.33 \quad 0.09 .54-5.790 .16 \quad 0.0$

COM= ALUMINUM WEIGHT DISTRIBUTION PLATE FOR BOXES '

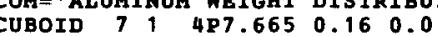


Table A.3 (continued)

UNIT 37

CYLINDER 911.562 .20 .0

UNIT 9

'NORTH SPLIT TABLE CORE'

CUBOID $0.130 .66-0.9477 .50 .083 .4750 .0$

COM='SOUTH SPLIT TABLE CORB'

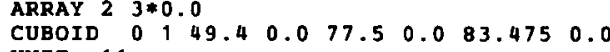

COM='PLEXIGLASS REFLECTOR SHEET WITHOUT TRIS, NORTH BOTTOM REFLECTOR'

CUBOID $412815.80002838 .7500280 .6150^{\circ}$

UNIT 12

COM='PLEXIGLASS REFLECTOR SHEET WITH TRIS, NORTH BOTTOM REPLECTOR'

CUBOID 5 I 2P15.8000 2P38.7500 2P0.6150

UNIT 13

COM OPPER PORTION NORTH BOTTOM REPLECTOR WITH TRIS'

CUBOID 512 P 15.8 2P38.75 288.24

COM=' LOWER PORTION NORTH BOTTOM REYLECTOR WITH TRIS'

CUBOID 5 1 2 2P15.8 2P38.75 2P3.69

UNIT 15 COM ' NORTH BOTTOM REFLECTOR INCLUDES REGULAR AND TRIS' ARRAY $3 \quad 3 * 0.0$

The

CUBOID $0112 \mathrm{P} 5,12 \mathrm{P2} 5 \mathrm{PPO}, 6150$

CUBOID 4 1 $44.3-5.12$ P 38.75 2PO.615

UNIT 17

COM='PLEXIGLAS SHEET BOTTOM SOUTH REFLECTOR WITH TRIS'

CUBOID $001285.1282 .52 P 0.6150$

CUBOID $5144.3-5.12 \mathrm{P38} .75 \quad 280.6150$

UNIT 18

COM=' LOWER PORTION SOUTH BOTTOM REPLECTOR WITH TRIS '

CUBOID 012 P 5.12 2P2.5 2 P8.85

CUBOID 5 1 $44.3 \quad-5.12$ 2P38.75 2 P8.85

UNIT 19

COM=' SOUTH BOTTOM REFLECTOR WITH REGULAR AND TRIS'

ARRAY $43 * 0.0$

COM=' BAST AND WEST REPLECTORS FOR MORTH REPLECTOR WITH TRIS CUBOID 512 P15.8 2P12.65 2P54.2825

UNIT 21

EAST AND WEST REFLECTORS FOR NORTH RETLECTOR ARRAY $5 \quad 3 * 0.0$

UNIT 22

COM= 'BAST AND WEST REFLCTORS FOR SOUTH REFLECTOR WITH TRIS CUBOID 5 I 2P24.700 2P12.65 2P54.2825

COM=. ARRAY TOR EAST AND WEST RETLECTORS TOR SOUTH REFIECTOR

ARRAY $6 \quad 3 * 0.0$

UNIT 24

COM= NORTH TOP REFLECTOR WITH TRIS

CUBOID 5 1 2 P28.35 2P64.05 2P12.1

UNIT 25

ARRAY $73 * 0.0$

COM=' SOUTH TOP REFLECTOR WTTH TRIS'

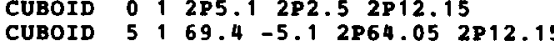

UNIT 27
COM='ARRAY FOR SOUTH TOP REPLECTOR
ARRAY $83 * 0.0$

UNIT 28 . 28 CUBOID $4,284.90002864 .05002854 .2825$

UNIT 29

COM='NORTH BND RELPBCTOR $5.2 \mathrm{CM}$ PORTION WITH TRIS

CUBOID 512 2P2.6 2P64.0500 2P54.2825

UNIT 30 COM=' NORTH END RELFECTOR $10.1 \mathrm{CM}$ PORTION WITHOUT TRIS' CUBOID 49285.052 P64.05 2P54.2825

UNIT 31 COM= ARRAY FOR NORTH END REPLECTOR'

ARRAY $93 * 0.0$

UNIT 32

COM=' SOUTH END REPLECTOR'

CUBOID 51 2P12.55 2P64.05 2P54.2825

UNIT 33

'ARRAY FOR SOUTH END REFLECTOR'

ARRAY $10 \quad 3 * 0.0$

COM=' NORTH CORE WITH BOTTOM REFLECTOR'

ARRAY 13 3* 0.0

UNIT

COM=' NORTH CORE WITH EAST AND WEST REPLECTOR'

ARRAY $143 * 0.0$

COM='NORTH CORE WITH END REFLECTOR'

ARRAY $15 \quad 3 * 0.0$

UNIT 42

COM=' NORTH CORE WITH TOP REFLECTOR'

ARRAY $16 \quad 3 * 0.0$

UNIT 46

' SOUTH CORB WITH BOTTOM REPLECTORS

ARRAY $20 \quad 3 * 0.0$

COM=' SOUTH CORE WITH BAST WEST REFLECTORS '

ARRAY $213 * 0.0$

JNIT 50

COM=' SOUTH CORE END REFLECTOR'

ARRAY $223 * 0.0$

COM=' SOUTH CORE WITH TOP REFLECTOR'

ARRAY $23 \quad 3 * 0.0$

UNIT 54

$\begin{array}{llllllll}\text { COM='AIR GAP' } & & & & & & & \end{array}$

GLOBAL

UNIT 55

ARRAY $25 \quad 3 * 0.0$

BND GBOMBTRY

READ ARRAY

ARA $=1$ NUX $=2$ NUY $=5$ NUZ $=9$

IIT TABLE CORE

FILL $10 R 7$

$10 \mathrm{R} 35$
$10 \mathrm{R} 7$

10R35

10R7
IOR35

10R7

1087 BND FILI 
Table A.3 (continued)

ARA $=2 \quad$ NUX $=3 \quad$ NUY $=5 \quad \mathrm{NUZ}=9$

ARA $=2$ NUX $=3$ NUY $=5$ NUZ $=9$
COM $=\cdot$ SOUTH SPLIT TABLE CORE'
PILL 6R7 6 8R7

$6 R 3534$ 8R 35

$6 R 768 R 7$

$6 R 35348 R 35$

6R7 $88 R 7$
$6 R 35348835$

$\begin{array}{ll}6 R 35 & 348 R 35 \\ 6 R 7 & 6 R 7\end{array}$

$6 R 35348 R 35$

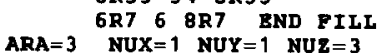

COM= ' NORTH BOTTOM REFLECTOR'

$\begin{array}{lllll}\text { FILL } & 14 & 11 & 13 & 3 \text { END FILI } \\ \text { ARA }=4 & \text { NUX }=1 & \text { NUY=1 }\end{array}$

ARA $=4$ NUXX $=1$ NUY $=1 \quad$ NUZ $=7$
COM $=$ ' SOUTH BOTTOM

$\begin{array}{llllllll}\text { FILL } & 18 & 16 & 17 & 16 & 16 & 17 & 17\end{array}$ END

ARA $=5 \quad$ NUX $=1 \quad$ NUY $=1 \quad$ NUZ $=1$

COM='BAST AND WEST WALLS OF NORTH RBPLECTOR'

PILL 20 BND FILI
ARA $=6 \quad$ NUX $=1$ NUY $=1 \quad$ NUZ $=1$

ARA $=6$ NUX $=1$ NUY $=1$ NUZ $=1$
COM $=$ 'BAST AND WEST WALLS OF SOUTH RETLECTOR'

FIL. 22 BND FII

$A R A=7 \quad$ NUX $=1 \quad$ NUY $=1 \quad$ NUZ $=1$

COM=' ARRAY FOR NORTH TOP REPLECTOR'

PILL 24 BND FILI

ARA $=8$ NUX $=1 \quad$ NUY $=1 \quad$ NUZ $=1$

COM=' ARRAY POR SOUTH TOP REFLECTOR'

FILL 26 END FILL
$A R A=9$ NUX $=3$ NUY $=1 \quad$ NUZ $=1$

COM=' ARRAY TOR NORTH END REFLECTOR'

PILL $28 \quad 2930$ BND FILL

RA $=10$ NUX $=1$ NUY $=1 \quad N U Z=1$

COM='ARRAY FOR SOUTH END REPLECTOR'

FILI 32 END PILL

COM=' COMBINATION OF NORTH CORE WTTH BOTTOM REFLECTOR'

FILL 159 BND FILL

$A R A=14 \quad N U X=1 \quad N U Y=3 \quad N U Z=1$

COM=' NORTH CORE WITH SIDE REFLECTORS

FILI 213821 END FILL

ARA $=15$ NOX $=2$ NOY $=1$ NUZ $=1$

COAL ' NORTH CORE WITH

PILI 3139 BND FILI

COM=' NORTH CORE WITH TOP REFLECTOR

FILI 4025 BND FILI

$A R A=20 \quad$ NXX $=1 \quad$ NUY $=1$ NUZ $=2$

COM='SOUTH CORE WITH BOTTOM REFLBCTOR'

FILL 1910 END FILI

COM=' SOUTH CORE WITH EAST WEST REFLECTORS '

FIIL $23 \quad 4823$ BND FILI

$A R A=22$ NUX $=2$ NUY $=1$ NUZ $=1$

COM=' SOUTH CORE WITH END REFLECTOR'

FILL 4933 BND FILL

COM=' COMBINATION OF CORB WITH TOP REFLECTOR'

CIIL 5027 :ND $F$

$A R A=25 \quad$ NUX $=3$ NUY $=1 \quad$ NUZ $=1$

COM= 'TOTAL

PILL 425452 END FILL

$A R A=26 \quad N U X=3 \quad N U Y=1 \quad N U Z=1$

COM='ASSBMBLY OF PURL BOX PRONT AND REAR

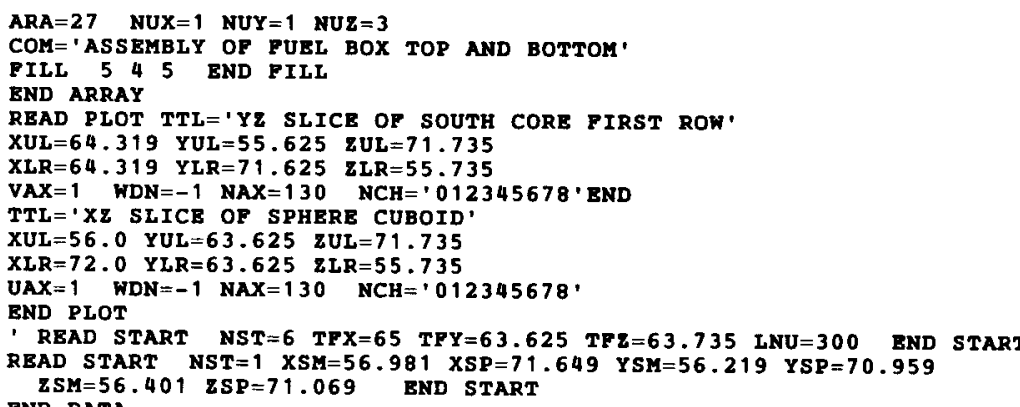

$A R A=27 \quad N U X=1 \quad N U Y=1 \quad N U Z=3$

(1)

PILL 545 END FILL

READ PLOT TTL='YZ SLICE OF SOUTH CORE FIRST ROW'

$X U L=64.319$ YUL $=55.625 \quad$ ZUL $=71.735$

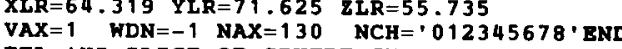

TTL $=1 X Z$ SLICB OF SPHERE CUBOID

$X I R=72.0$ YLR $=63.625$ in $=55.735$

UAX $=1$ WDN $=-1 \quad \mathrm{NAX}=130 \quad \mathrm{NCH}=+012345678$,

END PLOT

READ START NST $=6 \quad$ TPX=65 TPY $=63.625 \quad$ TP2 $=63,735$ LNU $=300$ END START READ START NST=1 XSM=56.981 XSP=79.649 YSM=56.219 YSP=70.959 ZSP $=71.069$ END START

END

CAR20

ROCKY FLATS CRITICALS NUREG/CR-2500 EXPERIMENT NUMBER ? (27 GROUP MODEL) PURL CUBE $15.28 \mathrm{CM}, \mathrm{U}(4.46) 308$ H/U=2.03, HI ENRICHED SPHERE DRIVEN

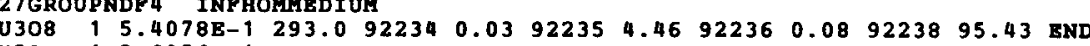
ARBM-BAGGIE $1.0 \quad 3 \quad 0 \quad 0001001 \quad 14.01 \quad 6012 \quad 84.98016 \quad 1.20 \quad 1 \quad 1.9134 \mathrm{~B}-2$ END $1.030021302799 .10260000 .5290000 .229 .53908-1$ ARBM-AL1100 $1.0 \quad 3 \quad 0 \quad 0 \quad 11302799.18 \quad 26000 \quad 0.5 \quad 29000 \quad 0.2220 .5390 \mathrm{E}-1$ ARBM-TAPE (VINYL) $\quad 1.077000010015 .926012 \quad 45.918016 \quad 10.82 \quad 17000$

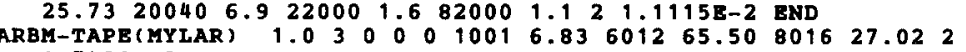
RBM-MODERATOR $1.18533000010017 .83601259 .49801632 .483=$ 1.1773 BND

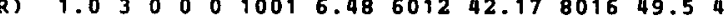
$3.7534 \mathrm{E}-3$ END

ARMY

PB-PLEX(TRIS) $1.088000110017 .16 \quad 601252.03 \quad 7014 \quad 0.16 \quad 8016 \quad 29.82$ 150319.02170001 .81350794 .260350812 .8405192757 END

ARBM-PLEX(PAPER)

ARBO

ARBM-PIEX (GLUE)

1.1648E-3 END 1.03000100111 .67601286 .2980161 .205

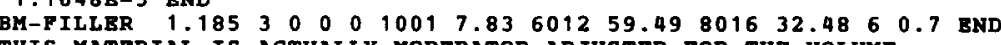

FACTION OF THE VOTLUALY MODERATOR ADJUSTED FOR THE VOLUM

ARBM-AI $1100 \quad 1.0 \quad 3 \quad 0 \quad 0 \quad 1 \quad 1302799.18 \quad 26000 \quad 0.529000 \quad 0.272 .715$ END

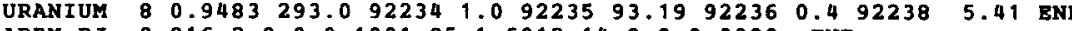
ARBM-PJ $0.81620000100185 .1601214 .980 .0089 \quad$ END

As 30491.0

ROCKY FLATS CRITICALS NUREG/CR-2500 EXPERTMENT NUMBER ? (27 GROUP MODEL 1. FUEL CUBE $15.28 \mathrm{CM}, \mathrm{U}(4.46) 308$ H/U=2.03, HI BNRICHED SPHERE DRIVEN READ PARM 
Table A.3 (continued)

UNIT 1

COM=' PUEL BOX WITH VOID AND PART OF AL BOX'

CUBOID $019.80 \quad 0.0 \quad 3.75 \quad 0.0 \quad 14.980 .0$

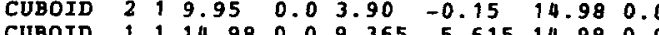

UNIT

COM=' PRONT OF FUEL BOX.

$\begin{array}{llllllllllll}\text { CUBOID } 2 & 1 & 0.15 & 0.0 & 5.615 & 0.0 & 14.98 & 0.0\end{array}$

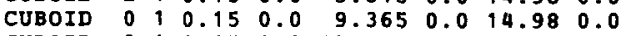

$\begin{array}{llllllllll}\text { CUBOID } 2 & 1 & 0.15 & 0.0 & 14.98 & 0.0 & 14.98 & 0.0\end{array}$

UNIT 3

COM=' REAR OF FUEL BOX

$\begin{array}{lllllllll}\text { CUBOID } 2 & 1 & 0.15 & 0.0 & 14.98 & 0.0 & 14.98 & 0.0\end{array}$

UNIT

COM $=$ ARRAY TO ASSEMBLE PART OF BOX

ARRAY $26 \quad 3 * 0$

UNIT 5

COM=' TOP AND BOTTOM OF BOX'

$\begin{array}{lllllllll}\text { CUBOID } & 0 & 1 & 9.80 & 0.0 & 3.750 & 0.0 & 0.15 & 0.0\end{array}$

GLOBAI

GLOBAL

COM='ARRAY TO ASSEMBLE TOP AND BOTTOM.

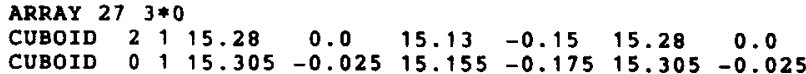

CUBOID,

COM= 1 FUET BOX

CM WALLS .05CM STACKING VOID

COBD 1687.49

CUBOID 0 1 6 P 7.6650

UNIT 8

COM='BOX FOR DRIVER

CYLINDER $910.3175 \quad 282.318$

$\begin{array}{lllll}\text { SPHERE } & 0 & 1 & 2.34 \\ \text { SPHERB } & 8 & 1 & 5 & -67\end{array}$

$\begin{array}{lrlll}\text { HOLE } & 36 & 0.0 & 0.0 & 2.3401\end{array}$

HOLE $100 \quad 0.0 \quad 0.0-2.3401$

CUBOID $0 \begin{array}{lllllll} & 8.71 & -6.62 & 2 P 7.665 & 7.365 & -7.965\end{array}$

$\begin{array}{lllll}\text { HOLE } & 37 & 0.0 & 0.0 & -7.965\end{array}$

COM='ALUMINUM WEIGHT DISTRIBUTION PLATE FOR SPECIAL BOX

$\begin{array}{llllllllll}C U B O I D & 0 & 9.80 & 0.0 & 3.750 & 0.0 & 0.16 & 0.0\end{array}$

$\operatorname{CUBOID}^{2}{ }^{7}$

COM= $=$ ALUMI UMY

$\begin{array}{lll}\text { CUBOID } 714 \text { P } 7.665 & 0.160 .0\end{array}$

36

DRIVER SUPPORT

CYLINDER $9010.3175 \quad 3.320 .0$

COM= ' DRIVER SUPPORT

$\begin{array}{llllll}C Y L I N D E R & 9 & 0.3175 & 0.0 & -3.32\end{array}$

UNIT 37

COM = ' DRIVER MOUNT'

CYLINDE

ARRAY $13 * 0$, 3 SPRT

T TABLB CORB

UNIT 10

COM='SOUTH SPLIT TABLE CORE

ARRAY 2 3 0.0
UNIT 11 CUBOID 4 $12 \mathrm{P} 15.80002 \mathrm{P} 38.7500 \quad 2 \mathrm{P0.6150}$

UNIT 12 12 M CUBOID $512 \mathrm{P} 15.80002 \mathrm{P38.7500} 2 \mathrm{P0} 0.6150$

UNIT 13

COM=' UPPER PORTION NORTH BOTTOM REFLECTOR WITH TRIS'

CUBOID 5 T 2P15.8 2 P38.75 2 P8.24

UNIT 14

COM=' LOWER PORTION NORTH BOTTOM

CUBOID 512 P 15.8 2P38.75 2P3.69

COM=' NORTH BOTTOM REFIECTOR INCLUDES REGULAR AND TRIS' ARRAY $3 \quad 3 * 0.0$

UNIT 16

COM='PLEXIGLAS SHEET BOTTOM SOUTH REPLECTOR WITHOUT TRIS

CUBOID 0 1 2 P5 .1 2 P2.5 2 P0.6150

CUBOID $4144.3-5.12 P 38.75 \quad 2 P 0.615$

COY ='PLEXIGLAS SHEET BOTTOM SOUTH REFLECTOR WITH TRIS

CUBOID $0,2 \mathrm{P} 5,1,2 \mathrm{P2}, 52 \mathrm{PO}, 5150$

CUBOID 5 1 $44.3-5.12838 .75 \quad 280.6150$

UNIT 18 COM=' LOWER PORTION SOUTH BOTTOM REFLECTOR WITH TRIS

CUBOID 0 , 12 P5, 12 P2.5 2 P8.85

CUBOID 5 T 4 .4. 3 -5.1 2 P 38.75 2P8.85

UNIT 19 .

ARRAY $4 \quad 3 * 0.0$

UNIT 20 COM=. BAST AND WEST REPLECTORS FOR NORTH REFLECTOR WITH IRIS' CUBOID 512 P15.8 2P12.65 2P54.2825

UNTT 21 COY 1 2P15 ARRAY 5 \% $3 * 0$

UNIT 22

COM='BAST AND WEST REFLECTORS FOR SOUTH REFLECTOR WITH TRIS' CUBOID $512 \mathrm{P} 24.7002 \mathrm{P} 12.652 \mathrm{P} 54.2825$

UNIT 23 COM='ARRAY FOR BAST AND WEST REFLECTORS FOR SOUTH REFLECTOR' ARRAY $6 \quad 3 * 0.0$

COM=' NORTH TOP REFLECTOR WITH TRIS'

CUBOID 512 2P28.35 2P64.05 2P12.15

UNIT 25 . ARRAY FOR NORTH TOP REPLECTOR'

ARRAY $73 * 0.0$

COMI ' SOUTH TOP REFLECTOR WITH TRIS'

CUBOID $012 \mathrm{P5}, 12 \mathrm{P2} .52 \mathrm{P} 12.15$

$\begin{array}{llllllll}\text { CUBOID } 5 & 1 & 69.4 & -5.1 & 2 \mathrm{P} 64.05 & 2 \mathrm{P} 12.15\end{array}$

UNIT 27

COM= A ARRAY FOR SOUTH TOP REFLECTOR'

ARRAY 8 3*0.0

UNIT 28 COM= NORTH END REFLECTOR $9.8 \mathrm{CM}$ PORTION WITHOUT TRIS'

CUBOID 4,2 P4.9000 2P64.0500 2P54.2825

UNIT 29

COM= ' NORTH END RELFECTOR 5.2 CM PORTIO
CUBOID 5,2 P2.6 2 P64.0500 2 P54.2825

UNIT 30 . NORTH END RELFECTOR $10,1 \mathrm{CM}$ PORTION WITHOUT TRIS

CUBOID $412 \mathrm{P5} .05 \quad 2864.05 \quad 2854.2825$ 
Table A.3 (continued)

UNIT 31

FOR NORTH END REFLECTOR

ARRAY 9 3*0.0

COM=' SOUTH BND REPLECTOR'

CUBOID 512 P12.55 2P64.05 2P54.2825

UNIT 33

FOR SOUTH END REFIECTOR

ARRAY $10 \quad 3 * 0.0$

COM='NORTH CORE WITH BOTTOM REPLBCTOR'

ARRAY $13 \quad 3 * 0.0$

UNIT 39

COM=' NORTH CORE WITH EAST AND WEST REFIECTOR'

ARRAY $14 \quad 3 * 0.0$

COM=' NORTH CORE WITH END REFLECTOR'

ARRAY $15 \quad 3 * 0.0$

UNIT 42

COM=' NORTH CORE WITH TOP REFLECTOR'

ARRAY $16 \quad 3 * 0.0$

UNIT

BOTTOM REFLECTORS

ARRAY $20 \quad 3 * 0.0$

COM=' SOUTH CORE WITH EAST WEST REPLECTORS

ARRAY $213 * 0.0$

UNIT 500 COM $=$ SOUTH CORE END REPLECTOR'

ARRAY $22 \quad 3 * 0.0$

I SOUTH CORE

ARRAY $23 \quad 3 * 0.0$

UNIT 54

COM= ' AIR GAP'

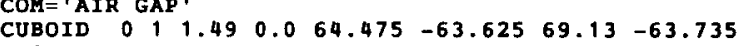

GLOBAL

UNIT 55

ARRAY $25 \quad 3 * 0.0$

END GEOMETRY

BAD ARRAY

$A R A=1 \quad N U X=2 \quad$ NUY $=5 \quad$ NUZ $=9$

NOX 2 NUY 5 NUE=9

FILI 1OR7

$10 \mathrm{R} 35$

$10 R 7$

IOR3

TORT

$10 R 7$ END FILL

$A R A=2$ NUX $=3$ NUY $=5$ NUZ $=9$

COM $=$ 'SOUTH SPLIT TABLE CORE

PILL $6 R 7$ 6 6 R 7

$6 R 35$ $348 R 35$
$6 R 7$ 6 $8 R 7$

$6 R 7 \quad 6 \quad 8 R 7$
$6 R 35 \quad 348 R 35$

$6 R 35$ 8R $8 R 35$

$6 R 35348 R 35$
$6 R 768 R 7$

6R7 6 BR $34 \quad 8 R 35$
$A R A=3 \quad N U X=1 \quad N U Y=1 \quad N U Z=3$

COM=' NORTH BOTTOM REPLECTOR'

$P I L A=4$ NUX 13 END FIL

COM= ' SOUTH BOTTOM REPLECTOR'

FILL $\begin{array}{lllllllll}18 & 16 & 17 & 16 & 16 & 17 & 17 & \text { END FIII }\end{array}$

COM='BAST AND WEST WALLS OF NORTH REFLECTOR

PILL 20 END FILI

$A R A=6 \quad N U X=1$ NUY $=1 \quad N U Z=1$

PILL 22 END TILI

COM = NUX $=1$ NUY $=1 \quad$ NUZ $=1$ PRPECTOR

PILL 24 END PILI.

ARA $=8$ NUX=1 NUY $=1$ NUZ $=1$

COM=' ARRAY FOR SOUTH TOP REFLECTOR'

PILL 26 END FILI

COM= ARRAY TOR NORTH $\quad 1$ NUD REPLECTOR

PILL $28 \quad 29 \quad 30$ END FILL

ARA $=10$ NUX $=1 \quad$ NUY $=1$ NU $Z=1$

COM='ARRAY FOR SOUTH END REFLECTOR'

FILL 32 END FILI

$A R A=13 \quad$ NOX $=1$ NUY $=1$ NUZ $=2$ CORE WITH BOTTOM PRPLECTOR

PILL 159 END

$A R A=14 \quad N U X=1 \quad N U Y=3 \quad N U Z=1$

COM=' NORTH CORE WITH SIDE REPLECTORS

FILL 213821 END FILL

COM=' NORTH CORE WITH BND REFLECTOR'

PILL 3139 END FILI

COM=' NORTH CORE WITH TOP REFLECTOR'

PILL 4025 BND FILL

$A R A=20 \quad N U X=1 \quad N U Y=1 \quad N U Z=2$

COM='SOUTH CORE WITH BOT

PILA 21 NUX 1 NUY $=3$ NUZ

COM= SOUTH

PILL 234823 BND 4 ILL

$A R A=22 \quad N U X=2 \quad N U Y=1 \quad N U Z=1$

COM='SOUTH CORE WITH END REFLECTOR'

PILL 4933 END FILL

$A R A=23 \quad$ NUX $=1$ NUY $=1 \quad$ NUZ $=2$

PILL 5027 BND FILL

ARA $=25$ NUX $=3$ NUY $=1$ NUZ $=1$

COM= 'TOTAL

FILL $4254 \quad 52$ END FIL

ARA $=26 \quad$ NUX $=3$ NUY $=1 \quad$ NUZ $=1$

COM=' ASSBMBLY OF FUBL BOX PRONT AND REAR'

PILL $2{ }^{2} 3 \quad$ END PILL

COM=' ASSEMBLY OF FUEL BOX TOP AND BOTTOM.

FILI 545 END FILL 
Table A.3 (continued)

READ PLOT TTL='YZ SLICE OF SOUTH CORE FIRST ROW'

$X U Z=64.319$ YUL $=55.625 \quad 2 U L=71.735$

$X L R=64.319 \quad$ YLR $=71.625 \quad 2 L R=55.735$

TTL= XY SLICE OF SPHERE CUBOID

$\mathrm{XUL}=56.0 \quad \mathrm{YUL}=63.625 \quad \mathrm{ZUL}=71.735$

$X L R=72.0 \quad Y L R=63.625$

UAX $=9$ WDN $=-1$ NAX $=130$ NCH $=1012345678$

END PLOT

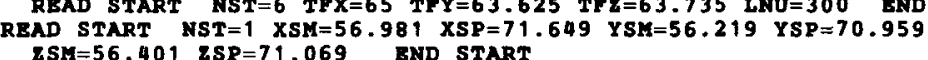

END DATA

SND 
Table A.4. Table 4 input data

\section{CAA0 3}

UO2 (NO3) 2 SOLN H/U-235=1379 BARE SPHERE CASB 3

27GROUPNDF 4 MULTIREGION

$\mathrm{U}-234 \quad 1 \quad 0 \quad 5.400-7$ END

$\mathrm{U}-236$ 1 $01.390-7$ END

$\mathrm{U}-238 \quad 1 \quad 0 \quad 2.811-6$ END

H $1006.640-2$ END

$\begin{array}{llllll}0 & 1 & 0 & 3.379-2 & \text { END } \\ N & 1 & 0 & 1.753-4 & \text { BND }\end{array}$

AI $\quad 21$ END

END COMP

134.6 NOEXTERMOD 234.92 NOEXTERMOD END ZONE UO2 (NO3) 2 SOLN $H / O-235=1379$ READ PARAM NPG $=600$ NUB $=$ YES FDN $=$ YES END PARAM READ GEOM

SPHERE 1134.6

END GBOM

BND DATA

\section{CAAO4}

UO2F2 SOLN H/U-235=76.1 H2O REFL SPHERE CASE 4

27GROUPNDF 4 MULTIREGION

$\mathrm{U}-234 \quad 1 \quad 0 \quad 8.795-6$ END

$\mathrm{U}-235$ 1 $008.327-4$ END

\begin{tabular}{lllll}
$\mathbf{U}$ \\
$\mathbf{U} 238$ & 1 & 0 & $4.449-6$ & BND \\
\hline
\end{tabular}

$\begin{array}{lllll}H & 1 & 0 & 6.337-2 & \text { BND } \\ 0 & 1 & 0 & 3.347-2 & \text { END }\end{array}$

$\begin{aligned} & 1 \\ & F\end{aligned} \quad 1.786-3$ END

$\begin{array}{llll}\text { AL } & 2 & 1 & \text { END } \\ \text { H2O } & 3 & 1 & \text { END }\end{array}$

END COMP

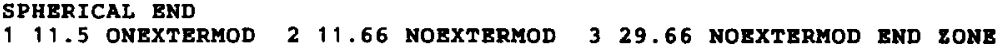

READ PARAM NPG $=600$ NUB $=$ YES FDN $=$ YBS BND PARAM

READ GEOM

SPKERE 1 1 11.5

SEPLECTOR 111.66

REPLETOR

READ BIAS ID $=50027$ END BIAS

END DATA

\section{CAA0 5
=CSAS 25}

UO2F 2 SOLN H/U-235=126.5 H2O REFL SPHERE CASE

7GROUPNDF 4 MULTIREGION

$-2351005.405-6$ END

U-236 $1002.734-6$ END

U-238 $1002.906-5$ END

$\begin{array}{lllll}H & 1 & 0 & 6.473-2 & \text { BND } \\ 0 & 1 & 0 & 3.346-2 & \text { END }\end{array}$

P $\quad 1001.098-3$ END

$\begin{array}{llll}\text { AL } & 2 & 1 & \text { BND } \\ \text { H2O } & 3 & 1 & \text { BND }\end{array}$

END COMP

SPHERICAL END

111.8 ONEXTERMOD 211.96 NOEXTERMOD 329.96 NOEXTERMOD END ZONE UO2P2 SOLN H/U-235 $=126.5$ H2O REFL SPHERE CASB 5

READ GBOM

SPHERE $11,111.8$

SPFLECTOR 111.96

BND GEOM

READ BIAS ID=500 27 END BIAS

CAA06

$=$ CSAS 25

UO2F2 SOLN H/U-235=1270 H2O REPL SPHERE CASE 6

27.ROUPNDF 4 MULTIREGION

U-235 $1005.530-7$ END

$\begin{array}{lllll}U-236 & 1 & 0 & 2.800-7 & \text { END }\end{array}$

U-238 $1002.971-6$ END

\begin{tabular}{lllll}
$H$ & 1 & 0 & $6.643-2$ & END \\
0 & 1 & 0 & $3.333-2$ & BND \\
\hline
\end{tabular}

$1001.122-4$ END

$\begin{array}{llll}\text { AL } & 2 & 1 & \text { END } \\ \text { H2O } & 3 & 1 & \\ & & \end{array}$

END COMP

SPHERICAL END

127.9 ONEXTERMOD 228.10 NOEXTERMOD 346.10 NOEXTERMOD END EONE UO2P2 SOLN H/U-235=1270 H2O REFL SPHERE CASB

READ PARAM NDG $=600$ NUB $=$ YES FDN=YES END PARAM

SPHERE $2,27.9$

REPLECTOR 323

END GEOM

READ BIAS ID $=50027$ END BIAS

END DATA 
Table A.4 (continued)

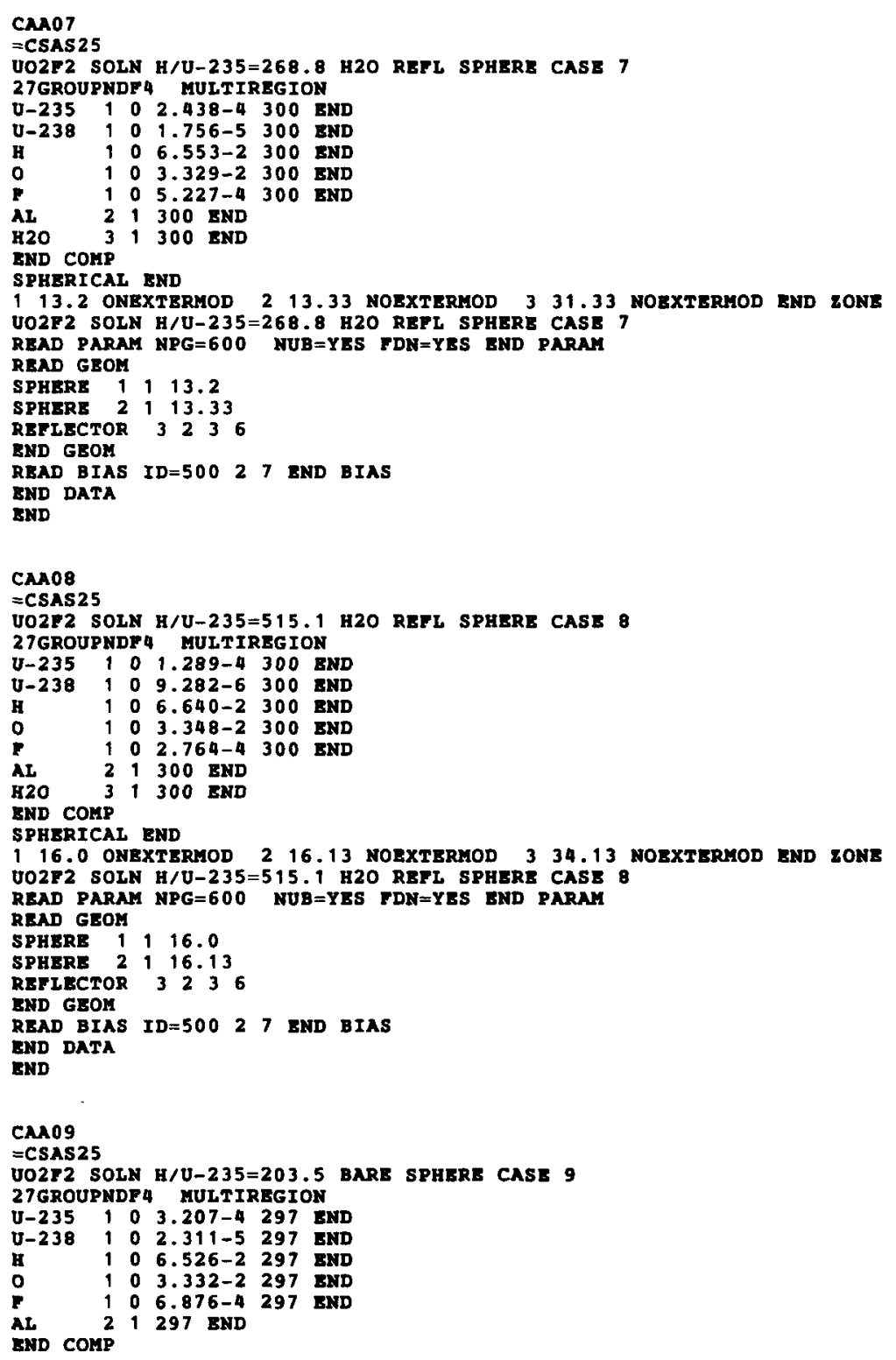

SPHERICAL END

16.0 NOEXTERMOD 216.13 NOEXTERMOD END ZONE READ PARAM NPG $=600$.

SPHERE

SPHERE 2116.13

END GEOM

END DAIA

CAA10

UO2F2 SOLN H/U-235=239.3 H2O REFL SPHERE CASE 10

27GROUPNDF 4 MULTIREGION

$\begin{array}{llllll}\mathbf{U}-235 & 1 & 0 & 2.735-4 & 359 & \text { END } \\ \mathbf{U}-238 & 1 & 0 & 1.970-5 & 359 & \text { BND }\end{array}$

$\begin{array}{llllll}0 & \\ H & 1 & 0 & 6.545-2 & 359 & \text { END } \\ 0 & 1 & 0 & 3.331-2 & 359 & \end{array}$

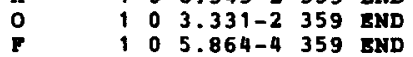

AL $\quad 21359$ END

BND COMP

SPHERICAI END

113.2 ONEXTERMOD 213.33 NOEXTERMOD 331.33 NOEXTERMOD END ZONE UO2F 2 SOLN H/U-235=239.3 H2O REFL SPHERE CASE 10

READ PARAM MPG $=600$ NUB=YES FDN=YES END PARAM

READ GEOM

SPHERE 11113.2

PETECTOR 3.33

END GEOM

READ BIAS ID $=50027$ END BIAS

SND DATA

$\cos 11$

CSAS25

UO2F2 SOLN H/U-235=468.2 H2O REFI SPHERE CASE 11

27GROUPNDF 4 MULTIRBGION

\begin{tabular}{lllllll}
$\mathrm{U}-235$ & 1 & 0 & $1.416-4$ & 361 & END \\
$\mathrm{U}-238$ & 1 & 0 & $1.020-5$ & 361 & BND \\
\hline & 1 & 0 & $0.630-2$ & 361 & END
\end{tabular}

$\begin{array}{llllll}\text { H } & 1 & 0 & 6.630-2 & 361 & \text { END } \\ 0 & 1 & 0 & 3.345-2 & 361 & \text { END }\end{array}$

F $103.036-4361$ END

AL $\quad$\begin{tabular}{llllll}
2 & 1 & 361 & END \\
\hline & 3 & 1 & 361 &
\end{tabular}

END COMP

SPHERICAL END

1 16.0 ONEXTERHOD 216.13 NOEXTERMOD 334.13 NOEXTERMOD END EONE UO2F2 SOLN H/U-235=468.2 H2O REFL SPHERE CASE 11

READ PARA

$\begin{array}{llll}\text { SPHERE } & 2 & 16.0 \\ & 16.13\end{array}$

REPLECTOR 323

END GEOM

ID=500 27 END BIAS

END DATA 
Table A.4 (continued)

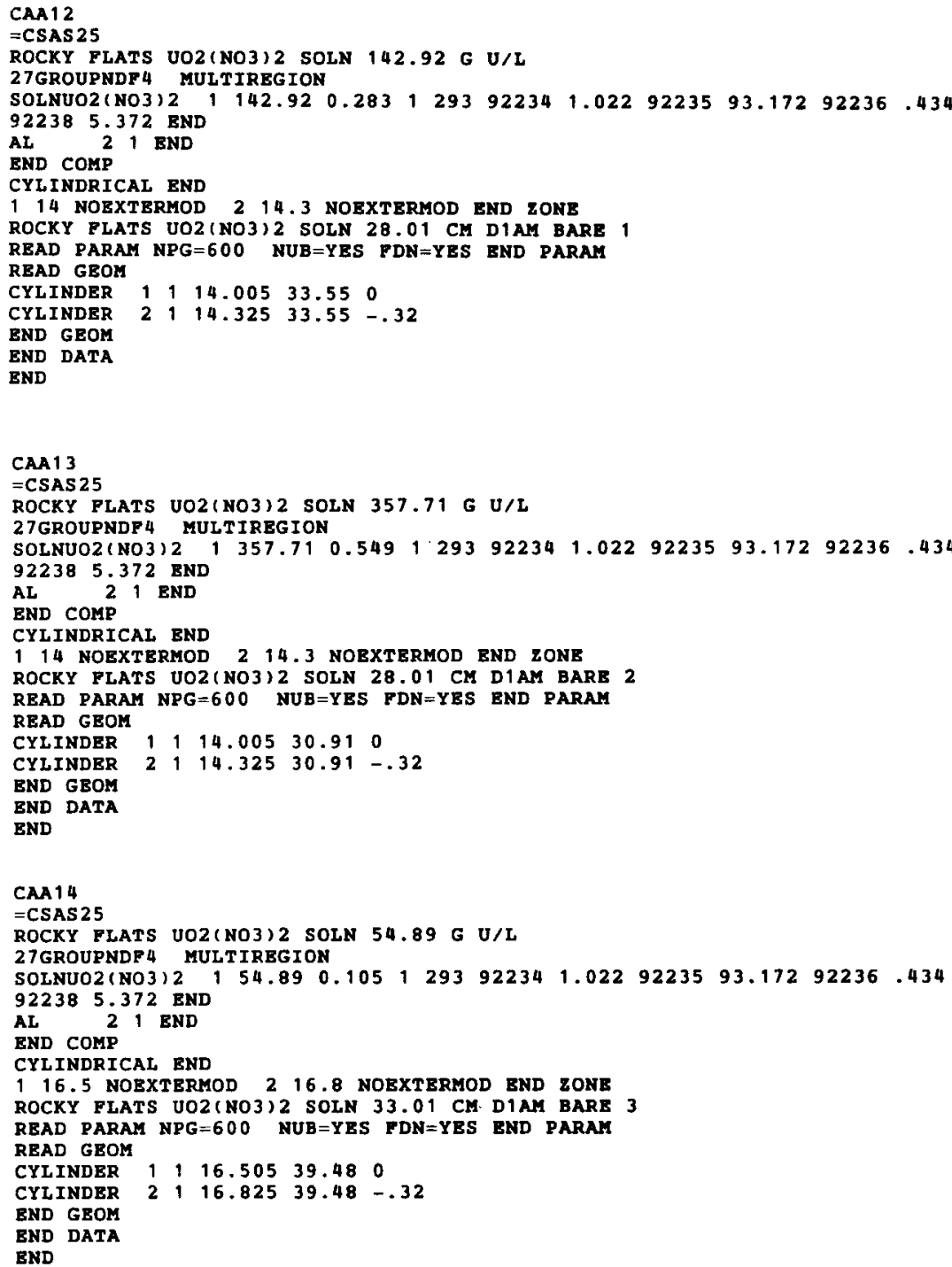


Table A.4 (continued)

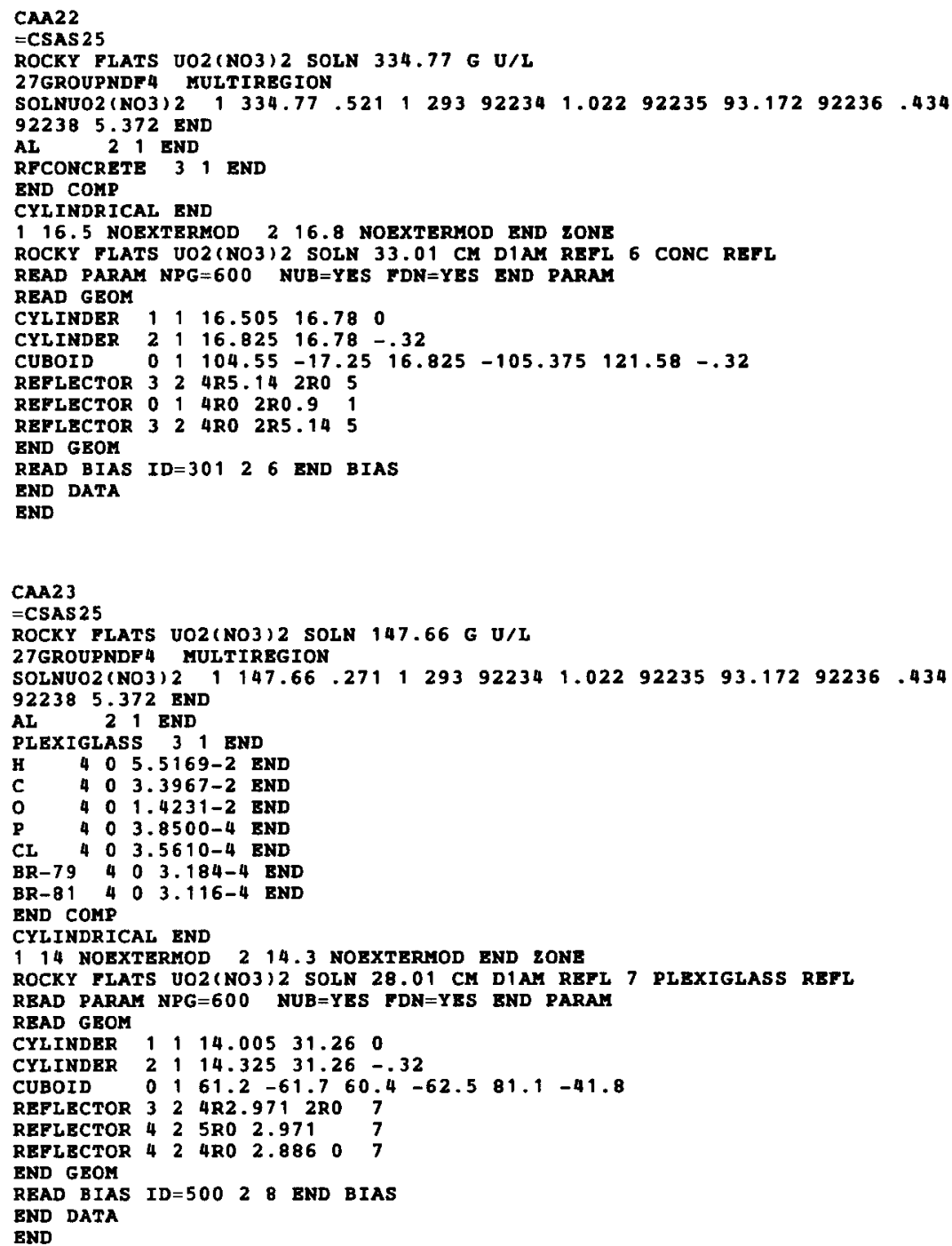

CAA25

ROCKY FLATS UO2(NO3) 2 SOLN $147.66 \mathrm{G} \mathrm{U/L}$

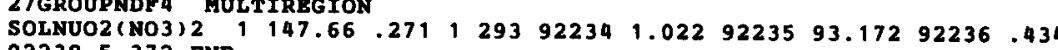
AL 21 END

AL 221 END

PLEXIGLASS 31 END

$\begin{array}{llll}H & 4 & 0 & 5.5169-2 \\ C & 4 & 0 & 3.3967-2 \\ \end{array}$

$0401.4231-2$ END

P $40 \begin{array}{llll}0.8500-4 & \text { END } \\ \text { CI } & 4 & 0 & 3.5610-4\end{array}$

BR-79 4 O $3.184-4$ END

BR-81 4 O $3.116-4$ END

END COMP

CYLINDRICAL END

116.5 NOEXTBRMOD 216.8 NOEXTERMOD END ZONE ROCKY FLATS UO2 (NO3) 2 SOLN 33.01 CM D1AM REFL 9 PLEXIGLASS REPL

READ GEOM

CYINDER 1116.50522 .780

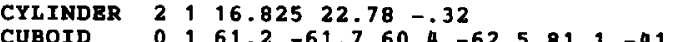

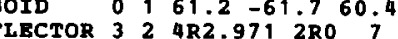

$\begin{array}{llllll}\text { REFLECTOR } & 4 & 2 & 5 R 0 & 2.971 & 7 \\ \text { REFLECTOR } & 4 & 2 & 4 R 0 & 2.886 & 0\end{array}$

$\begin{array}{lllllll}\text { REFLECTOR } & 4 & 2 & 4 R 0 & 2.886 & 0 & 7\end{array}$

BND GEOM

END BIAS ID $=50028$ BND BIAS

END 
Table A.4 (continued)

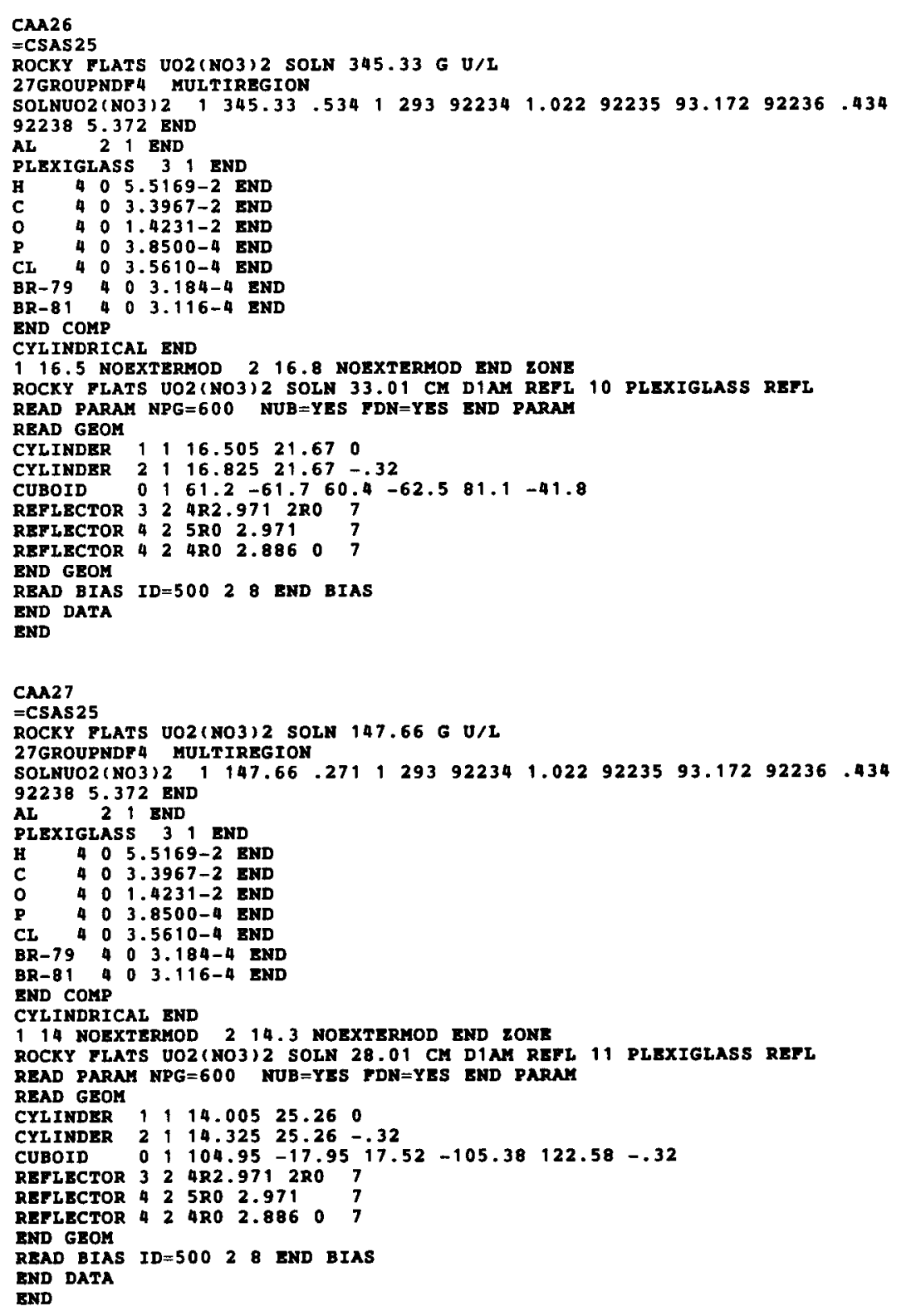

CAA2B
$=$ CSAS2

ROCKY PLATS UO2(NO3) 2 SOLN $345.33 \mathrm{G}$ U/L

27GROOPAD (NO3) 922385.372 END

PLEXIGLASS 31 END

H $405.5169-2$ END

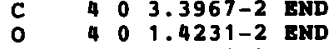

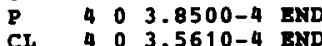

BR-79 4 O $3.184-4$ BND

BR-81 4 O $3.116-4$ END

GND COMP

CYLINDRICAL END

16.5 NOEXTERMOD 216.8 NOEXTERPMOD END ZONE

12 PLEXIGLASS REFu ROCKY PLATS U02(NO3)2 SOLN 33.01 CM D1AM REFA

READ GEOM

CYLINDER $11116.505 \quad 17.2$

CYLINDBR 2 1 $16.82517 .2-32$

$\begin{array}{lllllllll} & & & \end{array}$

REPLETOR 3 L 2 TR2.971 2RO

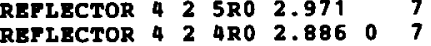

END GEOM 4 4RO 2.806 o

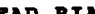

END DATA

CMA29

CSAS25

27GROUPADT

$\begin{array}{lllllllll} & \end{array}$

$92238 \quad 5.372$ END

AL TFCONCRETE 21 END 1 END

SND COMP

10.5 NOEXTERMOD 210.9 NOEXTERMOD EYD ZOME

ROCKY rLATS UO2(NO3) 2 SOIN 21.12 CM D1AM $4 \times 4$ ARRAY CONC REFL

READ PARAM NPG $=600$ NUB $=$ YES FDN=YES END PARAM

READ GEOM

CYLINDER $11110.56 \quad 27.150$

$\begin{array}{llllllll} & & \\ \text { CYIINDER } & 2 & 1 & 10.96 & 27 & 15 & -.32\end{array}$

CORE $\quad 0112 R-60.96-.32$

REFLECTOR 32 4R5.14 2RO 5

$\begin{array}{llllll}\text { REPLECTOR } & 0 & 1 & \text { 4RO } & \text { 2R1.25 } & 1 \\ \text { REPLECTOR } & 3 & 2 & \text { 4RO } & \text { 2R5.14 } & 5\end{array}$

END GEOM

READ ARRAY NUX $=4$ NUY=4 NUZ=1 END ARRAY

BAD BIAS ID=301 20 BID BIAS

END 


\section{Table A.4 (continued)}

CAA 30
$=C 5 A S 25$

ROCKY FLATS UOZ(NO3)2 SOLN $364.11 \mathrm{G} \mathrm{U} / \mathrm{L}$

$\begin{array}{llllllllll}\text { 27GROUPNDF } 4 & \text { MULTIREGION } & & & & & & & & \end{array}$

AI 3 (2)

AL RFONCRETE 21 END

CYLINDRICAL END

$1 \quad 10.5$ NOEXTERMOD 210.9 NOEXTERMOD END ZONB

ROCKY FLATS UO2 (NO3) 2 SOLN 21.12 CM D1AM 4 X4 ARRAY CONC REFL C2

\section{READ GEOM}

$\begin{array}{llllll}1 & 1 & 10.56 & 17.13 & 0\end{array}$

$\begin{array}{llllll}\text { CYLINDER } & 2 & 1 & 10.96 & 17.13 & -.32 \\ \text { CUBOID } & 0 & 1 & 4 P 15.24 & 121.58 \\ \text { COPE } & 0 & 1 & 2 R-60.96\end{array}$

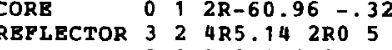

REFLECTOR 0 T 1 4R0 $2 \mathrm{R} 1.25$

REFLECTOR

READ ARRAY NUX $=4$ NUY $=4$ NUZ $=1$ END ARRAY

READ BIAS ID $=30126$ END BIAS

END DATA

CAM 1

CAA31
$=$ CSAS 25

ROCXY FLATS U02(NO3) 2 SOLN $83.49 \mathrm{G} \mathrm{U} / \mathrm{L}$

27GROUPNDF 4 MULTIREGION

SOLNUO2(NO3) $2 \quad 183.49 .151 \quad 129392234 \quad 1.0229223593 .17292236 \quad .434$

92385.372 END

RECONCRETE 3 , 3 END

BND COMP

CYLINDRICAL END

18 NOEXTERMOD 28.3 NOEXTERMOD END ZONB

ROCKY FLATS UO2 (NO3) 2 SOLN 16.12 CM D1AM $4 X 4$ ARRAY CONC REPL C3

READ PARAM

READ GEOM

CYLINDER 118.0651 .210

$\begin{array}{lllllll}\text { CYLINDER } & 2 & 1 & 8.38 & 51.21 & -31.32 \\ \text { CUBOID } & 0 & 1 & 4 P 15.24 & 121.58 & -.32\end{array}$

$\begin{array}{llllll}\text { CORE } & 0 & 1 & 2 R-60.96 & -.32\end{array}$

REFLECTOR 322 4R5.14 2R0 5

REPLECTOR 014 TRO 2R1.25

REPLECTOR 3

READ ARRAY NUX $=4 \quad$ NUY $=4 \quad$ NUZ $=1$ RND ARPAY

READ BIAS ID $=30126$ END BIAS

END DATA

RND
CAA 32
$=$ CSAS 25

ROCKY FLATS UO2(NO3) 2 SOLN 359.55 G U/L

SOLNUO2(NO3)2 $1359.55 .578 \quad 1 \quad 29392234 \quad 1.0229223593 .17292236 \quad .434$

AL 21 END

RFCONCRETE 3 , END

END COMP

CYLINDRICAL BND

18 NOEXTERMOD 28.3 NOEXTERMOD END 8 ONE

ROCKY FLATS UO2(NO3) 2 SOLN 16.12 CM D1AM 4 X4 ARRAY CONC REFL C4

READ PABAM

$18.06 \quad 31.82$

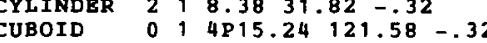

$\begin{array}{lllll}\text { CORE } & 0 & 1 & 2 R-60.96 & -.32\end{array}$

REFLECTOR 3 2 4R5.14 2RO 5

REPLECTOR 01 पRO 2R1.25 1

BND GEOM

READ ARRAY NUX $=4$ NUY $=4$ NUZ $=1$ END ARRAY

READ BIAS ID $=30126$ END BIAS

END DATA

$\operatorname{CAA} 33$

ROCKY PLATS UO2(NO3) 2 SOLN $76.09 \mathrm{G} \mathrm{U/L}$

$\begin{array}{llllllllll}\text { 27GROUPNDF } & \text { MULTIREGION } & & & & & & & & \end{array}$

AI

RFCONCRETE 3 END

BND COMP

CYLINDRICAL END

$1 \quad 10.5$ NOEXTERMOD 210.9 NOBXTERMOD END ZONE

ROCKY FLATS UO2(NO3) 2 SOLN 21.12 CM D1AM 2 X2 ARRAY CONC REPL C

READ GEOM

CYLINDER $111910.56 \quad 62.340$

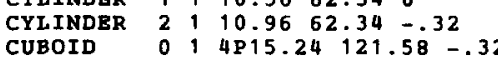

CUBOID $\quad 0 \quad 1 \quad 4 P 15.24 \quad 121.58-.32$

$\begin{array}{lllll}0 & 1 & 4 \mathrm{P} 15.24 & 121.58 \\ 0 & 1 & 2 \mathrm{R}-60 & -9.96 & -32 \\ 3 & 2 & -32\end{array}$

REFLECTOR 3 2 IRO 2R1.25

REFLECTOR 32 4RO 2R5.14 5

END GEOM

READ ARRAY NUX $=4$ NUY $=4$ NUZ $=1$

FR2 2R1 2R2 2R1 5R2 END FILI

END ARRAY

READ BIAS ID $=30126$ END BIAS

END DATA 
Table A.4 (continued)

$\operatorname{can} 34$

=CSAS25

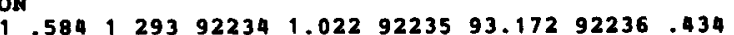
922385372 ( 1903

AL 21 END

SND COMP 110.5 NOEXTERMOD 2 10.9 NOEXTERMOD END ZONE
ROCKY YLATS UO2(NO3) 2 SOLN 21.12 CM D1AM 2 X2 ARRAY CONC RETI C6 READ PARAM NPG $=600$ NUB $=$ YES TDN=YES END PARAY

READ GEOM

CYLTMDER 1910.5631 .110

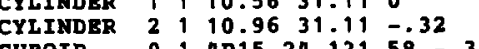

CUBOID

o 14 P 15.24 121.58 -.32

$012 R-60.96-.32$

REFLCTOR 322 MR5. 14 2RO 5

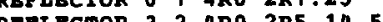

READ ARPAY $N U=4$ NUY $=4$ NUZ $=1$

FILI 5R2 2R1 2R2 2R1 5R2 END FILL

SND ARRAY

READ BIAS ID $=30126$ END BIAS

CAA35

ROCKY FLATS U02(NO3) 2 SOLN $359.55 \mathrm{G} \mathrm{U} / \mathrm{L}$

27GROUPNDEA MUTTIRBGION

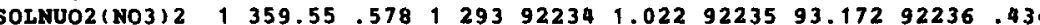
922385.372 END

AL CONCRETE 2 END 1 END

RED COMP

END COMP

8 NOEXTERMOD 28.3 NOEXTEPMOD END ZONE

ROCKY PLATS U02(NO3)2 SOLN 16.12 CM D1AM $2 \times 2$ ARRAY CONC RETL C7 READ PARAM NPG $=600$ NUB $=$ YES FDN=YES BND PARA

READ GEOM

CNIT 1

1 $18.06 \quad 104.040$

$\begin{array}{llllll}\text { CYLINDER } & 2 & 1 & 8.38 & 104.04 & -.32 \\ \text { CUBOID } & 0 & 1 & 4 P 15.24 & 121.58-.32\end{array}$

$014 P 15.24121 .58-.32$

CORE 0 O 1 2R-60.96 -.32

REFLECTOR 0 O 1 4RO $2 R 1.25$

RETLECTOR 32 4RO 285.165

READ ARRAY NUX $=4$ NUY $=4$ NUZ $=1$

READ ARRAY NUX=4 NUY $=4$ NUZ=1
FILL OR2 $2 R 1$ 2R2 $2 R 1$ 2R2 END FILL
END ARRAY

READ BIAS ID $=30126$ END BIAS

BND DATA
$\operatorname{cAs} 36$

ROCKY PLATS UO2(NO3)2 SOLN 359.55 G V/l

SOI.02(103) $2359.55 .578,29392234,0229223593.17292236 .434$

922385,372 EMD

ALCONCRETE 3 , 3 END

END COMP

CYLINDRICAL END

ROCTY ITATS UD 28.3 NOEXTERMOD END ZONE

U02 (NO3)2 SOLN 16.12 CM DIAY $2 \times 4$ ARRAY CONC REFI C8 READ PARAM NPG $=600$ NUB $=$ YES PDN $=$ YSS

READ GEOI

CYLINDER 118.0651 .450

CYIINDER 2 1 $8.3851 .45-.32$

CUBOID 2 O 1 apIs.24 $121.58-.32$

$\begin{array}{lllllll}\text { CUBOID } & 0 & 1 & 4 P 15.24 & 121.58 & -.32\end{array}$

CORE $0012 R-60.96-.32$

REFLECTOR 322 4R5.14 2RO 5

$\begin{array}{llllll}\text { REPLECTOR } & 0 & 1 & \text { ARO } & \text { 2R1.25 } \\ \text { REFIECTOR } & 3 & 2 & \text { ARO } & \text { 2R5.14 } & \end{array}$

END GEOM

NRAY NUX $=4$ NUY $=4$ NUZ $=1$

FILL 2R1 2R2 $3 Q^{4}$ END FILI

READ BIAS ID $=30126$ END BIAS

END DATA

CAM37

ROCKY FLATS U02(NO3)2 SOLN $60.32 \mathrm{G} \mathrm{U} / \mathrm{L}$

$\begin{array}{llllllllll}\text { 27GROUPNDFA MULTIREGION } & \\ \text { SOLMUO2(NO3)2 } & 160.32 .113 & 1 & 293 & 92234 & 1.022 & 92235 & 93.172 & 92236 & .434\end{array}$ 922385.372 END

AL 2 EXIGLASS 31 END

H $405.5169-2$ END

c $403.3967-2$ END

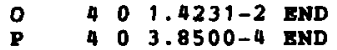

CL 40 3.5610-4 END

BR-79 4 \%

END COMP

CYLINDRICAL END

1 10.5 NOEXTERMOD 2 10.9 NOEXTERMOD END ZONE ROCXY FLATS VO2 (NO3)2 SOLN 21.12 CM D1AM $2 X 4$ ARRAY PLEXIGLASS RETL P1

READ GEO

CYLINDER $1110.56 \quad 31.760$

CYLINDER 2 1 $10.9631,76 \quad-.32$

CUBOID 0014 AP15.24 122.58

REFLECTOR O 1 4R.49 2RO 1

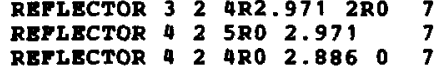

BND GEOM

READ ARRAY NUX $=4$ NUY $=4$ NUZ $=1$

END ARRAY

READ BIAS ID $=50028$ END BIAS

END DATA 
Table A.4 (continued)

CAA38

ROCKY PLATS UO2(NO3) 2 SOLN $355.94 \mathrm{G} \mathrm{U} / \mathrm{I}$

27GROUPNDF 4 MULTIREGION

SOLNUO2(NO3)2 $1355.94 .494 \quad 29392234 \quad 1.0229223593 .17292236 \quad .434$ 922385.372 END

AL 21 END

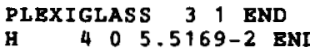

C $\quad 4 \quad 0 \quad 3.3967-2$ END

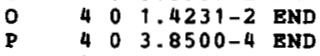

CL 4 O $3.5610-4$ END

BR-79 4 - $03.184-4$ END

BRD COMP

CYLINDPICAI END

$1 \quad 10.5$ NOBXTERMOD 210.9 NOEXTERMOD END ZONE

ROCKY FLATS U02(NO3)2 SOLN 21.12 CM D1AM 4 X4 ARRAY PLEXIGLASS REFL P2 READ PARAM NPG $=600$ HUE

$\begin{array}{llllll}\text { READ GEOM } & 10.56 \quad 18.82 & 0\end{array}$

$\begin{array}{lllllll}\text { CYLINDER } & 1 & 1 & 10.56 & 18.82 & 0 \\ \text { CYLINDER } & 2 & 1 & 10.96 & 18.82 & -.32\end{array}$

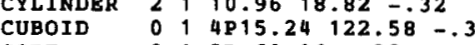

CORE $\quad 012 R-60.96-.32$

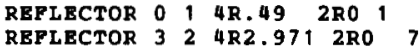

$\begin{array}{llll}\text { REPLECTOR } & 3 & 2 & 4 R 2.971 \\ \text { REFLECTOR } & 4 & 2 & 5 R O \\ 2.971 & 7\end{array}$

REFLECIOR 4 2 2 SRO 2.971 (

BND GEOM

READ ARRAY NUX $=4 \quad N U Y=4 \quad N U Z=1$

BND ARRAY

READ BIAS ID $=50028$ END BIAS

END DATA

CAA39
$=$ CSAS25

ROCXY FLATS UO2(NO3)2 SOLN $60.32 \mathrm{G} \mathrm{U} /$

77GROUPADF 4 MULTIREGION

SOLNUO2 (NO3) $2 \quad 160.32 .113 \quad 129392234 \quad 1.0229223593 .17292236 \quad .434$ 922385.372 END

AL 221 END

H $\quad 4005.5169-2$ END

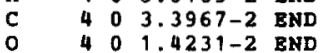

P $403.8500-4 \mathrm{BND}$

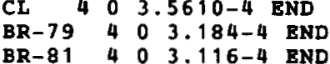

BND COMP

CYLINDRICAL BND

18 NOEXTERMOD 28.3 NOEXTERMOD END ZONE

ROCKY FLATS UO2 (NO3)2 SOLN 16.12 CM D1AM $4 X 4$ ARRAY PLEXIGLASS REFL P3

READ GBOM

118.0678 .400

$\begin{array}{lllll}\text { CYLINDBR } & 2 & 8.38 & 78.40-.32 \\ \text { CUBOID } & 0 & 4 P 15.24 & 422.58-.32 \\ \text { CORB } & 0 & 12 R-60.96-.32\end{array}$

CORB $\begin{array}{lllll}\text { REPLECTOR } & 0 & 1 & 4 R .49 & \text { 2RO } 1 \\ \text { REFLECTOR } & 3 & 2 & 4 R 2.971 & 2 R 0\end{array}$

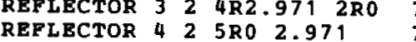
REFLECTOR $4 \begin{array}{llllll}4 & 4 R O & 2.886 & 0 & 7\end{array}$ END GBOM READ ARRAY NUX $=4 \quad N U Y=4 \quad N U Z=1$

READ BRAS

ID $=50028$ END BIAS END DATA

CAA40

ROCKY PLATS U02(NO3) 2 SOLN 355.94 G U/L

27GROUPNDP 4 MULTIREGION

SOLNUO2(NO3) $2 \quad 1355.94 .494 \quad 1 \quad 29392234 \quad 1.0229223593 .17292236 \quad .434$ $92238 \quad 5.372$ END

AL 21 END

405.5169 END

c $4 \quad 4003.3967-2$ END

$044001.4231-2$ END

P 4 4 0 3.8500-4 END

CL 79 - $3.5610-4$ BND

$\begin{array}{llll}B R-79 & 4 & 0 & 3.116-4 \\ B R & \text { END }\end{array}$

BND COMP

CYLINDRICAL BND

18 NOEXTERMOD 2 B.3 NOEXTRRMOD END ZONE

ROCKY FLATS UO2 (NO3) 2 SOLN 16.12 CM D1AM $4 X 4$ ARRAY PLEXIGLASS REFI P4 READ PARAM NPG $=600$ NUB $=$ YES FDN $=$ YES END PARAM

CYLINDER 118.0635 .560

CYLINDER 2 1 $8.38 \quad 35.56-.32$

CUBOID 0114 P $15.24 \quad 122.58-.32$

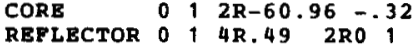

REFLECTOR 32 4R2.971 2R0 7

$\begin{array}{lllllll}\text { REFLECTOR } & 4 & 2 & \text { SRO } & 2.971 & & 7 \\ \text { REPLECTOR } & 4 & 2 & 4 R 0 & 2.886 & 0 & 7\end{array}$

END GBOM

READ ARRAY NUX=4 NUY $=4 \quad \mathrm{NUZ}=1$

END ARRAY

READ BIAS ID $=50028$ BND BIAS

END DATA

CAA4 1

$=\operatorname{CSAS} 25$

ROCKY FLATS UO2(NO3) 2 SOLN $60.32 \mathrm{G} \mathrm{U/L}$

$\begin{array}{llllllllll}\text { 27GROUPNDF4 } & \text { MULTIREGION } & & \end{array}$ 922385.372 END

AL 21 END

C $\quad 405.5169-2$ END

\begin{tabular}{lllll}
$C$ & 4 & 0 & $3.3967-2$ & END \\
0 & 4 & 0 & $1.4231-2$ & END \\
\hline
\end{tabular}

$\begin{array}{lllll}P & 4 & 0 & 3.8500-4 & \text { END } \\ C L & 4 & 0 & 3.5610-4 & \end{array}$

$B R-79 \quad 4$ O $3.184-4$ BND 
Table A.4 (continued)

END COMP

1 10.5 NOBXTERMOD 210.9 NOEXTERMOD END ZONE

ROCKY FLATS U02 (NO3) 2 SOLN 21.12 CM D1AM 2 22 ARRAY PLEXIGLASS REFL P5 READ PARAM NPG $=600$ NUB $=$ YES PDN $=$ YES END PARAM

READ GEOM

UNIT 1

$\begin{array}{lllllll} & & 10.56 & 102.29\end{array}$

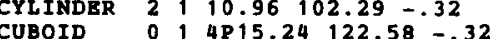

$15.24122 .58-.32$

$\begin{array}{llll}0 & 1 & 4 P 15.24 & 122.58 \\ 0 & 1 & 2 R-60.96 & -.32\end{array}$

REFLBCTOR O 1 4R.49 2RO 9

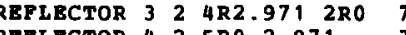

REPIBCTOR 42 4RO 2.88607

BND GEOI

FILI 5R2 2R1 2R2 2R1 5R2 END FILI

GND ARRAY

READ BIAS ID $=5002$ B END BIAS

SND DATA

$\operatorname{CAM} 42$

ROCKY FLATS UO2(NO3)2 SOLN $355.94 \mathrm{G} \mathrm{U/L}$

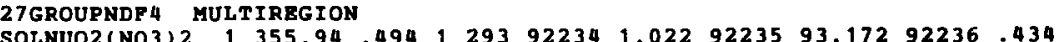
922385,372 END

AL 21 END

PLEXIGLASS 31 END

$\begin{array}{llllll}H & 4 & 0 & 5.5169-2 & \text { END } \\ \text { C } & 4 & 0 & 3.3967-2 & \text { END }\end{array}$

\begin{tabular}{lllll}
$C$ & 4 & 0 & $3.3967-2$ & END \\
0 & 4 & 0 & $1.4231-2$ & END \\
\hline & 4 & $3.4250-4$ & END
\end{tabular}

$P \quad 4 \quad 03.8500-4$ END

CL $403.5610-4$ BND

$\begin{array}{lllll}\mathrm{BR}-79 & 4 & 0 & 3.184-4 & \text { BND } \\ \mathrm{BR}-81 & 4 & 0 & 3.116-4 \\ \mathrm{ENDD}\end{array}$

END COMP

CYLINDRICAL END

210.9 NOEXTERHOD EMD ZONE

ROCKY FLATS UO2(NO3) 2 SOLN 21.12 CM D1AM $2 \times 2$ ARRAY PLEXIGLASS REFL 96 READ PARAM NPG $=600$ NUB $=$ YES PDN=YES END PARAM

READ GBOM

UNIT 1

CYLINDER $11110.56 \quad 33.200$

$\begin{array}{llllll}\text { CYLINDER } & 2 & 1 & 10.96 & 33.20 & -.32 \\ \text { CUBOID } & 0 & 1 & 4 P 15.24 & 122.58 & \end{array}$

$0 \quad 14 P 15.24122 .58-.32$

CUBOID

$14 P 15.24122 .58-.32$

CORE $0112 \mathrm{R}-60.96-.32$

REFLECTOR O 1 4R.49 2RO 1

REFIECTOR 32 4R2.971 2RO

RETIECTOR 4 2 $5 R 02.971$

RLETCTOR 4

READ APPAY NUX $=4$ MUY $=4$ NUZ $=1$

PILL 5R2 2R1 2R2 2R1 5R2 END FILI

END ARRAY
READ BIAS ID $=50028$ END BIAS

BND DATA

CAA4 3

$=$ CSAS 2

ROCKY FLATS UO2(NO3) 2 SOLN 355.94 G U/L

27GROUPNDF 4 MULTIREGION $922385.372 \mathrm{BND}$

AL 21 END

PLEXIGLASS 31 END

$\begin{array}{lllll}H & 4 & 5 & 5169-2 & \text { END } \\ \text { C } & 4 & 0 & 3.3967-2 & \text { END }\end{array}$

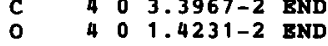

P $40 \begin{array}{lll}4.8500-4 & \mathrm{END}\end{array}$

BR-79 4 O 0 3.184-9

BR-81 4 O $3.116-4$ EN

END COMP

CYLINDRICAL END

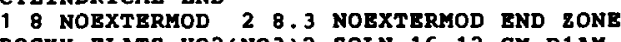

2 (NO3) 2 SOLN 16.12 CM D1AM $3 \times 2$ ARRAY PLEXIGLASS REFL P7 READ PARAM NPG $=600$ NUB $=$ YES FDN=YES END PARAM

GEOM

CYLINDER $118.06 \quad 89.780$

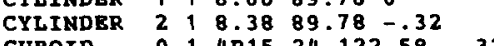

UNIT 2 OUT

CUBOID 0114 P 15.24122 .58

REFLECTOR 0 O

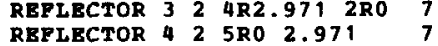

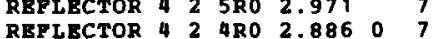

BND GEOM

READ ARRAY NUX $=4$ NUY $=4$ NUZ $=1$

PILI AR2 3R1 2 3R1 5R2 END FILI

END ARRAY

READ BIAS ID $=50028$ END BIAS

END DATA 
Table A.5. Table 5 input data

CASO 1

KENO-5 VALIDATION CASE A- 1

27 CSOUPNDF 4 MULTIREGION

URANIUM $1.9837 \quad 29392235 \quad 93.892238 \quad 6.2$ END

BND COMP

SPHERICAL END

8.73136 NOEXTERMOD END ZONE

KENO-5 VALIDATION CASE A- 1 NEA $=600$ FDN $=$ YES NUB $=$ YES END PARAM

READ GEOM

SPHERE 118.73136

BND GEOM

END DATA

CAS 02

$=$ CSAS25

KENO-5 VALIDATION CASE A-10

27GROUPNDF 4 MULTIREGION

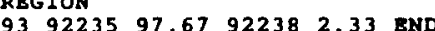

H2O 2 END

PLEXIGLASS

SPHBRICAL END

16.55 ONEEXTERMOD 212.7 NOEXTERMOD END ZONE

KENO-5 VALIDATION CASE A-10

READ PARAM TME $=60 \quad$ NPG $=600 \quad$ FDN $=Y E S \quad N U B=Y E S$ BND PARAM

READ GEOM

BOX TYPB $1,15.5537$

$\begin{array}{lllllllll}\text { CUBOID } & 2 & 1 & 12.7 & -12.7 & 12.7 & -12.7 & 6.5537 & -6.5537\end{array}$

BOX TYPE 2

$\begin{array}{lllllll}\text { CYLINDER } & 2 & 1 & 4.1275 & 1.27 & -1.27\end{array}$

CYLINDER $311,12.7,1.27-1.27$

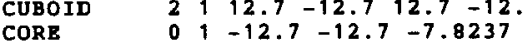

CYLINDER $24130.48 \quad 24.3337^{-39.5737}$

END GEOM

READ ARRAY NUX $=1 \quad$ NUY $=1 \quad$ NUZ $=2$ LOOP

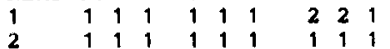

END ARRAY

BND DATA

CASO 3

$=$ CSAS 25

KENO-5 VALIDATION CASE A1

SOLNOP2(NO3)2 1346.73 .54212939223593 .172922386 .828 BND

SS 3042 END

BND COMP

CYLINDRICAL END

1 13.96 ONEEXTERMOD 214.28 ONEEXTEKMOD END ZONE

KENO-5 VALIDATION CASE A 11
READ PARAM NPG $=600$ FDN=YES NUB=YES BND PARAM

READ PARAM

CYLINDER $111013.96 \quad 14.465-14.465$

CYLINDER $2,14.2827 .135-15.105$

BND GEOM

END DATA
CASO 4

KENO-5 VALIDATION CASE A-2

ARBMUMO $17.0821001920009042000 \quad 101112939223593.292238 \quad 6.8$ END END COMP

CYLINDRICAL END

17.62 NOEXTERMOD END ZONE

READ PARAM NPG $=600$ PDN=YES NUB=YBS END PARAM

READ GEOM

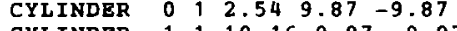

CYLINDER $\quad 1 \quad 1010.16 \quad 9.87-9.87$

CUBE

END GEOM

END

CAS 05

CSAS 25

RENO-5 VALIDATION CASE A-3

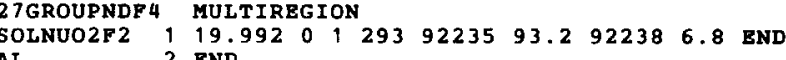

AL 2 END

END COMP

134.6 ONEEXTERMOD 234.92 NOEXTERMOD END ZONE

READ PARAM NPG $=600$ FDN $=$ YES NUB $=$ YES END PARAM

READ GEOM

SPHERE 1134.6

$\begin{array}{lllll}\text { CUBE } & 2 & 1 & 34.92 \\ & 0 & 1 & 35.0 & -35.0\end{array}$

END GBOM

BND DAT

CAS 06

KENO-5 VALIDATION CASE A-4

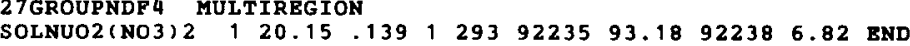

AL

END COMP

SPHERICAL END

134.6 ONEEXTERMOD 234.92 NOEXTERMOD END ZONE

KENO-5 VALIDATION CASB A-4
READ PARAM NPG $=600$ FDN $=$ YES NUB=YES END PARAM

SPHERE

$\begin{array}{lllll}\text { SPHERE } & 1 & 1 & 34.6 \\ \text { SPHERE } & 2 & 1 & 34.92\end{array}$

CUBE $\quad 0 \quad 1 \quad 35.0-35.0$

END GEOM

END DATA 
Table A.5 (continued)

CAs 07

=CSAS25
XENO-5 VALIDATION CASE A-5

27GROUPNDF4 MULTIREGION

URANIOH 1 iND

HND COMP

SPHERICAI END

1 12.7 ONEEXTERHOD 2 31. NOEXTERHOD END ZONE

KENO-5 VALIDATION CASE A-5

READ GEOY

HEAISPHERE 2 : 12.7

$\begin{array}{lllllllll}\text { HEMISPHERE } & 1 & 1 & 15.24 & & & \\ \text { CUBOID } & 2 & 1 & 31.00 & -31.00 & 31.00 & -31.00 & 31.00 & -16 .\end{array}$

EMD GEOA

END DATA

$\operatorname{CAS08}$
$=\operatorname{CSAS} 25$

VALIDATION CASE a-

27GPOUPNDYA MULTIREGIOM

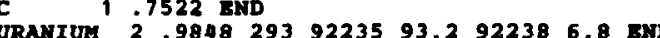

RMD cOMP

CYR COMP 2 .

1 8.89 NOEXTERHOD 2 13.97 TWOEXTERHOD 131.75 NOEXTERHOD END ZONE

READ PARAM NPG $=600$ FDN=YES NUB=YES END PARAM

READ GBOM

$\begin{array}{llllll} & & & & 3.425 & -3.425\end{array}$

CYLIMDER 1 1 $11.43 \quad 3.495-3.425$

CYLINDER 2 1 $13.973 .495-3.425$

CYLINDER $91131.7521 .275-21.205$

HND DATA

\section{CASO9}

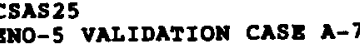

27GROUPNDF 4 MULTIREGION

(1) 922386 END

URANTUY 2 END

END COMP

OEXTERMOD END ZOME

17.62 NOEXTERMOD 252.02

KENO-5 VALIDATION CASE A-7

READ GBOM

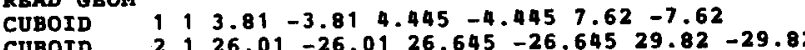

CUBE

$0,130.0-30.0$

END GBON
END DATA

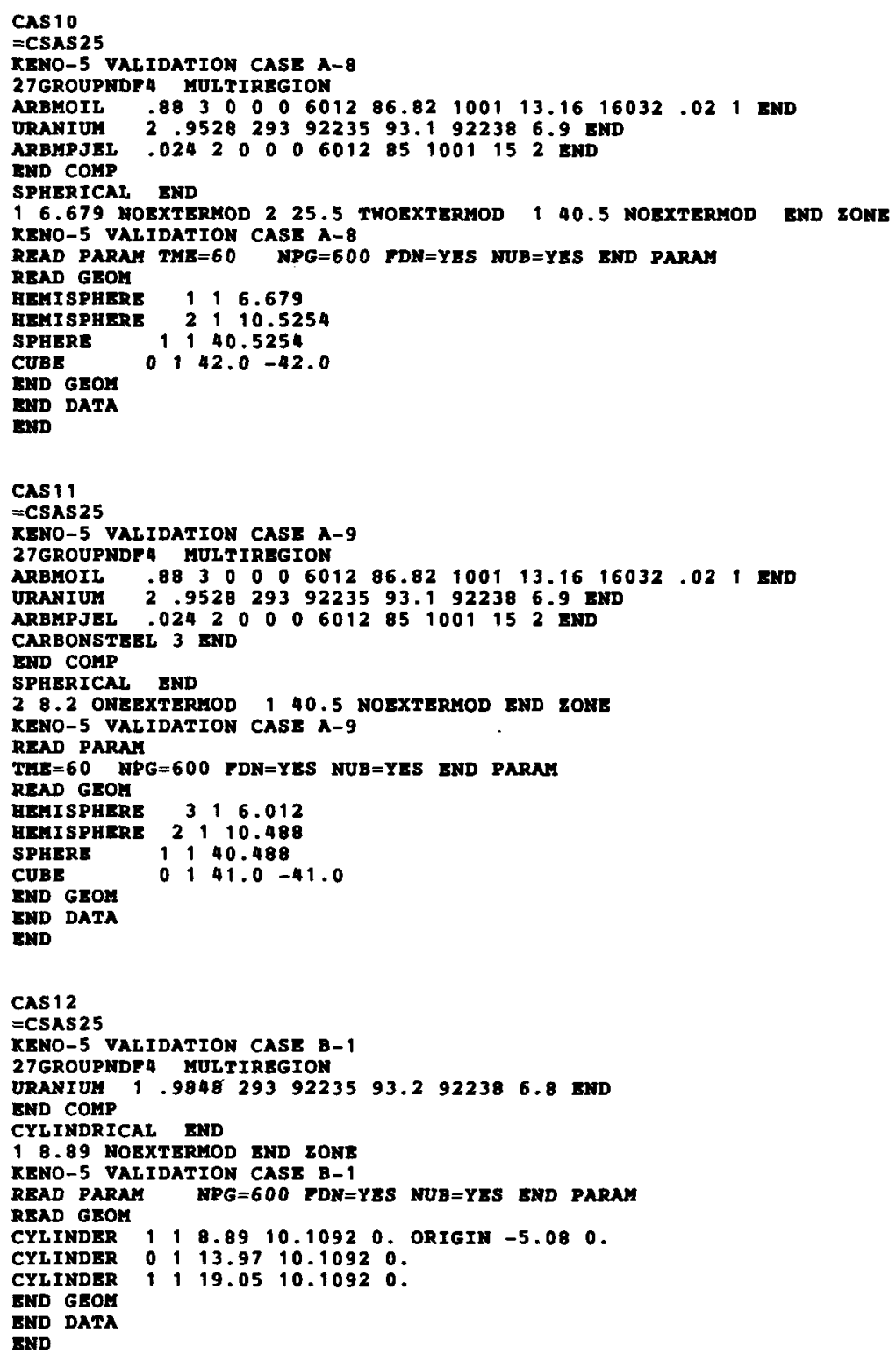

CAS 12
$=\operatorname{CSAS} 25$

KENO-5 VALIDATION CASE B- 1

27GROUPNDFA MULTIREGION

9223593.2922386 .8 ExD

END COMP

CYMD

89 NOEXTERMOD END ZONE

READ PARAM NPG $=600$ PDN=YES NUB=YES END PARAN

READ GEOM

CYLINDER 118.8910 .10920 . ORIGIN -5.080

CYLIYDER 119.9710 .1092 .

END GEOE

END DATA
SND 
Table A.5 (continued)

CAS 13
$=\operatorname{CSAS} 25$

KENO-5 VALIDATION CASE B-10

27GROUPNDF 4 MULTIREGION

SOLNUO2 (NO3)2 $163.30012939223592 .692238 \quad 7.4$ END

PLEXIGLASS 21 END

END COMP

19.52 ONEBXTERMOD 2 10.16 NOEXTERMOD END ZONE

KENO-5 VAL,TDATION CASE B-10

READ PARAM NPG $=600$ FDN=YSS NUB=YES END PARAM

READ GEOM

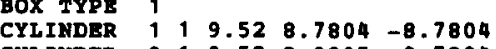

$\begin{array}{llllll}\text { CYLINDER } & 1 & 1 & 9.52 & 8.7804 & -8.7804 \\ \text { CYLINDER } & 0 & 1 & 9.52 & 8.9895 & -8.7809\end{array}$

CYLINDER $2110.169 .6295^{-8}-984$

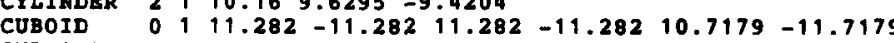

READ ARRAY NUX $=3$ NUY $=3$ NUZ $=3$ END ARRAY

END DATA

CAS 14
$=$ CSAS 25

KENAS25 VALIDATION CASE B-11

KTGOS VAJIDATION CASE B- 11

SOLNUO2(NO3) $2 \quad 1505092939223593.2922386 .8$ END

PLEXIGLASS 201 END

SS $304 \quad 3$ END

END COMP

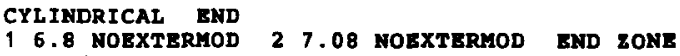

16.8 NOEXTERMOD 2 7.08 NOEXTERMOD END ZONE
XENO-5 VALIDATION CASE B-11

READ PARAM NPG $=600$ PDN=YES NUB=YES END PARAN

READ GEOM

BOX TYPB

CYIINDER $11106.81 .76-1.76$

CYLINDER $3197.08 \quad 1.76-2.04$

$\begin{array}{llllllll}1 & 15.0875 & -15.0875 & 15.0875 & -15.0875 & 1.76 & -2.0\end{array}$

$16.8 \quad 30.5-30.5$

CYLINDER $1196.8 \quad 30.5-30.5$

CYLINDER $3317.0896 .7-30.5$

$\begin{array}{lllllllll}\text { CUBOID } & 0 & 1 & 15.0875 & -15.0875 & 15.0875 & -15.0875 & 125.7 & -30.5\end{array}$

CORE $01-60.35-60.35-80.0$ $\begin{array}{lllllllll}\text { CUBOID } & 2 & 1 & 70.55 & -70.55 & 70.55 & -70.55 & 90.2 & -90.835 \\ \text { CUBE } & 0 & 1 & 95.0 & -95.0 & & & & \end{array}$

READ ARRAY NUX $=4$ NUY $=4$ NUZ $=2$ LOOP

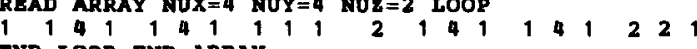

BND LOOP END ARRAY

BND DATA
CAS15

KENO-5 VALIDATION CASE B-12

TEROUPNDF 4 MULTIREGION

$\begin{array}{lll}.9829 & 293 \\ 2 & .8296 \text { END }\end{array}$

POLYETHYLENE 3 1

SND

5.747 ONEEXTERMOD 210.92 NOEXTERMOD END ZONE

KENO-5 VALIDATION CASE B-12

READ PARAM TME $=60$ NPG=600 FDN=YES NUB=YES END PARAM

READ GEOM

CYX TYPB

$\begin{array}{llllll}1 & 1 & 5.747 & 3.9699 & -4.107\end{array}$

CUBOID $2110.9195-10.9195,0.9195-10.9195 \quad 7.8755-7.8755$

CUBOID $\quad 0 \quad 1 \quad 15.047-15.047 \quad 15.047-15.04712 .0035-12.0035$

$\begin{array}{lllllllll}3 & 1 & 45.344 & -45.344 & 45.344 & -45.344 & 39.247 & -39.247\end{array}$

READ ARRAY NUX=2 NUY=2 NUZ=2 END ARRAY

END DATA

\section{CAS 16
$=$ CSAS2}

KENO-5 VALIDATION CASE B-13

SOLNUO2 (NO3) $2 \quad 1415012939223592.692238 \quad 7.4$ END

PLEXIGLASS 21 END

END COMP

CYLINDRICAL END

1 9.52 ONEEXTERHOD 2 10.16 NOEXTERMOD END ZONE

KENO-5 VALIDASION CASE B-13 NOEXTERMOD BND

READ PARAM

CYAT GER

CYLINDER $019.528 .7804-8.7804$

CUBOID $0115.495^{9}-15.495^{-9}$ is.495 $-15.49514 .9646-14.7554$

READ ARRAY NUX $=4$ NUY $=4$ NUZ $=4$ END ARRAY 
Table A.5 (continued)

CAS 17

KENO-5 VALIDATION CASE B-14

27GROUPNDF 4 MULTIREGION

SOLNUO2(NO3) $2 \quad 1415012939223592.6 \quad 92238 \quad 7.4 \quad$ END PLEXIGLASS 2 I 1 END

PARAFYIN

CYLINDRICAL END

19.52 ONEEXTERMOD 210.16 NOEXTERMOD END ZONE

READ PARAM TME $=60$ NPG $=600$ FDN=YES NUB=YES END PARAM

READ GEOM

CYLINDER $1119.528 .7804-8.7804$

CYLINDER $0019.528 .9896 \quad-8.7804$

CUBOID $\quad 0 \quad 1 \quad 18.425 \quad-18.425 \quad 18.425 \quad-18.425 \quad 17.8946 \quad-17.6854$

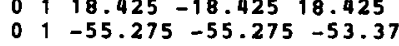

CUBOID

$3170.515-70.51570 .515-70.515 \quad 68.61-68.61$

END GBOM

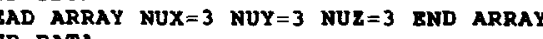

END DAT

CAS 18
$=$ CSAS 25

KENO-5 VALIDATION CASE B-15

SOLNUO2(N03)2 1 415 $012939223592.692238 \quad 7$ (4ND PLEXIGLASS 2,1 END

END COMP

CYLINDRICAL BND

19.52 ONEBXTBRMOD 210.16 NOEXTERMOD END ZONE

READ PARAM TME $=60$ NPG $=600$ PDN=YES NUB=YSS END PARAM

READ TROM

CYLINDER $199.528 .7804-8.7804$

CYLINDER $019.528 .9896-8.7804$

CYLINDBR $21010.169 .6296-9.4204$

$\begin{array}{lllllll}\text { CUBOID } & 0 & 1 & 14.54 & -14.54 & 14.54 & -14.54 \\ \text { CORE } & 0 & 1 & -0096 & -13.800\end{array}$

$2144.89-44.8944 .89-44.8942 .985-42.985$

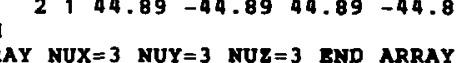

BND

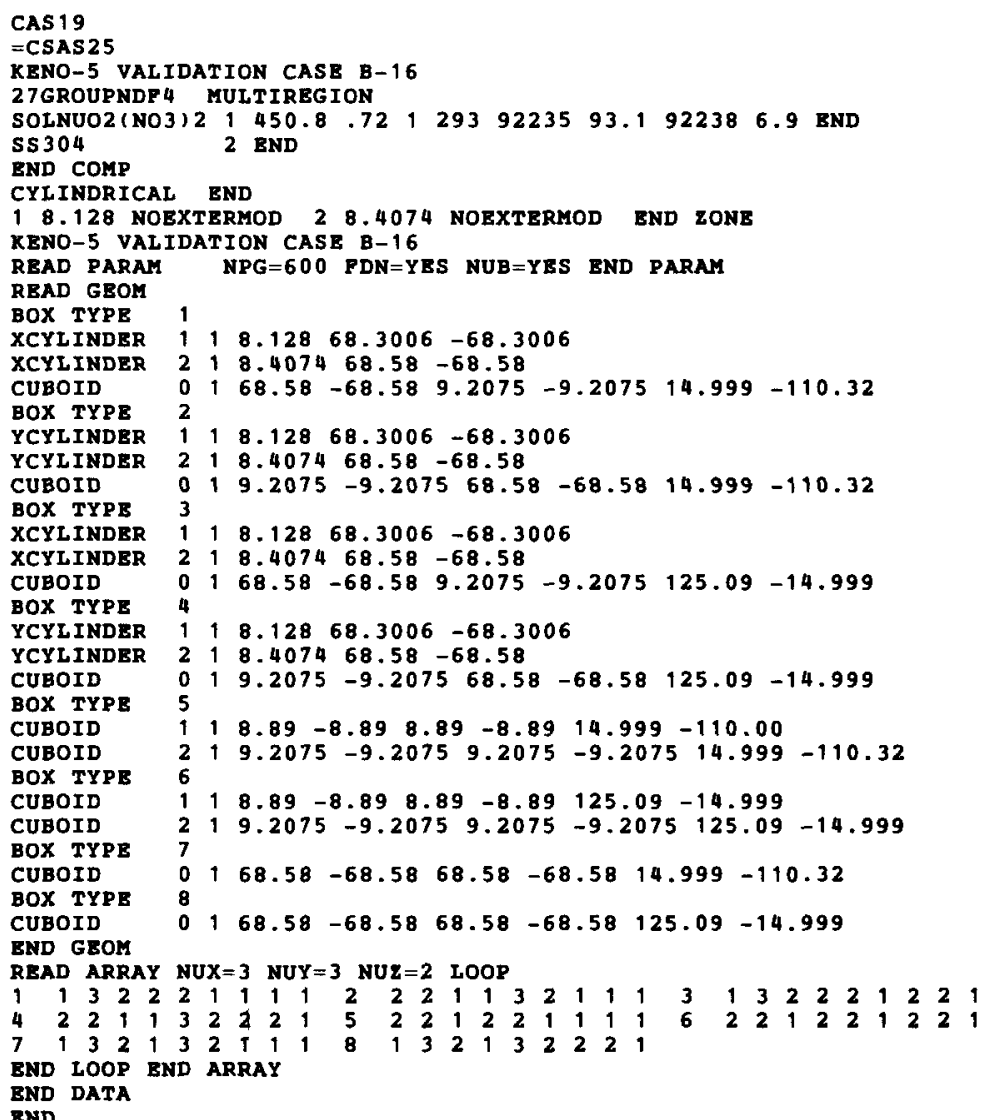

CAS19

KENO-5 VALIDATION CASB B-16

27GROUPNDPU MULTIREGTON

SOLNUO2(NO3) $21450.8 \quad 72112939223593.1922386 .9$ END 55304

BND COMP

CYLINDRICAL END

18.128 NOEXTERMOD 28.4074 NOEXTBRMOD END ZONE

1

READ GBOM

BOX TYPE

XCYLINDER $11198.128 \quad 68.3006-68.3006$

XCYLINDER 2 I 9.128

0
0

YCYLINDER $1418.128 \quad 68.3006 \quad-68.3006$

$\begin{array}{llllllllll} & & & \\ \text { YCYLINDER } & 2 & 1 & 8.4074 & 68.58 & -68.58 & & & & \end{array}$

$\begin{array}{lllllll}\text { XCYLINDER } & 1 & 1 & 8.128 & 68.3006 & \mathbf{6} & -68.3006\end{array}$

XCYLINDER 2 1 $8.4074-68.58-68.58$

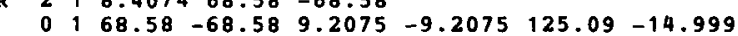

YCYLINDER $1418.128 \quad 68.3006 \quad-68.3006$

YCYLINDER $2018.4074 \quad 68.58-68.58$

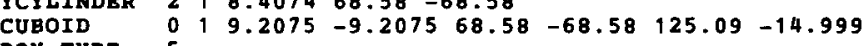

$\begin{array}{llllllllll} & & & & \\ \text { CUBOID } & 1 & 1 & 8.89 & -8.89 & 8.89 & -8.89 & 14.999 & -110.00\end{array}$

2. $9.2075-9.2075$ 9.2075 $-9.207514 .999-110.32$

COX TYPE

CUBOID

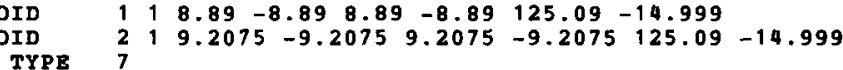

$\begin{array}{lllllllll}\text { BOX TYPB } & 7 & & 68.58 & -68.58 & 68.58 & -68.58 & 14.999 & -110.32\end{array}$

COX TYPE

$\begin{array}{ll}7 \\ 0.58-68.5868 .58-68.58 & 14.999-110.32\end{array}$

CORRAY NUX $=3$ NUY $=3$ NUY $=2$ LOOP

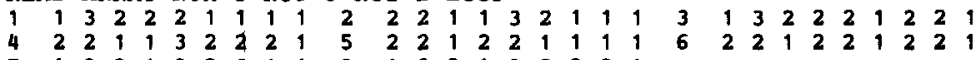

$\begin{array}{llllllllll}7 & 1 & 3 & 2 & 1 & 3 & 2 & \top & 1 & 1\end{array}$

END LOOP END ARRAY

END 
Table A.5 (continued)

CAs20

$=$ CSAS 25

VALIDATION CASB B-17

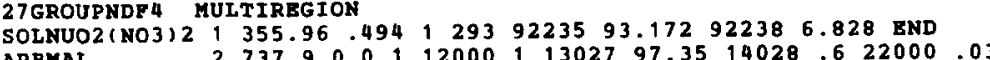

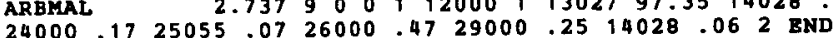

24000.1725055 .07

PIBXIGLASS

CND COMP

10.56 NOEXTERMOD 210.96 NOEXTERMOD END ZONE

XENO-5 VALIDATION CASE $B-17$
READ PARAM TME $=60 \quad$ NPG $=600 \quad$ YDN $=$ YES NUB $=$ YBS BND PARAM

READ GROM

CYLINDER $11110.56 \quad 9.41 \quad-9.41$

CYLINDER $00110.56 \quad 109.69-9.41$

$\begin{array}{lllllllll}\text { CUBOID } & 0 & 1 & 15.24 & -15.24 & 15.24 & -15.24 & 113.17 & -9.73\end{array}$ $\begin{array}{llll}1 & 15.24 & -15.24 & 15.24\end{array}$

REPLECTOR 01480.49281

CUBE $\quad 3182.05-82.05$

END GEOM

ARRAY NUX=4 NUY =4 END ARRAY

BND DATA

\section{CAS21}

XENO-5 VALIDATION CASE B-18

27GROUPNDF4 MULTIREGION

SOLNUO2 (NO3) $2 \quad 364.11 .58492939223593 .17292238 \quad 6.828$ END

ARBMAL $\quad 2.7379000191200091302797 .3514028 \quad 6 \quad 22000.03$

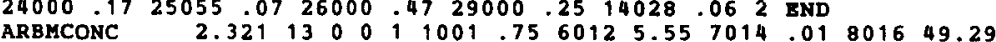

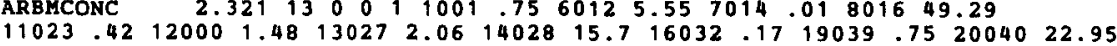
$22000.0526000 \quad .82 \quad 3$ END END COMP

CYLINDRICAL END

10.56 NOEXTBRMOD 210.96 NOEXTERMOD END ZONE

KBNO-5 VALIDATION CASE B-1

READ PARAM TMB $=60 \quad$ NPG $=600 \quad$ FDN $=$ YBS NUB $=$ YES END PARAM

READ GEOM

CYLINDER $1110.568 .565-8.565$

CYLINDER $00110.56 \quad 110.535-8.565$

CYLINDER $2110.96 \quad 110.535-8.865$

CUBOID

BOX TYPE

CYLINDER

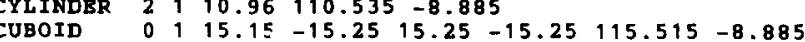

TYPE

CYLINDBR

$10.560 .565-8.565$

$\begin{array}{llllllll}0 & 1 & 15.25 & -15.25 & 15.25 & -15.25 & 115.515 & -8.885\end{array}$

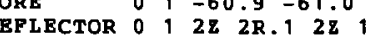

$\begin{array}{lllllllll} & & 86.6 & -86.6 & 86.8 & -86.8 & 87.9 & -87.9\end{array}$
READ ARRAY NUX $=4$ NUY $=4$ LOOP

END LOOP END ARRAY

END DATA

CAS 22

KENO-5 VALIDATION CASB B-2

URANIUM $1.98482939223593 .292238 \quad 6.8$ END

END COMP

CYLINDRICAL END

18.89 NOEXTERMOD END ZONE

READ PARAM NPG $=600$ PDN $=$ YES NUB=YES END PAPAM

$\begin{array}{lllllllll}\text { CUBOID } 1 & 1 & 0.3434 & -12.4434 & 6.35 & -6.35 & 6.49 & -6.49\end{array}$

CYLINDER $0011913.97 \quad 6.49-6.49$

CYLINDBR

END GEOM

END DATA

CAS 23

KENO-5 VALIDATION CASE B-3

27GROUPNDF 4 MULTIREGION

URANIUM $1.9823129392235 \quad 93.292238 \quad 6.8$ END

END COMP

END ZONE

READ VALIDATION CASE B-3 READ GEOM

UNIT 1

$\begin{array}{llllll}\text { CUBOID } 11 & 2 \mathrm{P} 6.3515 & 1.2685 & -3.8115 & 13.377 & 13.058\end{array}$

$\begin{array}{lllllll}\text { CUBOID } & 1 & 286.3515 & 6.3515 & \mathbf{3} .8115 & 13.377 & 13.058\end{array}$

CUBOID

CYLINDER 114.555512 .9180$.

UNIT 3

CYLINDER $1195.761 \quad 13.4750$.

UNIT 4

552512.9690 .

ARRAY

$$
1-4.573-4.5730 \text {. }
$$

UNIT 7ER 114.554512 .9740$.

CYLINDER 1115.749513 .4750$.

CYLINDER

UNIT 9

COM $=$ ' CENTERPIEC

ARRAY

UNIT 10

TOM OF UNIT 5

$\begin{array}{lllll}\text { CUBOID } & 1 & 1 & 2 P 3.818 .13 & -4.5738 .911^{0} \\ \text { CUBOID } & 0 & 1 & 2 P 4.5738 .13 & -4.573^{8} 8.91^{\circ}\end{array}$ 
Table A.5 (continued)

UNIT 11

COM= TOP OP UNIT 5

CYLINDER 114.5734 .3190

cuborp $0.1284 .5738 .13-4.5734 .3190$

OF CENTERPIBCE,

CYLINDER 115.7572 .6900$. ORIGIN $-.593-.593$

CUBOID

UNIT 13

COK='MIDDLE OF CENTERPIBCE'

CUBOID

COM=I TOP OF CENTERPIECB

HEMISPHERE 116.082 ORIGTY -.268 .2680 .

CUBOID

14 P6.35 6.0820.

CUBOID

$\begin{array}{llllllll}\text { CUBOID } 0 & 1 & 4825.0 & 15.0 & -2.0\end{array}$

HOLE $10.0-17.306 \quad 0.15$

HOLE 3

HOLE $4 \quad-9.394 \quad 10.9990 .156$

HOLE $5 \quad 0.015 .400 \quad 0.29$

HOLE $6 \quad 9.727 \quad 10.8030 .134$

HOLE 716.2241 .4190 .140

$\begin{array}{llccc}\text { HOLE } & 8 & 11.931 & -9.322 & 0.087 \\ \text { HOLE } & 9 & 0.0 & 0.0 & -1.755\end{array}$

END GEOY

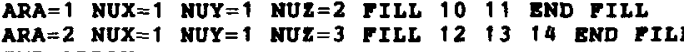

BND ARRAY

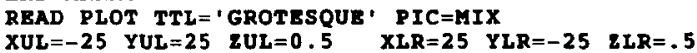

$X U L=-25 \quad$ YUL $=25 \quad 2 U L=0.5 \quad X$

$X U L=-25 \quad Y U L=25 \quad$ ZUL $=2 \quad$ XLR=25 YLR=-25 $\quad$ LLR=2 $\quad$ END $X U L=-25 \quad$ YUL $=25 \quad$ ZUL $=9.5 \quad$ XLR $=25 \quad$ YLR $=-25 \quad$ \&LR $=9.5 \quad$ END

$X U L=-.593 \quad Y U L=-25 \quad 2 U L=15 \quad X L R=-.593 \quad$ YLR $=25 \quad$ ZLR $=-2$

UAX $=0 \quad$ VAX $=1 \quad$ VDN $=0$ WDN $=-1 \quad$ NAX $=130$ END

BND PLOT
BND DATA

END
CAs24

KENO-5 VALIDATION CASE B-4

27GROUPNDF4 MULTIREGION

URANIUM $1.9848 \quad 2939223593.2922386 .8$ END

SS304 2 END

INCONEL

CYI INDRICAL END

2.254 NOEXTERMOD 15.74 NOBXTERMOD END $20 \mathrm{NE}$

KENO-5 VALIDATION CASE B-4

READ PARAM NPG=600 FDN=YBS NUB=YBS PLT=NO END PARAM READ GEOM

ONTT

IS RODS IN FUEL,

CYLINDER 2,2542 P2.691

COM='SS ROD \& INCONEL SLEEVE'

CYLINDER $210.254 \quad 2 P .987$

$\begin{array}{lllll}\text { CYLINDER } & 3 & 1 & .381 & \text { 2P. } 987\end{array}$

UNIT 3

COM='A6 CYLINDER E SUPPORT RODS.

CYLINDER $1115.7405 \quad 2 P 2.69$

HOLB $\quad-4.27352 \mathrm{R}$

CUBOID $\quad 0 \quad 1 \quad 4$ P7.7165 2P4.667

HOLE $\quad 2-4.27350-3.679$

HOLE 2 - 2.2735 O 3.679

HOLE $24.27350-3.679$

END GEOM

READ ARRAY

NUX $=4$ NUY $=4$ NUZ=4 FILI P3 END PILL

SND ARRAY

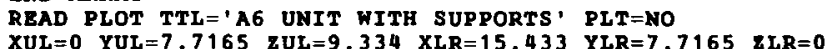

$X U L=0 \quad Y U L=7.7165 \quad$ ZUL $=9.334 \quad X L R=$

BND DATA

CAs25

$=$ CSAS 25

KENO-5 VALIDATYON CASB B-5

URANIUM 1 ,

END COMP

CYLINDRICAL END

14.56 NOEXTERMOD END ZONE

KENO-5 VALIDATION CASE B-5

作

READ GBOM

CYLINDER

$\begin{array}{lllll}1 & 4.558 & 2.16 & -2.16\end{array}$

$\begin{array}{lllll}1 & & 558 & 2.16 & -2.16\end{array}$

$\begin{array}{lllllllll}\text { CYLINDER } & 1 & 1 & 5.747 & 4.851 & -4.851 & & & \\ \text { CUBOID } & 0 & 1 & 5.8554 & -5.8554 & 5.8554 & -5.8554 & 4.9771 & -4.9771\end{array}$

LND GEOM

ARRAY NUX $=2$ NUY $=2$ NUZ=2 BND ARRAY

BND DATA 
Table A.5 (continued)

CAS 26
$=$ CSAS 25

KBNO-5 VALIDATION CASE B- 6

GROUPNDF MULTIREGIOH

$9223593.292238 \quad 6.8$ END

PARAFFI

CYLINDRICAL END

14.56 NOEXTERMOD BND ZONB

READ PARAM TME $=60$ NPG $=600$ FDN=YES NUB=YES END PARAY

READ GBOM

CYLINDER $1114.558 \quad 8.641-8.641$

CUBOID $\quad 0 \quad 19.7212-9.7212 \quad 9.7212$

CORE $01-19.4424-19.4424-27.6082$

REFLECTOR 201 6R15.2 1

END GEOM

NUX $=2$ NUY $=2$ NUZ $=2$ BND ARPAY

BND

CAS27

KENO-5 VALIDATION CASE B-7

27GROUPNDP" MULTIREGION

URANIUM $1 \quad 9848 \quad 2939223593.292238 \quad 6.8$ END

PARAFFIN 2 END

BND COMP

CYLINDRICAL END

MOD END ZONB

PEAD PARAM TME $=60 \quad$ NPG $=600 \quad$ FDN=YES NUB=YES END PARAK

READ GEOM

$\begin{array}{lllllll}\text { CYLINDER } & 1 & 1 & \mathbf{4} .558 & \mathbf{2} .160 & \mathbf{2} .160\end{array}$

$\begin{array}{lllllll}\text { CYLINDER } & 0 & 1 & 5.747 & 2.160 & \mathbf{2} .160 \\ \text { CYLINDER } & 1 & 1 & 5.747 & 4.85 & -4.85\end{array}$

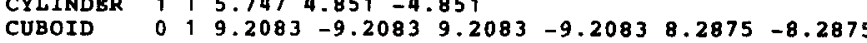

CORB $01-18.4166-18.4166-16.575$

REFLECTOR $2+6 R 15.2$ i

END GEOM

READ ARRAY NUX=2 NUY=2 NUZ=2 END ARRAY

END DATA

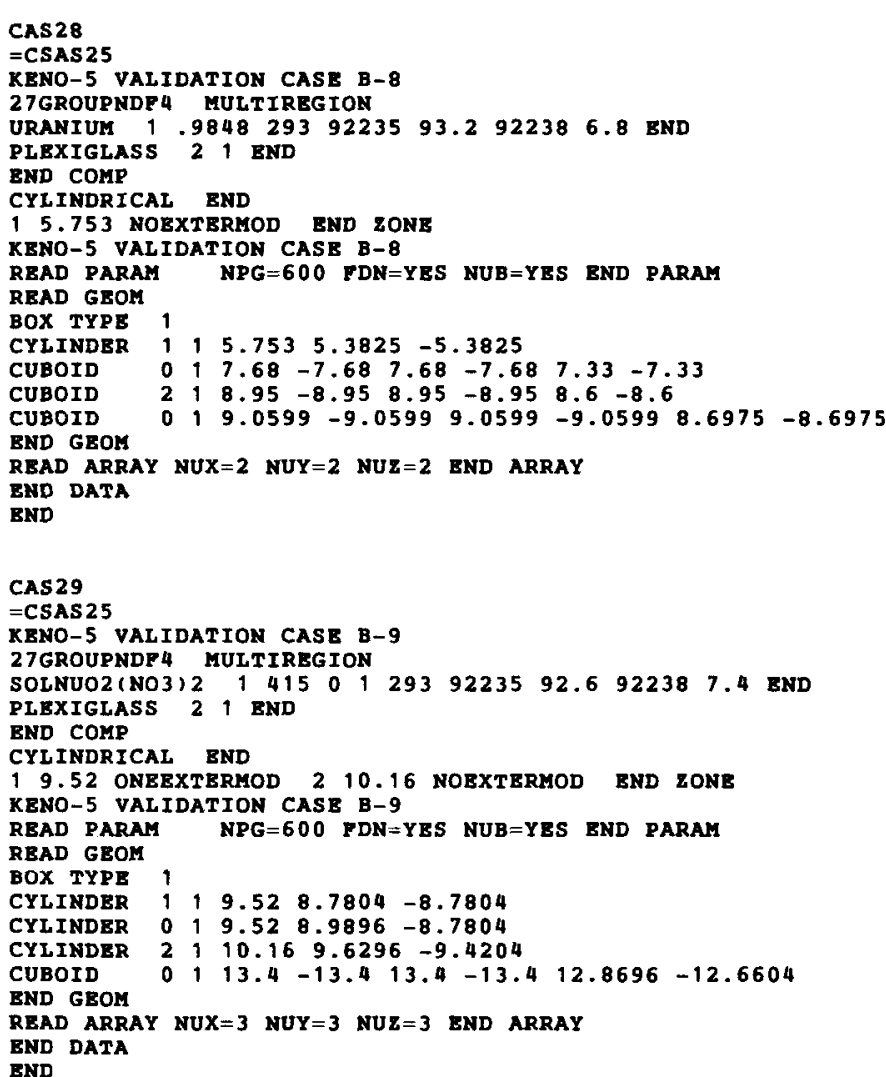


Table A.5 (continued)
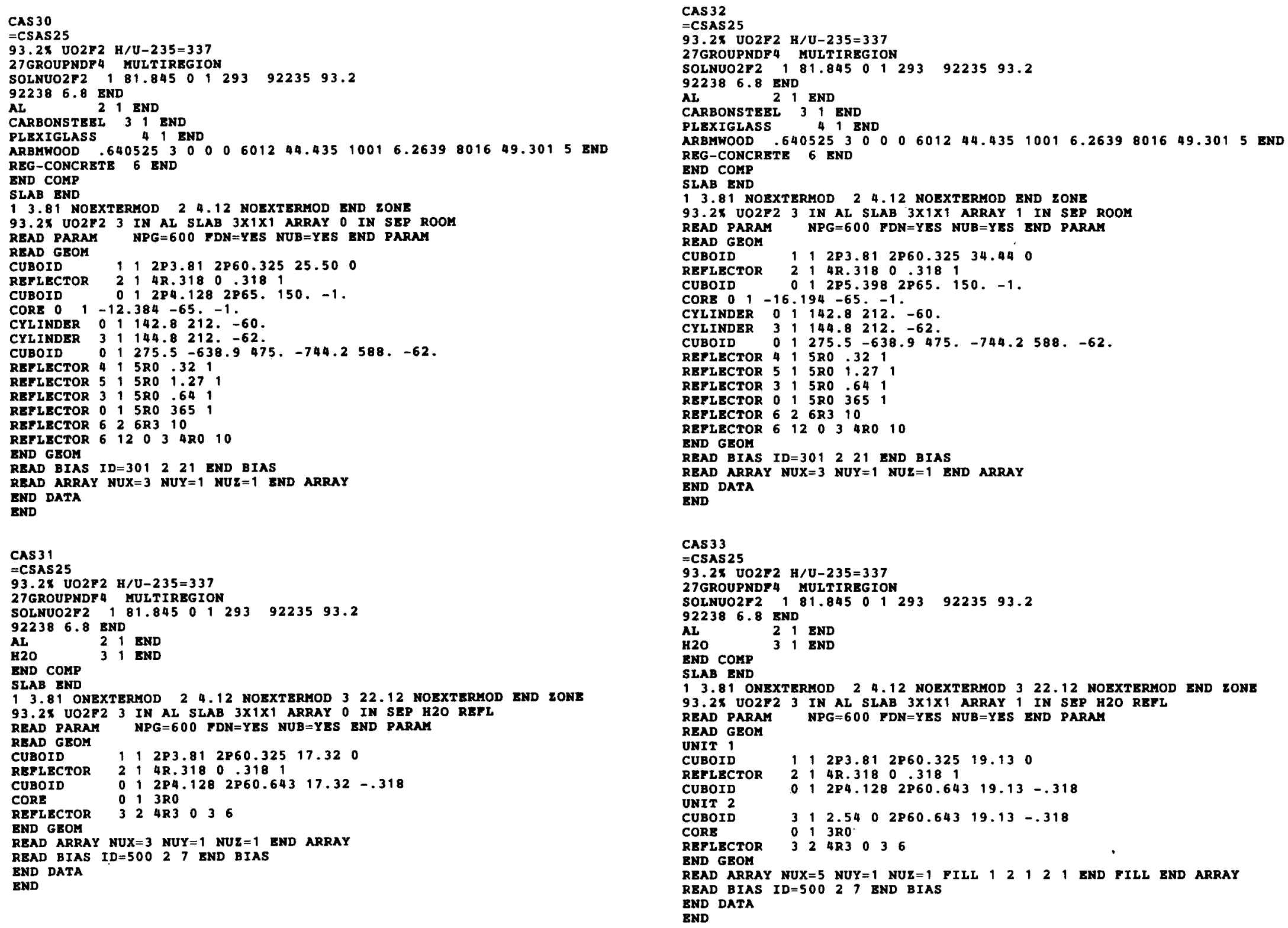
Table A.5 (continued)

CAS34

93.2X UO2F 2 H $/ U-235=337$

SOLNUO282, 81.845012939223593 .2

$\begin{array}{lll}92238 & 6.8 & \text { END } \\ \text { AL } & 2 & 1 \text { END }\end{array}$

AL CARBONSTEEL 31 END 1 END

\section{$418 \times D$}

ARBMHOOD $.640525 \quad 30000601244.4351001 \quad 6.26398016 \quad 49.3015$ END

REG-CONCREI

BND COMP

1 3.81 NOEXTERMOD 24.12 NOEXTERMOD END ZONE

93.2X UO2F2 3 IN AL SLAB $3 \times 1 \times 1$ ARRAY 3 IN SEP ROO

CUBOID $\quad 1 \quad 1283.81 \quad 2860.325 \quad 58.78$

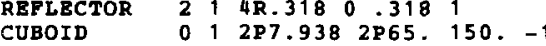

CORE $01-23.814-65 .-1$.

CYLINDER $01142.8212 .-60$.

CUBOID $001275.5-638.9475,-744.2588,-62$.

REPLECTOR 4 1 1 5RO

REFLECTOR 5 1 1 5RO 1.27

$\begin{array}{llllll}\text { REFLECTOR } & 3 & 1 & 5 R 0 & .64 \\ \text { REPLECTOR } & 0 & 9 & 5 R 0 & 365\end{array}$

$\begin{array}{llllll}\text { REPLECTOR } & 0 & 1 & 5 R 0 & 365 \\ \text { REFLECTOR } & 6 & 2 & 6 R 3 & 10\end{array}$

$\begin{array}{lllllll}\text { REPLECTOR } & 6 & 2 & 6 R 3 & 6 R & 10 & \\ \text { REPLECTOR } & 6 & 12 & 0 & 3 & 4 R 0 & 10\end{array}$

RENDETOR 6

PEAD BIAS ID $=301221$ RND BIAS

READ ARRAY NUX $=3$ NUY $=1$ NUZ $=1$ END ARRAY

END DATA

CAS 35

$93.2 \times \mathrm{UO2F} 2 \mathrm{H} / \mathrm{U}-235=337$

SOLNUO2F2, $181.84501293 \quad 9223593.2$

$\begin{array}{llll}92238 & 6.8 & \text { END } & \\ A L & 2 & 1 & \text { END }\end{array}$

$\mathrm{H} 2 \mathrm{O}$

END COMP

13.81 ONEXTERMOD 24.12 NOBXTERMOD 322.12 NOBXTERMOD BND ZONE 93.2X UO2F2 3 IN AL SLAB $3 \times 1 \times 1$ ARRAY 3 IN SBP H2O REFL

READ PARAM

READ GEOM

CUBOID

$\begin{array}{lllllll}1 & 1 & 2 P 3.81 & 2860.325 & 32.92\end{array}$

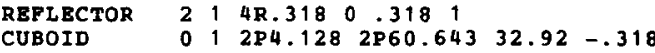

UNIT 2

CUBOID

$\begin{array}{lllllll}3 & 1 & 7.62 & 0 & 2 \mathrm{P} 60.643 \quad 32.92 & -.318\end{array}$

CORE
REFLECTO

$\begin{array}{lllllll}3 & 2 & 4 R & 0 & 3 & 6\end{array}$

READ ARRAY NUX=5 NUY=1 NUZ=1 FILL $1 \begin{array}{llllll}1 & 2 & 1 & 2 & 1 & \text { END PILI END ARRAY }\end{array}$ READ BIAS ID $=50027$ END BIAS

READ BIAS
CAS 36
$=\operatorname{CSAS} 25$

$93.2 \times$ U02F 2 H $/ U-235=337$

SOLNUO2F2 $181.84501293 \quad 9223593.2$

922386.8 END

CARBONSTEEL 1 END

PLEXIGLASS 319

ARBMWOOD $.640525 \quad 300006012 \quad 44.435 \quad 10016.26398016 \quad 49.3015$ END REG-CONCRETE 6 BND

END COMP

SLAB END

3.81 NOEXTERMOD 24.12 NOEXTERMOD END 2 TOME

$93.2 \times$ UO2F2 3 IN AL SLAB $3 \times 1 \times 1$ ARRAY 4.5 IN SEP ROOM

READ PARAM NPG $=600$ FDN=YBS NUB=YBS END PARAM

$\begin{array}{llllllll}\text { CUBOID } & 1 & 1 & 2 P 3.81 & 2 P 60.325 & 85.52 & 0\end{array}$

$\begin{array}{llllllll}\text { REFLECTOR } & 2 & 1 & 4 R .318 & 0 & 318 & 1 & \\ \text { CUBOID } & 0 & 1 & 2 \mathrm{P} 9.843 & 2 \mathrm{P} 65 . & 150 . & -1\end{array}$

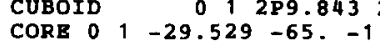

CYLINDER $0 ; 142.8212 .-60$

CYLINDER 31144.0212 .62$.

$\begin{array}{llllllllll} & & & \\ \text { CUBOID } & 0 & 1 & 275.5 & -638.9 & 475 & -744.2 & 588, & -62 .\end{array}$

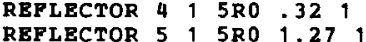

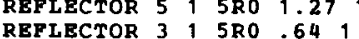

$\begin{array}{llllll}\text { REFLECTOR } & 3 & 1 & 5 R 0 & 64 & 1 \\ \text { REFLBCTOR } & 0 & 1 & 580 & 365 & 1\end{array}$

REFLECTOR $6 \begin{array}{lllll}6 & 6 R 3 & 10\end{array}$

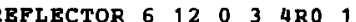

END GEOM

READ BIAS ID $=301$

READ ARRAY NUX $=3$ NUY $=1$ NUZ $=1$ END ARRAY

END DATA

CAS 37
$=$ CSAS 25

93. $2 x$ UO2F2 H $/ U-235=337$

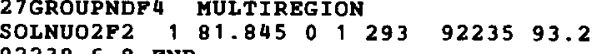

$\begin{array}{lllll}92238 & 6.8 & \text { END } & \\ A L & 2 & \text { END }\end{array}$

BND COMP

1 3.81 ONEXTERMOD 24.12 NOEXTERMOD 322.12 NOBXTERMOD END ZONE 3.25 UO2F2 3 IN AL SLAB $3 \times 1 \times 1$ ARRAY 4.5 IN SEP H2O RBPL

READ GBOM

$\begin{array}{llll}1 & 2 P 3.812 P 60.325 & 61.060\end{array}$

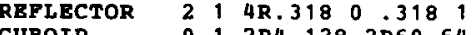

$\begin{array}{llllll}0 & 1 & 2 \mathrm{P} 4.128 & 2 \mathrm{P} 60.643 & 61.06 & -.318\end{array}$

$\begin{array}{lllllllll}3 & 1 & 11.43 & 0 & 2 \mathrm{P} 60.643 \quad 61.06 & -.318\end{array}$

$\begin{array}{lllll}3 & 1 & 11.0 & 4 & 0 \\ 0 & 1 & 3 \mathrm{R} 0 & & 286 \\ 3 & 2 & 4 \mathrm{R} 3 & 0 & 3\end{array}$

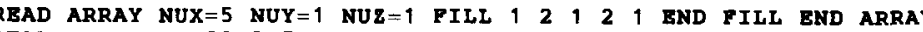
READ BIAS ID $=50027$ END BIAS BND DATA 
Table A.5 (continued)

CAS42
$=$ CSAS25

$93.2 \times$ บ0252 H/U-235=337

$\begin{array}{lllll}\text { 27GROUPNDF4 } & \text { MUITIREGION } & & \\ \text { SOLNUO2F2 } 181.845 & 0 & 1293 & 92235 & 93.2\end{array}$

$\begin{array}{llll}92238 & 6.8 & \text { END } & \\ \text { AL } & 2 & 1 & \text { END }\end{array}$

AL COMP

13.81 NOEXTERMOD 24.12 NOEXTERMOD END ZONE

$93.2 \times$ UO2F2 3, 6 IN AL SLABS $2 \times 1 \times 1$ ARRAY 2 IN SEP

READ PARAM NPG $=600$ FDN $=$ YES NUB $=$ YES END PARAM

READ GEOM

CUBOID

$\begin{array}{llllll}1 & 12 \mathrm{P} 3.812 \mathrm{P} 60.325 & 32.39 & 0\end{array}$

$\begin{array}{lllllll}\text { REFLECTOR } & 2 & 1 & 4 R .318 & 0 & .318 & 1 \\ \text { CUBOID } & 0 & 1 & 2 P 4.128 & 2 P 65 . & 150 . & -1 .\end{array}$

CUBOID

cusoro

CUBOID

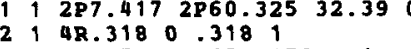

CUBOTD

$2 P 7.7352865,150,-1$.

END GEOM

$015.0802865 .150 .-1$.

READ ARRAY NUX=3 NUY=1 NUZ=1 FILL 23,1 END FILL END ARRAY END DATA

\section{CAS43}

$=$ CSAS 25

$93.2 \times$ UO2F2 H/U-235=337

27GROUPNDF 4 MULTIREGION $293 \quad 9223593.2$

922386.8 END

IND COMP

END COMP

13.81 NOBXTERMOD 24.12 NOEXTEPMOD END ZONE

93.2X UO2F2 3,6 IN AL SLABS 2 X1X1 ARRAY 30 IN SEP

READ PARAM

READ GROM
UNIT 1

CUBOID

$\begin{array}{lllllll}\text { REFLECTOR } & 2 & 1 & 4 R .318 & 0 & 318 & 3 \\ \text { CUBOID } & 0 & 1 & 2 P 4.128 & 2865 & 150 & -1\end{array}$

UNIT 2

$112 \mathrm{PP} .4172 \mathrm{P} 60.32592 .480$

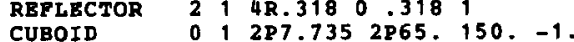

UNIT 3

$0176.202865 .150 .-1$.

UNTE

READ ARRAY NUX=3 NUY=1 NUI=1 FILI 231 END FILI END ARRAY END DATA

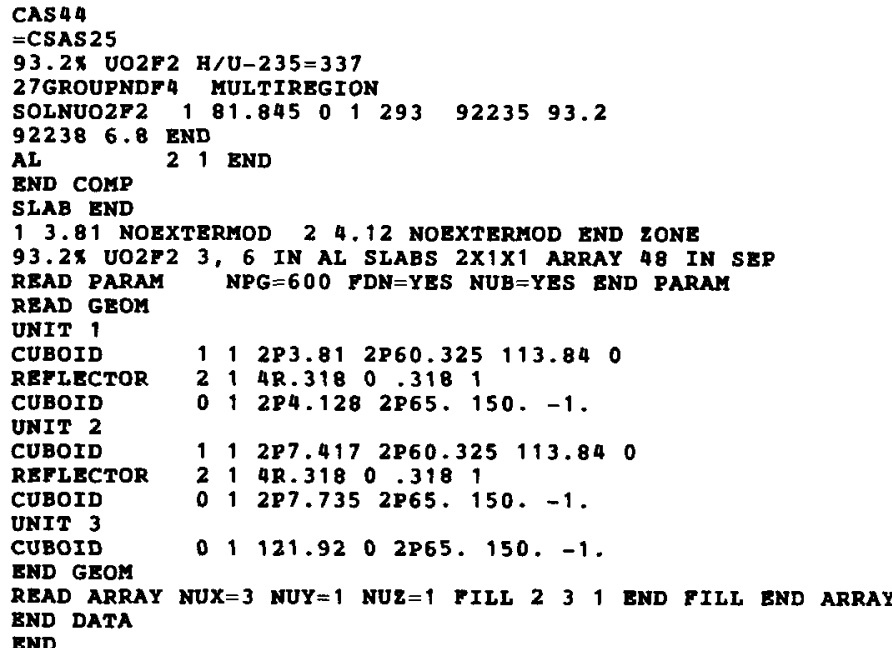

\section{CAS 45
$=$ CSAS 25}

93.28 UO2F2 H/U-235=337

SOLNUO2P2 $181.84501293 \quad 9223593.2$

\section{$\begin{array}{llll}9238 & 6.8 & \text { END } & \\ 2 & 1 & \text { END }\end{array}$}

END COMP

13.81 NOEXTERMOD 2 4.12 NOEXTERMOD END ZONE

33.2X UO2P2 $3,6,3$ IN AL SLABS $3 \times 1 \times 1$ ARRAY 0 IN SE

$\begin{array}{lllll} & 12 \mathrm{P} 3.81 & 2 \mathrm{P} 60.325 & 19.63\end{array}$

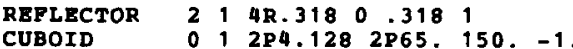

$\begin{array}{lllll}\text { ONIT } 2 & 12 \mathrm{P} 7.417 & 2 \mathrm{P} 60.325 & 19.63\end{array}$

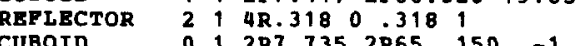

READ ARRAY NUX=3 NUY=1 NUZ=1 FILI 121 END FILL END ARRAY

END DATA 
Table A.5 (continued)

\section{CAS 46
$=$ CSAS2}

93.2X VO2F2 H/U $-235=337$

27 GROUPNDF 4 MULTIREGIOH

SOLNUO2P2 $181.84501293 \quad 9223593.2$

$\begin{array}{llll}92238 & 6.8 & \text { END } & \\ A L & 2 & 1 & \text { END }\end{array}$

BND COMP
SLAB END

13.81 NOEXTERMOD 24.12 NOEXTERMOD END EONE

$93.2 \times$ VO2P2 $3,6,3$ IN AL SLABS $3 \times 1 \times 1$ ARRAY 10 IN SEP

READ PARAM NPG $=600$ PDN=YES NUB=YES END PARAM

READ GBOM

CUBOID

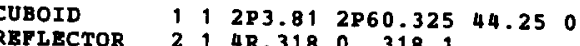

CUBOID $\quad 0$,

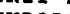

$\begin{array}{llllllllll}\text { CUBOID } & 1 & 1 & 2 P 7.417 & 2 P 60.325 & 44.25 & 0\end{array}$

CUBOID

CUBOID

1287.735 2P65. 150. -1

BND GEON

$0125.402865 .150 .-1$

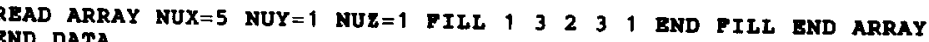
END DATA

\section{CAS 47
$=$ CSAS 25}

93.28 UO2F2 H/U-235=337

27GROUPNDF4 HULTIREGTON

SOLNUO2F2 181.845092939223593 .2

$\begin{array}{llll}92238 & 6.8 & \text { END } \\ \text { AL } & 2 & 9 \text { END }\end{array}$

END COMP

1 3.81 NOEXTERMOD 24.12 NOEXTERMOD END ZONB

93.28 U02F2 3, 6, 3 IN AL SLABS $3 \times 1 X 1$ ARRAY 20 IN SEP

ES END PARA

READ GEOM

$\begin{array}{lllllllll} & & & & & & & & \end{array}$

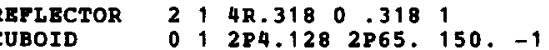

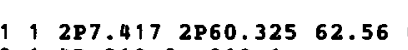

CUBOID

cusoin

UNIT

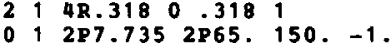

CUBOID

$0150.802855 .150 .-1$.

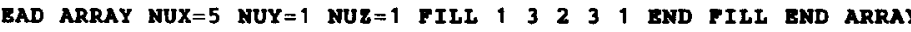
GND DATA
CAS 48
$=$ CSAS 25

$93.2 \times$ UO2F2 H $/ U-235=337$

SOLNUO2F2 $181.84501293 \quad 9223593.2$

$\begin{array}{llll}9238 & 6.8 & \text { END } & \\ \text { AL } & 2 & 1 & \text { END }\end{array}$

AND COMP

3.81 NOEXTERMOD 24.12 NOEXTERMOD END ZONE

93.2X U02P2 3,6, 3 IN AL SLABS 3X1X1 ARRAY 32 IN SEP

READ PARAM NPG $=600$ FDN=YES NUB=YES END PARAN

READ GEOM

CUBOID

$\begin{array}{lllllll} & 1 & 2 P 381 & 2 P 60.325 & 81.560\end{array}$

CUBOID $0112 \mathrm{P} 4.128$ 2P65. $150 .-1$

UNIT 2 CUBDIDCTOR

$\begin{array}{llllllll}\text { CUBOID } & 1 & 1 & 287.417 & 2 P 60.325 & 81.56\end{array}$

CUBOID $01287.7352865,150,-1$

UNIT 3

CUBOI

$0181.2802865 .150 .-1$

READ ARPAY NUX=5 NUY=1 NUZ=1 FILL $\begin{array}{llllllll}1 & 3 & 2 & 3 & 1 & \text { END FILI END ARRA }\end{array}$ END DATA

\section{CAS49}

93.2Х บ02F2 H/U-235=337

7GROUPNDP4 MULT

SOLNUO2F2 $181.845 \quad 0 \quad 1293 \quad 9223593.2$

$\begin{array}{lll}92238 & 6.8 & \text { END } \\ \text { AL } & 2 & 1\end{array}$

BND COMP

7.62 NOEXTERMOD 27.94 NOEXTERMOD END ZONE

93.28 U02F2 6 . 3 IN AL SLABS $2 \times 1 \times 1$ ARRAY 12 IN SEP

READ PARAM NPG $=600$ FDN $=Y$ YS NUB=YES END PARAM

READ GBOM

CUBOID

$\begin{array}{lllll} & 1 & 2 P 3.81 & 2860.325 & 58.190\end{array}$

CUBOID $\quad 0 \quad 12$ 2P4.128 2 P65. $150 .-1$

NIT 2

CUBOI:

CUBOID

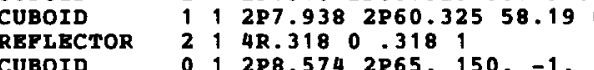

CUBOID $0130.4802265 .150 .-1$.

BND GBO\&

READ ARRAY NUX=3 NUY=1 NUZ=1 FILL 231 END FILL END ARRAY END DATA 
Table A.5 (continued)

CAS 50
$=C S A S 25$

93.2X UO2F2 H/U-235=337

$\begin{array}{llll}27 \text { GROUPNDF } 4 & \text { MULTIRBGION } \\ \text { SOLNUO2P2 } 81.84501293 \quad 92235 & 93.2\end{array}$

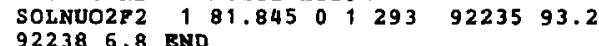

92386.8 BND 2 END

END COKP

17.62 NOEXTERMOD 27.94 NOEXTERMOD END $20 \mathrm{ONE}$ 93.2\% U02F2 6 \& 3 IN AL SLABS $2 \times 1 \times 1$ ARRAY 18 IN SBP READ PARAM NPG $=600$ PDN=YES NUB $=$ YES END PARAM

READ GEOM

CUBOID

$\begin{array}{llllllll} & 1 & 2 P 3.81 & 2 P 60.325 & 68.3 & 0\end{array}$

CUBOID 0 1 12 2P4.128 2P65. 150. -1 .

UNIT 2

CUBOID

$\begin{array}{lllll}\text { CUBOID } & 2 & 1 & 2 \mathrm{P} .318 & 2 \mathrm{P} 60.32568 .30^{\circ} \\ \text { CUBOID } & 1 & 1 & 2 \mathrm{P} 7.9382 \mathrm{P} 60.32568 .3\end{array}$

REPLECTOR

CUBOID

CUBOID

$8.5742 P 65.150,-1$

END GEOM
READ ARRAY NUX=3 NUY $=1$ NUZ=1 FILL 231 END FILL END ARRAY

$0145.7202865 .150 .-1$. BND DATA

CAS51

$=$ CSAS 25

93.2X บO2P2 H/U-235=337

SOLNUO2F2, B1.845 $01293 \quad 9223593.2$

922386.8 END

AL

\section{BND COMP}

STAB BND 7.62 NOBXTBRMOD 27.94 NOEXTERMOD END ZONE

93.28 UO2P2 6 \& 3 IN AL SLABS $2 \times 1 \times 1$ ARRAY 30 IN SBP

READ PARA

READ GBOM

CUBOID $1112 \mathrm{P3} .812 \mathrm{P} 60.32583 .110$

CUBOID

$12 \mathrm{P3} .812 \mathrm{P} 60.325$

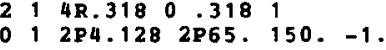

CUBOID

REFLECTOR

CUBOID

उNIT 3

$212 \mathrm{P} .3182 \mathrm{P} 60.32583 .110$

CUBOID

$\begin{array}{llll}1 & 287.938 & 2 P 60.32583 .110\end{array}$ 1 4R. 3180.318

$2 P 8.574$ 2P65. $150 .-1$.

MRRAY NUX=3 NUY=1 NUE=1 FILL 231 END FILL END ARRAY BND DATA

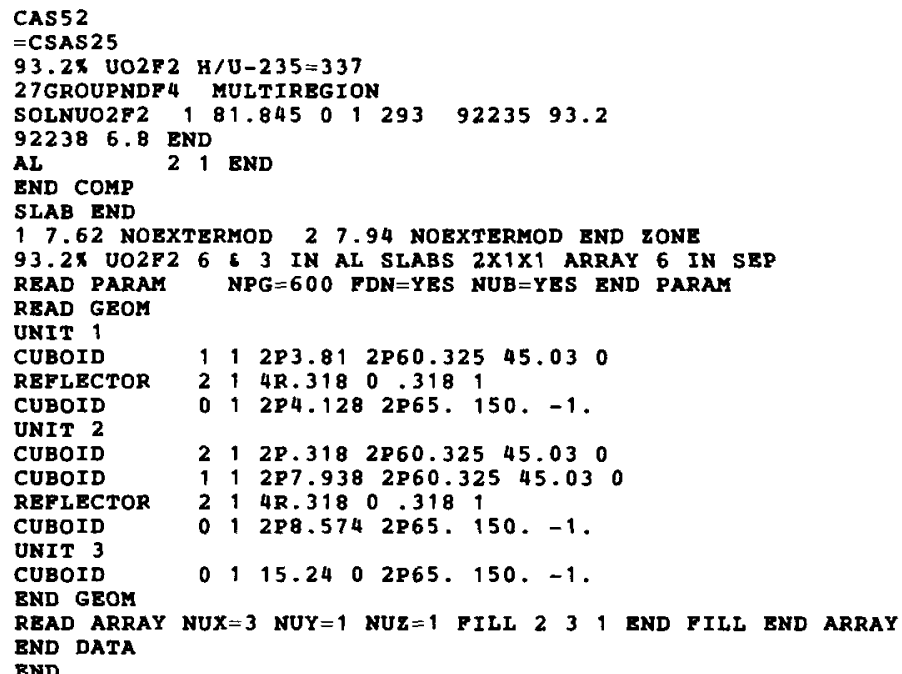

UNIT 2

$\begin{array}{lllllll} & 2 & 12 P .318 & 2 P 60.325 & 45.030\end{array}$

$2,4 R .3180 .318,145.030$

CUBOID $0,2 P 8.5742 \mathrm{P} 65.150 .-1$

$\begin{array}{llllllll}\text { UNIT } 3 & \\ \text { CUBOID } & 0 & 1 & 15.24 & 0 & 2 \mathrm{P} 65.150 .-1\end{array}$

RED GEOM ARRAY NUX $=3$ NUY $=1$ NUZ $=1$ FILL 23,1 END FILL END ARRAY BND DATA

CAs 53

93.2X UO2F2 H/U-235=337

SOLNUO2P2 $181.845 \quad 01293 \quad 92235 \quad 93.2$

92238 6.8 2 END

AND COMP

SLAB BND
17.62 NOEXTERMOD 27.94 NOEXTERMOD END ZONE

93. $2 \times$ UO2P2 6 IN AL SLAB $2 \times 1 \times 1$ ARRAY 15 IN SEP

READ PARAM NPG $=600 \quad \mathrm{PDN}=\mathrm{YBS}$ NUB $=\mathrm{YES}$ END PARA

READ GEOM

UNIT 1

$\begin{array}{llllllll} & 1 & 287.417 & 2 P 60.325 & 44.88 & 0\end{array}$

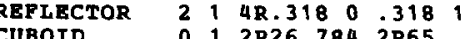

65. 150, -1 .

CUBOUD $212 \mathrm{P} .3182 \mathrm{P} 60.32544 .880$

CUBOID $\quad 112$ 2P7.938 2P60.325 44.880

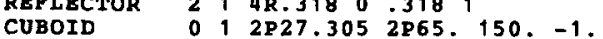

CUBOID

READ ARRAY NUX=2 NUY=1 NUZ=1 FILL 12 END FILL END ARRAY

END DATA 
Table A.5 (continued)

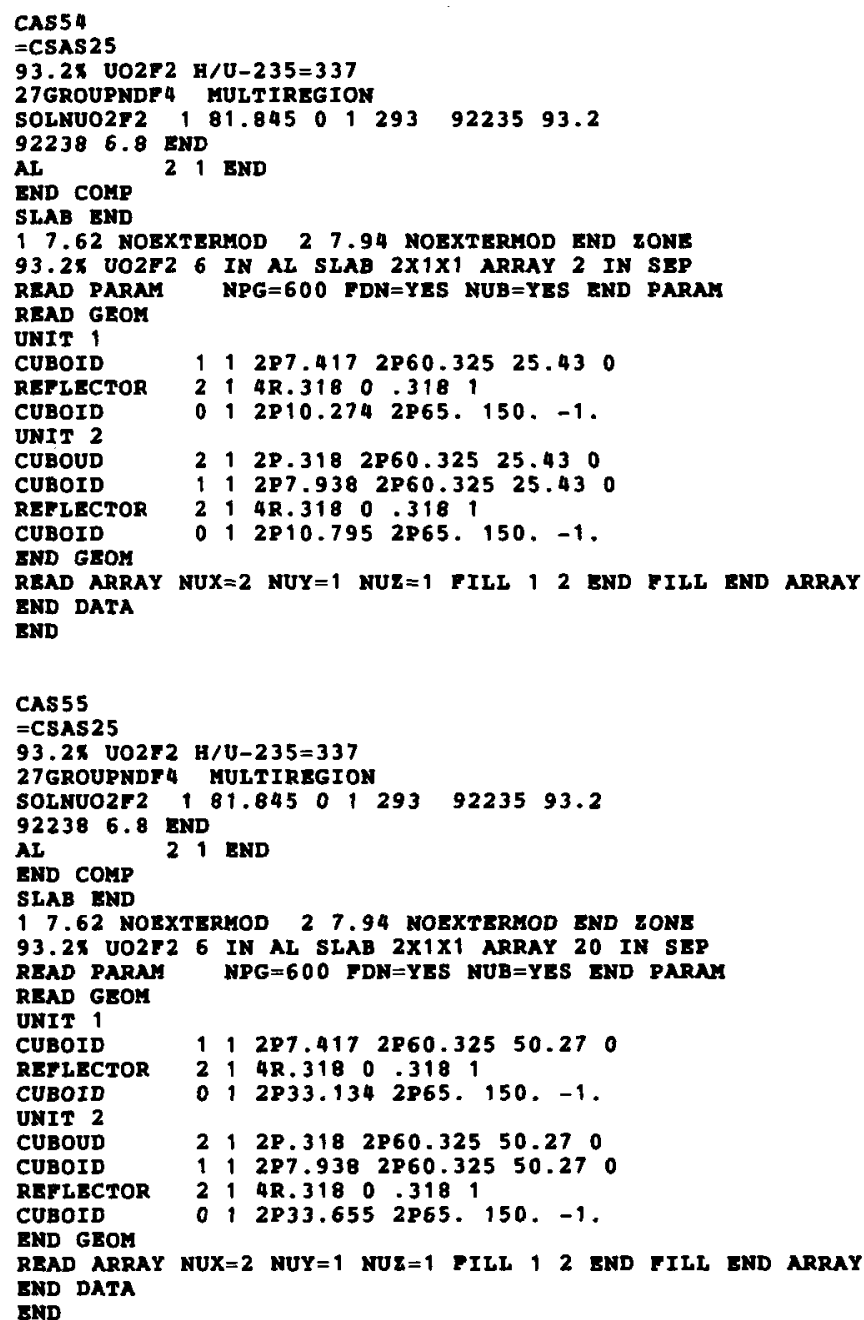

CAs56

$=$ CSAS25

93.2X $002 \mathrm{P} 2 \mathrm{H} / \mathrm{U}-235=337$

SOLNUO2F2 $181.845 \quad 01293 \quad 9223593.2$

922386.8 END

\section{AL}

SLAB END

SLAB END

93.2X UO2F2 6 IN AL SLAB 2X1X1 ARRAY 30 IN SEP

ES END PARAM

READ GEOA

CUBOID $11287.4172860 .325 \quad 59.720$

21 4R. 3180.318

CUBOID 012 2P45.834 2P65. 150. -1 .

CUBOUD $212 P .3182 P 60.325 \quad 59.720$

CUBOID $112 P 7.9382 P 60.32559 .720$

$\begin{array}{lllllll}\text { REFLECTOR } & 2 & 1 & 4 R .318 & 0 & 318 & 1 \\ \text { CUBOID } & 0 & 1 & 2 P 46.355 & 2865 & 150 . & -1 .\end{array}$

READ ARRAY NUX=2 NUY=1 NUZ=1 FILL 12 END PILL END ARRAY BND DATA

\section{CAs57}

$=\operatorname{csas} 25$

$93.2 \times$ บ02F2 H/U-235=337

SOLNUO2F2 181.845 O 12939223593.2

922386.8 END

END
21 END

SLD COMP

17.62 NOEXTERMOD 27.94 NOEXTERMOD END ZONE

93.2X U02F2 6 IN AL SLAB $2 X 1 X 1$ ARRAY 48 IN SEP

READ PARAM

UNIT 1

$\begin{array}{llllllll}\text { CUBOID } & 1 & 1 & 2 \mathrm{P} 7.417 & 2 \mathrm{P} 60.325 & 73.23 & 0\end{array}$

REPLECTOR 2148.31800 .3181

6.694 2P65. $150 .-1$

CUBOUD $212 P .3182860 .32573 .230$

REPLECTOR 211 AR. 318

$\begin{array}{llllll}\text { CUBOID } & 0 & 1 & 2 P 69.215 & 2 P 65.150, & -1 .\end{array}$

READ ARRAY NUX=2 NUY=1 NUZ=1 FILI 12 END FILI END ARRAY END DATA 
Table A.5 (continued)

CAS58

$93.2 \times$ U02F2 $\mathrm{H} / \mathrm{U}-235=337$

27GROUPNDP4 MULTIREGION

SOLNUO2F2 181.845 O $1293 \quad 9223593.2$

922386.8 END

BND COMP

17.62 NOEXTEPMOD 27.94 NOEXTERMOD END ZONE

READ PARAM NPG=600 FDN=YES NUB=YES BND PARA

CUBOID

$\begin{array}{lllll}1 & 1 & 2 \mathrm{P} 7.417 & 2 \mathrm{P} 60.325 & 32.79\end{array}$

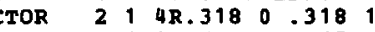

OUBOID $12 P 15.3542 P 65.150,-1$.

$2,2 P .318 \quad 2 P 60.325 \quad 32.790$

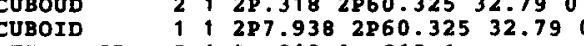

$214 R .31800318$

$12 P 15.8752865,150 .-1$.

READ ARRAY NUX=2 NUY=1 NUZ=1 FILL 12 END FILI END ARRAY END DATA

CAS 59
$=$ CSAS 25

$=$ CSAS25
$93.2 \times$ บO2F $2 \mathrm{H} / \mathrm{U}-235=337$

SOLNUO2F2 $181.84501293 \quad 9223593.2$

92238 6.8 END

AL

SLAB END

17.62 NOEXTERMOD 27.94 NOEXTERMOD END ZONE

93.2X VO2P2 6 IN AI SLAB 2X1X1 ARRAY 66 IN SEP

READ GEOM

UNIT 1

CUBOID

$\begin{array}{lll}1 & 2 \mathrm{P} 7.4172 \mathrm{P} 60.32582 .12\end{array}$

REFECTOR 2 : 4 4R.31800.318

$012 P 91.5542 P 65,150 .-1$.

$212 \mathrm{PP} 3182 \mathrm{P} 60.32582 .120^{\circ}$

REFLECTOR 214 R.318 0.318

CUBOID 012 2P92.075 2P65. 150, -1 .

END GEOM
READ ARRAY NUX=2 NUY=1 NUZ=1 FILL 12 END FILI END ARRAY

END DATA

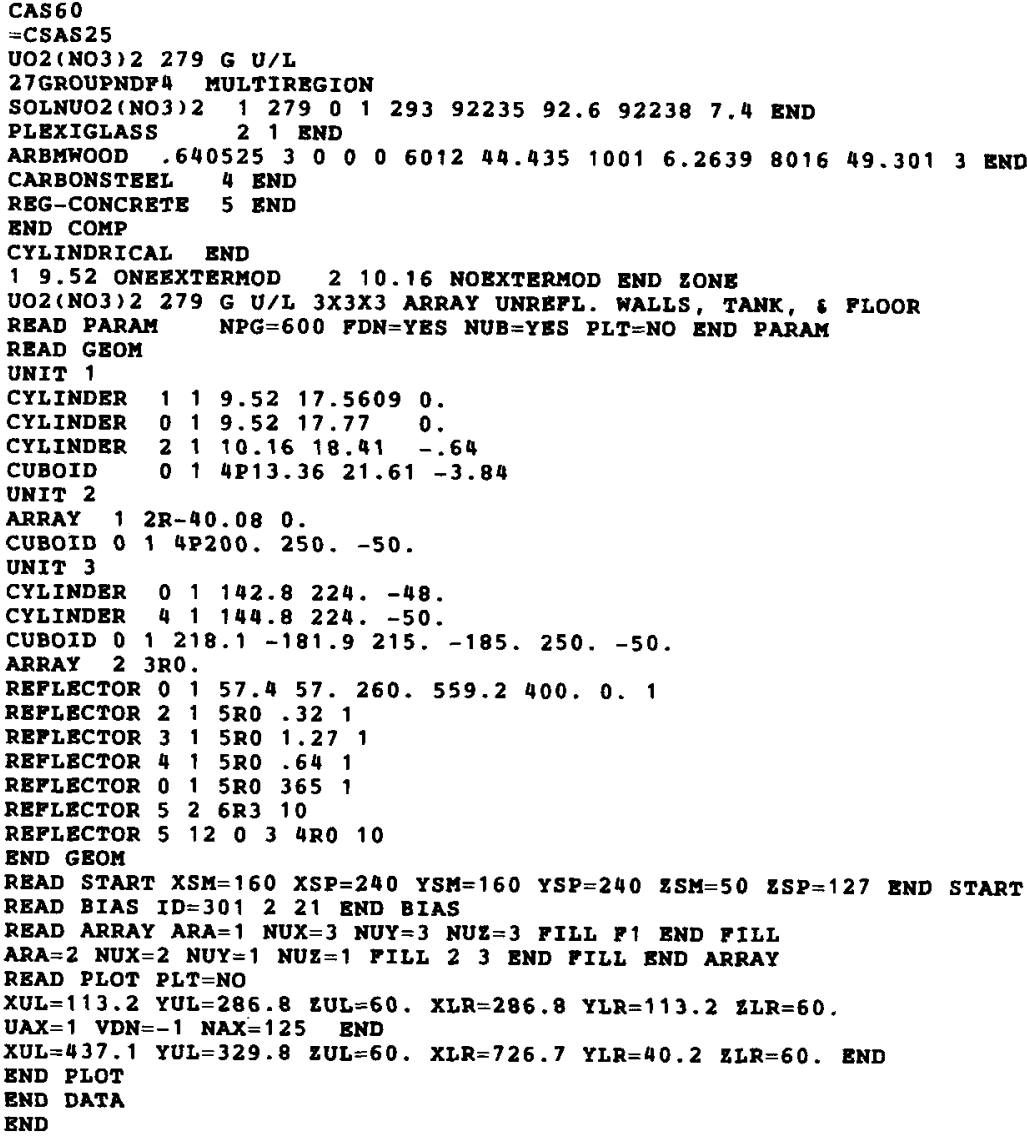


Table A.5 (continued)

CAS61

CSAS25 279 G UI

27GROUPNDP 4 MULTIREGION

SOLNUO2(NO3)2 $1279012939223592.692238 \quad 7.4$ END

LEXIGLASS 21 EIO

. CARBONSTBEL 4 END

END COMP

CYLINDRICAL END

19.52 ONEEXTERMOD 2 10.16 NOEXTERMOD END ZONE

UO2 (NO3) 2279 G U/L 2X2X2 ARRAY UNREFL. WALLS, TANK, FLOOR

READ PARAM

READ GBOA

$\begin{array}{llllll}\text { CYL INDER } & 1 & 1 & 9.52 & 17.5609 & 0 \\ \text { CYLINDER } & 0 & 1 & 9.52 & 17.77 & 0\end{array}$

$\begin{array}{lllll}1 & 9.52 & 17.77 & 0 .\end{array}$

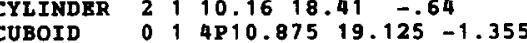

UNIT 2

NIT 2 2R-21.75 0.

CUBOID 014 P200. 250 . -50

CIT 3

CYLINDER 001 142.8 224. -48 .

CUBOID o $1218.1-181.9215,-185,250 .-50$

ARRAY $2380 ; 157.457 .260 .559 .2400 .0 .1$

REPLECTOR 2 T SRO .32 1

RER

REFLECTOR O 9 5RO 365

REFLECTOR $\begin{array}{lllll}5 & 2 & 6 R 3 & 10\end{array}$

$\begin{array}{llllll} & \end{array}$

END GEOM

READ START XSM=178 XSP=222 YSM=178 YSP=222 $2 \mathrm{SM}=50 \quad$ 2SP=9 1 END START

READ ARRAY ARA $=1$ NUX $=2$ NUY $=2$ NUZ $=2$ FILL $F 1$ END FILL

READ PIOT

$X U L=113.2$ YUL=286.8 $\quad$ ZUL $=60 . \quad X L R=286.8 \quad$ YLR=113.2 $\quad \mathrm{ZLR}=60$

$\mathrm{UAX}=1$ VDN $=-1$ NAX $=125$ SND.$X$

. END BND PLOS

END
CAS62
$=$ CSAS25

JO2(NO3) 2415 G U/L

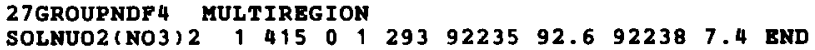

AREMICLASS 121 END 2939223592.6

REG-CONCRETE 5 END

END COMP

CYLINDRICAL BND
19.52 ONBEXTERMOD 210.16 NOEXTERMOD END ZONB

UO2 (NO3) $2415 \mathrm{G}$ U/L $5 \times 5 \times 5$ ARRAY UNRTEL HALTS TANK, FLOOR

作

READ GEOM

$\begin{array}{lllllll}\text { CYLINDER } & 1 & 1 & 9.52 & 17.5609 & 0 \\ \text { CYLINDER } & 0 & 1 & 9.52 & 17.77 & 0\end{array}$

$\begin{array}{llllll}\text { CYLINDER } & 2 & 1 & 10.16 & 18.41 & -.64 \\ \text { CUBOID } & 0 & 1 & 4817.36 & 25.61^{-7} & -7.8\end{array}$

CNIT 2

CUBOID

CYLINDER $001142.8224 .-48$.

CULOID 0 1 $218.1-181.9215 .-185.250 .-50$.

ARRAY 2 3R0; $57,457,260,559.2400 .0,1$

REFLECTOR 2 1 $5 R 0^{4}: 321$

REFLECTOR 4 H 1 5R0 .64 - 1

REPLECTOR 0013 5RO 365

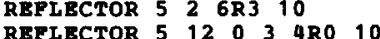

REFLECTOR 512 O 12 3 4 RO 10

BND GEOM

START XSM=113 XSP=287 YSM=113 YSP=287 ZSM=50 ZSP=151 END START READ BIAS ID=301 221 END BIAS

READ ARRAY ARA=1 NUX=5 NUY=5 NUZ=5 PILL F1 END FILL

READ PLOT

$X U L=113.2 \quad Y U L=286.8 \quad 2 U L=60 . \quad X L R=286.8 \quad$ YLR=113.2 $\quad$ ZLR=60 .

$x$ Nat $=113.2$ Y

$X L R=726.7 \quad Y L R=40.2 \quad \geq L R=60$. END

END PLOT

END 
Table A.5 (continued)

CAS63

UO2 (NO3) 2415 G U/L

27GROUPNDF4 HULTIREGION

SOLNUO2(NO3) $2 \quad 1415 \quad 012939223592.6 \quad 92238 \quad 7.4$ END

ARBMWOOD $640525 \quad 3 \quad 000601244.43510016 .26398016 \quad 49.3013$ BND CARBONSTBEL 4 END

REG-CONCR

CYLINDRICAI END

9.52 ONEEXTERMOD 2 10.16 NOEXTRRMOD END ZONE

UO2 (NO3) 2415 G U/L 3X3X3 ARRAY UNREFL. WALLS, TANK, G FLOOR

READ PARAM

READ GBOM

CYLINDER $1919.52 \quad 17.56090$

$\begin{array}{llllll}\text { CYLINDER } & 1 & 9 & 9.52 & 17.5609 & 0 . \\ \text { CYLINDER } & 0 & 1 & 9.52 & 17.77 & 0 .\end{array}$

$\begin{array}{llllll}\text { CYLINDER } & 2 & 1 & 10.16 & 18.41 & -.64 \\ \text { CUBOID } & 0 & 1 & 4 P 13.40 & 21.65 & -3.88\end{array}$

ARRAY $12 R-40.20$

CUBOID OO 4 4P200. $250,-50$

CYLINDER $01142.8224 .-48$.

CYLINDER $41144.8224 .-50$.

CUBOID $011218.1-181.9215 .-185.250,-50$.

ARRAY 2 3RO;

TOR $0157,457,260,559.2400,0.1$

REFLECTOR 2 1 5 5RO 321

REPLECTOR 4 5R0 154,

REPLECTOR $0115 R 0365$

REFLECTOR 521 6R3 365

REFLECTOR 5 12 12 O 3 4RO 10

BND GEOM

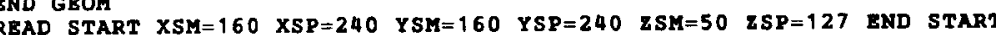

READ BIAS ID $=301221$ EHD BIAS

READ ARRAY ARA 1 NUX $=3$ NUY $=3$ NUZ $=3$ FILL $P 1$ END FILL

READ PLOT

$X U L=113.2 \quad Y U L=286.8 \quad 2 U L=60 . \quad X L R=286.8 \quad Y L R=113.2 \quad 2 L R=60$.

UAX $=1 \quad$ VDN $=-1 \quad$ NAX $=125 \quad$ END $\quad X L R=726,7 \quad$ YLR $=40.2 \quad$ zLR $=60 . \quad$ END

XUL $=437.1$
BND PLOT

BND DAT
CAS64

UO2(NO3) 2415 G U/L

27GROUPNDF4 MULTIREGION

SOLNUO2(NO3) 2 1 415012939223592.6922387 .4 END

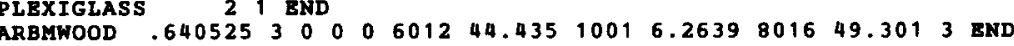

CARBONSTEEL 4 END

REG-CONCR

CYLINDRICAL END

19.52 ONBEXTERMOD 2 10.16 NOEXTERMOD END ZONE

U02(NO3) 2415 G U/L 4X4X4 ARRAY UNREPL. WALLS, FLOOR, C TANK

RBAD PARA

RBAD GBOM

CYLINDER 1919.5217 .56090$.

$\begin{array}{lllllll}\text { CYLINDER } & 2 & 1 & 10.16 & 18.41 & -.64 \\ \text { CUBOID } & 0 & 1 & 4 P 15.495 & 23.745 & -5.975\end{array}$

ARRAY $12 R-61.980$.

CYLINDER $4011142.8224 .-48$.

CUBOID $01218.1-181.9215,-185.250,-50$

ARRAY 23 3R0 157.457 .260 .559 .2400 .0 .1

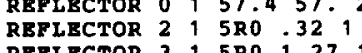

REPLECTOR 4 , 5 R0 641

REFLECTOR $0115 R 0 \quad 3651$

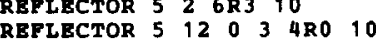

BND GBOK

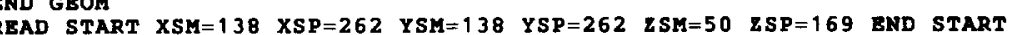

READ BIAS ID $=301221$ END BIAS

READ ARRAY ARA $=1$ NUX $=4$ NUY $=4$ NUZ $=4$ FILL $F 1$ END FILL

READ PLOT

$X U L=113.2 \quad Y U L=286.8 \quad 2 U L=60 . X L R=286.8 \quad Y L R=113.2 \quad 2 L R=60$.

UAX $=1 \quad$ VDN $=-1 \quad$ NAX $=125$ BND
$X U L=437.1 \quad$ YUL $=329.8 \quad$ zUL $=60 . \quad X L R=726.7 \quad$ YLR $=40.2 \quad$ ZLR $=60$. END

END PLOT

END 
Table A.5 (continued)

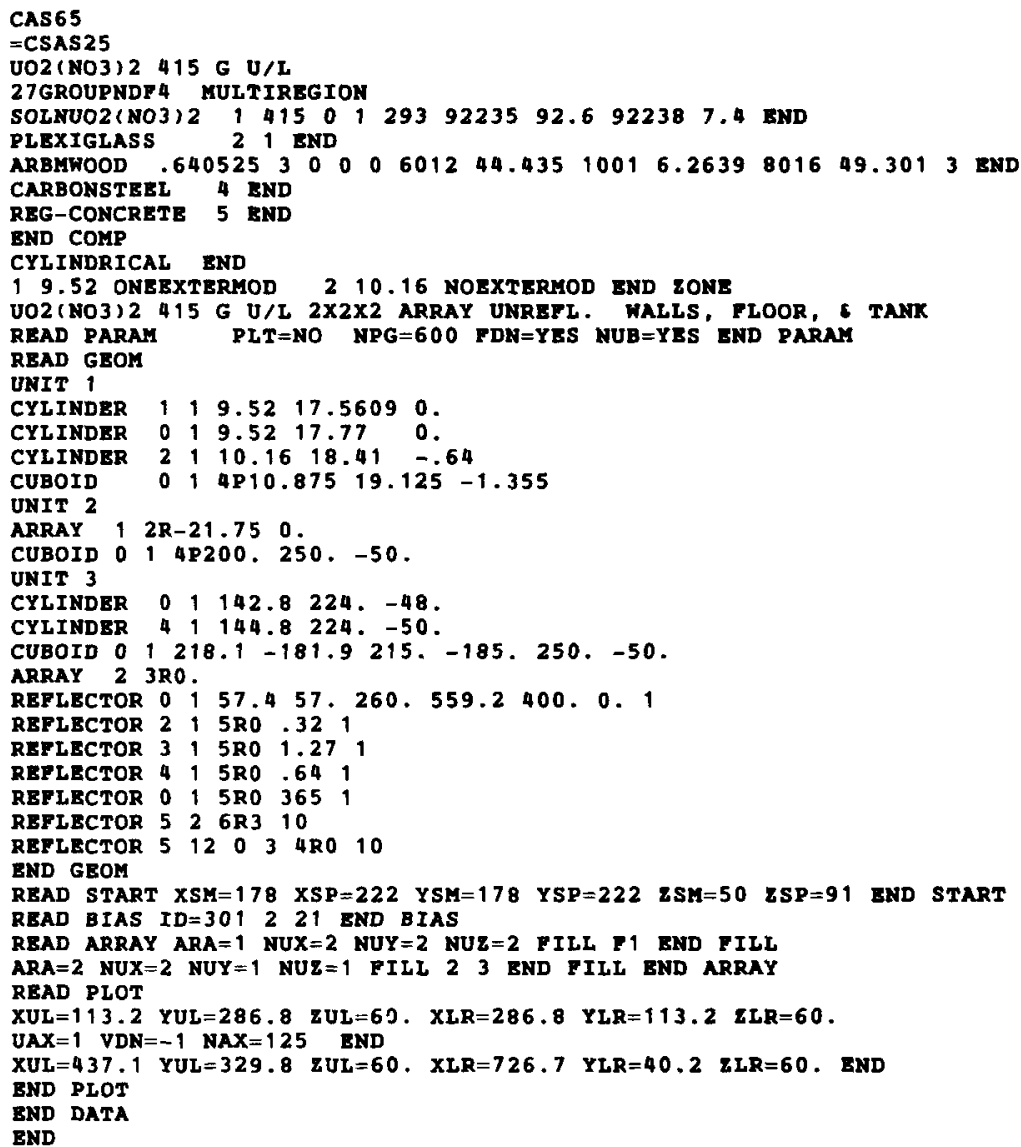


Table A.5 (continued)

CAS 68

$=$ CSAS25
UO2(NO3) 2415 G U/L

UO2(NO3) 2415 G U/L

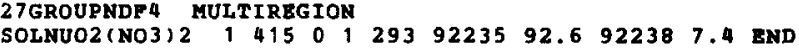

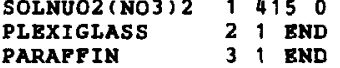

19.52 ONEBXTERMOD 210.16 NOEXTBRMOD END ZONE

UO2(NO3)2 $415 \mathrm{G} \mathrm{U} / \mathrm{L} 3 \times 3 \times 3$ ARRAY $15.24 \mathrm{CM}$ PAR. BOT., $1.27 \mathrm{CM}$ PAR.

READ PARAM

CYLINDER 1190.5217 .56090$.

CYIINDER $019.52 \quad 17.77 \quad 0$.

$\begin{array}{lllllll}\text { CXLINDER } & 2 & 1 & 10.16 & 18.41 & -.64 \\ \text { CUBOID } & 0 & 1 & 4 P 15,10 & 23.35 & -5.5\end{array}$

CUBOID, 01

REFLECTOR 32 5R 1.27 O

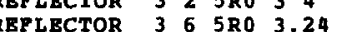

BND GROM

26 END BIA

ARRAY NUX $=3$ NUY $=3$ NUZ=3 END ARRAY

BND DATA

CAS69

$=\operatorname{csas} 25$

JO2 (NO3) 2415 G U/L

SOLNUO2 (NO3) $2 \quad 1415012939223592.6922387 .4$ END $\begin{array}{llll}\text { PLEXIGLASS } & 2 & 1 & \text { BND } \\ \text { PARAFTIN } & 3 & 1 & \text { END }\end{array}$

CYLINDRICAL END

9.52 ONEEXTERMOD 2 10.16 NOEXTERMOD END ZONE

U02 (NO3) 2415 G U/L 3X3X3 ARRAY 15.24 CM PAR 5 PC, PLEX 1 FC

RBAD PARAM

CYLINDER $1119.52 \quad 17.56090$

CYLINDER $0019.52 \quad 17.77 \quad 0$

CYIINDER 2 $19.1698 .41-564$

CORB 0 1 3 RO

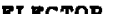

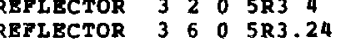

REFLECTOR $2 \begin{array}{llllll}2 & 2 & 3 & 5 R 0 & 4\end{array}$

REFLECTOR $2563.24 \quad 5 R 0$

BND GEOM

RAD ID $=40026$ END BIAS

D ARRAY NUX $=3$ NUY $=3$ NUZ $=3$ END ARRAY

END
CAS70

UO2 (NO3) 2415 G U/L

27GROUPNDF 4 MULTIRBGION

SOLNUO2(NO3)2 1415012939223592.6922387 .4 END

$\begin{array}{llll}\text { PLEXIGLASS } & 2 & 1 & \text { END } \\ \text { PARAFTIN } & 3 & 1 & \text { END }\end{array}$

PARAFTIN

CYLINDRICAL END

19.52 ONEEXTERMOD 210.16 NOEXTERMOD BND ZONE

UO2(NO3)2 $415 \mathrm{G} \mathrm{U} / \mathrm{L} 3 \times 3 \times 3$ ARRAY $15.24 \mathrm{CM}$ PAR, BOT. $3.81 \mathrm{cM}$ PAR. READ PARAM NPG 600 PDN=YBS NUB=YES BND PARAM

READ GEOM

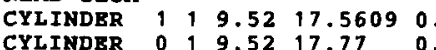

CYLTHPR 211091510.410.

CUBOID 014 4P17.295 $25.545-7.77$

$13 \mathrm{RO}$

REPLECTOR 3 2 5 5R3.89 0

$\begin{array}{llllll}\text { REFLECTOR } & 3 & 2 & 5 R 0 & 3 & 4 \\ \text { REPLECTOR } & 3 & 6 & 5 R 0 & 3.24\end{array}$

END GEOM

BND GEOM

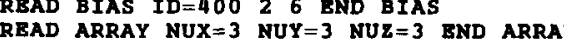

END DATA

CAS71

JO2(NO3)2 415 G U/L

SOLNUO2 (NO3)2 $14415012939223592.6 \quad 92238 \quad 7.4 \quad$ END

$\begin{array}{lllll}\text { PLEXIGLASS } & 2 & 1 & \text { END } \\ \text { PARAFPIN } & 3 & 1 & \text { END }\end{array}$

PARATPIN

CYLINDRICAL END

19.52 ONEEXTERMOD 210.16 NOEXTERMOD END $20 \mathrm{NE}$

UO2(NO3) 2415 G U/L 3X3X3 ARRAY $15.24 \mathrm{CM}$ PAR. BOT., $7.62 \mathrm{CM}$ PAR. READ PARAM NPG $=600$ PDN $=$ YES NUB $=$ YES END PARAM

READ GEOM

CYLINDER $00199.52 \quad 17.56090$

CYLINDER $2,10.16 \quad 18.41,3354$

CUBOID 0 1 4 P18.085 $26.335 \quad-8.565$

CORE $0 \quad 13 \mathrm{RO}$.

$\begin{array}{lllllll}\text { REFLECTOR } & 3 & 2 & 5 R 3 & 0 & 2 \\ \text { REPLECTOR } & 3 & 4 & 5 R 1.62 & 0\end{array}$

RERLECTOR 3 4 5R1.62 0

REPLECTOR 3 6 5 5R0 33.24

RND GBOM

READ BIAS ID $=40026$ BND

READ ARRAY NUX $=3$ NUY $=3$ NUZ $=3$ END ARRAY

READ ARRAY
END DATA
BND 
Table A.5 (continued)

CAS72

UO2(NO3) $2415 \mathrm{G} \mathrm{U/L}$

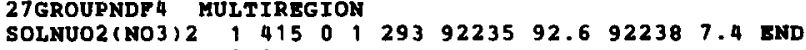
PLEXIGLASS 21 END

PARAFPIN

CYLINDRICAL END

19.52 ONEEXTERHOD 2 10.16 NOEXTERMOD END ZONE บO2(NO3) $2415 G$ G/2 $3 \times 3 \times$ ? READ PARAM NPG $=600 \quad$ FDN=YES NUB=YES END PARAY

READ GBOM

CYLINDER $1199.52 \quad 17.56090$.

CYLINDER 2 2 $10.16 \quad 18.41 \quad-.64$

CUBOID 014 P $14.54 \quad 22.79-5.02$

$\begin{array}{lcccc}\text { CORE } & 1 & \text { 3RO } & & \\ \text { REFLECTOR } & 2 & 2 & 6 R 1.27 \text {, }\end{array}$

END GEOM

BAAD ID $=40026$ END BIAS

$=3$ END ARRAY

END DATA

CAS73

บO2(NO3)2 $415 \mathrm{G} \mathrm{U/L}$

27GROUPNDP 4 GULTIREGTON

$\begin{array}{lllllllll}\text { 27GROUPNDL } & \end{array}$

$\begin{array}{llll}\text { PLEXIGLASS } & 2 & 1 & \text { END } \\ \text { PARAFFIN } & 3 & 1 & \text { BND }\end{array}$

parargens

CYLINDRICAL END

19.52 ONEEXTERHOD 210.16 NOEXTERMOD END ZONE

U02 (N03)2 $415 \mathrm{G} \mathrm{V} / \mathrm{L} 3 \times 3 \times 3$ ARRAY $1.27 \mathrm{CM}$ PARAPFIM

READ PARAM NPG $=600$ PDN $=$ YES NUB $=$ YES END PARAM

CYLINDER $1919.52 \quad 17.56090$.

CYLINDER 019.5217 .770 .

CUBOID 2170.16 $19.419-.64$

CORE

$\begin{array}{llll} & \end{array}$

BND GEOH

40026 END BIAS

促

BND DATA

END
CAS74

UO2(NO3) $2415 \mathrm{G} \mathrm{U} / \mathrm{L}$

27GROUPNDF 4 MULTIREGION

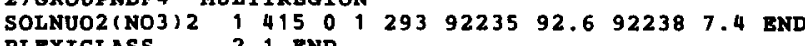
PLEXIGLASS 21 END

PARA COMP

CYLINDRICAL END

19.52 ONBEXTBRMOD 2 10.16 NOBXTERMOD END ZONE

UO2(N03)2 415 G U/L 3X3X3 ARRAY 15.24 CK PARAFFIN REFL. READ PARAM NPG $=600$ FDN=YBS NUB=YES END PARAM

READ GBOM

CYIINDER 019.5217 .56090

CUBOID 0 I 4 P $18.42526 .675-8.905$

CORE 0 i 3RO.

$\begin{array}{llllll}\text { REFLECTOR } & 3 & 2 & 6 R 3 & 4 \\ \text { REFLECTOR } & 3 & 6 & 6 R 3.24 & 1\end{array}$

\section{603.24}

READ BIAS ID $=40026$ END BIAS

READ ARRAY NUX $=3$ NUY $=3$ NUZ $=3$ END ARRAY

BND DATA

CAS75

UO2(NO3) 2415 G U/L

27GROUPNDF 4 MULTIREGION

SOLNUO2(NO3) 2 1 415012939223592.6922387 .4 END

$\begin{array}{llll}\text { PLEXIGLASS } & 2 & 1 & \text { END } \\ \text { PARAFFIN } & 3 & 1 & \text { END }\end{array}$

PARAFFIN

CYD COMP

19.52 ONEEXTERMOD 210.16 NOEXTERMOD END ZONE

PATAM 4 G U/L 3X3X3 ARRAY 3.81 CM PARATPI

READ PARAM NPG $=600 \quad$ FDN=YES NUB=YBS END PARAM

CYLINDER $1119.52 \quad 17.56090$

CYIINDER $2,10.15,18.41$ -

CUBOID 014 4P17.005 25.255 -7.485

CORE O 1 3RO

RBPLECTOR

BND GEOM

READ BIAS ID $=40026$ END BIAS

IVX $=3$ NUY $=3$ MUE $=3$ END ARRAY

END 
Table A.5 (continued)

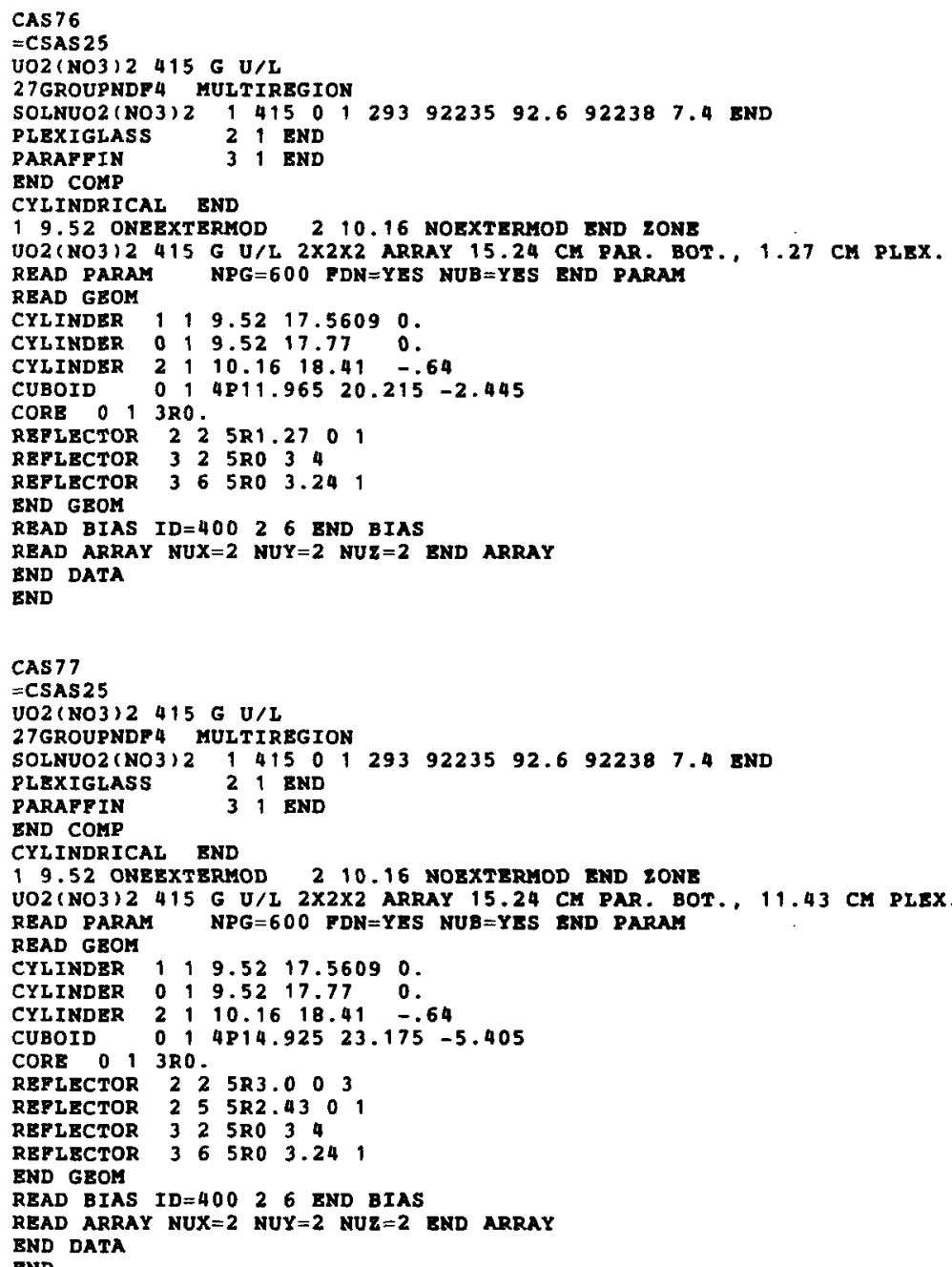


Table A.5 (continued)

CAS 80
$=$ CSAS 25

UO2(NO3)2 $415 \mathrm{G} \mathrm{U} / \mathrm{L}$

27GROUPNDF" MULTIREGION

SOLNUO2 (NO3) 2 192012939223592.6922387 .4 END

PLEXIGLASS 251 END

PARAFriN

19.52 ONEEXTERMOD 210.16 NOEXTERMOD END ZONE

UO2 (NO3) $2415 \mathrm{G} U / \mathrm{L} 2 \times 2 \times 2$ ARPAY $15.24 \mathrm{CH}$ PAR, BOT $4.45 \mathrm{CM}$ PLEX.

READ PARAM

READ GEOA

CYLINDER 1199.5217 .56090$.

CYLINDBR 2 i $10.16 \quad 18.41,-.64$

CUBOID 014 ,

CORE $0013 \mathrm{RO} 0$

REPLECTOR 2 2 3 5R1.45 O 1

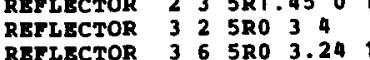

END GEOM

READ BIAS ID $=40026$ END BIAS

READ BIAS ID=400 2 6 6 END BIAS
READ ARRAY NUX=2 NUY $=2$ NUZ=2 END ARRAY
END DATA

CAs8 1

$=\operatorname{csis25}$

UO2(MO3) 2415 G U/L

27 GROUPNDF 4 MULTIREGION

SOLwUO2(N03)2 $11415012939223592.692238 \quad 7.4$ END

PLEXIGLASS 2 , END

RMD COMP

CYIINDRICAL END

END 210.16 NOEXTERMOD END ZONE

UO2 (NO3)2 $415 \mathrm{G} \mathrm{U} / \mathrm{L} 2 \times 2 \times 2$ ARRAY $15.24 \mathrm{CM}$ PAR. BOT., 6.35 CM PLEX.

READ PARAM

READ GEOM

$\begin{array}{lllll}0 & 1 & 9.5217 .77 & 0\end{array}$

$\begin{array}{llllll}\text { CYLINDER } & 2 & 1 & 10.16 & 18.41 & -.64 \\ \text { CUBOID } & 0 & 1 & 4 P 14.48 & 22.73-4.96\end{array}$

CORE $0 \quad 1$ 3RO

REFLECTOR 225253.0001

REPLECTOR 2 3 5 R3.350

PEPLECTOR 365803.24

END GEOM

READ BIAS ID $=400$
2 GEAD ARRAY NUX $=2$ WUY $=2$ NUZ $=2$ END ARRAY

END DATA

\section{CAS82}

UO2(NO3) 2 4 $\mathrm{G} U /$

27GROUPNDPA MULTIREGION 2939223592.6922387 .4 END

PLEXIGLASS 21 END

PARAFYI

CYLINDRICAL END

10.16 WORxTEROD END zONE

O2 (NO3) $2415 \mathrm{G} U / \mathrm{L}$ 2 10.16 NOBXTERMOD END 2ONE $1.27 \mathrm{CM}$ PAR.

READ PARAM

READ GEOM

CYIINDER $1119.52 \quad 17.56090$

CYLINDER 21

CUBOID 014 Pi2.09 $20.34^{-.64} \mathbf{- 2 . 5 7}$

CORE 0 1 13 3RO.

REFLECTOR $3258 R 1.27001$

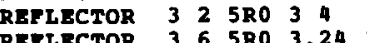

END GEOH

READ BIAS ID $=40026$ END BIAS

READ ARRAY NUX $=2$ NUY $=2$ NUZ $=2$ END ARRAY

END DATA

CASB3

UO2(NO3) 2415 G U/L

MUNF 4 MULTIREGIO

27. \begin{tabular}{llll} 
PLEXIGLASS & 2 & 1 END \\
PARAPTIN & 3 & 9 & END \\
\hline
\end{tabular}

PARATPIN

CYLINDRICAL END

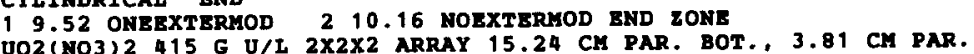

ORAD

READ PARA

CYLINDER 1119.5217 .56090$.

CYLINDER 0019.5217 .770$.

$\begin{array}{llllll}\text { CYLINDER } & 2 & 1 & 10.16 & 18.41 & -.64 \\ \text { CUBOID } & 0 & 1 & 4813.79 & 22.04-4.27\end{array}$

REFLECTOR $33_{3}$ i $5 R 3.81 \quad 0 \quad 1$

REFLECTOR 332 5RO 334

REPD GEOM

READ ARRAY NUX $=2$ NUY $=2$ WUZ $=2$ END ARRAY

END DATA 
Table A.5 (continued)

CAS84

UO2 (NO3)2 $415 \mathrm{G} \mathrm{U} / \mathrm{L}$

27GROUPNDF 4 MULTIREGIO

SOLNUO2(NO3) $2 \quad 1415 \quad 0129392235 \quad 92.6 \quad 92238 \quad 7.4$ END PIEXIGLASS 21 END

END COMP

CYLINDRICAL BND

19.52 ONEEXTERMOD 210,16 NOEXTREMOD END ZONE

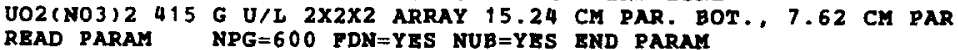

READ PARAM

READ GEOM

CYLINDER 0019.5217 .56090$.

CYLIMDER 2110.1618 .41

CUBOID 0 1 4 P $14.51522 .765-4.995$

CORE $O$

3RO.

$\begin{array}{llllllll}\text { REFLECTOR } & 3 & 2 & 5 R 3 & 0 & 2 \\ \text { REFLECTOR } & 3 & 4 & 5 R 1.62 & 0\end{array}$

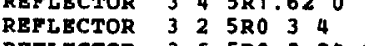

$\begin{array}{llll} & \\ & \end{array}$

BND GBOM

READ BIAS ID $=40026$ END BIAS

READ ARRAY NUX $=2$ WUY $=2$ NUZ $=2$ END ARRAY

END DATA

CAS85

UO2(NO3) $2415 \mathrm{GU} / \mathrm{L}$

SOLNUO2(NO3) 2 1415012939223592.6922387 .4 END

PLEXIGLASS 21 BND

PND COMP

CYLINDRICAL END

UO2(NO3) 2415 G U/L 2X2X2 ARRAY 1.27 CM PLEXIGLASS REFL.

READ PARA

CYLINDER $1919.52 \quad 17.56090$.

CYLINDER O

$\begin{array}{llllll}\text { CYLINDER } & 2 & 1 & 10.16 & 18.41 & -.64 \\ \text { CUBOID } & 0 & 1 & 4 P 11.66 & 19.91 & -2.14\end{array}$

CORE 01 3R

$226 R 1.271$

RBAD ARRAY NUX $=2$ NUY $=2$ NUZ $=2$ END ARRAY

BND DATA
CAS86
$=$ CSAS25

27GROUPNDP 4 MULTIREGION

SOLMUO2 (NO3) 21501293922359259223874 END $\begin{array}{llll}\text { PLEXIGLLASS } & 2 & 1 & \text { END } \\ \text { PARAFIIN } & 3 & 1 & \text { BND }\end{array}$

END COMP

CYLINDRICAL BND

19.52 ONBEXTERMOD 210.16 NOEXTERMOU BND ZONE UO2(NO3)2 415 G U/L 2X2X2 ARRAY 1.27 CM PARAFFIN REFL.

READ PARAM

CYLINDER

CYLINDER 019.5217 .56090

$\begin{array}{llllll}\text { CYLINDER } & 2 & 1 & 10.16 & 18.41 & -.64 \\ \text { CUBOIDD } & 0 & 1 & 4 P 19.80 & 20.05-2.28\end{array}$

CUBOID

CORE 0 I 3RO

6R1.27 1

GETE

26 BND BIAS

END DATA

CAS87

UO2(NO3)2 $415 \mathrm{G} \mathrm{U} / \mathrm{L}$

27GROUPNDF 4 MULTIREGION

U-235 $1009.846-4$ END

O-238 $107.769-5$ END

$\begin{array}{lllll}\mathbf{H} & 1 & 0 & 5.809-2 & \text { END } \\ \mathrm{N} & 1 & 0 & 2.131-3 & \text { END } \\ 0 & 1 & 0 & 3.756-2 & \text { END }\end{array}$

$\begin{array}{lrrrr}0 & 1 & 0 & 3.756-2 & \text { END } \\ \text { PLEXIGLASS } & 2 & 1 & \text { END } \\ \text { PARAFFIN } & 3 & 1 & \text { END }\end{array}$

BND COMP

CYLINDRICAL END

210.16 NOEXTERMOD END ZOMB

RO2(NO3)2 415 G U/L 2X2X2 ARRAY 15.24 CM PARAFYIN REPL.

READ GEOM

$\begin{array}{lllllll}\text { CYLINDER } & 1 & 1 & 9.52 & 17.5609 & 0 . \\ \text { CYLINDER } & 0 & 1 & 9.52 & 17.77 & 0 .\end{array}$

21.0216 18.41 0.64

0 4P14.655 22.905 -5.135

CORE O 1 3RO

REPLECTOR $3256 R 3 \quad 4$

BND GBOM

READ BIAs $1 D=400$

作

END DATA 
Table A.5 (continued)

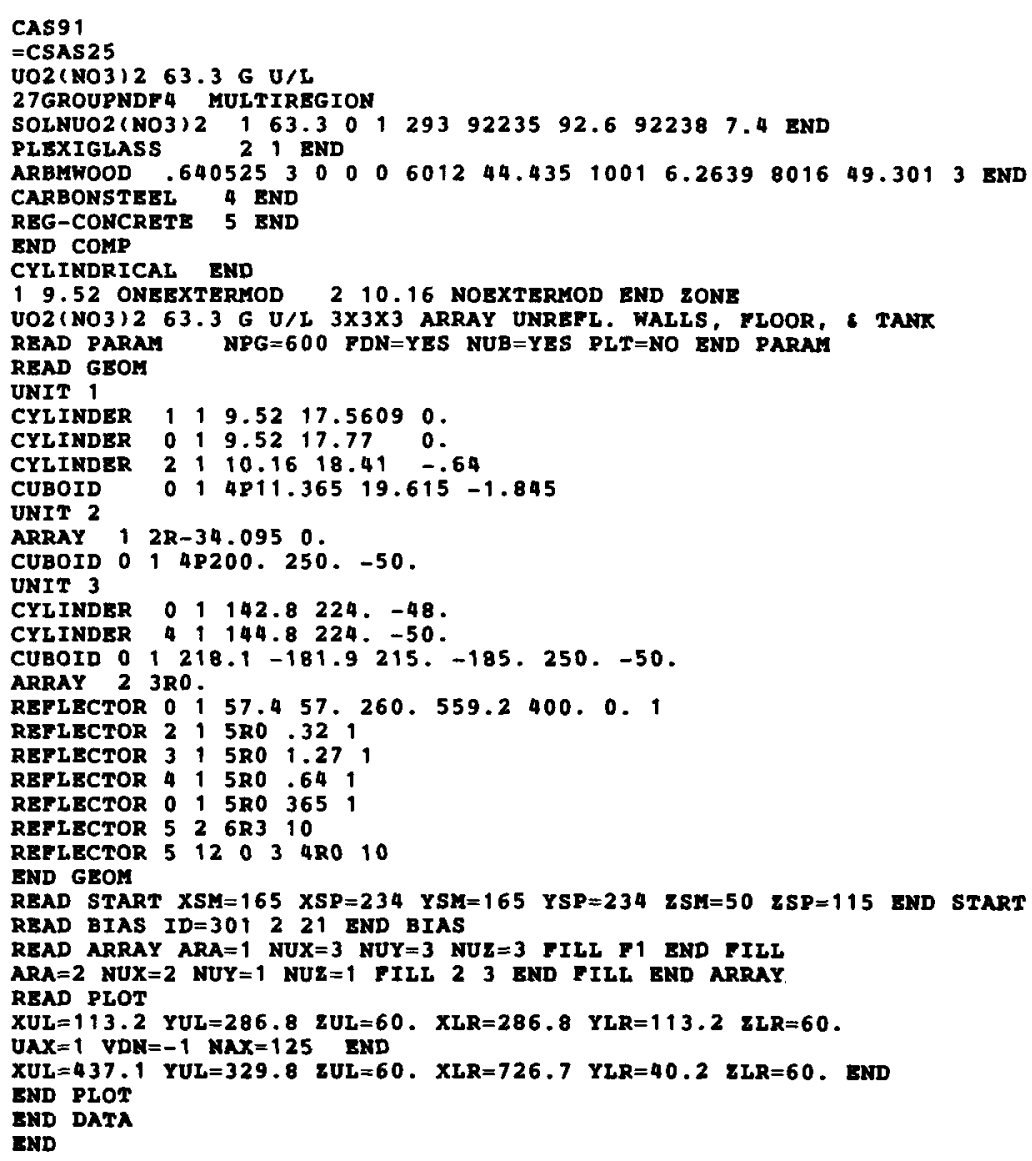


Table A.6. Table 6 input data

CAS 01
$=$ CSAS25

ETA EXPERIMENTS EXP 1

27GROUPNDF 4 INPHOMMEDTUY

$\begin{array}{lllll}\text { U-233 } & 1 & 0 & 0.0 & \text { END } \\ \mathbf{U - 2 3 4} & 1 & 0 & 0.00538-4 & \text { END } \\ \mathbf{U - 2 3 5} & 1 & 0 & 0.48006-4 & \text { END } \\ \mathbf{U - 2 3 6} & 1 & 0 & 0.00138-4 & \text { END } \\ \mathbf{U - 2 3 8} & 1 & 0 & 0.02807-4 & \text { END } \\ \mathbf{N} & 1 & 0 & 1.869-4 & \text { END } \\ \mathbf{H} & 1 & 0 & 0.066228 & \text { END } \\ 0 & 1 & 0 & 0.033736 & \text { END } \\ \text { B-10 } & 1 & 0 & 0.0 & \text { END } \\ \text { B-11 } & 1 & 0 & 0.0 & \text { END } \\ \text { TH-232 } & 1 & 0 & 0.0 & \text { END } \\ \text { AL } & 2 & 1 & & \text { END } \\ \text { SS316 } & 3 & 1 & & \text { END } \\ \text { CARBONSTEEL } & 4 & 1 & & \text { END }\end{array}$

\section{END COMP}

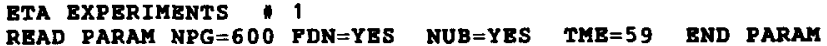

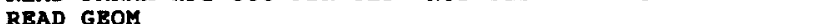

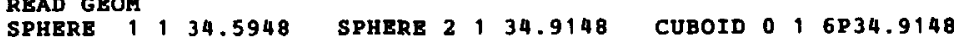
END GEOM READ START NST $=1$ BND START END DATA

\section{CAS 02}

ETA EXPERIMENTS EXP 2
27GROUPNDF 4 INPHOMMEDIUM

\begin{tabular}{lllll} 
27GROUPNDF4 & \multicolumn{4}{l}{ INPHOMMEDIUM } \\
U-233 & 1 & 0 & 0.0 & \\
U-234 & 1 & 0 & $0.00631-4$ & END \\
U-235 & 1 & 0 & $0.56206-4$ & END \\
U-236 & 1 & 0 & $0.00163-4$ & END \\
U-238 & 1 & 0 & $0.03281-4$ & END \\
N & 1 & 0 & $2.129-4$ & END \\
H & 1 & 0 & 0.066148 & END \\
0 & 1 & 0 & 0.033800 & END \\
B-10 & 1 & 0 & $0.01029-4$ & END \\
B-11 & 1 & 0 & $0.04166-4$ & END \\
TH-232 & 1 & 0 & 0.0 & END \\
AL & 2 & 1 & & END \\
SS316 & 3 & 1 & & END \\
CARBONSTEEL & 4 & 1 & & BND
\end{tabular}

RTA EXPERIMENTS PARAM NPG $=600$ FDN=YES NUB=YES TME=59 END PARAM

READE 134.5948 SPHERE 2134.9148 CUBOID 016 P 34.9148

END GEOM READ START NST $=1$ END START

BND DATA

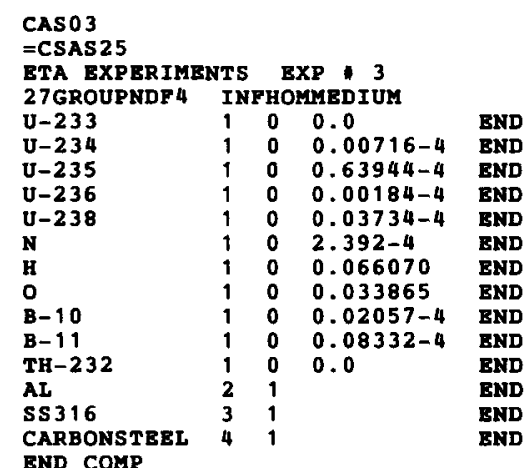

CARBONSTB

ETA BXPERIMENTS
READ PARAM NPG $=600$ PDN=YES NUB=YES TME=59 END PARAM

READ GEOM

SPHERE 1134.5948 SPHERE 2134.9148 CUBOID 016 6P34.9148 END GEOM READ START NST $=1$ END START END DATA

CASO 04
$=$ CSAS25

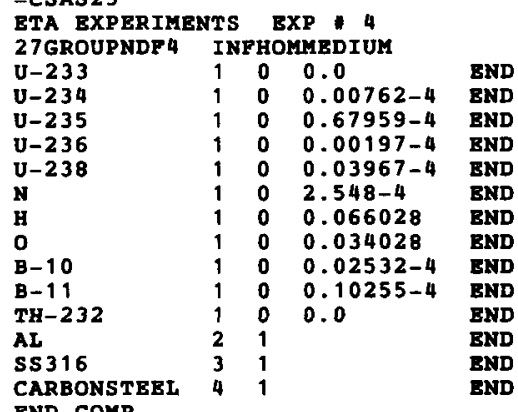

CARBONSTEEL

RTA EXPERIMENTS 4 READ PARAM NPG $=600$ FDN=YES NUB=YBS TME=59 END PARAM

READ PARAM NPG $=600$ FDN=YSS NUB=YBS TME=S

SPHBRE 1134.5948 SPHERE 2134.9148 CUBOID 016 P34.9148 END GEOM READ START NST $=1$ END START END DATA 
Table A.6 (continued)

CAS 05

ETA EXPERTMENTS EXP 15

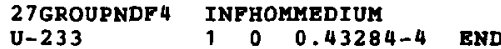

$\mathrm{U}-234$

$U-235$

$\mathrm{U}-236$

N

B- 10

B-11

AL

1
2
3
4

CAS 06

BTA EXPERIMENTS EXP 16

$\mathrm{U}-233 \quad 100.45120-4$ BND

$\mathrm{U}-235$

$100.00744-4$ BND

$\begin{array}{lll}1 & 0 & 0.0001 \\ 1 & 0 & 0.0\end{array}$

$\mathrm{U}-238$

8-10

B-11

AL

0.0 END

$\begin{array}{lll}0 & 0.00291-4 & \text { END } \\ 0 & 1.224-4 & \text { END }\end{array}$

0.066345

$1000.033621 \quad$ BND

CARBONSTERT

$0.00263-4$ END

$0.01066-4$ END

$\begin{array}{lll}0 & 0.000205-3 & \text { END } \\ 1 & \text { END } \\ 1 & \text { END }\end{array}$

END COMP

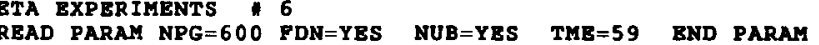

READ PARAM NPG $=600$ FDN=YES NUB=YES TME $=59$ BND PARAM

SPHERE 1134.5948 SPHERE 2134.9148 CUBOID 016834.9148 BND GEOM READ START NST $=1$ END START

BND DATA

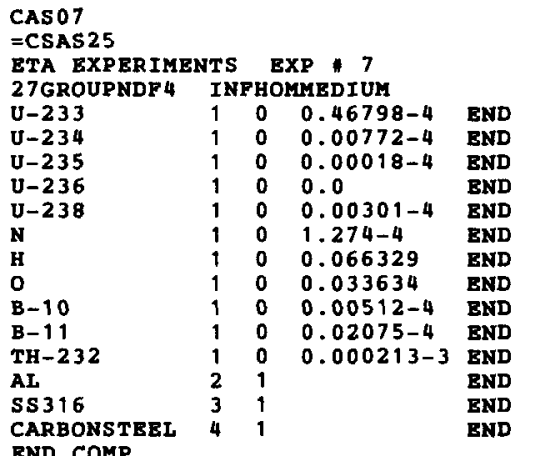

END COMP

ETA EXPERIMENTS 7 7 $=$ YES NUB=YES TME $=59$ END PAPAM

SPHERE 1134.5948 SPHERE 2134.9148 CUBOID 016 P 34.9148 END GEOM READ START NST $=1$ END START

END DATA

CAS 08

ETA EXPERIMENTS EXP * 8

27GROUPNDF 4 INFHOMMEDIUM

$\begin{array}{lllll}\mathrm{U}-233 & 1 & 0 & 0.48455-4 & \text { END } \\ \mathrm{U}-234 & 1 & 0 & 0.00801-4 & \text { END }\end{array}$

$\begin{array}{lllll}U-235 & 1 & 0 & 0.00801-4 & \text { END } \\ U-236 & 1 & 0 & 0.0021-4 & \text { BND }\end{array}$

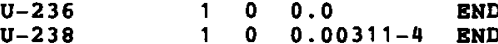

B-10

$1.319-4$ END

$1.319-4$
0.066315

0.033646 END

$0.00758-4$ END

$\begin{array}{lllll}\text { B-11 } & 1 & 0 & 0.03069-4 & \text { END } \\ \text { TH-232 } & 1 & 0 & 0.000221-3 & \text { BND } \\ \text { AL } & 2 & 1 & & \text { END } \\ \text { SS } 316 & 3 & 1 & & \text { END }\end{array}$

$\begin{array}{llll}\text { AL } & & & \\ \text { SS } 316 & 3 & 1 & \text { END } \\ \text { CARBONSTEEL } & 4 & 1 & \text { END }\end{array}$

BND COMP

END

ETA BXPERIMENTS 8 \% 8 TES NUB=YES TME=59 END PARAM

$\begin{array}{llllllll} & \end{array}$ END GEOM READ START NST $=1$ END START

END DATA 
Table A.6 (continued)

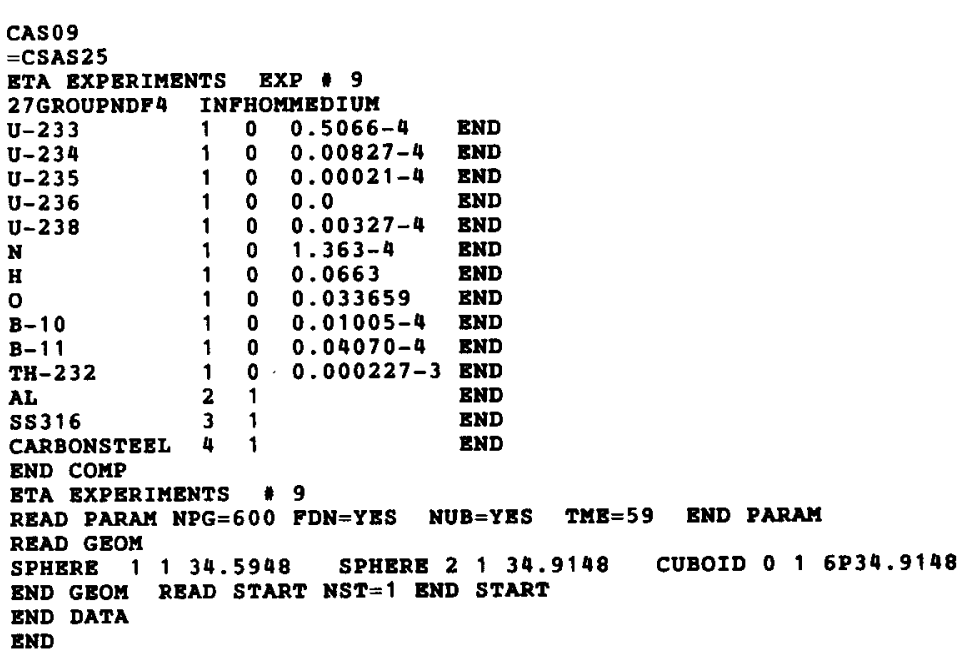

\section{CAs 10}

\begin{tabular}{|c|c|c|c|}
\hline $\begin{array}{l}\text { ETA EXPERIME } \\
\text { 27GROUPNDF4 }\end{array}$ & $\begin{array}{l}\text { NTS } \\
\text { INF }\end{array}$ & $\begin{array}{r}\text { EXP } 10 \\
\text { HOMESDIUR }\end{array}$ & \\
\hline $\mathrm{U}-233$ & 1 & 00.0 & END \\
\hline v-234 & & $0.00409-4$ & END \\
\hline$v-235$ & & $0.36185-4$ & END \\
\hline$v-236$ & & $0.00220-4$ & BND \\
\hline$U-238$ & & $0.01985-4$ & END \\
\hline N & & $1.116-4$ & END \\
\hline $\boldsymbol{n}$ & & $0 \quad 0.066394$ & \\
\hline & & 0.033592 & END \\
\hline$B-1$ & 1 & 0.0 & END \\
\hline B-11 & & 0.0 & END \\
\hline TH-232 & 1 & 0.0 & END \\
\hline AL & 2 & 1 & END \\
\hline SS316 & 3 & 1 & END \\
\hline CARBONSTBEL & 4 & 1 & END \\
\hline
\end{tabular}

ETA BXPERIMENTS 10

READ PARAM NPG=600 FDN=YES NUB=YES THE=59 END PARAM

SPHERE 1161.0108 SPHERE 2161.7808 CUBOID 016 P61.7808 END GEOM READ START NST=1 END START

END DATA

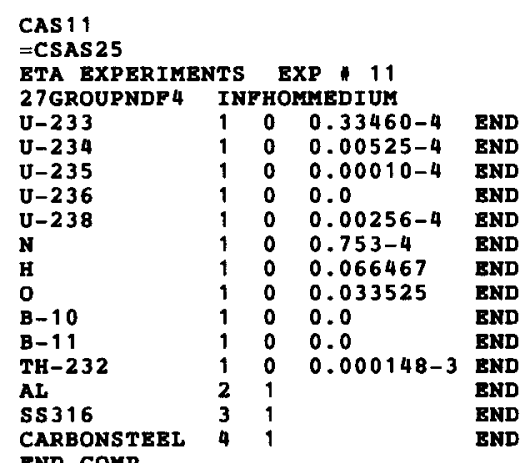

END COMP

READ PARAM NPG $=600$ FDN=YES NUB=YES TME=59 END PARAM

READ GEOM 191.0108 SPHEPE 2161.7808 CUBOID 016861.7808

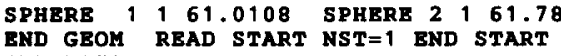
BND DATA

\section{CAS 12}

ETA BXPERIMENTS BXP 12

27GROUPNDF 4 INFHOMMEDIUM

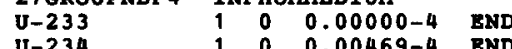

100

$\begin{array}{llll}U-236 & 0 & 0.00243-4 & \text { END }\end{array}$

U-238 $1100.02271-4$ END

$1 \quad 1 \quad 00.066345$

100.033624

$\begin{array}{llll}0-10 & 1 & 0 & 0.0 \\ 8-11 & 1 & 0 & 0.0\end{array}$

$100.000000-3$ EMD

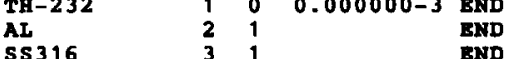

CARBONSTEEL 4 1

CND COMP

TA EXPERIMENTS 12

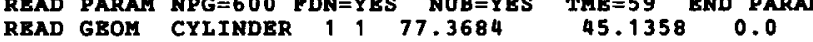

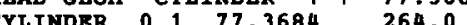

$\begin{array}{llllll}\text { CYLINDER } & 3 & 1 & \mathbf{7 7 . 4 9 8 4} & 264.13 & -0.13\end{array}$

CUBOID $\quad 0 \quad 1 \quad 4 P 77.4984 \quad 264.13 \quad-0.13$

BND GEOH

READ START NST $=1$ END START

BND DATA 
Table A.6 (continued)

CAS 13

ETA EXPERTMENTS EXP 13

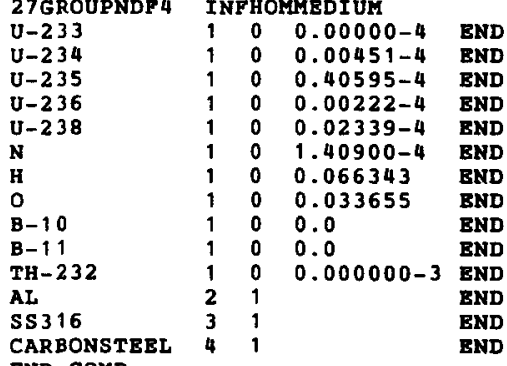

\section{CARBONTE}

BTA EXPERIMBNTS 13

READ PARAM NPG $=600$ PDN $=Y$ YBS NUB=YBS TME $=59$ BND PARAM

READ GEOM CYLINDER $11 \% \mathbf{7 7 . 3 6 8 4}$

$\begin{array}{llllll}\text { CYLINDER } & 0 & 1 & 77.3684 & 264.0 & 0.0 \\ \text { CYLINDER } & 3 & 1 & 77.4984 & 264.13 & -0.13\end{array}$

CUBOID 01 4P77.4984 $264.13 \quad-0.13$

READ START NST $=1$ END START

END

CAS 14

BTA BXPERTMENTS EXP 14

\begin{tabular}{|c|c|}
\hline $\begin{array}{l}\text { GROUPND } \\
233 \\
234 \\
235 \\
236 \\
238\end{array}$ & 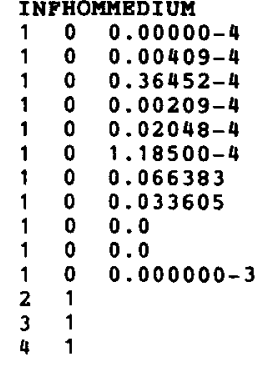 \\
\hline
\end{tabular}

ETA EXPRRIMENTS 14

READ PARAM NPG $=600$ PDN $=Y$ YES NUB $=Y B S \quad T M E=59$ END PARA

$\begin{array}{lllll} & \end{array}$

$\begin{array}{llllll}\text { CYIINDER } & 0 & 77.3684 & 264.0 & 0.0 \\ \text { CXIINDER } & 3 & 1 & 77.4984 & 264.13 & -0.13\end{array}$

CUBOID 0114 aP77.4984 $264.13 \quad-0.13$

BND GBOM

READ START NST $=1$ END START

END DATA
CAS15
$=$ CSAS25

ETA EXPERTMENTS EXP 15

27GROUPNDF 4 INFHOMMEDIUM

U-233 $11000.00000-4$ BND

$\begin{array}{llll}0-234 \\ U-235 & 1 & 0 & 0.00397-4 \\ 0-236 & \text { BND }\end{array}$

$\begin{array}{lllll}U-236 & 1 & 0 & 0.00194-4 & \text { END }\end{array}$

$\begin{array}{lllll}U-238 & 1 & 0 & 0.01962-4 & \text { END }\end{array}$

N $100 \quad 1.20800-4$ END

$\begin{array}{lllll}\text { H } & 1 & 0 & 0.066389 & \text { BND } \\ 0 & 1 & 0 & 0.033609 & \text { END }\end{array}$

$\begin{array}{lllll}\text { B-10 } & 1 & 0 & 0.0 & \text { END } \\ \text { B-11 } & 1 & 0 & 0.0 & \text { END }\end{array}$

TH-232 $100.000000-3 \mathrm{END}$

$\begin{array}{llll}\text { AL } & 2 & 1 & \text { END } \\ \text { SS 316 } & 3 & 1 & \text { END } \\ \text { CARBONSTEEL } & 4 & 1 & \text { END }\end{array}$

BND COMP

READ PARAM NPG $=600$ FDN $=Y$ YS NUB $=$ YES TME $=59$ END PARAM

READ GEOM CYIINDER 1177.3684

$\begin{array}{llllll} & 77.3684 & 264.0 & 0.0\end{array}$

$\begin{array}{llllll}\text { CYLINDER } & 3 & 1 & 77.4984 & 264.13 & -0.13 \\ \text { CUBOID } & 0 & 1 & 4777.4984 & 264.13 & -0.13\end{array}$

END GEOI

READ START NST $=1$ END START

END DATA

CAS 16

ETSAS25 EXPERIMENTS EXP 16

\begin{tabular}{lllll}
27 GROUPNDF & \multicolumn{5}{l}{ INFHOMEDIUM } & \\
U-233 & 1 & 0 & $0.00000-4$ & END \\
U-234 & 1 & 0 & $0.00384-4$ & END \\
U-235 & 1 & 0 & $0.33519-4$ & END \\
U-236 & 1 & 0 & $0.00186-4$ & END \\
U-238 & 1 & 0 & $0.01924-4$ & END \\
N & 1 & 0 & $1.24400-4$ & END \\
H & 1 & 0 & 0.066391 & END \\
0 & 1 & 0 & 0.033615 & END \\
E-10 & 1 & 0 & 0.0 & END \\
B-11 & 1 & 0 & 0.0 & END \\
Th-232 & 1 & 0 & $0.000000-3$ & END \\
AL & 2 & 1 & & END \\
SS316 & 3 & 1 & & END \\
CARBONSTEE & 4 & 1 & & END
\end{tabular}

END COMP

ETA EXPERIMENTS 16
READ PARAM NPG $=600$ FDN=YES NUB=YES TME=59 END PARAM

READ GEOM CYLINDER $1177.3684 \quad 203.2762 \quad 0.0$

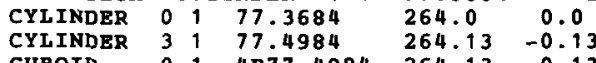

$\begin{array}{lllllll}\text { CUBOID } & 0 & 1 & 4877.4984 & 264.13 & -0.13\end{array}$

END GEOM

READ START NST $=1$ END START

END DATA 
Table A.6 (continued)

CAS 17

=CSAS 25
BTA EXPERIMENTS EXP, 17

$\begin{array}{lllll}\text { 27GROUPNDF } & \text { INFHOMMEDUM } & \\ \text { U-233 } & 1 & 0 & 0.36517-4 & \text { END } \\ \text { U-234 } & 1 & 0 & 0.00556-4 & \text { END } \\ \text { U-235 } & 1 & 0 & 0.00000-4 & \text { END } \\ \text { U-236 } & 1 & 0 & 0.00000-4 & \text { END } \\ \text { U-238 } & 1 & 0 & 0.00410-4 & \text { END } \\ \text { N } & 1 & 0 & 0.82600-4 & \text { END } \\ \text { H } & 1 & 0 & 0.0656439 & \text { END } \\ 0 & 1 & 0 & 0.033539 & \text { END } \\ \text { B-10 } & 1 & 0 & 0.0 & \text { END } \\ \text { B-11 } & 1 & 0 & 0.0 & \text { END } \\ \text { TH-232 } & 1 & 0 & 0.000037-3 & \text { END } \\ \text { AL } & 2 & 1 & & \text { END } \\ \text { SS316 } & 3 & 1 & & \text { END } \\ \text { CARBONSTEEL } & 4 & 1 & & \text { END } \\ \text { END COMP } & & & \end{array}$

CARBONSTE

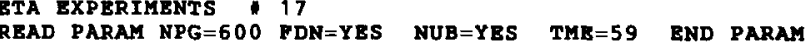
READ GEOM CYLINDER $1177.3684 \quad 49.50460 .0$ CYLINDER $00177.3684 \quad 264.0 \quad 0.0$ $\begin{array}{llllll}\text { CULINDER } & 3 & 1 & 77.4984 & 264.13 & -0.13 \\ \text { CUBOID } & 0 & 1 & 4877.4984 & 264.13 & -0.13\end{array}$

BND GrOM

READ START NST $=1$ END START

RND DATA

\section{CAS 18
$=\operatorname{CSAS} 25$}

ETA EXPERIMENTS EXP 18

27GROUPNDF 4 INFHOMMEDIUY

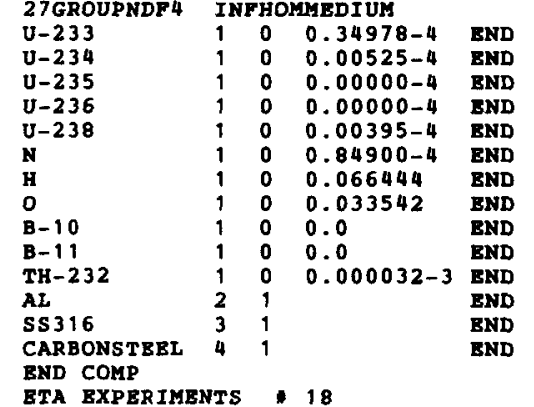

\section{BND COMP}

READ PARAM NPG $=600$ PDN $=$ YES NUB $=$ YES TME $=59$ END PARAM READ GBOM CYLINDER $1177.3684 \quad 59.2074 \quad 0.0$ $\begin{array}{llllll}\text { CYLINDER } & 0 & 9 & \mathbf{7 7 . 3 6 8 4} & \mathbf{2 6 4 . 0} & \mathbf{0 . 0}\end{array}$ $\begin{array}{lllllll}\text { CYLINDER } & 3 & 1 & 77.4984 & 264.13 & -0.13 \\ \text { CUPOID } & 0 & 1 & 4 P 77.4984 & 264.13 & -0.13\end{array}$

\section{END GBO}

READ START NST $=1$ BND START

END

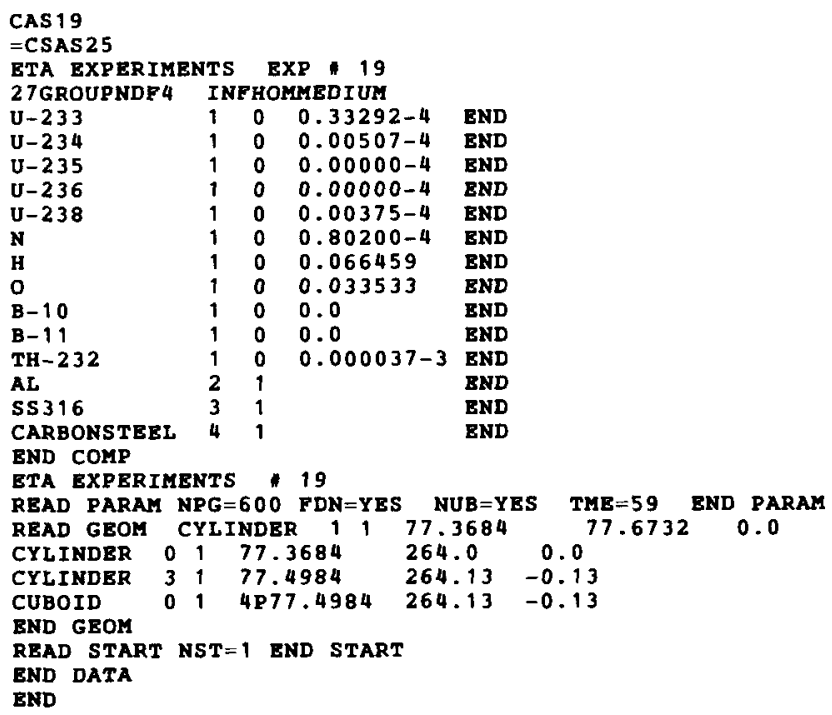

END DATA

CAS20
=CSAS 25

BTA EXPERIMENTS EXP 20

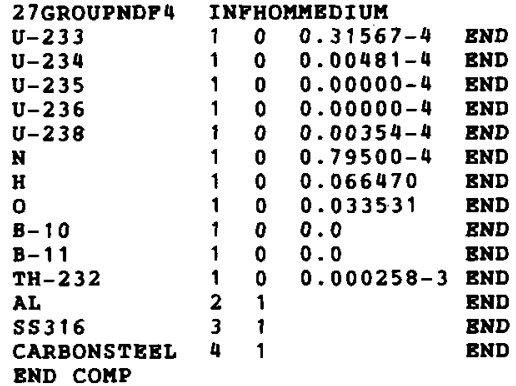

ETA EXPERIMENTS 20

READ PARAM NPG $=600$ PDN=YBS NUE=YBS TME $=59$ END PARAM

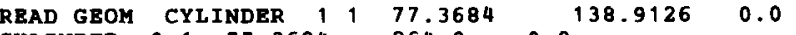

CYLINDER $\begin{array}{llllll} & 0 & 77.3684 & 264.0 & 0.0\end{array}$

$\begin{array}{llllll}\text { CYLINDER } & 3 & 1 & 77.4984 & 264.13 & -0.13 \\ \text { CUBOID } & 0 & 1 & 4 P 77.4984 & 264.13 & -0.13\end{array}$

END GEOM 1 TST

END DATA 
Table A.6 (continued)
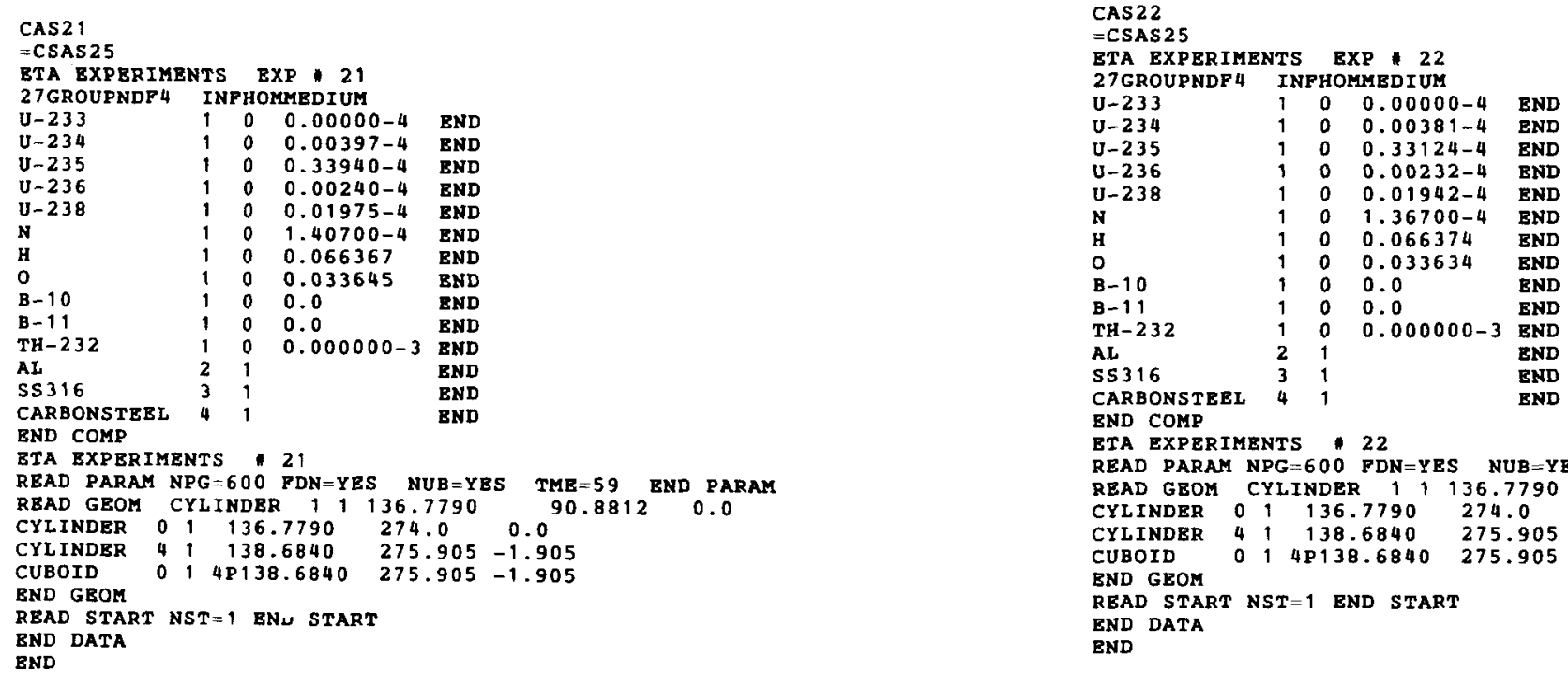

BND COMP

ETA EXPERIMENTS 22 TES NUB $=$ YES TME $=59$ END PARAM READ GEOM CYLINDER $11136.7790 \quad 122.4280 \quad 0.0$ CYLINDER 01 T36.7790 274.0

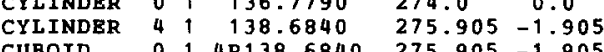

READ START NST $=1$ END START

END DATA

END

CAS 23 


\section{Appendix B \\ PLOT OF AVERAGE $\mathrm{K}_{\text {eff }}$ BY GENERATION SKIPPED FOR VALIDATION CASES}

Plots of average $\mathrm{k}_{\text {eff }}$ by generation skipped for the validation cases appear in the same order as listed in Tables 1-6 of Sect. 1 of this report.

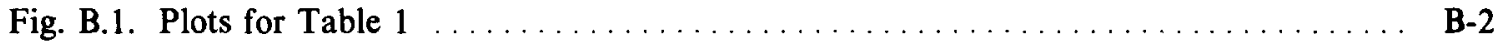

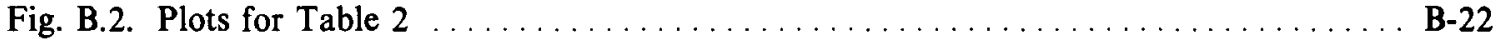

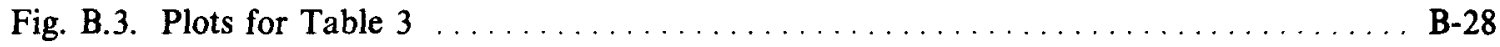

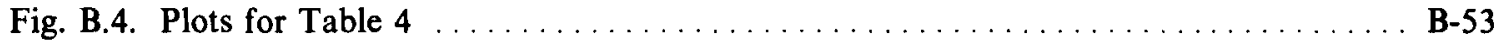

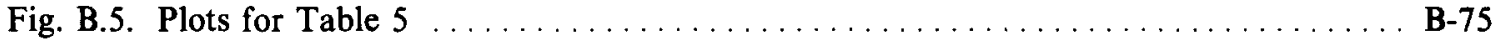

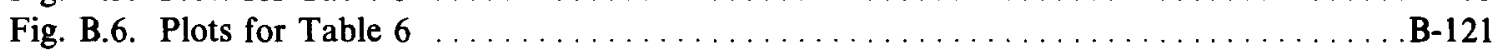




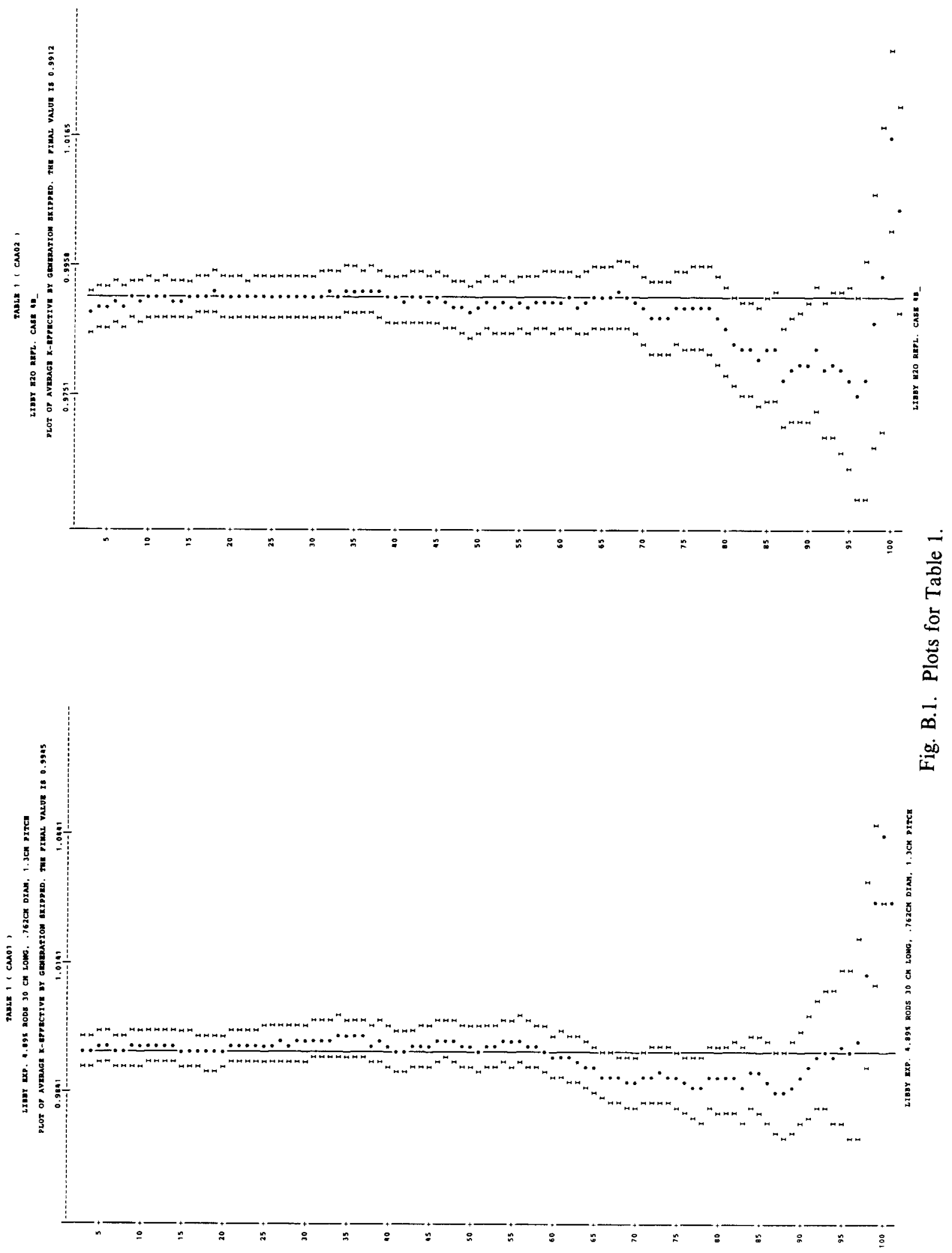




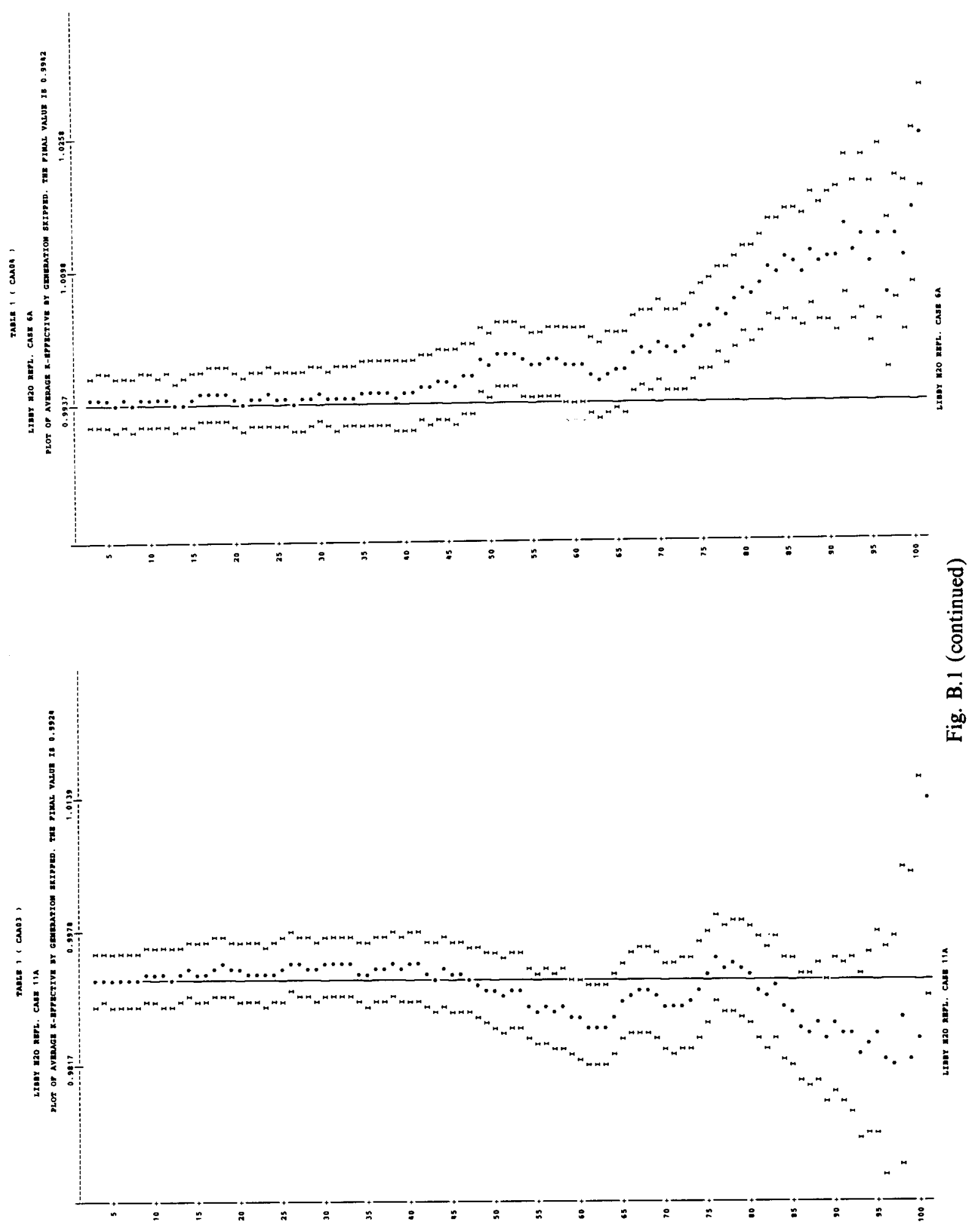




\section{B-4}
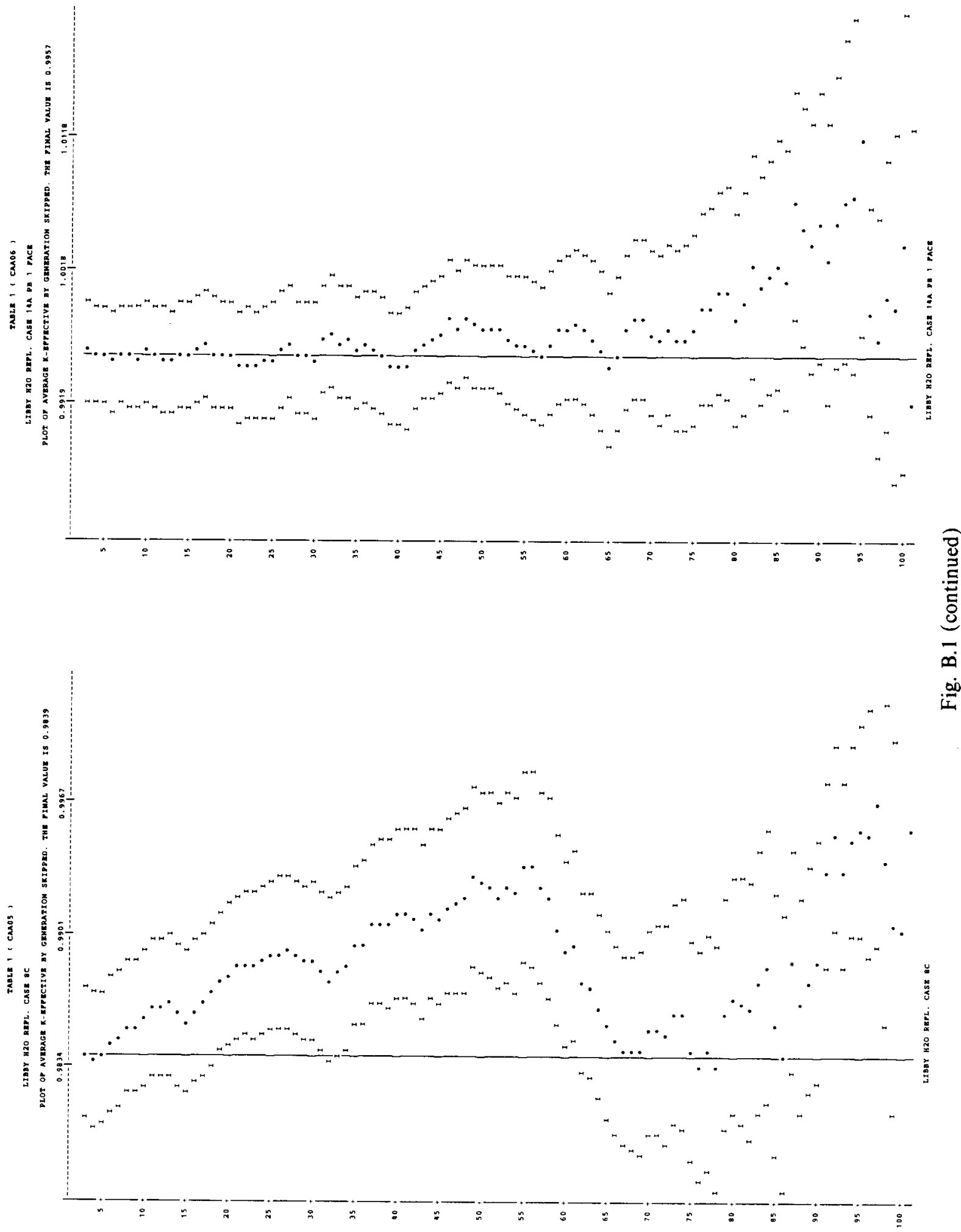
B-5

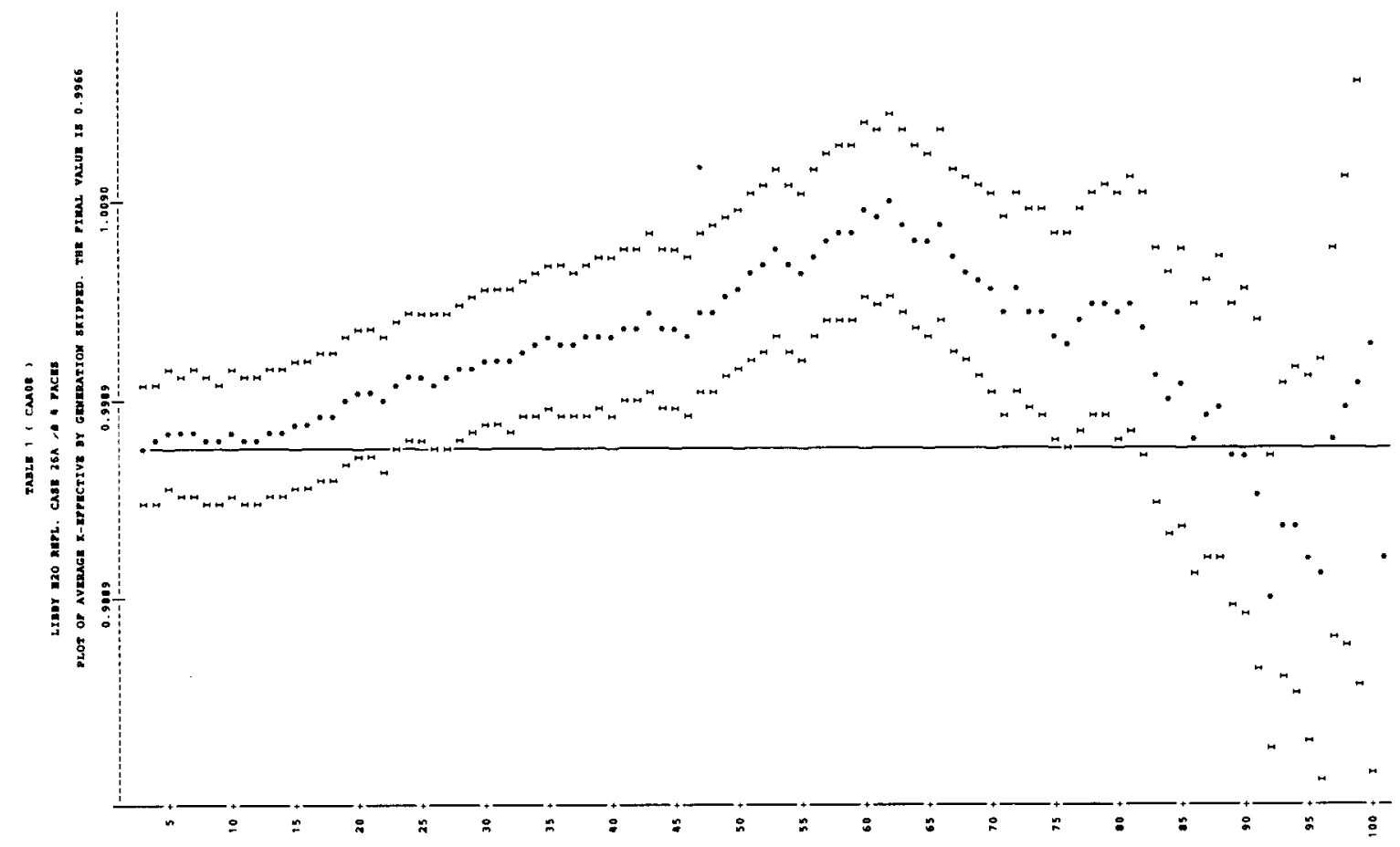

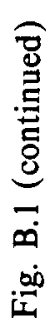

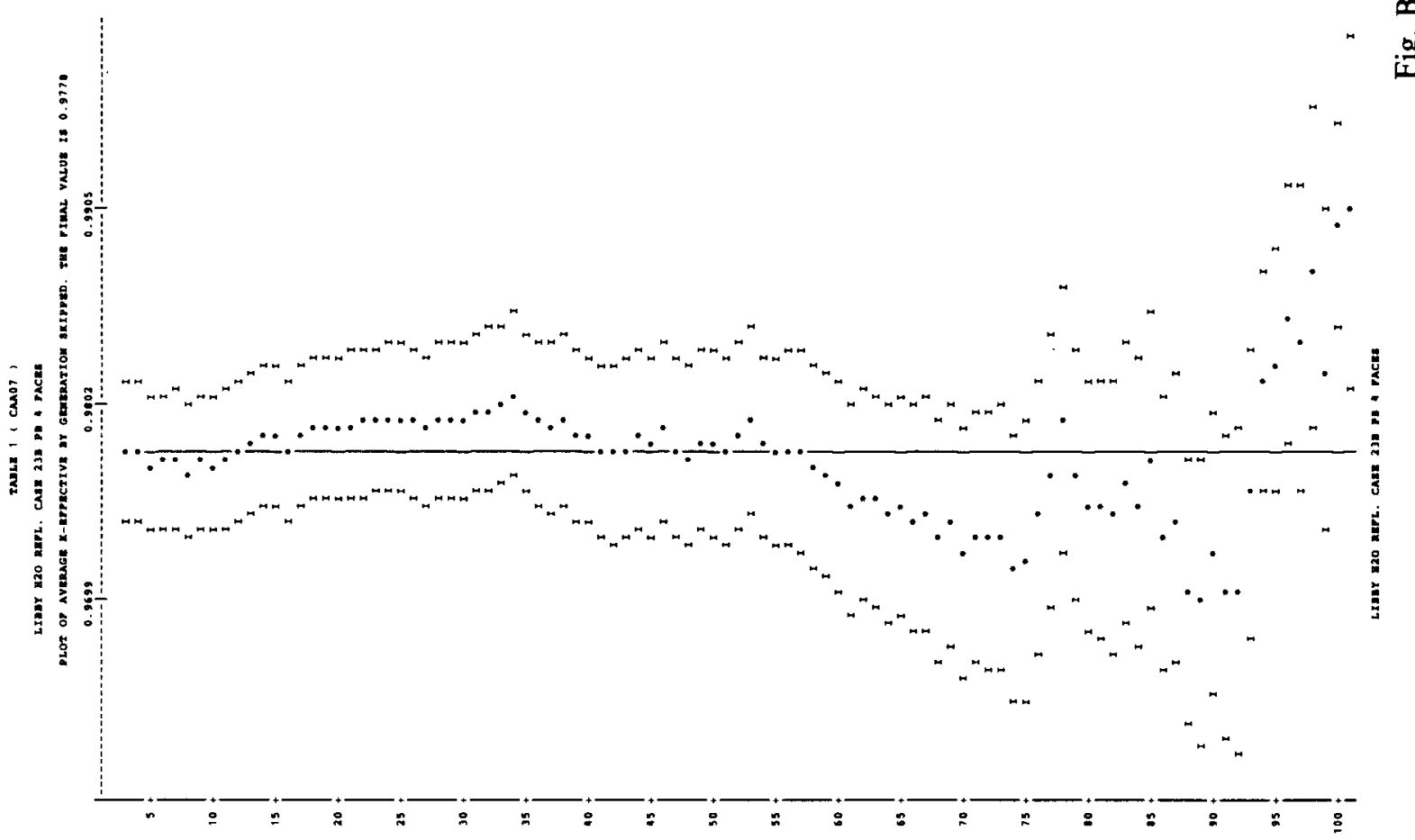


B-6
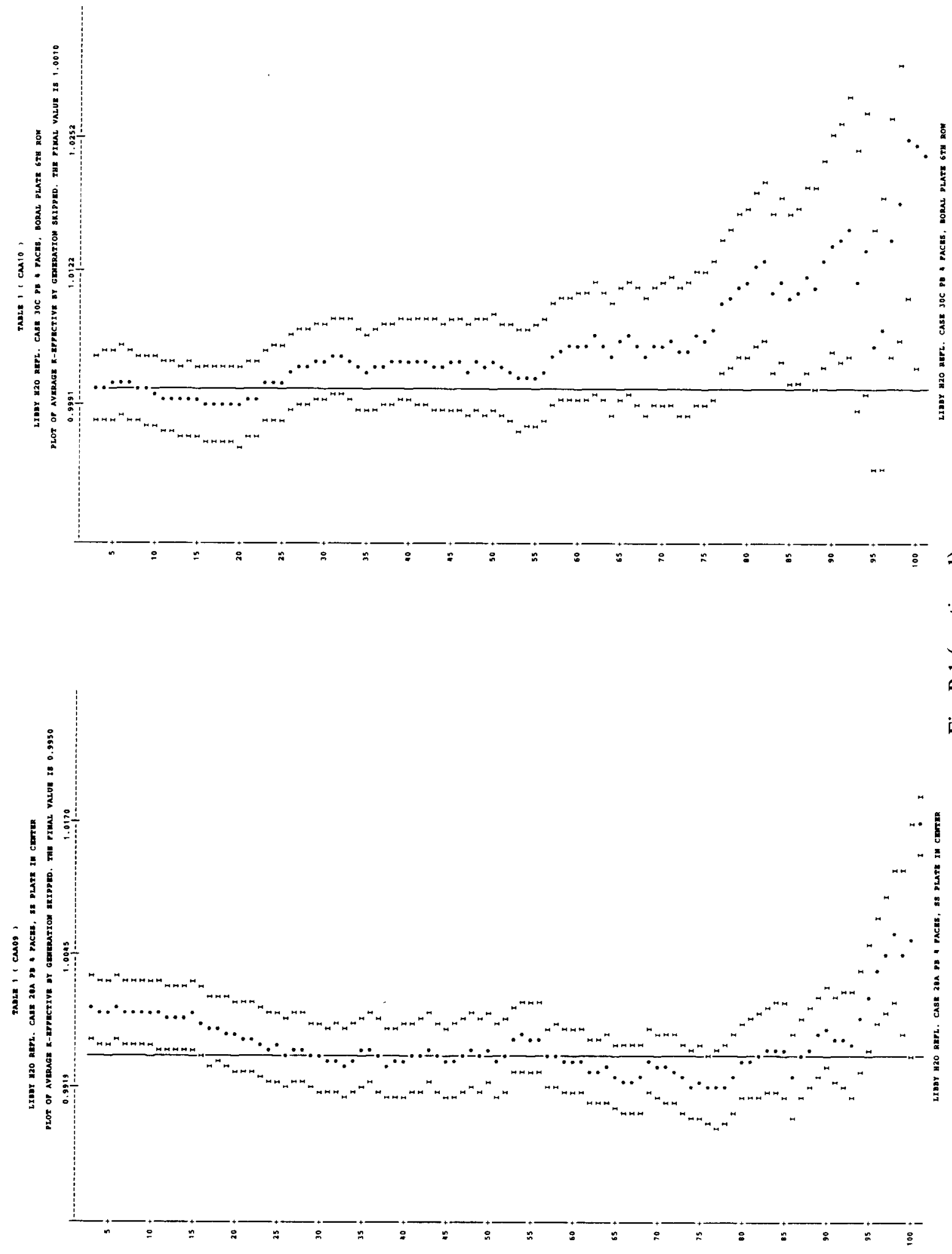


\section{B-7}
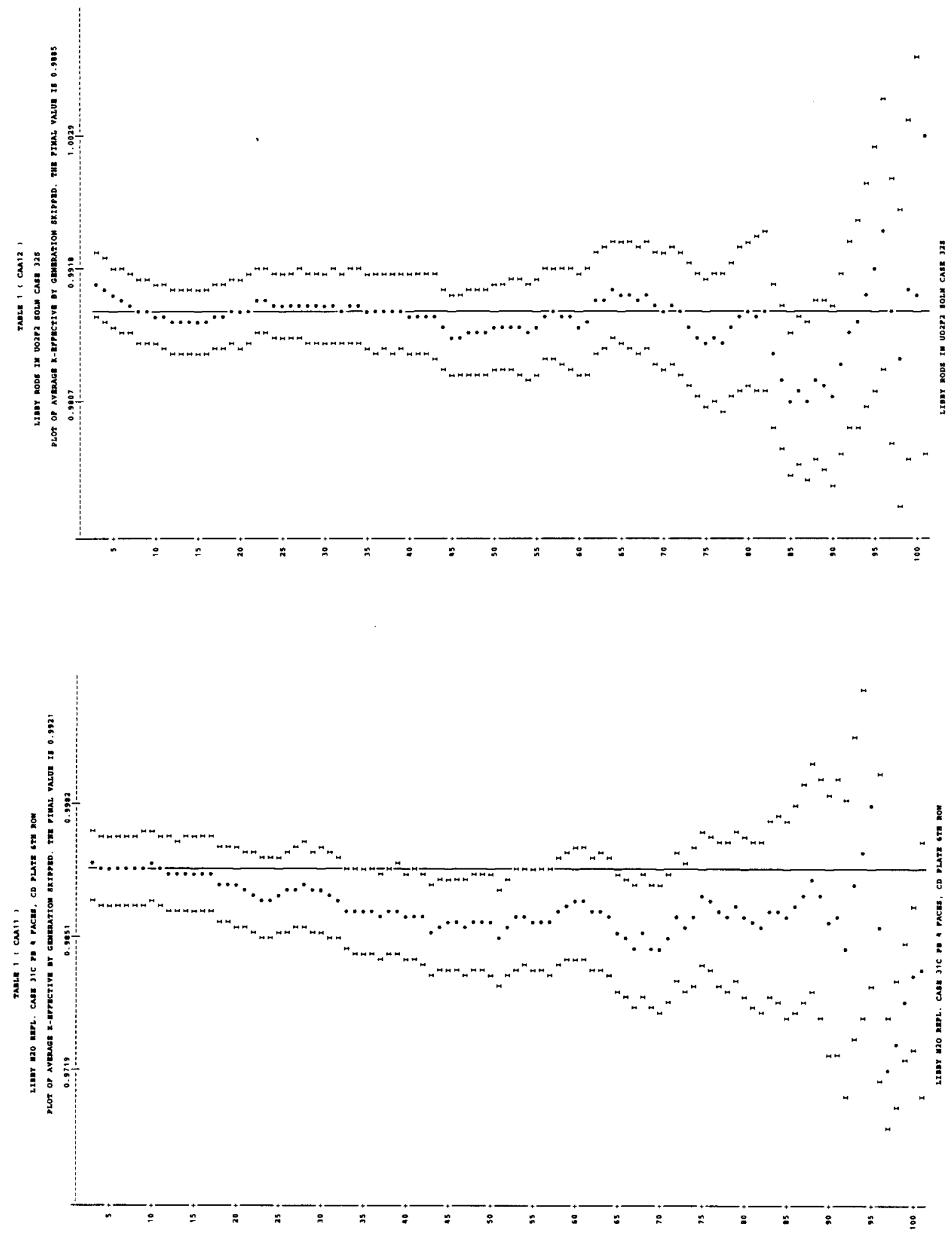

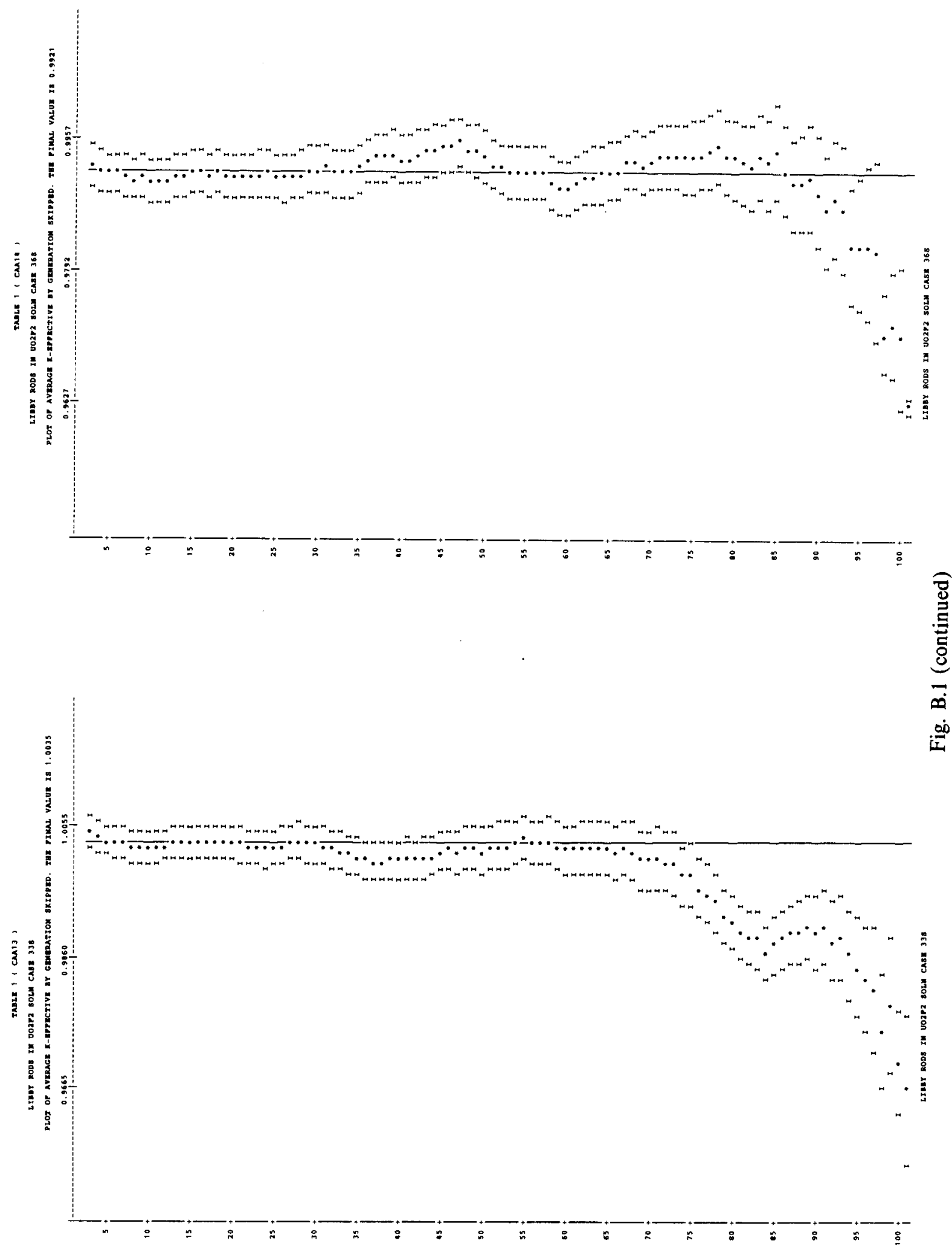


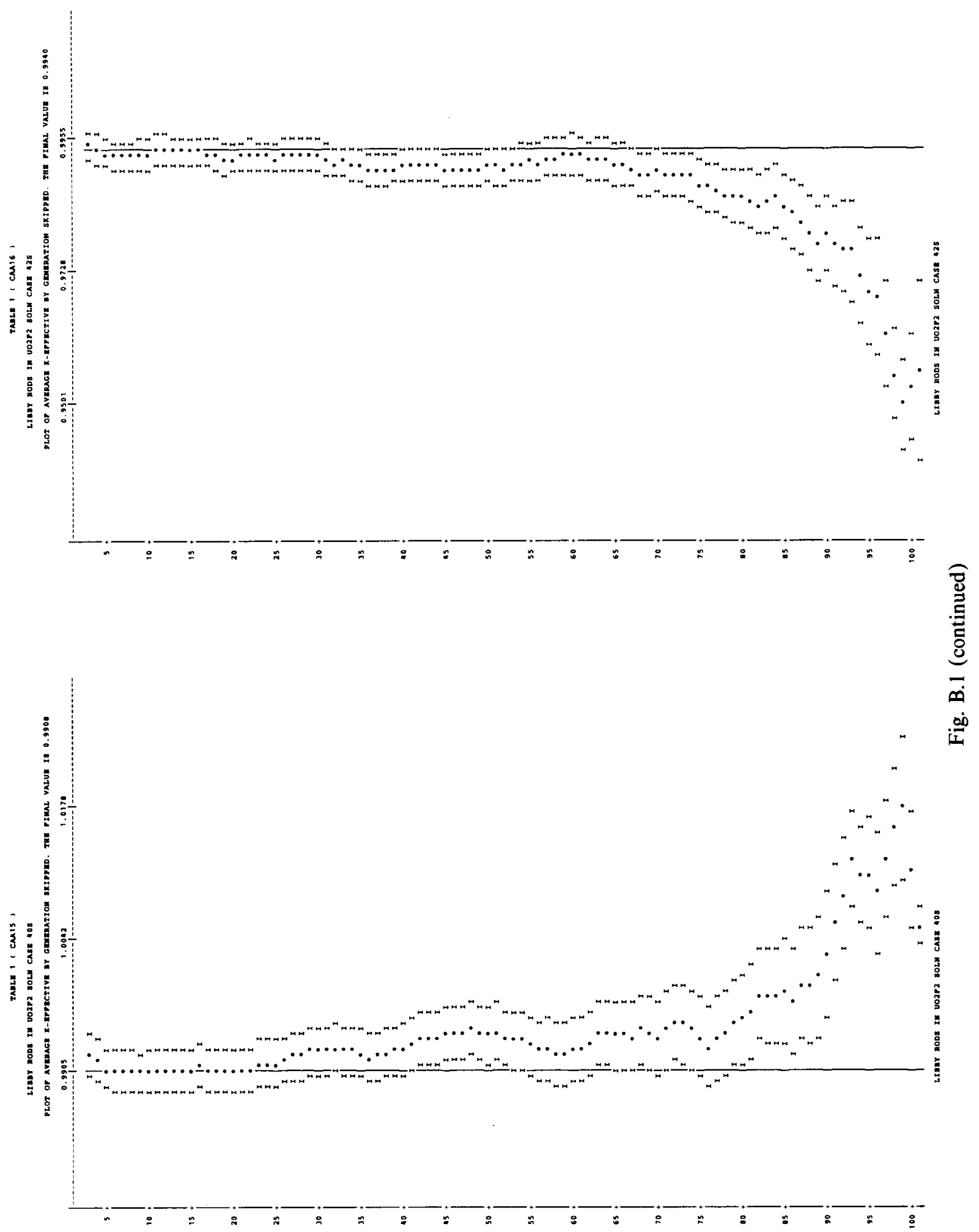


B-10
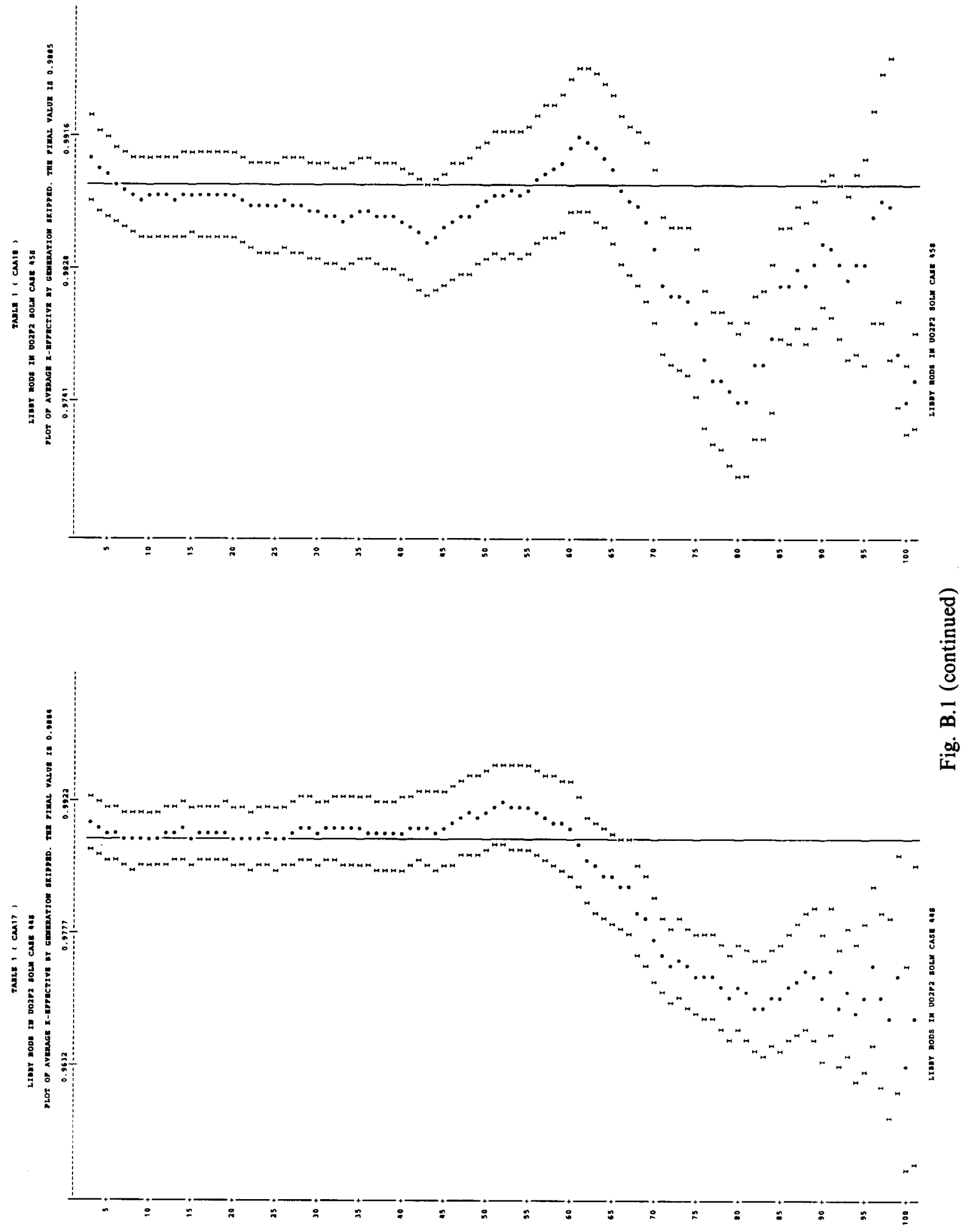
B-11

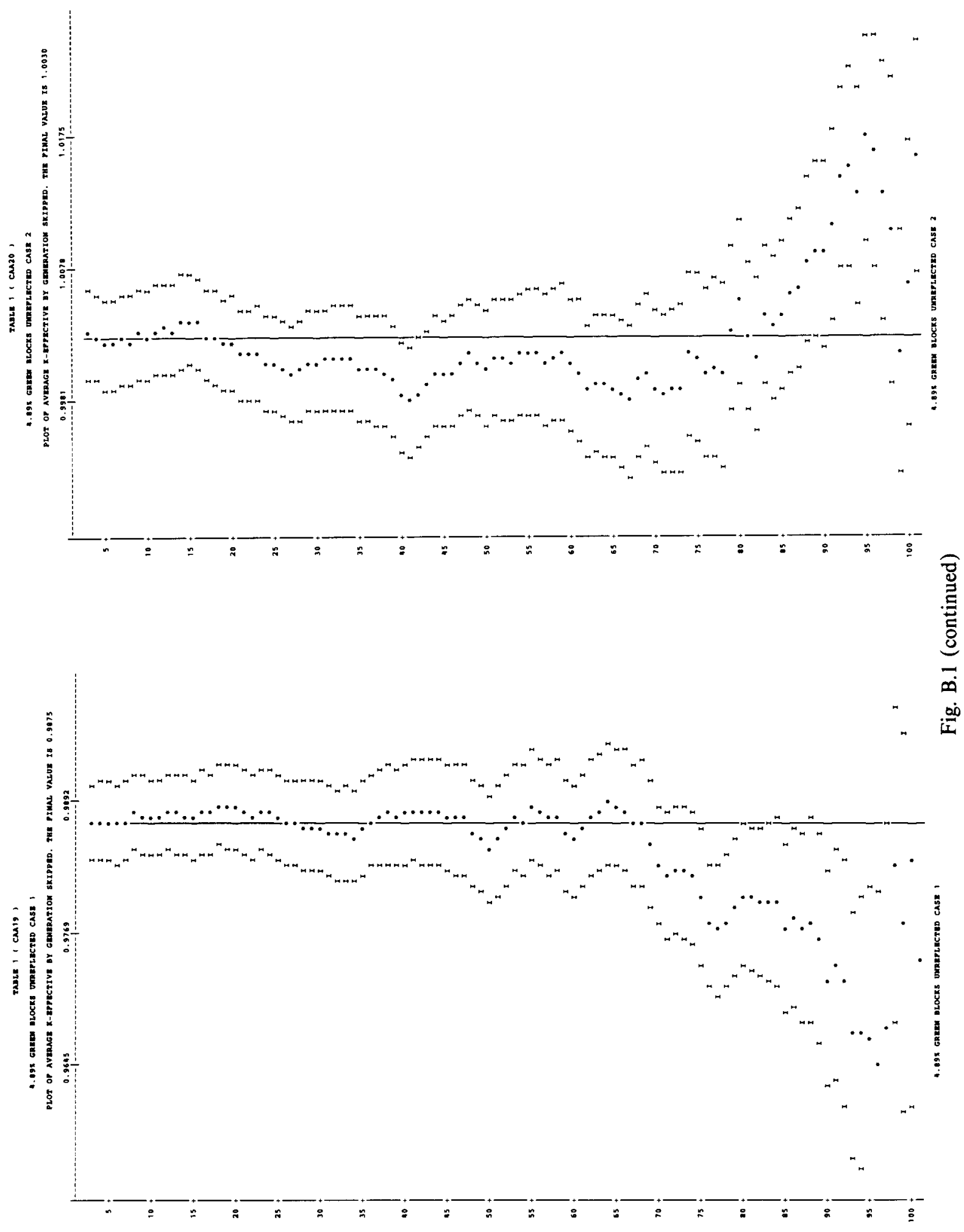




\section{B-12}
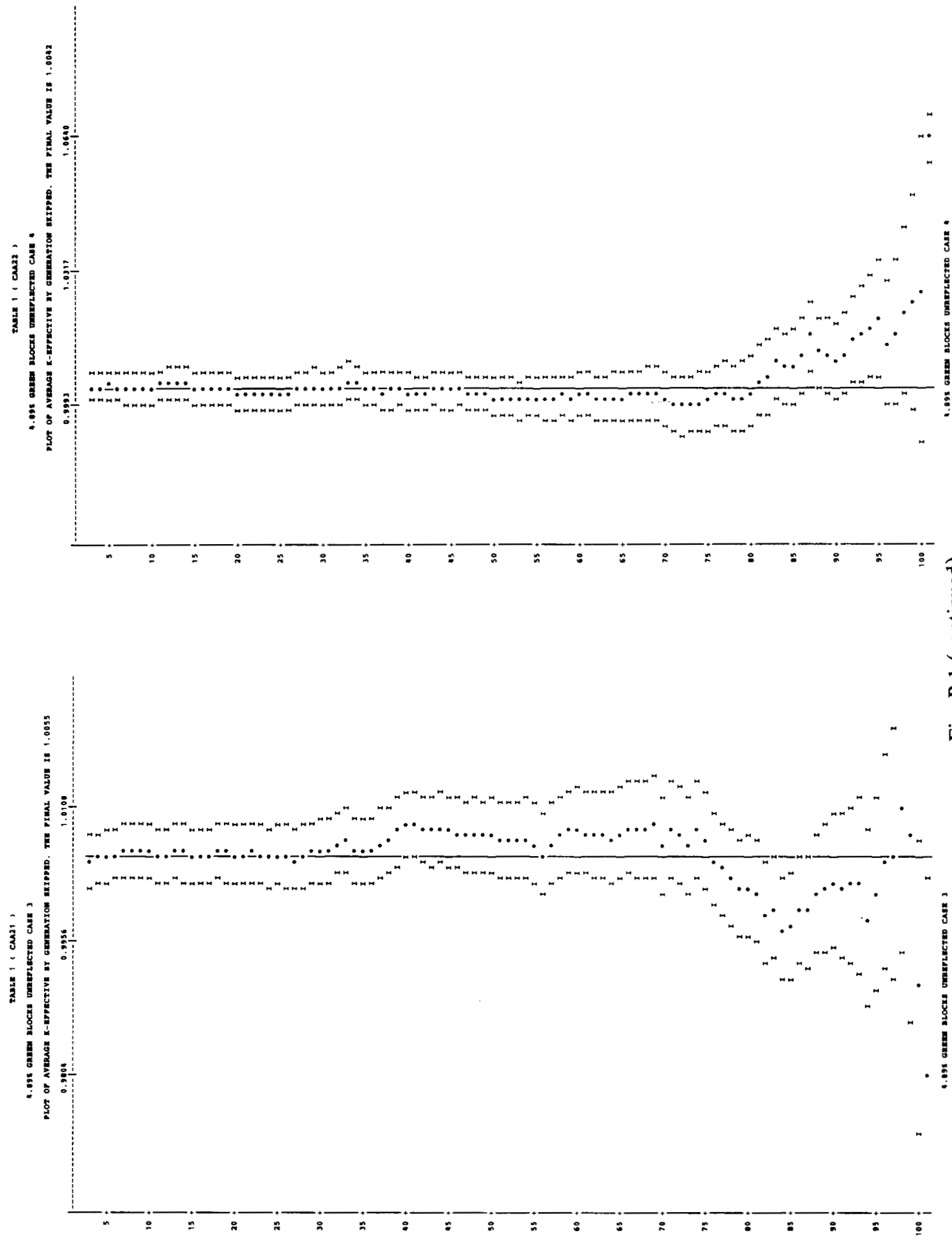
B-13
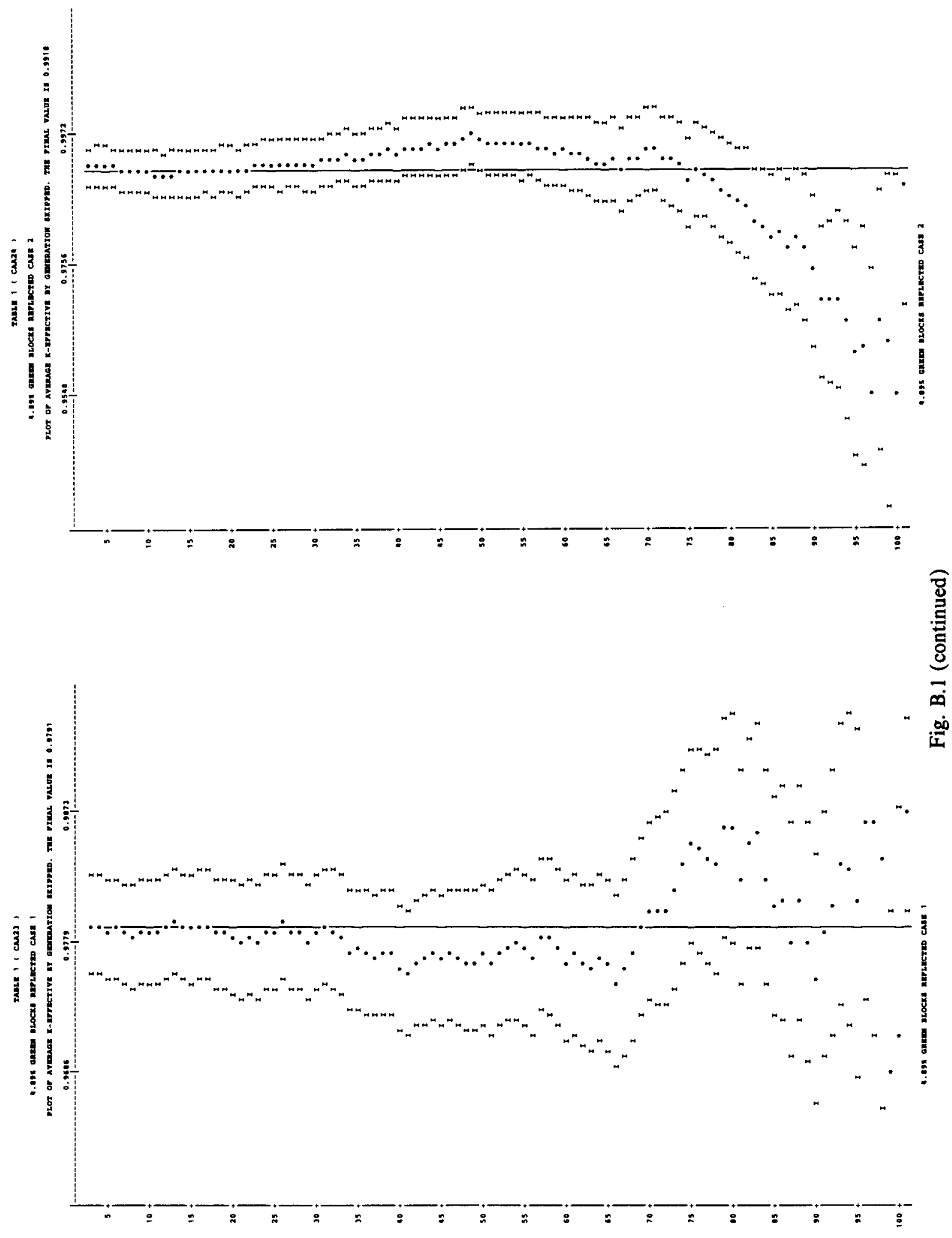
B-14

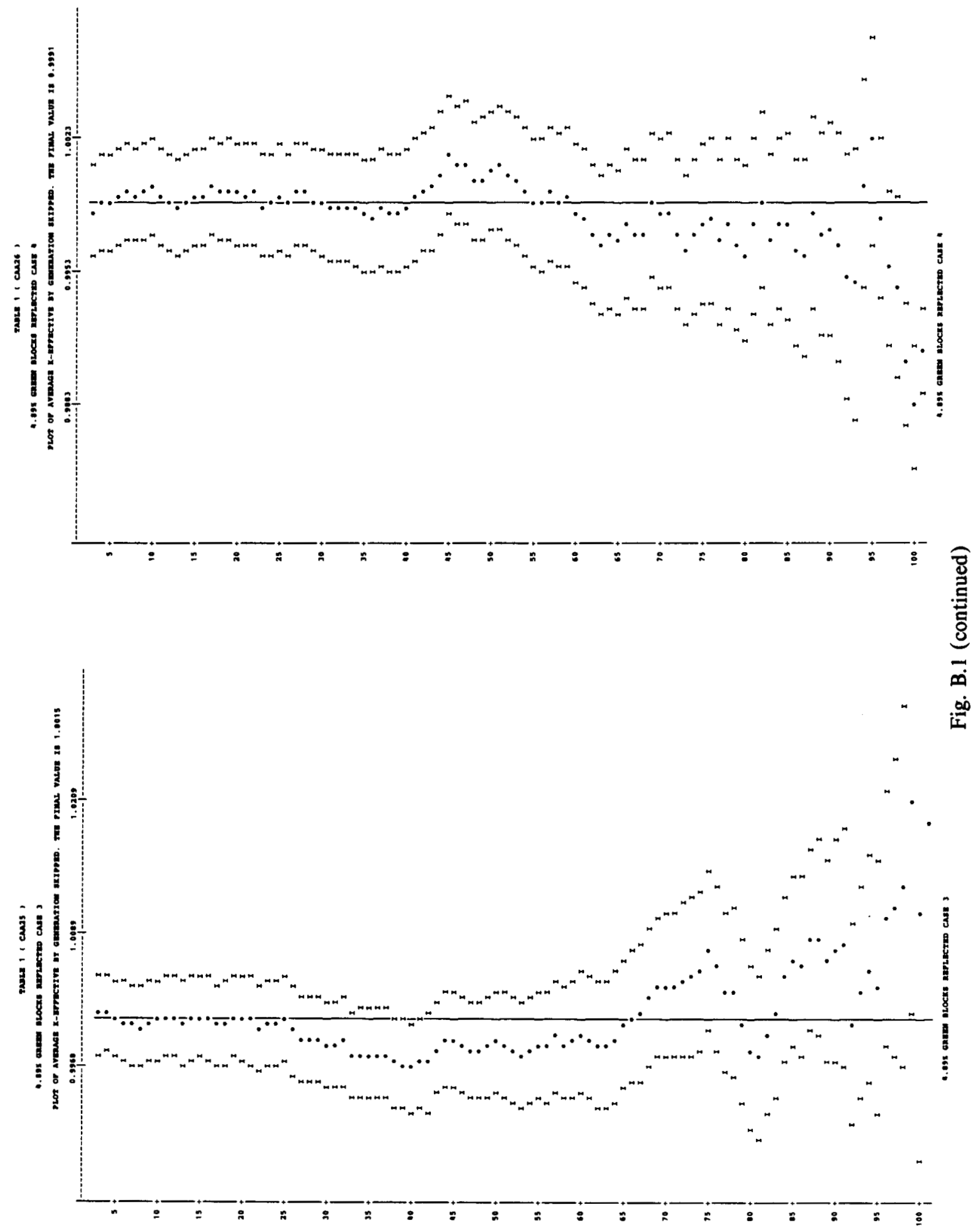


B-15

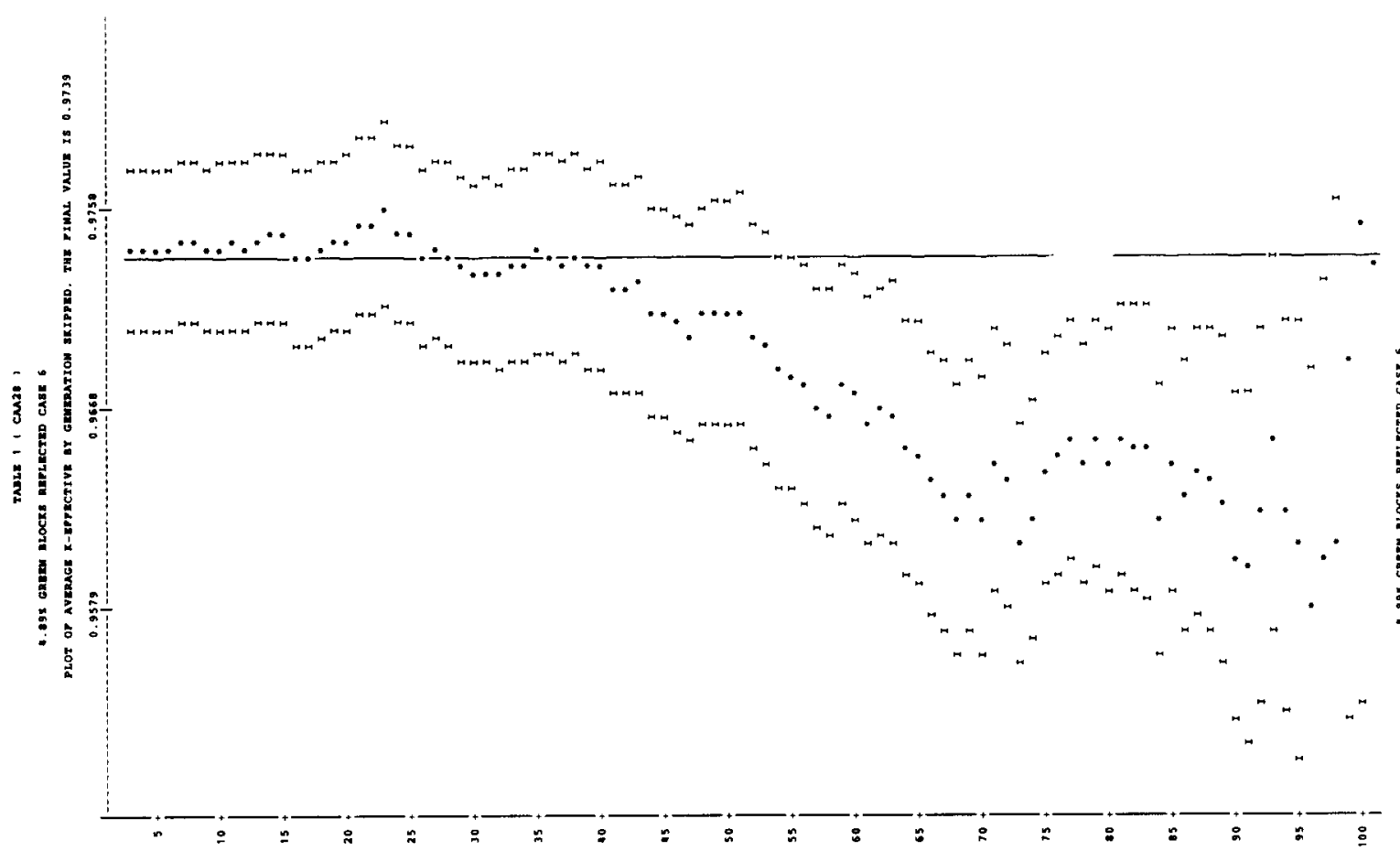

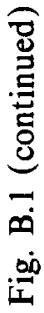

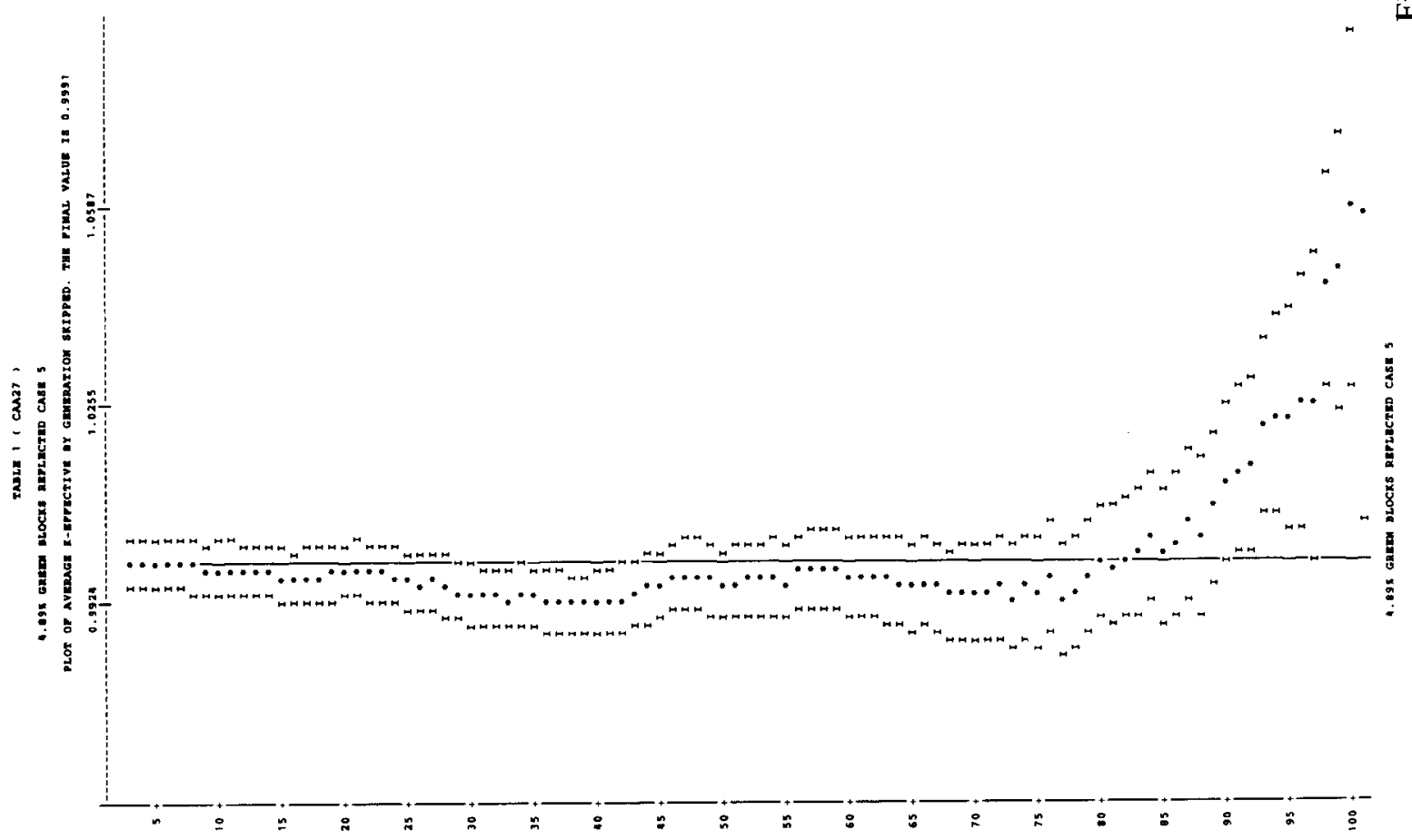




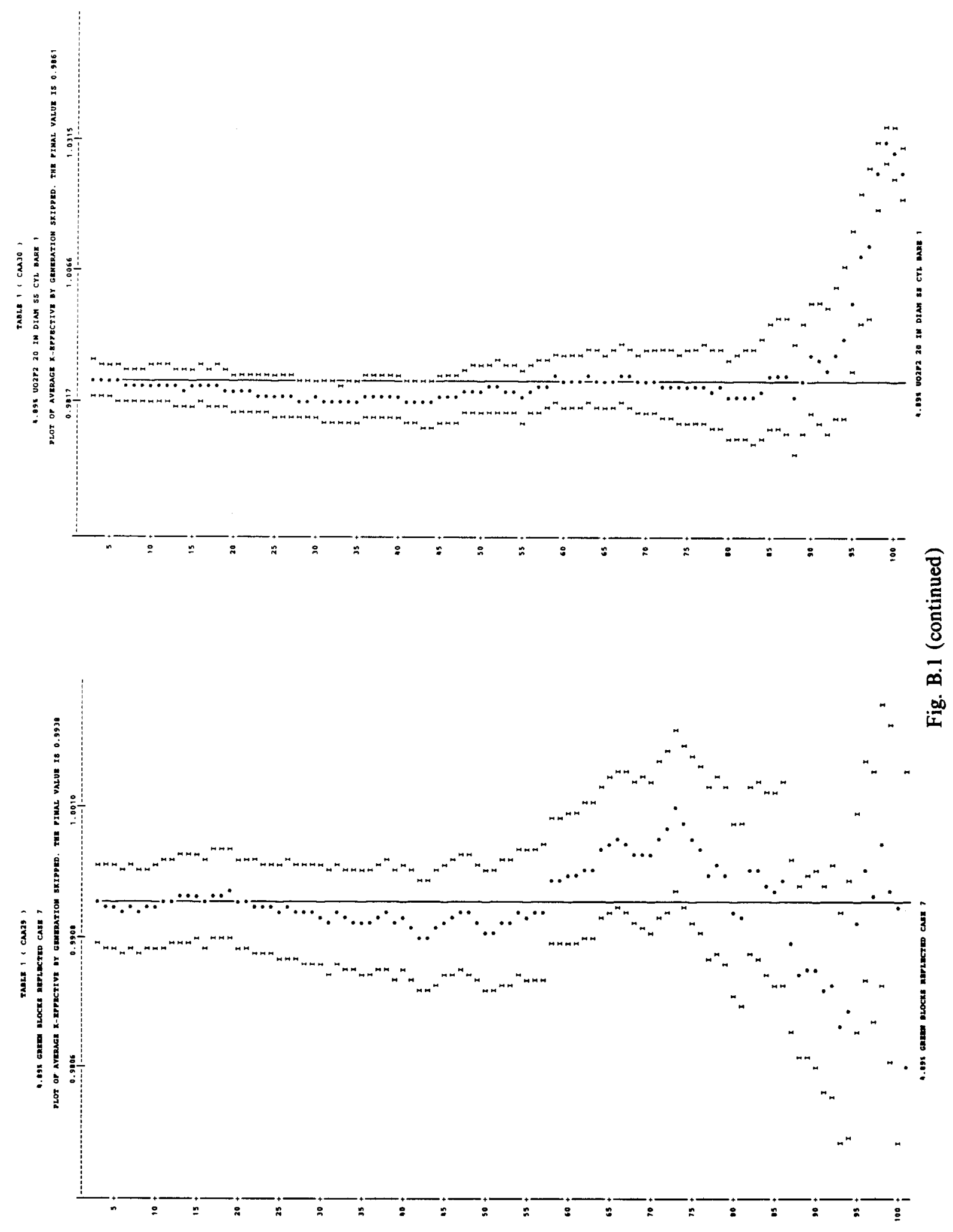


B-17

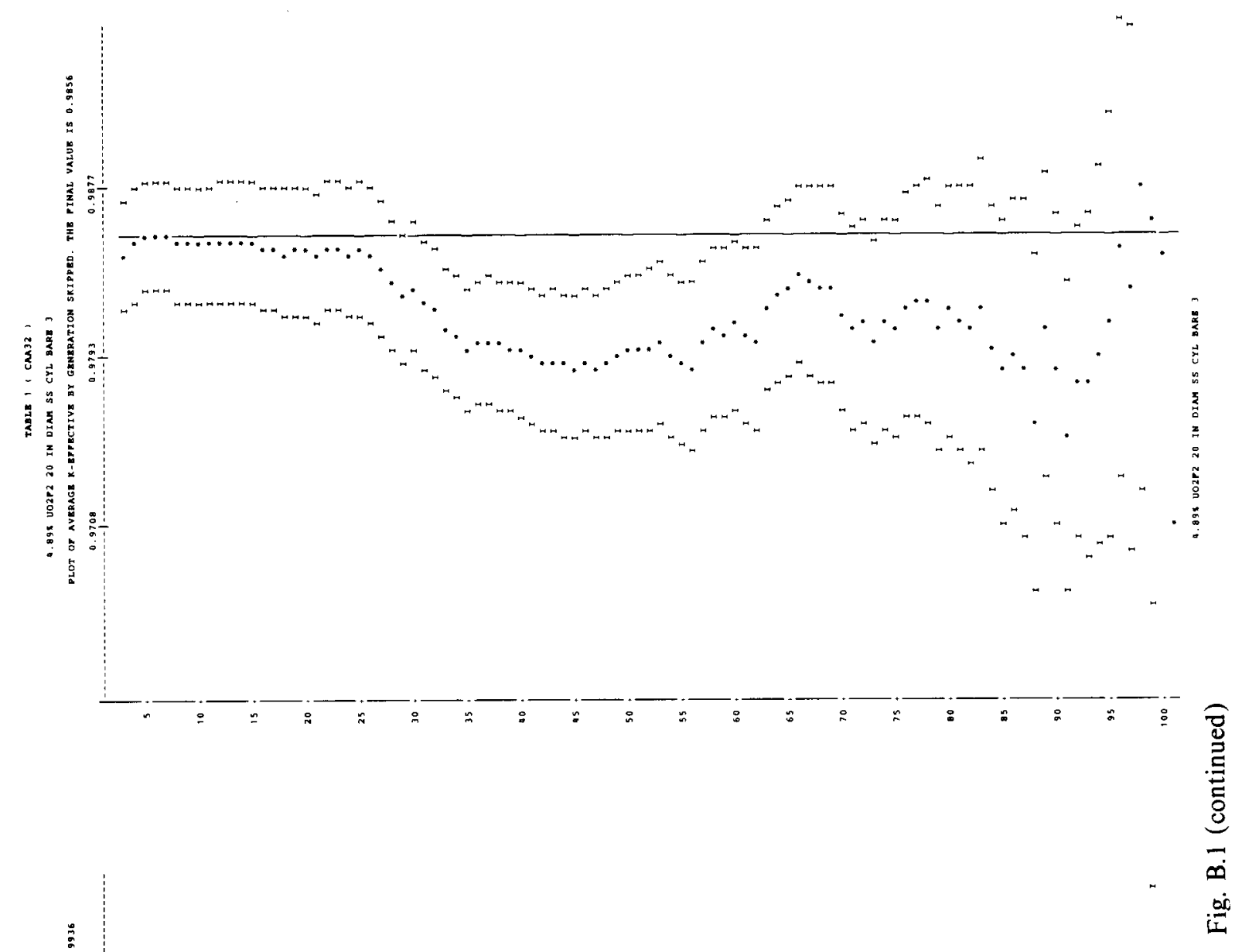




\section{B-18}

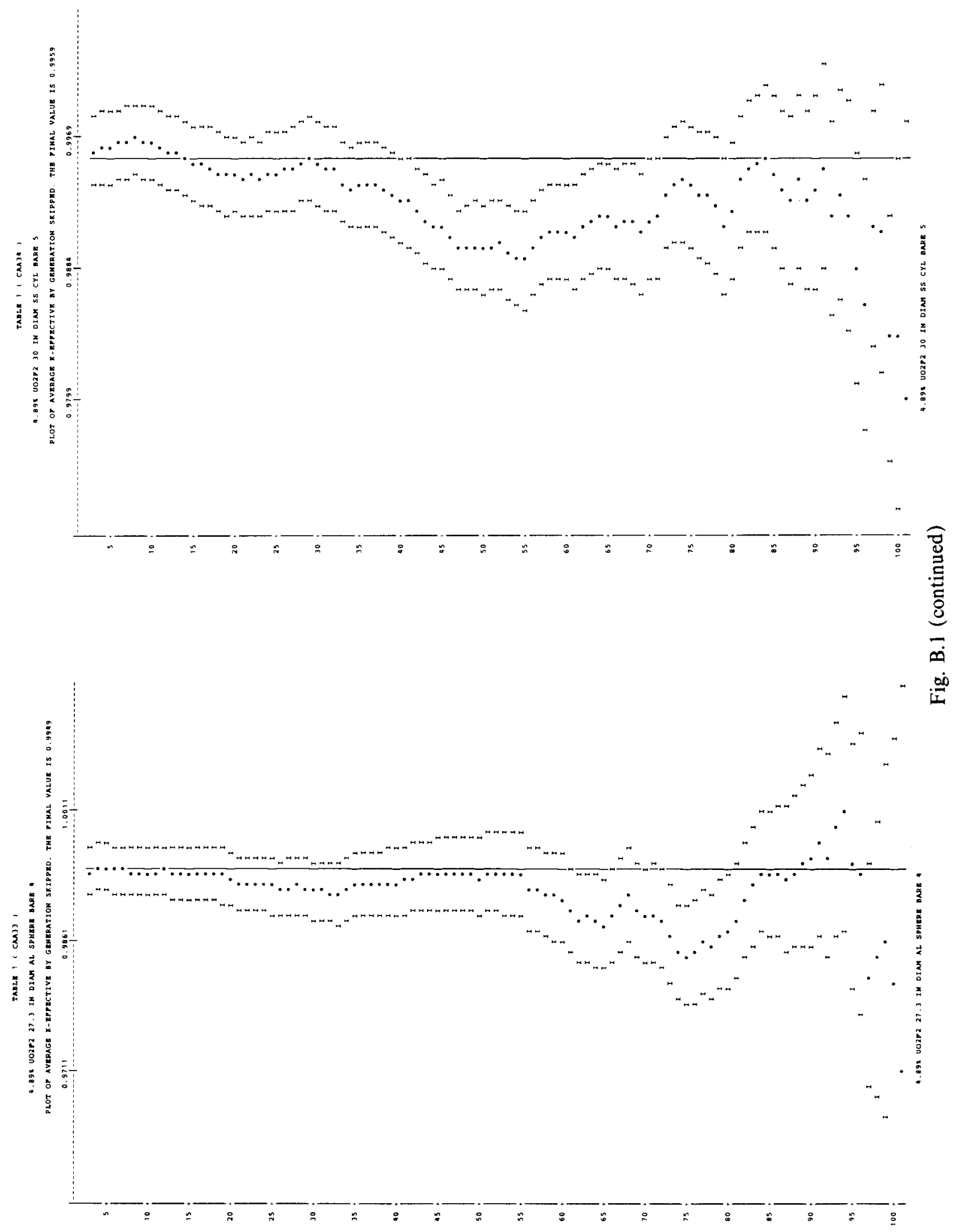


B-19
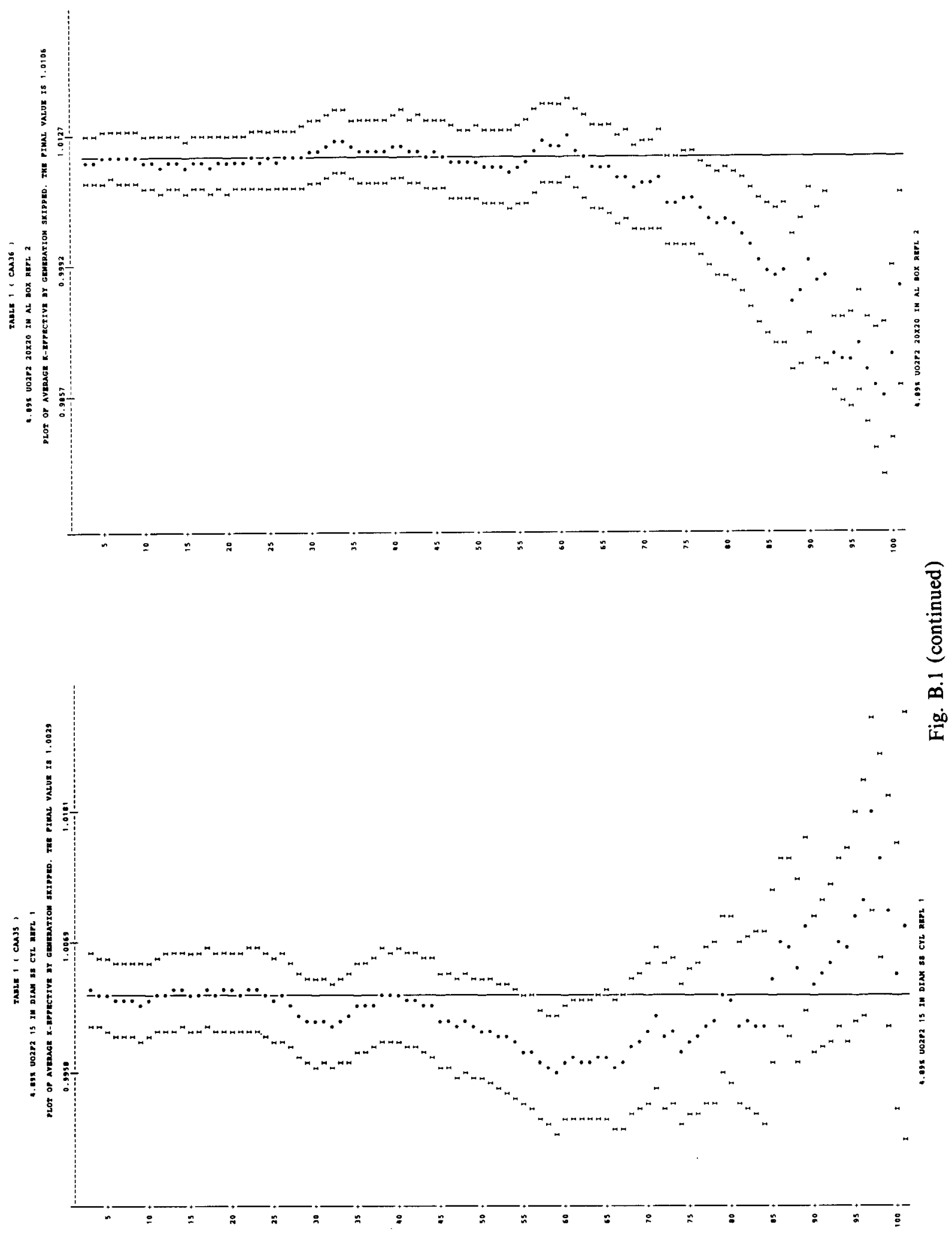

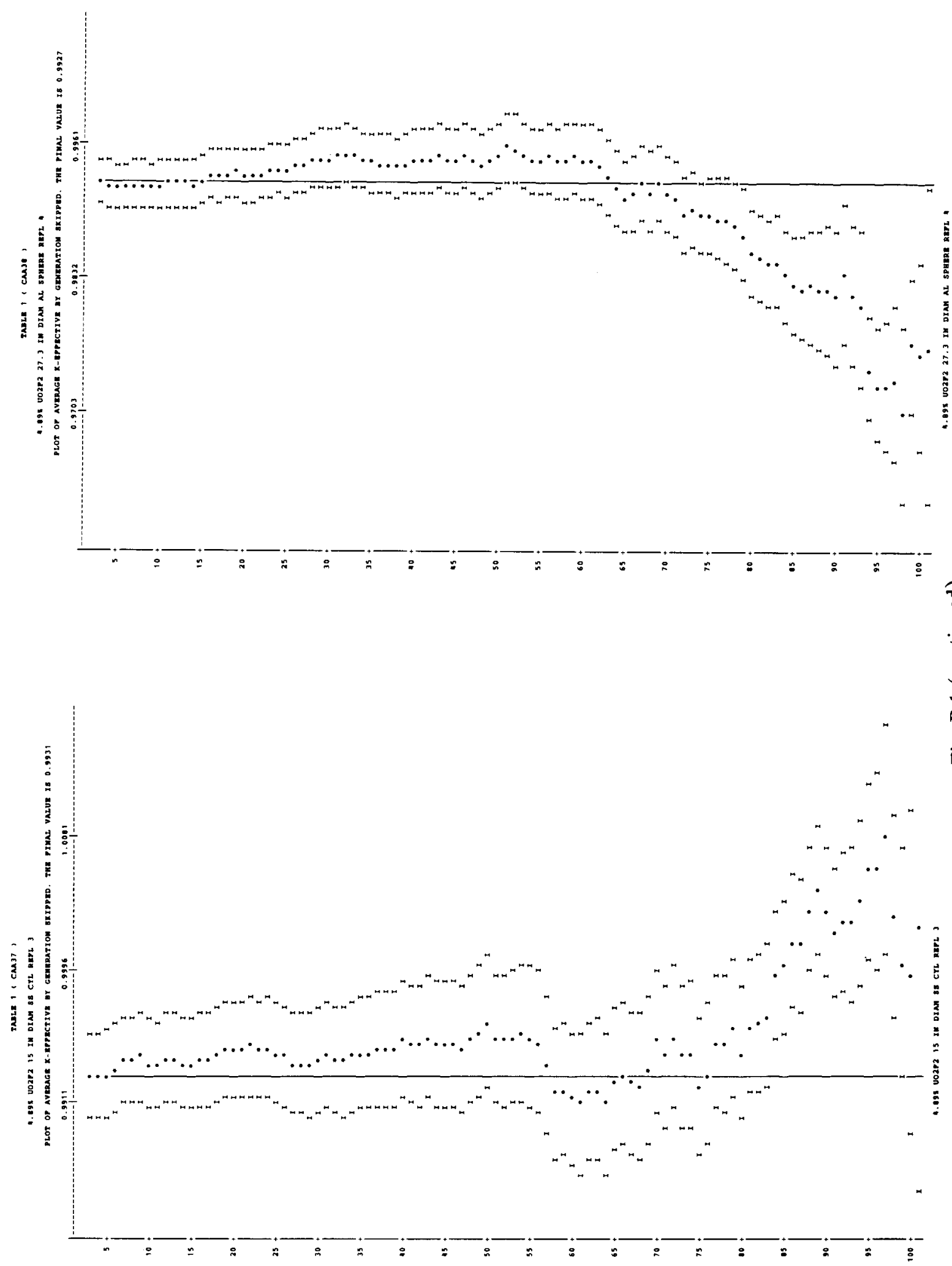
B-21

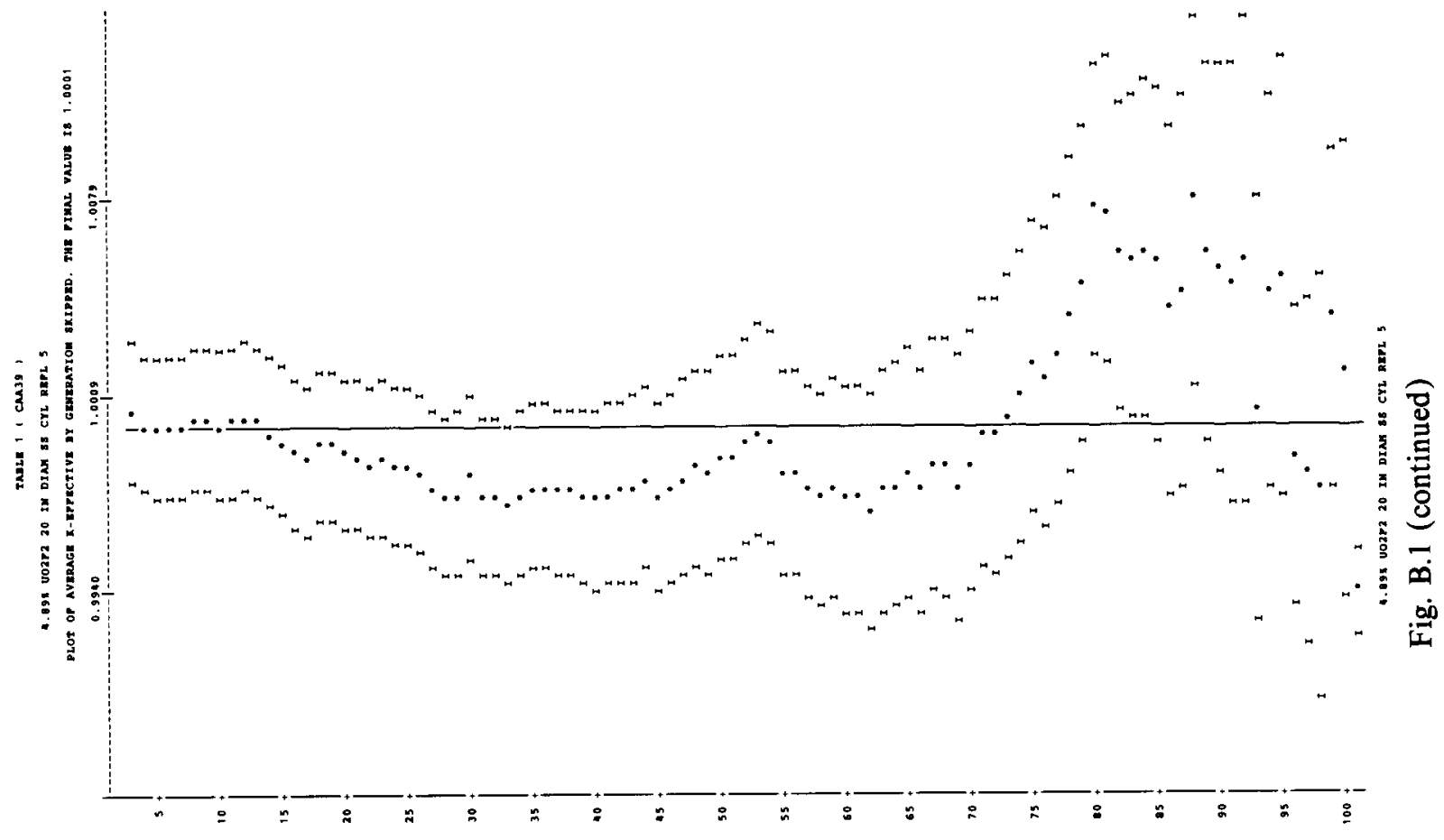




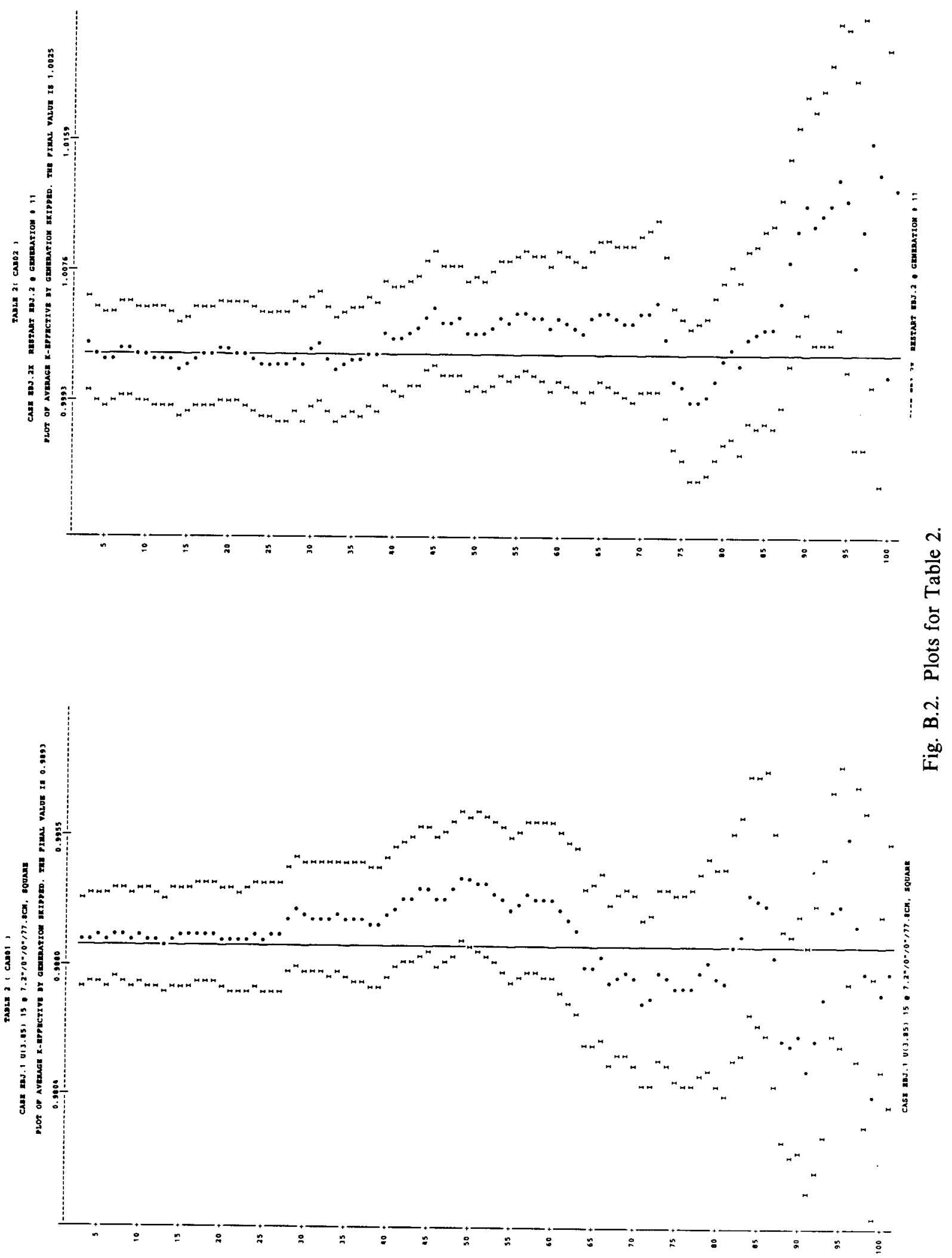




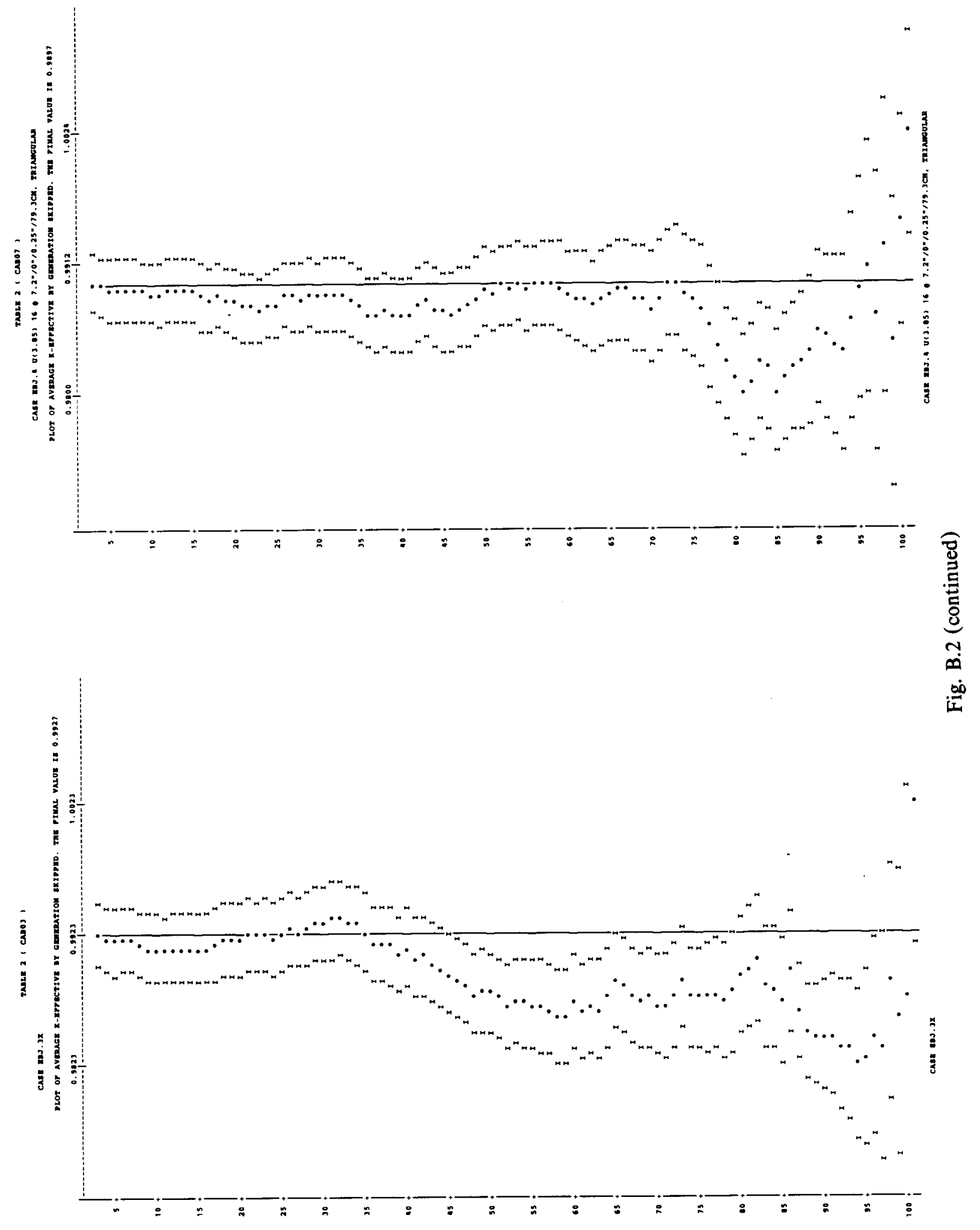



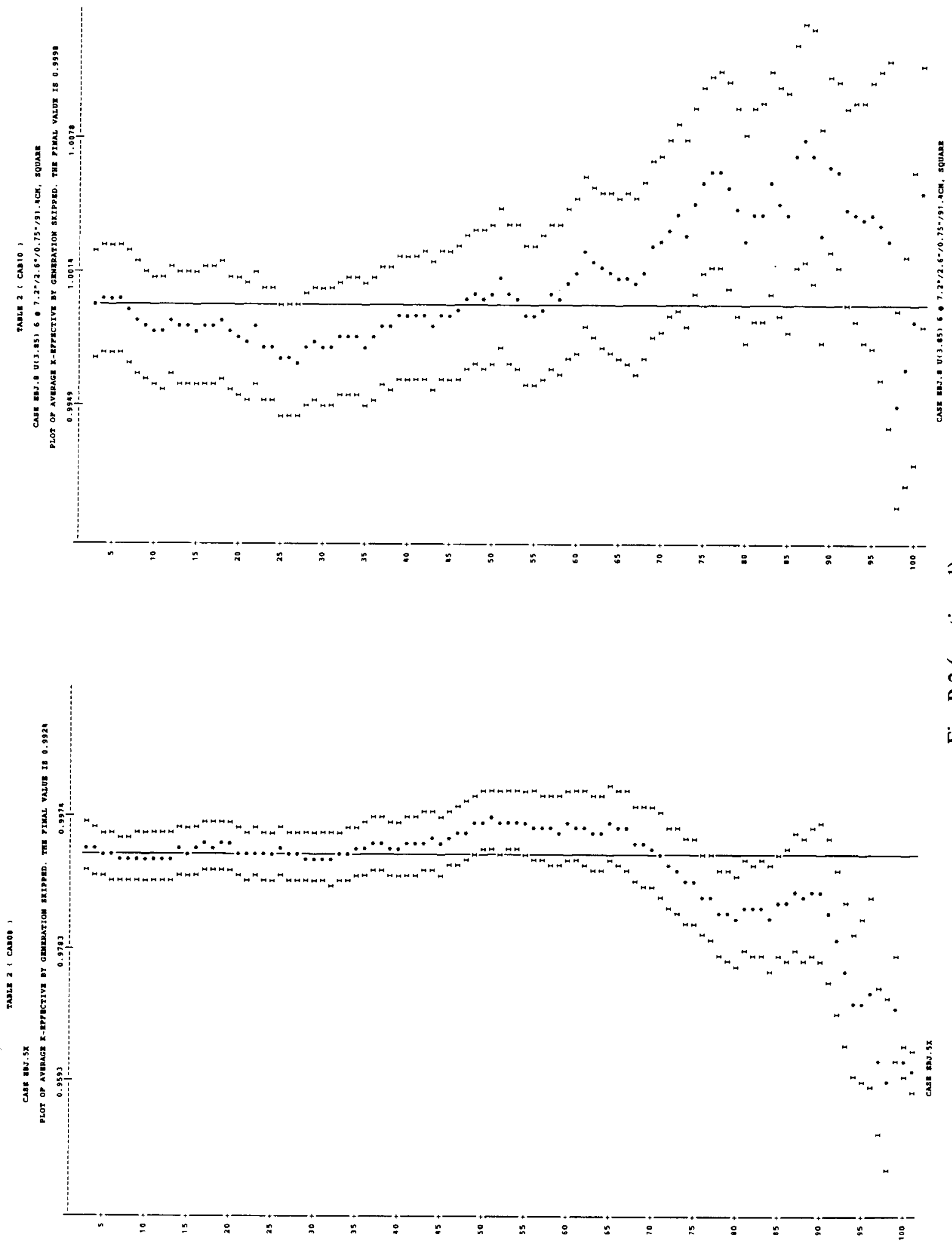

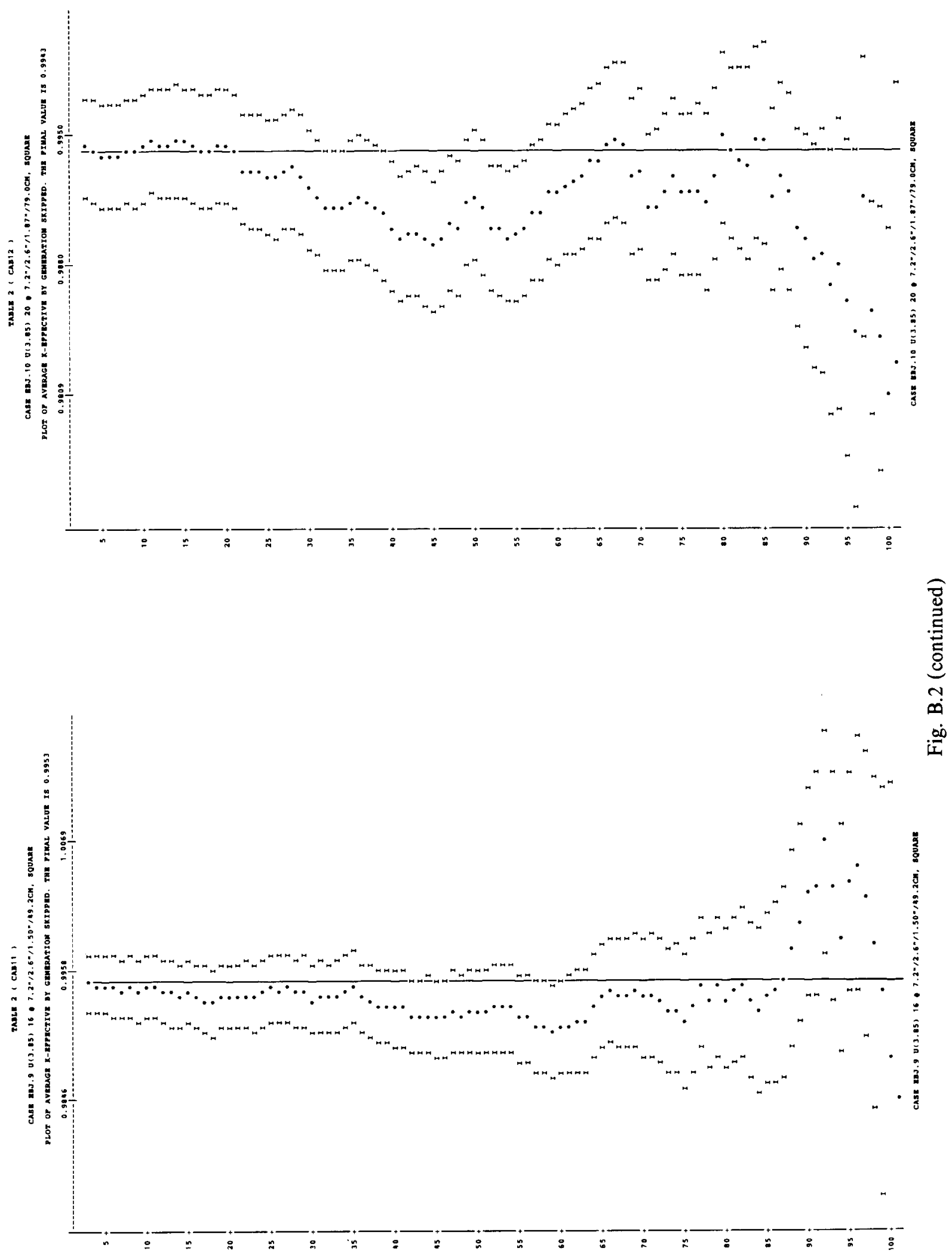

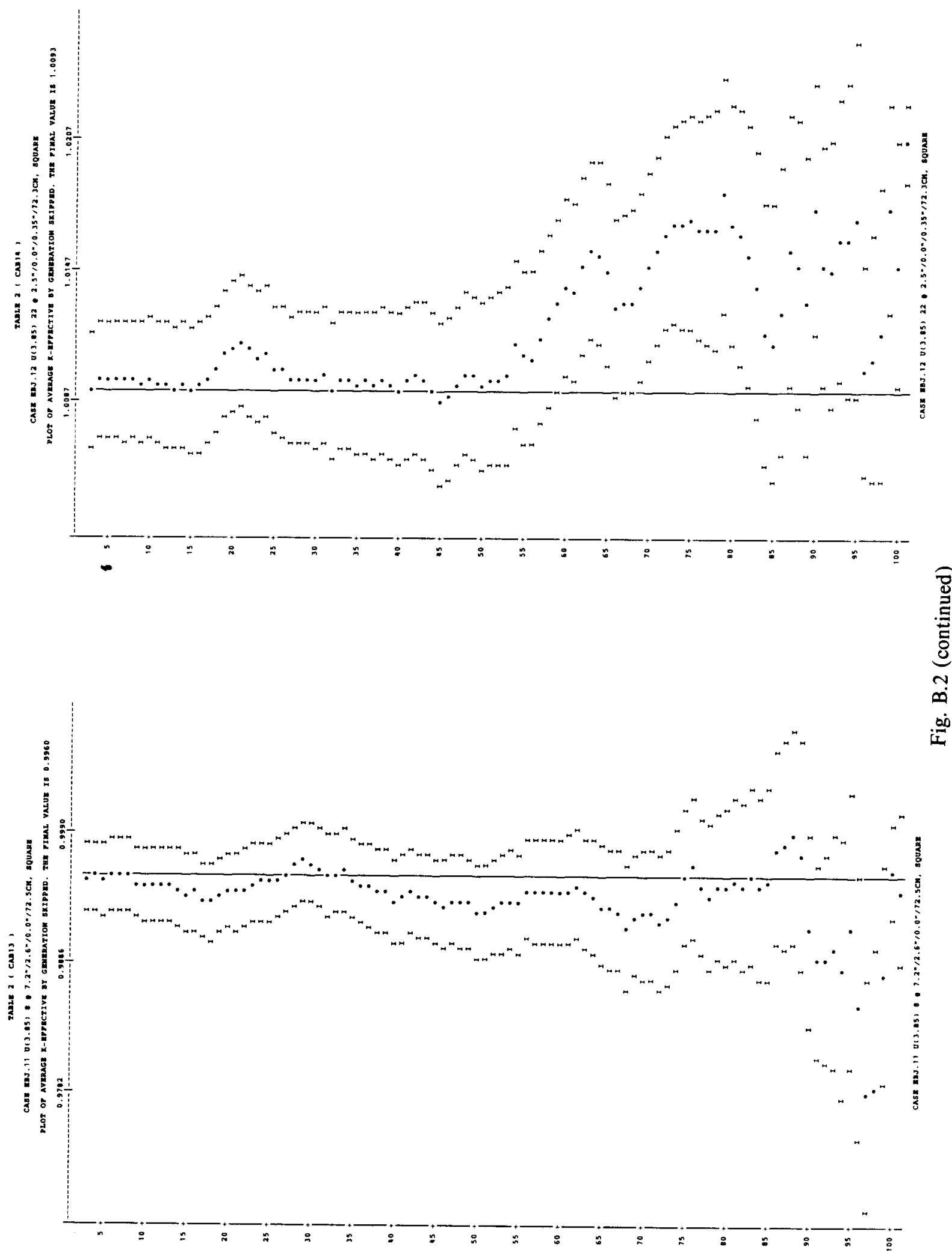


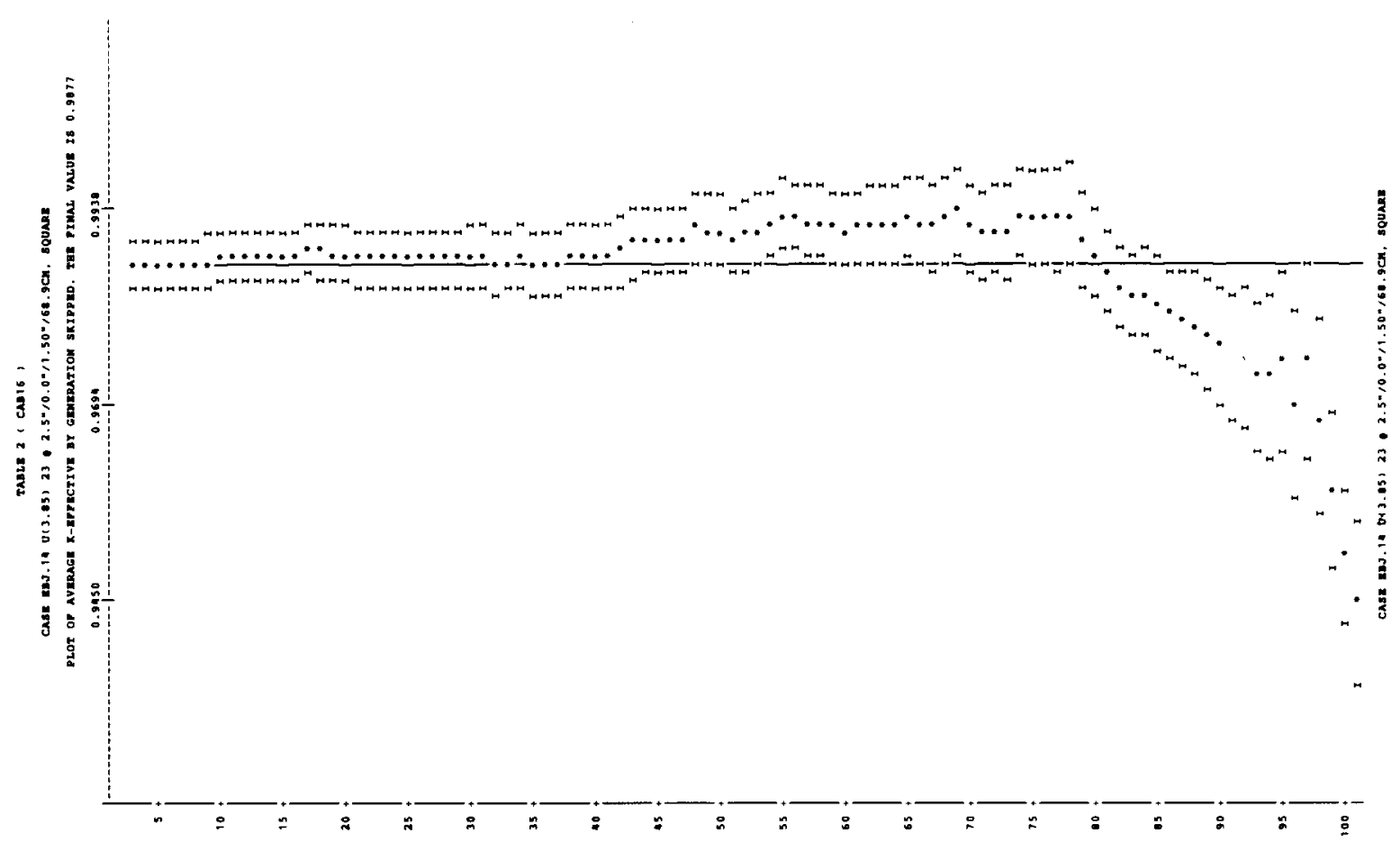

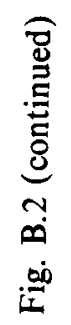

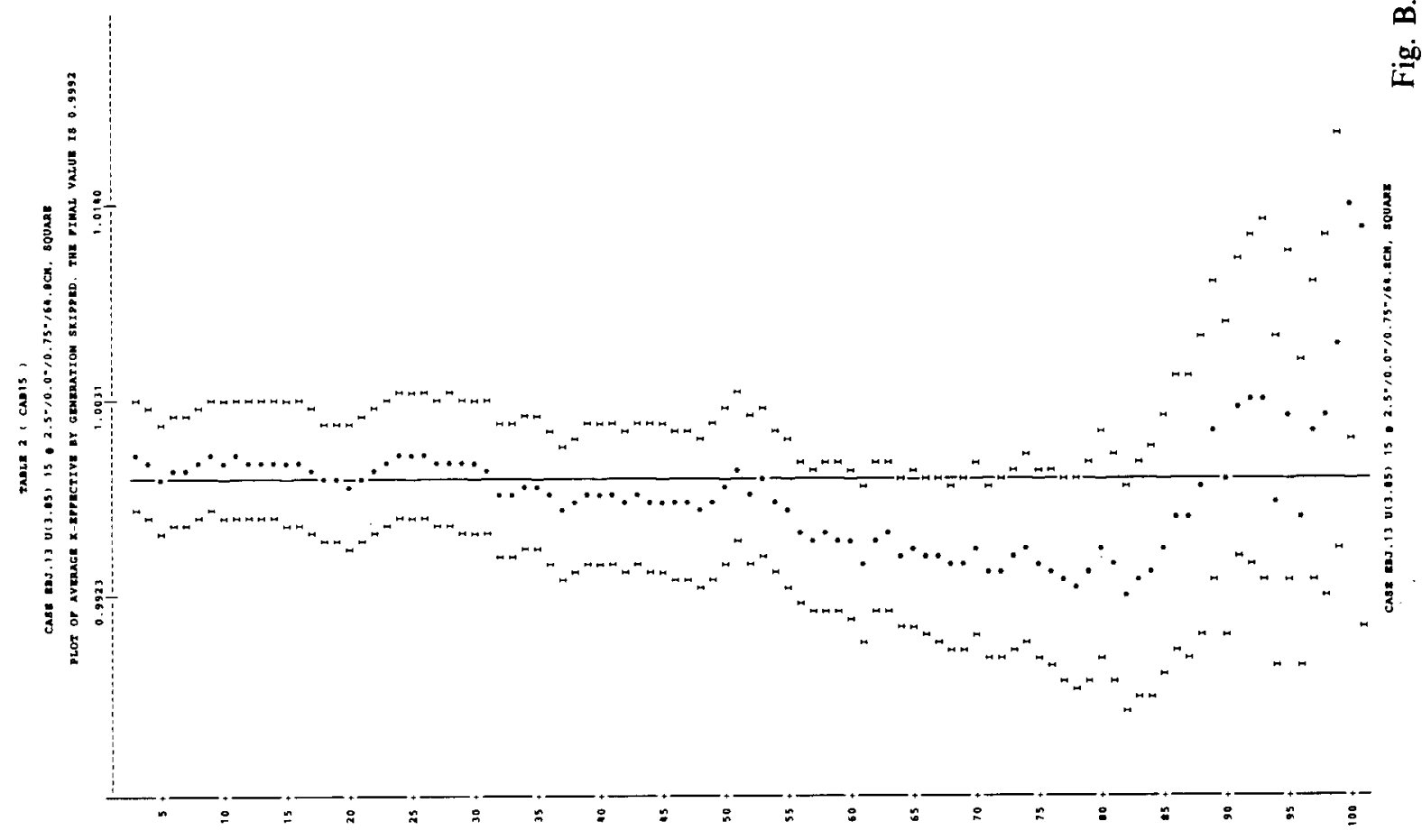


B-28

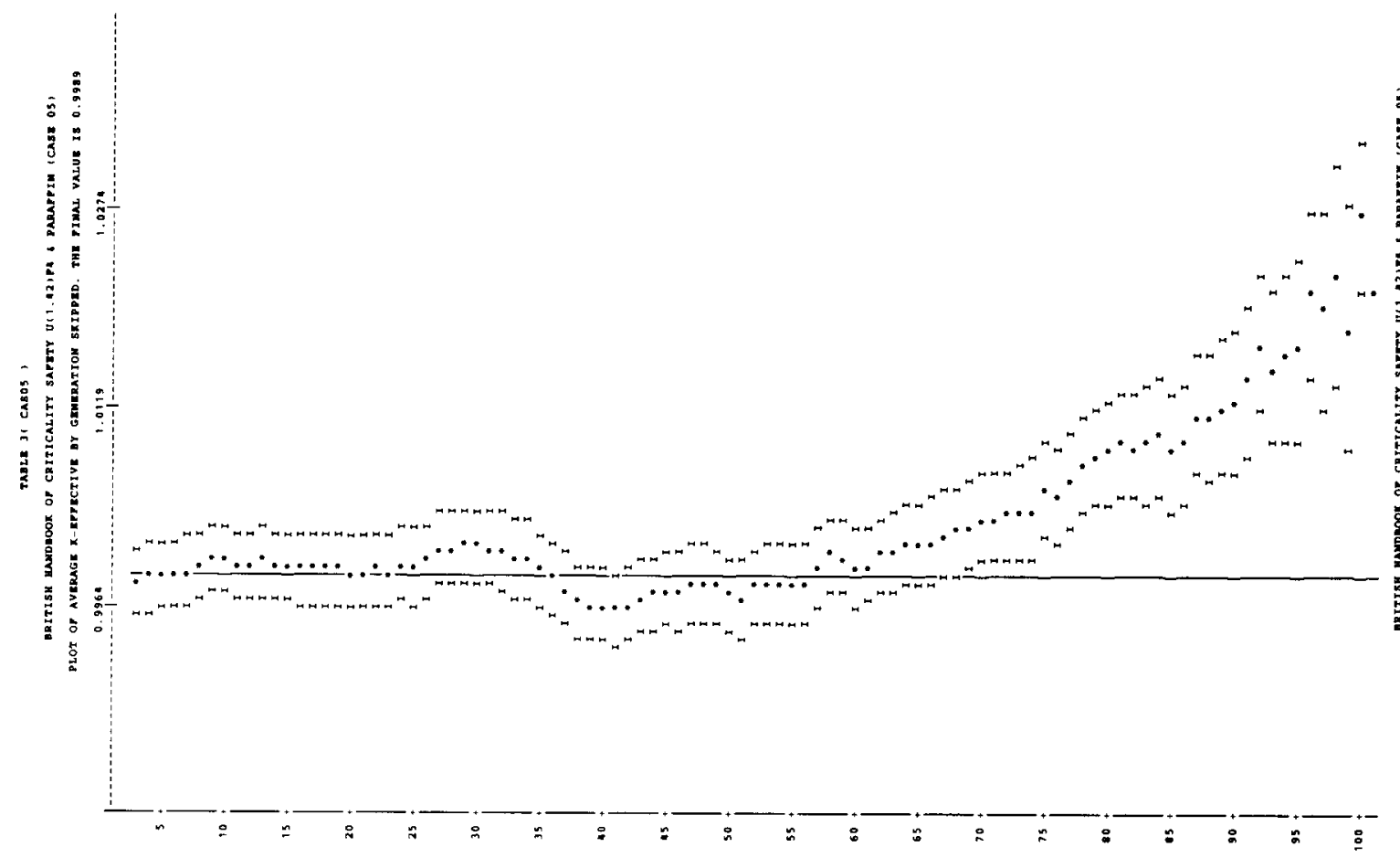

$m$
$\frac{0}{0}$
$\stackrel{\pi}{0}$
$\stackrel{0}{0}$
$\frac{0}{0}$

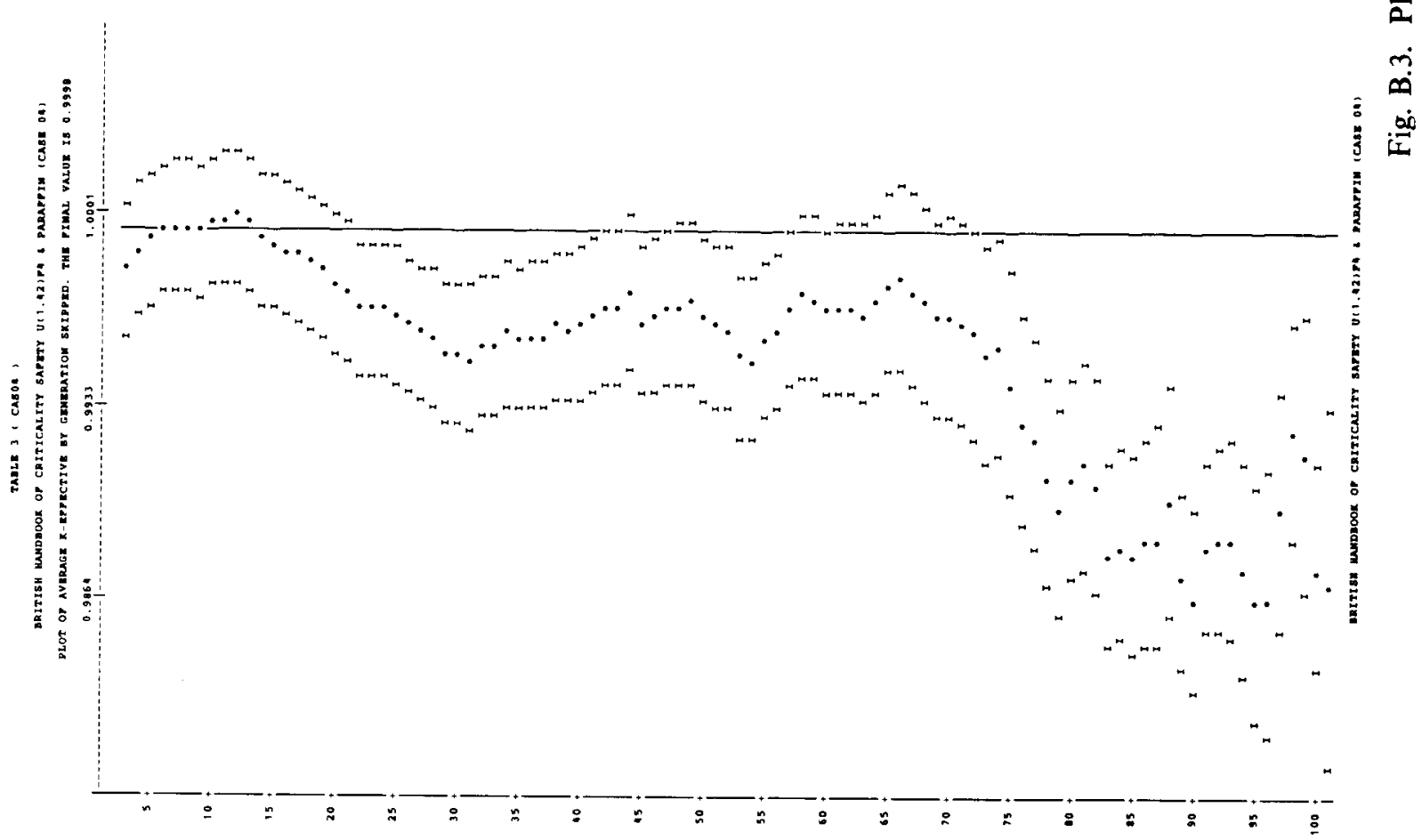



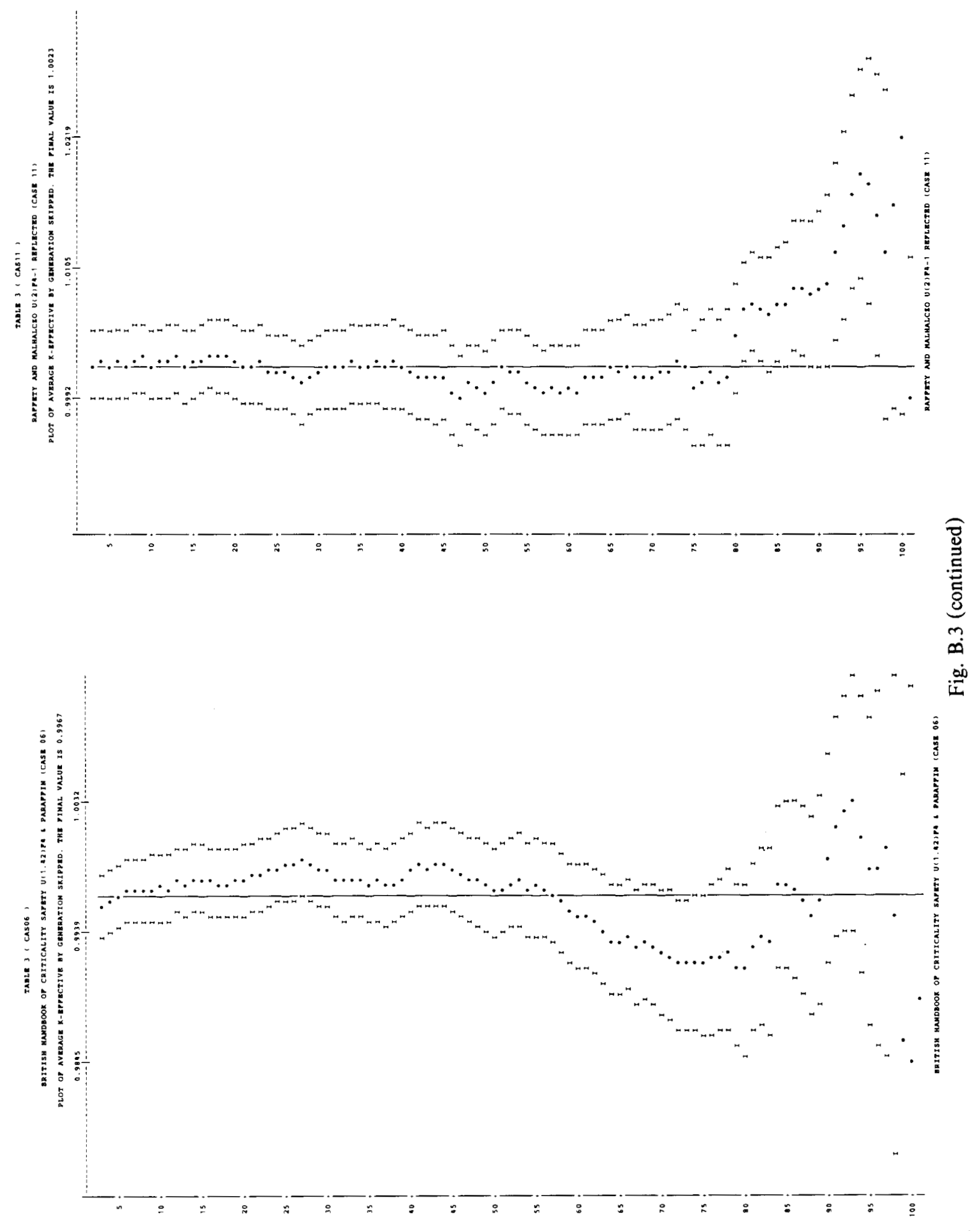

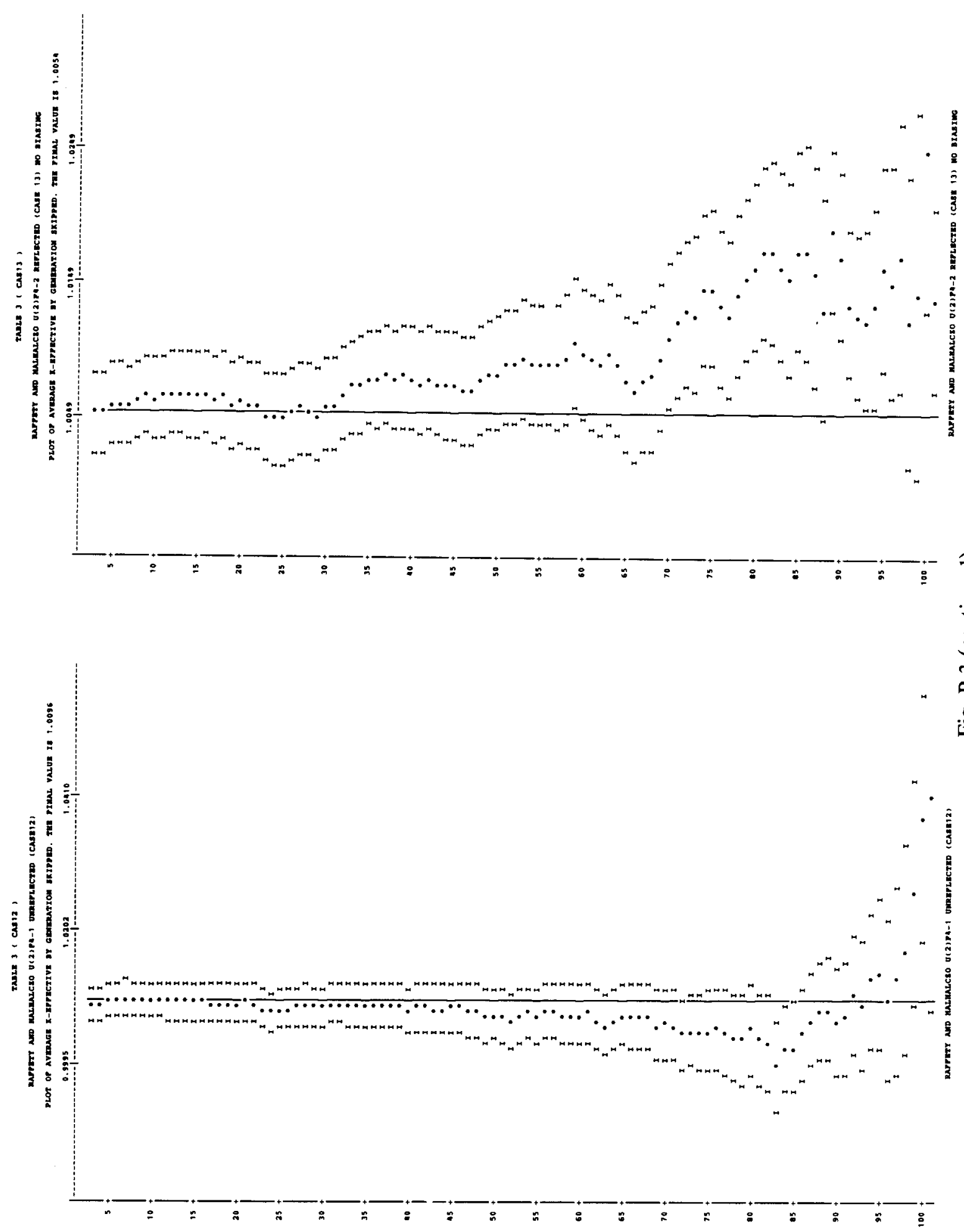
B-31

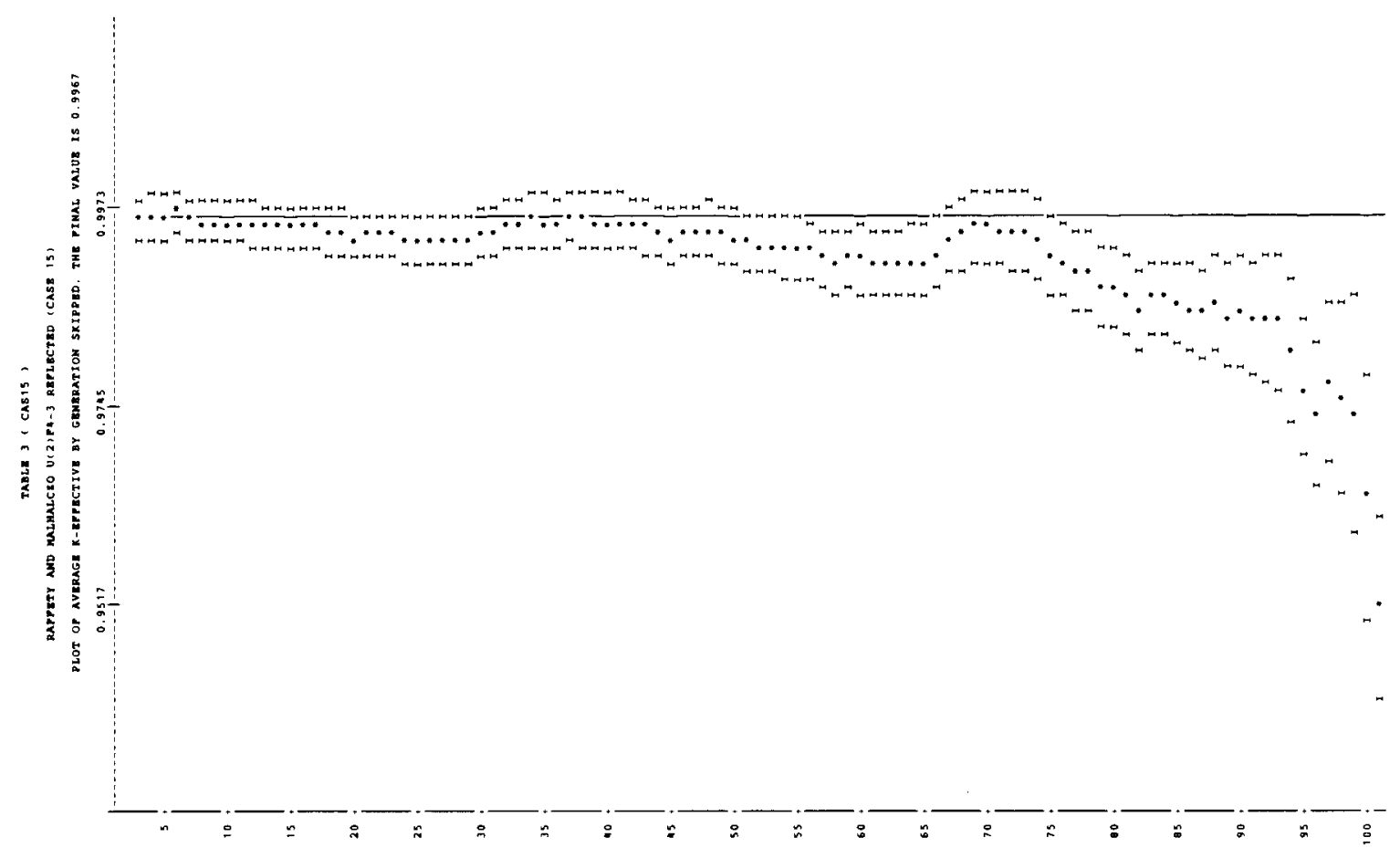

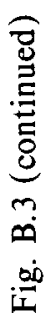

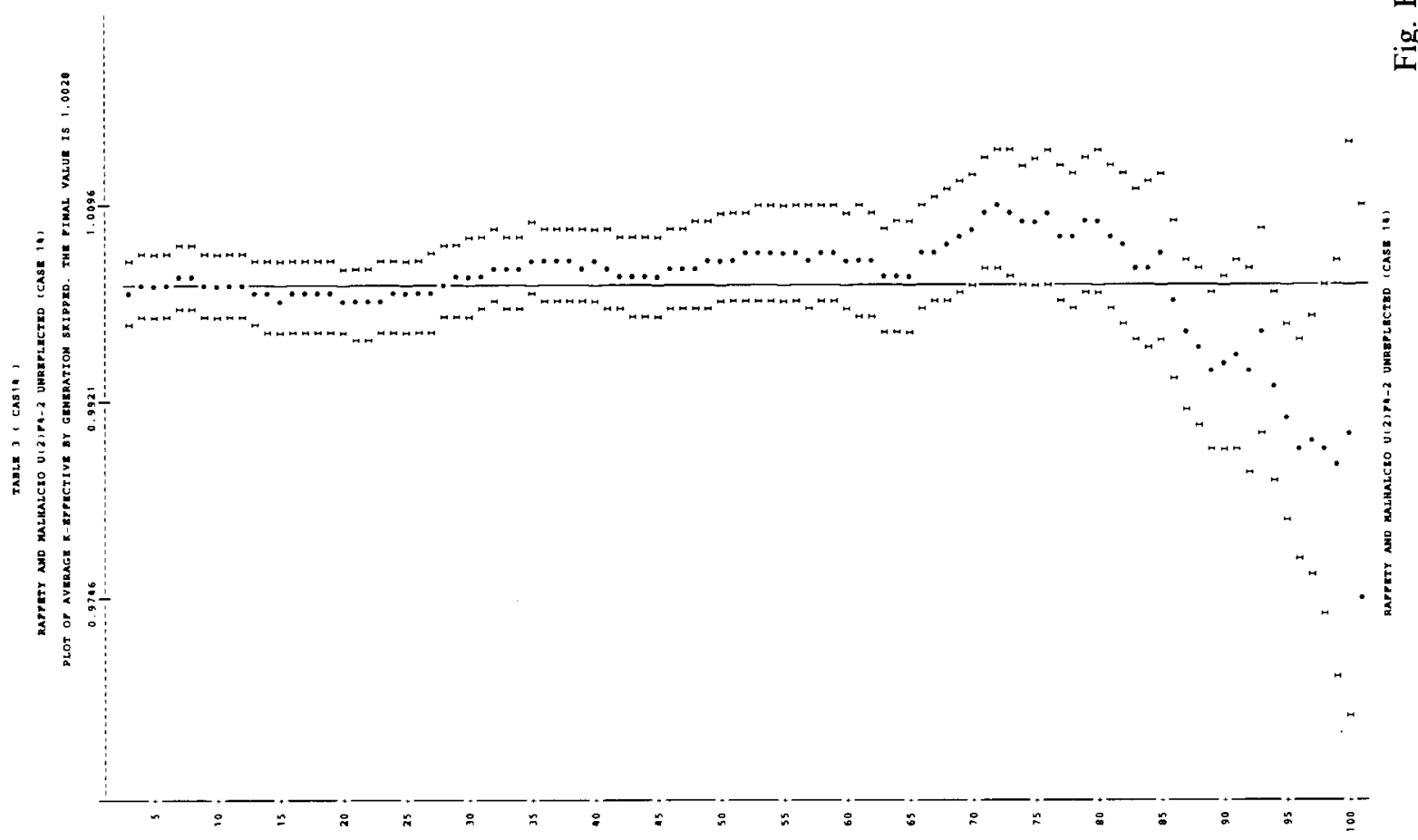



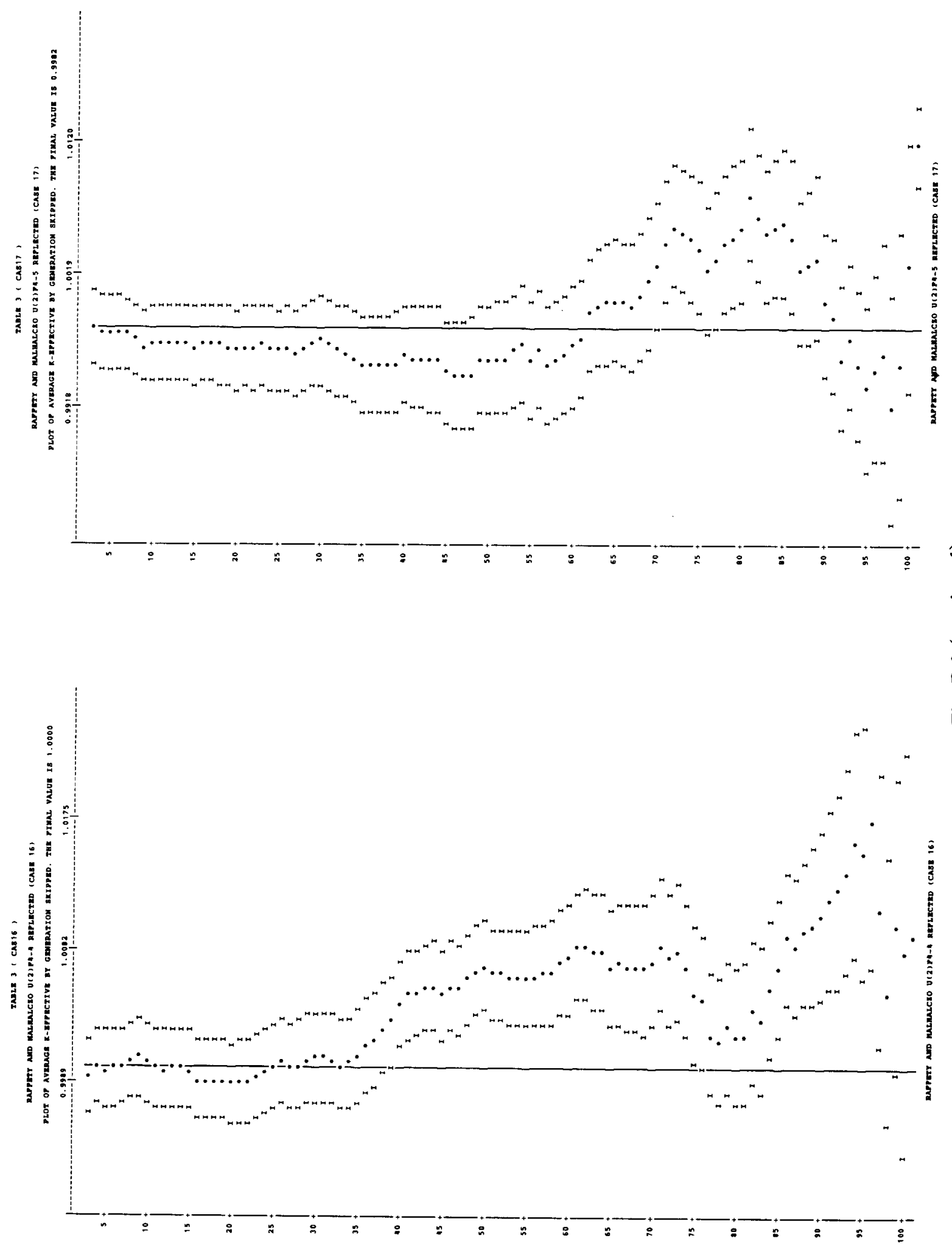

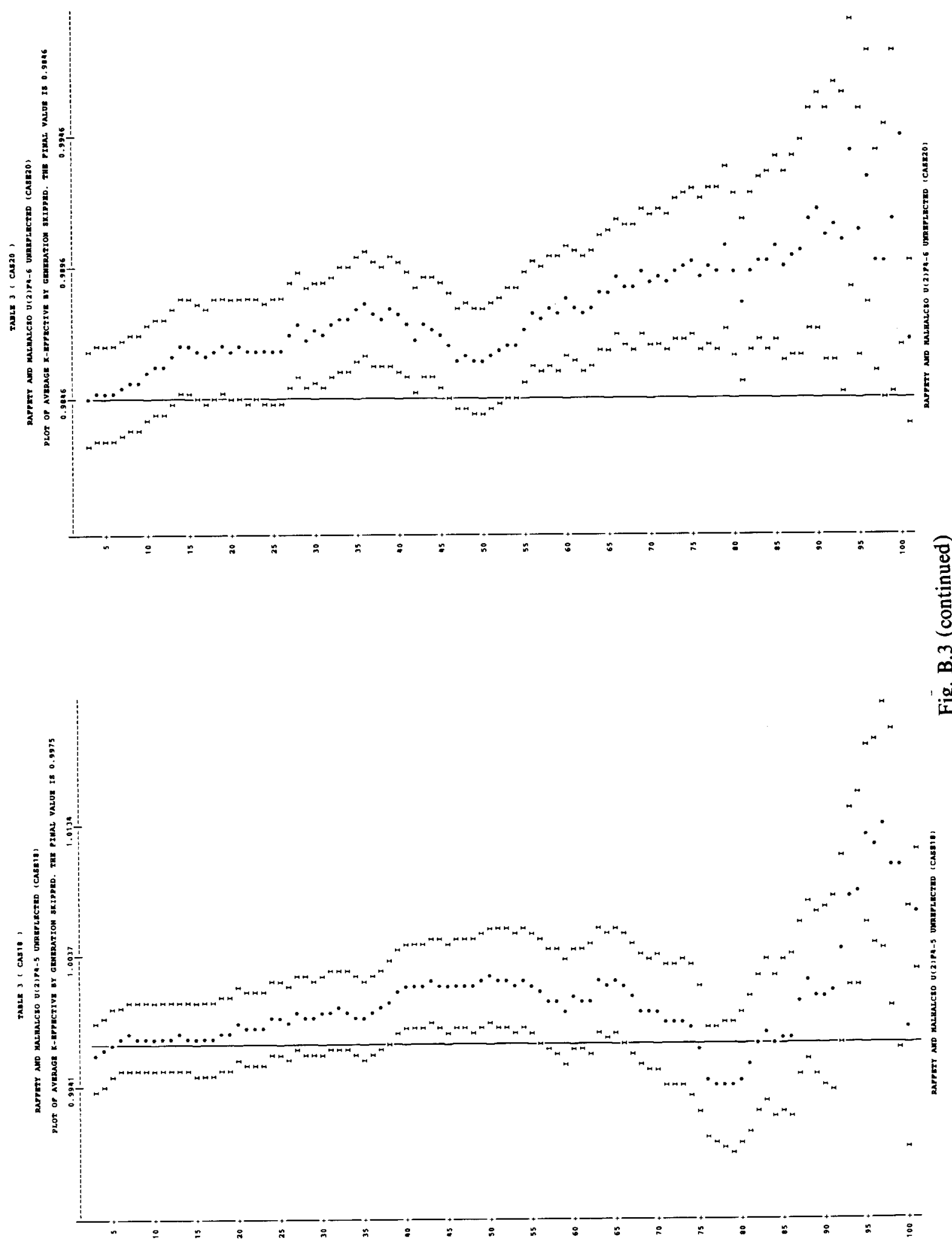


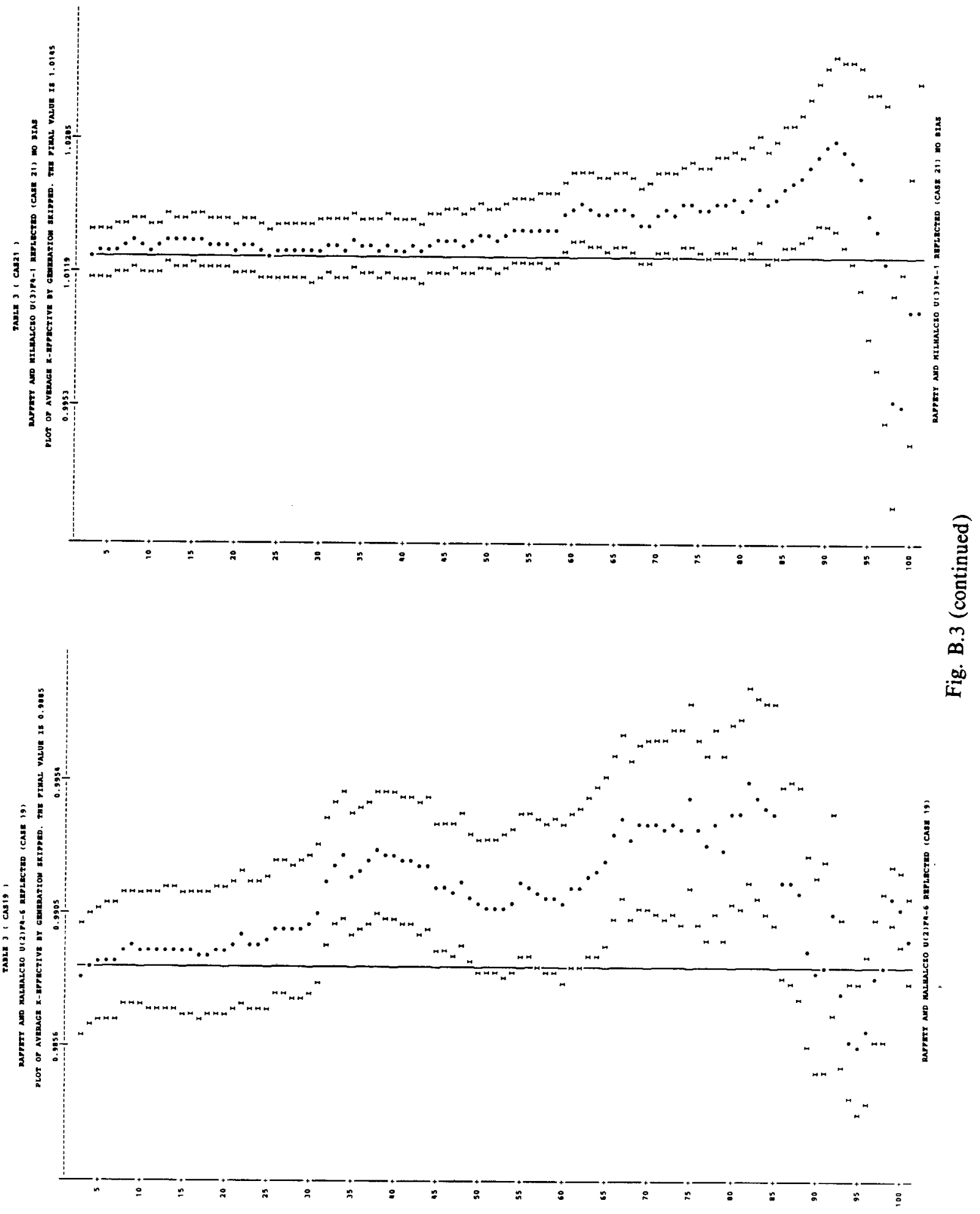


B-35

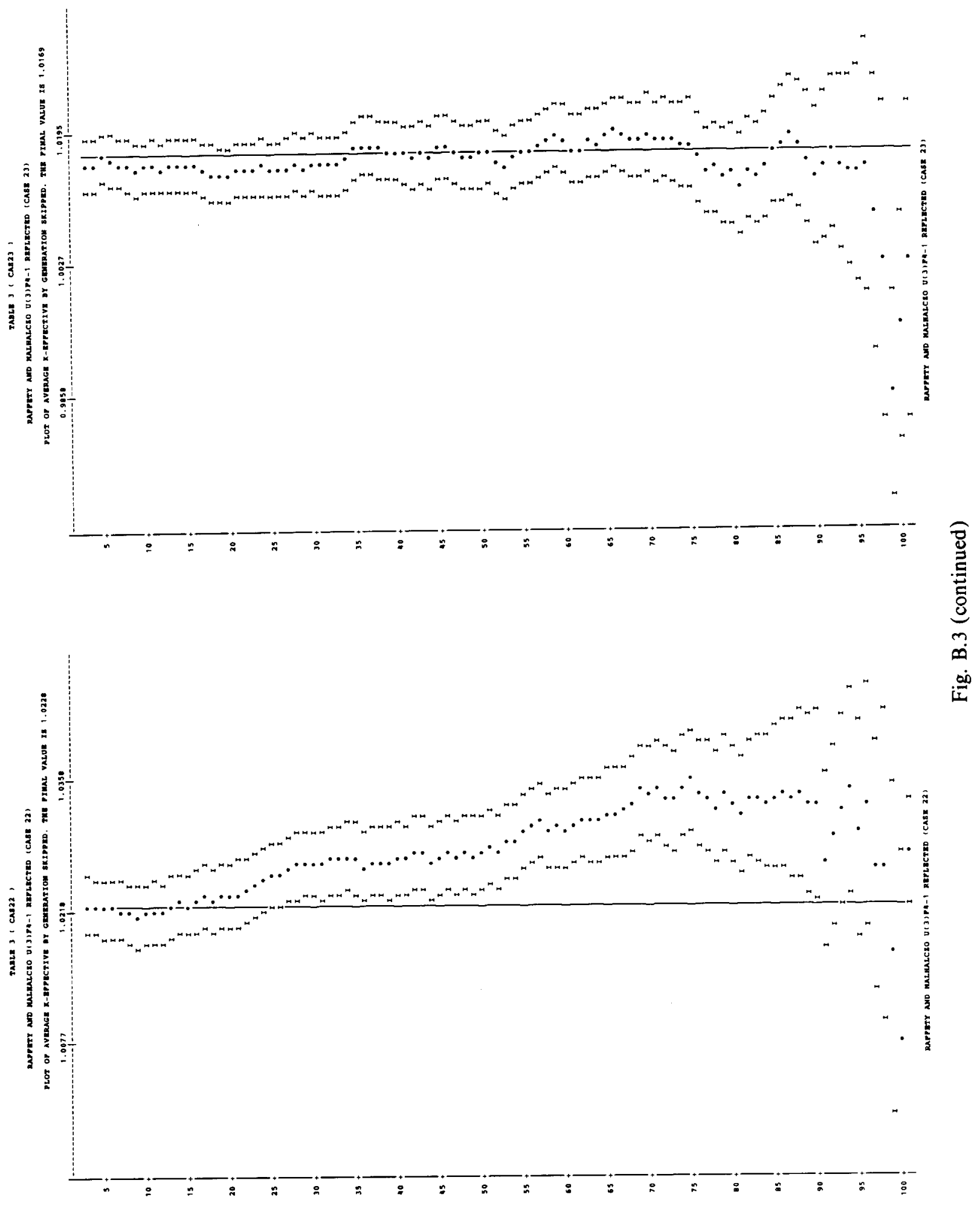



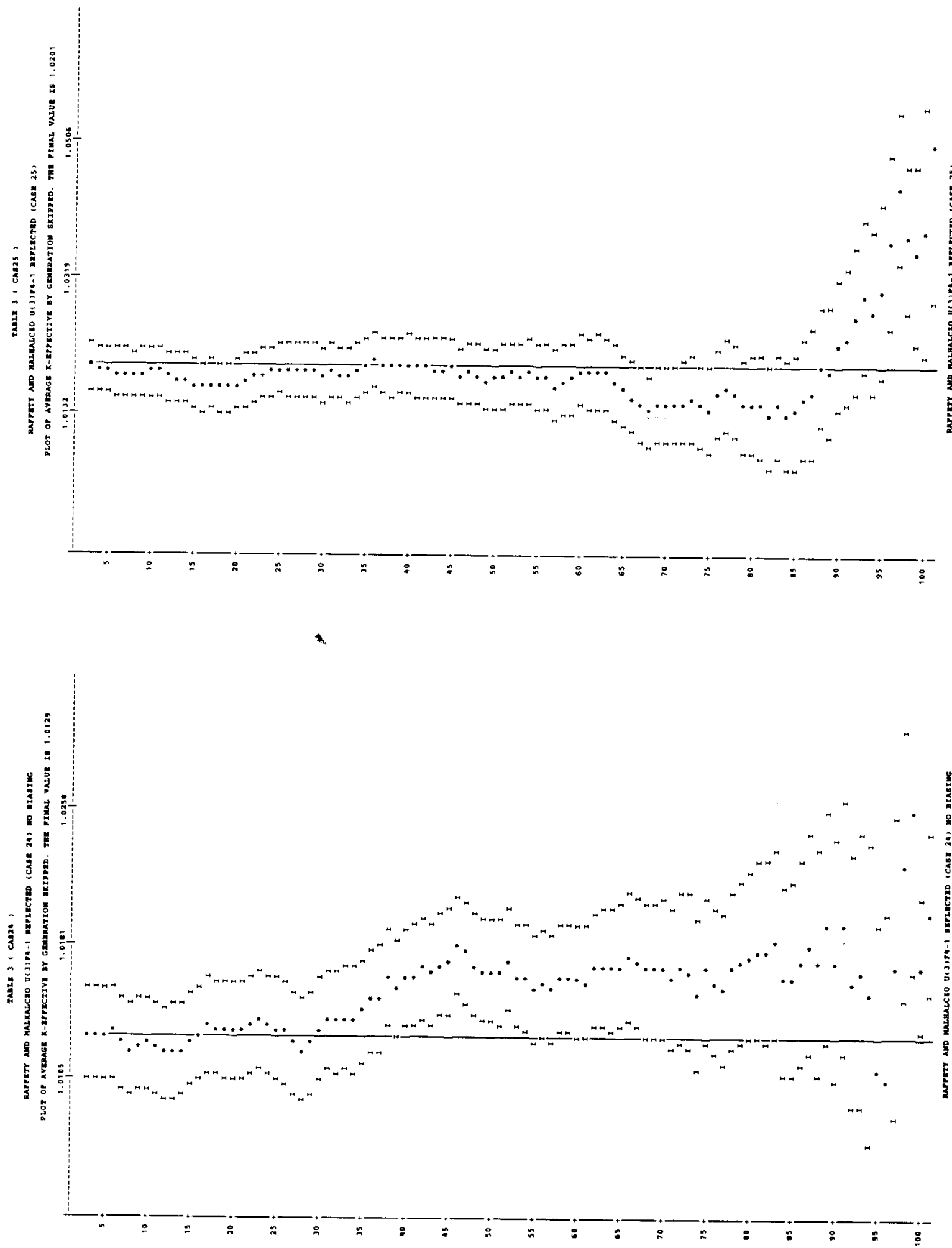


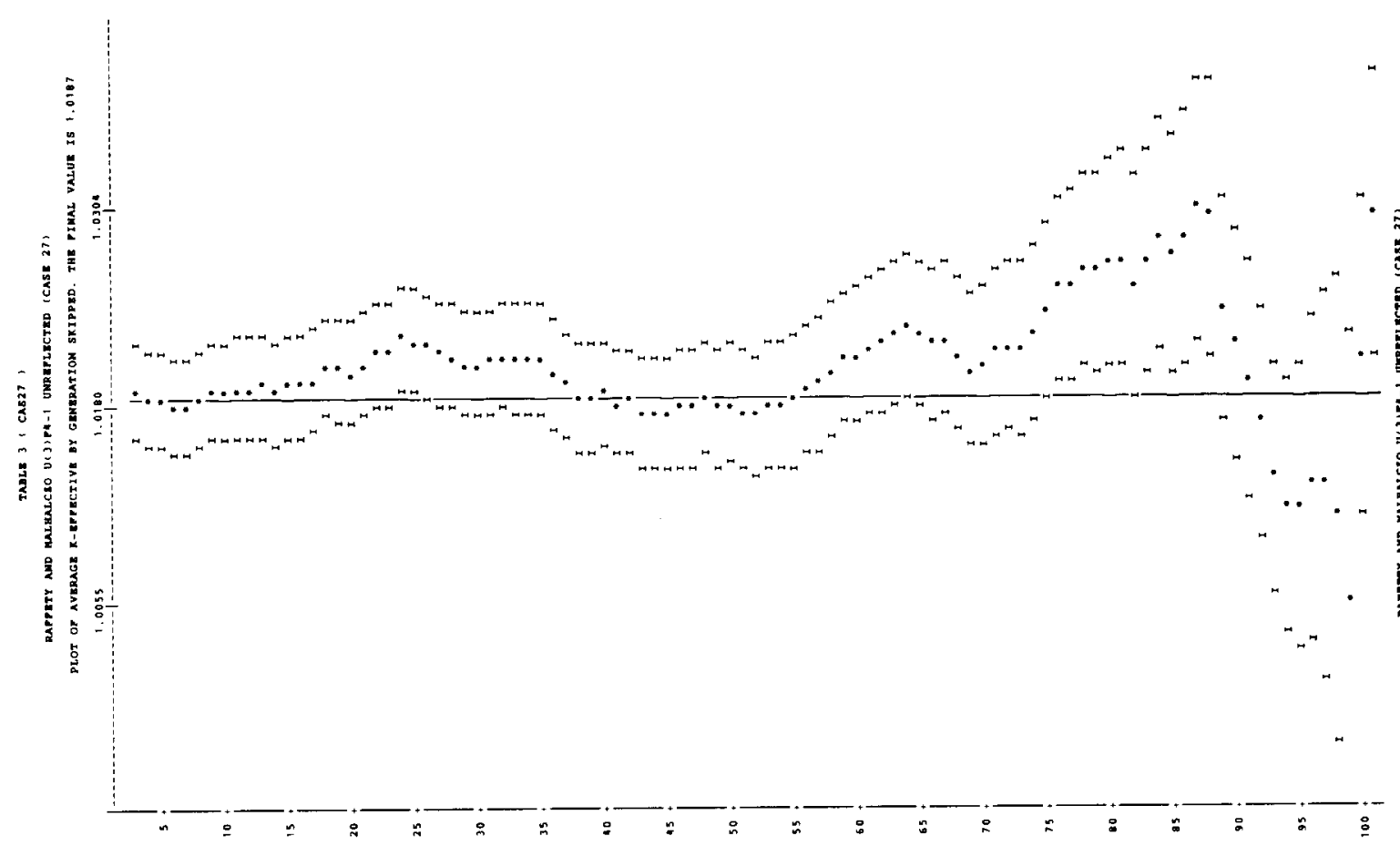

可

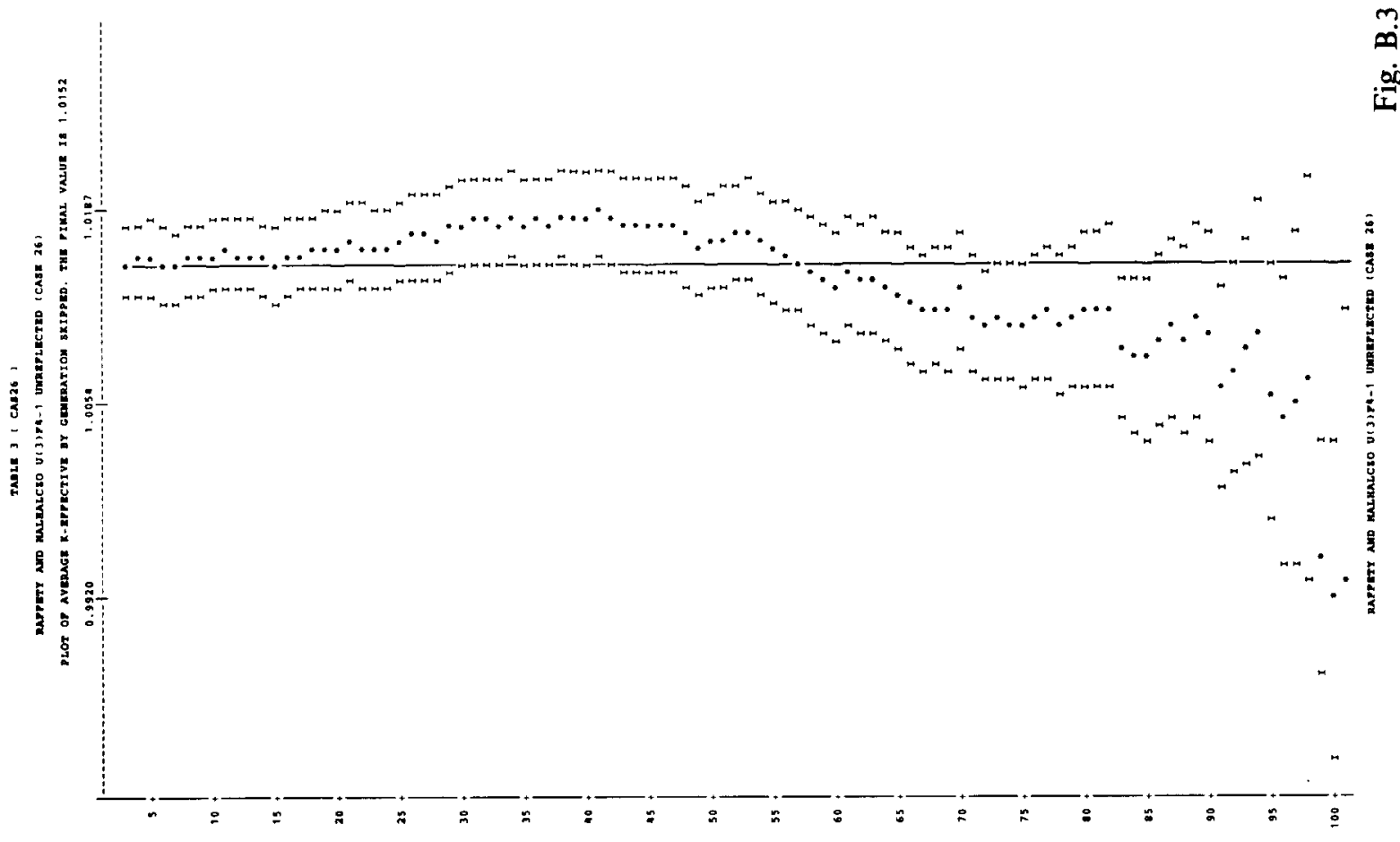



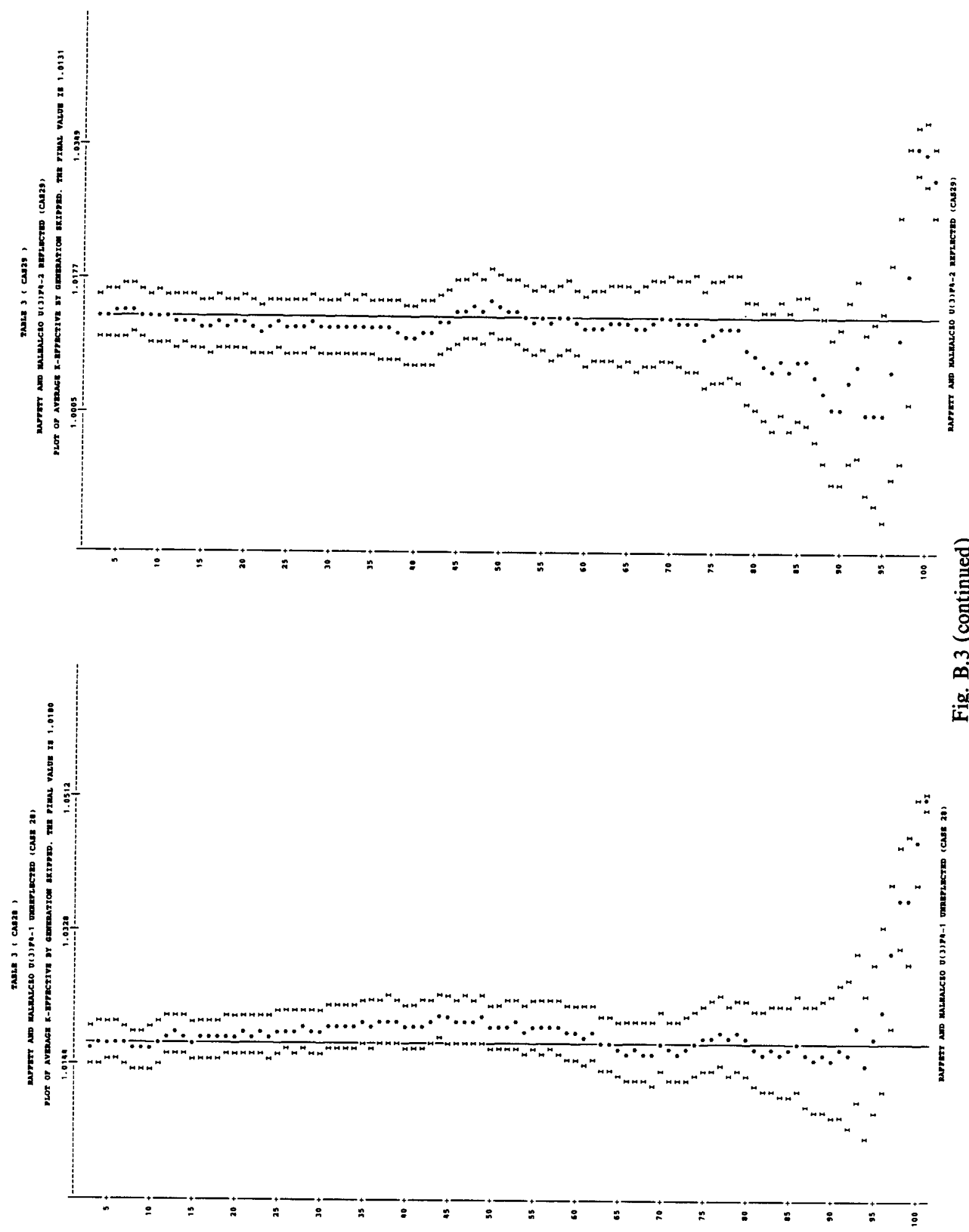


\section{B-39}
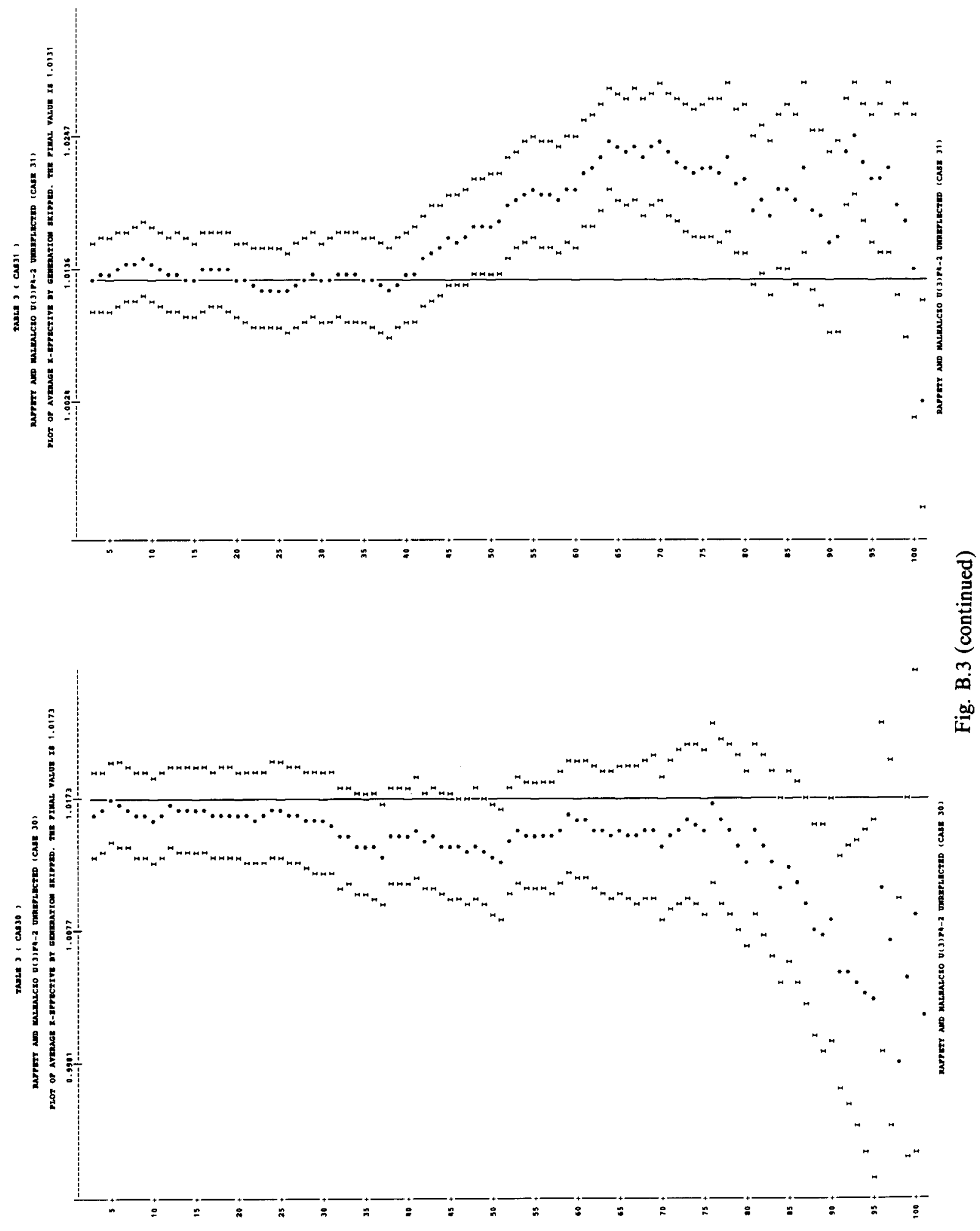

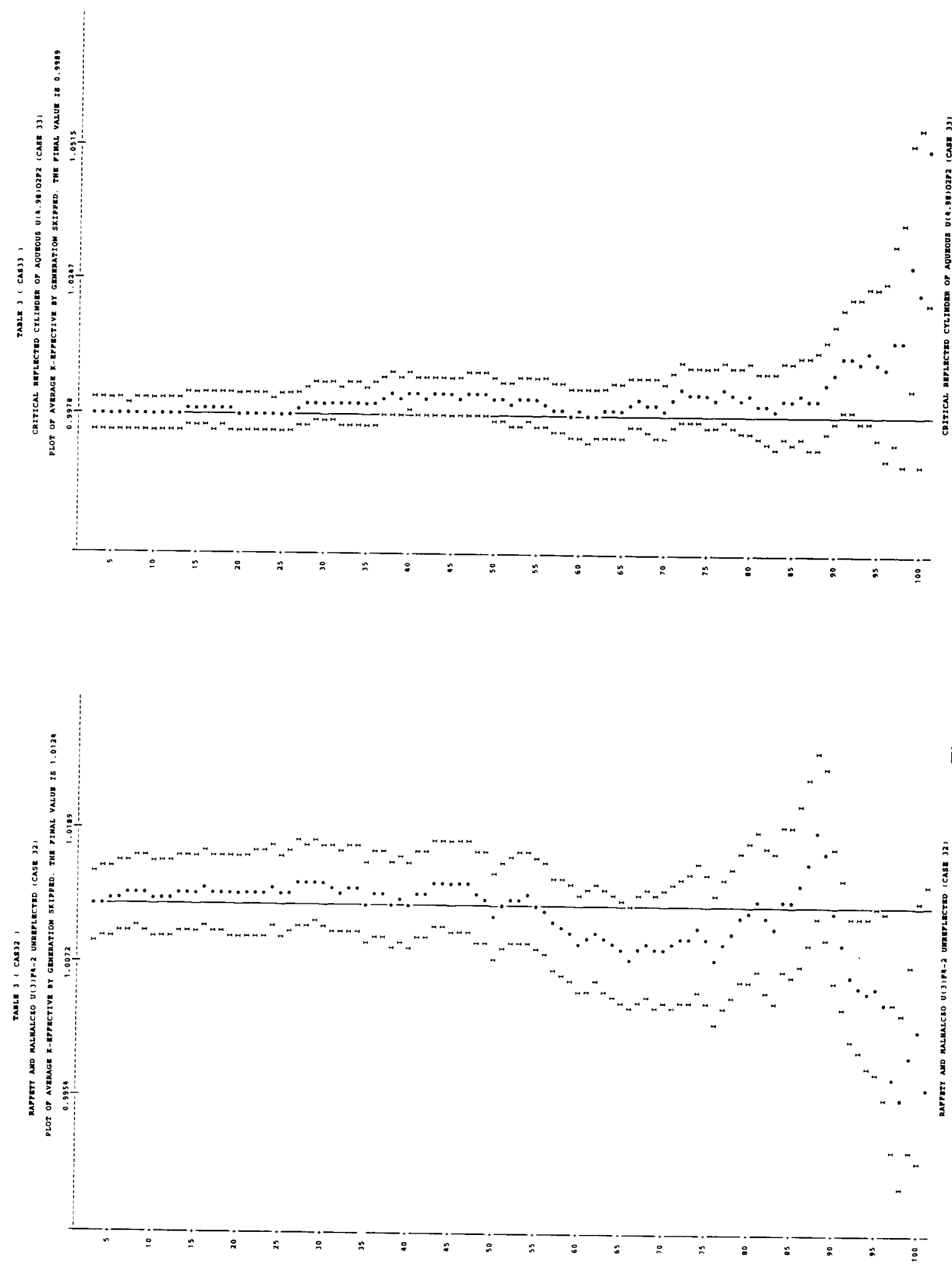


\section{B-41}
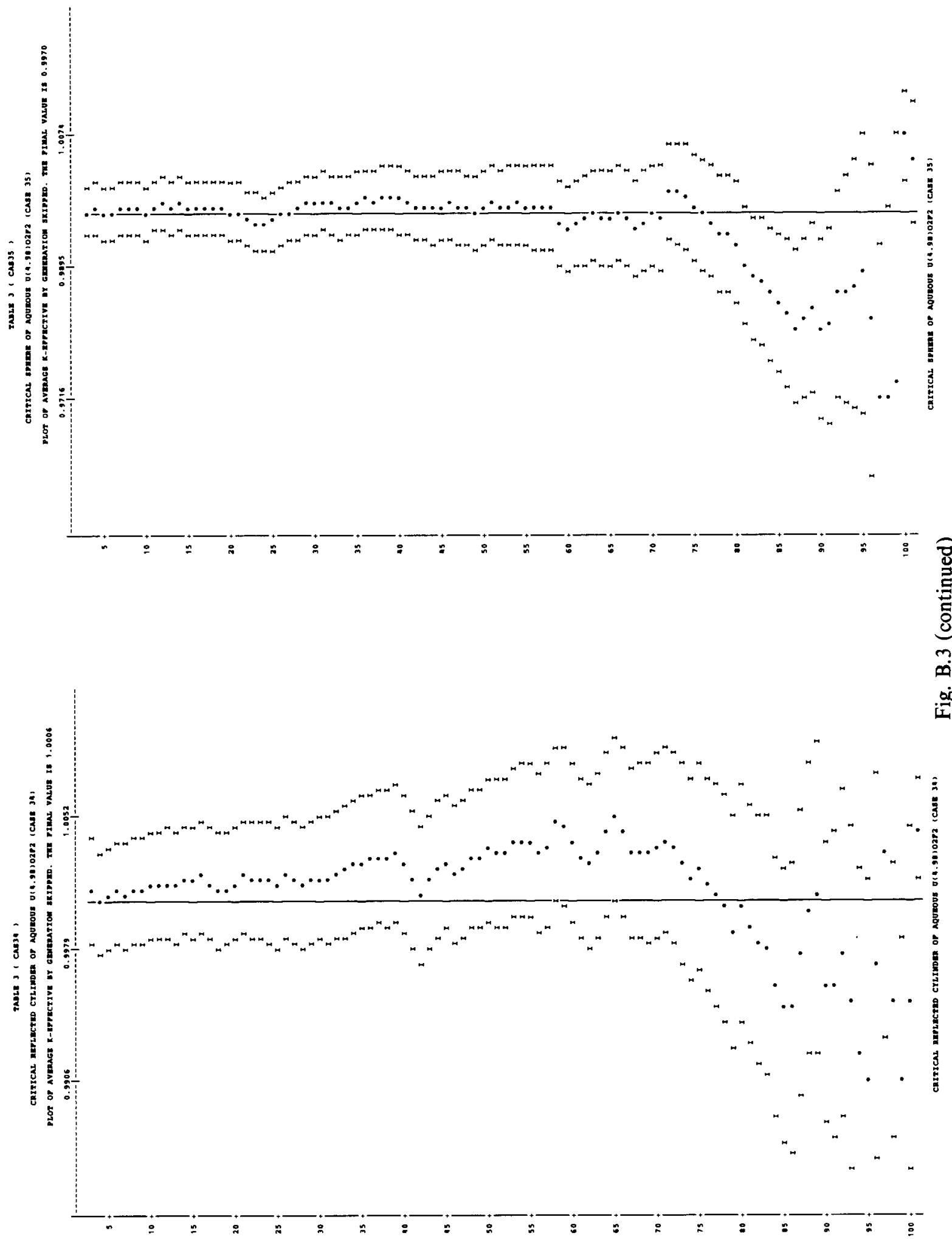


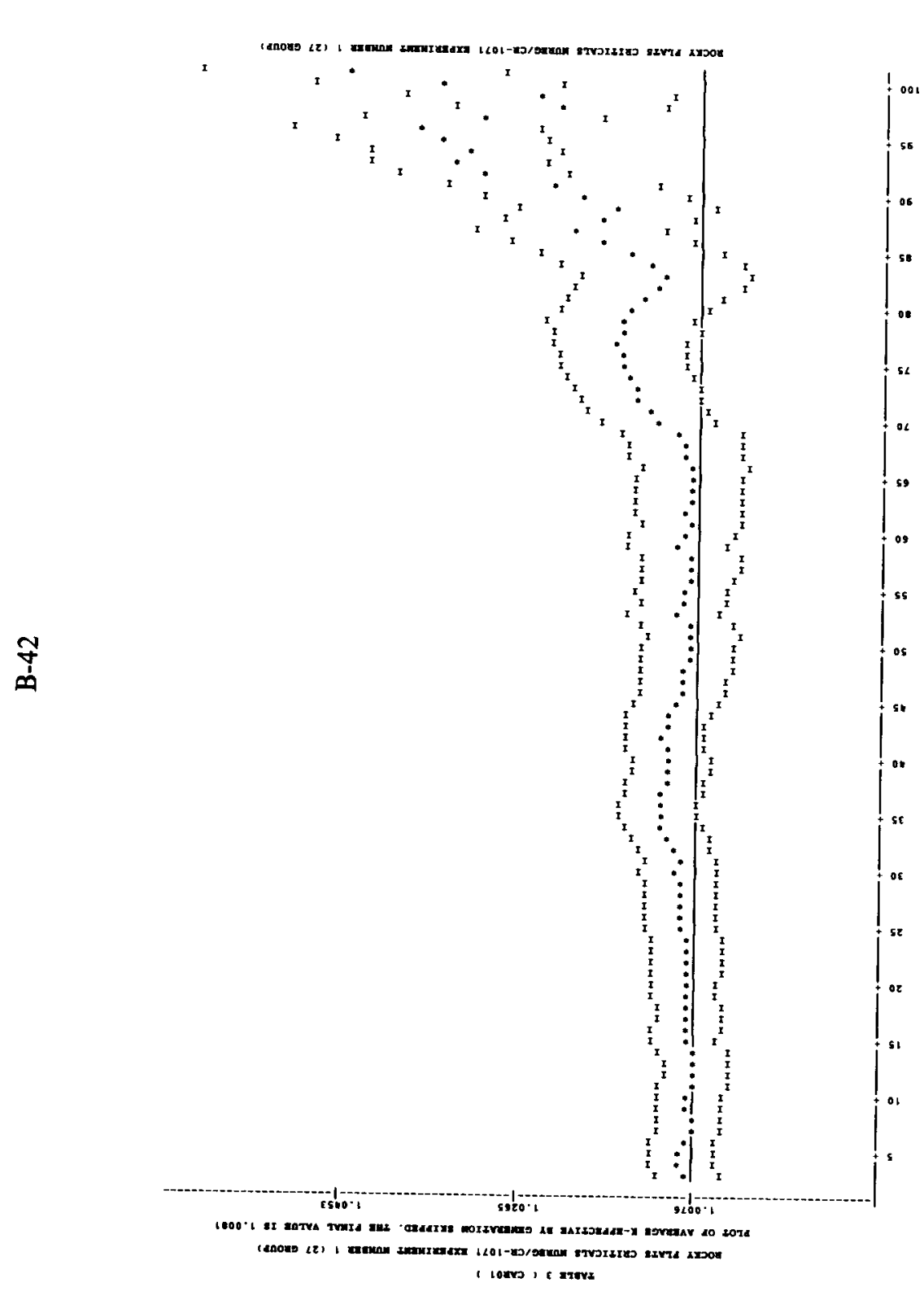

(panu!̣uos) $\mathcal{\varepsilon} \cdot \mathrm{g} \cdot 8$

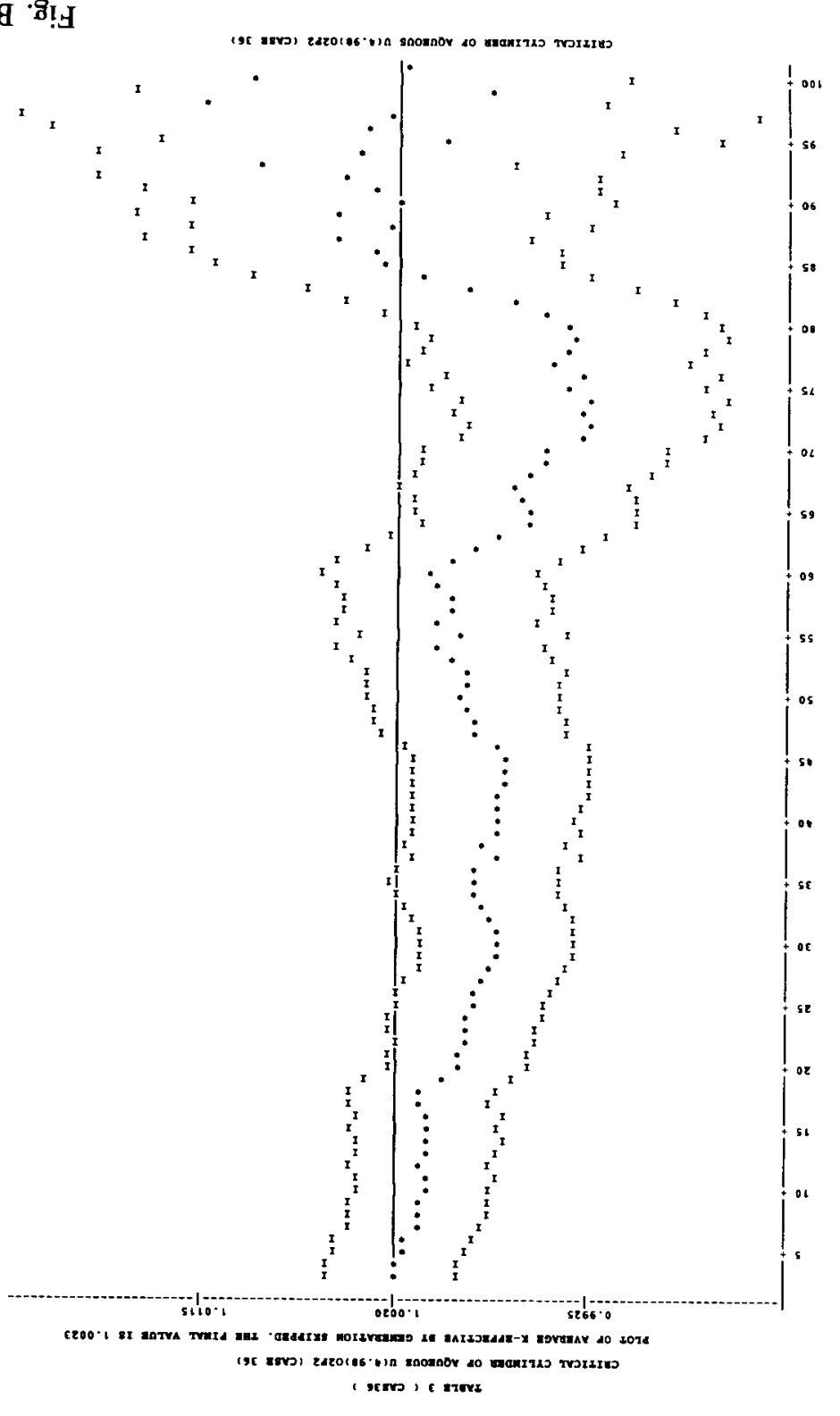



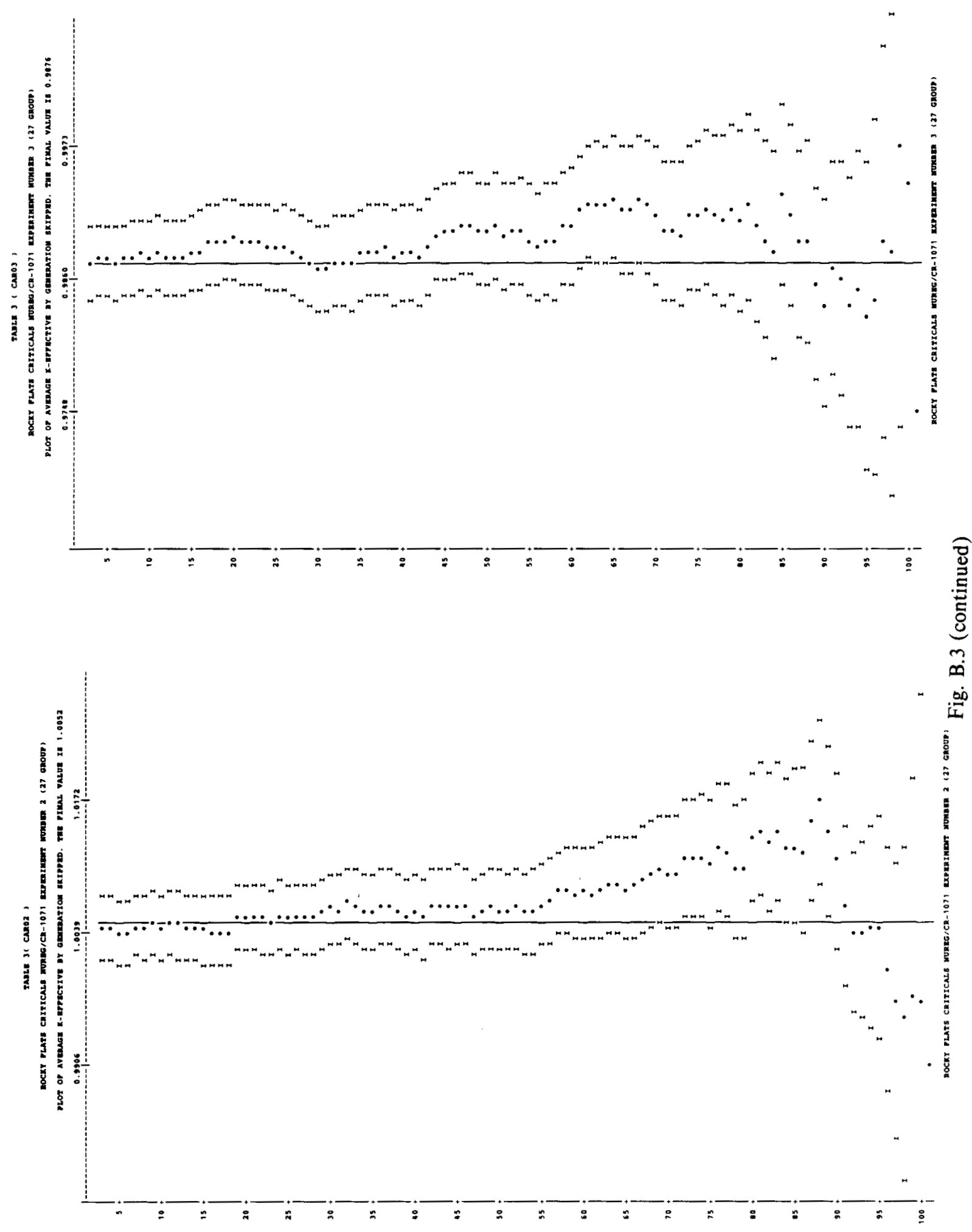


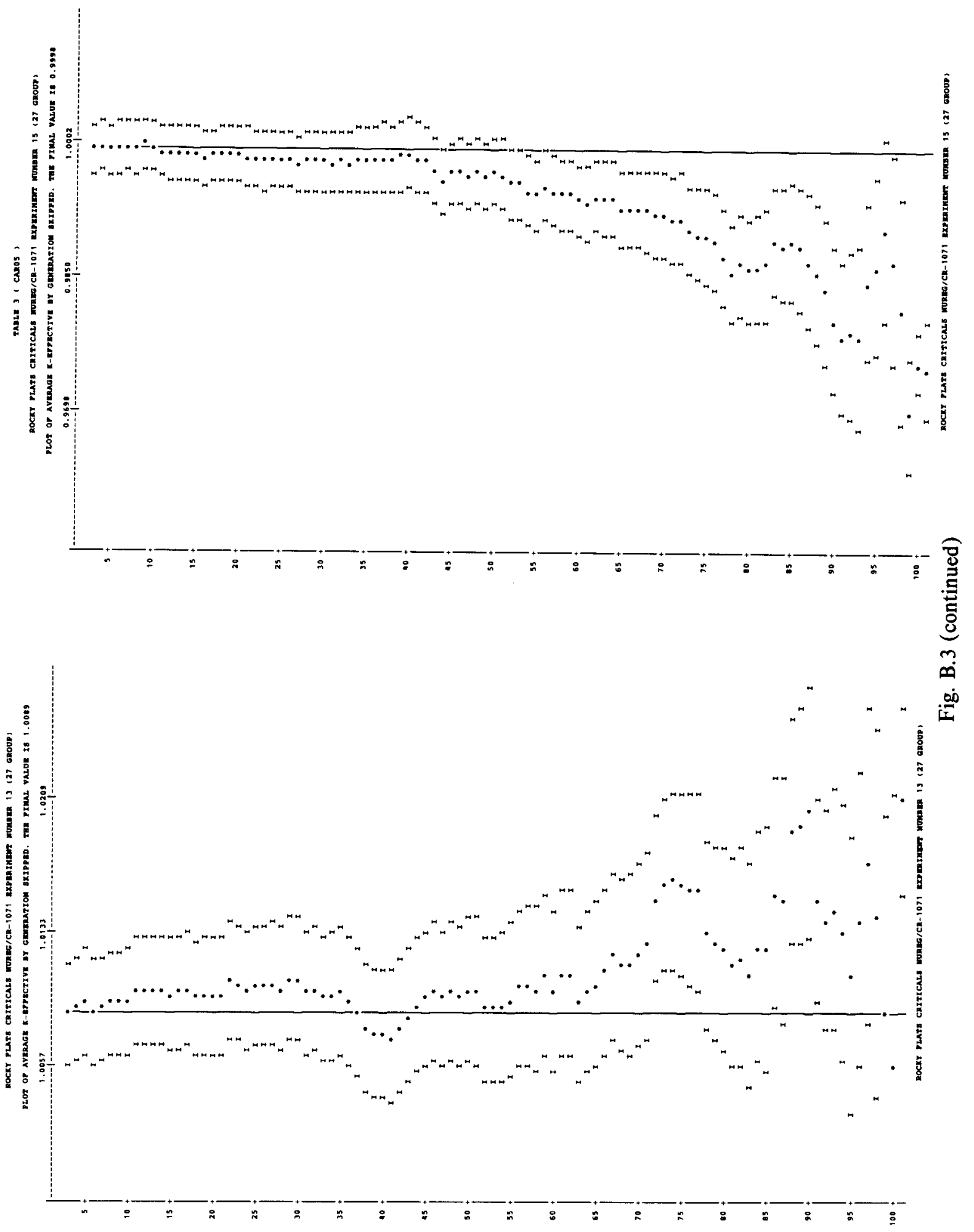



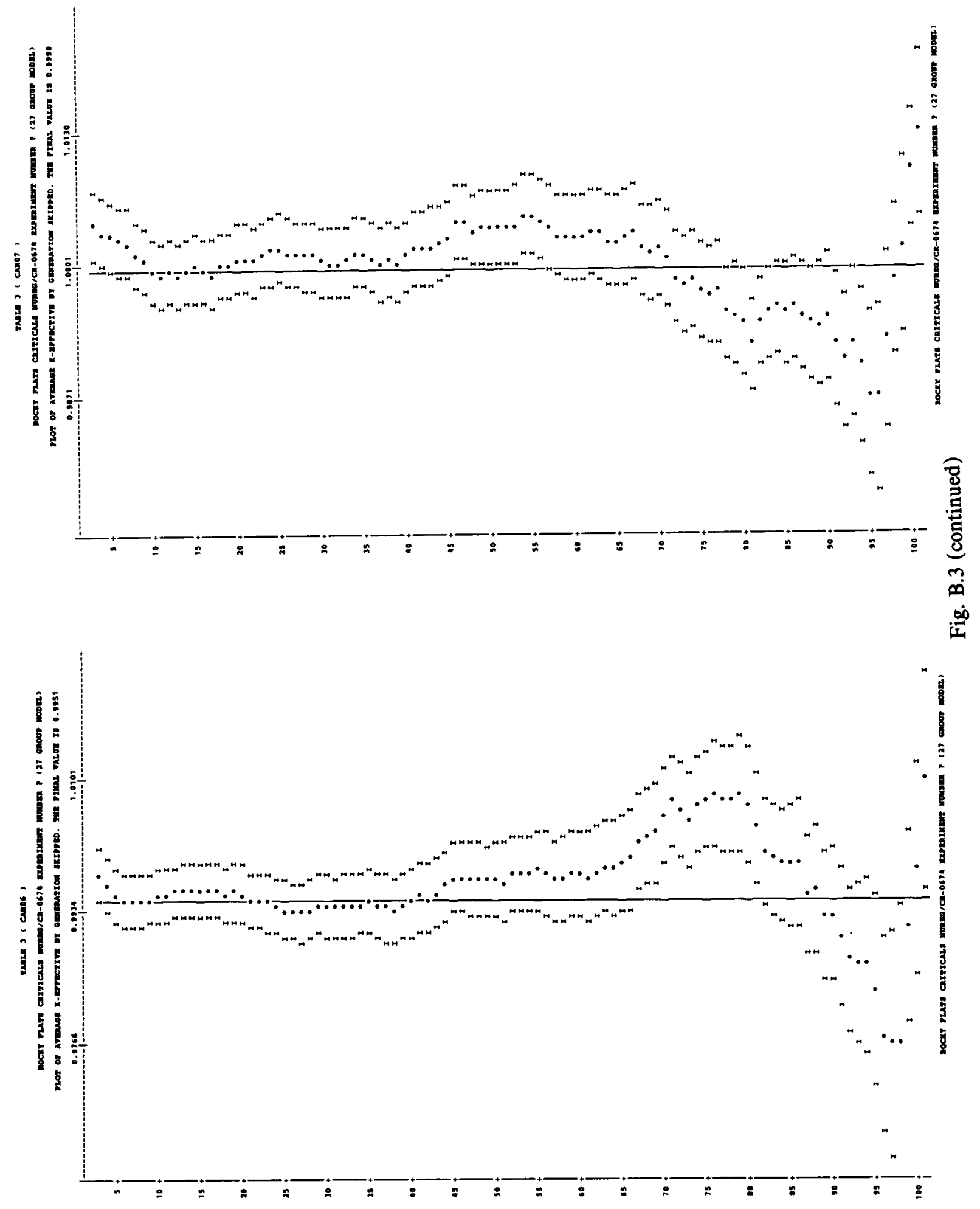


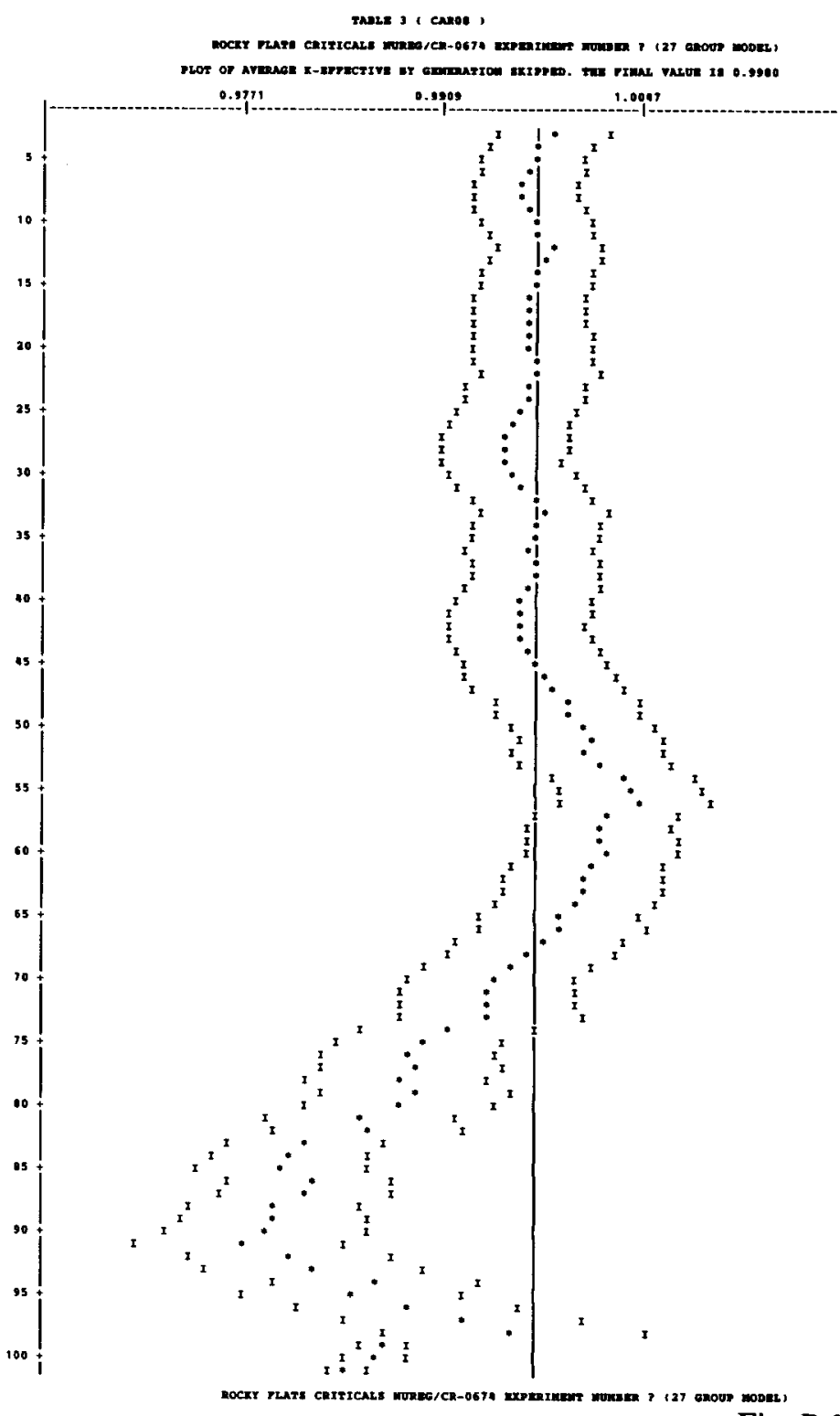

Fig. B.3 (continued)

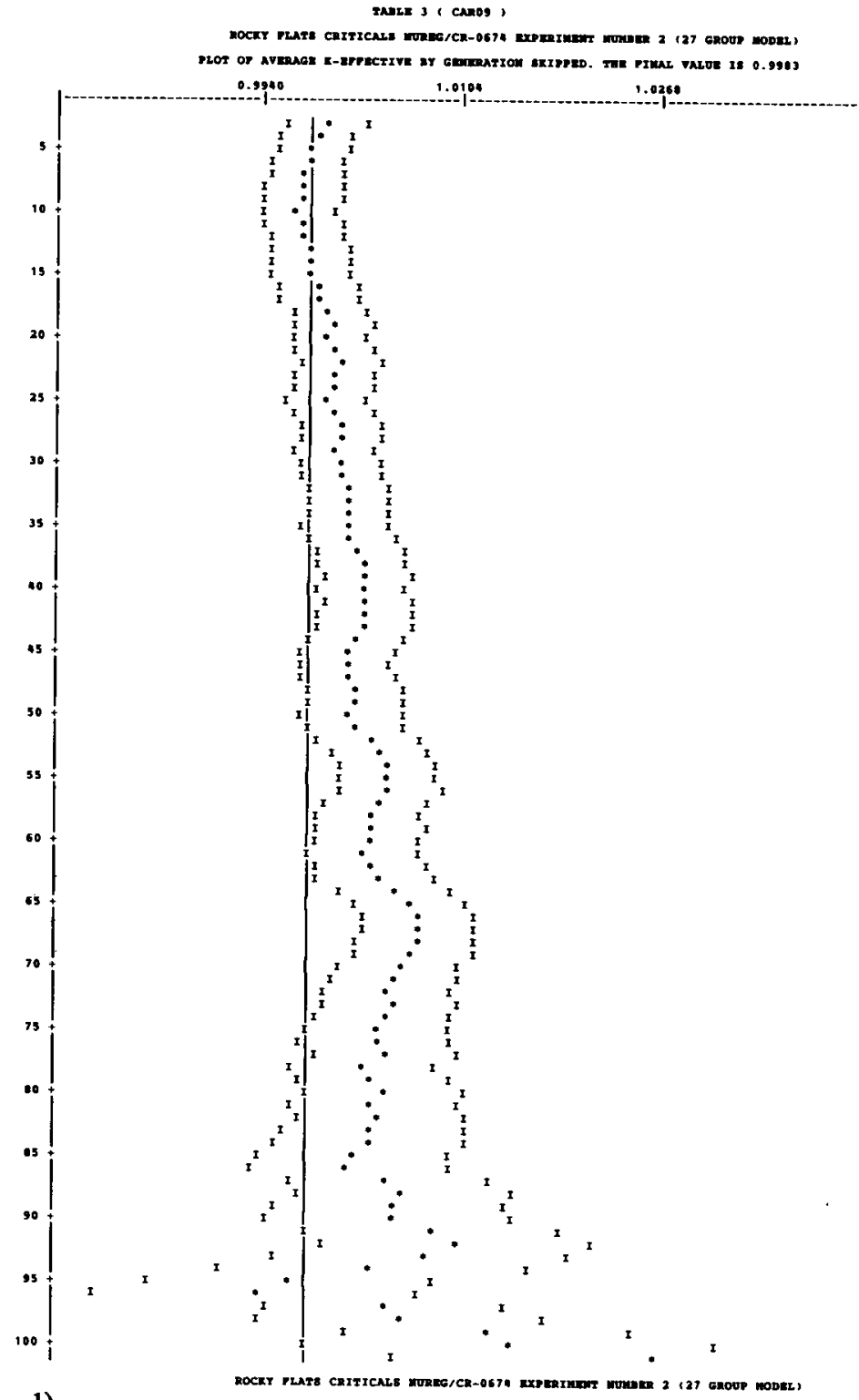




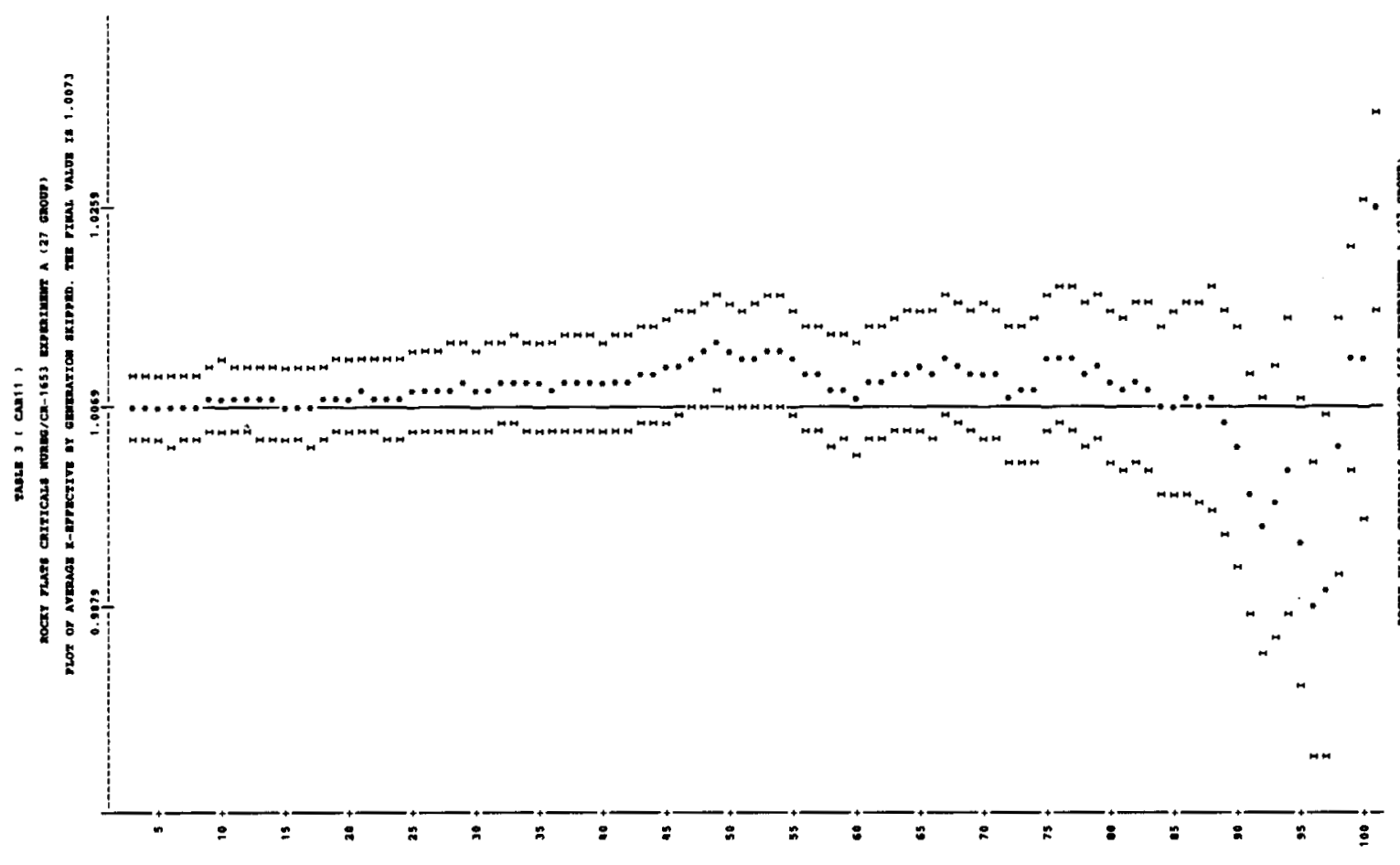

욜

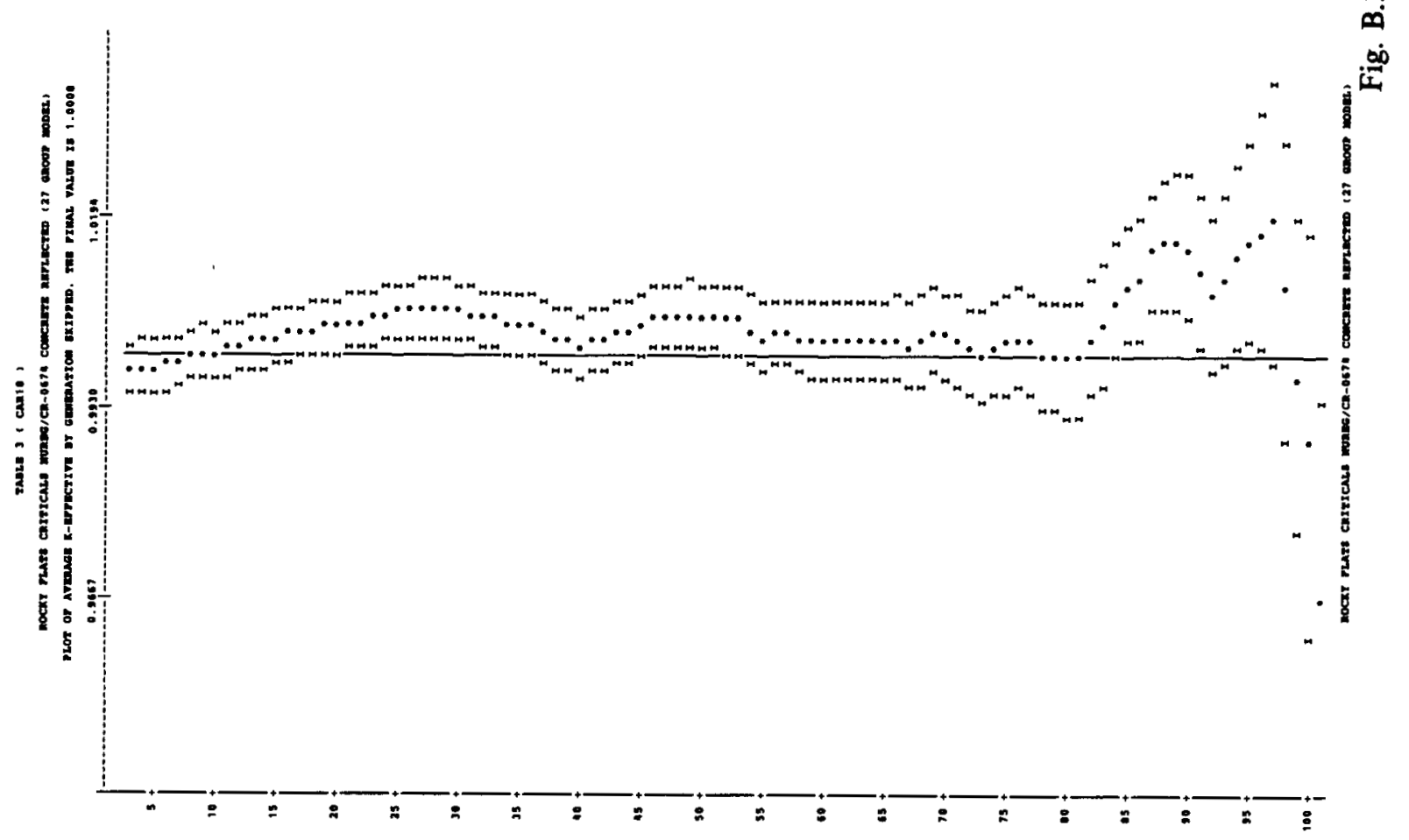



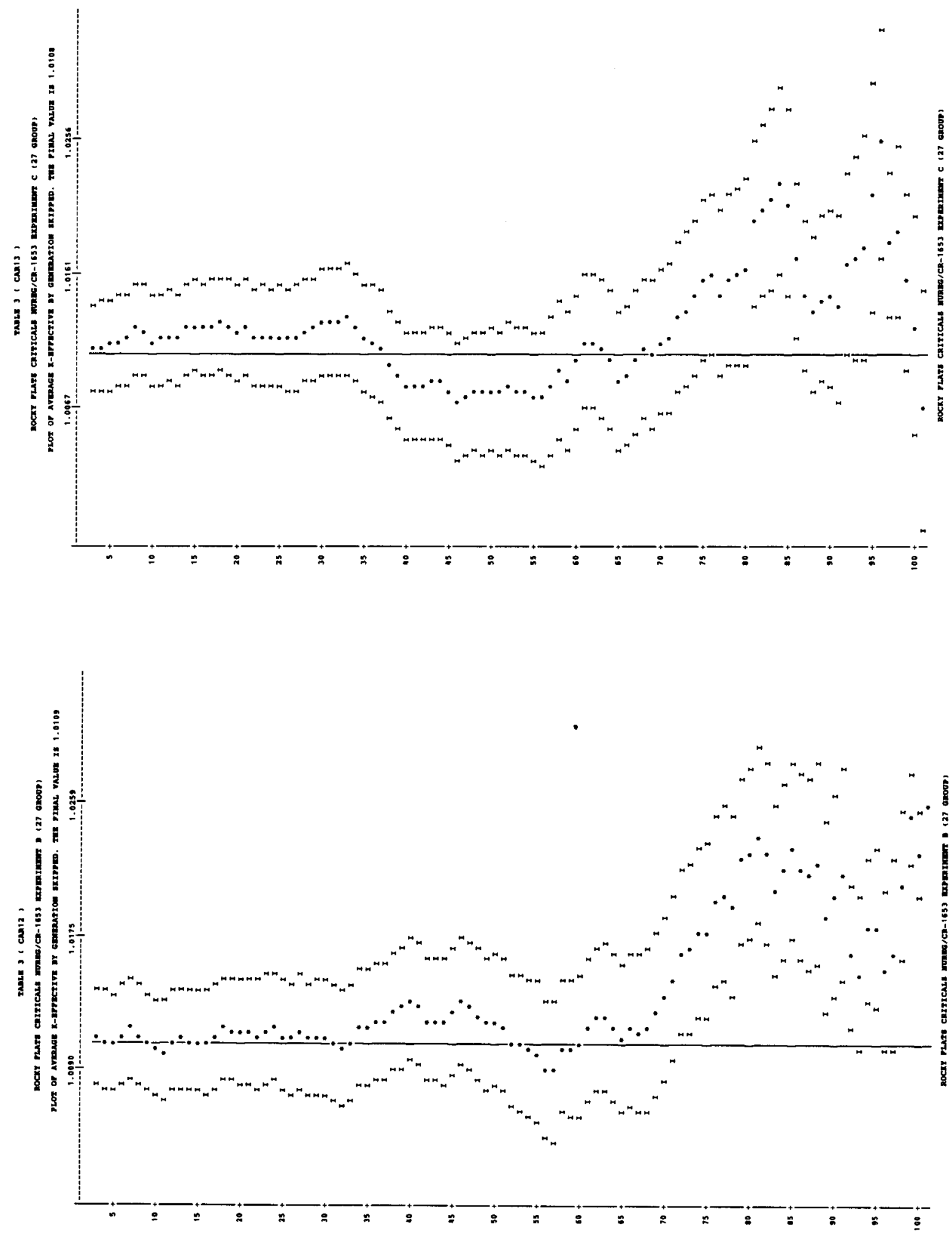

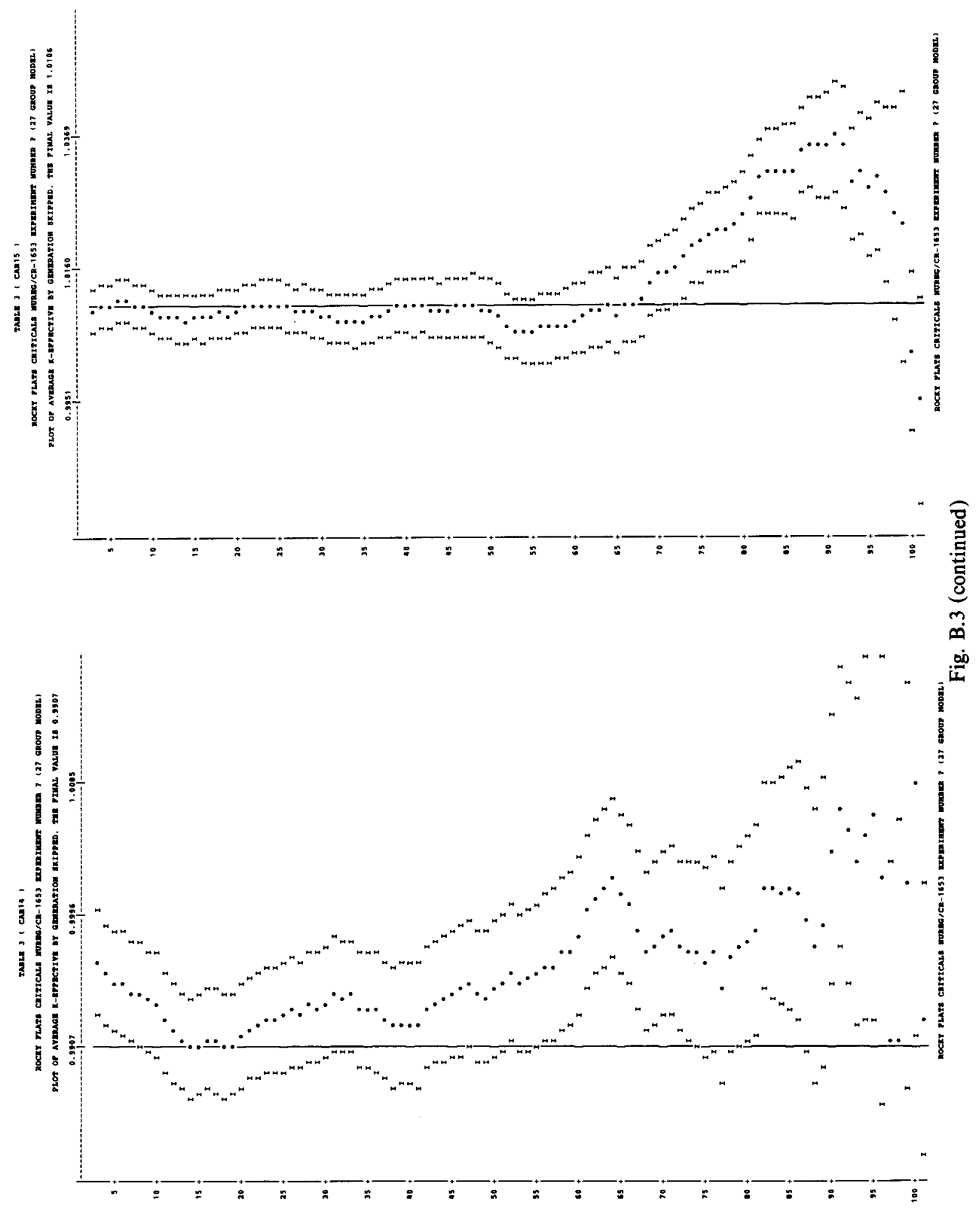


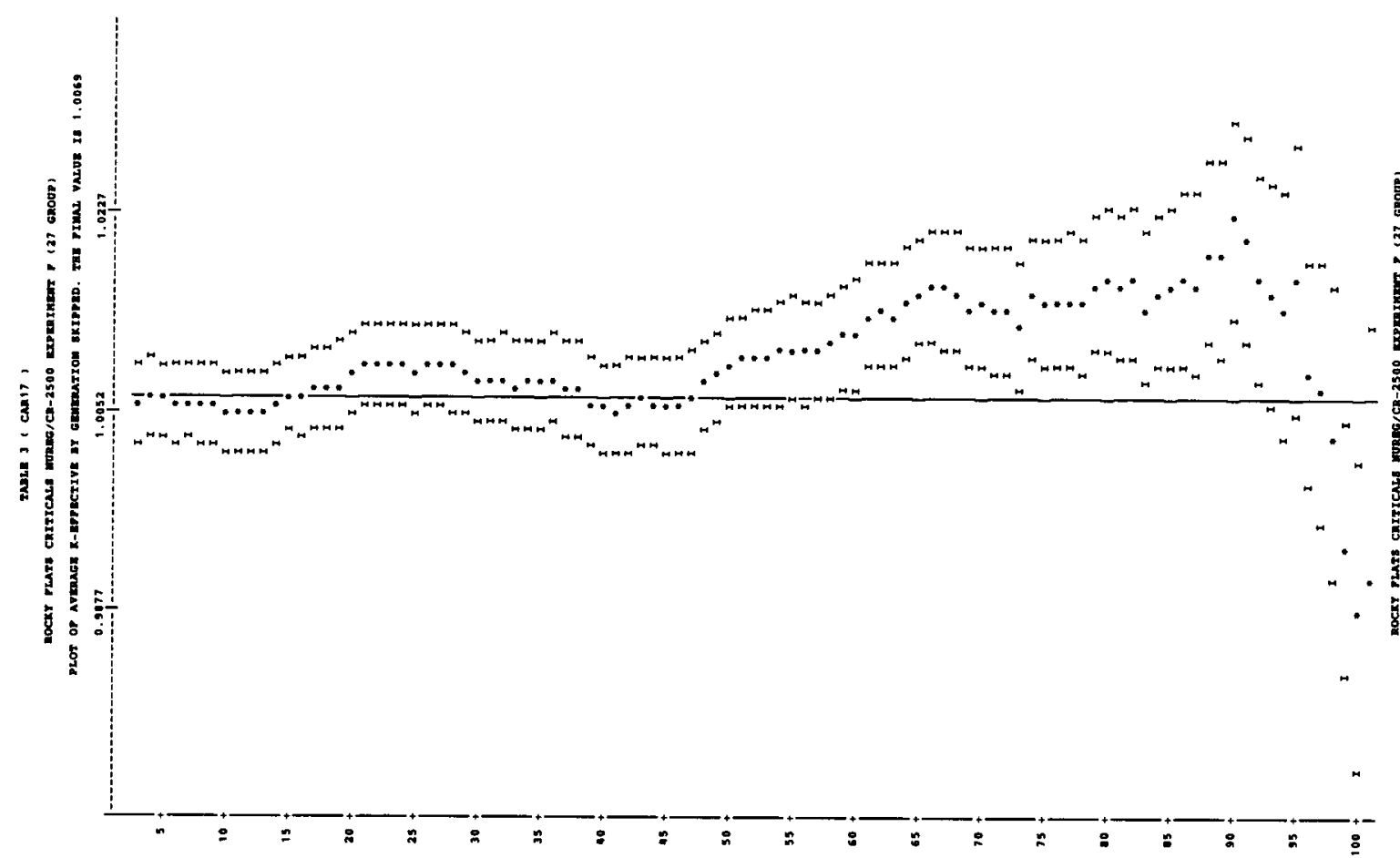

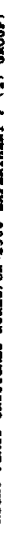

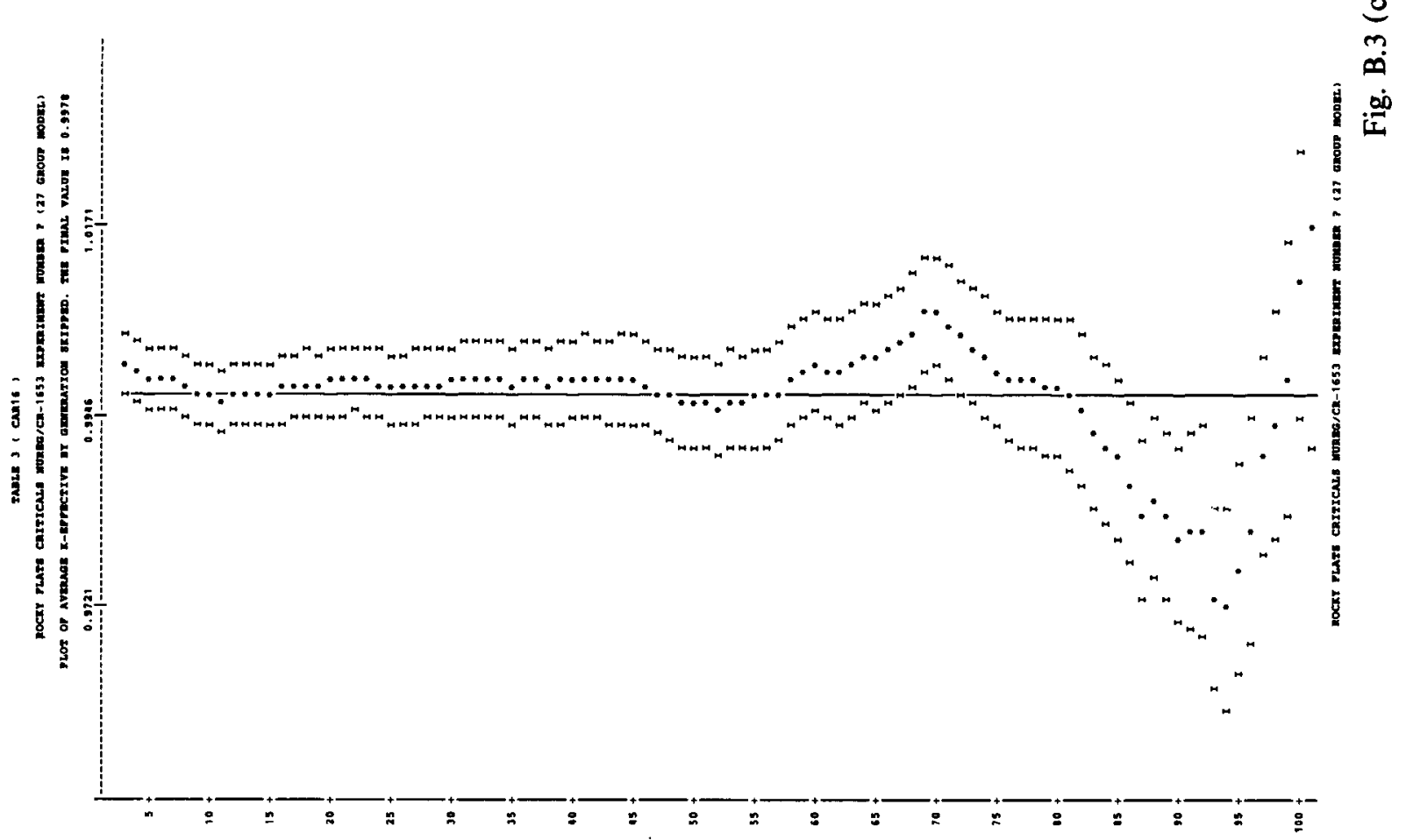




\section{B-51}
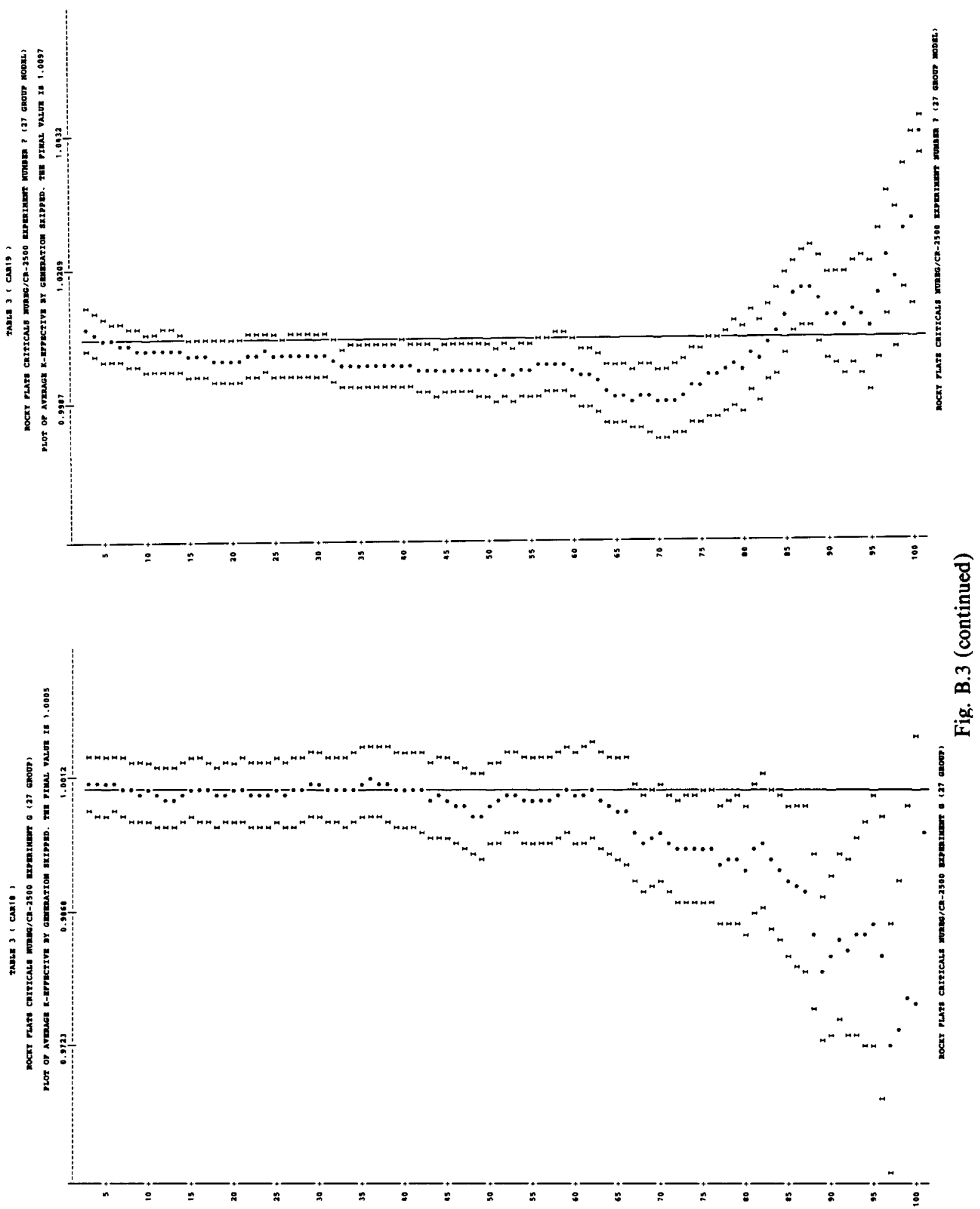
B-52

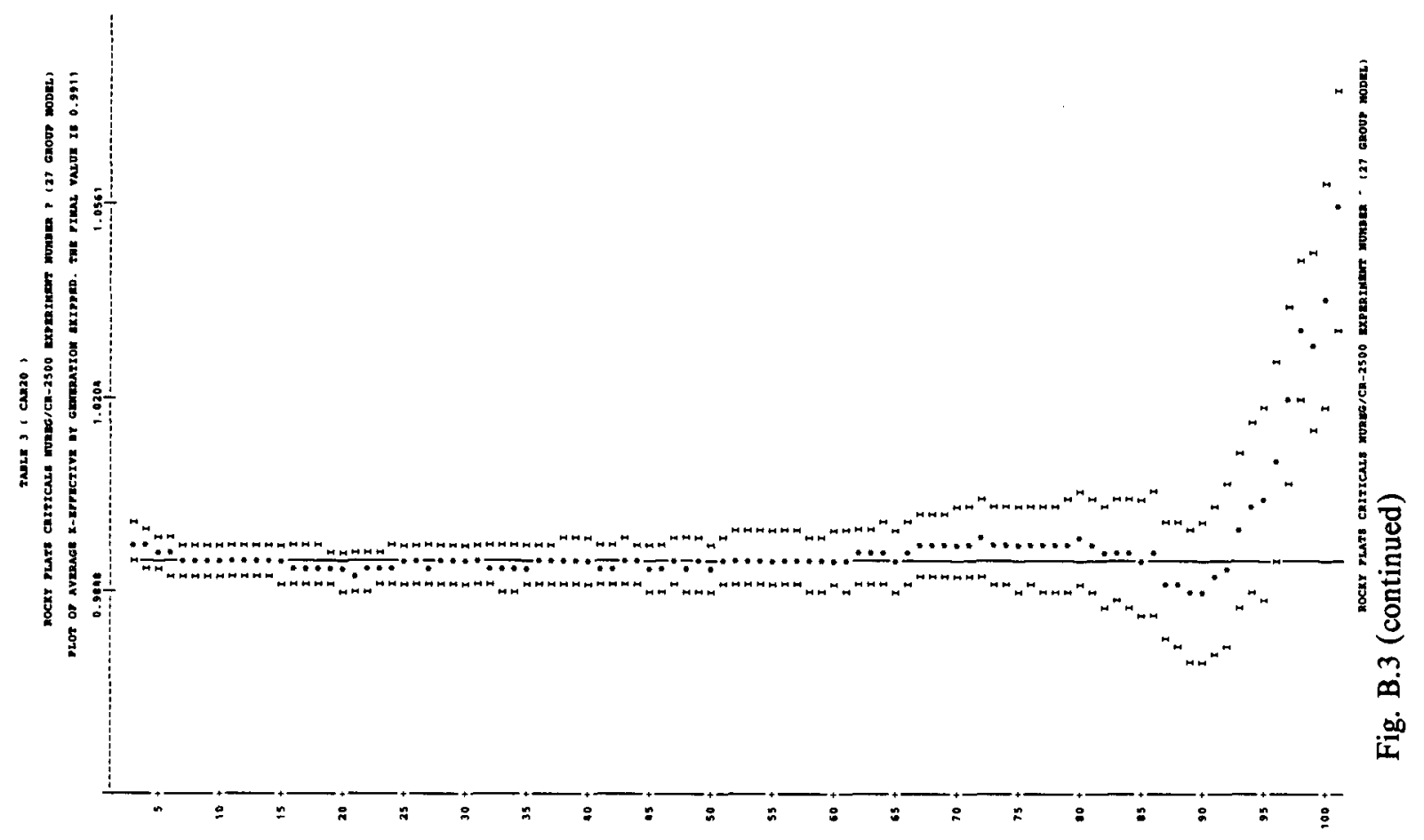


B-53

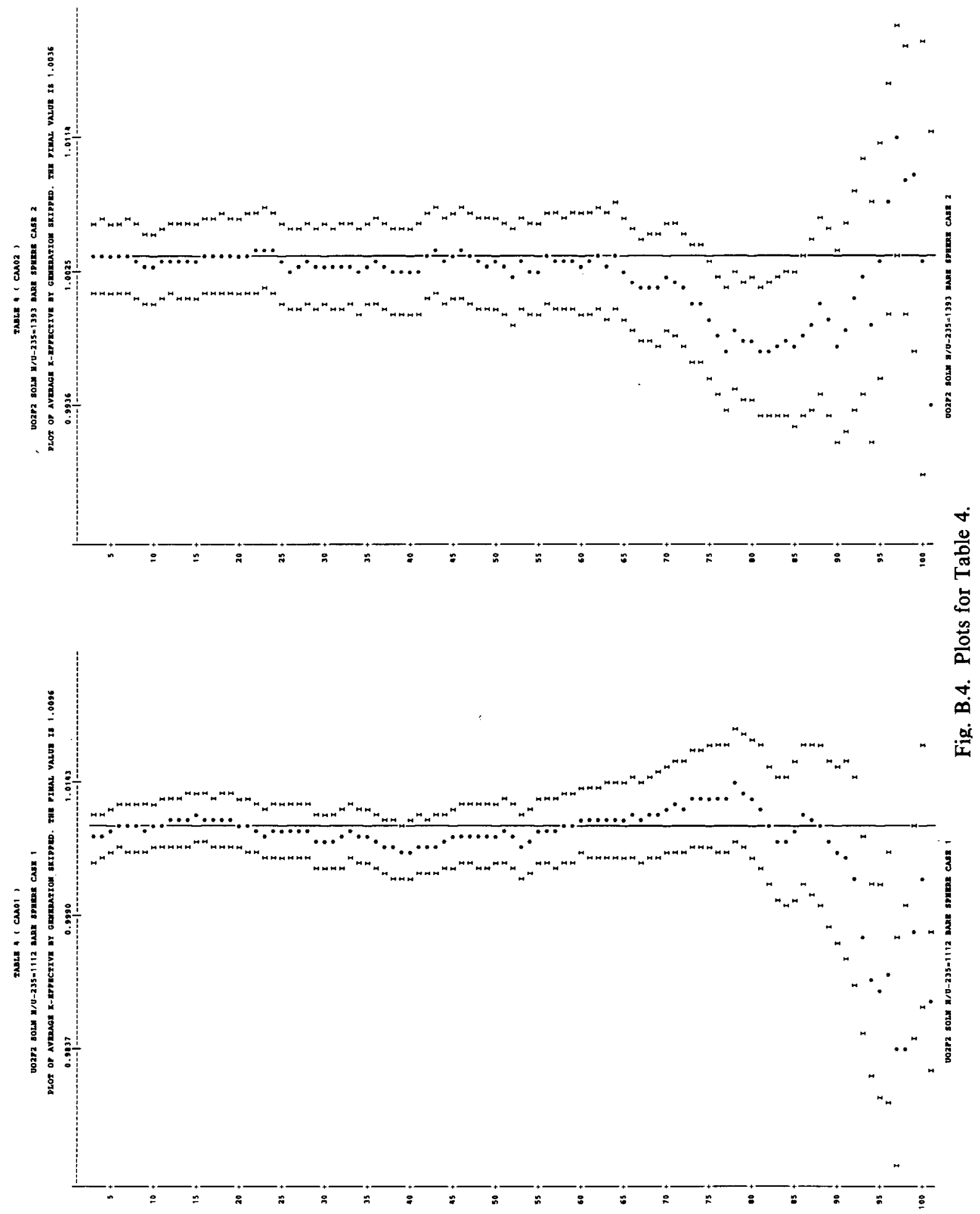


B- 54

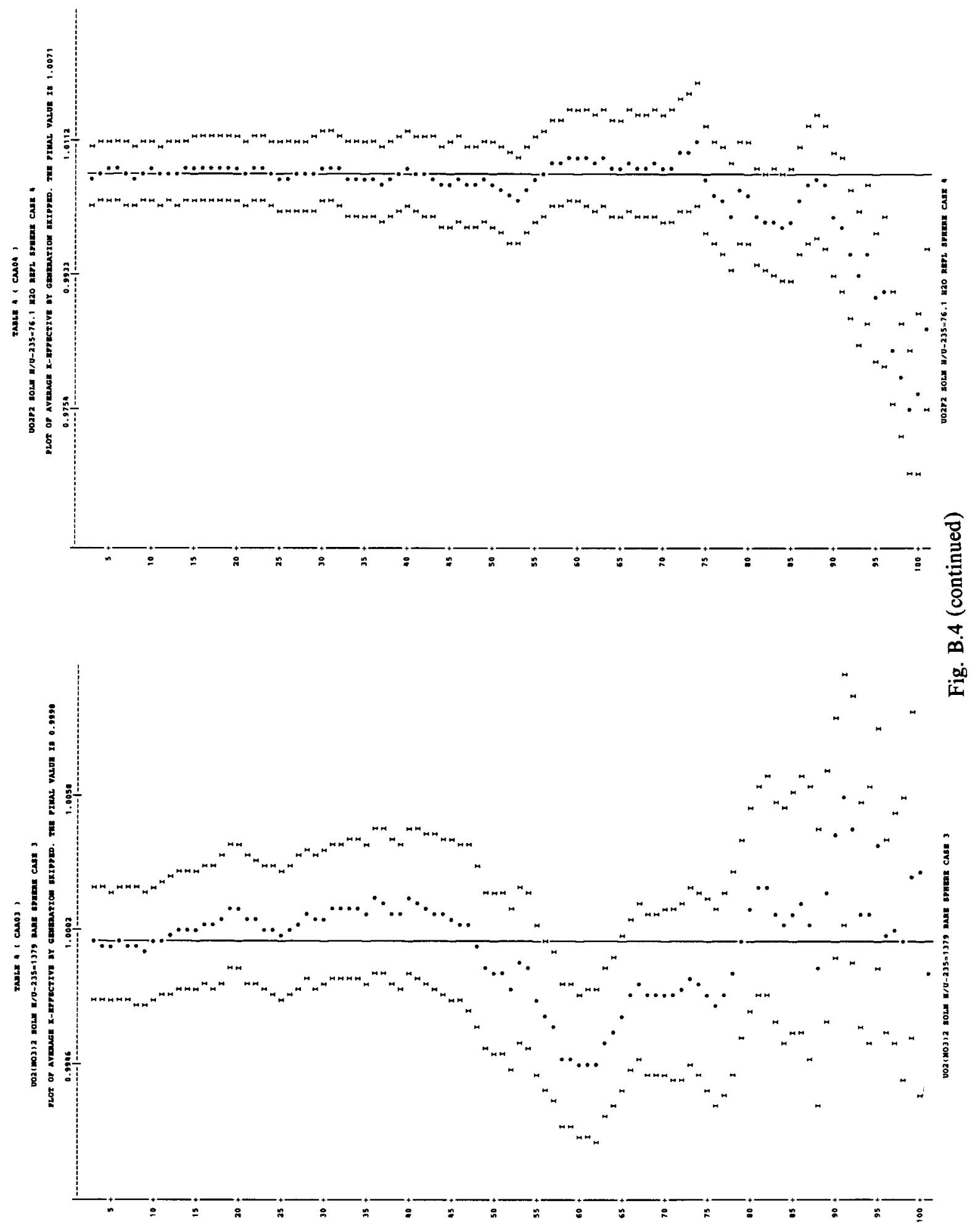




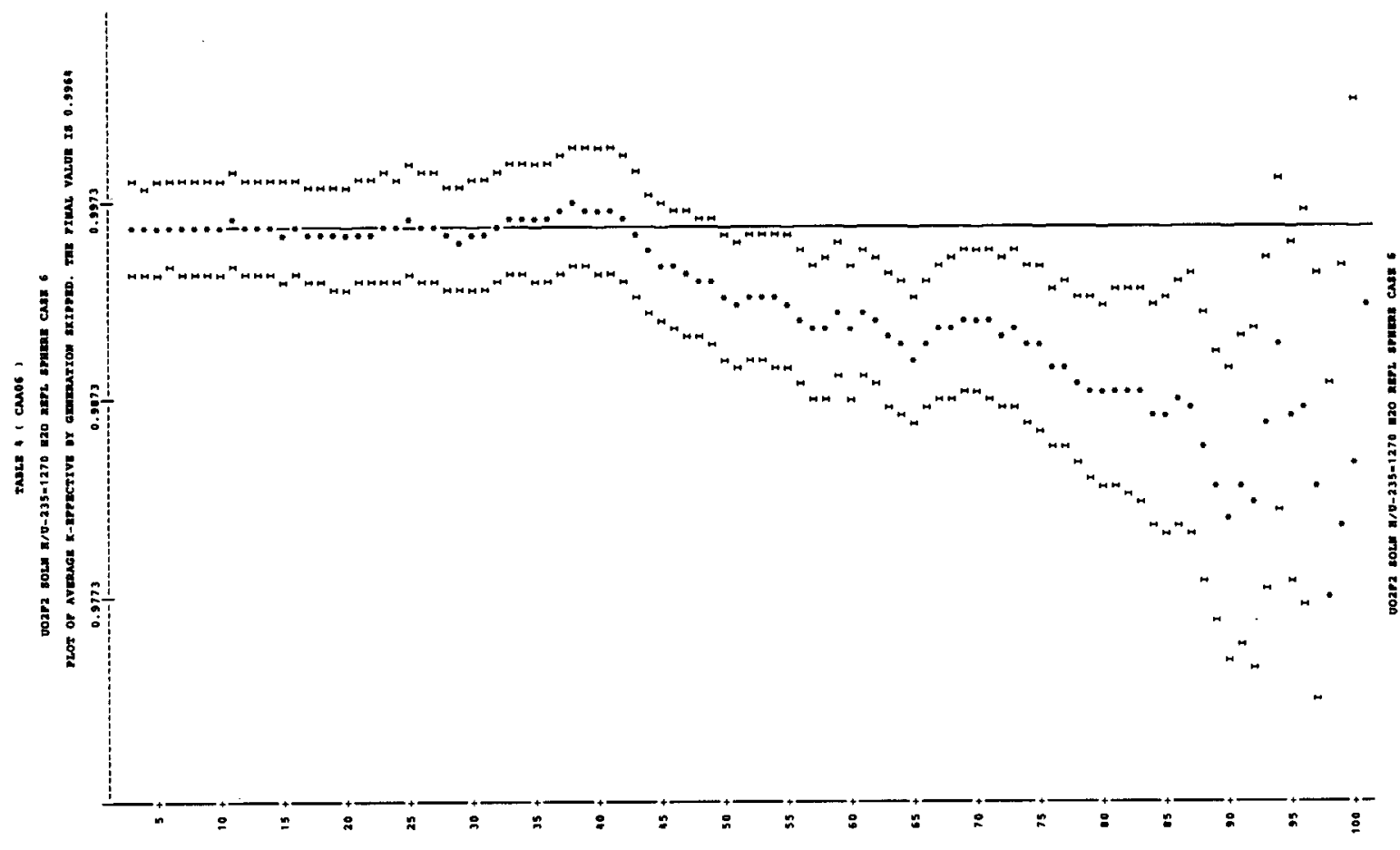

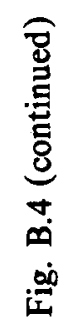

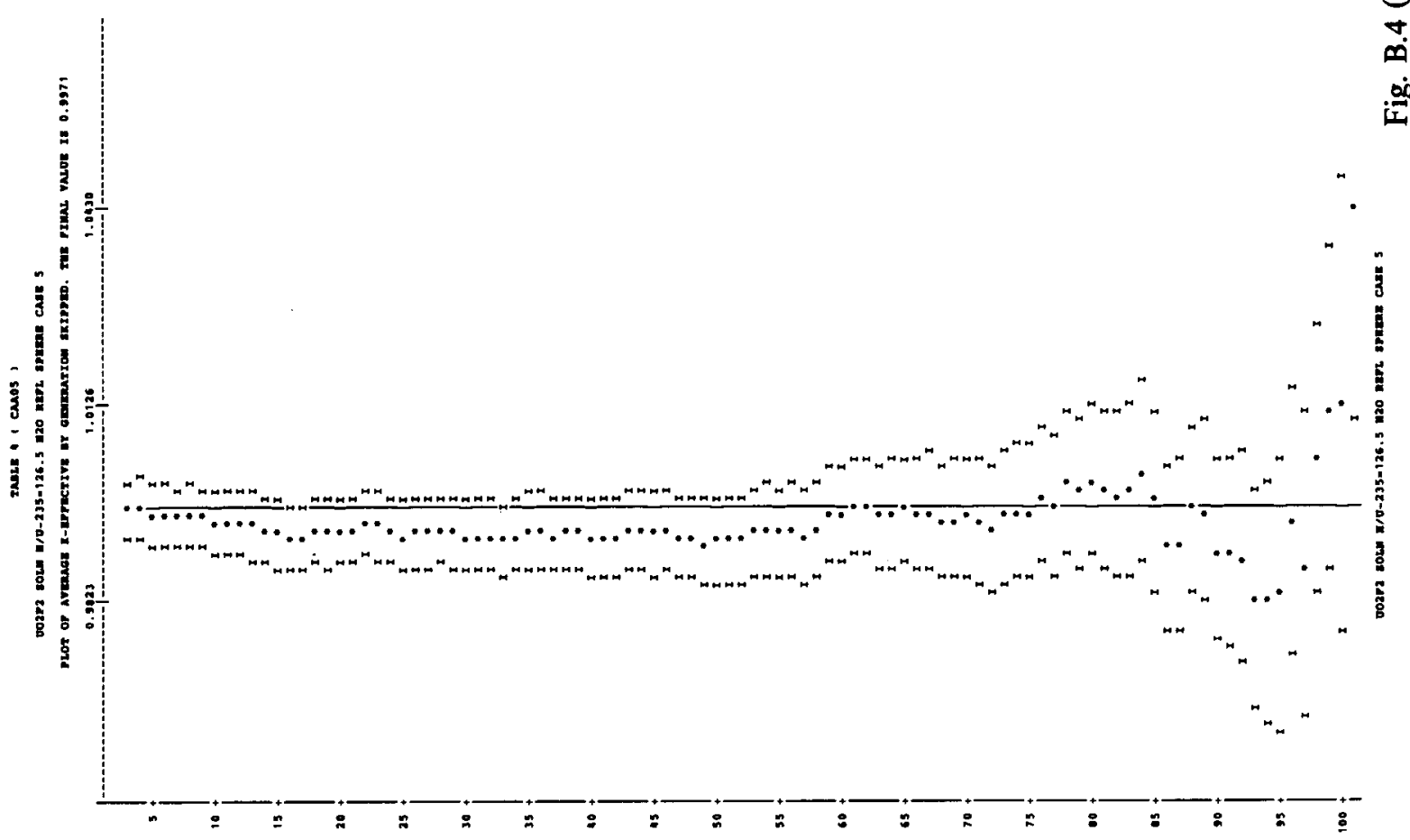


B-56

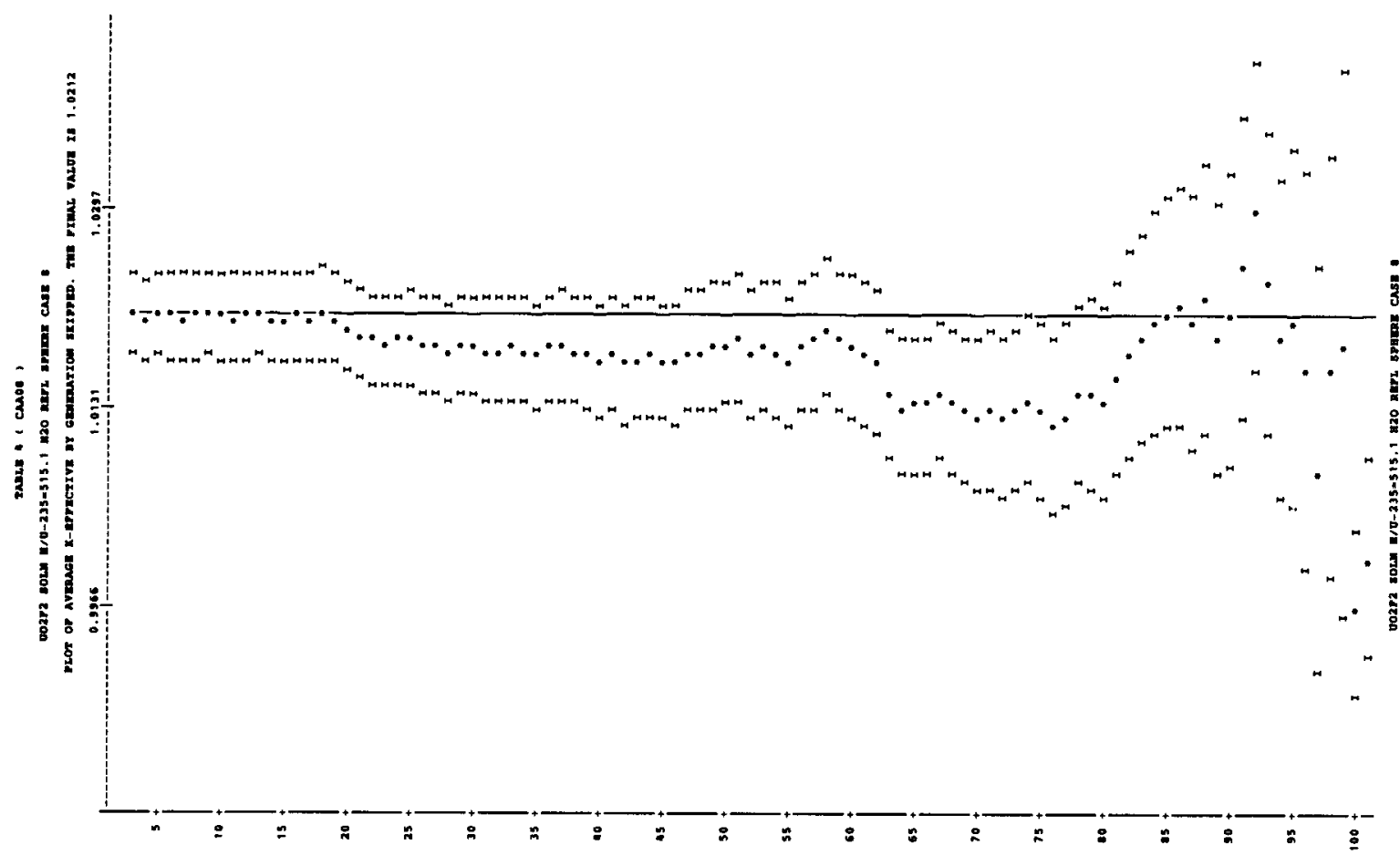

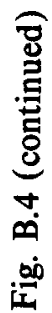

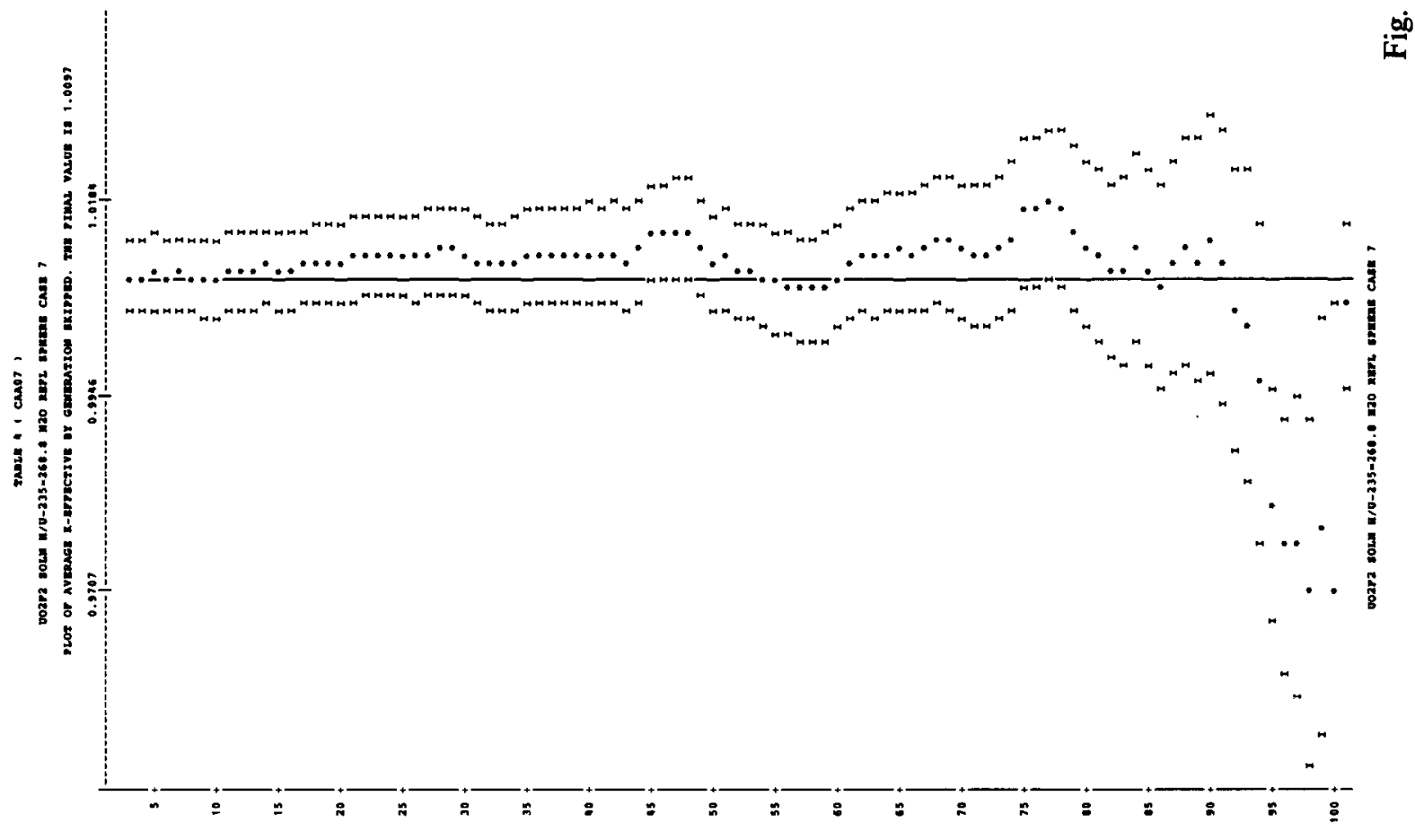



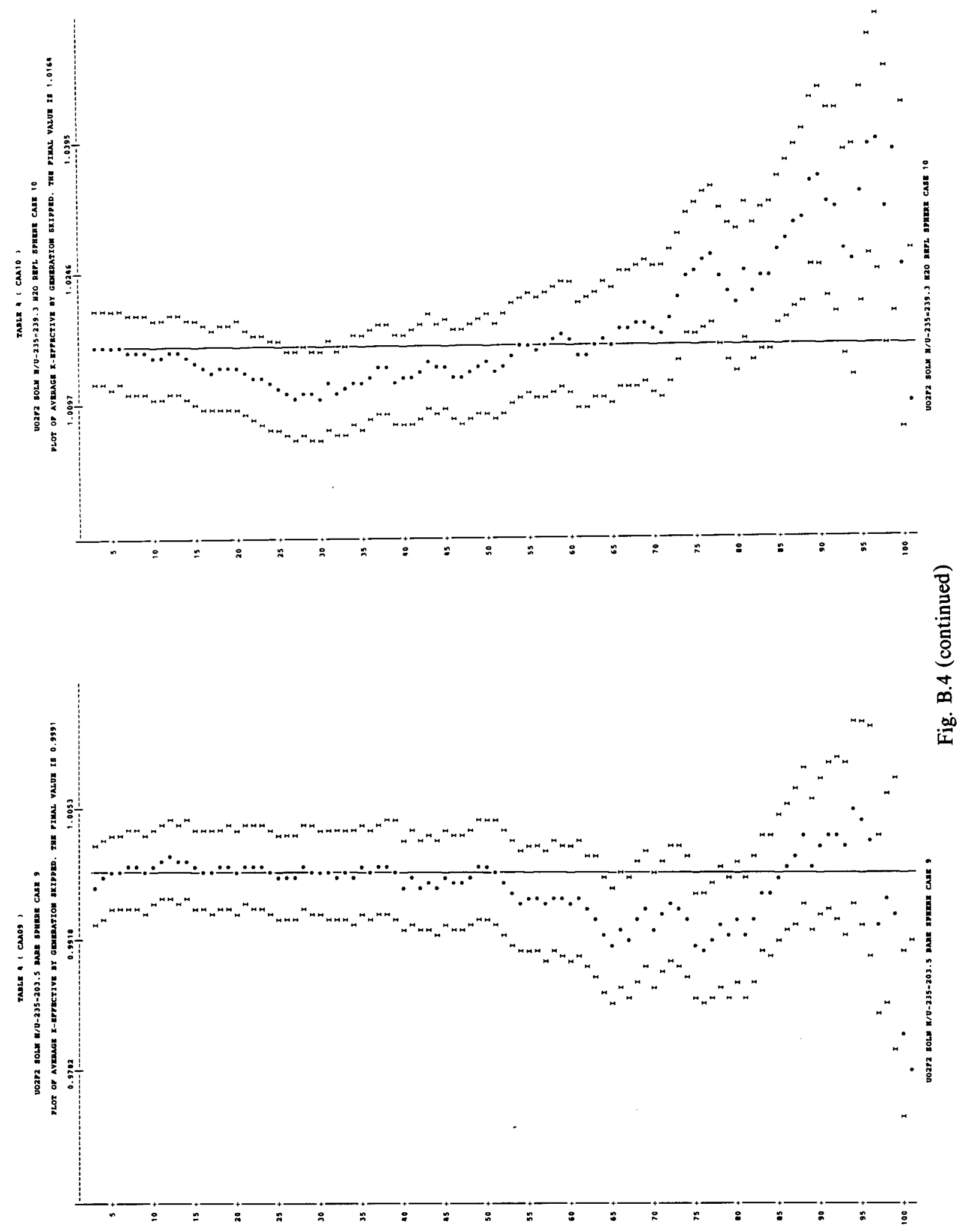


\section{B- 58}

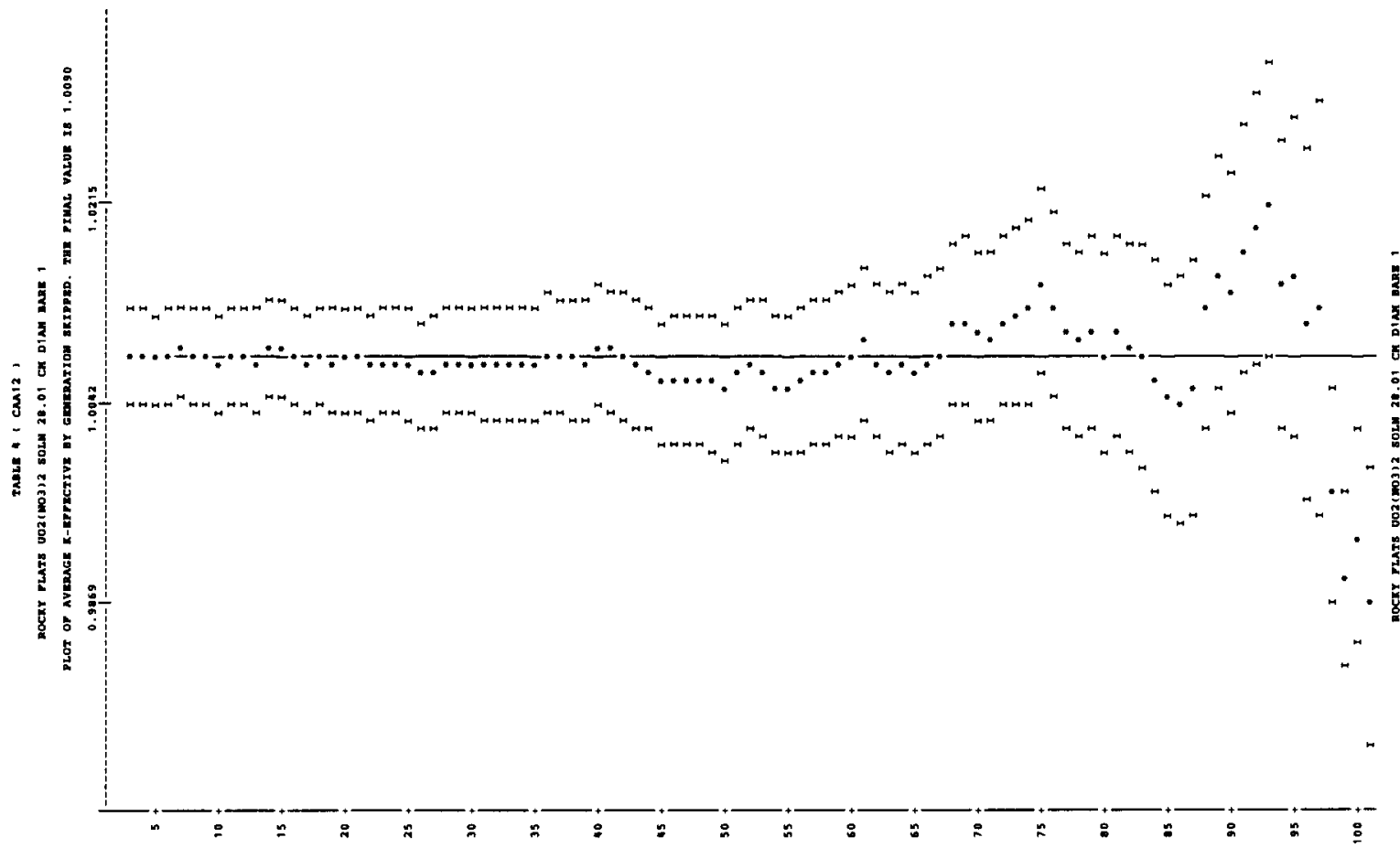

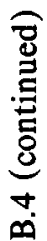

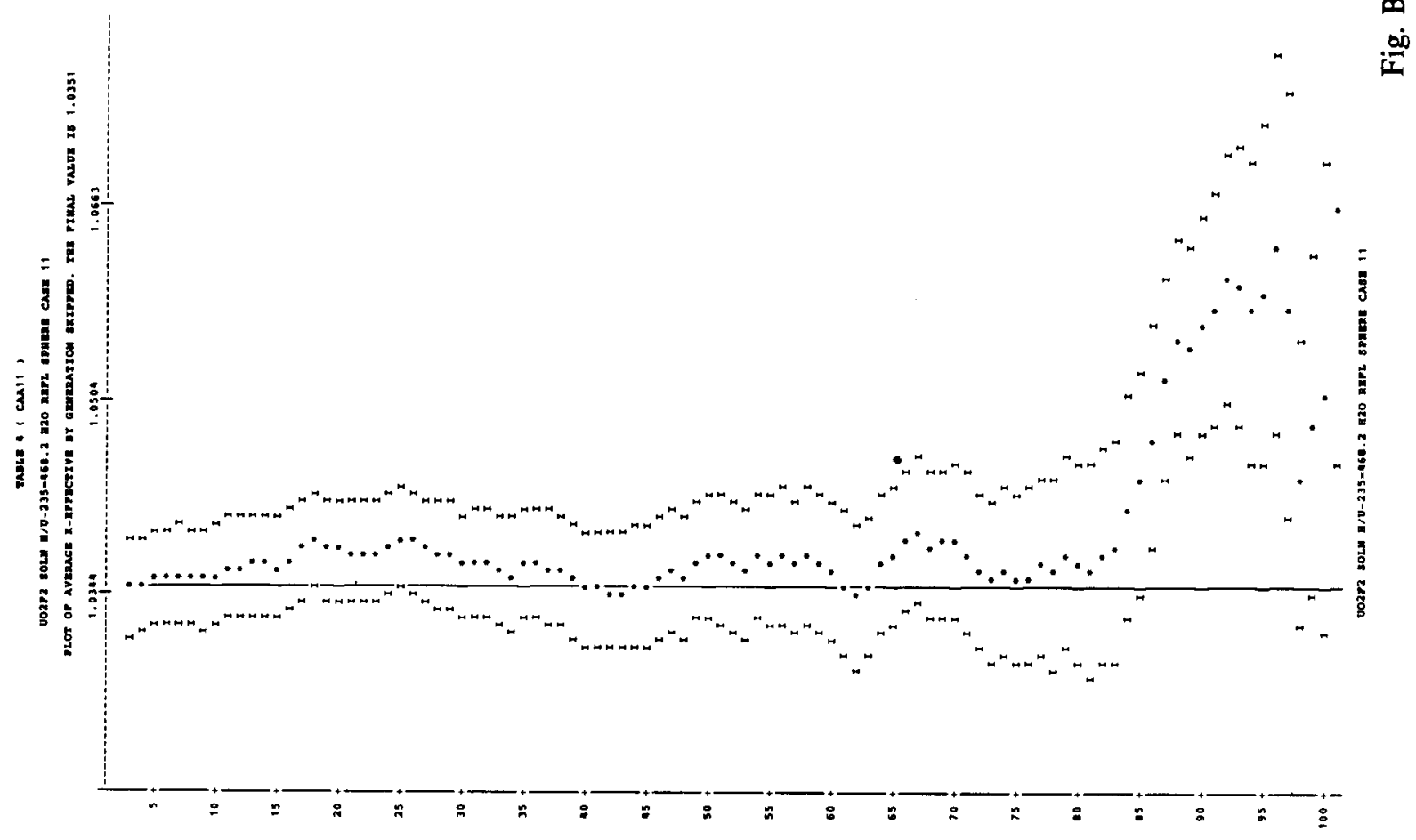


B-59

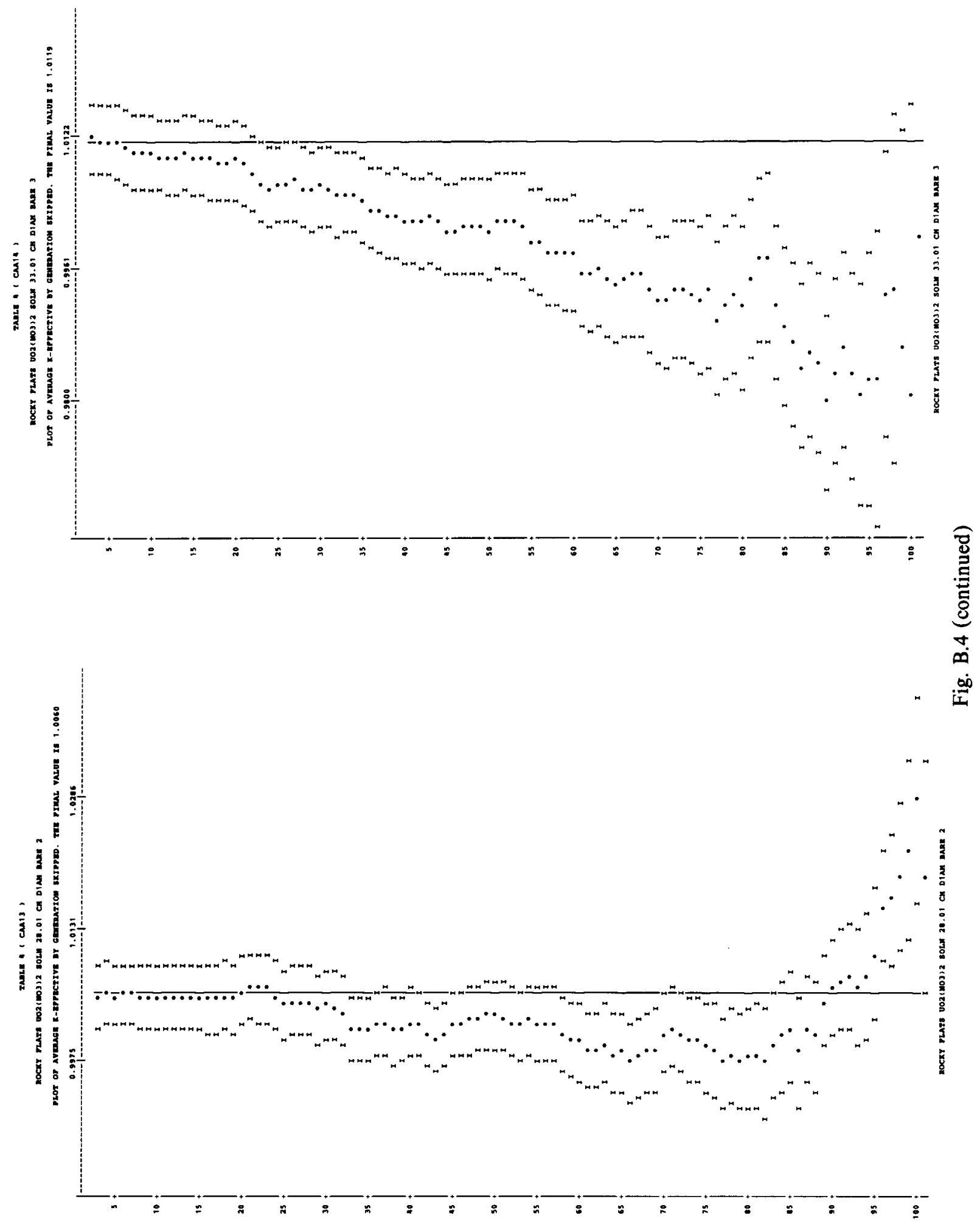


B- 60
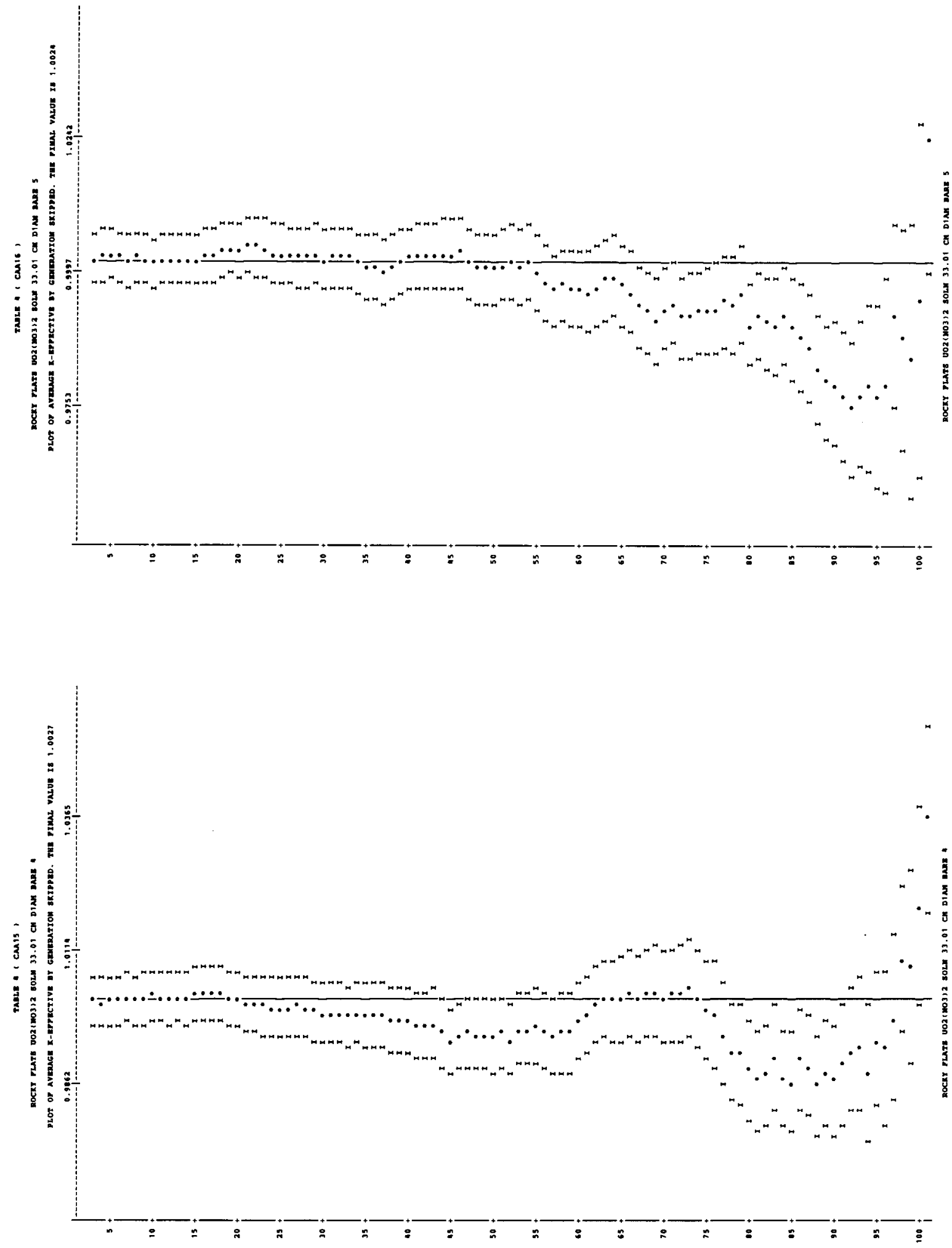


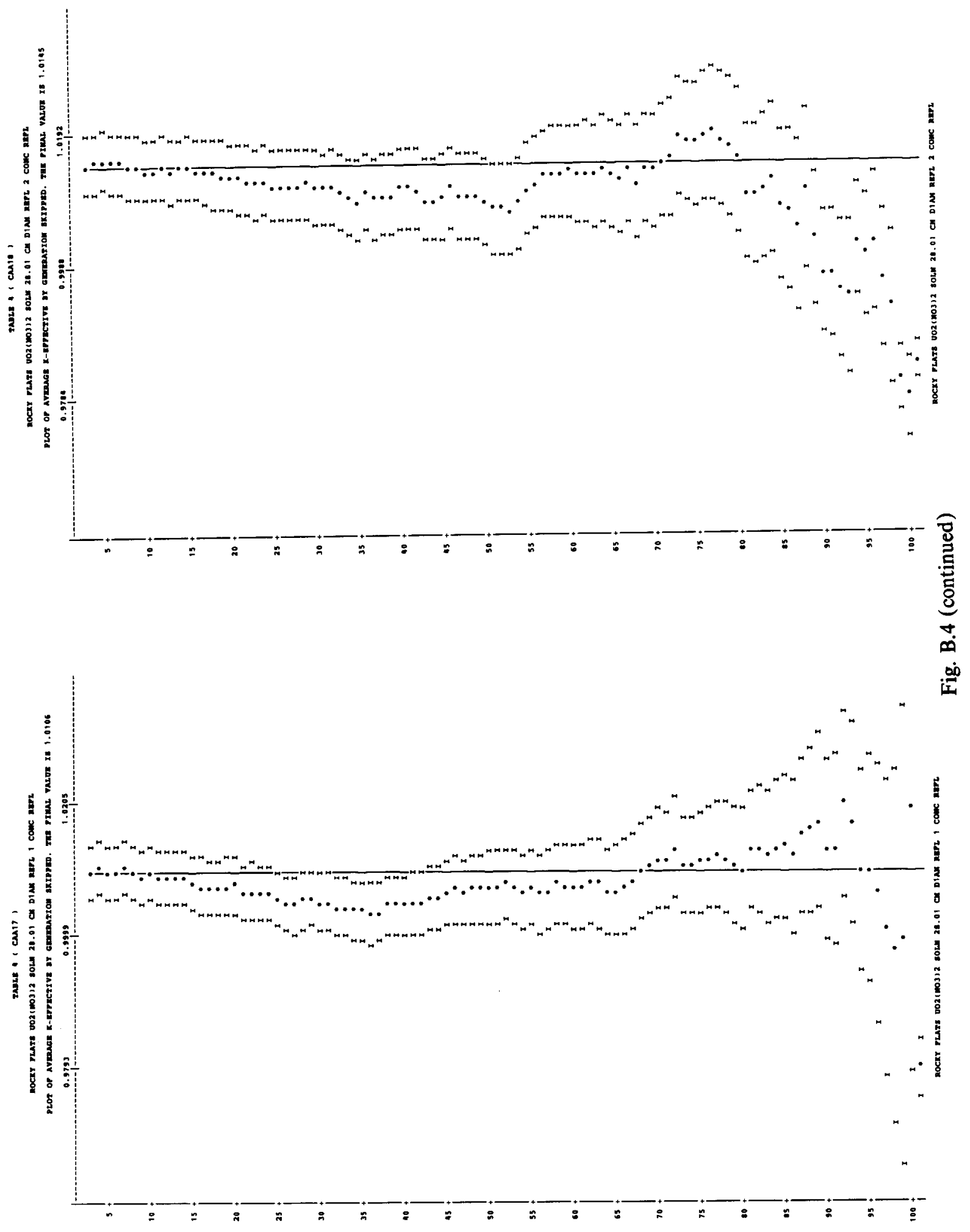



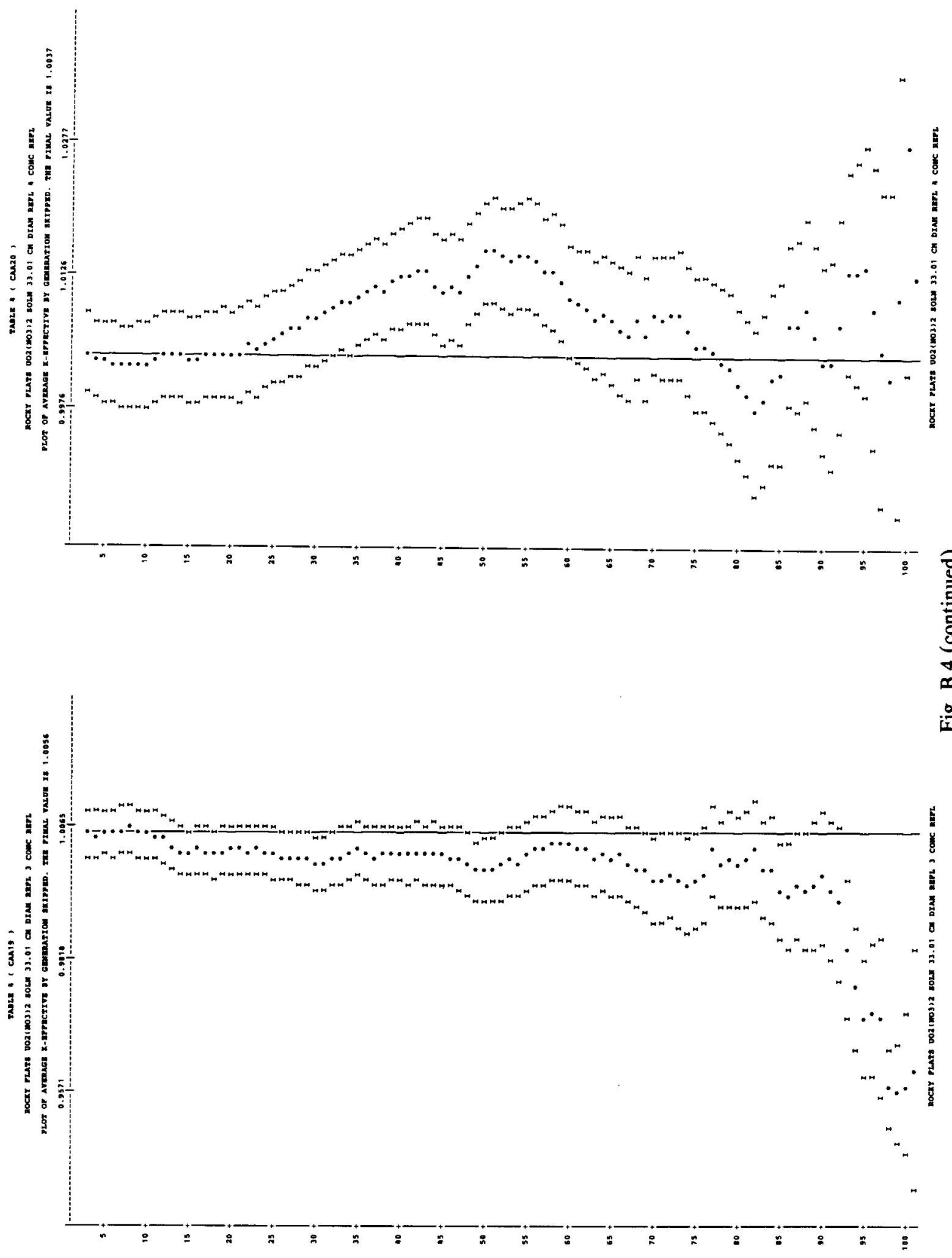


\section{B-63}

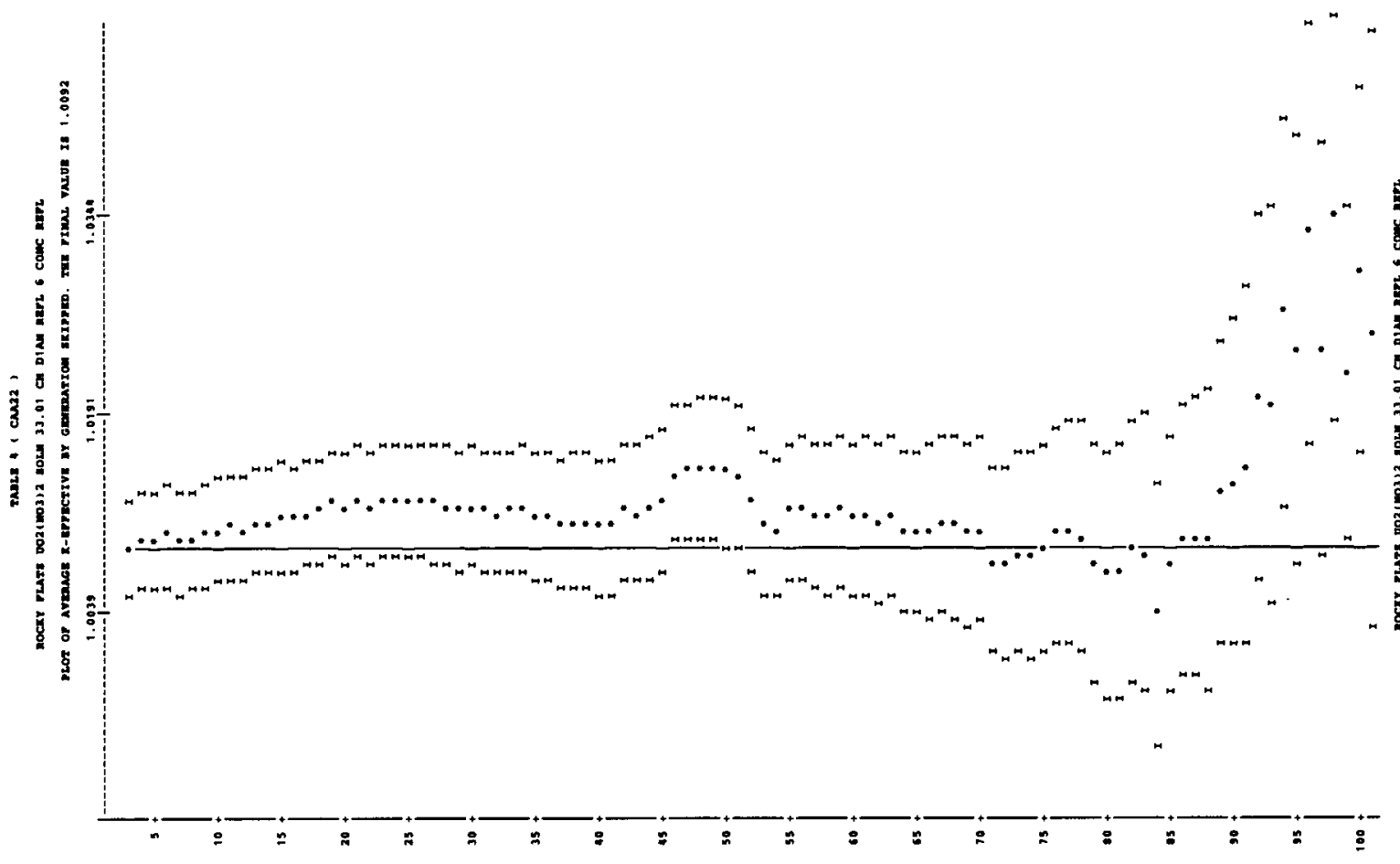

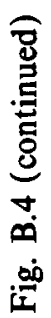

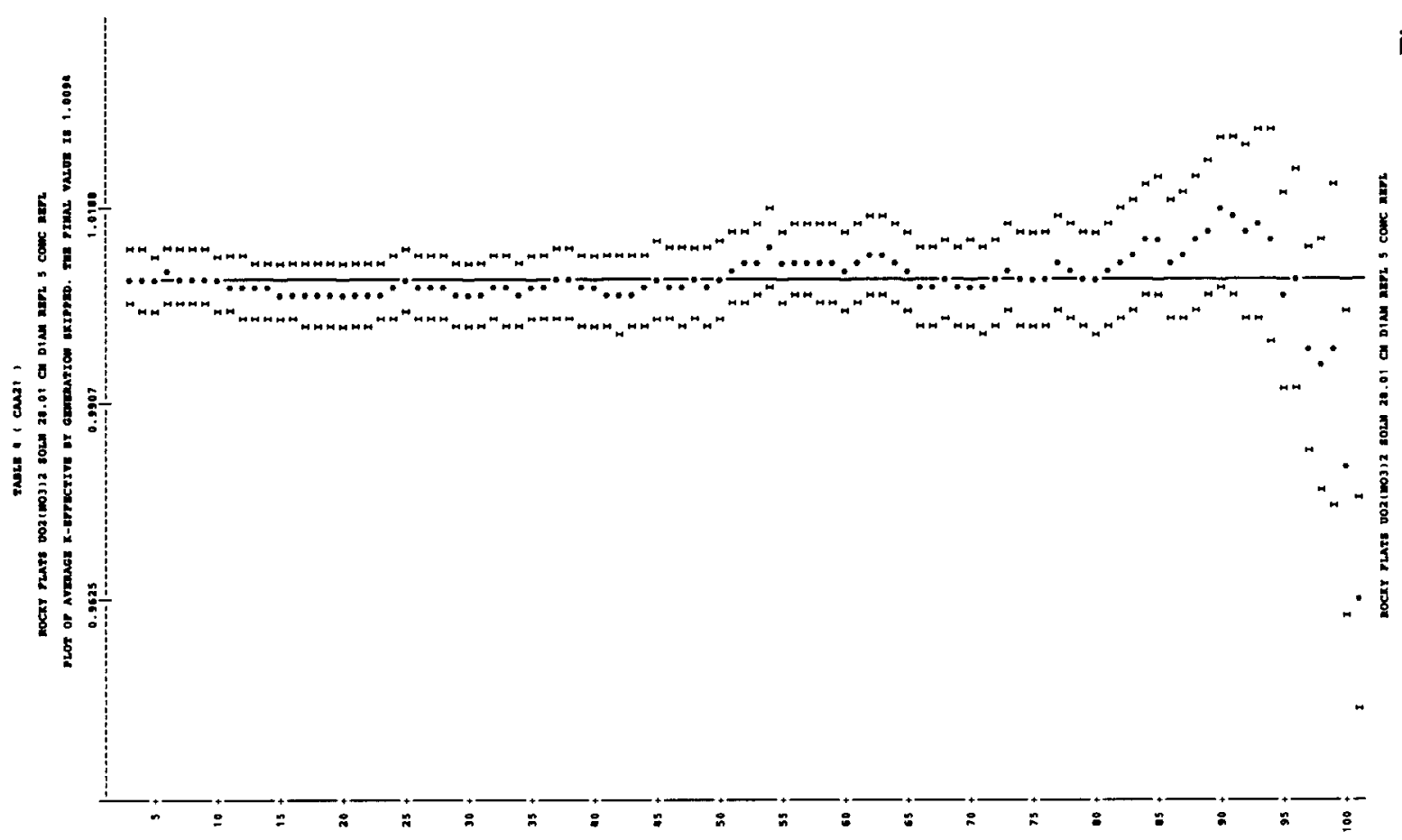



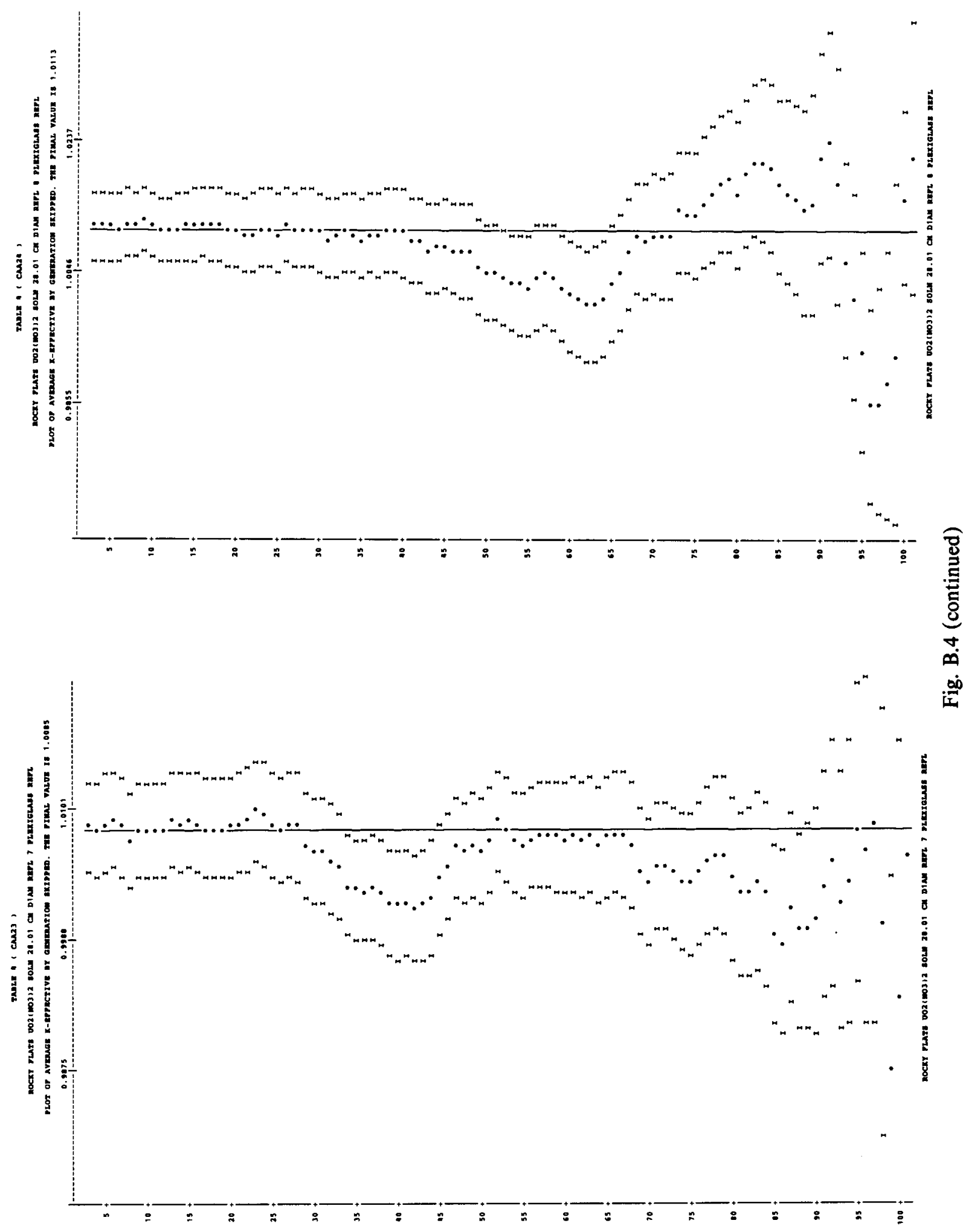

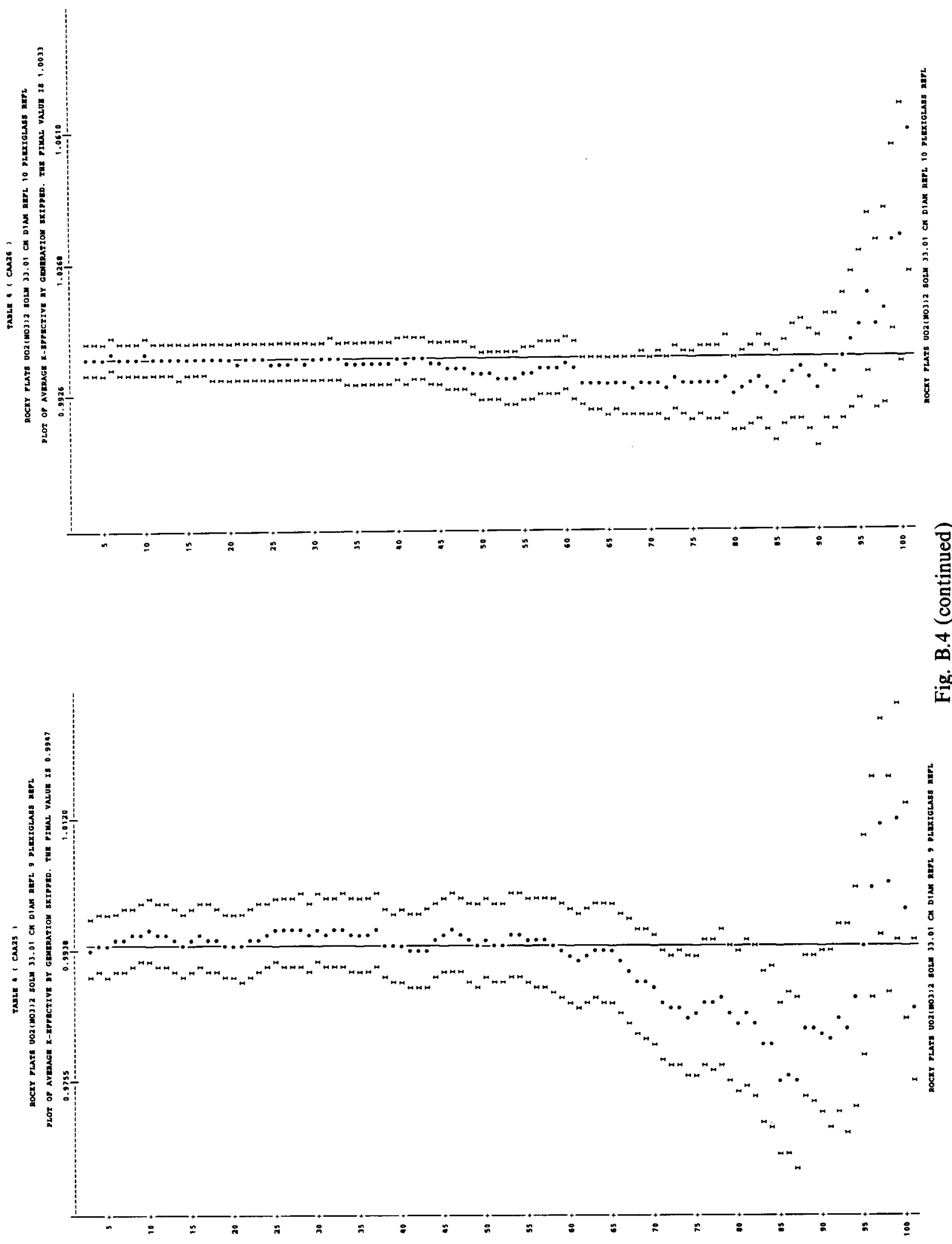
B-66

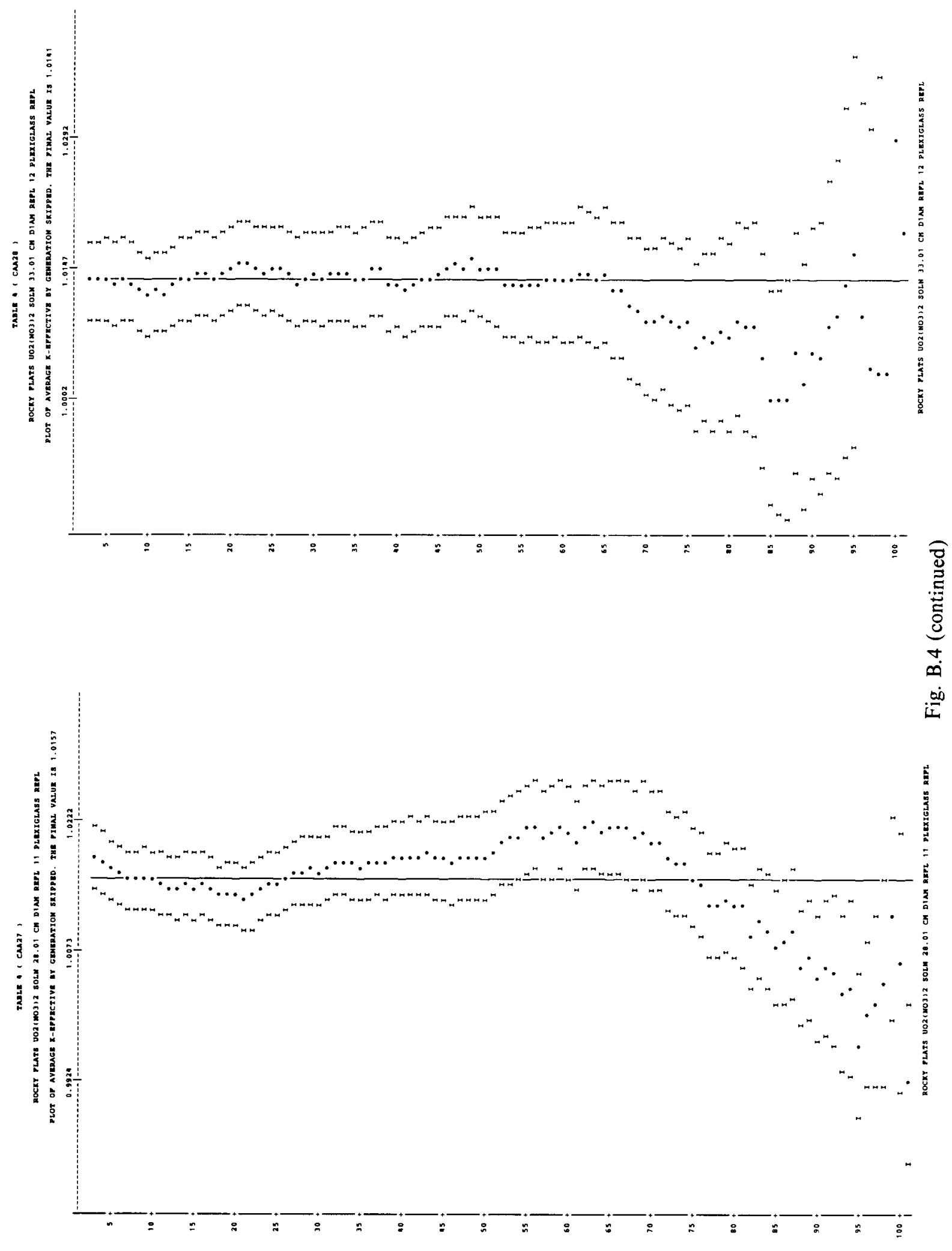



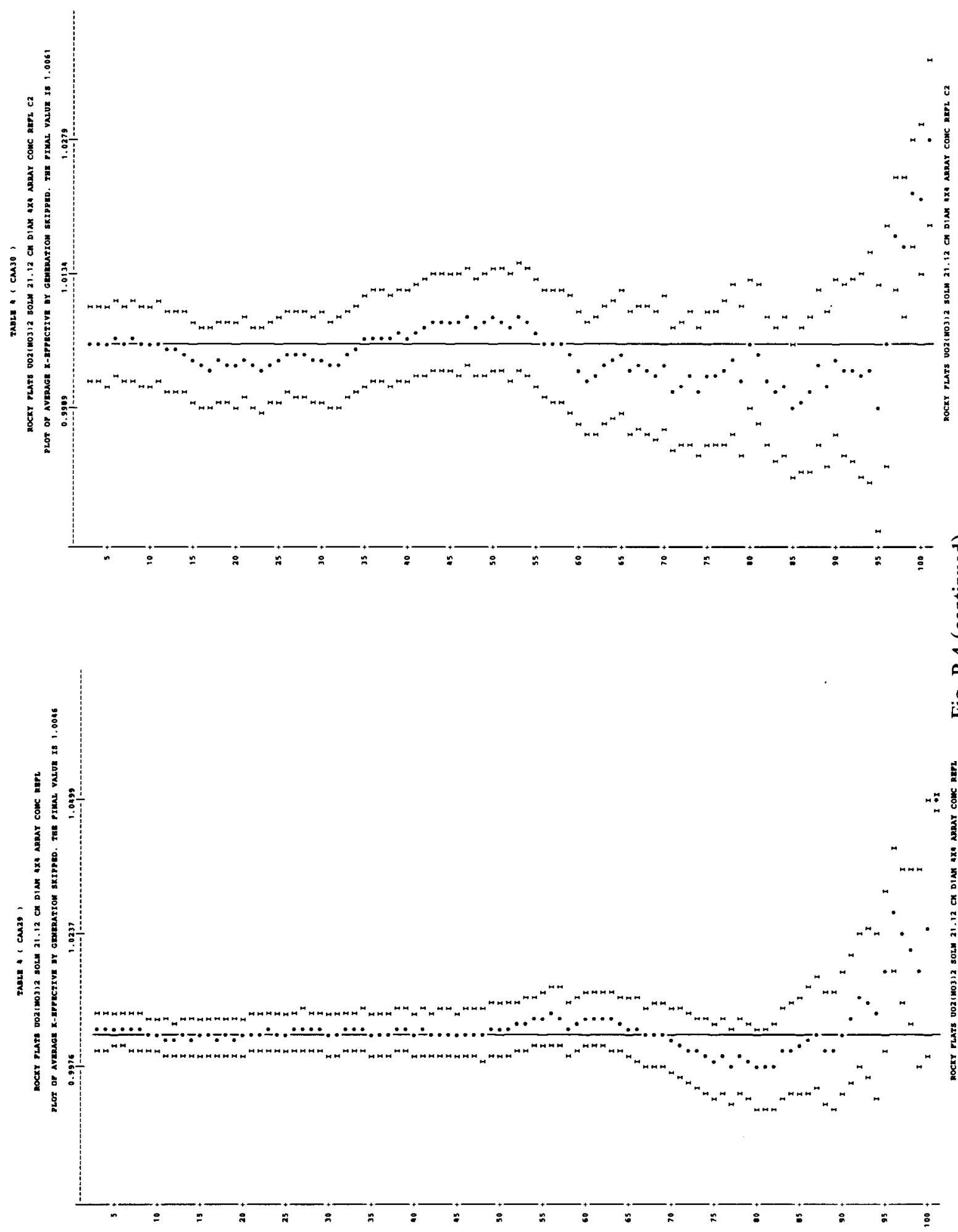

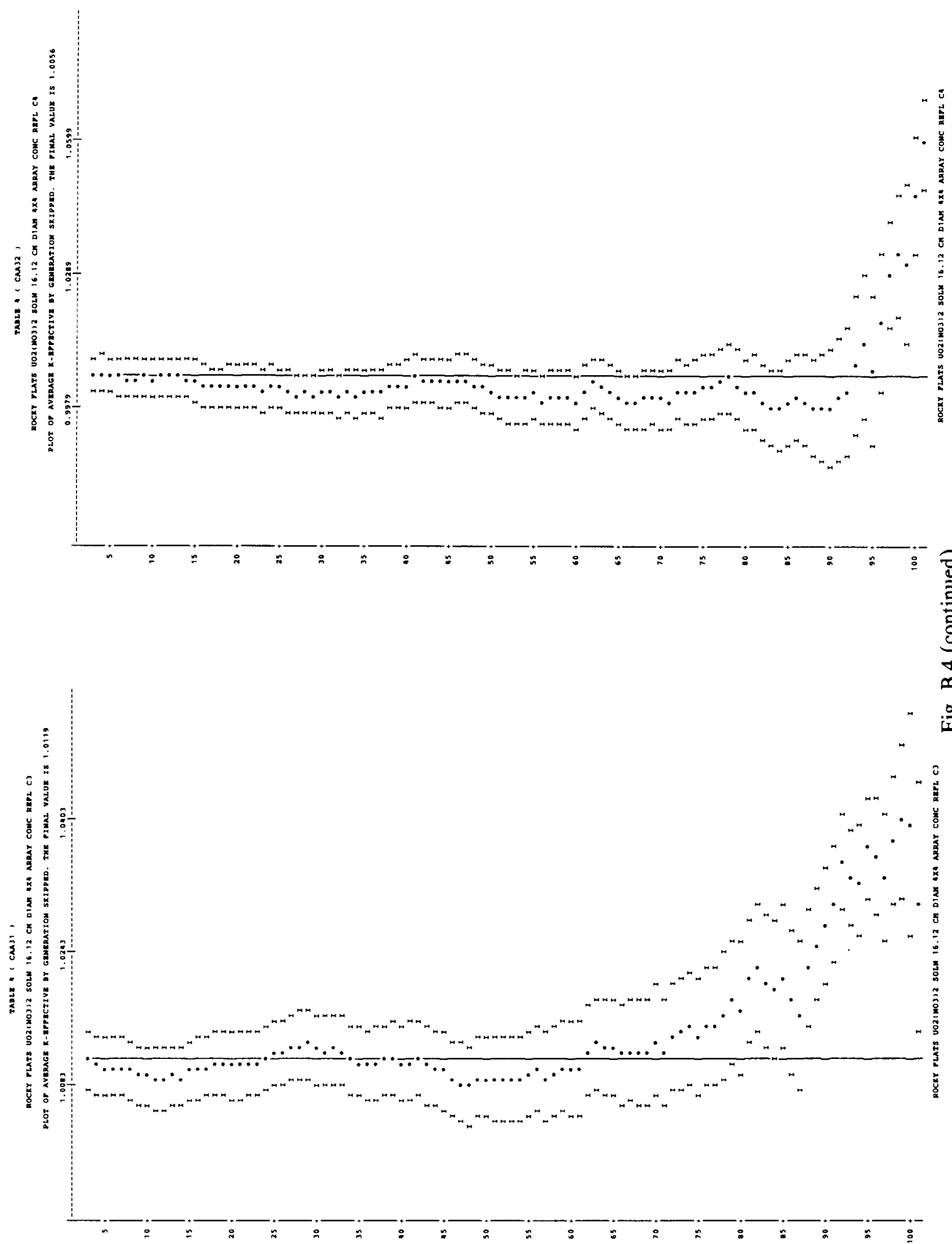


\section{B-69}
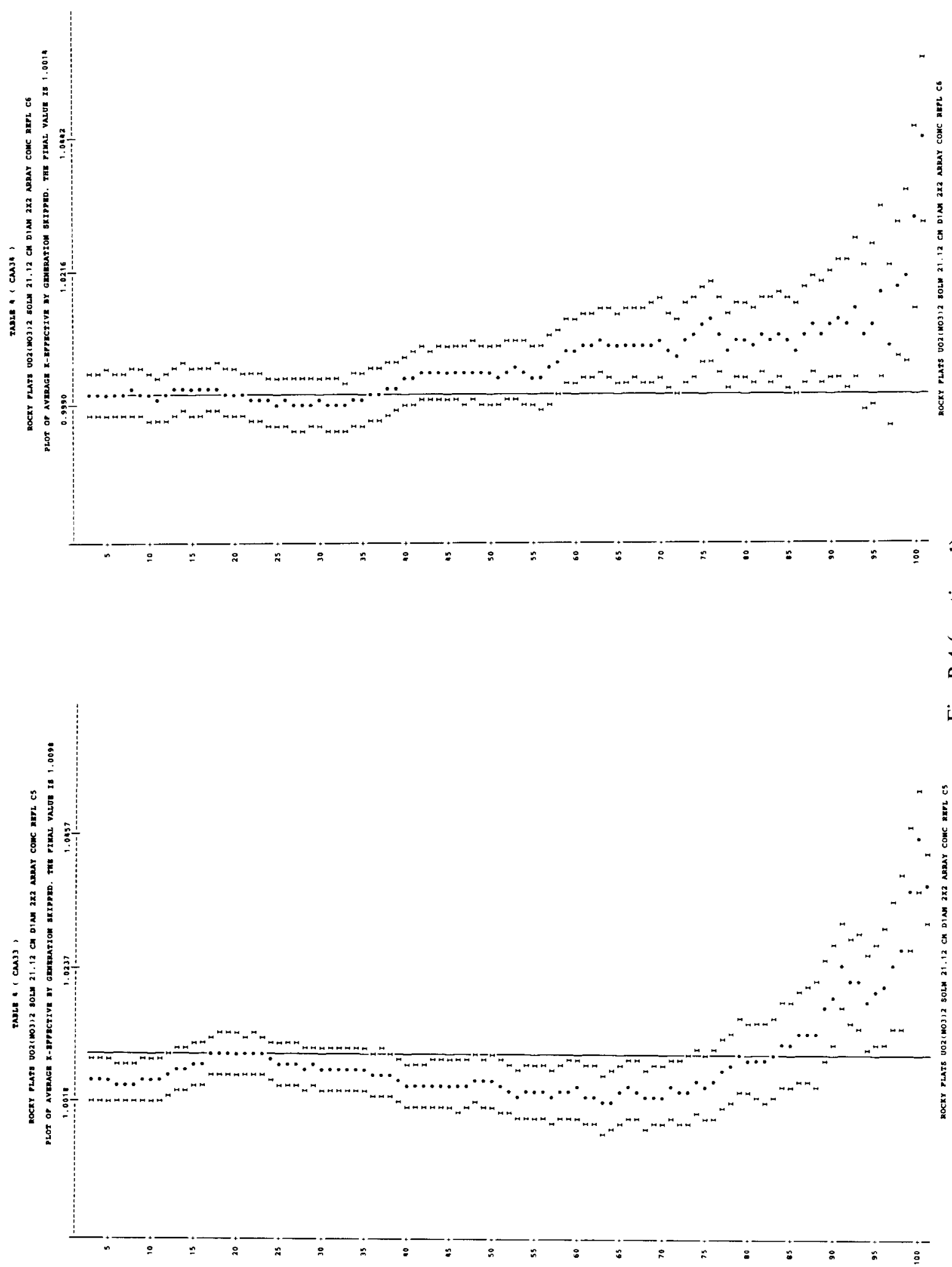
B-70
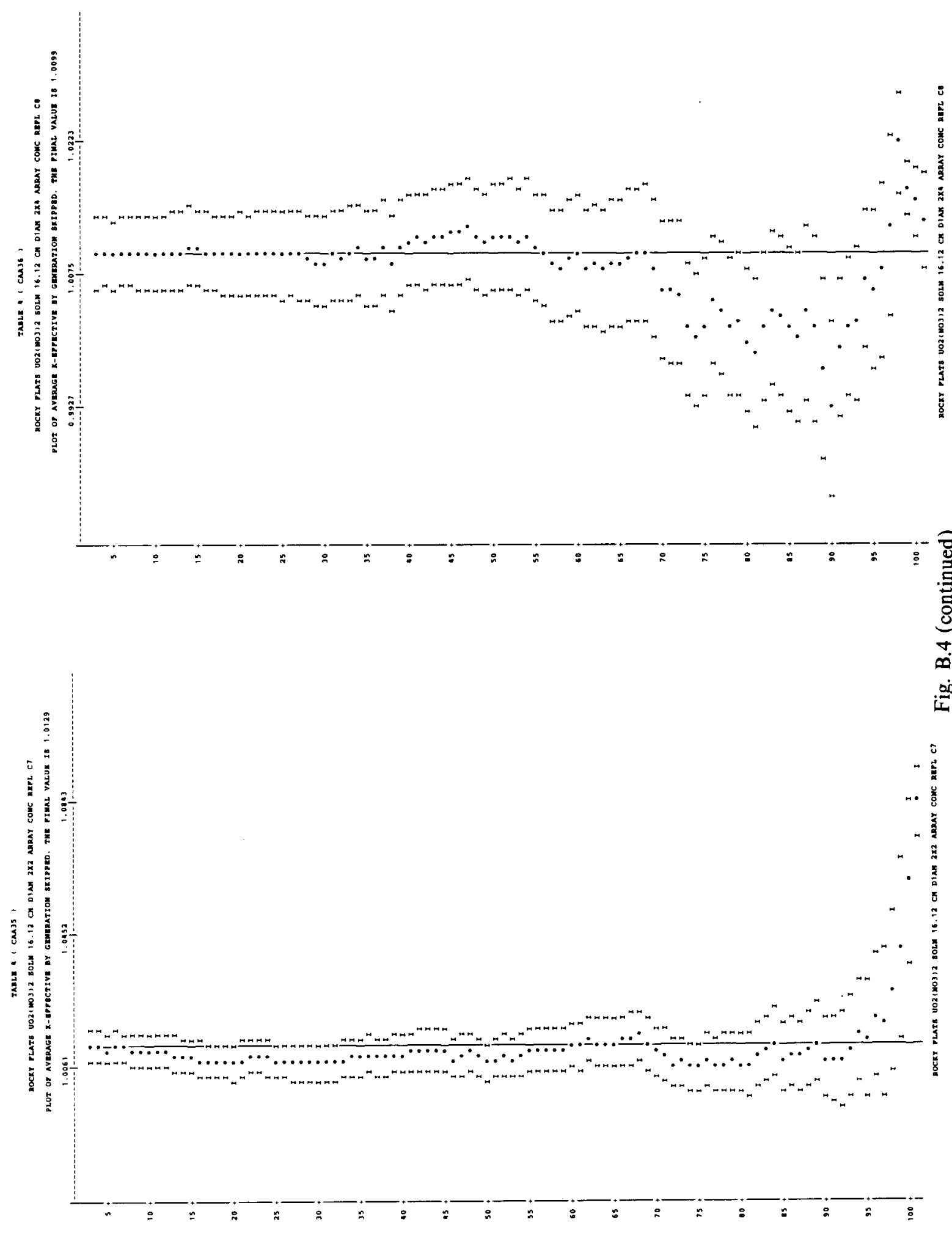
B-71

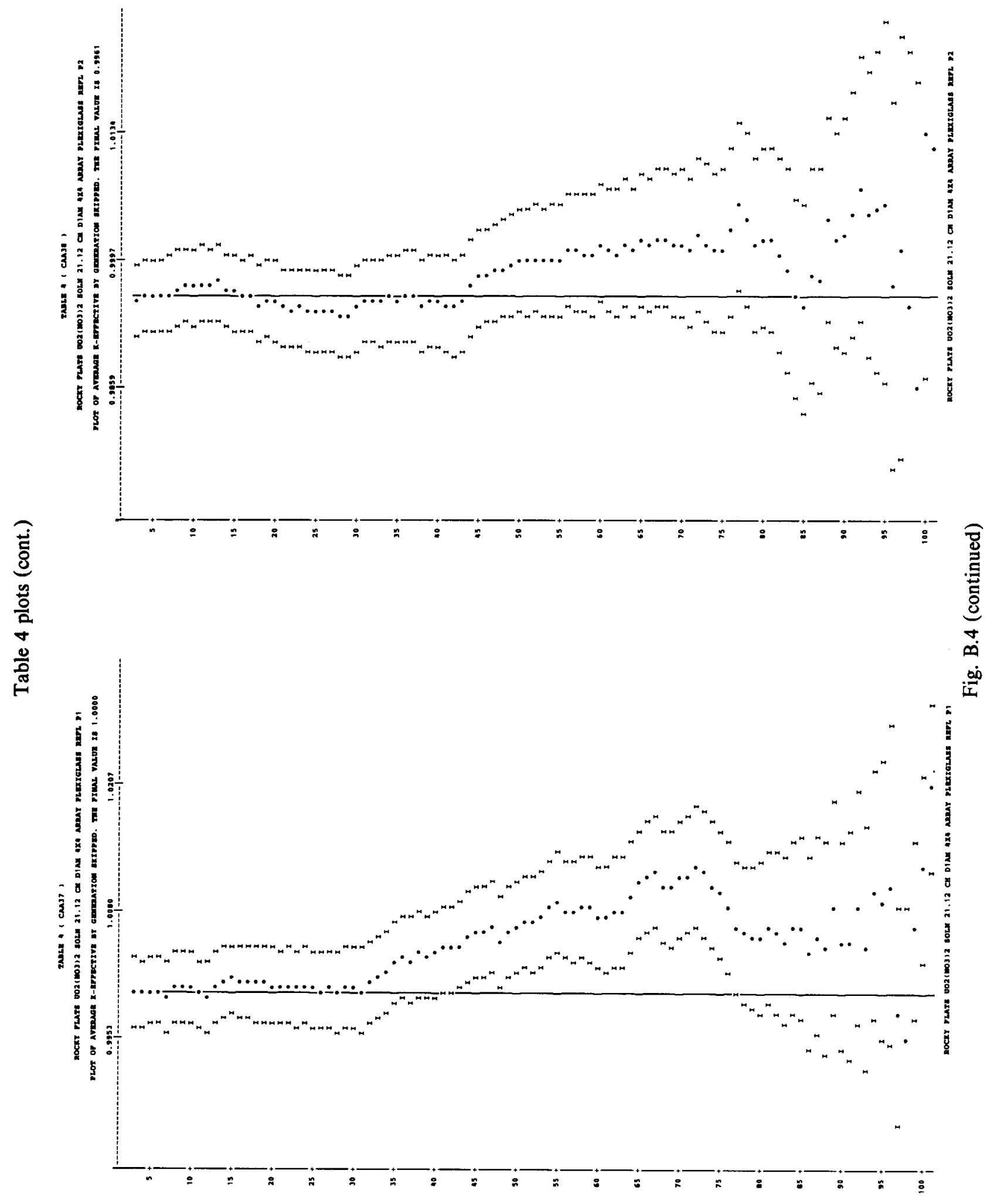



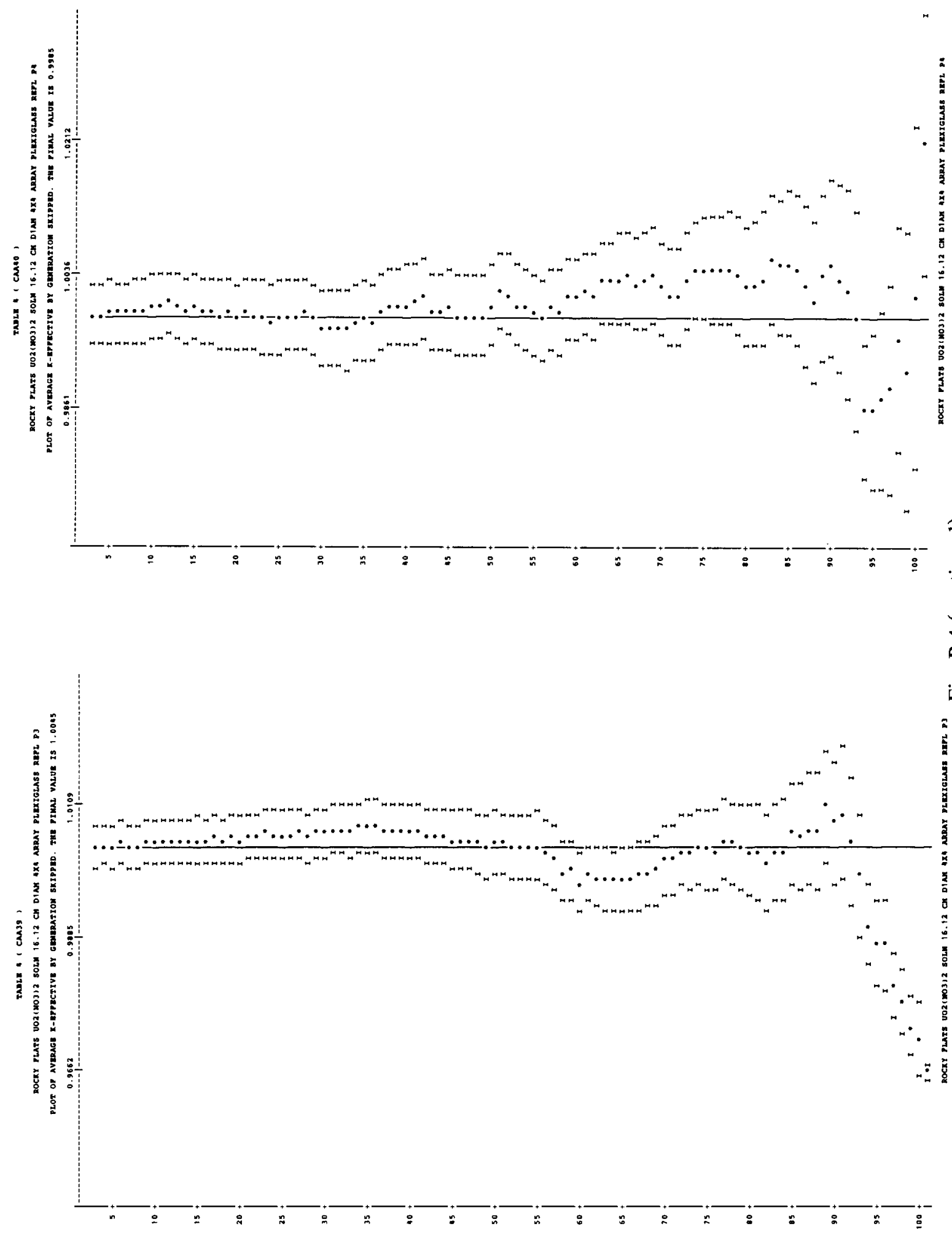

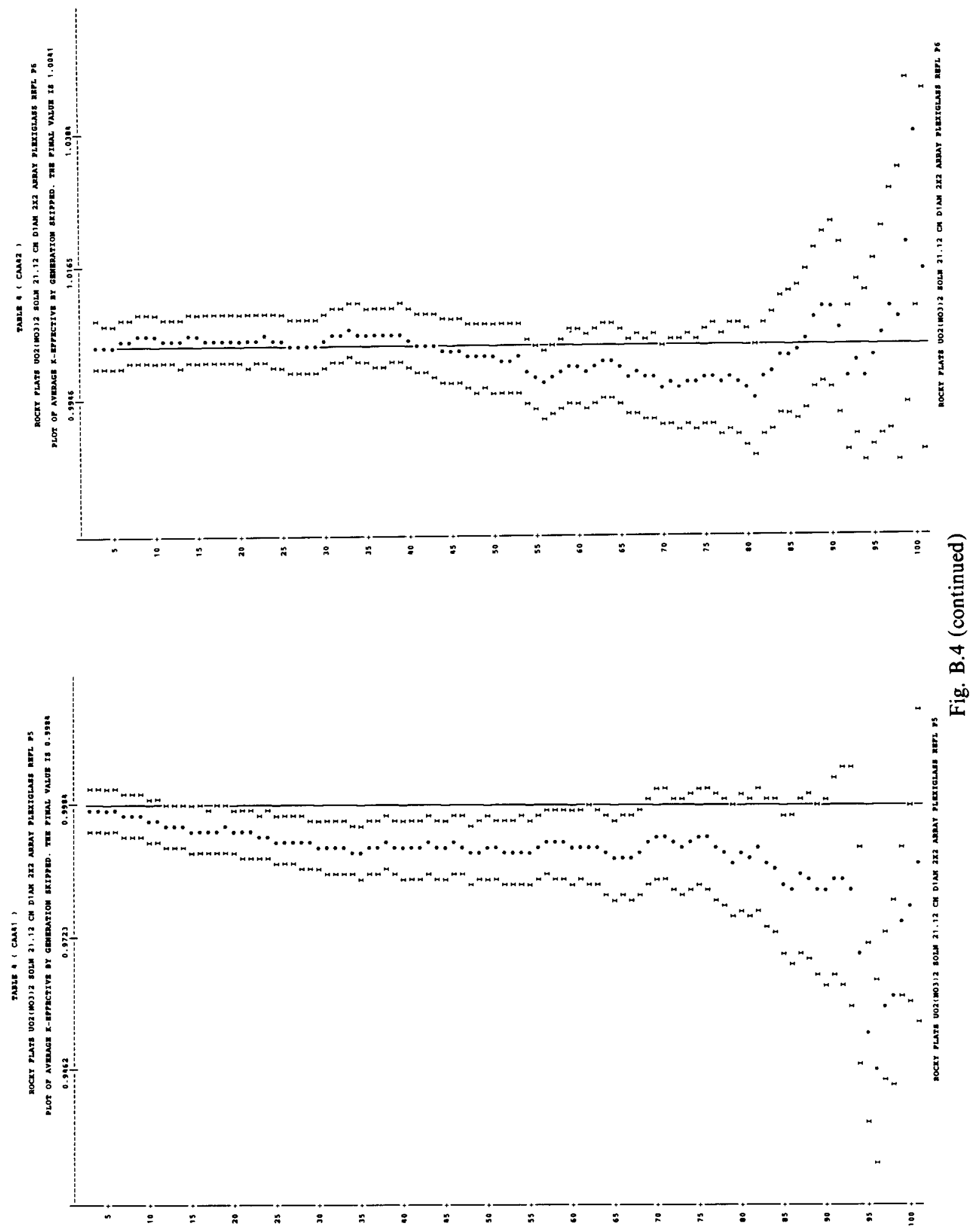


\section{B-74}

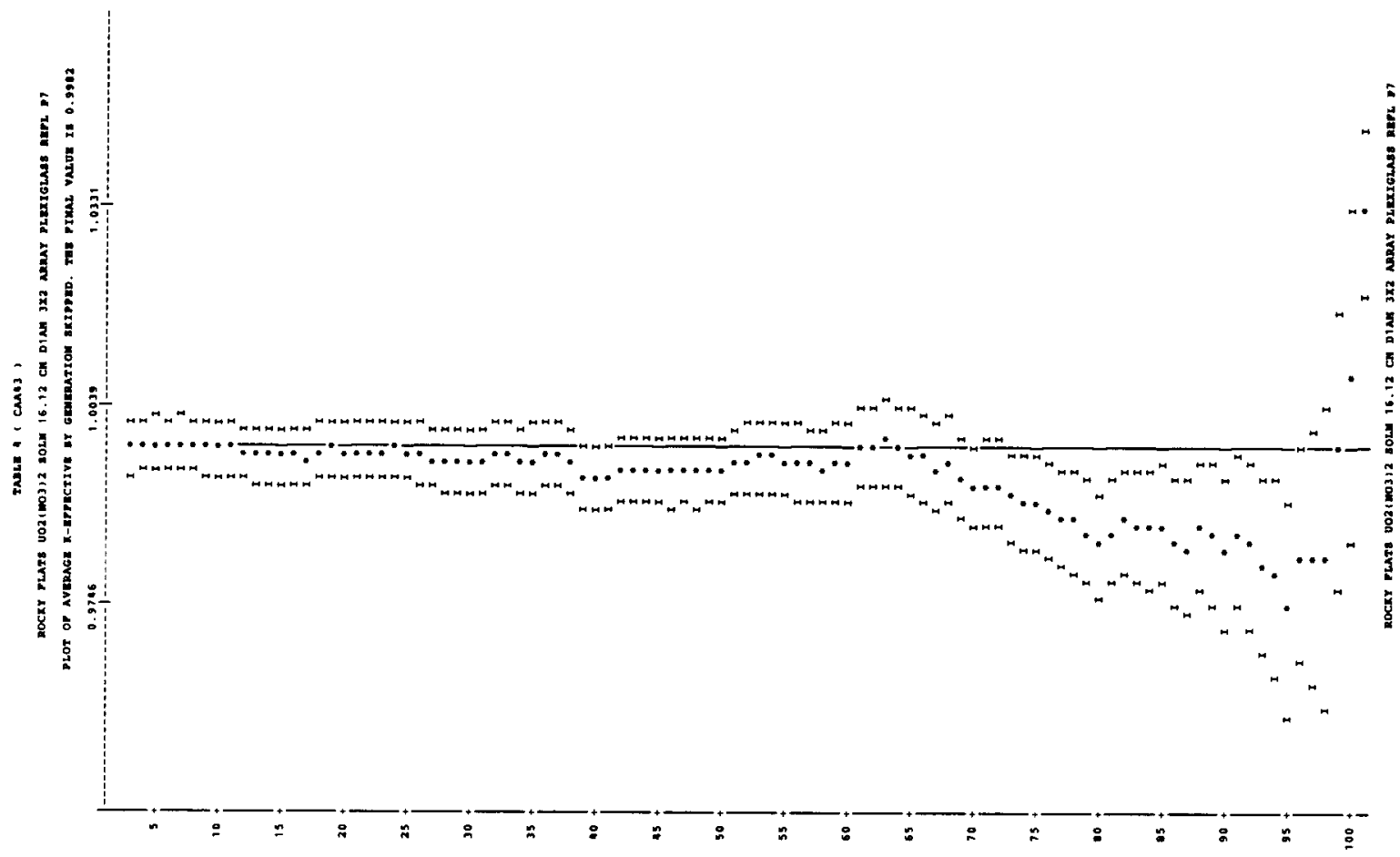



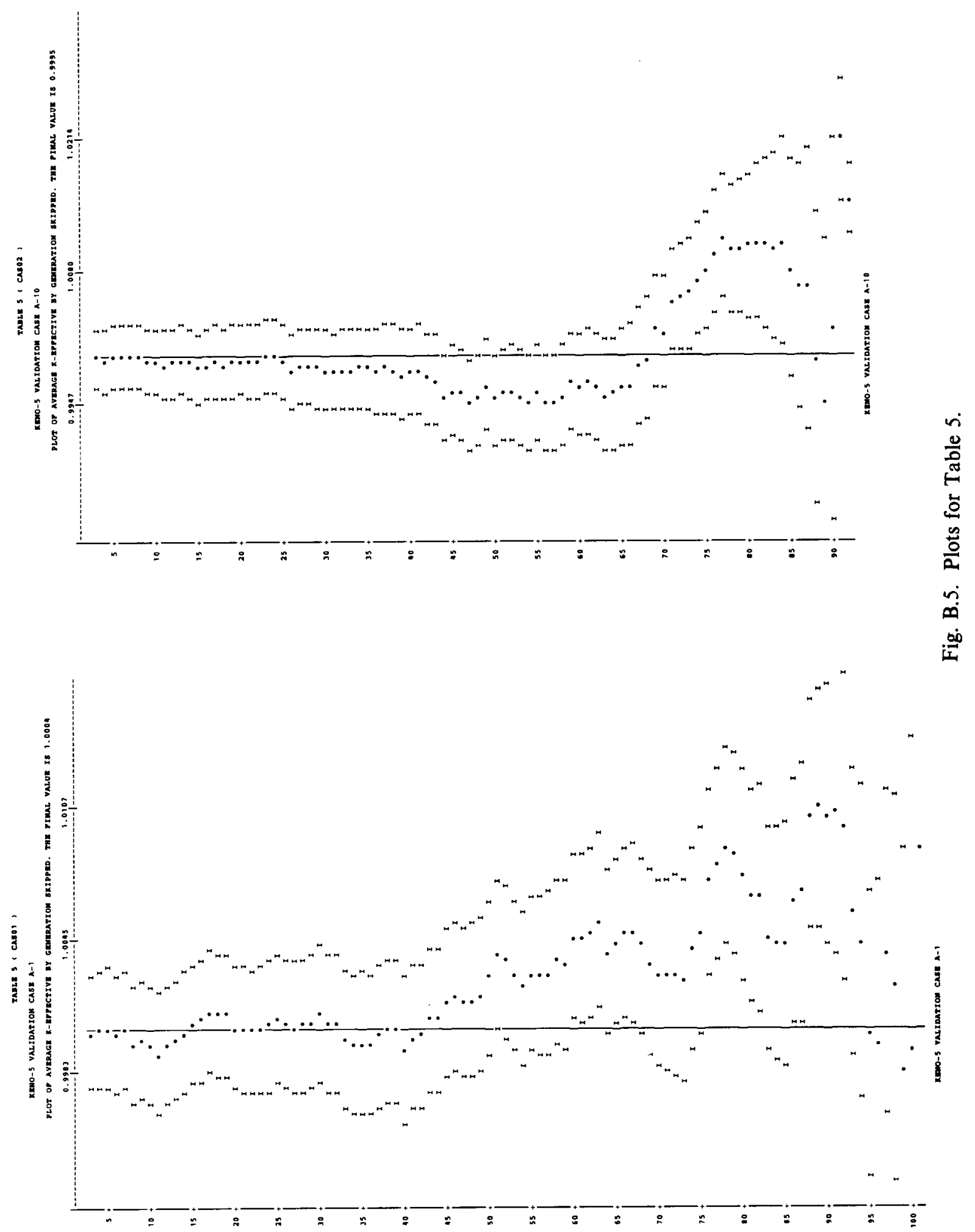


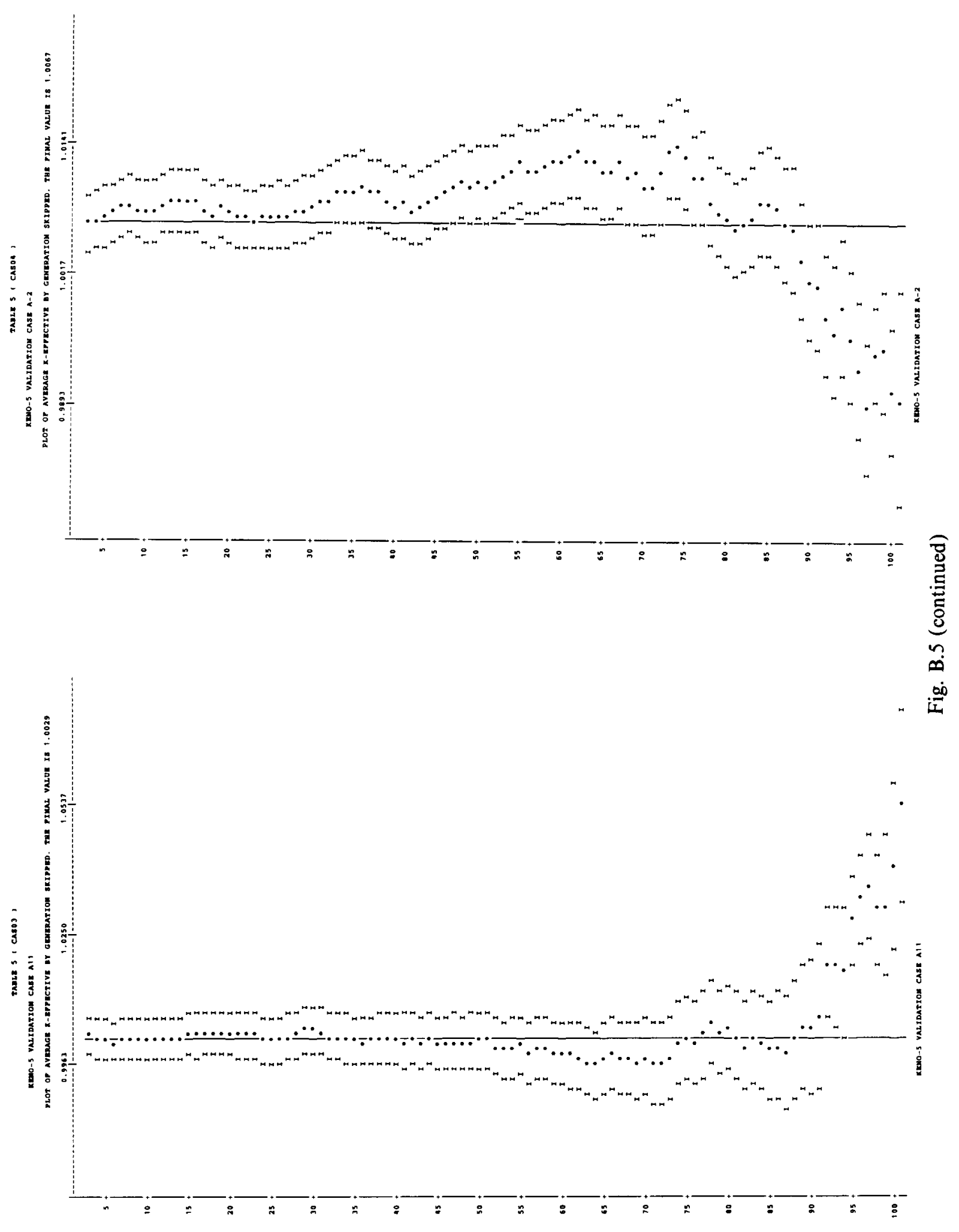




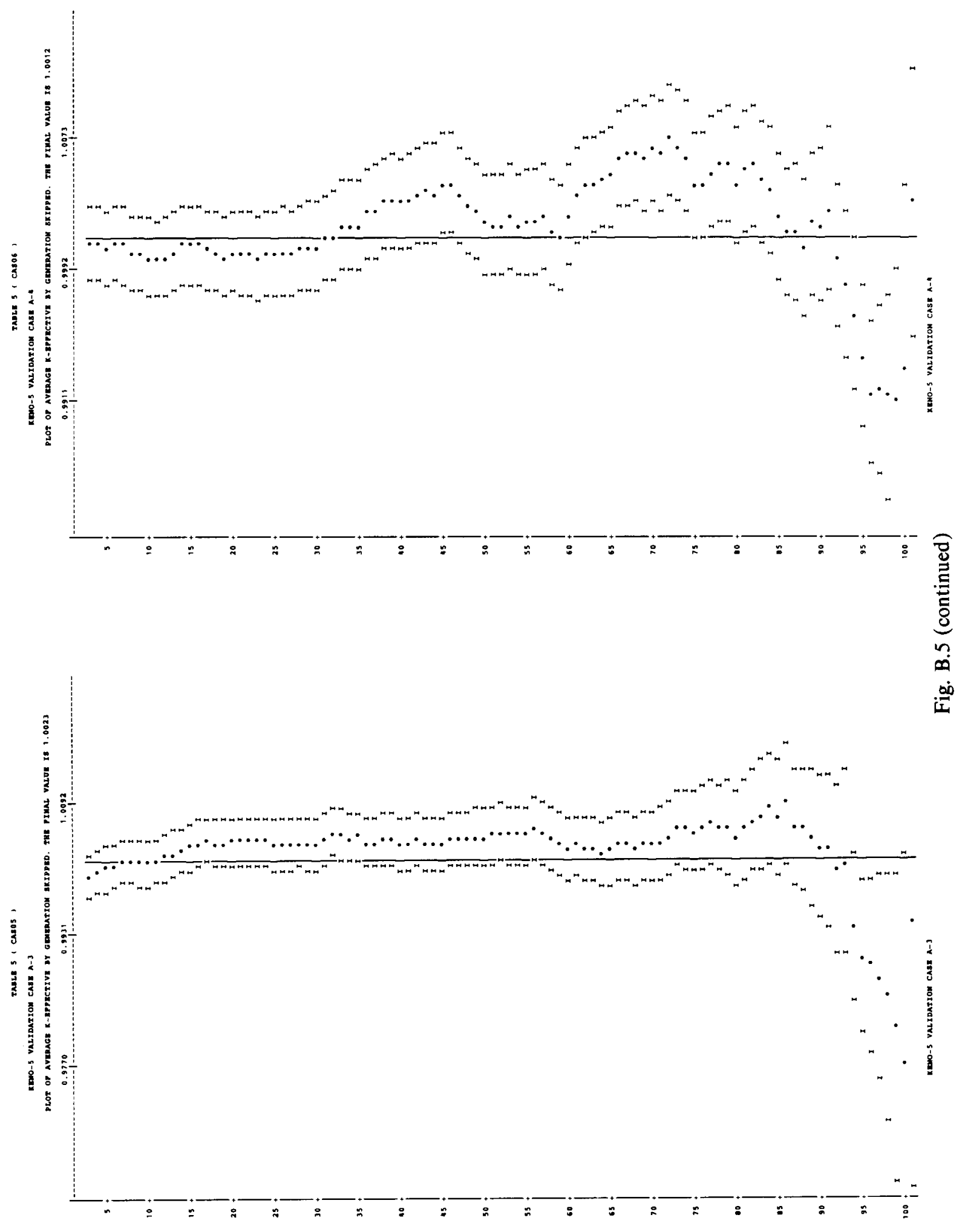




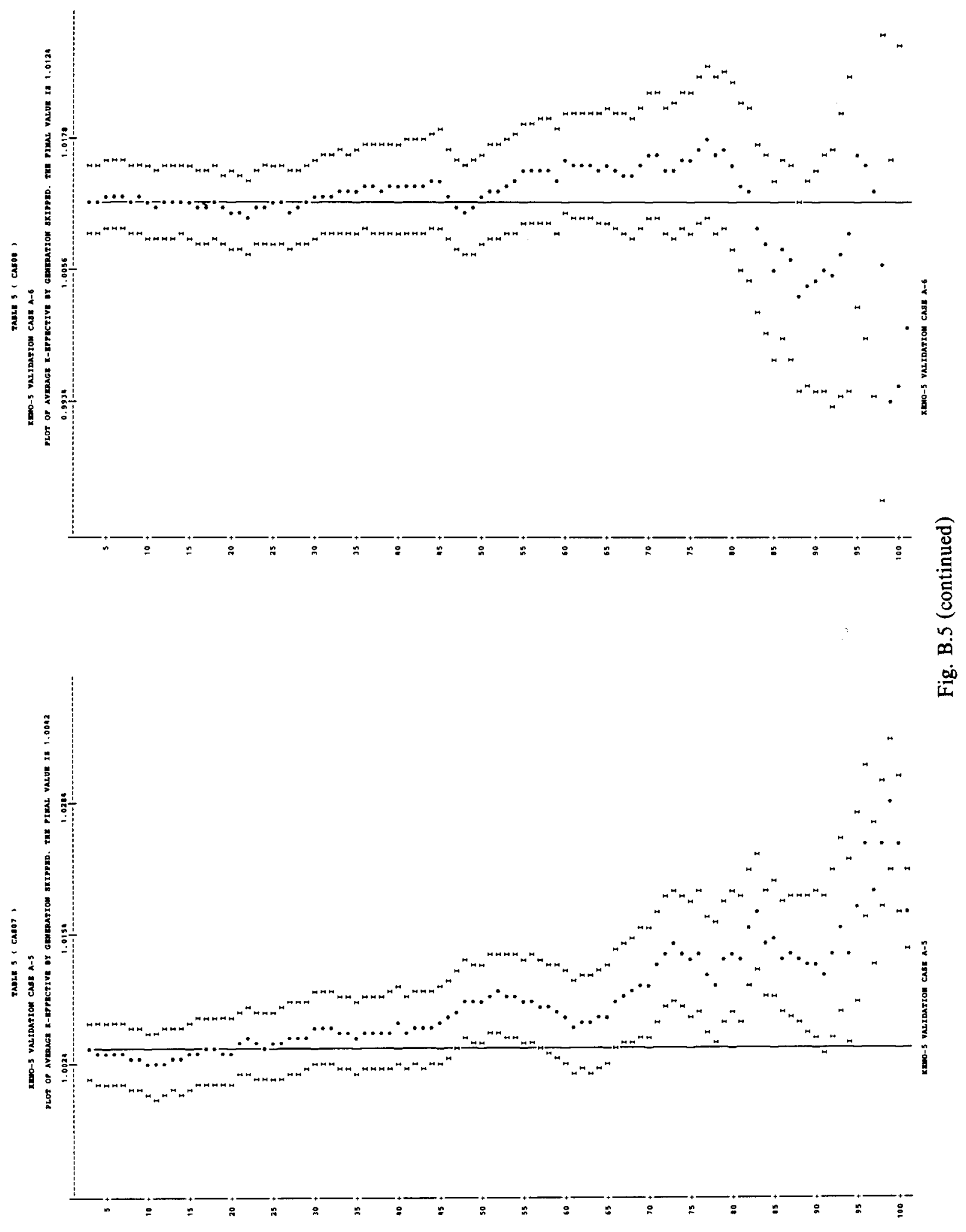



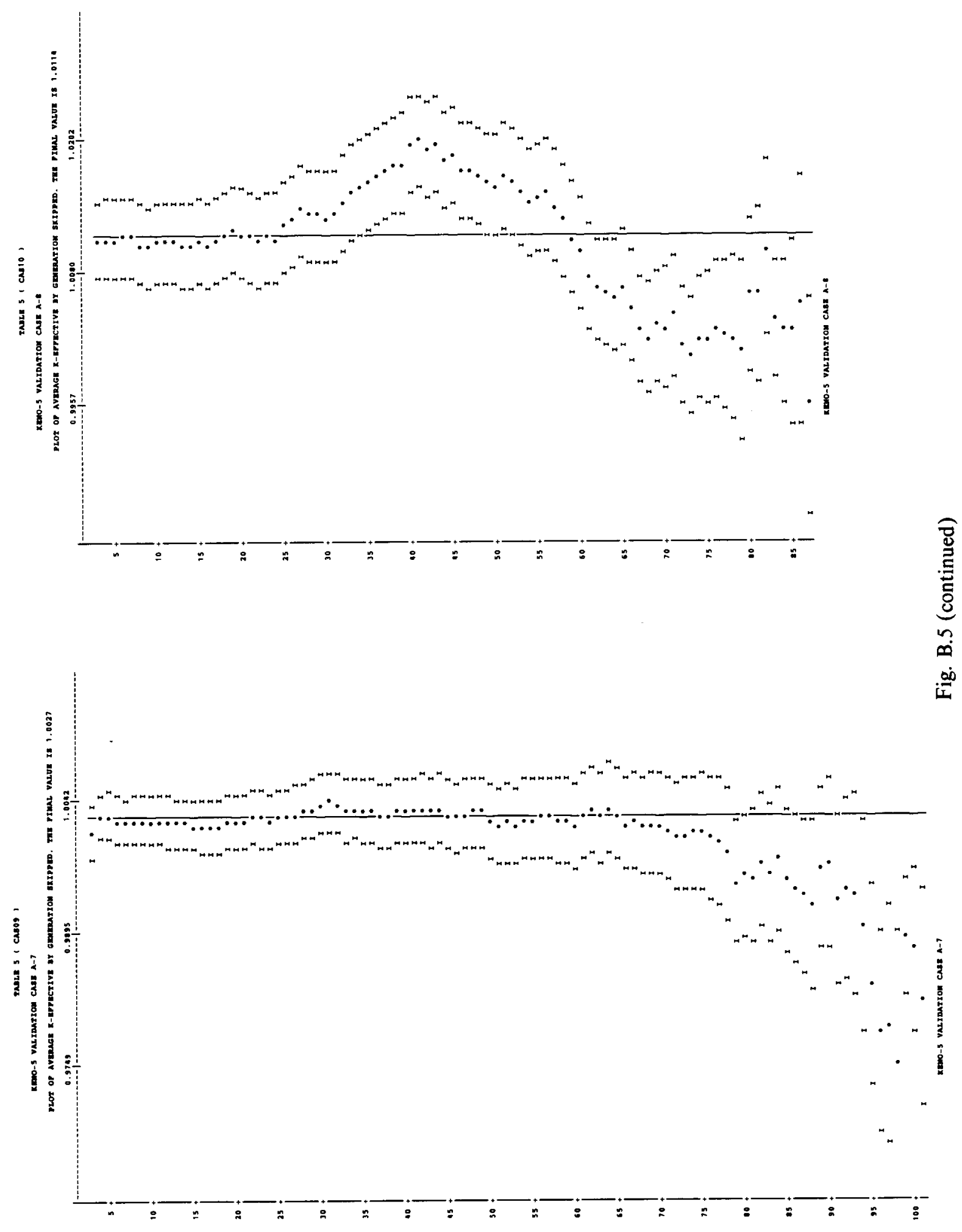


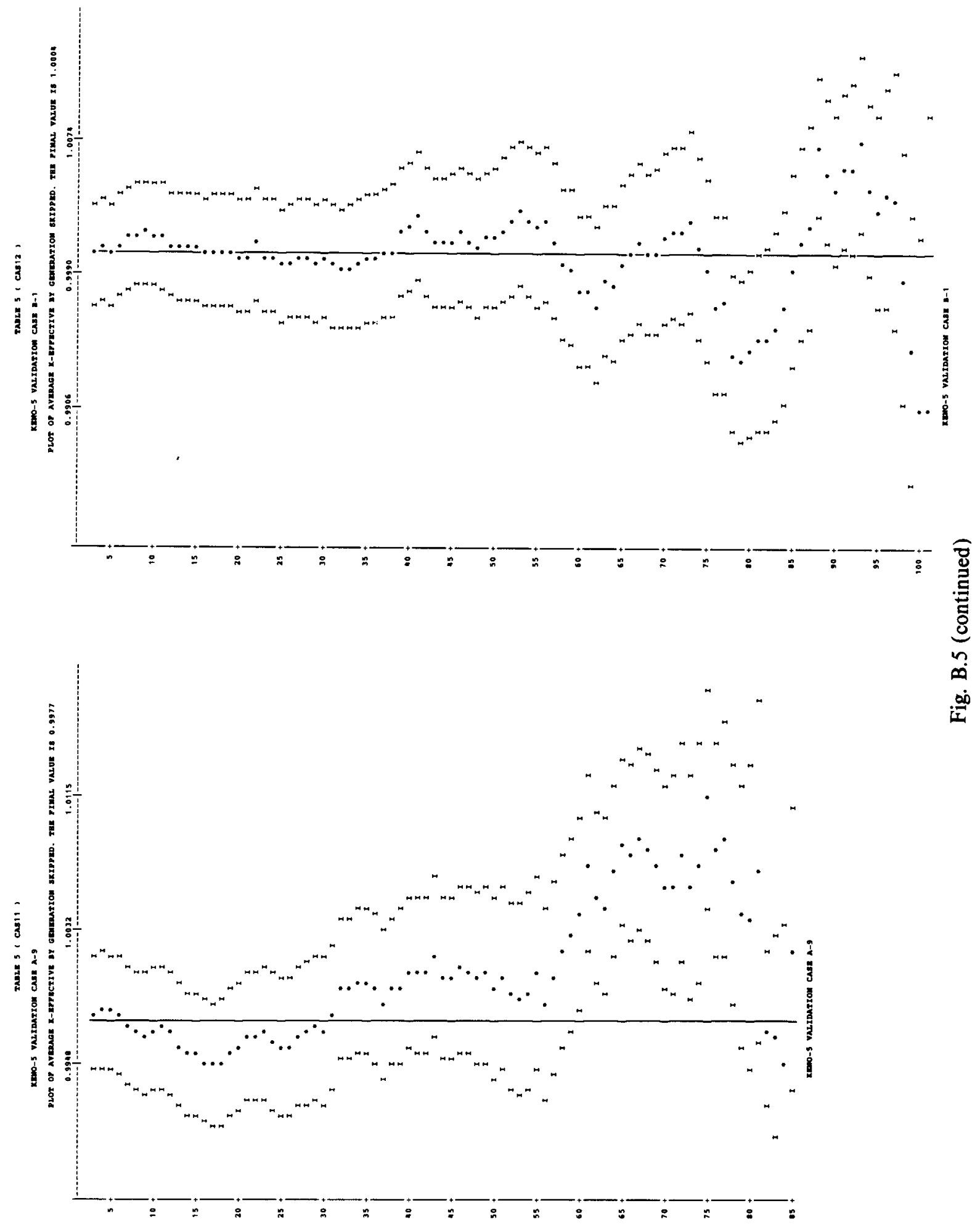


B-81

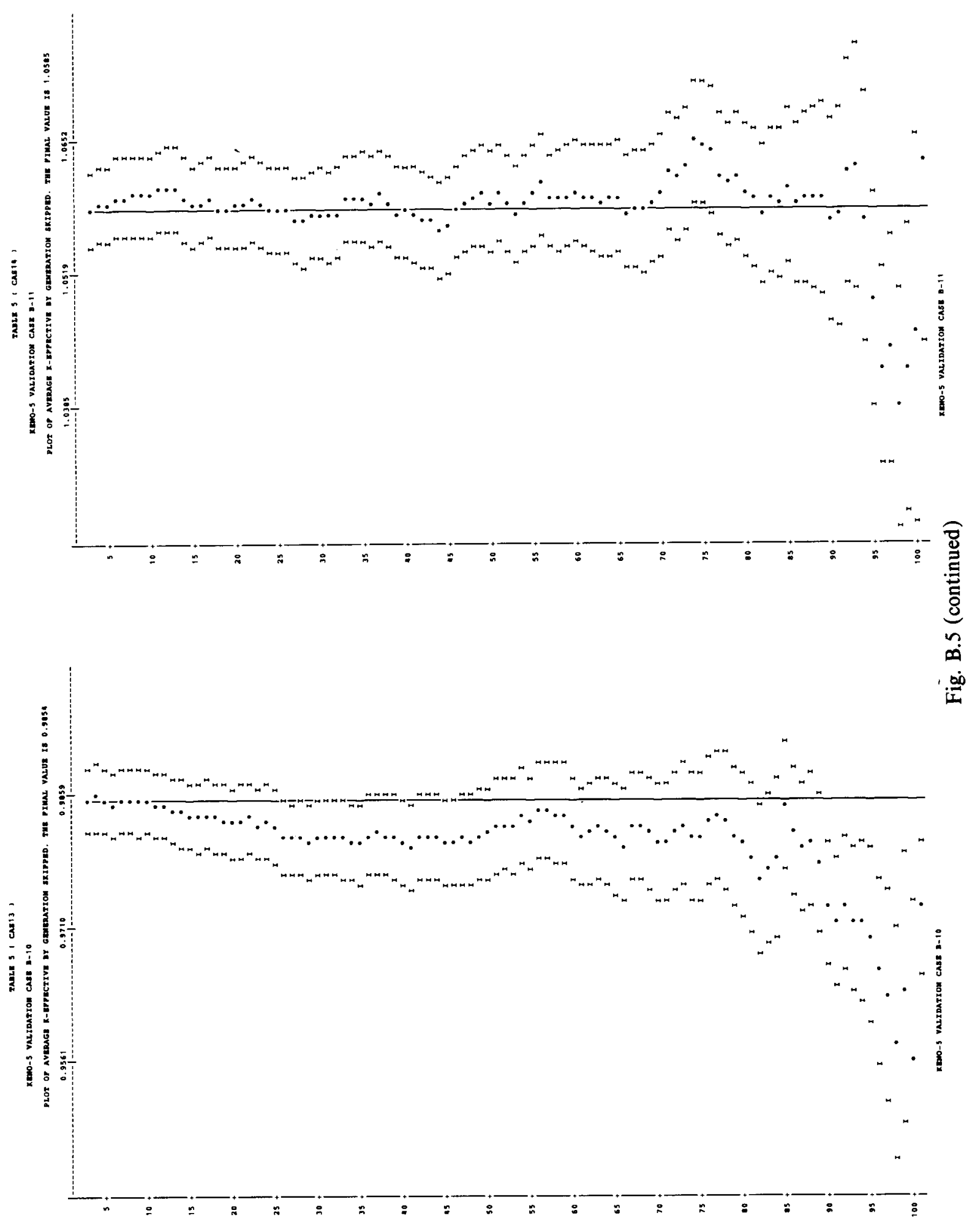


B-82

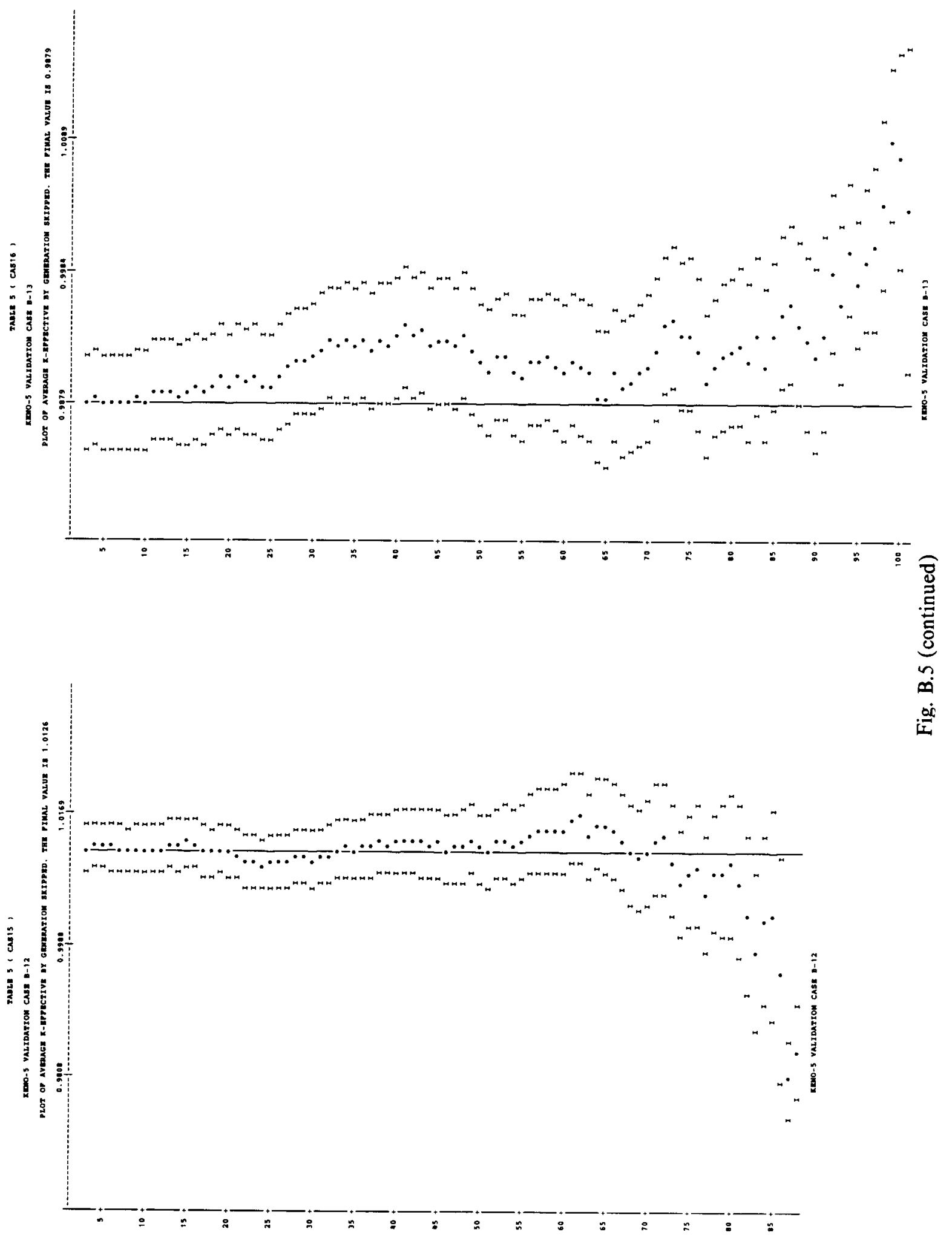




\section{B-83}

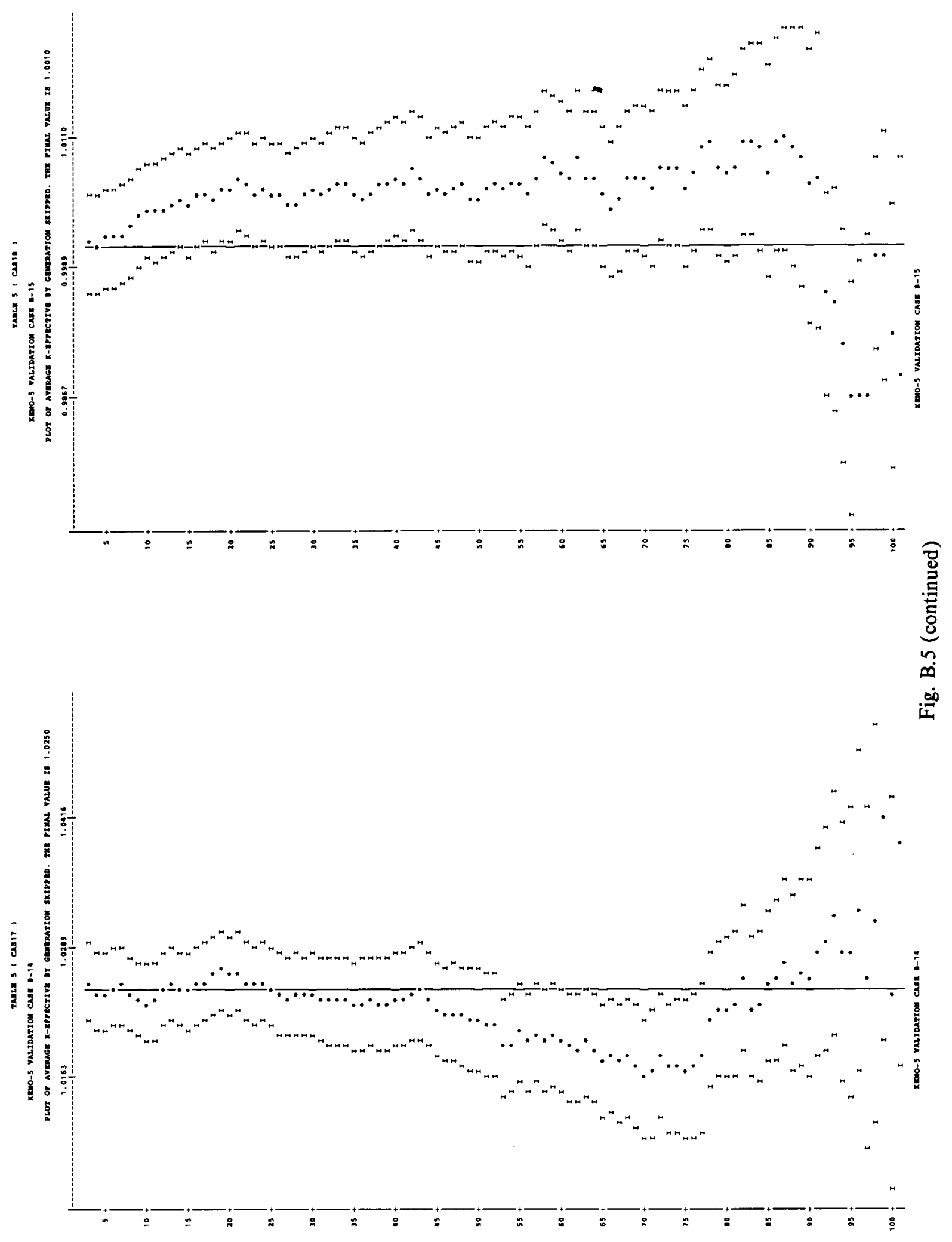


B-84
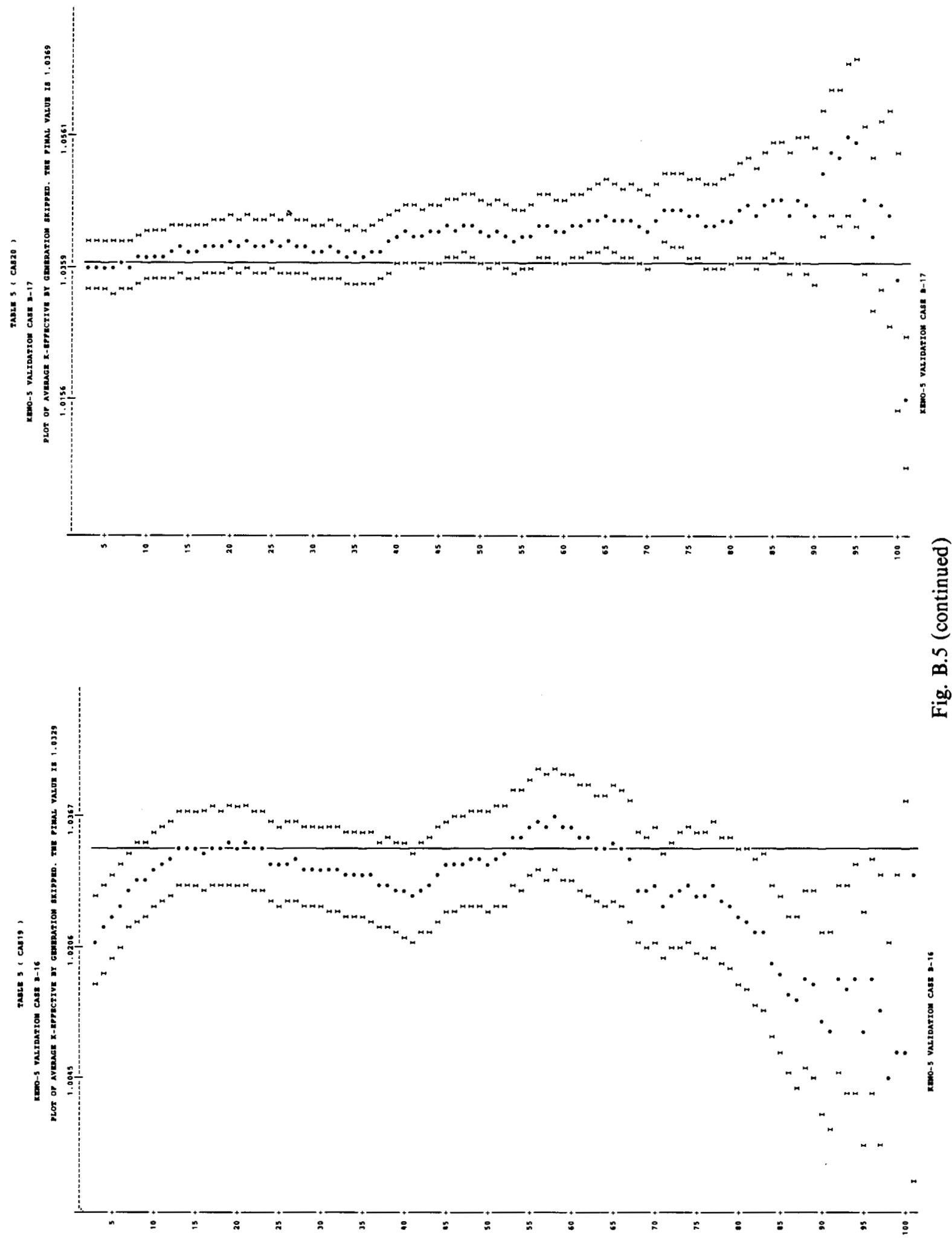

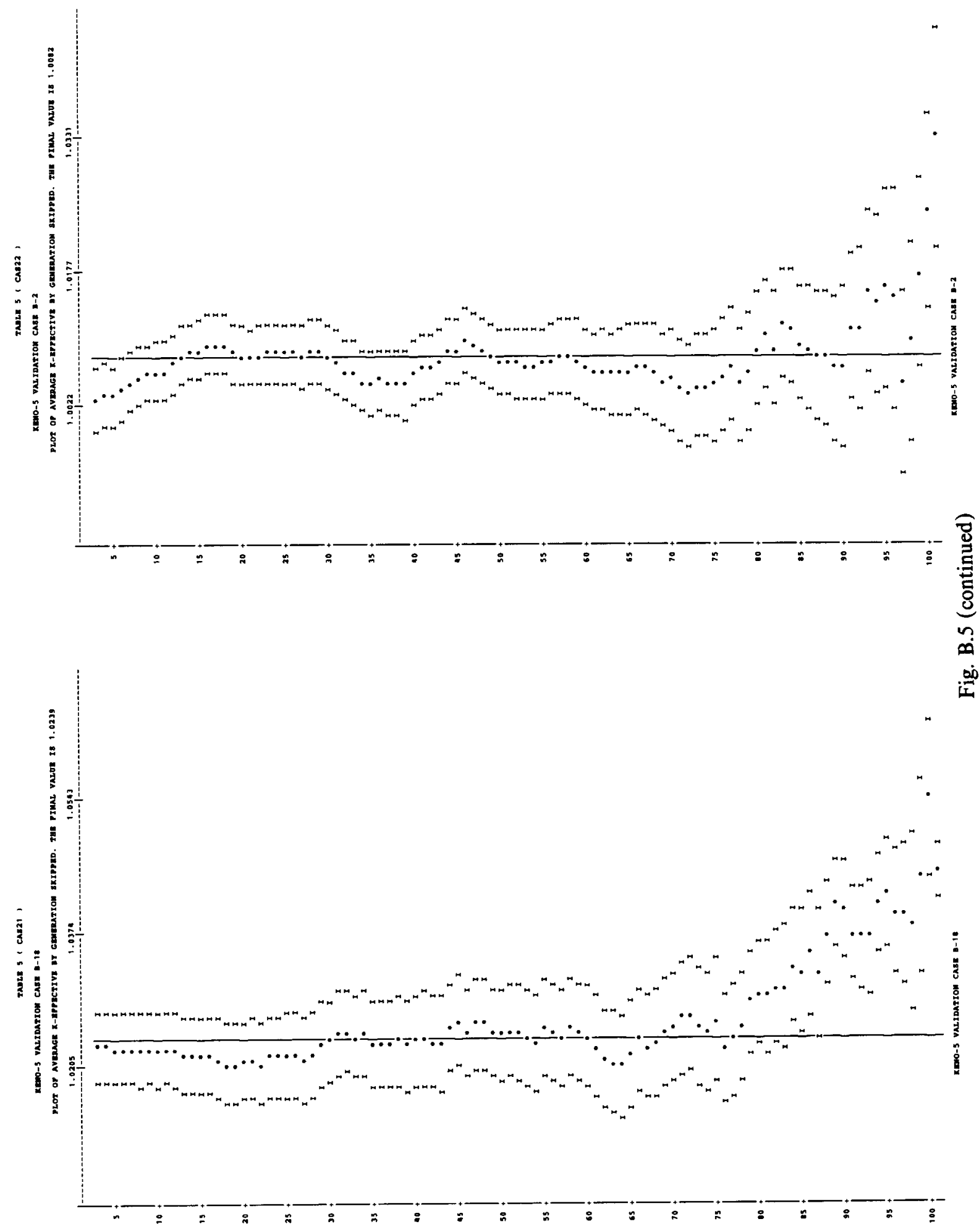

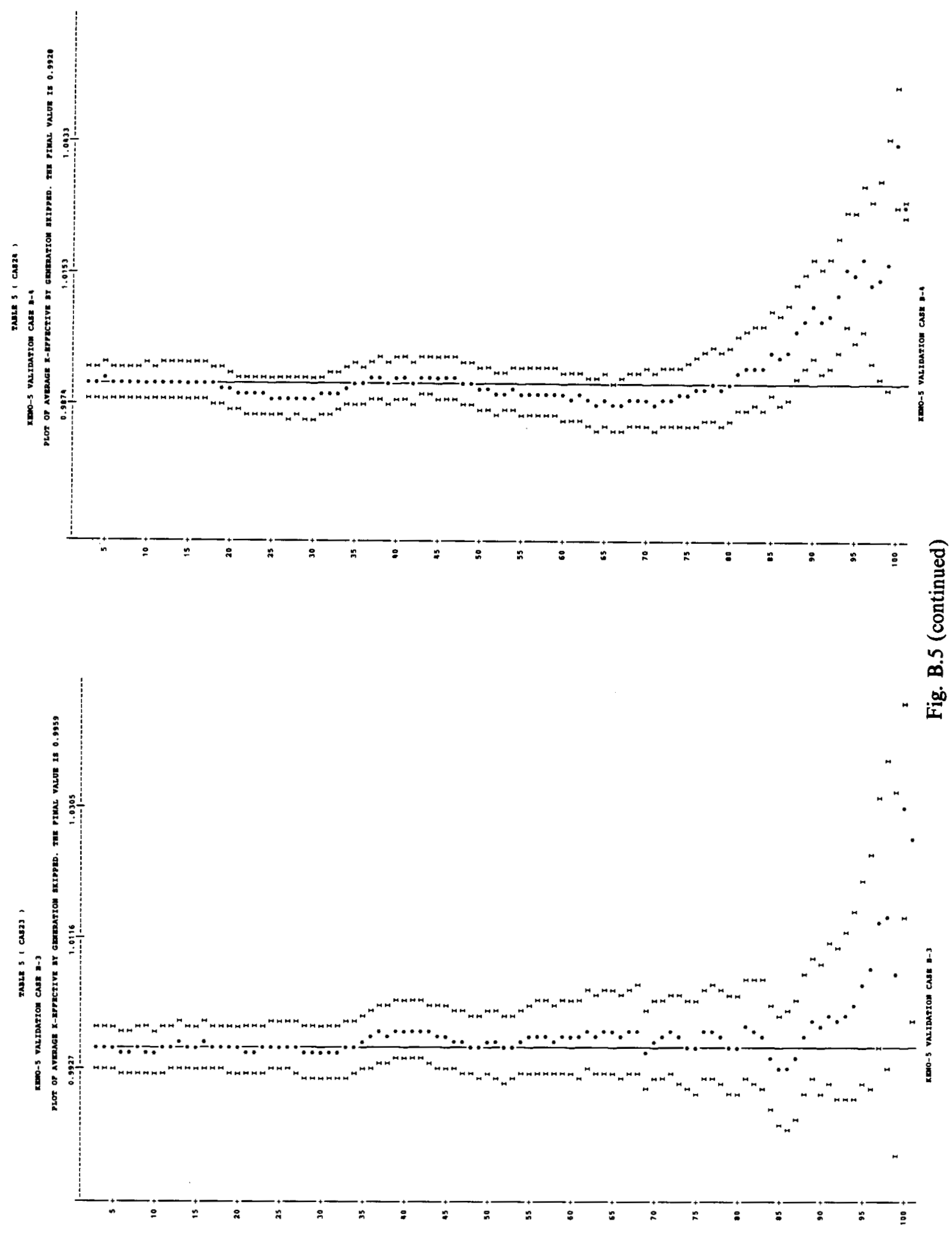


\section{B-87}

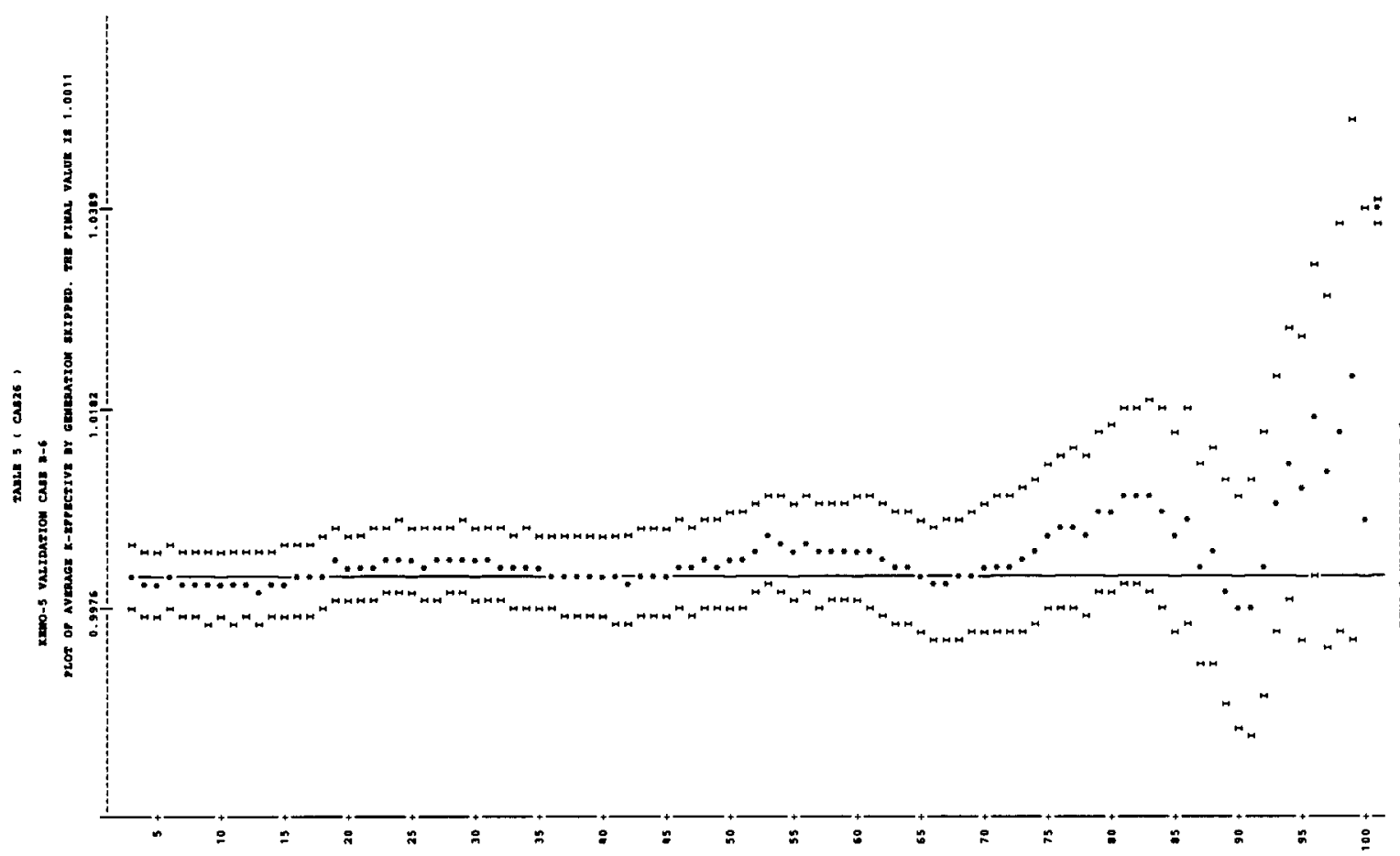

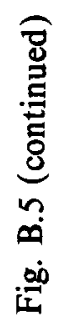

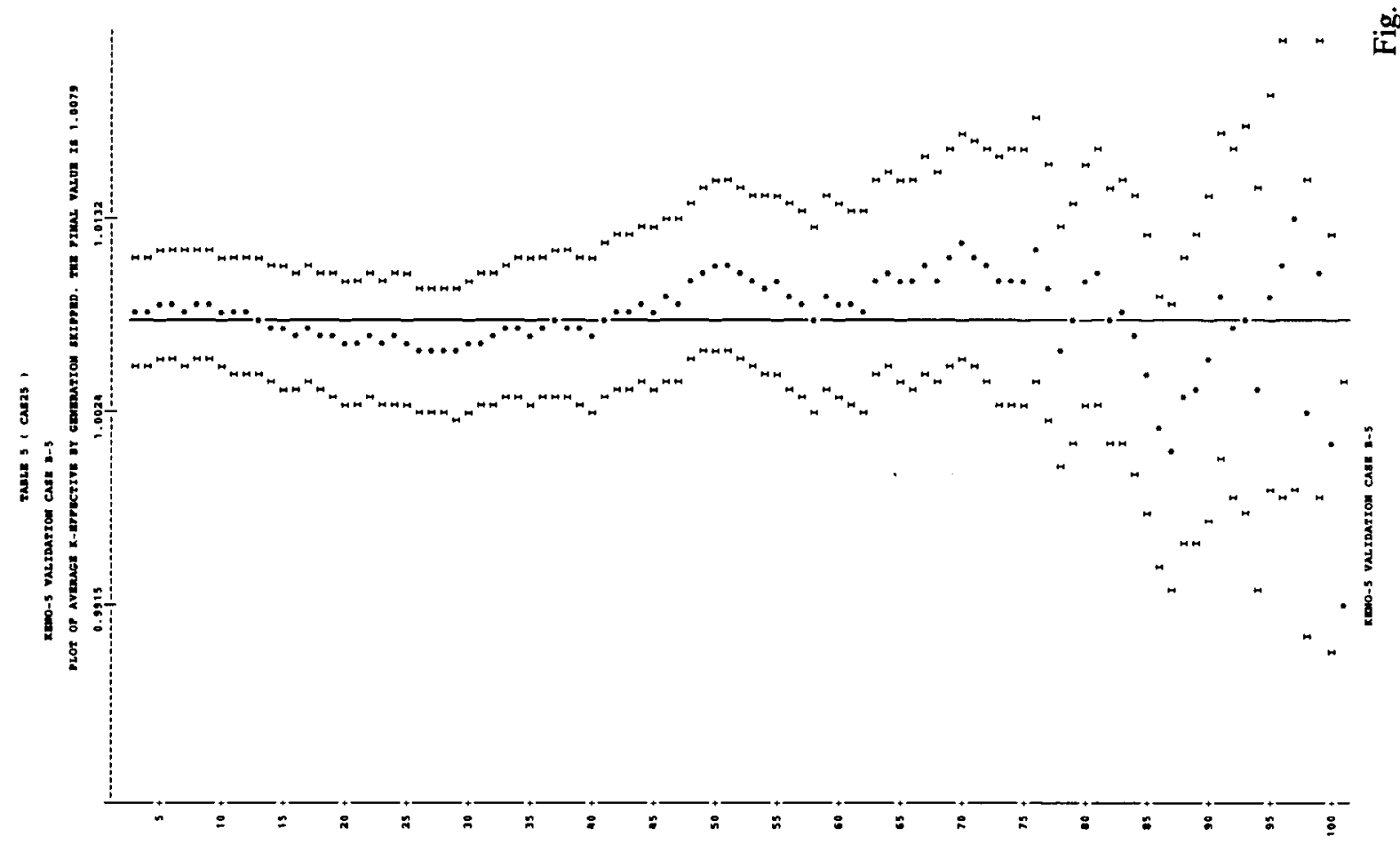




\section{B- 88}

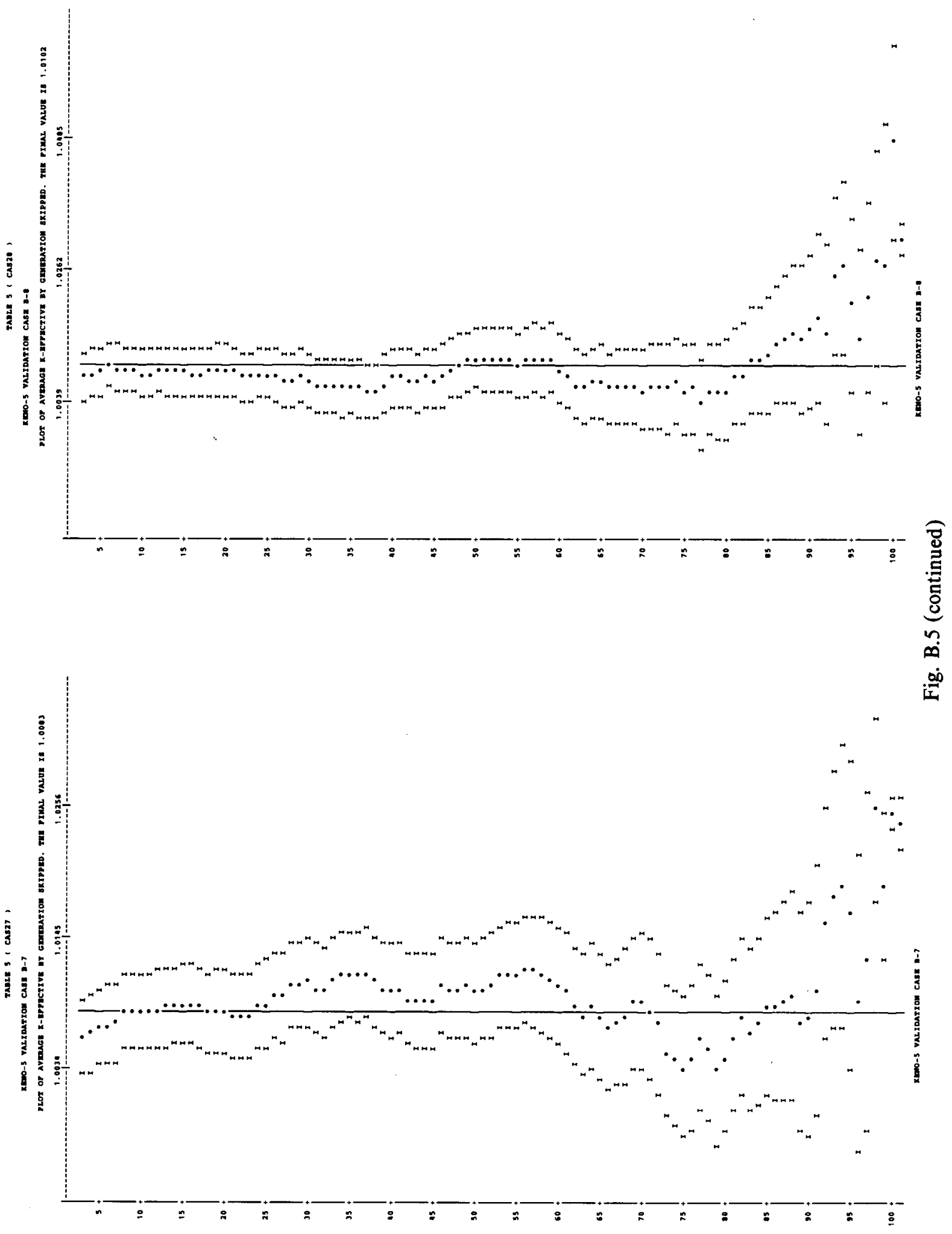




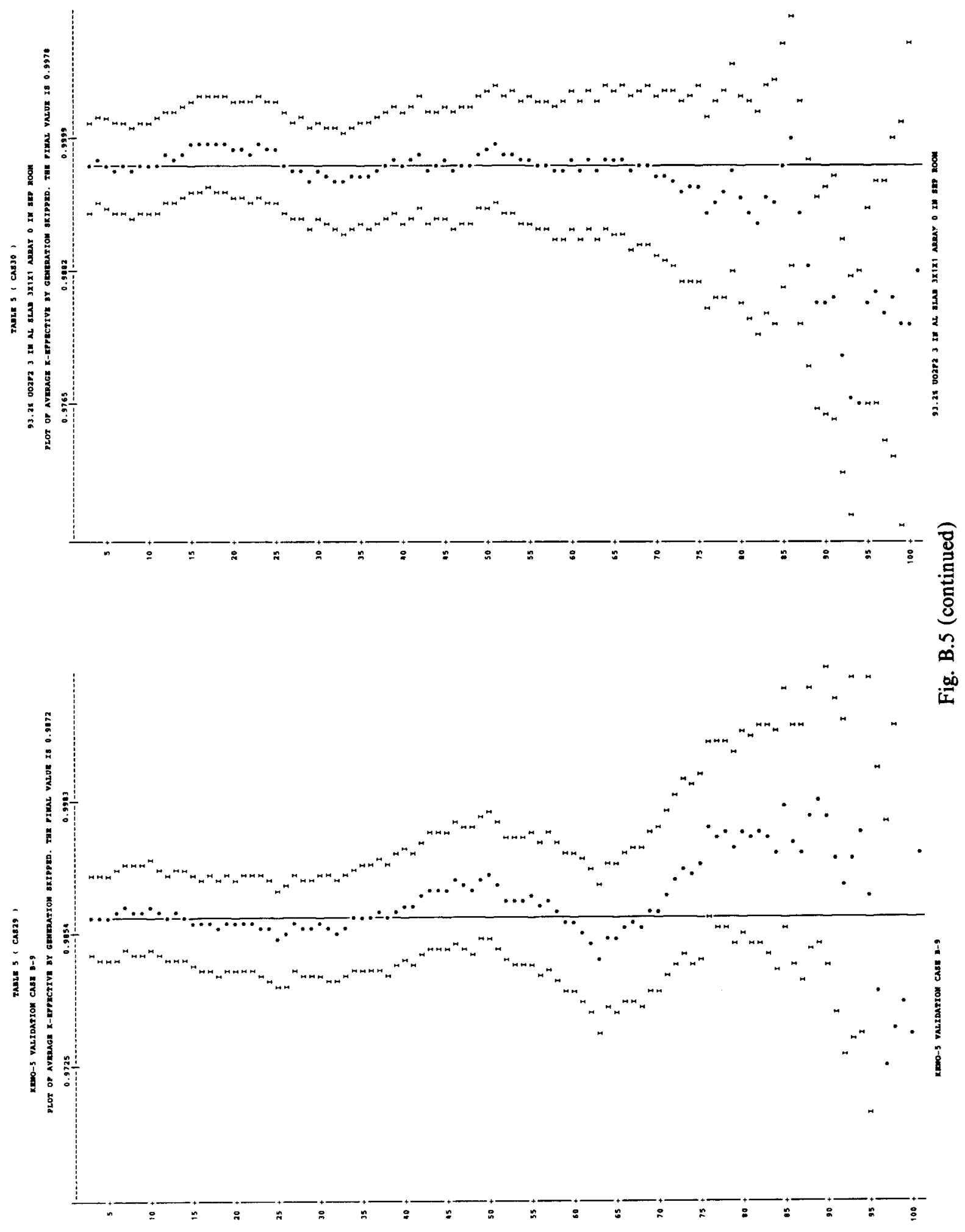



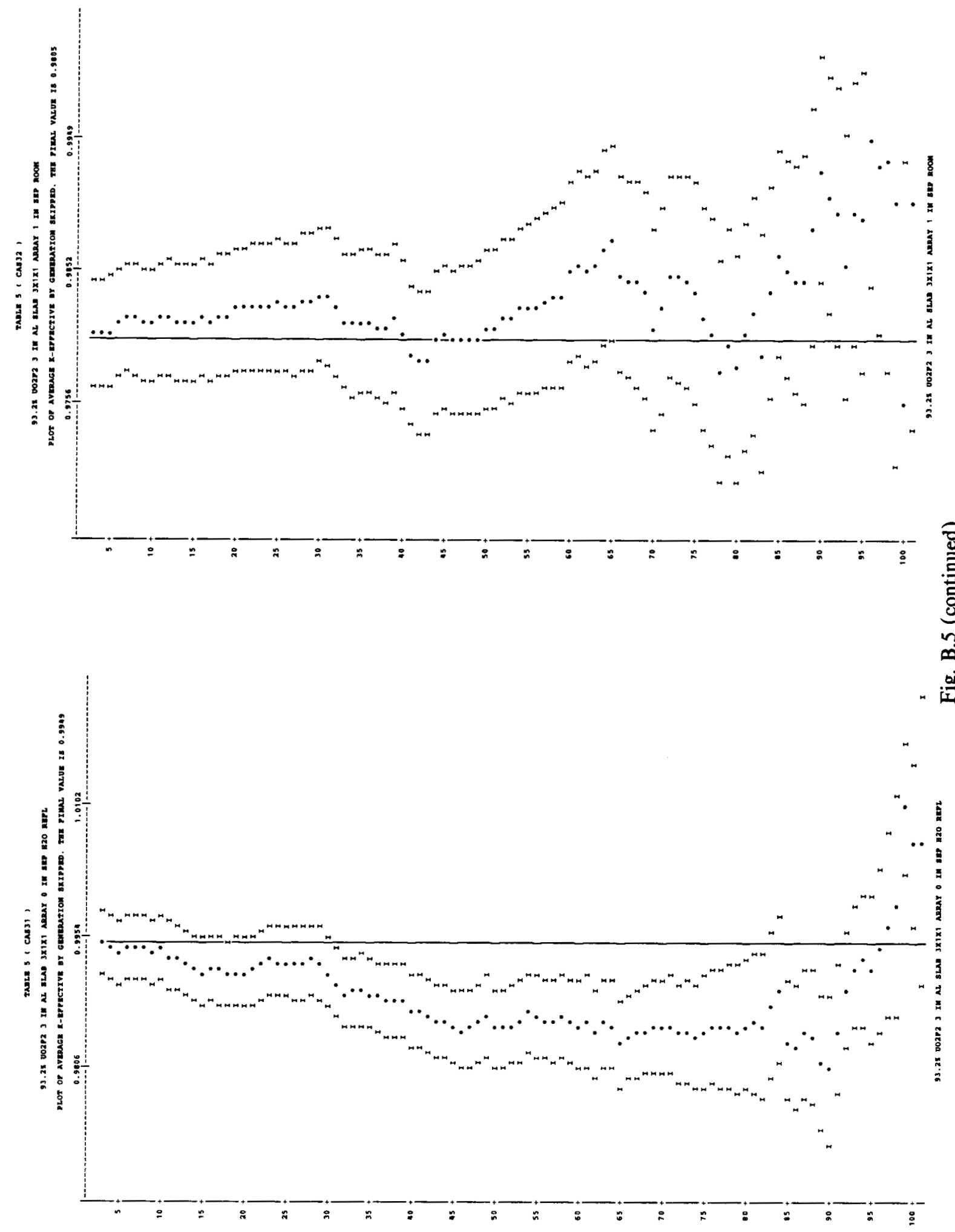

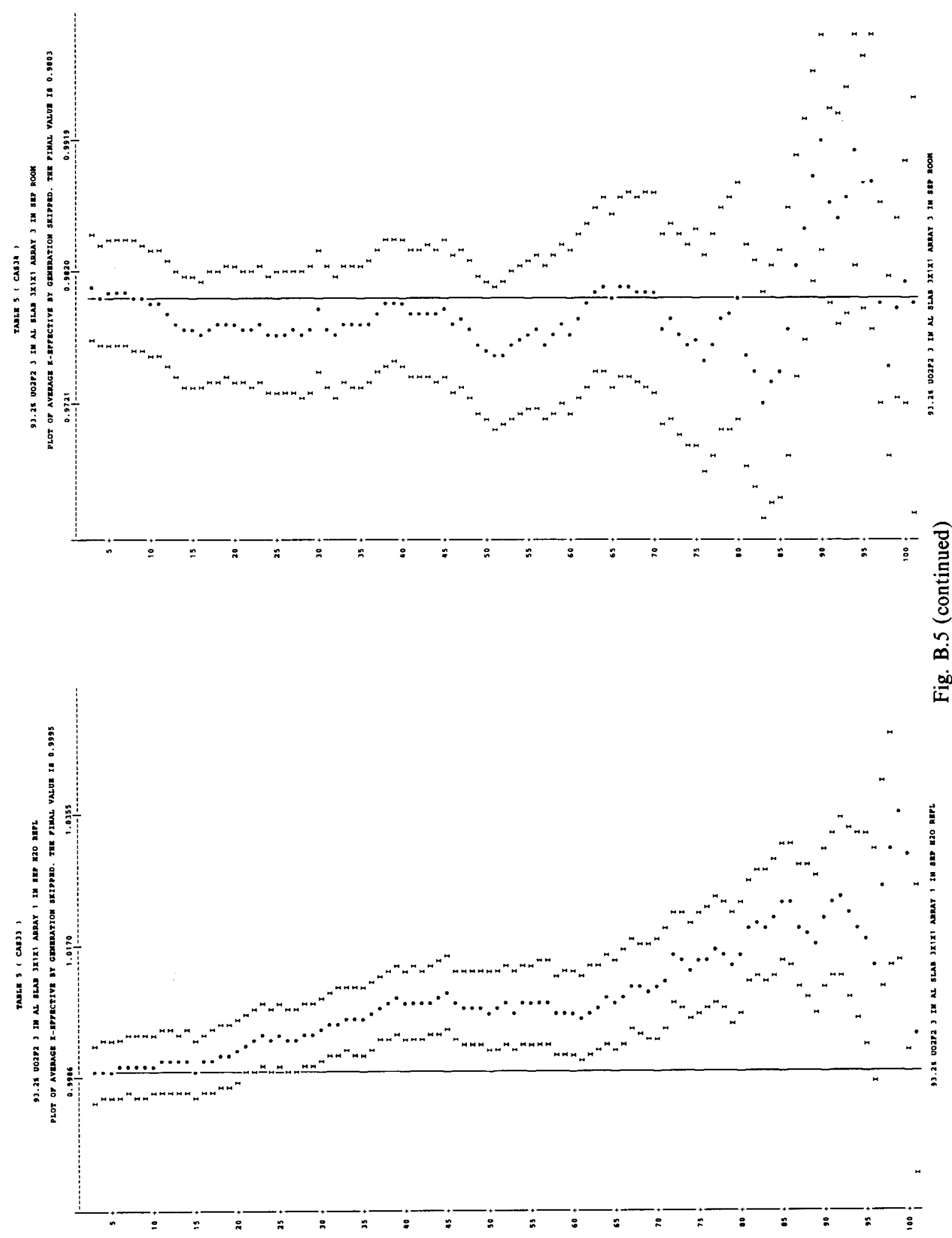


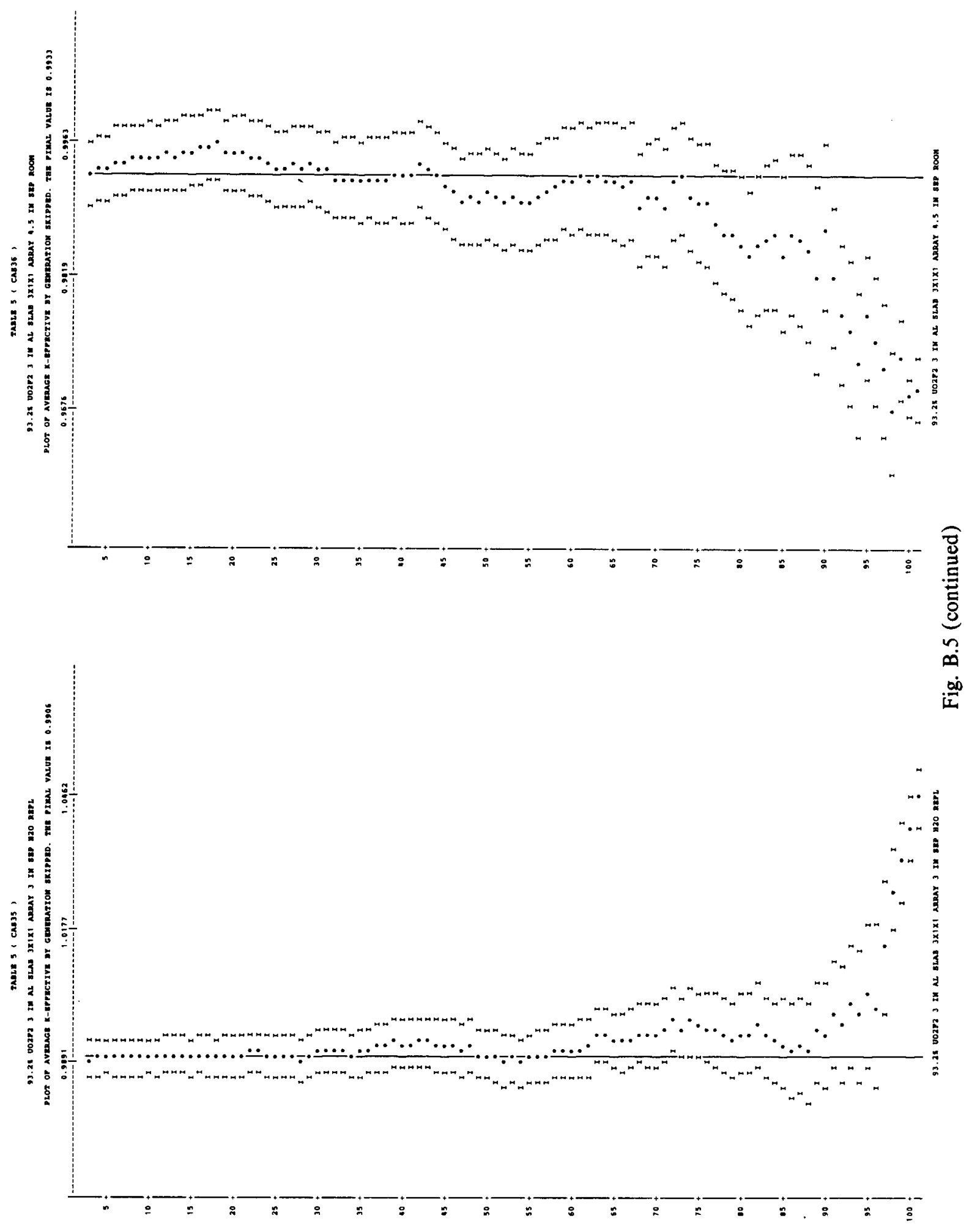



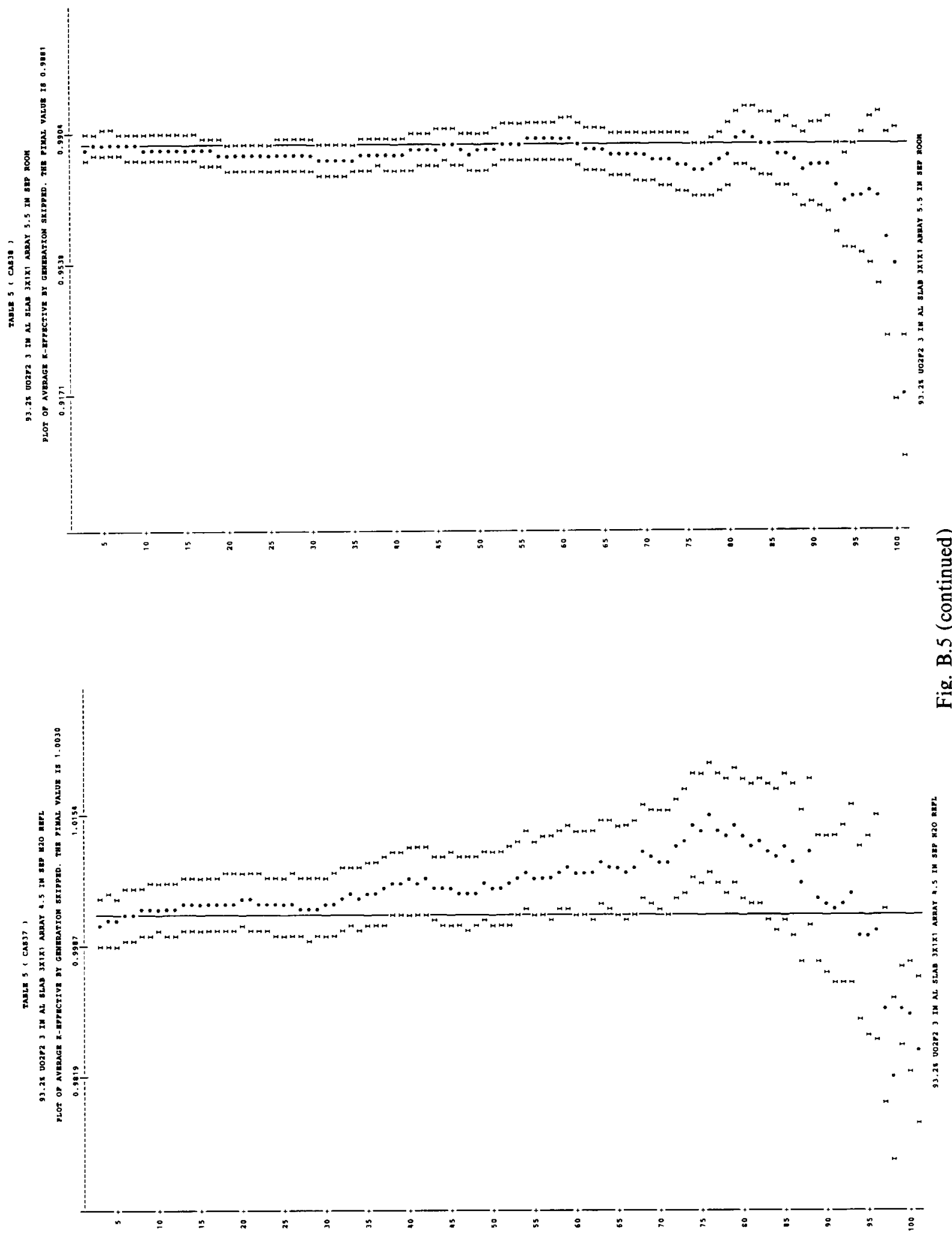


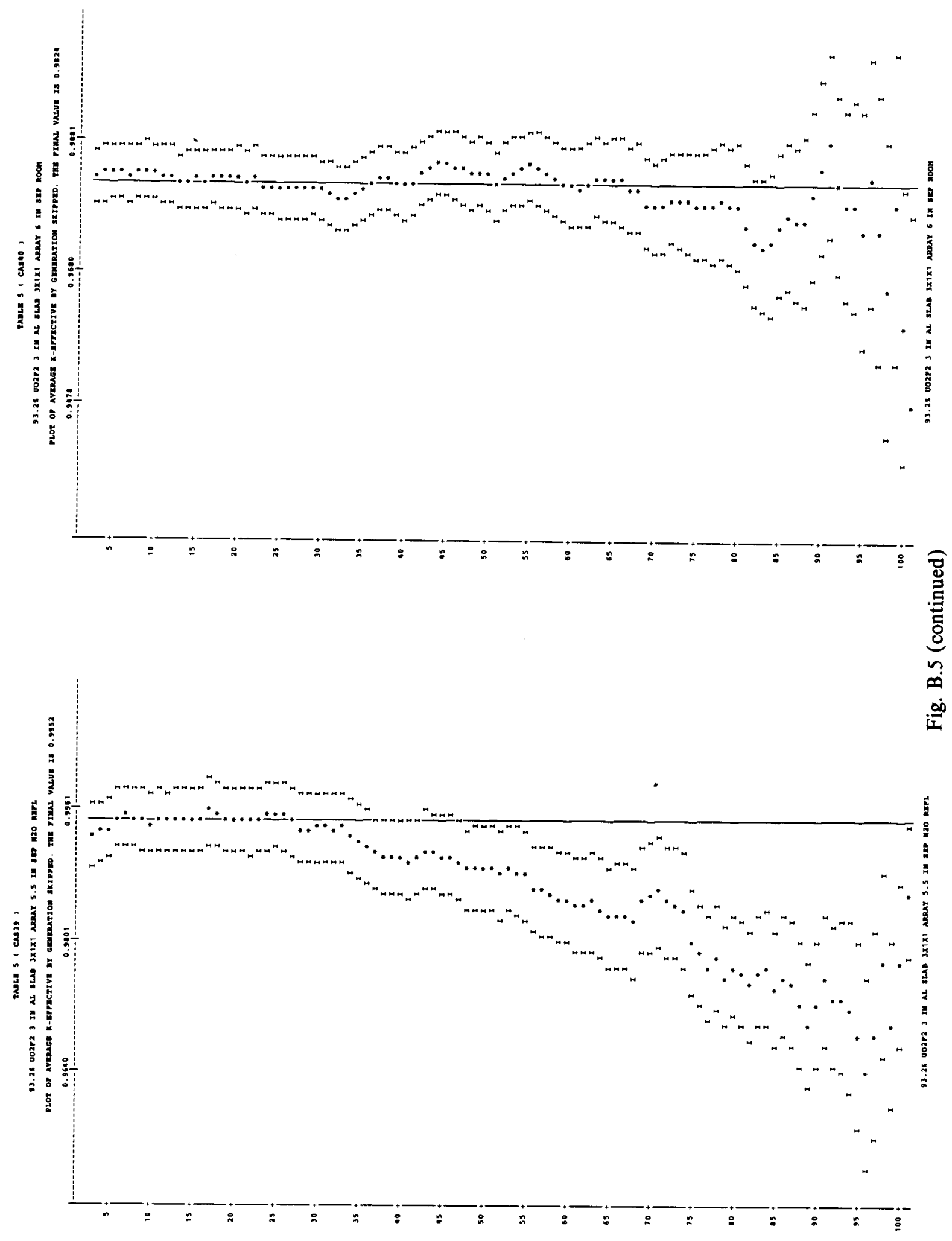



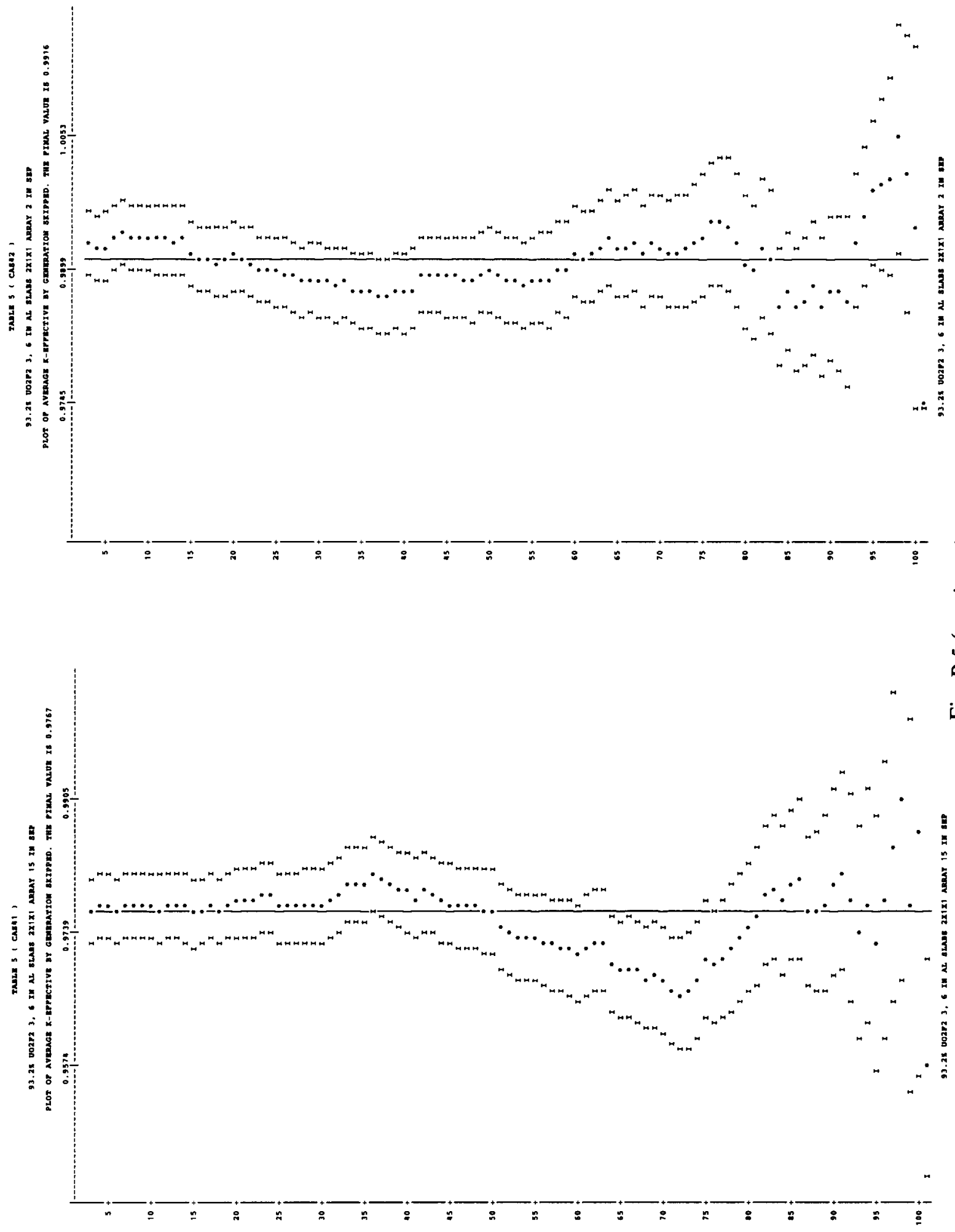


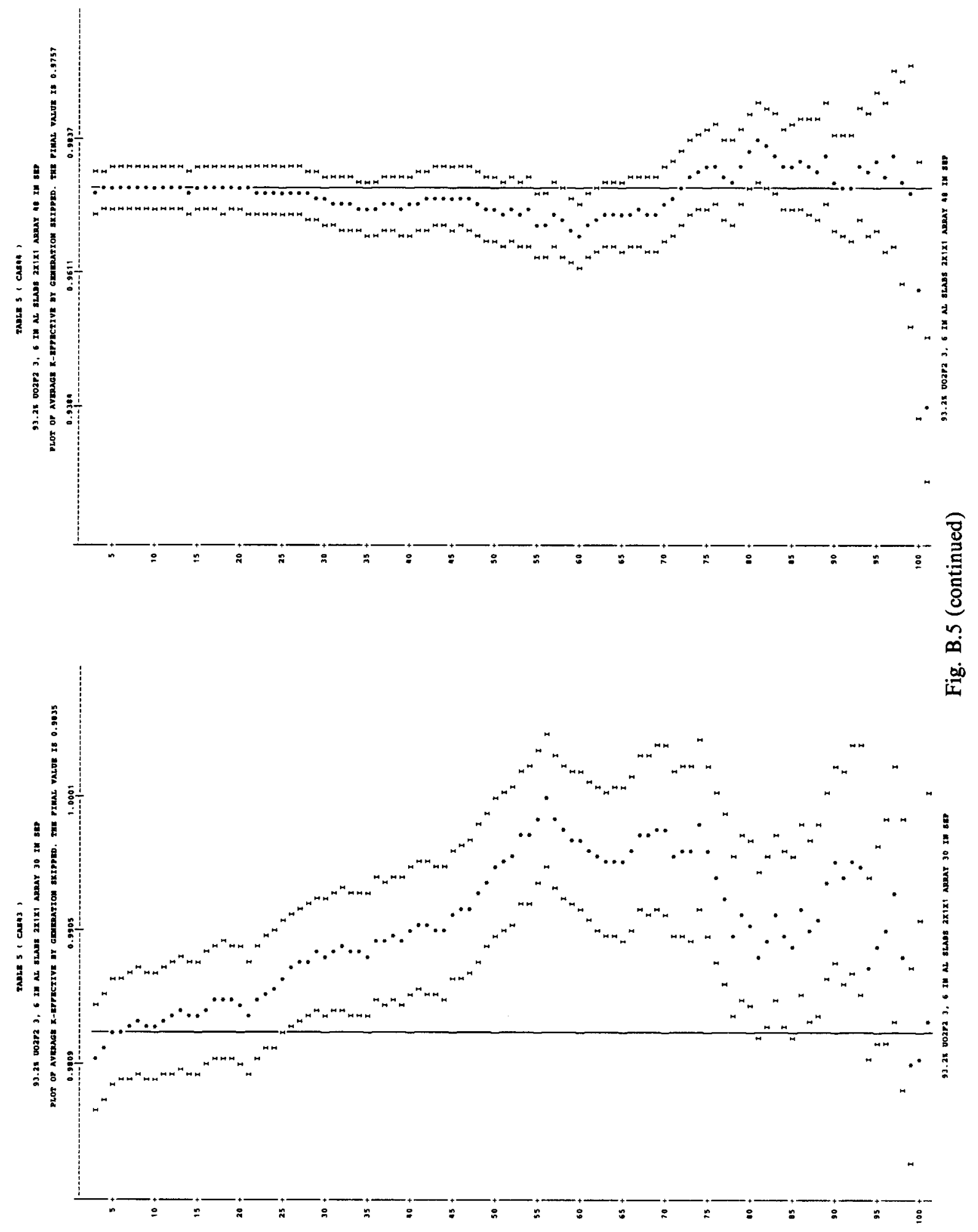



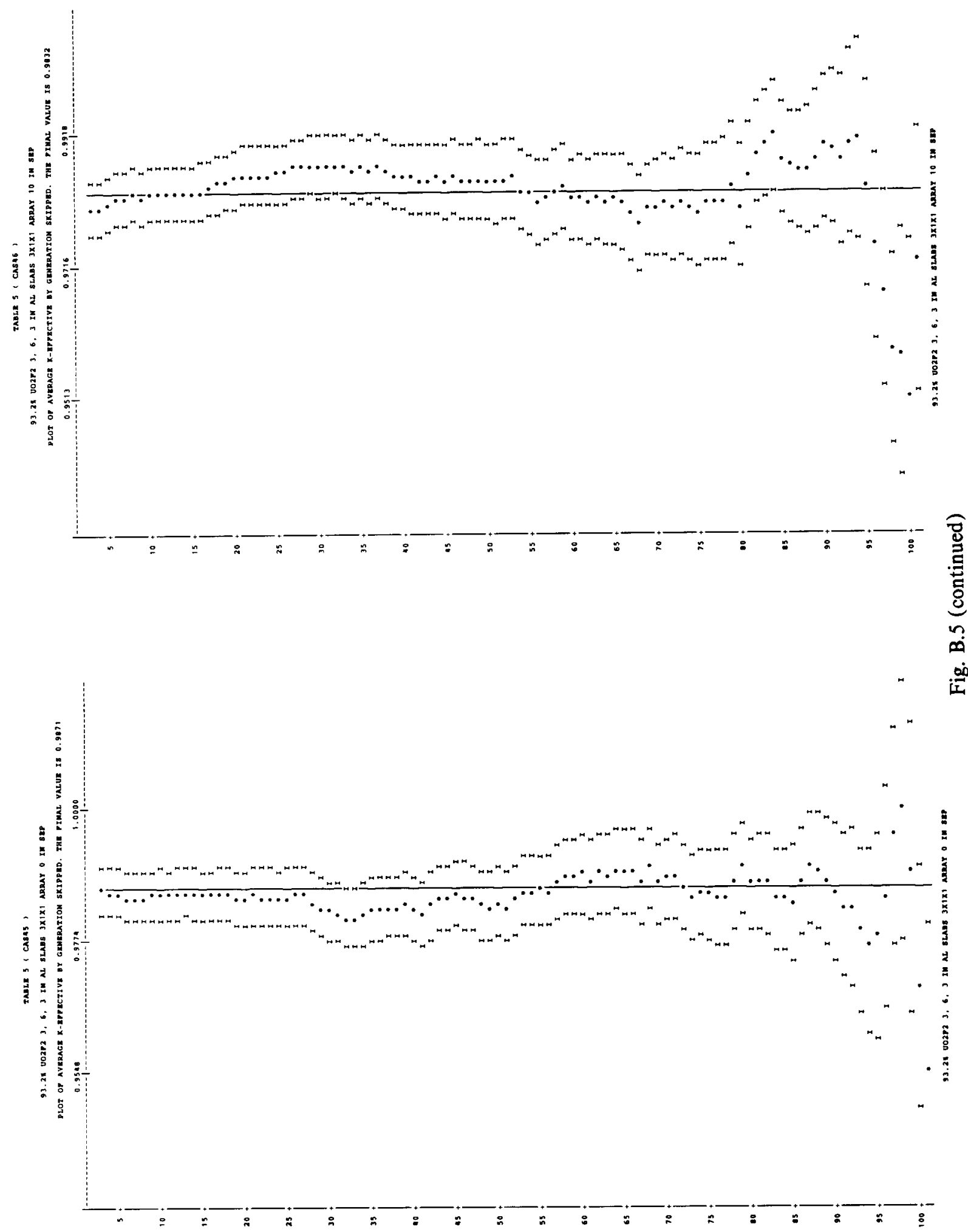

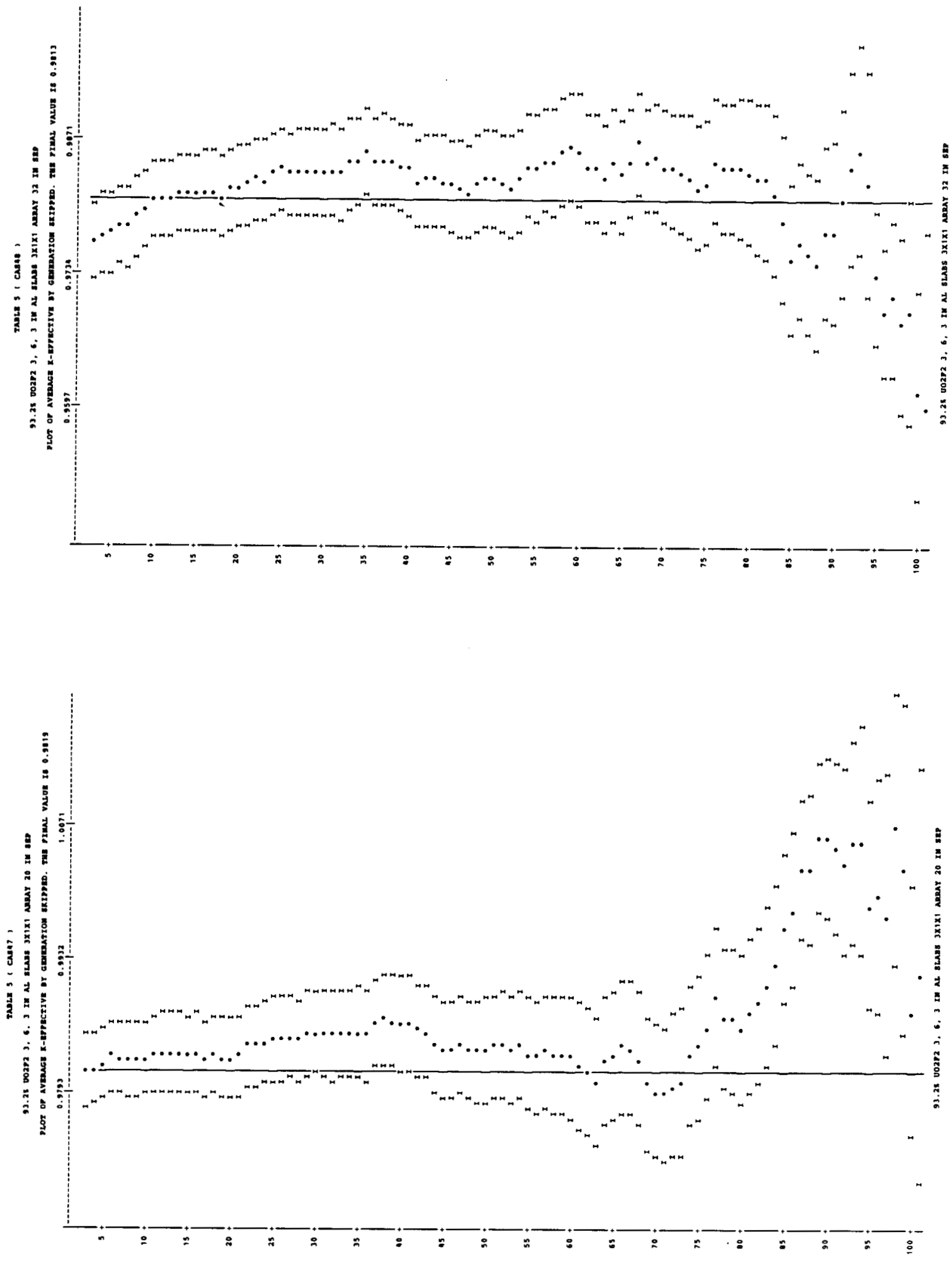

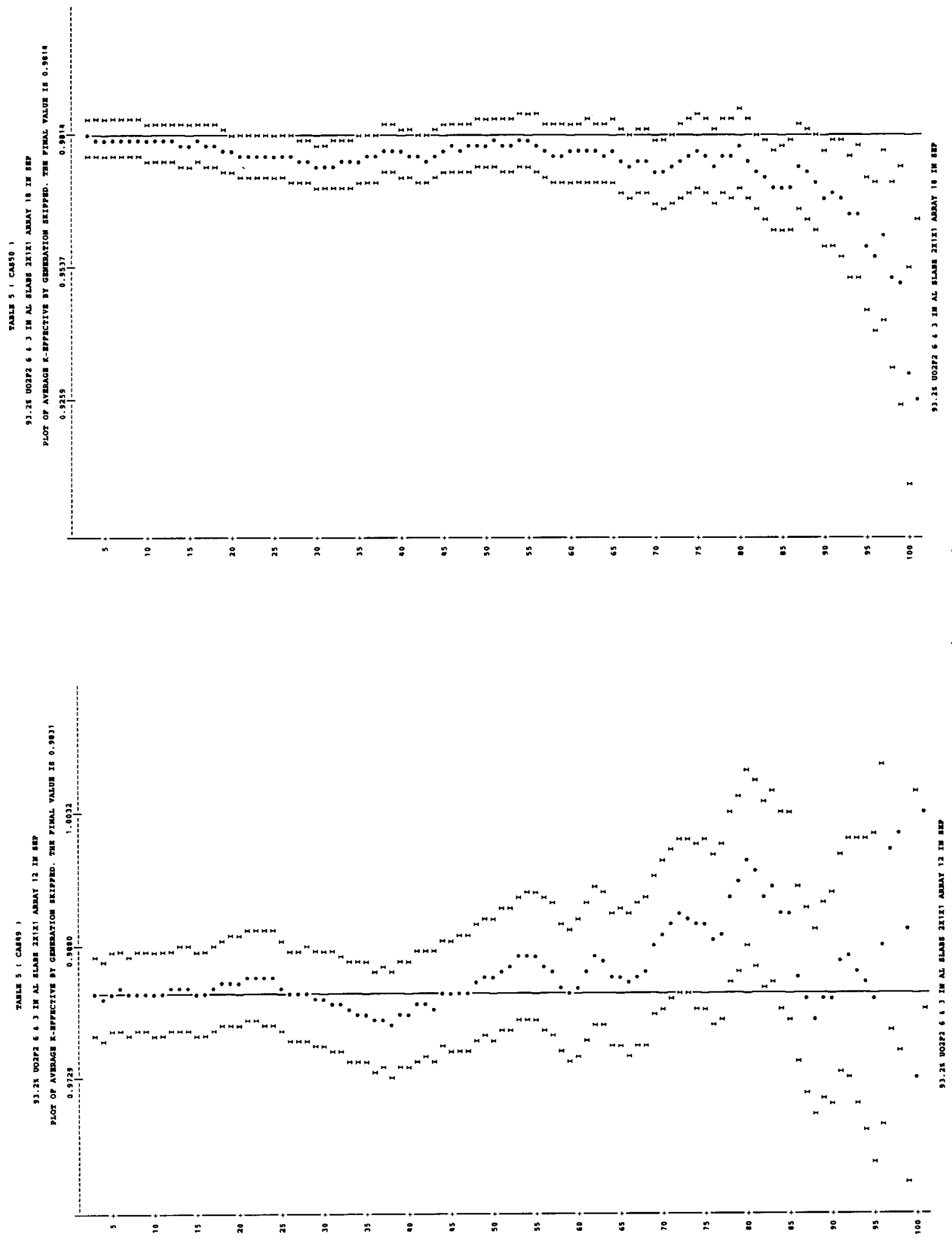

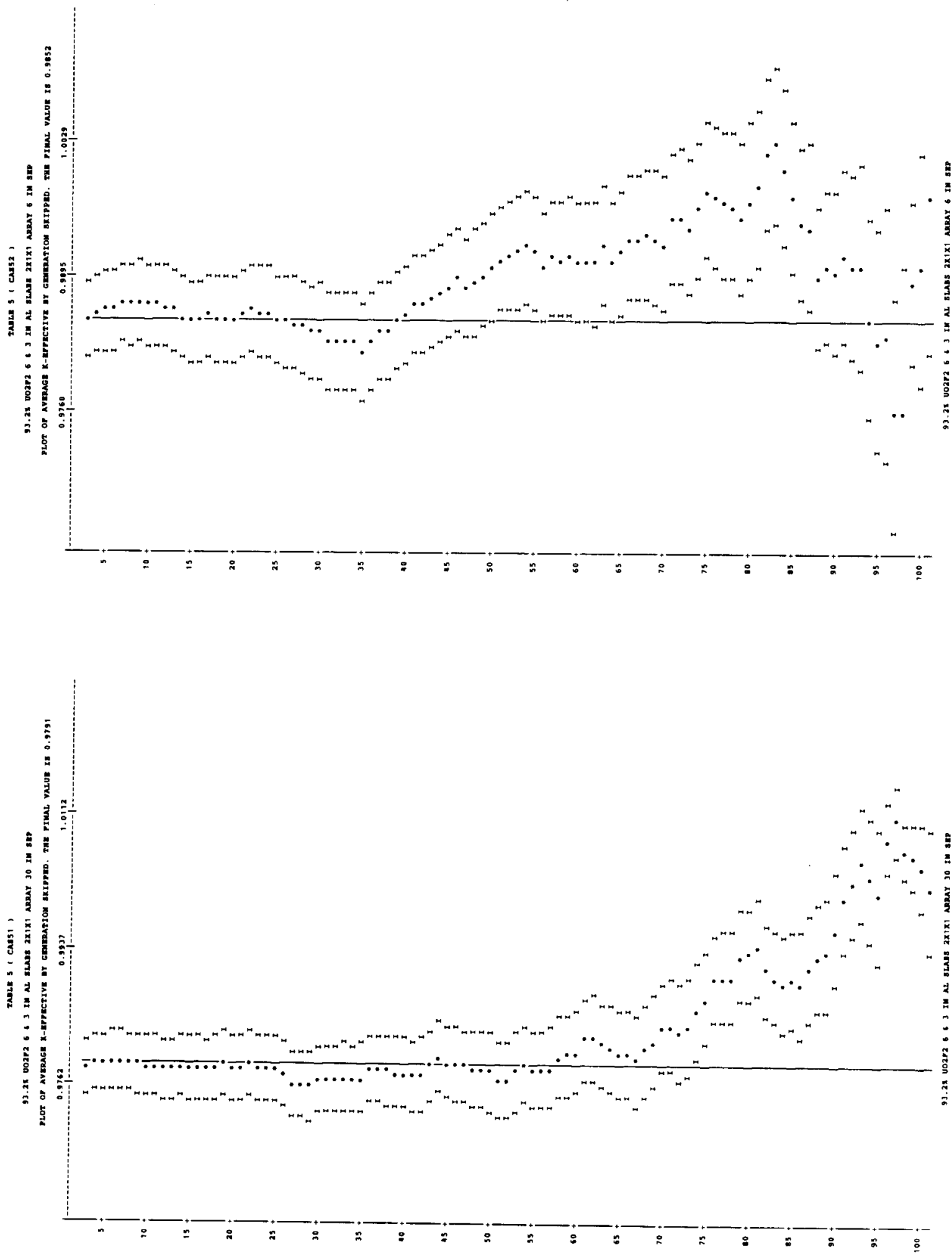
B-101
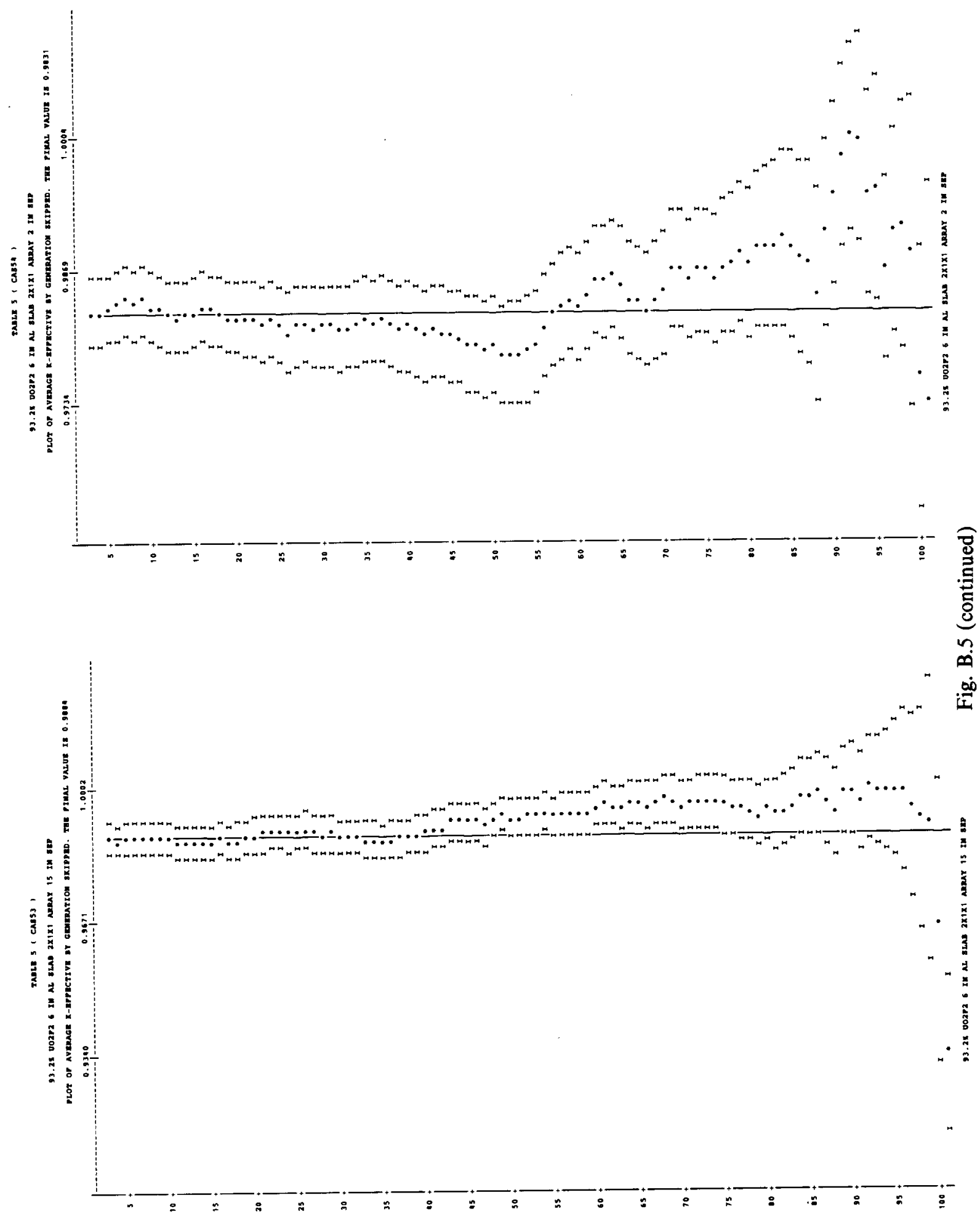


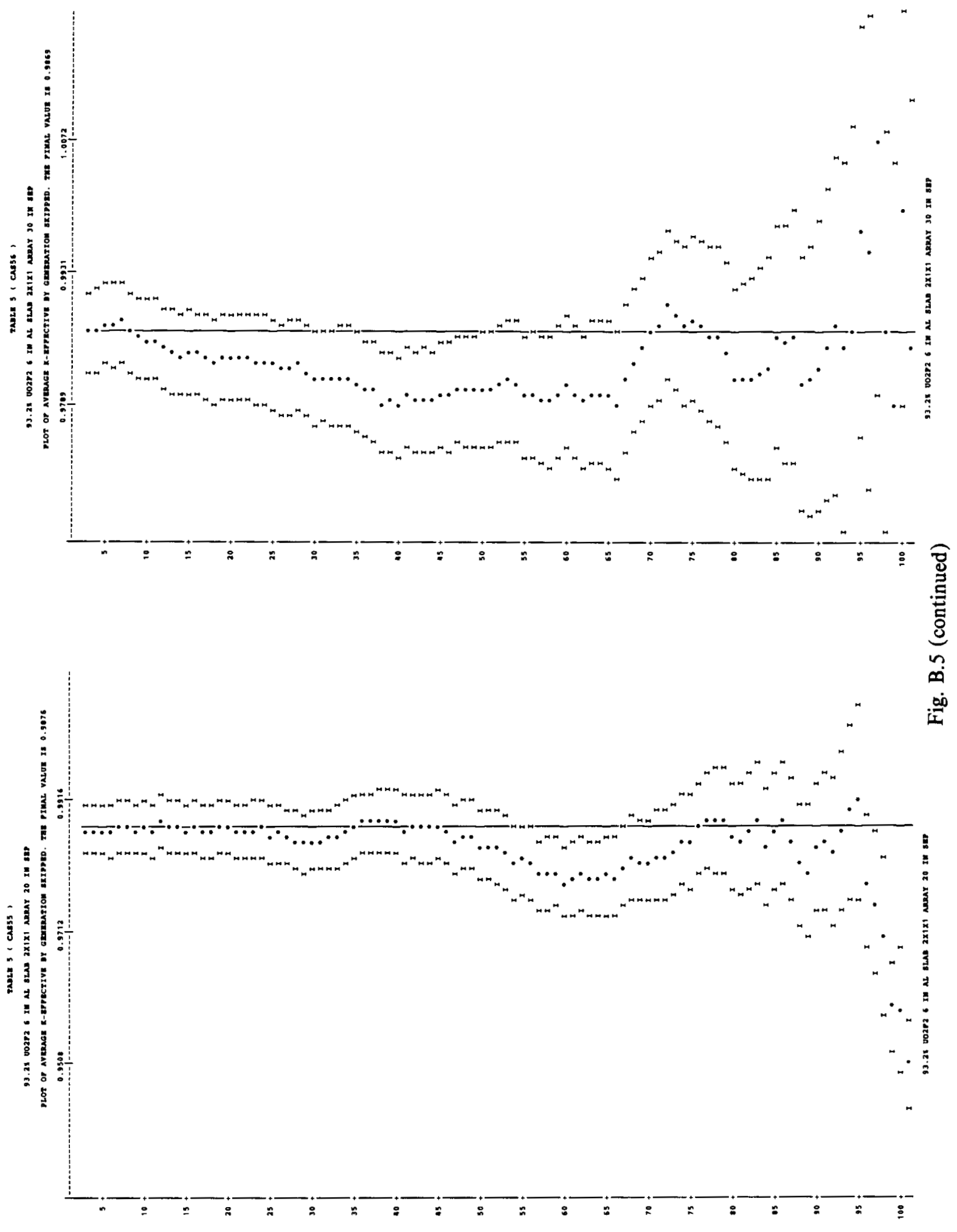


B-103
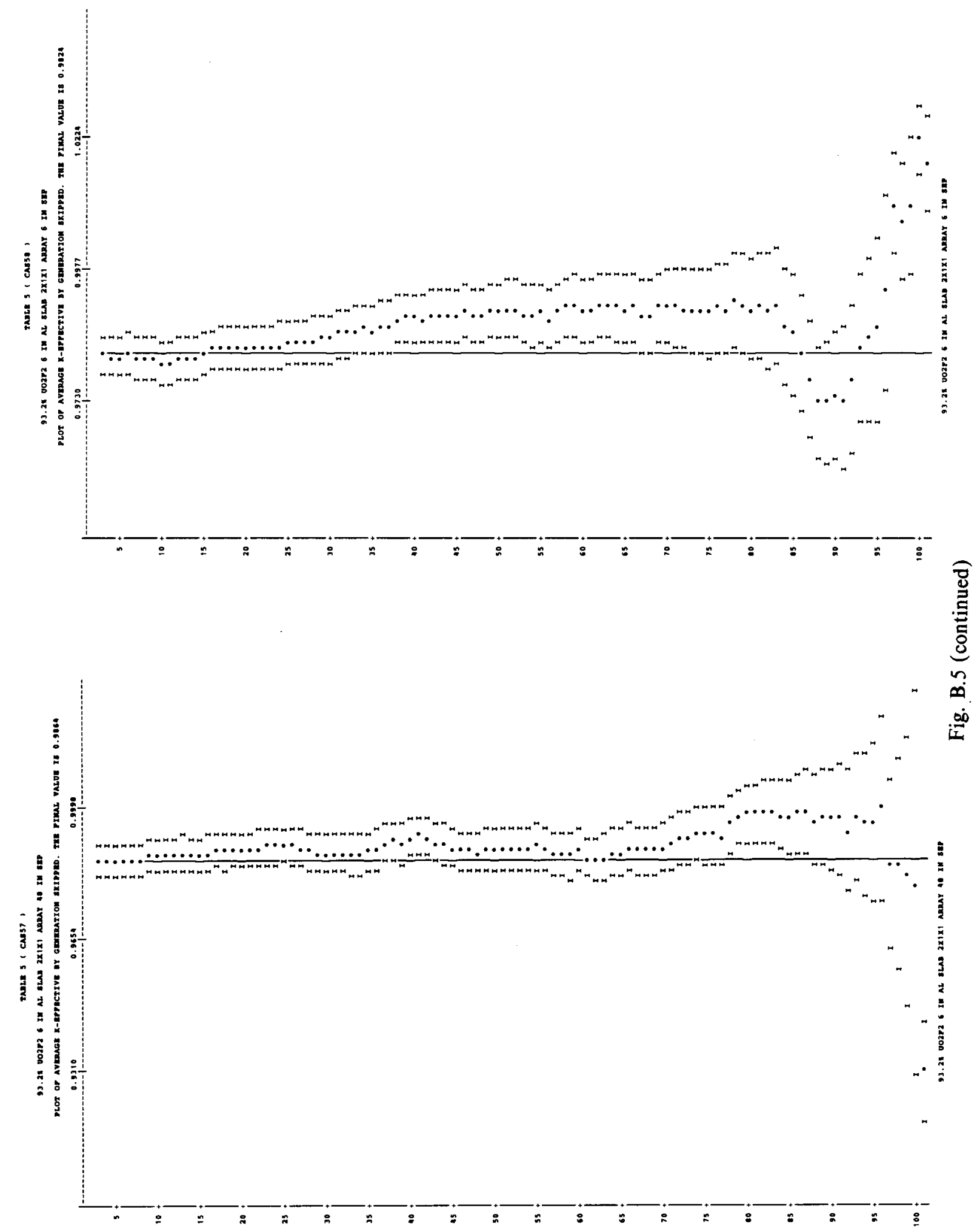

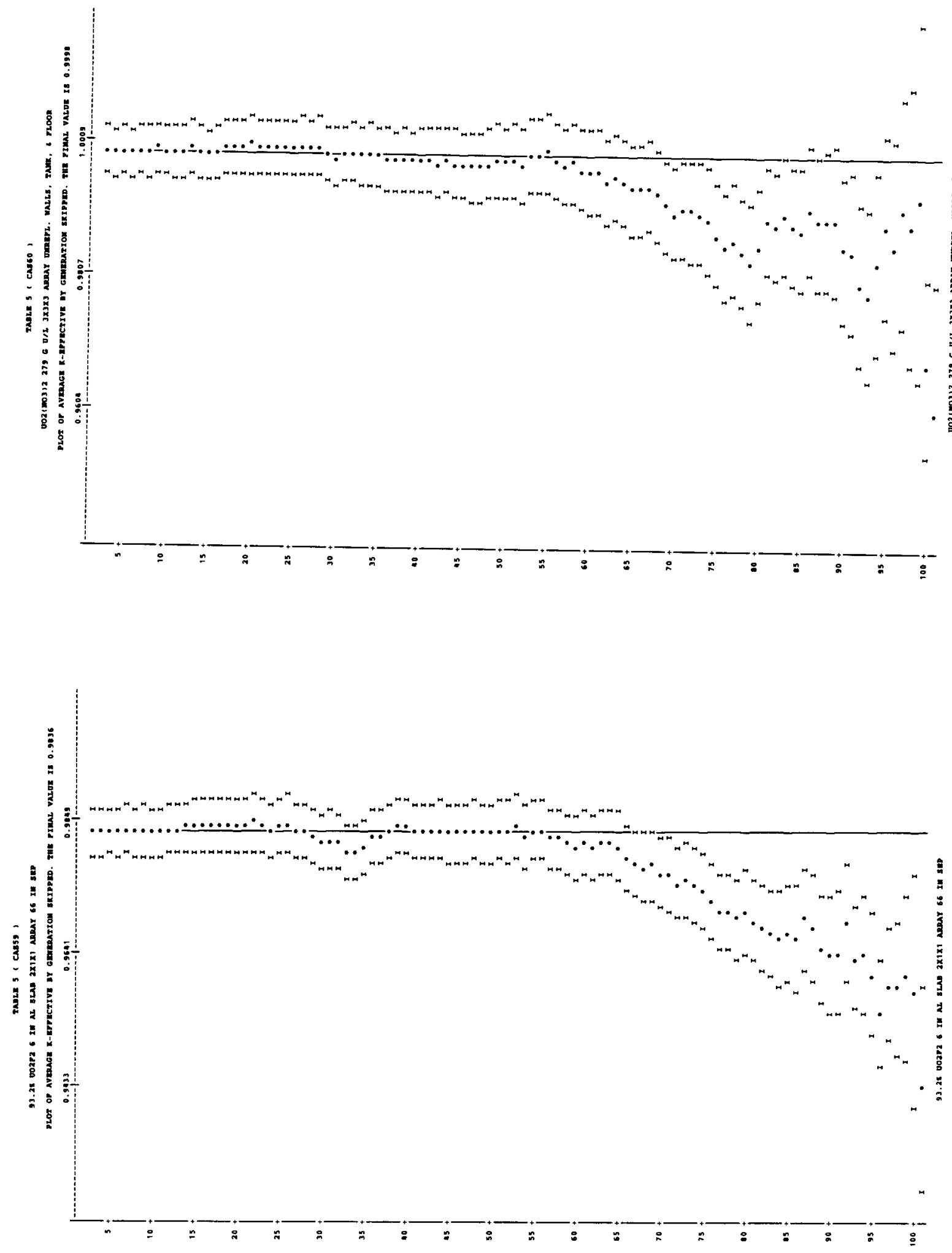


\section{B-105}
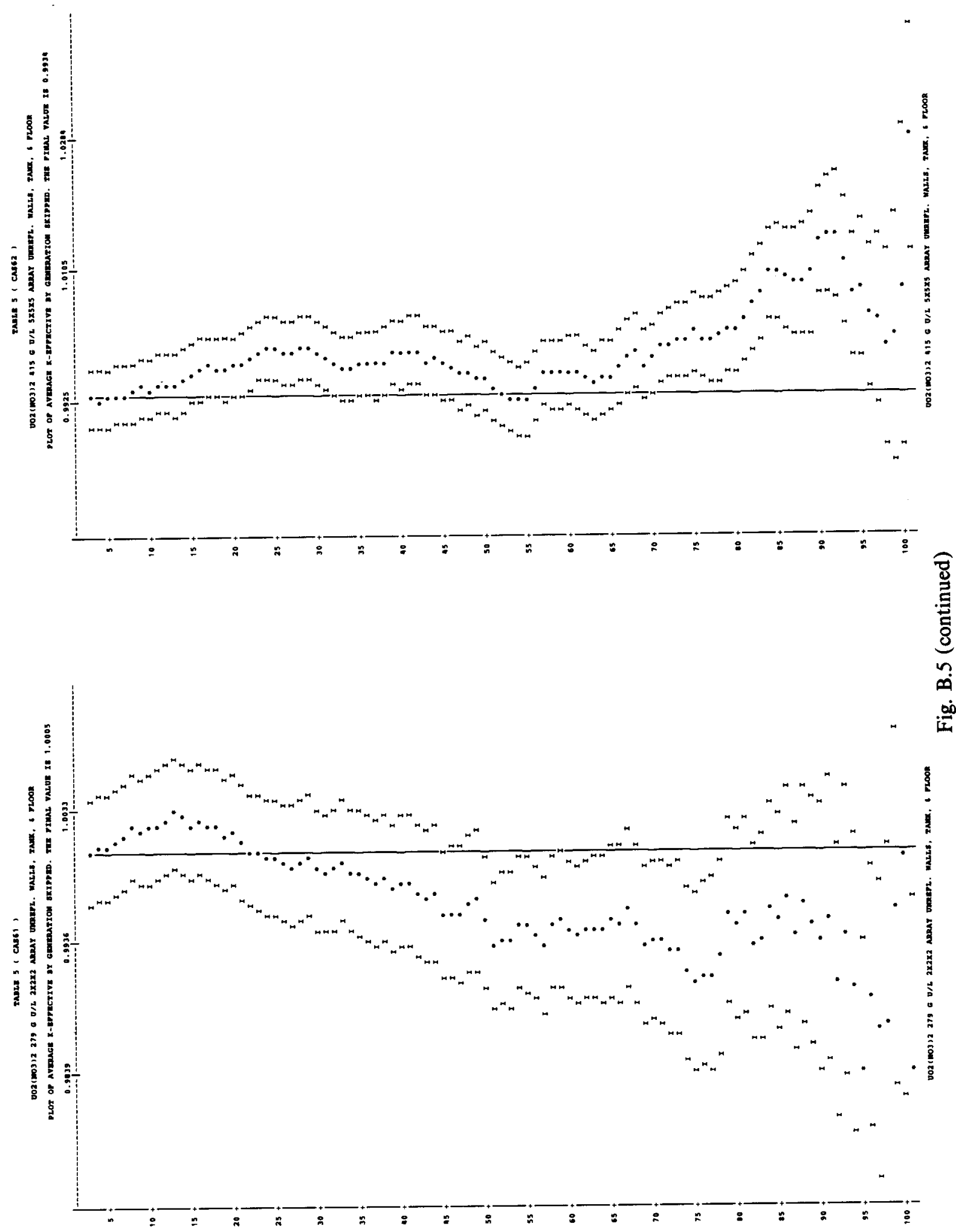

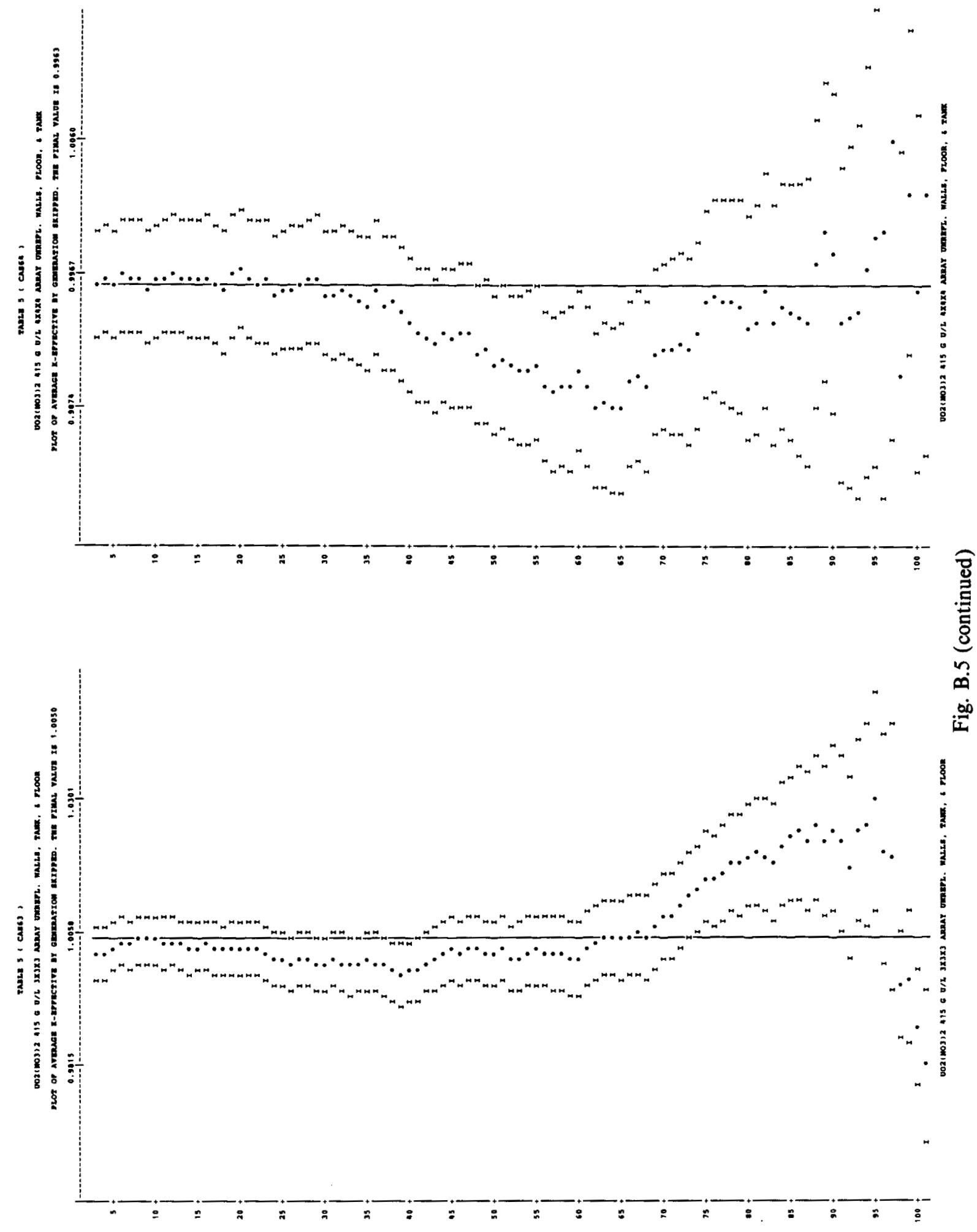


\section{B-107}
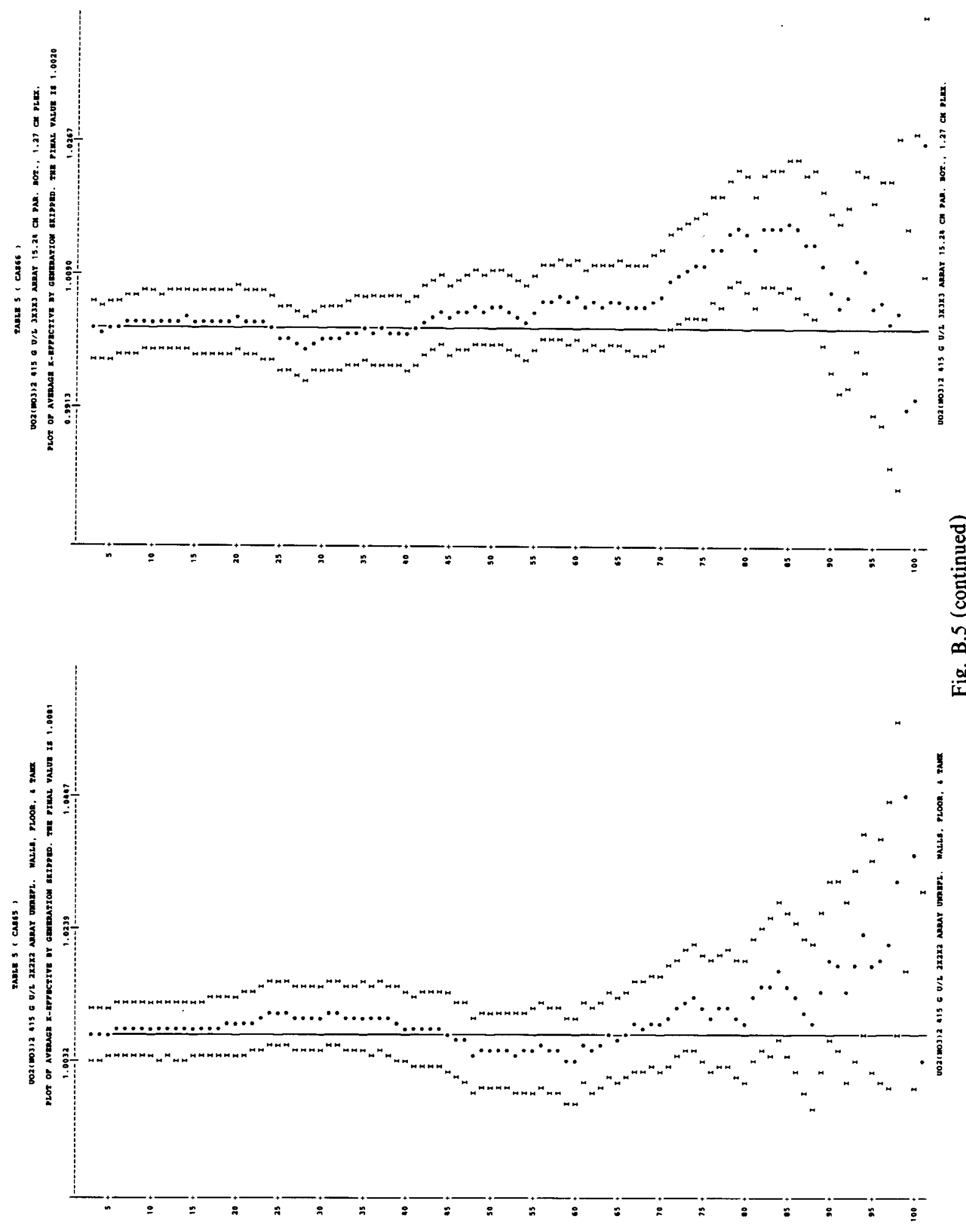


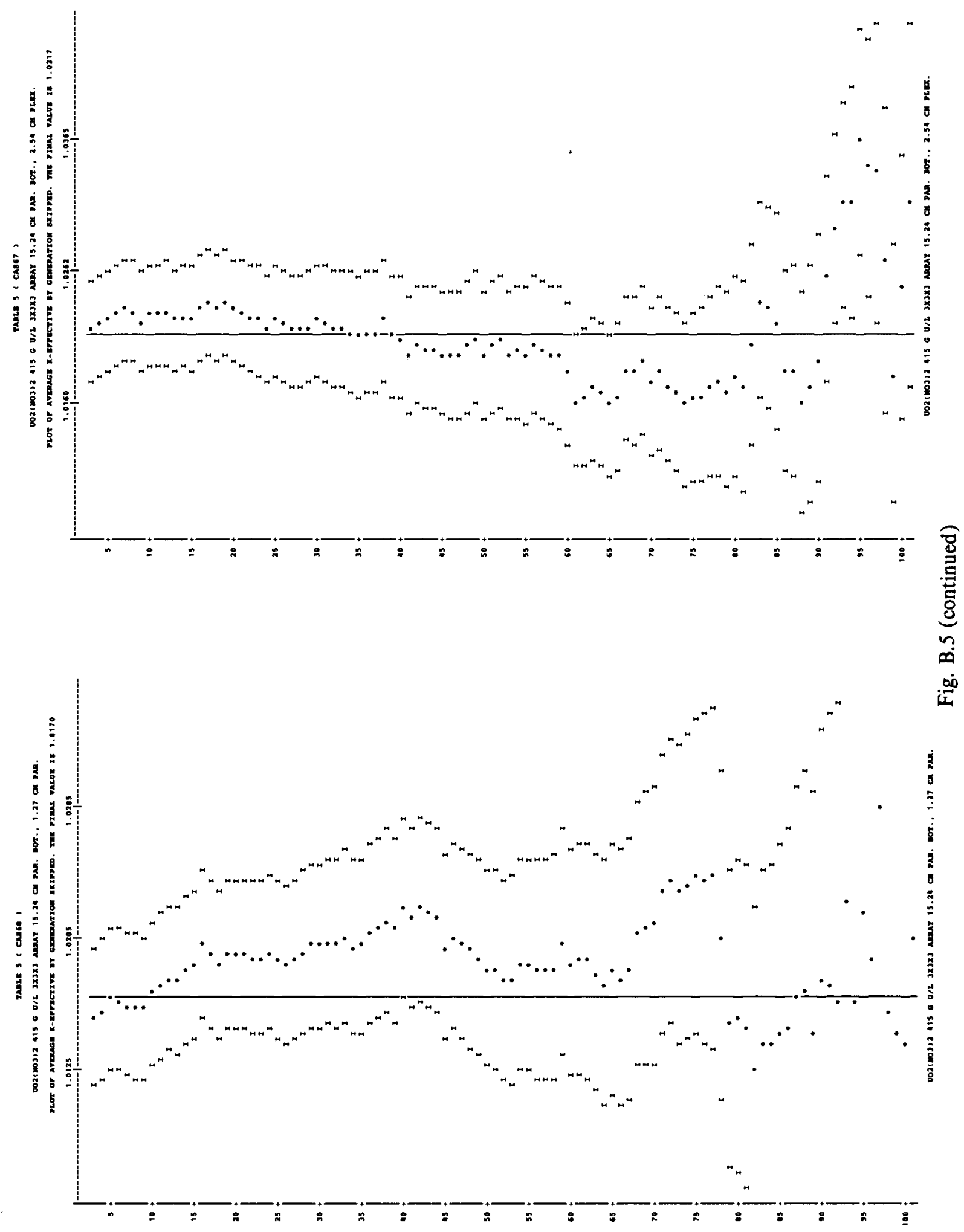



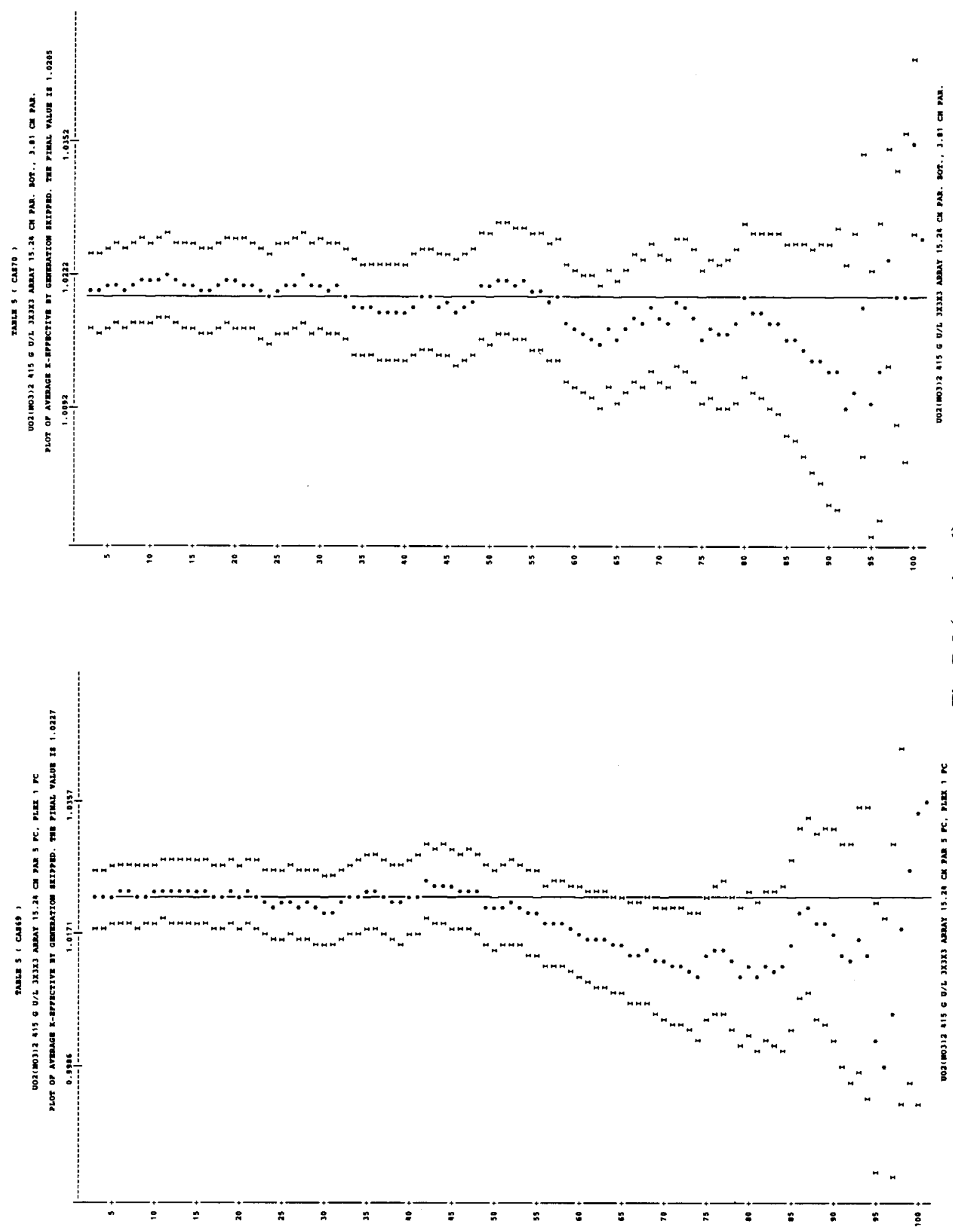
B-110

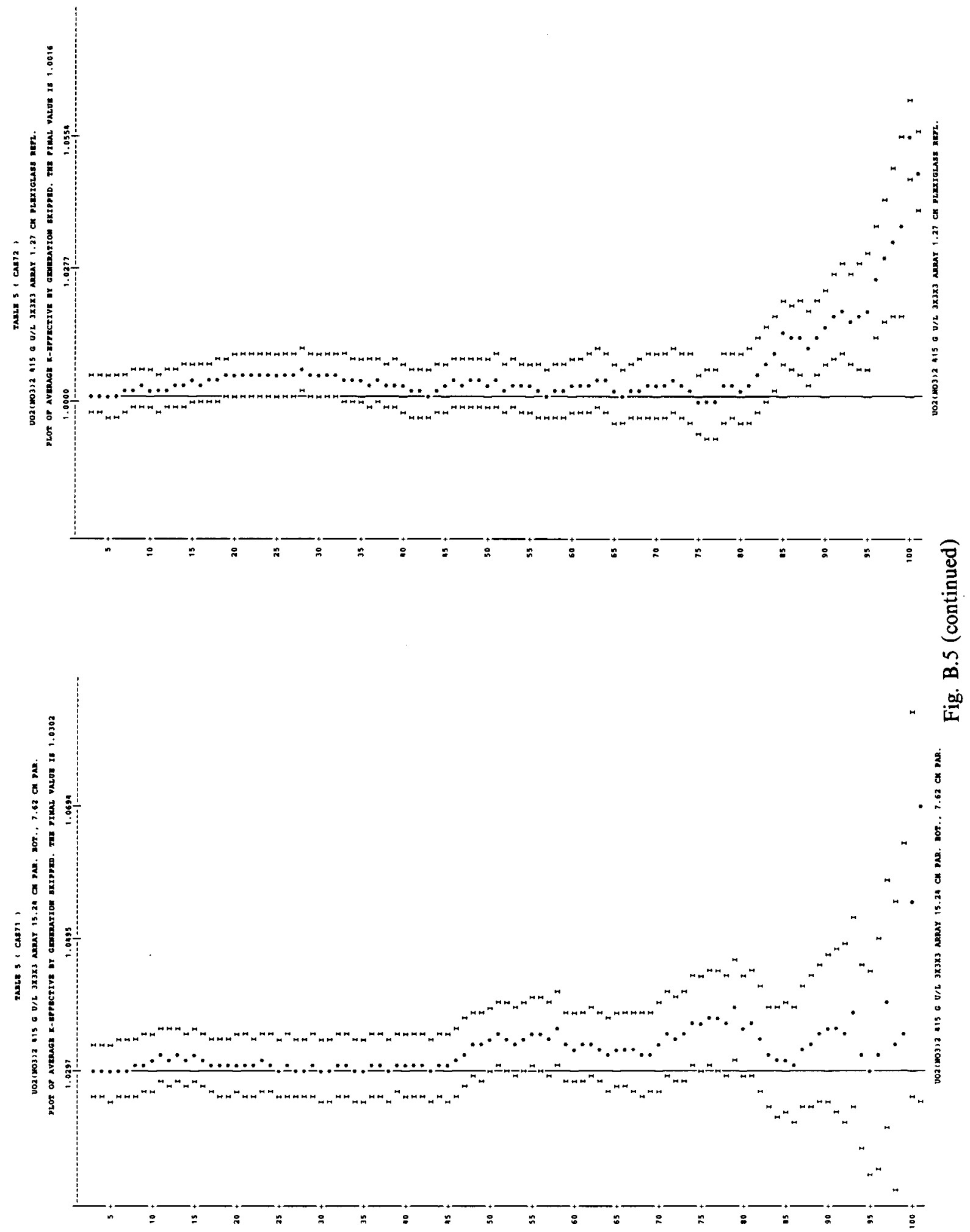




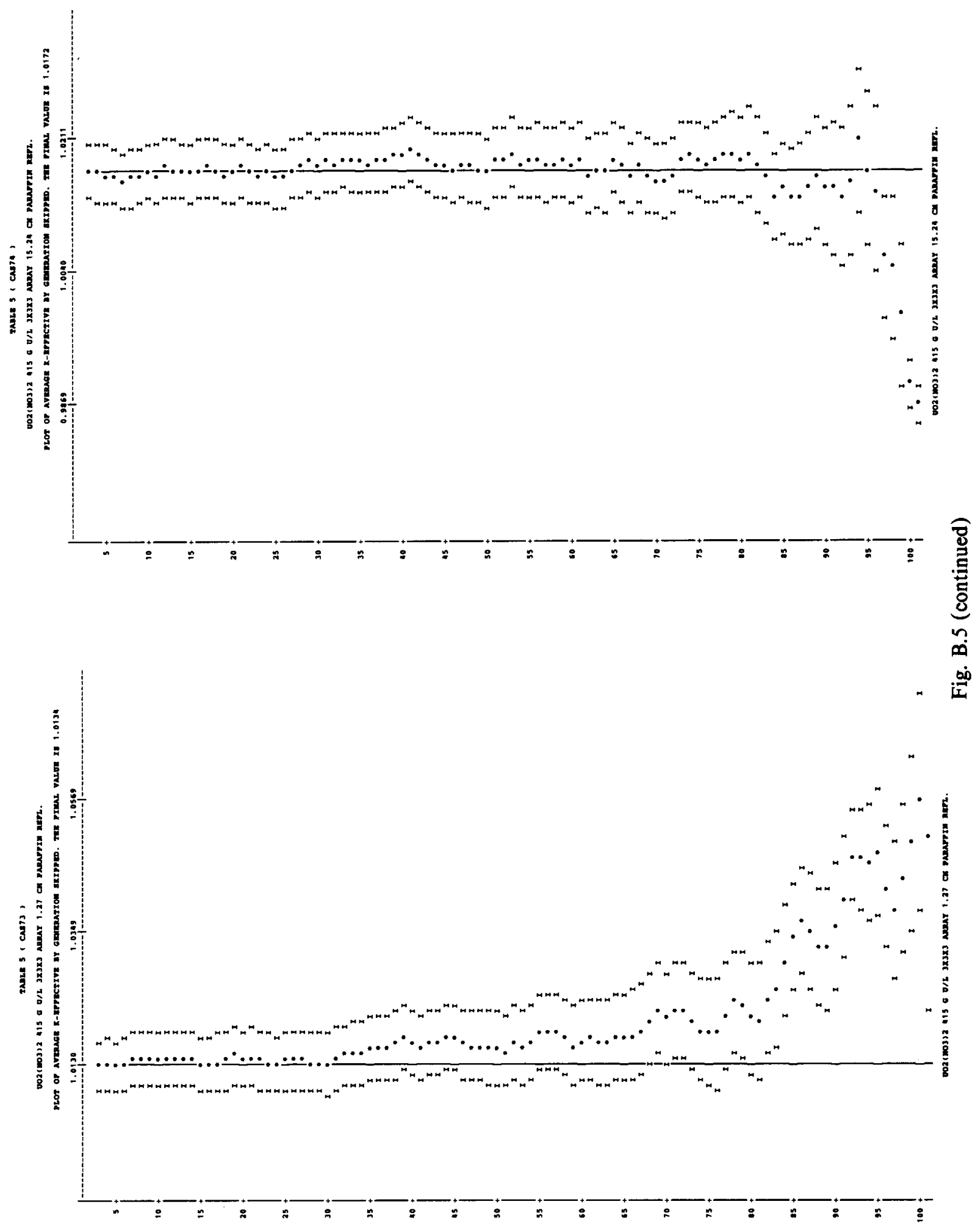



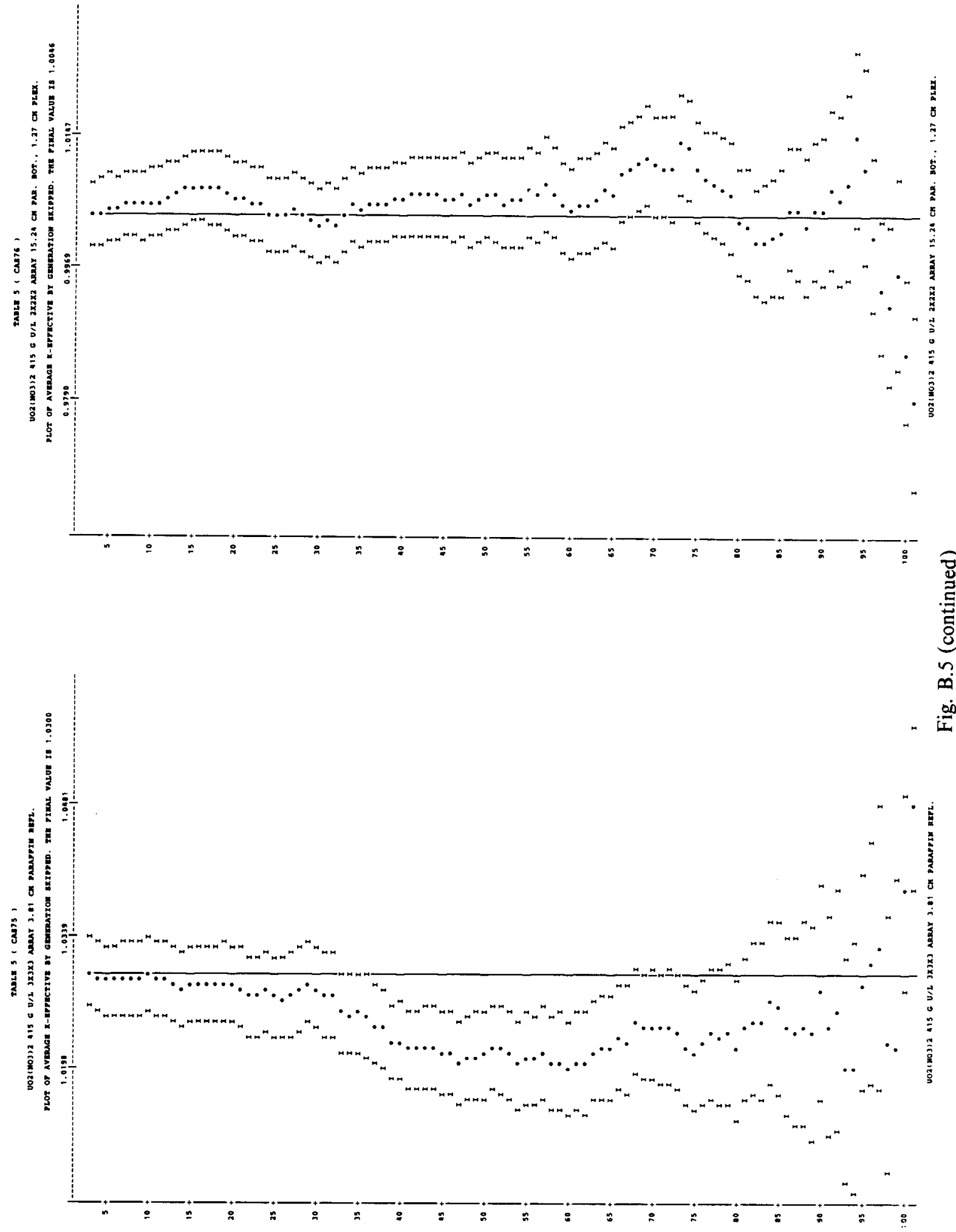
B-113
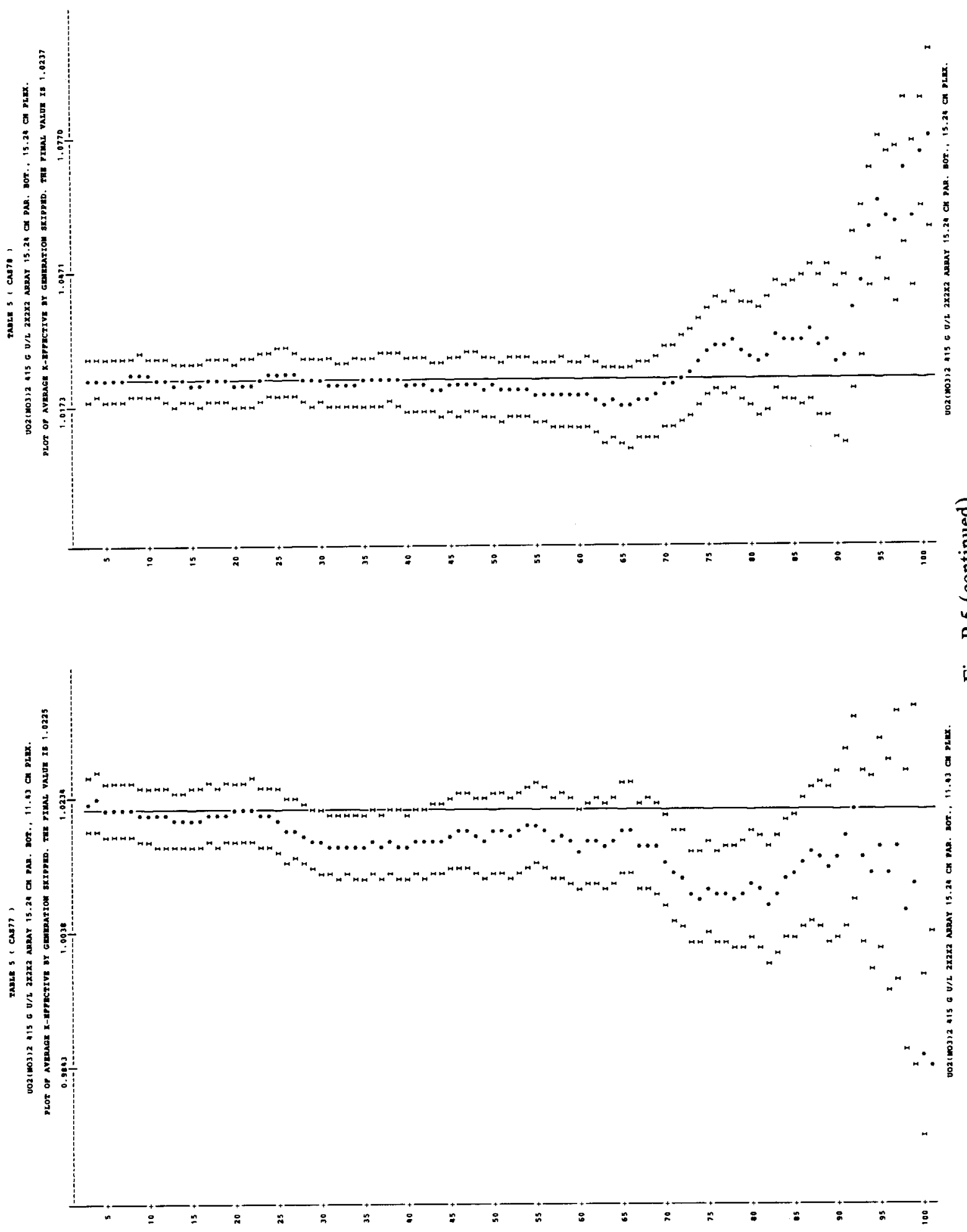


\section{B-114}
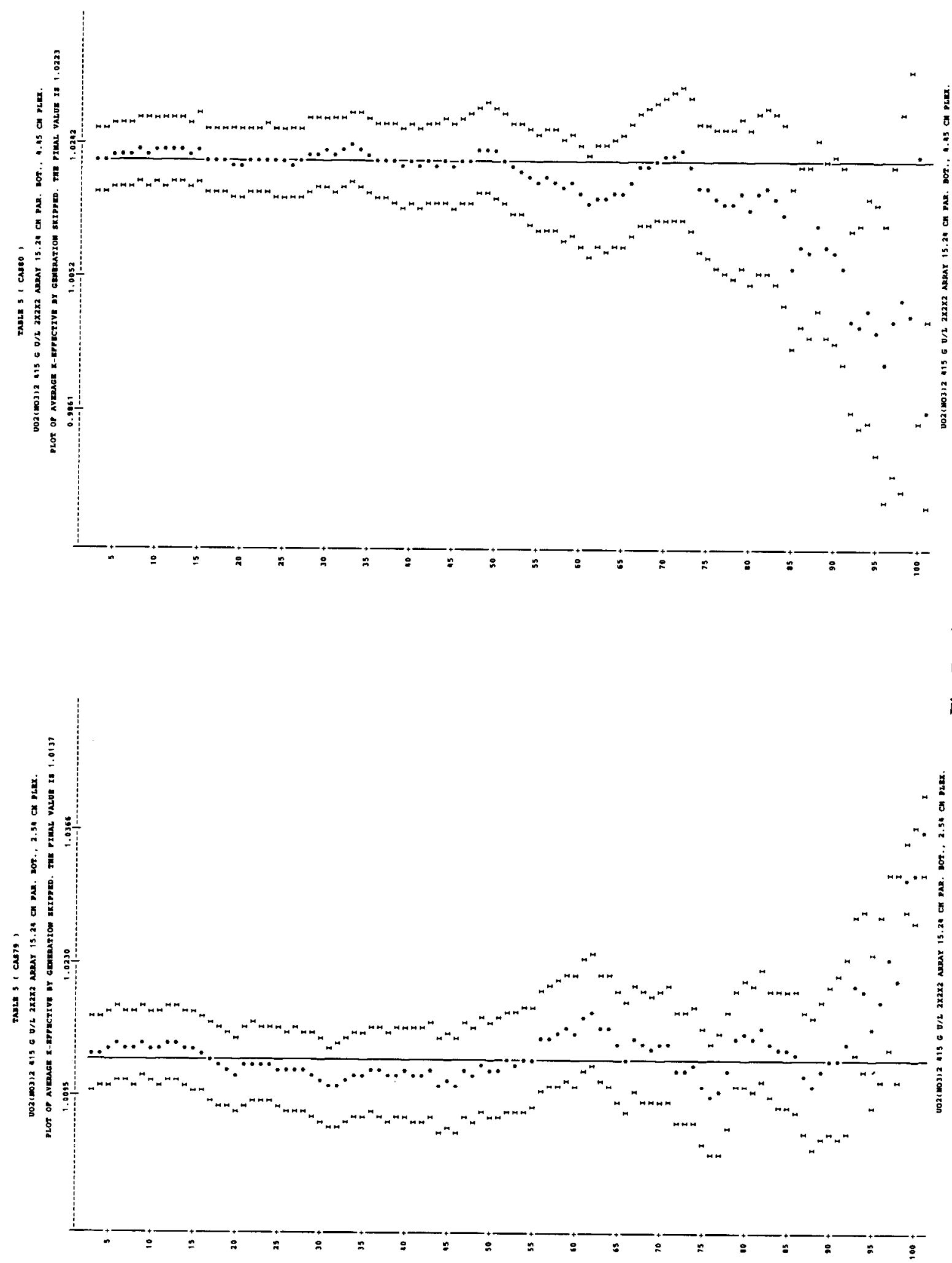


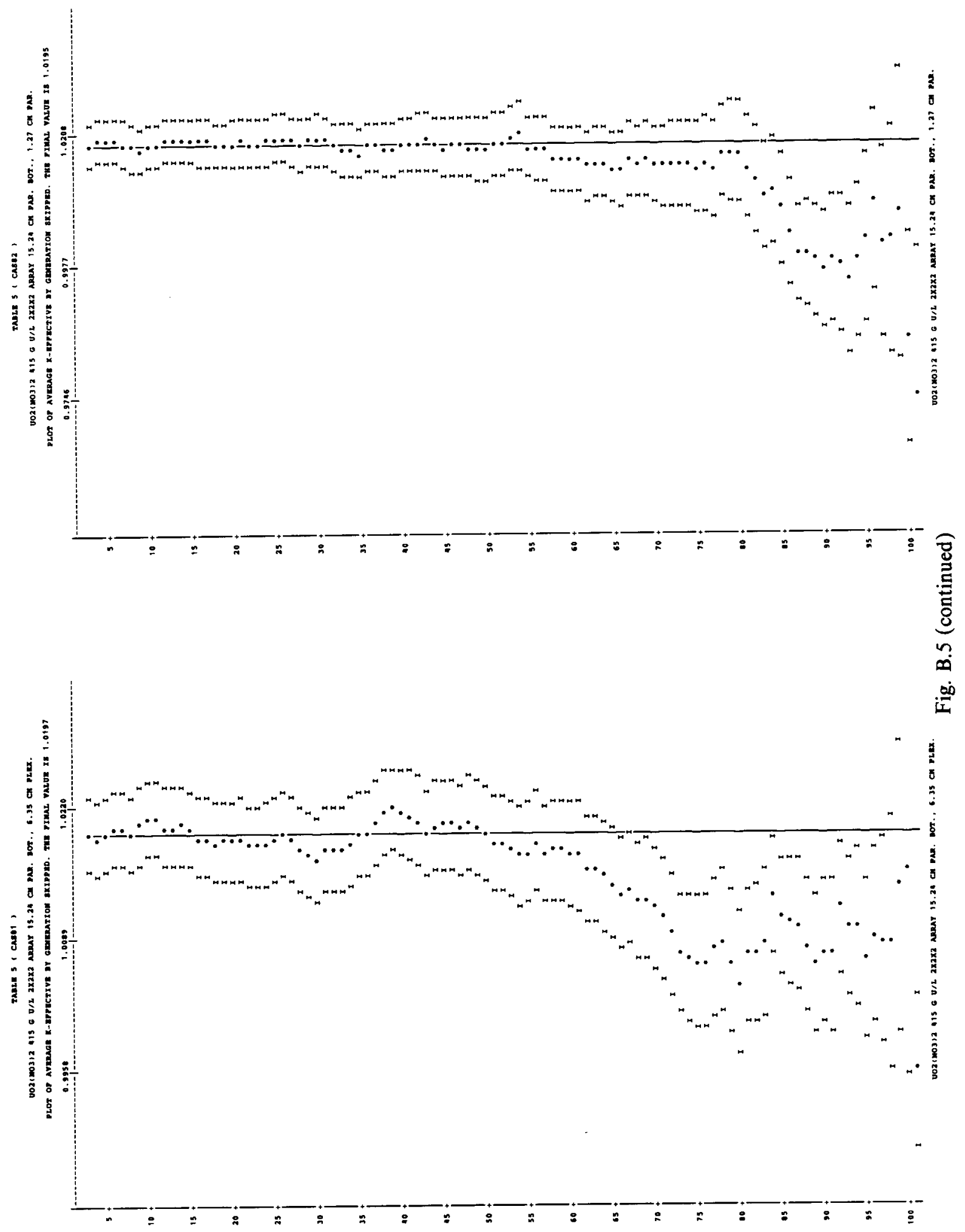




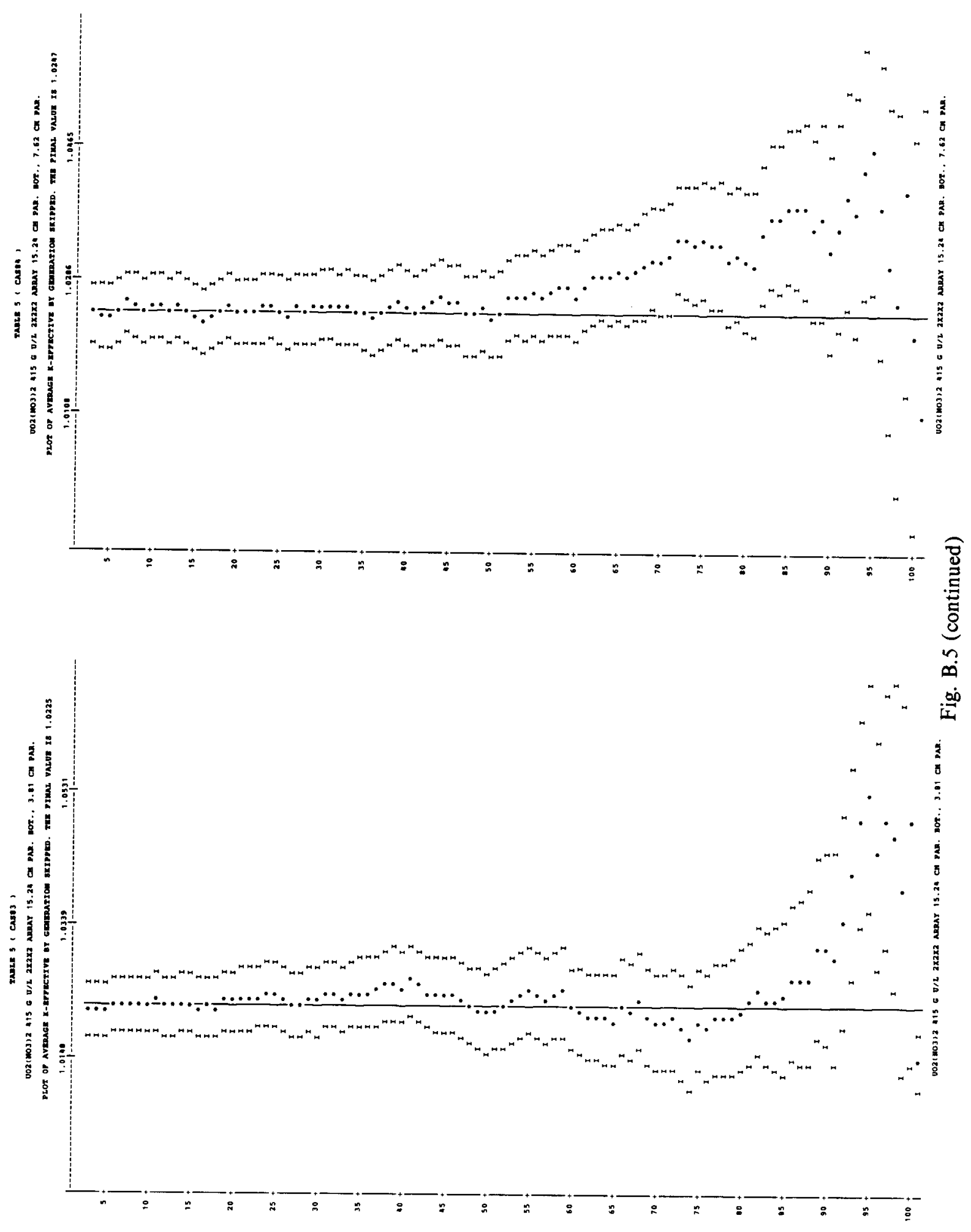


B-117

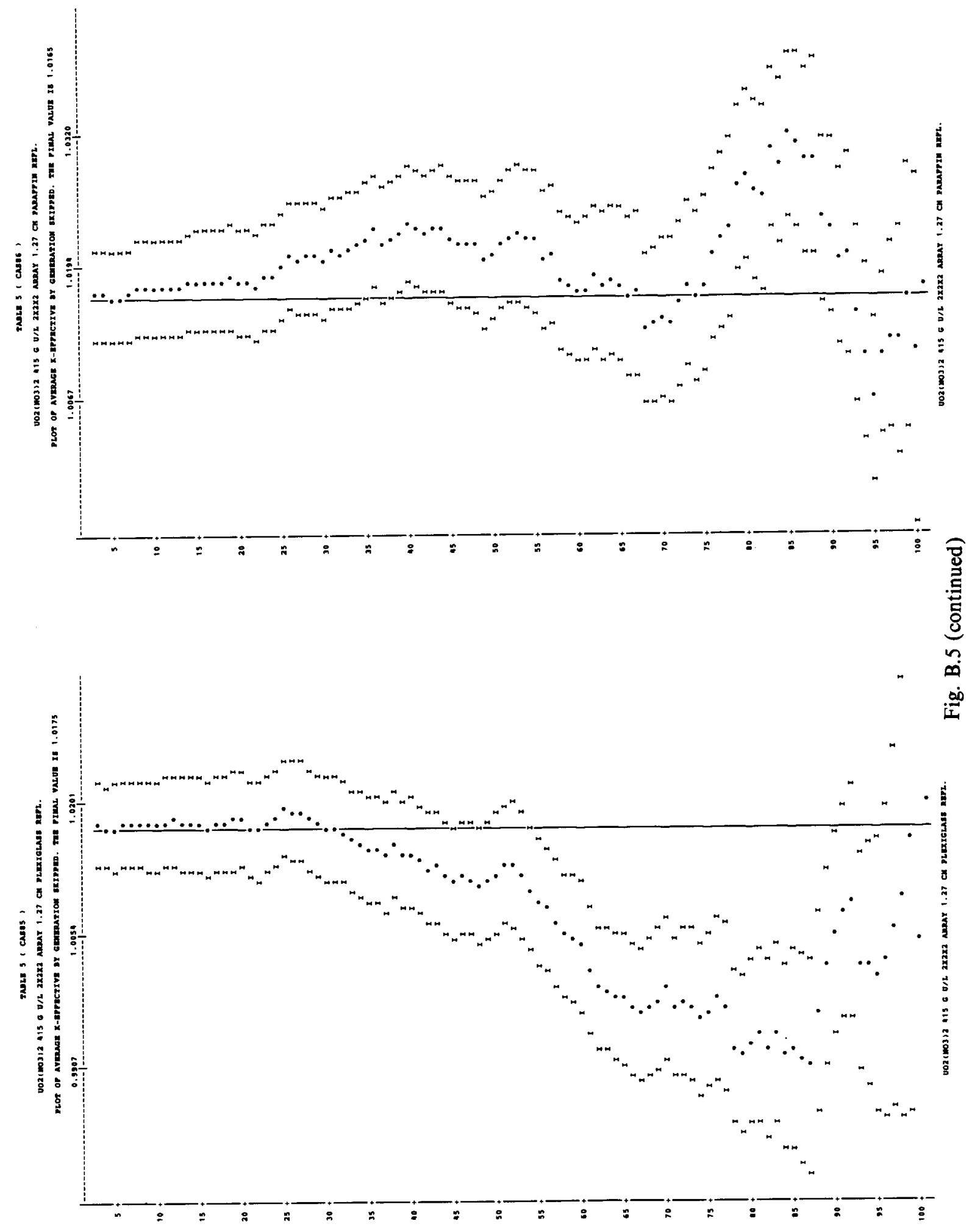




\section{B-118}
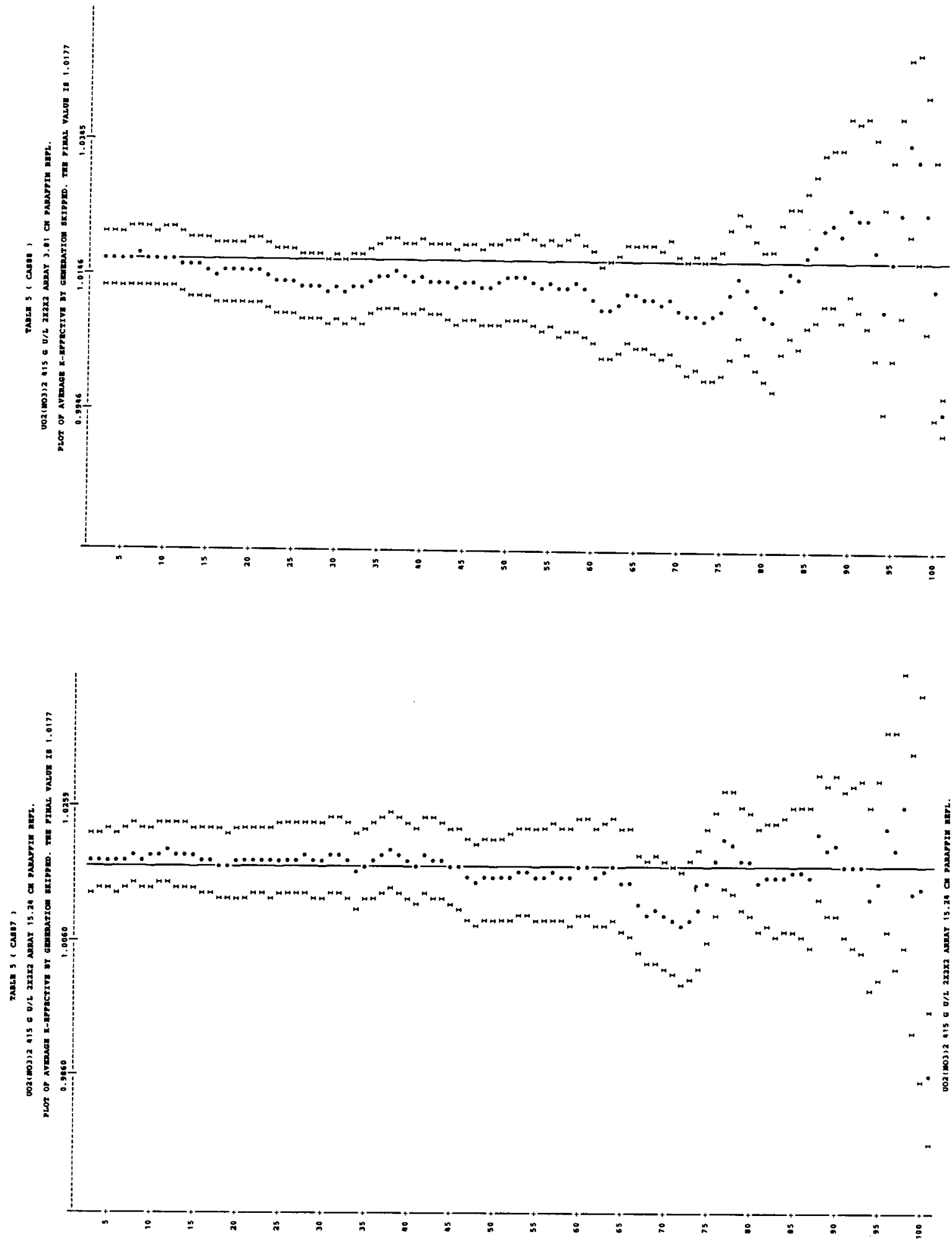


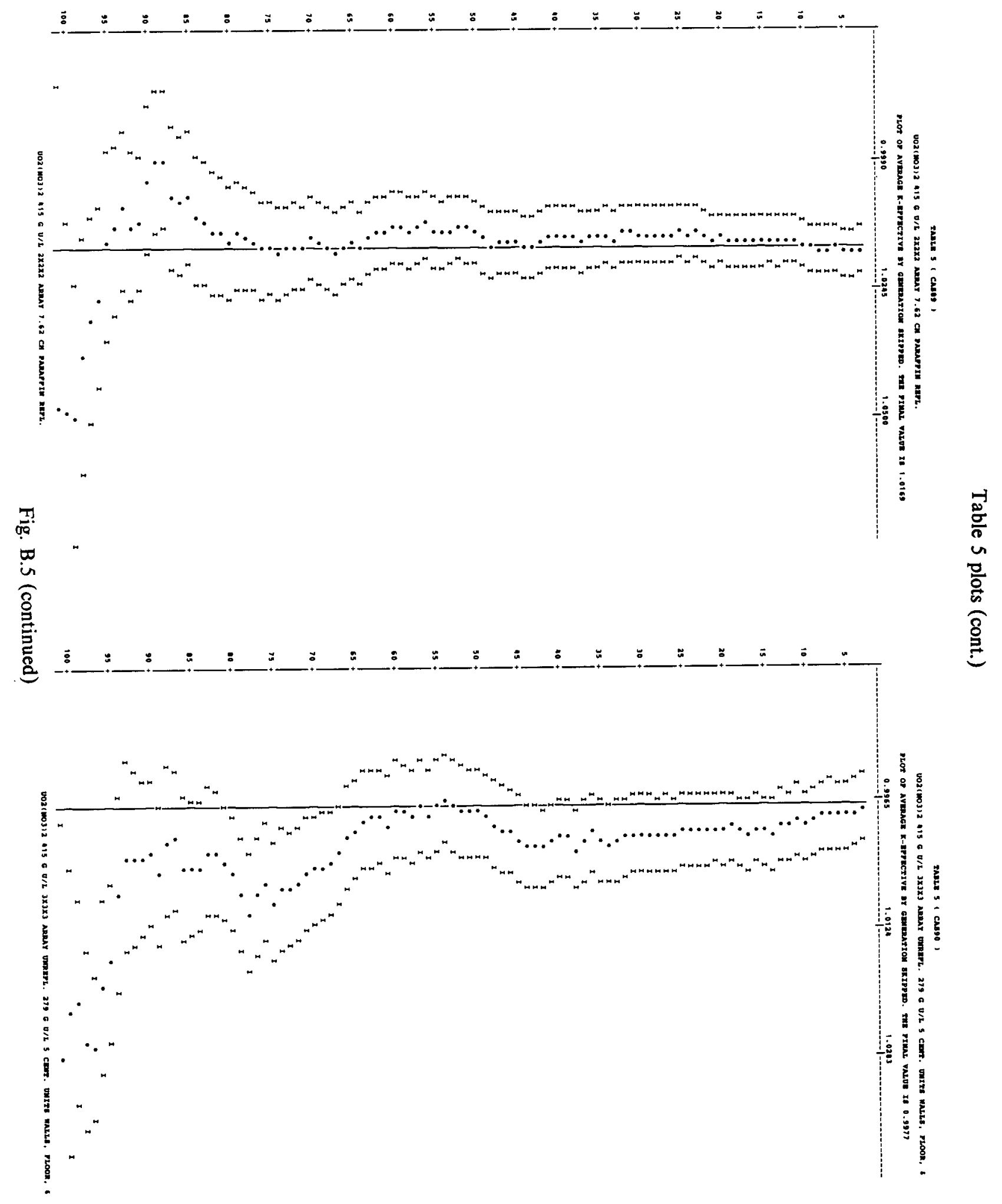


B- 120

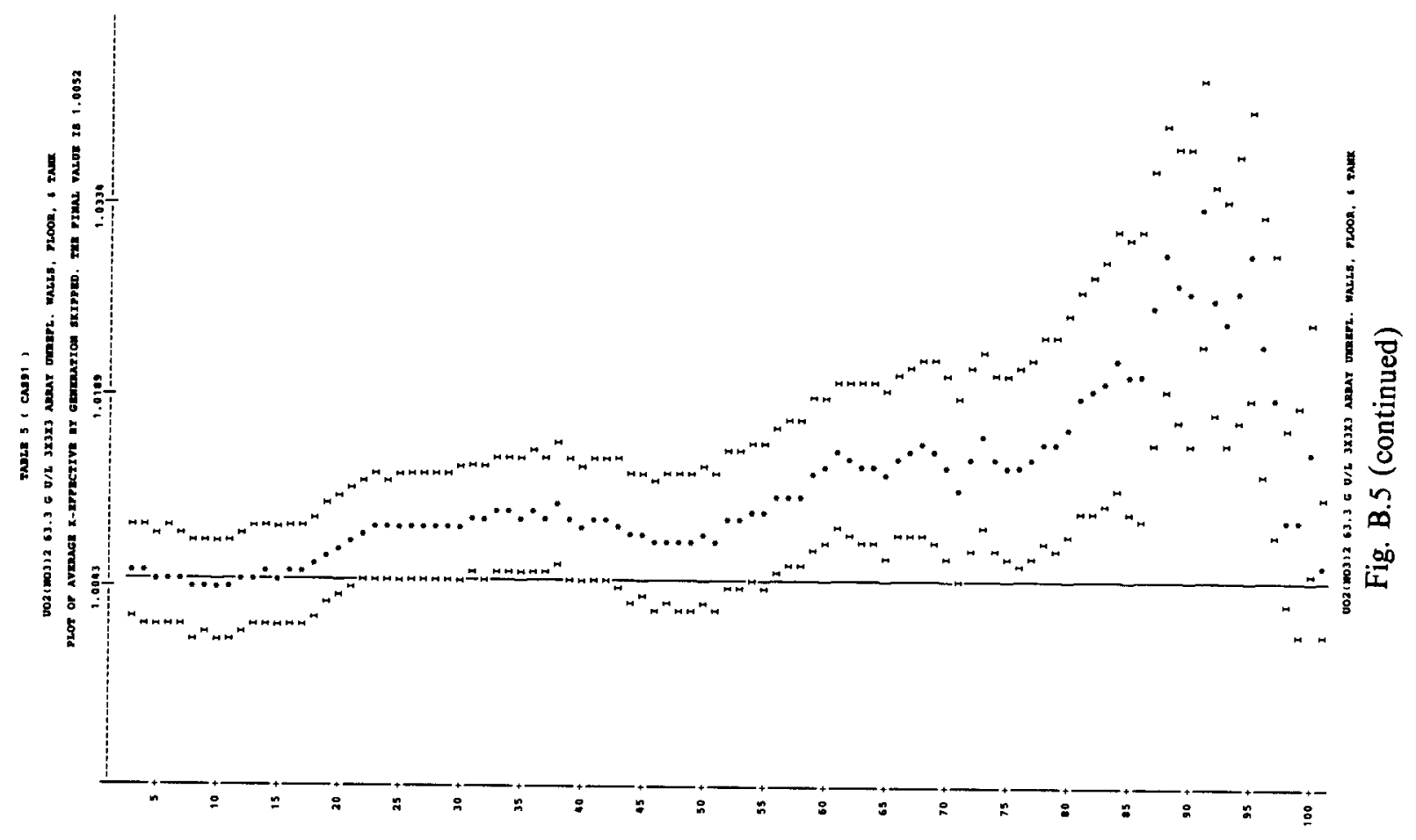



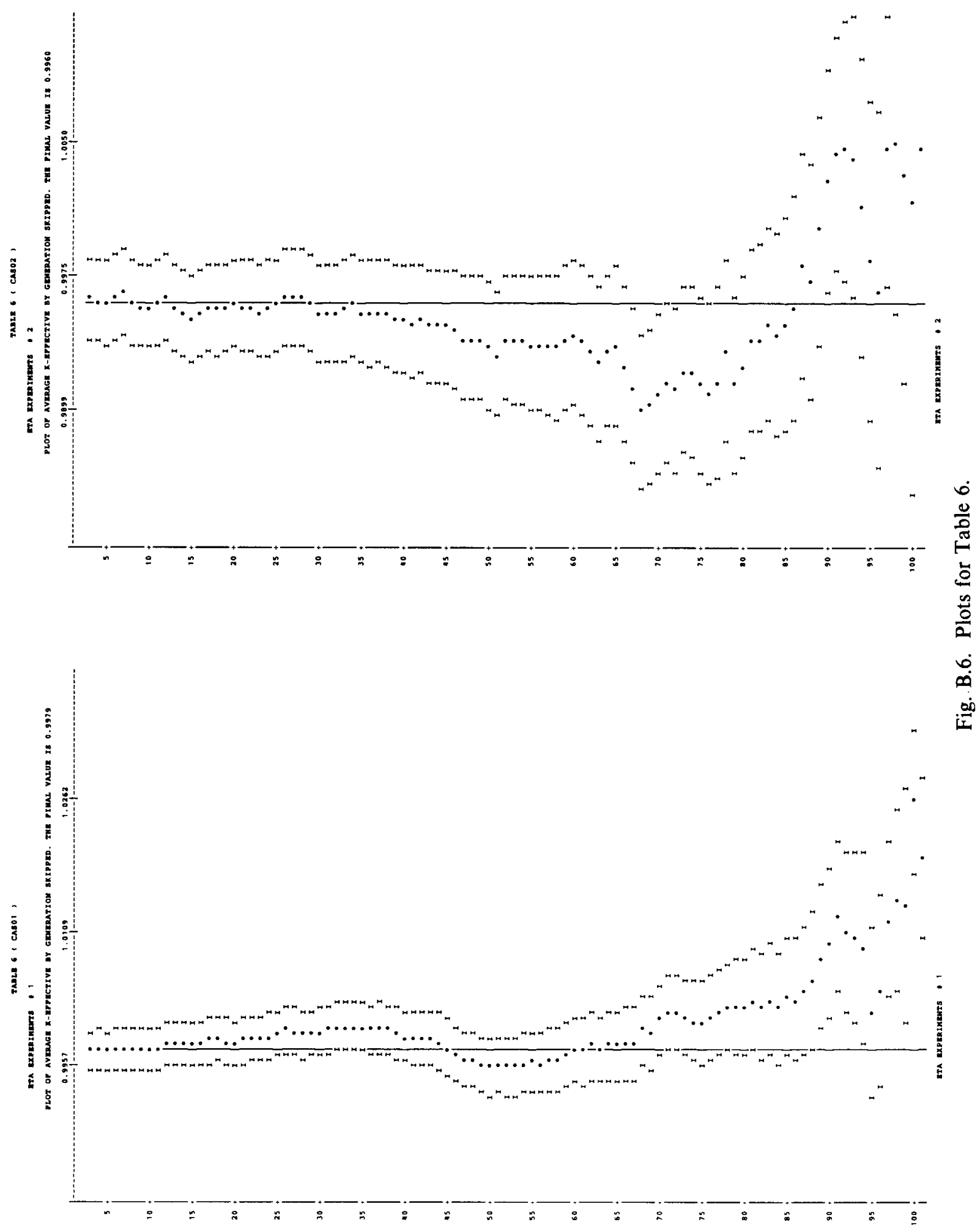
B-122

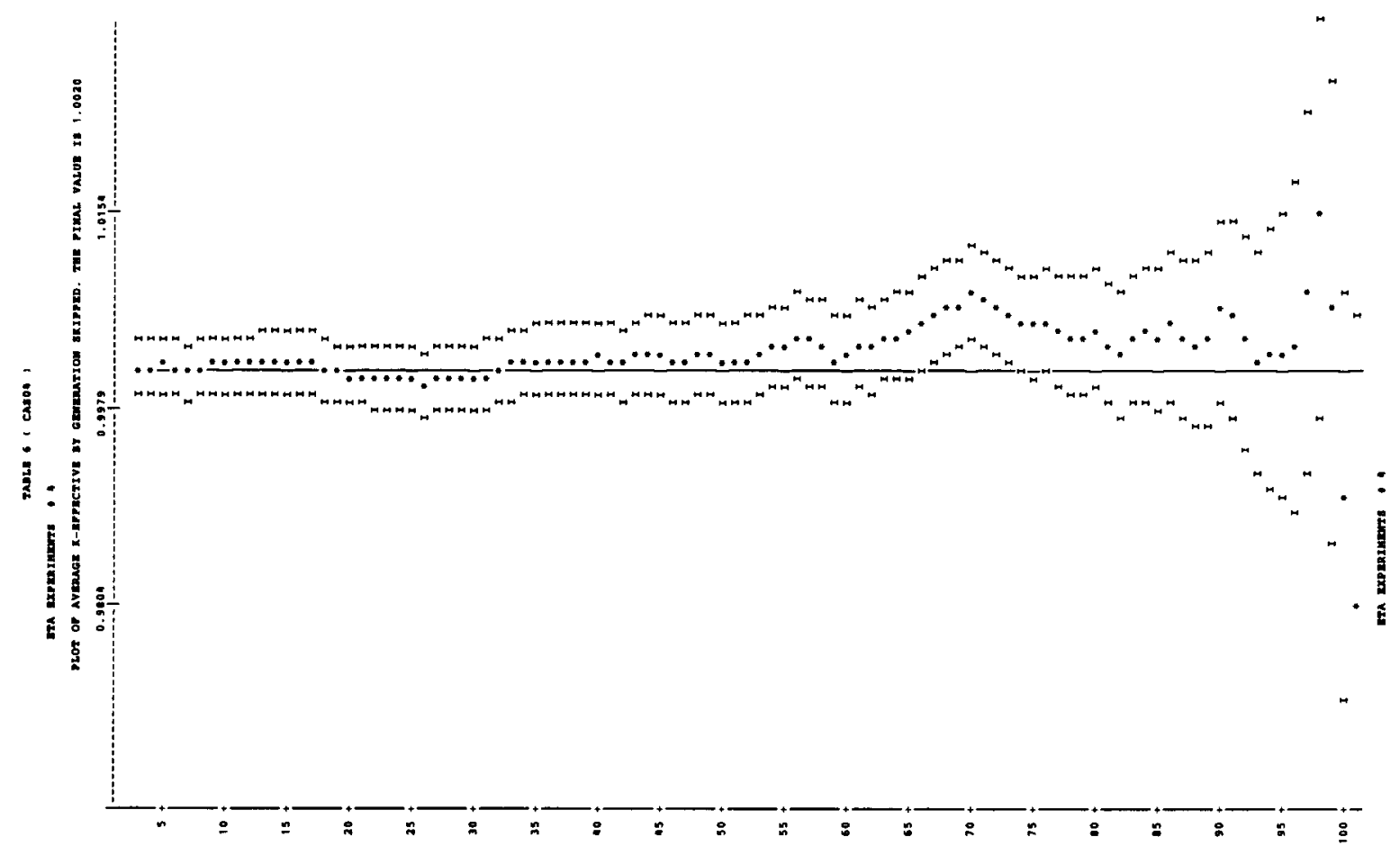

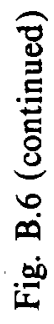

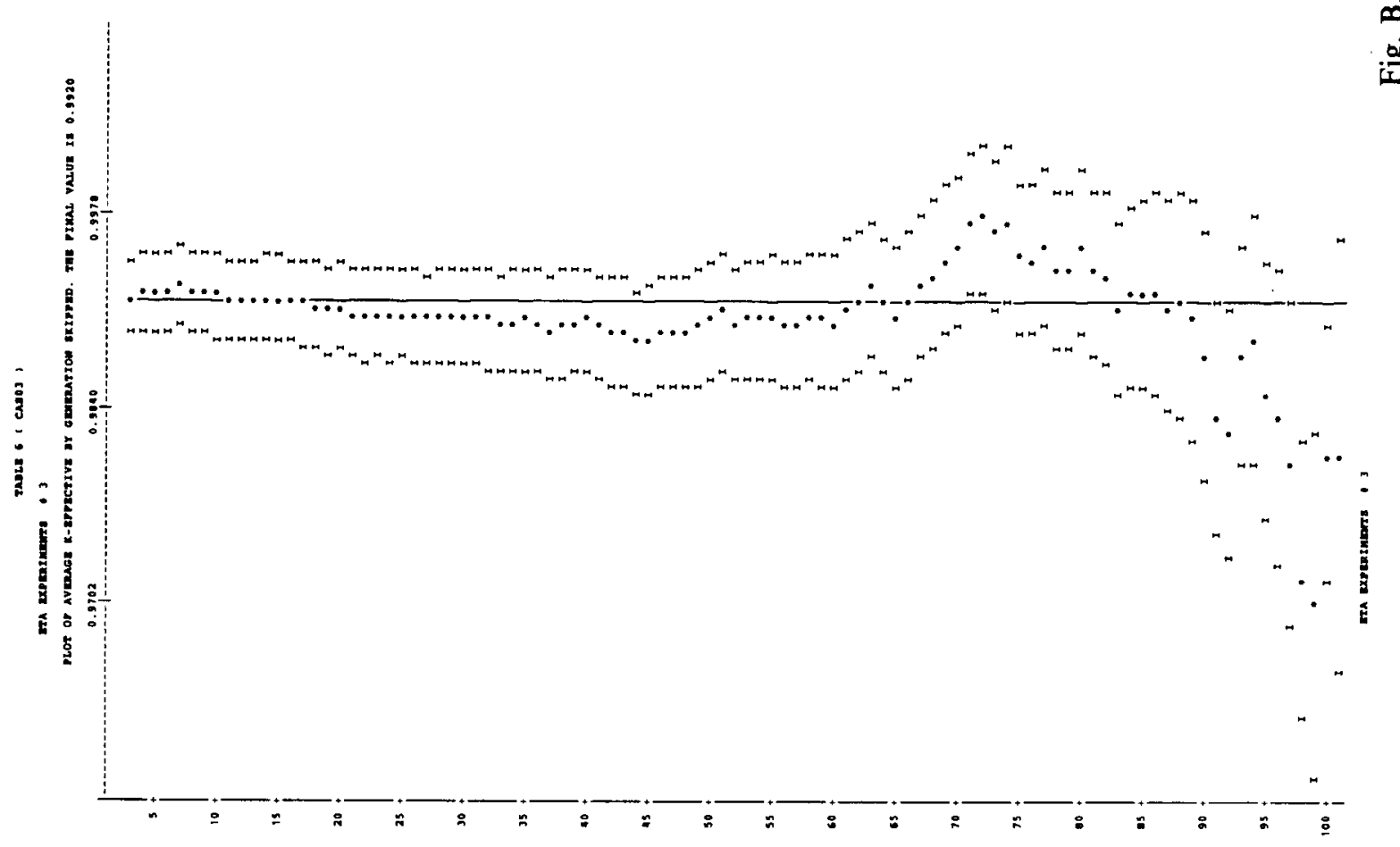



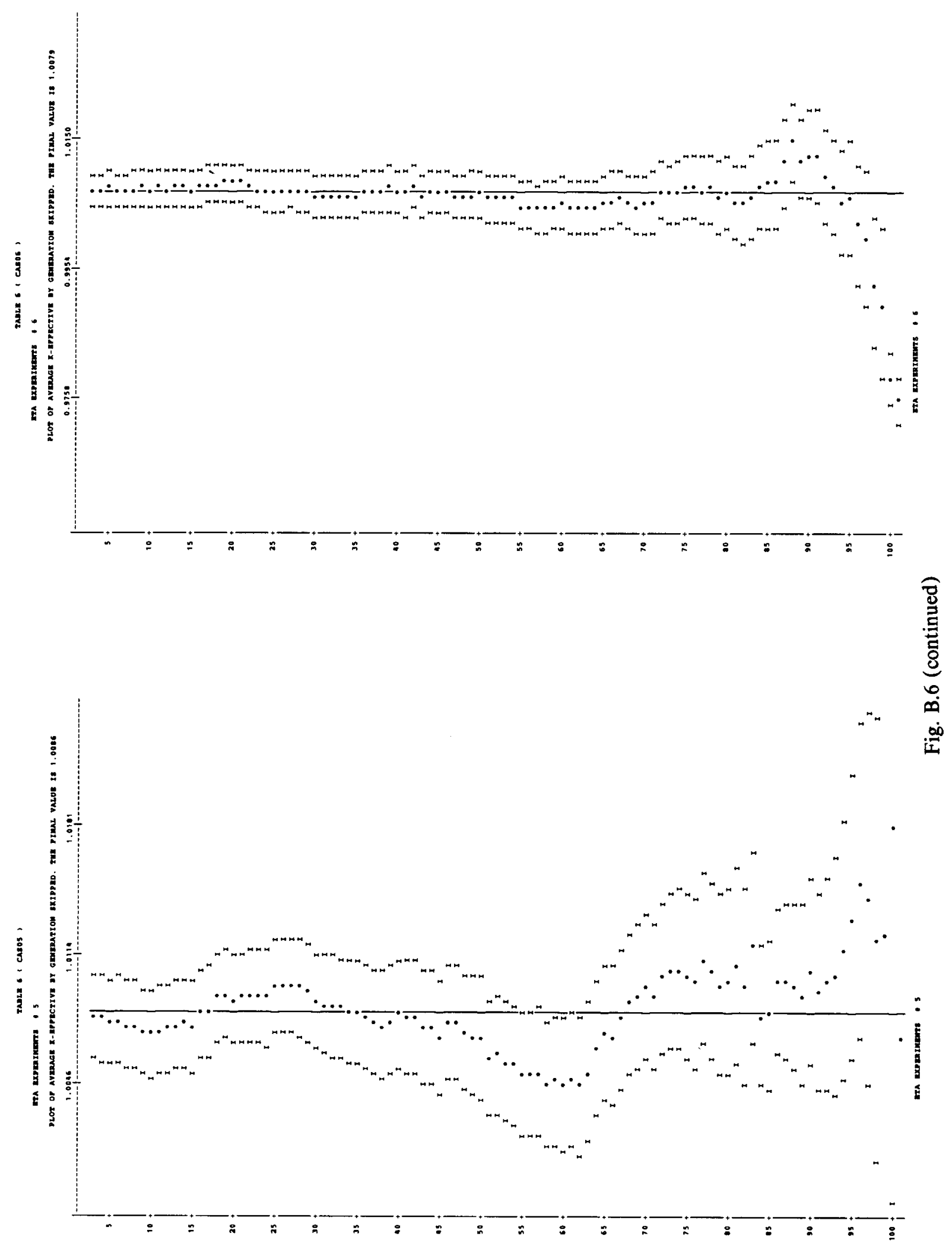


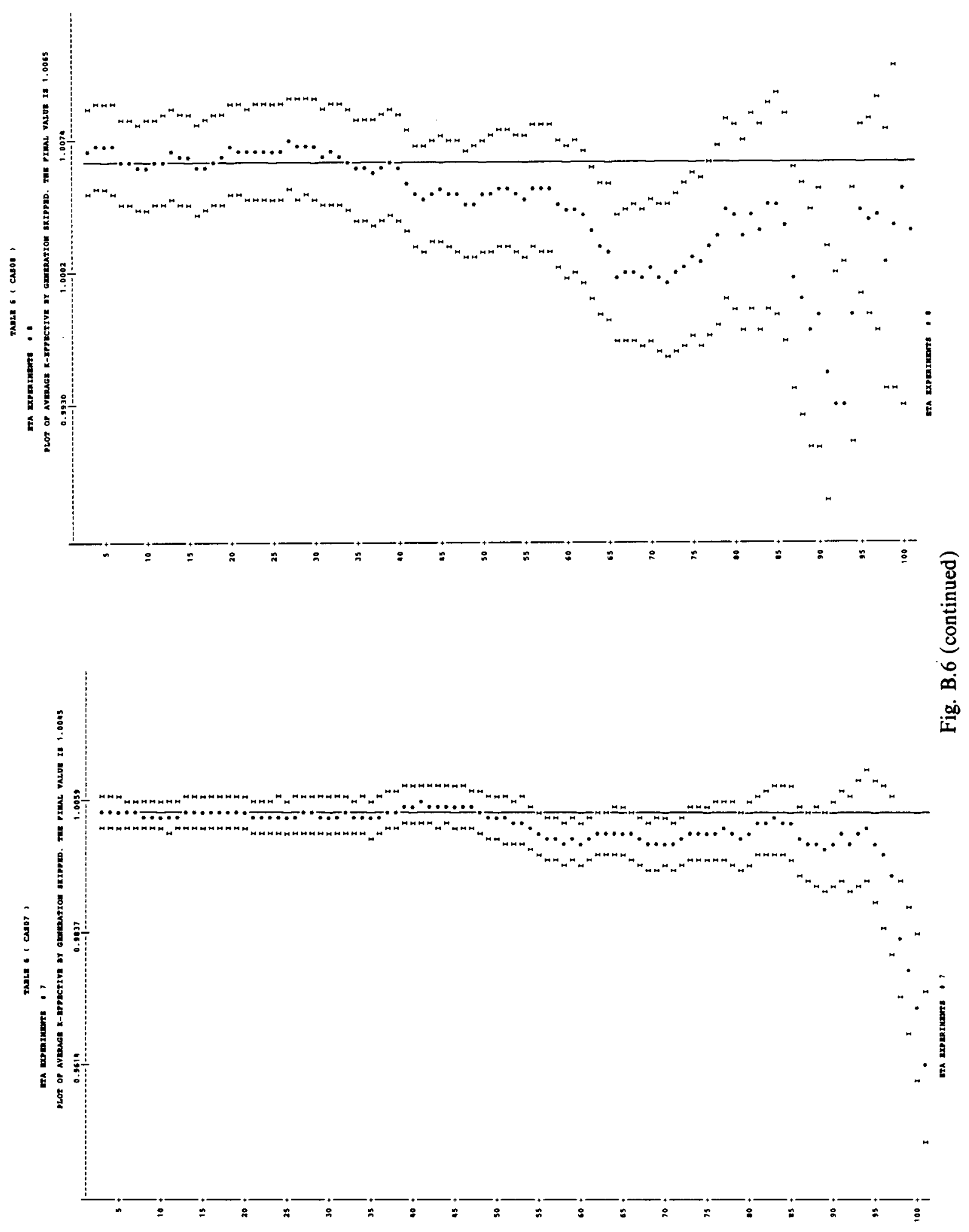




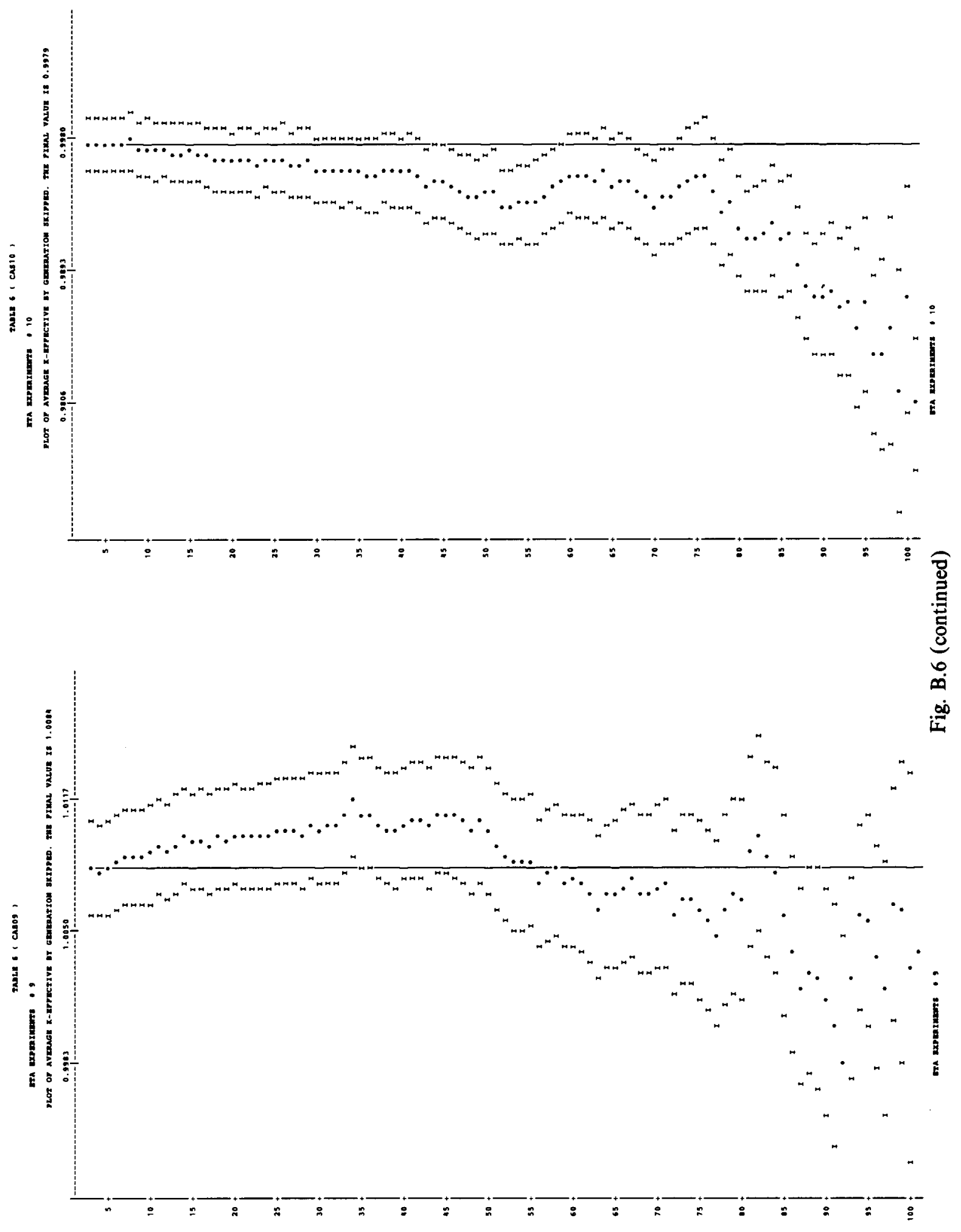


B- 126

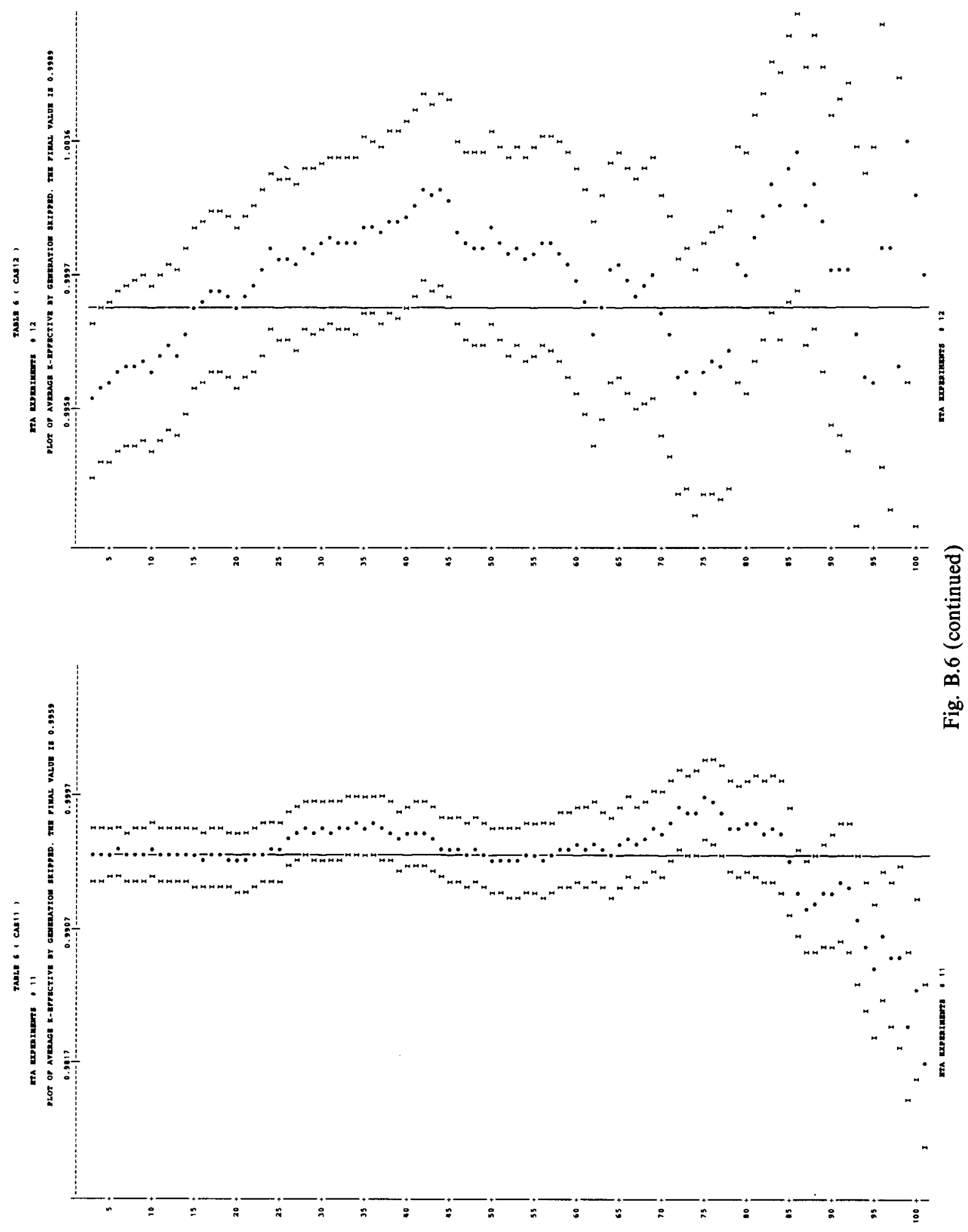


B-127

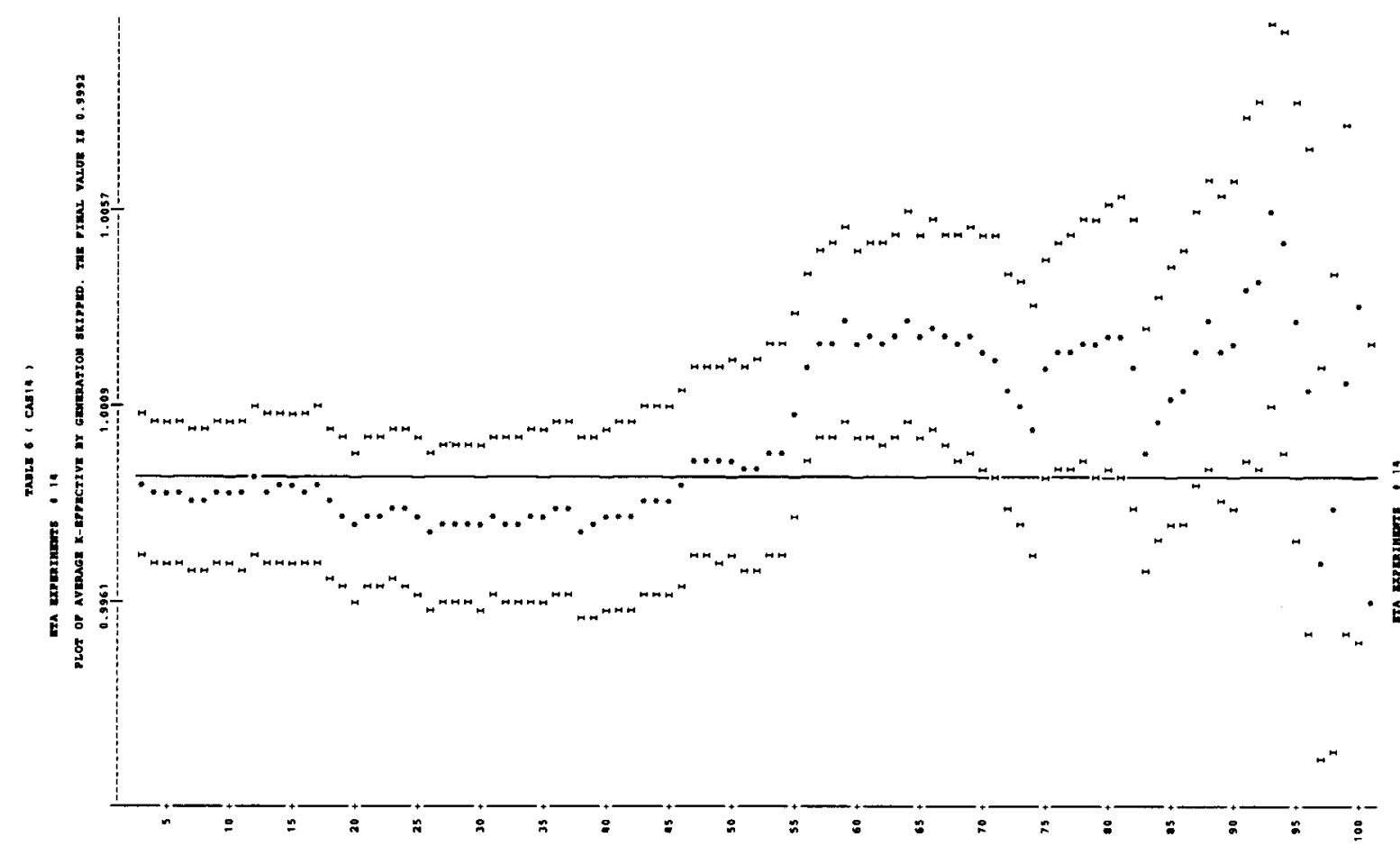

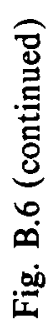

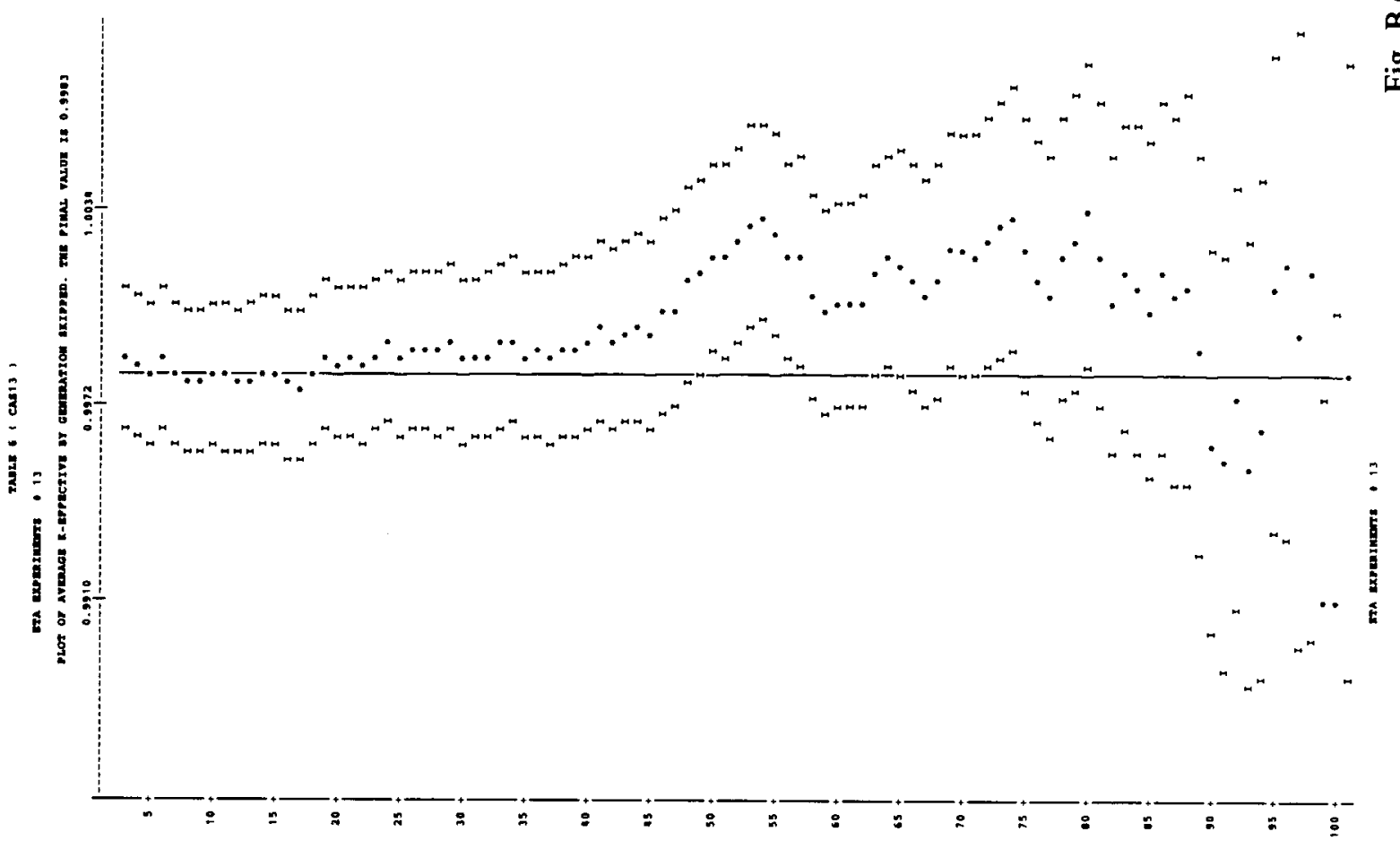




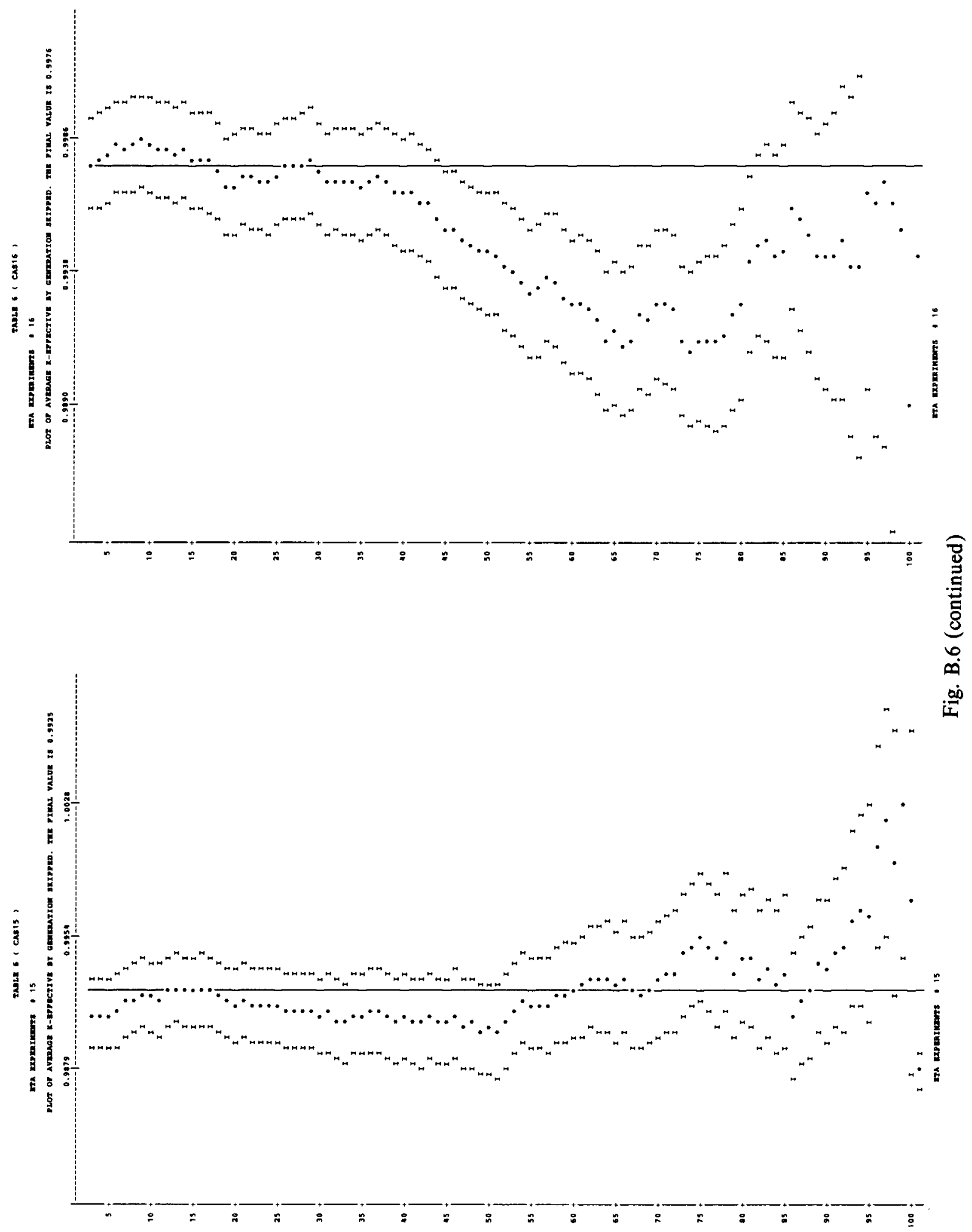




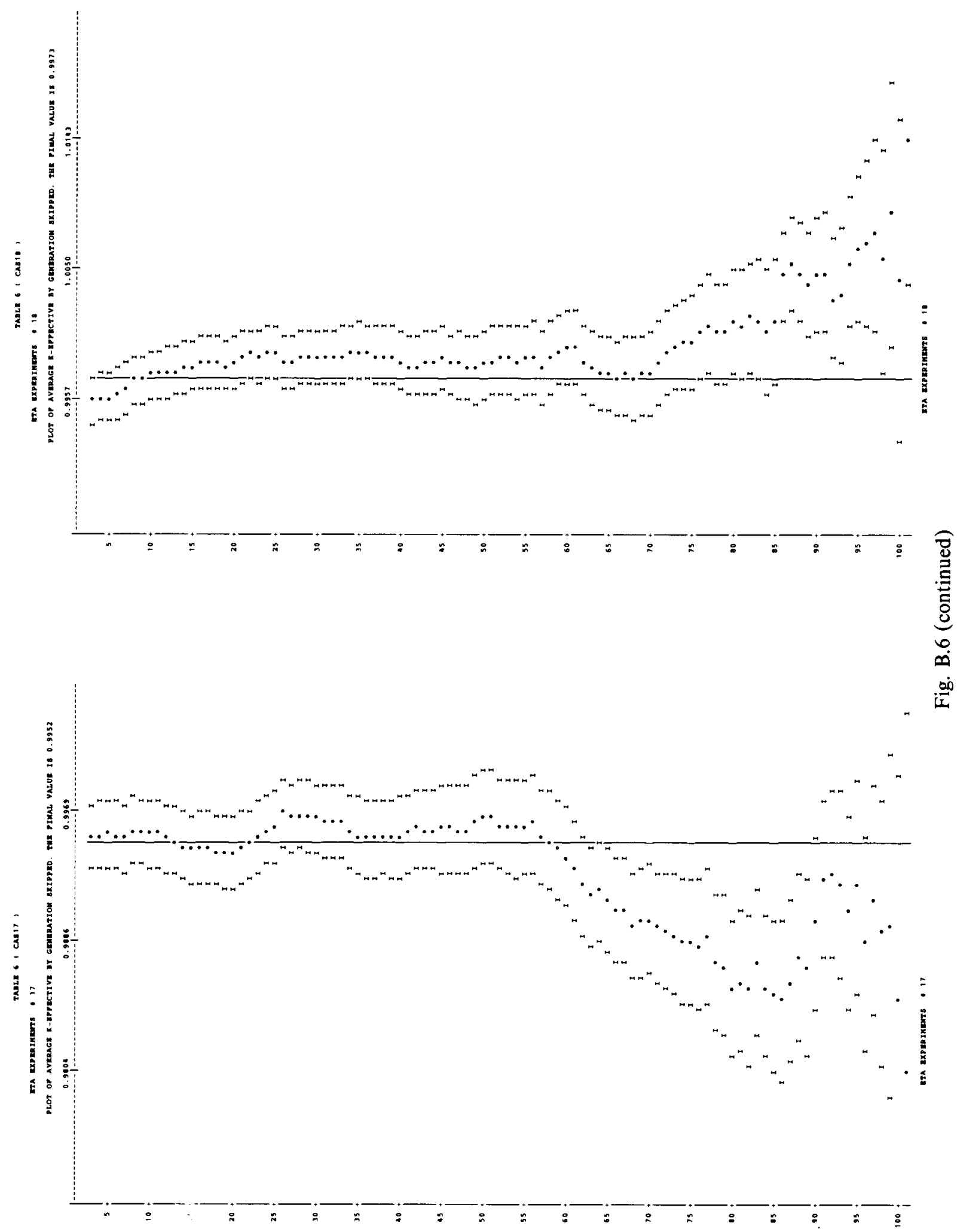


B-130

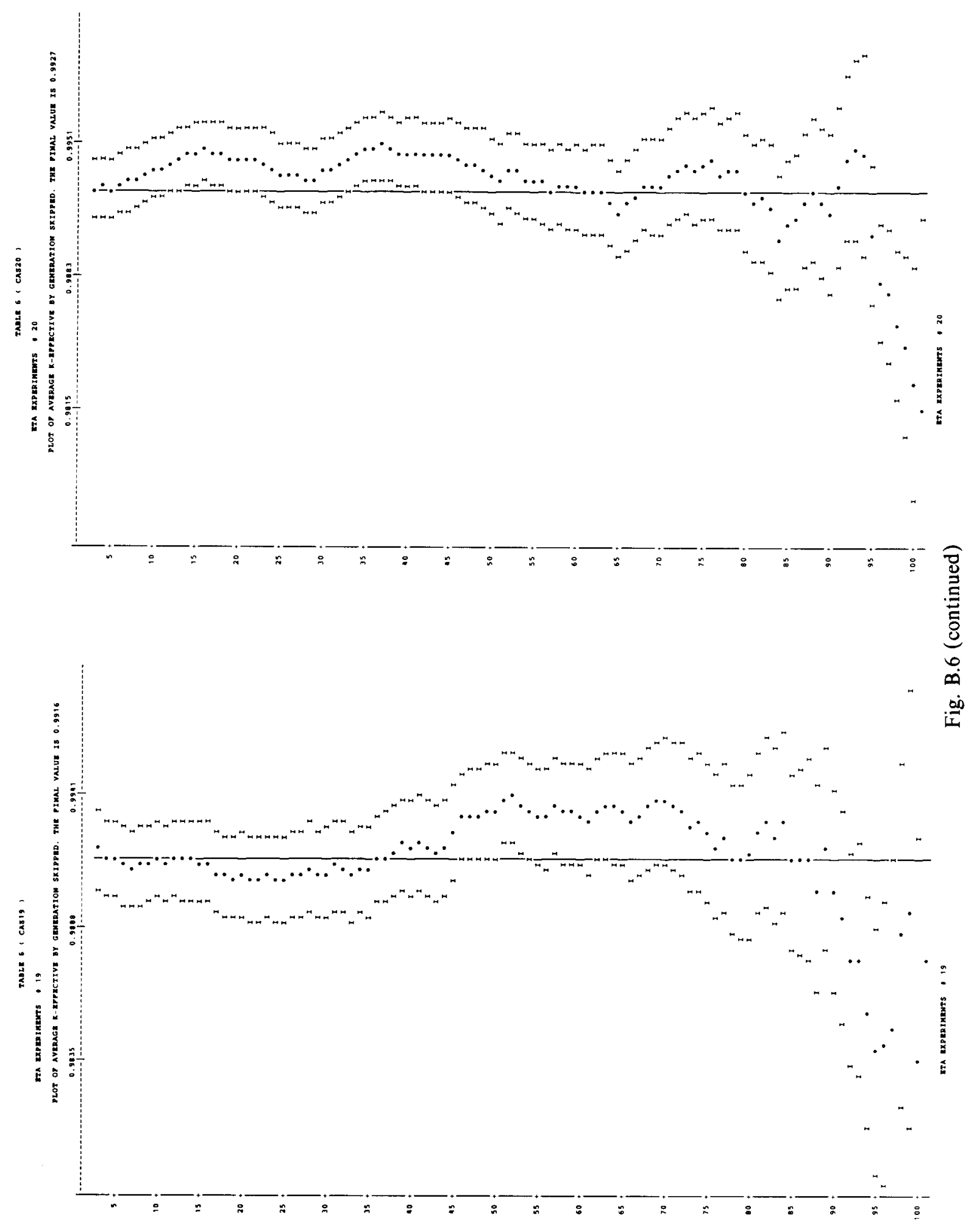



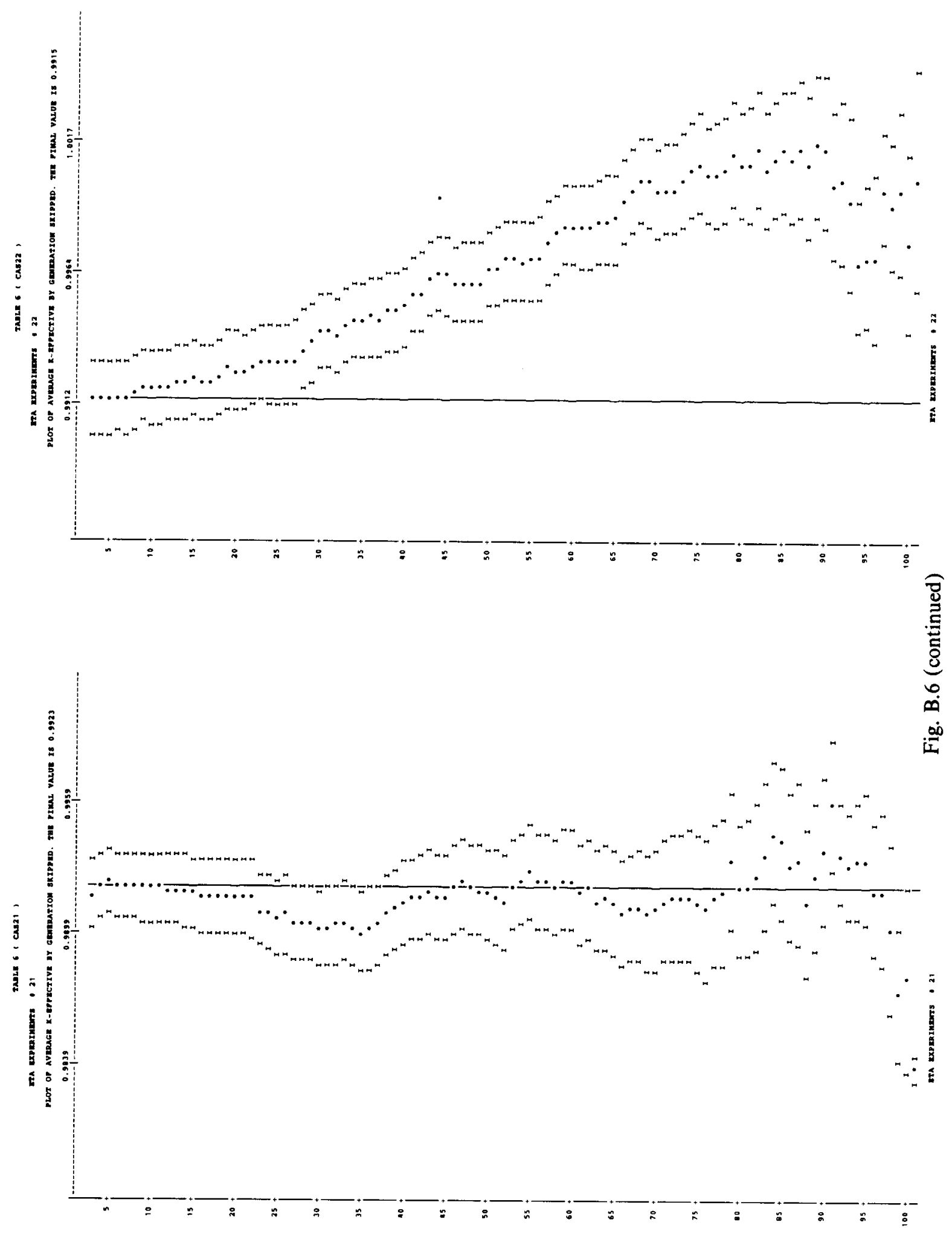
B-132

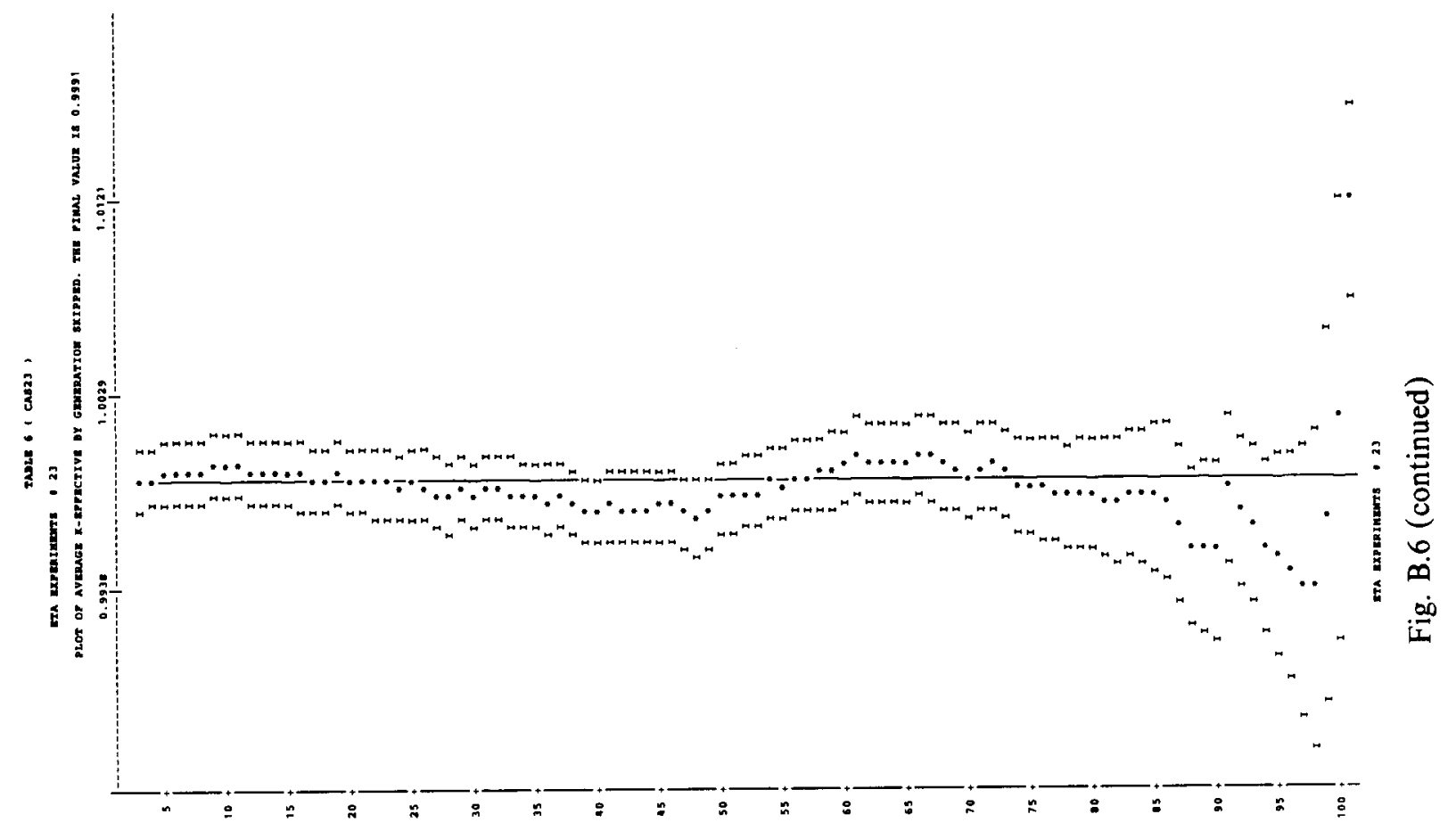




\section{Appendix C}

\section{CALCULATED RESULTS AND EXPERIMENTAL PARAMETERS}

In order to analyze the data from the validation calculations, the experiments were parameterized. The results could then be categorized in a number of ways to test for trends and bias. Table C.1 contains the parameters which were used for data analysis for each experiment.

Key terms are as follows:

RUN - Sequential number assigned to experiments.

MEMB - Case number which corresponds to Tables 1-6.

KEFF - KENO calculated $k$.

SDM - KENO calculated standard deviation.

GBAR - Average energy group of neutron causing fission.

ENRLEV - Enrichment level (high, intermediate, low).

ASSAY - Fuel enrichment.

REFLCOND - Reflection condition (reflected, partially reflected, unreflected).

REFL - Reflector material.

SYSGEOM - Overall system geometry (array, single unit).

UNITGEOM - Principal geometry of unit (cylinder, slab, sphere).

FUELMIX - Indicator as to whether the system was considered primarily heterogeneous or homogeneous.

FUEL - Primary form of fissile material.

FMATL - Principal fissile nuclide in fuel.

REF - Reference number for reference which describes the critical experiment. 
Table C.1. Calculational results and experimental parameters

\begin{tabular}{|c|c|c|c|c|c|c|c|c|c|c|c|c|c|c|}
\hline RUN & MEMB & KEFF & SDM & GBAR & ENRLEV & ASSAX & REFLCOND & RBFL & SYSGBOM & UNITGBOM & FUBLMIX & FUEL & PMATL & REF \\
\hline 1 & CAA0 1 & 0.9945 & 0.0036 & 21.2098 & I & 4.89 & $\mathbf{R}$ & H2O & A & CY & HET & METAL & U235 & 14 \\
\hline 2 & $\mathrm{CAAO} 2$ & 0.9912 & 0.0030 & 21.7488 & L & 4.89 & $\mathbf{R}$ & н20 & A & CY & HET & METAL & U235 & 14 \\
\hline 3 & CAAO3 & 0.9924 & 0.0031 & 22.8842 & I & 4.89 & $\mathbf{R}$ & H2O & $\mathbf{A}$ & CY & HET & METAL & U235 & 14 \\
\hline 4 & CAAO 4 & 0.9942 & 0.0032 & 23.1207 & L & 4.89 & R & $\mathrm{H} 2 \mathrm{O}$ & $\ddot{A}$ & $\mathrm{CY}$ & HET & METAL & U235 & 14 \\
\hline 5 & CAAO5 & 0.9839 & 0.0034 & 23.2252 & I & 4.89 & $\mathbf{R}$ & H2O & A & CY & HET & METAL & U235 & 14 \\
\hline 6 & CAA0 6 & 0.9957 & 0.0036 & 21.1295 & L & 4.89 & $\mathbf{R}$ & COMP & A & CY & HET & METAL & U235 & 14 \\
\hline 7 & CAA07 & 0.9778 & 0.0036 & 21.4048 & I & 4.89 & $\mathbf{R}$ & PB & A & CY & HET & METAL & U235 & 14 \\
\hline 8 & CAAOB & 0.9966 & 0.0030 & 22.8577 & L & 4.89 & R & PB & A & CY & HET & METAL & U235 & 14 \\
\hline 9 & CAA09 & 0.9950 & 0.0029 & 22.7496 & L & 4.89 & $\mathbf{R}$ & PB & A & $C Y$ & HET & METAL & U235 & 14 \\
\hline 10 & CAA 10 & 1.0010 & 0.0032 & 20.9158 & L & 4.89 & $\mathbf{R}$ & PB & A & CY & HET & METAL & U235 & 14 \\
\hline 11 & CAA 11 & 0.9921 & 0.0033 & 20.8889 & L & 4.89 & $\vec{R}$ & PB & A & $\mathrm{CY}$ & HET & METAL & U235 & 14 \\
\hline 12 & CAA 12 & 0.9885 & 0.0026 & 24.0050 & L & 4.89 & $\mathbf{R}$ & U02F2 & A & $C Y$ & HET & METAL & U235 & 14 \\
\hline 13 & CAA 13 & 1.0035 & 0.0022 & 24.3601 & I & 4.89 & R & U02F2 & A & $c Y$ & HET & METAL & บ235 & 14 \\
\hline 14 & CAA 14 & 0.9921 & 0.0025 & 24.4837 & L & 4.89 & $R$ & U02F2 & $\mathbf{A}$ & $\mathrm{CY}$ & HET & METAL & U235 & 14 \\
\hline 15 & CAA 15 & 0.9908 & 0.0020 & 24.1875 & L & 4.89 & $\mathbf{R}$ & UO2F2 & A & CY & HET & METAL & U2.35 & 14 \\
\hline 16 & CAA 16 & 0.9940 & 0.0025 & 24.1867 & L & 4.89 & $\mathbf{R}$ & UO2F2 & A & CY & HET & METAL & U235 & 14 \\
\hline 17 & CAA 17 & 0.9884 & 0.0029 & 24.2535 & I & 4.89 & $\mathbf{R}$ & U02F 2 & $\mathbf{A}$ & cy & HET & METAL & บ235 & 14 \\
\hline 18 & CAA 18 & 0.9885 & 0.0025 & 24.3097 & I & 4.89 & $\mathbf{R}$ & UO2F2 & A & CY & HET & METAL & U235 & 14 \\
\hline 19 & CAA 19 & 0.9875 & 0.0035 & 23.3587 & L & 4.89 & U & & $\mathrm{s}$ & SL & ном & U308 & U235 & 15 \\
\hline 20 & CAA2O & 1.0030 & 0.0031 & 24.0125 & L & 4.89 & U & & $\mathbf{s}$ & SL & ном & U308 & U235 & 15 \\
\hline 21 & CAA21 & 1.0055 & 0.0029 & 24.5659 & $\mathbf{L}$ & 4.89 & U & & s & st & ном & U 308 & U235 & 15 \\
\hline 22 & CAA22 & 1.0042 & 0.0034 & 24.2557 & I & 4.89 & u & & s & sI & ном & U 308 & U235 & 15 \\
\hline 23 & CAA23 & 0.9791 & 0.0036 & 23.3846 & L & 4.89 & $\mathbf{R}$ & H2O & $\mathbf{s}$ & SL & HOM & U308 & U235 & 15 \\
\hline 24 & CAA24 & 0.9918 & 0.0033 & 23.6607 & $\mathbf{L}$ & 4.89 & R & $\mathrm{H} 2 \mathrm{O}$ & s & SI & HOS & U308 & U235 & 15 \\
\hline 25 & CAA25 & 1.0015 & 0.0035 & 24.1830 & L & 4.89 & $\ddot{R}$ & H2O & s & SL & ном & U308 & U235 & 15 \\
\hline 26 & CAA26 & 0.9991 & 0.0025 & 24.6252 & L & 4.89 & $\mathbf{R}$ & н20 & $\mathbf{s}$ & SL & ном & U308 & U235 & 15 \\
\hline 27 & CAA27 & 0.9991 & 0.0040 & 22.8645 & L & 4.89 & $\mathbf{R}$ & H2O & $\mathbf{s}$ & SL & Hом & U308 & U235 & 15 \\
\hline 28 & CAA28 & 0.9739 & 0.0035 & 21.6194 & $\mathbf{L}$ & 4.89 & $\ddot{R}$ & H2O & s & SL & ном & บ 308 & U235 & 15 \\
\hline 29 & CAA29 & 0.9938 & 0.0031 & 24.3746 & L & 4.89 & $\mathbf{R}$ & H2O & $s$ & SL & HOM & U308 & บ235 & 15 \\
\hline 30 & CAA3O & 0.9861 & 0.0032 & 24.2794 & L & 4.89 & $\mathbf{u}$ & & s & $c Y$ & ном & U02F2 & U235 & 15 \\
\hline 31 & CAA31 & 0.9936 & 0.0032 & 24.2837 & $\mathbf{L}$ & 4.89 & $\mathbf{u}$ & & $s$ & SL & ном & U02F2 & U235 & 15 \\
\hline 32 & CAA32 & 0.9856 & 0.0028 & 24.5312 & $\mathbf{L}$ & 4.89 & $\mathbf{U}$ & & $\mathrm{s}$ & $\mathrm{CY}$ & HOM & U02F2 & บ235 & 15 \\
\hline 33 & CAA33 & 0.9949 & 0.0027 & 24.7174 & L & 4.89 & $\mathbf{u}$ & & $\mathbf{s}$ & SP & HOM & U02F 2 & U235 & 15 \\
\hline 34 & CAA34 & 0.9959 & 0.0022 & 24.7148 & L & 4.89 & u & & s & CY & HOM & U02F 2 & U235 & 15 \\
\hline 35 & $\operatorname{CAN} 35$ & 1.0029 & 0.0030 & 24.3638 & I & 4.89 & $\mathbf{R}$ & н20 & $\mathbf{s}$ & $\mathrm{CY}$ & ном & U02F 2 & U235 & 15 \\
\hline 36 & CAA36 & 1.0106 & 0.0026 & 24.3983 & I & 4.89 & $\mathbf{R}$ & H2O & s & SI & Hом & U02F2 & 0235 & 15 \\
\hline 37 & CAA37 & 0.9931 & 0.0027 & 24.5838 & L & 4.89 & $\mathbf{R}$ & H2O & $\mathbf{s}$ & $C Y$ & ном & U02F 2 & U235 & 15 \\
\hline 38 & CAA 38 & 0.9927 & 0.0022 & 24.7829 & $\mathbf{L}$ & 4.89 & $\mathbf{R}$ & H2O & s & SP & Hом & vo2F 2 & U235 & 15 \\
\hline 39 & CAA39 & 1.0001 & 0.0025 & 24.7326 & $\mathbf{L}$ & 4.89 & R & H2O & s & CY & ном & U02F2 & U235 & 15 \\
\hline
\end{tabular}


Table C.1 (continued)

\begin{tabular}{|c|c|c|c|c|c|c|c|c|c|c|c|c|c|c|}
\hline RUN & MEMB & KEFF & SDM & GBAR & BNRL BV & ASSAY & REFLCOND & REFL & SYSGEOM & UNITGEOM & FUELMIX & PUEL & FMATL & REF \\
\hline 40 & CABO 1 & 0.9893 & 0.0026 & 14.4590 & $\mathbf{z}$ & 3.85 & $\mathbf{R}$ & H2O & $\mathbf{A}$ & CY & HET & METAL & U235 & 16 \\
\hline 41 & СABO2 & 1.0025 & 0.0029 & 15.9174 & L & 3.85 & $\mathbf{R}$ & H2O & A & CY & HET & METAL & U235 & 16 \\
\hline 42 & $\mathrm{CABO} 3$ & 0.9927 & 0.0025 & 16.7417 & L & 3.85 & $\mathbf{R}$ & H2O & A & CY & HET & METAL & v235 & 16 \\
\hline 43 & CAB 07 & 0.9897 & 0.0025 & 13.4583 & L & 3.85 & $\mathbf{R}$ & H2O & $\mathbf{A}$ & CY & HET & METAL & U235 & 16 \\
\hline 44 & САB 08 & 0.9924 & 0.0032 & 15.6574 & L & 3.85 & $\vec{R}$ & H2O & A & CY & HET & METAL & U235 & 16 \\
\hline 45 & CABO9 & & . & & L & 3.85 & $\mathbf{R}$ & H2O & $\mathbf{A}$ & CY & HET & METAI & U235 & 16 \\
\hline 46 & CAB 10 & 0.9998 & 0.0024 & 16.8740 & L & 3.85 & $\mathbf{R}$ & H2O & $\mathbf{A}$ & CY & HET & METAL & U235 & 16 \\
\hline 47 & CAB 11 & 0.9953 & 0.0025 & 17.1558 & $\mathbf{L}$ & 3.85 & $\vec{R}$ & H2O & $\mathbf{A}$ & CY & HET & METAI & U235 & 16 \\
\hline 48 & CAB 12 & 0.9943 & 0.0028 & 17.5492 & L & 3.85 & $\mathbf{R}$ & H2O & $\mathbf{A}$ & CY & HET & METAL & U235 & 16 \\
\hline 49 & CAB 13 & 0.9960 & 0.0028 & 15.8499 & L & 3.85 & $\mathbf{R}$ & H2O & A & CY & HET & METAI & U235 & 16 \\
\hline 50 & CAB 14 & 1.0093 & 0.0026 & 17.1937 & $\mathbf{L}$ & 3.85 & $\mathbf{R}$ & H2O & $\mathbf{A}$ & CY & HET & METAL & U235 & 16 \\
\hline 51 & CAB 15 & 0.9992 & 0.0030 & 18.5920 & L & 3.85 & $\ddot{R}$ & $\mathrm{H} 2 \mathrm{O}$ & $\mathbf{A}$ & CY & HET & METAL & U235 & 16 \\
\hline 52 & CAB 16 & 0.9877 & 0.0029 & 19.6705 & L & 3.85 & $\mathbf{R}$ & H2O & $\mathbf{A}$ & CY & HET & METAL & U235 & 16 \\
\hline
\end{tabular}


Table C.1 (continued)

\begin{tabular}{|c|c|c|c|c|c|c|c|c|c|c|c|c|c|c|}
\hline RUN & MEMB & KBFP & SDM & GBAR & ENRLEV & ASSAY & REFLCOND & REFI & SYSGEOM & UNITGEOM & FUELMIX & FUEL & FMATL & REF \\
\hline 53 & CASO 4 & 0.9998 & 0.0022 & 23.2902 & I & 1.40 & U & & $\mathrm{s}$ & SI & ном & UP4 & 0235 & 17 \\
\hline 54 & CAS 05 & 0.9989 & 0.0026 & 23.2900 & I & 1.40 & $\mathbf{u}$ & & $\mathbf{s}$ & SI & ном & UF 4 & 0235 & 17 \\
\hline 55 & CAS 06 & 0.9967 & 0.0022 & 23.2874 & $\mathbf{L}$ & 1.40 & $\mathbf{u}$ & & $\mathbf{s}$ & SI & ном & UP4 & U235 & 17 \\
\hline 56 & CAS 11 & 1.0023 & 0.0030 & 22.5151 & I & 2.00 & $\mathbf{R}$ & PARA & $\mathbf{s}$ & SL & ном & UF 4 & U235 & 18 \\
\hline 57 & CAS 12 & 1.0096 & 0.0026 & 22.2578 & I & 2.00 & u & & $\mathbf{s}$ & SI & ном & UF 4 & U235 & 18 \\
\hline 58 & CAS 13 & 1.0054 & 0.0029 & 23.2545 & I & 2.00 & $\mathbf{R}$ & PARA & $\mathbf{s}$ & SI & ном & UF4 & U235 & 18 \\
\hline 59 & CAS 14 & 1.0028 & 0.0027 & 23.0477 & I & 2.00 & $\mathbf{u}$ & & $\mathbf{s}$ & SI & ном & UF 4 & U235 & 18 \\
\hline 60 & CAS 15 & 0.9967 & 0.0024 & 23.6820 & I & 2.00 & $\mathbf{R}$ & PARA & $\mathbf{s}$ & SI & ном & UP 4 & U235 & 18 \\
\hline 61 & CAS 16 & 1.0000 & 0.0026 & 23.9033 & I & 2.00 & $\mathbf{R}$ & PARA & $\mathbf{s}$ & SI & ном & UP 4 & U235 & 18 \\
\hline 62 & CAS 17 & 0.9982 & 0.0027 & 24.1189 & I & 2.00 & $\mathbf{R}$ & PARA & $\mathbf{s}$ & SI & ном & UF 4 & U235 & 18 \\
\hline 63 & CAS 18 & 0.9975 & 0.0024 & 24.0427 & I & 2.00 & U & & $\mathbf{s}$ & SL & ном & UF 4 & U235 & 18 \\
\hline 64 & CAS 19 & 0.9885 & 0.0020 & 24.4577 & $\bar{L}$ & 2.00 & $\mathbf{R}$ & PARA & $\mathbf{s}$ & SI & ном & UP 4 & U235 & 18 \\
\hline 65 & CAS20 & 0.9846 & 0.0018 & 24.4312 & I & 2.00 & U & & $\mathbf{s}$ & SI & Hом & UR4 & $\mathbf{U} 235$ & 18 \\
\hline 66 & CAS21 & 1.0145 & 0.0030 & 22.2473 & L & 3.00 & $\mathbf{R}$ & PARA & $\mathbf{s}$ & SI & ном & UF 4 & U235 & 18 \\
\hline 67 & CAS 22 & 1.0228 & 0.0030 & 22.2309 & I & 3.00 & $\mathbf{R}$ & PARA & $\mathbf{s}$ & SI & ном & UF4 & U235 & 18 \\
\hline 68 & CAS23 & 1.0169 & 0.0032 & 22.2164 & I & 3.00 & $\mathbf{R}$ & PARA & s & SL & ном & UP4 & U235 & 18 \\
\hline 69 & CAS24 & 1.0129 & 0.0026 & 22.2283 & $\mathbf{L}$ & 3.00 & $\mathbf{R}$ & PARA & $\mathbf{s}$ & SI & ном & UF4 & U235 & 18 \\
\hline 70 & CAS25 & 1.0201 & 0.0032 & 22.2284 & I & 3.00 & $\mathbf{R}$ & PARA & $\mathbf{s}$ & SI & HOM & UR 4 & U235 & 18 \\
\hline 71 & CAS26 & 1.0152 & 0.0025 & 21.7760 & I & 3.00 & u & & s & SI & ном & UF 4 & U235 & 18 \\
\hline 72 & CAS 27 & 1.0187 & 0.0030 & 21.7904 & L & 3.00 & u & & $\mathbf{s}$ & SI & ном & UF4 & U235 & 18 \\
\hline 73 & CAS28 & 1.0180 & 0.0028 & 21.7835 & L & 3.00 & U & & s & SI. & ном & UF 4 & U235 & 18 \\
\hline 74 & CAs29 & 1.0131 & 0.0030 & 23.5158 & I & 3.00 & $\mathbf{R}$ & POLY & $\mathbf{s}$ & SI & ном & UP4 & U235 & 18 \\
\hline 75 & CAS 30 & 1.0173 & 0.0030 & 23.2846 & $\mathbf{E}$ & 3.00 & U & & s & SI & ном & UF 4 & U235 & 18 \\
\hline 76 & CAS 31 & 1.0131 & 0.0030 & 23.2908 & I & 3.00 & u & & $\mathbf{s}$ & SL & ном & UF 4 & U235 & 18 \\
\hline 77 & CAS 32 & 1.0124 & 0.0030 & 23.2869 & E & 3.00 & U & & s & SI & ном & UP 4 & U235 & 18 \\
\hline 78 & CAS 33 & 0.9989 & 0.0030 & 24.2166 & I & 4.98 & $\mathbf{R}$ & COMP & $\mathbf{s}$ & CY & ном & UO2F 2 & U235 & 19 \\
\hline 79 & $\operatorname{cAs} 34$ & 1.0006 & 0.0028 & 24.2332 & I & 4.98 & $\mathbf{R}$ & COMP & $\mathbf{s}$ & CY & HOM & vo2F2 & U235 & 19 \\
\hline 80 & CAS 35 & 0.9970 & 0.0034 & 24.2310 & I & 4.98 & 0 & & $s$ & SP & нох & vo2F 2 & U235 & 20 \\
\hline 89 & CAS 36 & 1.0023 & 0.0032 & 24.2333 & I & 4.98 & v & & $\mathbf{s}$ & CY & ном & UO2F2 & U235 & 21 \\
\hline 82 & CAR01 & 1.0081 & 0.0034 & 21.4024 & I & 4.46 & $\mathbf{R}$ & PIEXX & $\mathbf{s}$ & SL & HET & U308 & U235 & 22 \\
\hline 83 & CARO2 & 1.0052 & 0.0031 & 21.3916 & I & 4.46 & $\mathbf{R}$ & PLEX & s & SL & HET & U308 & U235 & 22 \\
\hline 84 & CAR03 & 0.9876 & 0.0030 & 19.6444 & I & 4.46 & $\mathbf{R}$ & PLEX & $\mathbf{s}$ & SI & HET & U308 & U235 & 22 \\
\hline 85 & CARO 4 & 9.0089 & 0.0029 & 21.2115 & I & 4.46 & $\mathbf{R}$ & conc & s & SL & HET & U 308 & U235 & 22 \\
\hline 86 & CAR05 & 0.9998 & 0.0027 & 19.1840 & L & 4.46 & $\ddot{R}$ & conc & $\mathrm{s}$ & SL & HET & U 308 & U235 & 22 \\
\hline 87 & CAR06 & 0.9951 & 0.0031 & 12.8911 & I & 4.46 & $\mathbf{R}$ & PLEX & $\mathbf{s}$ & SL & HOX & U308 & U235 & 23 \\
\hline 88 & CARO7 & 0.9998 & 0.0032 & 18.3330 & I & 4.46 & $\mathbf{R}$ & PLEX & s & SI & HOM & U308 & U235 & 23 \\
\hline 89 & CAROB & 0.9980 & 0.0036 & 19.2715 & I & 4.46 & $\mathbf{R}$ & PIEX & $\mathbf{s}$ & sL & HOX & U 308 & U235 & 23 \\
\hline 90 & CAROG & 0.9983 & 0.0030 & 19.3540 & I. & 4.46 & R & PIEX & s & SL & HOM & U308 & U235 & 23 \\
\hline 91 & CAR10 & 1.0008 & 0.0034 & 17.7171 & L & 4.46 & $\mathrm{R}$ & cONC & $\mathbf{s}$ & SL & HOM & U308 & U235 & 23 \\
\hline 92 & CARI1 & 1.0073 & 0.0032 & 21.5573 & L & 4.46 & R & PIEX & s & SI & HET & U308 & U235 & 24 \\
\hline 93 & CAR12 & 1.0109 & 0.0031 & 20.2060 & L & 4.46 & $\mathbf{R}$ & PIEX & s & SI & HET & U308 & U235 & 24 \\
\hline 94 & CAR13 & 1.0108 & 0.0032 & 20.1720 & L & 4.46 & $\mathbf{R}$ & PLEX & $s$ & SI & HET & บ 308 & U235 & 24 \\
\hline 95 & CAR14 & 0.9907 & 0.0034 & 18.5482 & L & 4.46 & $\mathbf{R}$ & PLEX & s & si & нок & U308 & U235 & 24 \\
\hline 96 & CAR15 & 1.0106 & 0.0033 & 18.5995 & $\mathbf{L}$ & 4.46 & R & PLEX & s & SL & HOM & U308 & U235 & 24 \\
\hline 97 & CAR16 & 0.9978 & 0.0036 & 19.2434 & L & 4.46 & $\mathbf{R}$ & PLEX & $\mathbf{s}$ & sL & HOM & U308 & U235 & 24 \\
\hline 98 & CAR 17 & 1.0069 & 0.0033 & 20.7665 & L & 4.46 & $\mathbf{R}$ & PLEX & $\mathbf{s}$ & sL & HBT & U308 & U235 & 25 \\
\hline 99 & CAR18 & 1.0005 & 0.0030 & 21.8233 & L & 4.46 & $\mathbf{R}$ & PIEX & $\mathbf{s}$ & sL & HET & U 308 & U235 & 25 \\
\hline 100 & CAR 19 & 1.0097 & 0.0032 & 18.2384 & L & 4.46 & $\mathbf{R}$ & PLEX & $\mathbf{s}$ & sL & HоМ & U308 & U235 & 25 \\
\hline 101 & CAR2O & 0.9911 & 0.0031 & 18.4058 & L. & 4.46 & $\mathbf{R}$ & PLEX & $\mathbf{s}$ & sL & HOM & U308 & U235 & 25 \\
\hline
\end{tabular}


Table C.1 (continued)

\begin{tabular}{|c|c|c|c|c|c|c|c|c|c|c|c|c|c|c|}
\hline RUN & MEMB & KBFF & SDM & GBAR & BNRLEV & ASSAY & REFLCOND & RBPL & SYSGBOM & UNITGBOM & FUELMIX & FUEL & PMate & REP \\
\hline 102 & CAAO1 1 & 1.0096 & 0.0026 & 24.9405 & н & 93.20 & $\mathbf{v}$ & & $\mathbf{s}$ & SP & ноя & vo2F 2 & U235 & 26 \\
\hline 103 & CAA02 & 1.0036 & 0.0023 & 25.0019 & H & 93.20 & u & & s & SP & ном & טO2P2 & U235 & 26 \\
\hline 104 & CAAO3 & 0.9998 & 0.0023 & 24.9968 & н & 93.20 & $\mathbf{u}$ & & $\mathbf{s}$ & SP & Hом & NITRATE & U235 & 26 \\
\hline 105 & CAAO 4 & 1.0071 & 0.0039 & 22.5453 & H & 93.20 & $\mathbf{R}$ & H2O & $\mathbf{s}$ & SP & ном & v02F2 & U235 & 26 \\
\hline 106 & CAAO5 & 0.9971 & 0.0046 & 23.4178 & H & 93.20 & $\mathbf{R}$ & H2O & $s$ & SP & ном & U02F2 & U235 & 26 \\
\hline 107 & CAAOE & 0.9964 & 0.0023 & 24.9948 & H & 93.20 & $\mathbf{R}$ & H2O & $\mathbf{s}$ & SP & ном & vo2F2 & U235 & 26 \\
\hline 108 & CAA 07 & 1.0097 & 0.0043 & 24.2629 & H & 93.20 & $\mathbf{R}$ & н2O & s & SP & ном & vo2F2 & U235 & 27 \\
\hline 109 & CAA08 & 1.0212 & 0.0033 & 24.6833 & H & 93.20 & $\mathbf{R}$ & H2O & $\mathbf{s}$ & SP & ном & U02F2 & U2 35 & 27 \\
\hline 110 & CAA09 & 0.9991 & 0.0039 & 23.7419 & н & 93.20 & $\mathbf{u}$ & & $\mathbf{s}$ & SP & ном & บ02P2 & U2 35 & 27 \\
\hline 111 & CAA 10 & 1.0164 & 0.0042 & 24.1535 & H & 93.20 & $\mathbf{R}$ & $\mathbf{H 2 O}$ & $\mathrm{s}$ & SP & ном & U02F2 & U235 & 27 \\
\hline 112 & CAA 11 & 1.0351 & 0.0039 & 24.6366 & H & 93.20 & $\mathbf{R}$ & H2O & $\mathbf{s}$ & SP & ном & U02F2 & U2 35 & 27 \\
\hline 113 & CAA 12 & 1.0090 & 0.0039 & 23.6132 & H & 93.20 & v & & $\mathbf{s}$ & CY & ном & NITRATE & U235 & 28 \\
\hline 114 & CAA 13 & 1.0060 & 0.0035 & 21.6046 & H & 93.20 & $\mathbf{u}$ & & s & CY & ном & NITRATE & U235 & 28 \\
\hline 115 & CAA 14 & 1.0119 & 0.0042 & 24.5733 & H & 93.20 & $\mathbf{u}$ & & $\mathrm{s}$ & $\mathrm{CY}$ & ном & NITRATE & U235 & 28 \\
\hline 116 & CAA 15 & 1.0027 & 0.0046 & 23.6688 & H & 93.20 & u & & s & CY & HON & NITRATE & U235 & 28 \\
\hline 117 & CAA 16 & 1.0024 & 0.0045 & 21.5956 & H & 93.20 & $\mathbf{u}$ & & $s$ & CY & ном & NITRATE & U235 & 28 \\
\hline 118 & CAA 17 & 1.0106 & 0.0041 & 23.6234 & н & 93.20 & $\mathbf{R}$ & CONC & $s$ & CY & HOM & NITRATE & U235 & 28 \\
\hline 119 & CAA18 & 1.0145 & 0.0044 & 21.8900 & н & 93.20 & $\mathbf{R}$ & CONC & $\mathrm{s}$ & CY & ном & NITRATE & U235 & 28 \\
\hline 120 & CAA19 & 1.0056 & 0.0043 & 23.6210 & н & 93.20 & $\mathbf{R}$ & CONC & $\mathbf{s}$ & CY & ном & NITRATE & U235 & 28 \\
\hline 121 & CAA2O & 1.0037 & 0.0044 & 21.8675 & H & 93.20 & $\mathbf{R}$ & CONC & $\mathrm{s}$ & CY & HOM & NITRATE & U235 & 28 \\
\hline 122 & CAA21 & 1.0094 & 0.0040 & 23.6508 & H & 93.20 & $\mathbf{R}$ & CONC & $\mathbf{s}$ & CY & ном & NITRATE & U235 & 28 \\
\hline 123 & CAA22 & 1.0092 & 0.0037 & 21.9725 & H & 93.20 & $\mathbf{R}$ & CONC & $s$ & $C Y$ & HOM & NITRATE & U235 & 28 \\
\hline 124 & CAA23 & 1.0085 & 0.0040 & 23.6011 & H & 93.20 & $\mathbf{R}$ & PLEX & s & CY & ном & NITRATE & U235 & 28 \\
\hline 125 & CAA24 & 1.0113 & 0.0047 & 21.8062 & $\mathbf{H}$ & 93.20 & $\mathbf{R}$ & PLEX & $\mathrm{s}$ & CY & HOM & NITRATE & U235 & 28 \\
\hline 126 & CAA25 & 0.9947 & 0.0041 & 23.5969 & H & 93.20 & $\mathbf{R}$ & PLEX & s & CY & ном & NITRATE & U235 & 28 \\
\hline 127 & CAA26 & 1.0033 & 0.0040 & 21.8159 & H & 93.20 & R & PLEX & $\mathrm{s}$ & CY & ном & NITRATE & U235 & 28 \\
\hline 128 & CAA27 & 1.0157 & 0.0034 & 23.6729 & H & 93.20 & $\mathbf{R}$ & PLEX & $s$ & $\mathbf{C Y}$ & ном & NITRATE & U235 & 28 \\
\hline 129 & CAA28 & 1.0141 & 0.0043 & 22.0524 & н & 93.20 & $\mathbf{R}$ & PLEX & s & $\mathrm{CY}$ & HOM & NITRATE & U235 & 28 \\
\hline 130 & CAA29 & 1.0046 & 0.0033 & 24.4591 & H & 93.20 & $\mathbf{R}$ & cONC & A & $\mathrm{CY}$ & ном & NITRATE & U235 & 28 \\
\hline 131 & CAA30 & 1.0061 & 0.0041 & 21.9559 & H & 93.20 & $\mathbf{R}$ & CONC & A & CY & Hом & NITRATE & U235 & 28 \\
\hline 132 & CAA31 & 1.0119 & 0.0035 & 24.3045 & н & 93.20 & $\mathbf{R}$ & CONC & A & $\mathbf{C Y}$ & HOM & NITRATE & U235 & 28 \\
\hline 133 & CAA32 & 1.0056 & 0.0041 & 21.9640 & H & 93.20 & $\mathbf{R}$ & CONC & A & CY & ном & NITRATE & U235 & 28 \\
\hline 134 & CAA 33 & 1.0098 & 0.0033 & 24.3539 & H & 93.20 & R & coNc & A & CY & Hов & NITRATE & U235 & 28 \\
\hline 135 & $\operatorname{can} 34$ & 1.0014 & 0.0038 & 21.7453 & H & 93.20 & $\mathbf{R}$ & CONC & $\mathbf{A}$ & CY & HOM & NITRATE & U235 & 28 \\
\hline 136 & CAA35 & 1.0129 & 0.0045 & 21.9253 & H & 93.20 & $\mathbf{R}$ & CONC & A & CY & HOM & NITRATE & U235 & 28 \\
\hline 137 & CAA36 & 1.0099 & 0.0039 & 21.9359 & H & 93.20 & $\mathbf{R}$ & CONC & $\mathbf{A}$ & CY & ном & NITRATE & U235 & 28 \\
\hline 138 & CAA 37 & 1.0000 & 0.0034 & 24.5738 & H & 93.20 & $\mathbf{R}$ & PLEX & A & CY & HоM & NITRATE & U235 & 28 \\
\hline 139 & CAA38 & 0.9961 & 0.0038 & 22.1639 & H & 93.20 & $\mathbf{R}$ & PLEX & A & CY & HOM & NITRATE & U235 & 28 \\
\hline 140 & CAA39 & 1.0045 & 0.0034 & 24.5916 & H & 93.20 & $\mathbf{R}$ & PLEX & A & CY & Ном & NITRATE & U235 & 28 \\
\hline 141 & CAA40 & 0.9985 & 0.0039 & 22.2004 & н & 93.20 & $\mathbf{R}$ & PLEX & A & CY & ном & NITRATE & U235 & 28 \\
\hline 142 & CAA4 1 & 0.9984 & 0.0040 & 24.5568 & H & 93.20 & $\mathbf{R}$ & PLEX & A & CY & HOM & NITRATE & U235 & 28 \\
\hline 143 & CAA4 2 & 1.0041 & 0.0036 & 21.9131 & H & 93.20 & $\mathbf{R}$ & PLEX & $\boldsymbol{A}$ & CY & HOM & NITRATE & U235 & 28 \\
\hline 144 & CAA 43 & 0.9982 & 0.0038 & 22.1281 & H & 93.20 & $\mathbf{R}$ & PLEX & $\mathbf{A}$ & $\mathrm{CY}$ & HOM & NITRATE & U235 & 28 \\
\hline
\end{tabular}


Table C.1 (continued)

\begin{tabular}{|c|c|c|c|c|c|c|c|c|c|c|c|c|c|c|}
\hline RUN & MEMB & KEFF & SDM & GBAR & BNRLEV & ASSAY & REPLCOND & REPL & SYSGEOM & UNITGBOM & FUELMIX & FUEL & FMATL & REF \\
\hline 145 & CASO1 & 1.0004 & 0.0027 & 4.8613 & $\mathbf{H}$ & 93.80 & u & & $\mathbf{s}$ & SP & ном & METAL & U235 & 29 \\
\hline 146 & CASO2 & 0.9995 & 0.0031 & 8.5157 & H & 97.67 & $\mathbf{R}$ & H2O & $\mathbf{s}$ & SP & ном & METAL & U235 & 35 \\
\hline 147 & CAS 03 & 1.0029 & 0.0044 & 21.7017 & н & 93.20 & $\mathbf{u}$ & & s & CY & ном & NITRATB & U235 & 28 \\
\hline 148 & CASO4 & 1.0067 & 0.0028 & 4.9879 & $\mathbf{H}$ & 93.20 & $\mathbf{u}$ & & $\mathbf{s}$ & $C Y$ & ном & ALLOY & U235 & 30 \\
\hline 149 & CASO5 & 1.0023 & 0.0027 & 25.0041 & $\boldsymbol{H}$ & 93.20 & $\mathbf{u}$ & & $\mathbf{s}$ & SP & ном & U02F 2 & U235 & 26 \\
\hline 150 & CAS06 & 1.0012 & 0.0022 & 24.9987 & $\mathbf{H}$ & 93.20 & U & & s & SP & ном & NITRATE & U235 & 31 \\
\hline 151 & CASO7 & 1.0042 & 0.0030 & 11.7017 & н & 93.50 & $\mathbf{R}$ & $\mathrm{H} 2 \mathrm{O}$ & $\mathbf{s}$ & SP & HET & METAL & U235 & 32 \\
\hline 152 & CAS08 & 1.0124 & 0.0031 & 6.4136 & H & 93.20 & $\mathbf{R}$ & c & $\mathbf{s}$ & CY & HET & METAL & U235 & 33 \\
\hline 153 & CASOS & 1.0027 & 0.0025 & 4.9072 & н & 94.00 & $\mathbf{R}$ & U238 & $\mathrm{s}$ & SL & ном & METAL & 0235 & 32 \\
\hline 154 & CAS 10 & 1.0114 & 0.0034 & 10.4697 & H & 93.10 & $\mathbf{R}$ & OIL & $\mathbf{s}$ & SP & HET & METAL & U235 & 33 \\
\hline 155 & CAS 11 & 0.9977 & 0.0035 & 9.4346 & н & 93.10 & $\mathbf{R}$ & OIL & $\mathbf{s}$ & SP & HET & METAL & U235 & 34 \\
\hline 156 & CAS 12 & 1.0004 & 0.0031 & 4.8365 & H & 93.20 & u & & $x$ & CY & ном & METAL & U235 & 36 \\
\hline 157 & CAS 13 & 0.9854 & 0.0035 & 24.5321 & H & 92.60 & u & & $\mathbf{A}$ & $C Y$ & ном & NITRATE & U235 & 38 \\
\hline 158 & CAS 14 & 1.0585 & 0.0038 & 21.1722 & H & 93.20 & $\mathbf{R}$ & PLEX & $\mathbf{A}$ & MIX & ном & NITRATE & U235 & 39 \\
\hline 159 & CAS 15 & 1.0126 & 0.0030 & 8.2822 & H & 93.20 & $\mathbf{R}$ & POLY & A & $\mathbf{C Y}$ & HET & METAL & U235 & 40 \\
\hline 160 & CAS 16 & 0.9879 & 0.0037 & 21.4089 & H & 92.60 & u & & $\mathbf{A}$ & CY & ном & NITRATE & U235 & 38 \\
\hline 161 & CAS 17 & 1.0250 & 0.0037 & 22.1149 & $\mathbf{H}$ & 92.60 & $\mathbf{R}$ & PARA & $\ddot{A}$ & $C Y$ & ном & NITRATE & U235 & 38 \\
\hline 162 & CAS 18 & 1.0010 & 0.0045 & 21.5958 & H & 92.60 & $\mathbf{R}$ & PLEX & $\boldsymbol{A}$ & $C Y$ & HOM & NITRATE & 0235 & 38 \\
\hline 163 & CAS19 & 1.0329 & 0.0046 & 20.7978 & ห & 93.10 & u & & A & CY & ном & NITRATE & U235 & 41 \\
\hline 164 & CAS20 & 1.0369 & 0.0037 & 22.2455 & $\therefore$ & 93.20 & $\mathbf{R}$ & PLEX & A & CY & ном & NITRATE & U235 & 28 \\
\hline 165 & CAS21 & 1.0239 & 0.0043 & 21.9649 & H & 93.20 & $\mathbf{R}$ & CONC & $\mathbf{A}$ & CY & ном & NITRATE & U235 & 28 \\
\hline 166 & CAS 22 & 1.0082 & 0.0034 & 4.8329 & $\mathbf{H}$ & 93.20 & v & & M & $C Y$ & Hом & MBTAL & U235 & 36 \\
\hline 167 & CAS23 & 0.9959 & 0.0030 & 4.8188 & H & 93.20 & U & & $\mathbf{M}$ & $\operatorname{MIX}$ & ном & METAL & U235 & 36 \\
\hline 168 & CAS 24 & 0.9928 & 0.0033 & 4.8267 & B & 93.20 & u & & $\ddot{A}$ & $\mathrm{CY}$ & ном & MBTAL & U235 & 37 \\
\hline 169 & CAS 25 & 1.0079 & 0.0030 & 4.8332 & H & 93.20 & v & & A & CY & HOM & METAL & U235 & 37 \\
\hline 170 & CAS26 & 1.0011 & 0.0033 & 10.0912 & н & 93.20 & $\mathbf{R}$ & PARA & A & CY & HOM & METAL & U235 & 37 \\
\hline 171 & CAS 27 & 1.0083 & 0.0031 & 10.3812 & н & 93.20 & $\mathbf{R}$ & PARA & $\mathbf{A}$ & CY & ном & METAL & U235 & 37 \\
\hline 172 & CAS28 & 1.0102 & 0.0037 & 7.6142 & H & 93.20 & $\mathrm{v}$ & & $\mathbf{A}$ & $\mathrm{CY}$ & HET & METAL & U235 & 37 \\
\hline 173 & CAs29 & 0.9872 & 0.0041 & 21.4050 & H & 92.60 & u & & A & $C Y$ & ном & NITRATE & U235 & 38 \\
\hline 174 & $\operatorname{CAS} 30$ & 0.9978 & 0.0038 & 24.2763 & н & 93.20 & u & & s & SL & ном & UO2F 2 & U235 & 43 \\
\hline 175 & CAS 31 & 0.9949 & 0.0035 & 24.3936 & H & 93.20 & $\mathbf{R}$ & H2O & $\mathbf{s}$ & SL & ном & U02F 2 & U235 & 43 \\
\hline 176 & CAS 32 & 0.9805 & 0.0039 & 24.2603 & н & 9.3 .20 & v & & A & SL & ном & vo2F 2 & U235 & 43 \\
\hline 177 & $\operatorname{CAS} 33$ & 0.9995 & 0.0039 & 24.5095 & H & 93.20 & $\mathbf{R}$ & H2O & A & SL & ном & UO2F 2 & U235 & 43 \\
\hline 178 & $\operatorname{CAS} 34$ & 0.9803 & 0.0039 & 24.2515 & H & 93.20 & u & & A & SL & ном & v02F2 & U235 & 43 \\
\hline 179 & CAS 35 & 0.9906 & 0.0036 & 24.5440 & H & 93.20 & $\mathbf{R}$ & H2O & A & SL & ном & U02F2 & บ235 & 43 \\
\hline 180 & CAS 36 & 0.9933 & 0.0035 & 24.2518 & H & 93.20 & u & & $\ddot{A}$ & SL & HOM & U02F 2 & ข235 & 43 \\
\hline 181 & CAS 37 & 1.0030 & 0.0031 & 24.5294 & н & 93.20 & $\mathbf{R}$ & H2O & $\mathbf{A}$ & SL & HОМ & UO2F 2 & U235 & 43 \\
\hline 182 & $\operatorname{CAS} 38$ & 0.9881 & 0.0035 & 24.2464 & н & 93.20 & u & & $\mathbf{A}$ & SL & ном & vo2F2 & บ235 & 43 \\
\hline 183 & CAS 39 & 0.9952 & 0.0035 & 24.5228 & н & 93.20 & $\mathbf{R}$ & H 20 & A & SL & ном & UO2F 2 & U235 & 43 \\
\hline 184 & CAS40 & 0.9824 & 0.0041 & 24.2482 & н & 93.20 & u & & A & SL & ном & UO2F 2 & U235 & 43 \\
\hline 185 & CAS41 & 0.9767 & 0.0038 & 24.2608 & H & 93.20 & U & & $\mathbf{A}$ & SL & ном & UO2F 2 & U235 & 43 \\
\hline 186 & $\operatorname{CAS} 42$ & 0.9916 & 0.0037 & 24.2631 & н & 93.20 & u & & $\mathbf{A}$ & SL & ном & U02F 2 & บ235 & 43 \\
\hline 187 & CAS 43 & 0.9835 & 0.0037 & 24.2624 & н & 93.20 & u & & A & SL & ном & U02F2 & U235 & 43 \\
\hline 188 & CAS 44 & 0.9757 & 0.0035 & 24.2571 & н & 93.20 & u & & A & SL & ном & U02F 2 & U235 & 43 \\
\hline 189 & CAS 45 & 0.9871 & 0.0040 & 24.2721 & H & 93.20 & u & & $\mathbf{s}$ & SL & ном & UO2F 2 & U235 & 43 \\
\hline 190 & $\operatorname{CAS} 46$ & 0.9832 & 0.0040 & 24.2529 & H & 93.20 & u & & A & st & ном & U02F2 & บ235 & 43 \\
\hline 191 & CAS47 & 0.9819 & 0.0037 & 24.2521 & H & 93.20 & U & & A & SL & ном & U02F 2 & U235 & 43 \\
\hline 192 & $\operatorname{CAS} 48$ & 0.9813 & 0.0039 & 24.2524 & H & 93.20 & u & & A & SL & ном & U02F 2 & U235 & 43 \\
\hline 193 & CAS49 & 0.9831 & 0.0045 & 24.2540 & н & 93.20 & u & & A & SL & ном & U02F 2 & U235 & 43 \\
\hline 194 & $\operatorname{CAS} 50$ & 0.9814 & 0.0038 & 24.2552 & н & 93.20 & v & & A & SL & ном & U02F2 & U235 & 43 \\
\hline 195 & CAS5 1 & 0.9791 & 0.0036 & 24.2558 & H & 93.20 & $\mathbf{u}$ & & $\mathbf{A}$ & SL & ном & U02F 2 & บ235 & 43 \\
\hline 196 & CAS 52 & 0.9852 & 0.0038 & 24.2583 & H & 93.20 & $\mathrm{u}$ & & A & SL & ном & UO2F2 & U235 & 43 \\
\hline 197 & CAS 53 & 0.9884 & 0.0039 & 24.2670 & н & 93.20 & u & & $\mathbf{A}$ & SL & ном & vo2F 2 & U235 & $4 \underline{3}$ \\
\hline
\end{tabular}


Table C.1 (continued)

\begin{tabular}{|c|c|c|c|c|c|c|c|c|c|c|c|c|c|c|}
\hline RUN & MEMB & KEFF & SDM & GBAR & BNRLEV & ASSAY & REFLCOND & REFI & SYSGEOM & UNITGEOM & FUELMIX & FUEL & PMATL & REF \\
\hline 198 & CAS 54 & 0.9831 & 0.0034 & 24.2588 & H & 93.20 & U & & A & SL & ном & UO2F2 & v235 & 43 \\
\hline 199 & CAS 55 & 0.9876 & 0.0038 & 24.2616 & н & 93.20 & U & & A & SL & ном & UO2F 2 & U235 & 43 \\
\hline 200 & CAS 56 & 0.9869 & 0.0042 & 24.2615 & H & 93.20 & U & & $\mathbf{A}$ & SL & HOM & UO2F2 & U235 & 43 \\
\hline 201 & CAS 57 & 0.9864 & 0.0041 & 24.2662 & н & 93.20 & U & & $\mathbf{A}$ & SL & HOM & v0282 & U235 & 43 \\
\hline 202 & CAS 58 & 0.9824 & 0.0037 & 24.2548 & н & 93.20 & u & & $\mathbf{A}$ & SL & Hом & VO2F2 & U235 & 43 \\
\hline 203 & CAS59 & 0.9836 & 0.0036 & 24.2658 & H & 93.20 & U & & $A$ & SI & ном & UO2F 2 & U235 & 43 \\
\hline 204 & CAS 60 & 0.9998 & 0.0036 & 22.5166 & H & 92.60 & U & & A & $C Y$ & HоM & NITRATE & U235 & 38 \\
\hline 205 & CAS61 & 1.0005 & 0.0038 & 22.5263 & н & 92.60 & u & & A & CY & ном & NITRATE & U235 & 38 \\
\hline 206 & CAS 62 & 0.9934 & 0.0039 & 21.4562 & H & 92.60 & u & & A & $C Y$ & HOM & NITRATE & U235 & 38 \\
\hline 207 & CAS 63 & 1.0050 & 0.0044 & 21.4410 & H & 92.60 & U & & A & CY & ном & NITRATE & U235 & 38 \\
\hline 208 & CAS6 64 & 0.9963 & 0.0038 & 21.4417 & H & 92.60 & $\mathrm{u}$ & & $\mathbf{A}$ & CY & HOM & NITRATE & U235 & 38 \\
\hline 209 & CAS 65 & 1.0081 & 0.0039 & 21.4918 & H & 92.60 & $\mathbf{U}$ & & $\mathbf{A}$ & $C Y$ & HOM & NITRATE & ข235 & 38 \\
\hline 210 & CAS66 & 1.0020 & 0.0037 & 21.6613 & н & 92.60 & p & PLEX & A & CY & ном & NITRATE & 0235 & 38 \\
\hline 211 & CAS67 & 1.0217 & 0.0038 & 21.8258 & H & 92.60 & $\mathbf{P}$ & PLEX & $\mathbf{A}$ & CY & HOM & NITRATE & U235 & 38 \\
\hline 212 & CAS68 & 1.0170 & 0.0042 & 21.7269 & H & 92.60 & P & PLEX & A & CY & ном & NITRATE & U235 & 38 \\
\hline 213 & CAS69 & 1.0227 & 0.0040 & 22.1217 & $\mathbf{H}$ & 92.60 & $\mathbf{R}$ & PARA & A & $C Y$ & ном & NITRATE & U235 & 38 \\
\hline 214 & CAS70 & 1.0205 & 0.0039 & 22.0406 & н & 92.60 & $P$ & PARA & $\mathbf{A}$ & CY & ном & NITRATE & U235 & 38 \\
\hline 215 & CAS71 & 1.0302 & 0.0040 & 22.1075 & н & 92.60 & $\mathbf{P}$ & PARA & $\mathbf{A}$ & CY & HOM & NITRATE & U235 & 38 \\
\hline 216 & CAS 72 & 1.0016 & 0.0038 & 21.5822 & H & 92.60 & $\mathbf{P}$ & PLEX & $\mathbf{A}$ & CY & ном & NITRATE & 0235 & 38 \\
\hline 217 & CAS73 & 1.0134 & 0.0041 & 21.6538 & н & 92.60 & $\mathbf{P}$ & PARA & A & CY & нок & NITRATE & บ235 & 38 \\
\hline 218 & CAS74 & 1.0172 & 0.0035 & 22.1229 & H & 92.60 & $\mathbf{R}$ & PARA & A & CY & HOM & NITRATE & U235 & 38 \\
\hline 219 & CAS75 & 1.0300 & 0.0037 & 22.0009 & H & 92.60 & $\mathbf{P}$ & PARA & A & CY & ном & NI TRATE & U235 & 38 \\
\hline 220 & CAS76 & 1.0046 & 0.0044 & 21.6952 & H & 92.60 & $\mathbf{P}$ & PLEX & A & $C Y$ & HOM & NITRATE & U235 & 38 \\
\hline 221 & CAS77 & 1.0225 & 0.0040 & 22.1244 & н & 92.60 & $\mathbf{P}$ & PLEX & A & CY & ном & NITRATE & U235 & 38 \\
\hline 222 & CAS78 & 1.0237 & 0.0045 & 22.1373 & H & 92.60 & R & PLEX & $\mathbf{A}$ & CY & HOM & NITRATE & U235 & 38 \\
\hline 223 & CAS79 & 1.0137 & 0.0036 & 21.8230 & H & 92.60 & $\mathbf{P}$ & PLEX & A & CY & ном & NITRATE & U235 & 38 \\
\hline 224 & CAS 80 & 1.0223 & 0.0045 & 21.9906 & н & 92.60 & P & PLEX & A & CY & HOM & NITRATE & U235 & 38 \\
\hline 225 & CAS81 & 1.0197 & 0.0037 & 22.0513 & H & 92.60 & P & PLEX & A & CY & ном & NITRATB & 0235 & 38 \\
\hline 226 & CAS82 & 1.0195 & 0.0038 & 21.7696 & H & 92.60 & $\mathbf{P}$ & PLEX & $\mathbf{A}$ & CY & ном & NITRATE & U235 & 38 \\
\hline 227 & CAS 83 & 1.0225 & 0.0039 & 22.0273 & В & 92.60 & p & PLEX & A & CY & HOH & NITRATE & U235 & 38 \\
\hline 228 & CAS 84 & 1.0247 & 0.0041 & 22.1092 & H & 92.60 & $p$ & PLEX & A & CY & ном & NITRATE & U235 & 38 \\
\hline 229 & CAS 85 & 1.0175 & 0.0046 & 21.6264 & : & 92.60 & $\mathbf{P}$ & PLEX & $\mathbf{A}$ & CY & HOM & NITRATE & U235 & 38 \\
\hline 230 & CAS 86 & 1.0165 & 0.0042 & 21.6814 & H & 92.60 & P & PARA & A & CY & HOM & NITRATE & U235 & 38 \\
\hline 231 & CAS 87 & 1.0177 & 0.0042 & 22.1061 & н & 92.60 & $\mathbf{R}$ & PARA & A & CY & HOM & NITRATE & U235 & 38 \\
\hline 232 & CAS 88 & 1.0177 & 0.0041 & 22.0003 & H & 92.60 & $P$ & PARA & $\mathbf{A}$ & CY & ном & NITRATE & U235 & 38 \\
\hline 233 & CAS 89 & 1.0169 & 0.0046 & 22.1067 & H & 92.60 & P & PARA & A & CY & HOM & NITRATE & 0235 & 38 \\
\hline 234 & $\operatorname{CAs} 90$ & 0.9977 & 0.0042 & 21.7689 & H & 92.60 & U & & A & CY & ном & NITRATE & U235 & 38 \\
\hline 235 & CAS 91 & 1.0052 & 0.0036 & 24.5355 & н & 92.60 & u & & $\mathbf{A}$ & CY & ном & NITRATE & U235 & 38 \\
\hline
\end{tabular}


Table C.1 (continued)

\begin{tabular}{|c|c|c|c|c|c|c|c|c|c|c|c|c|c|c|}
\hline RUN & MEMB & KEFF & SDM & GBAR & ENRLEV & ASSAY & REFLCOND & REYL & SYSGEOM & UNITGEOM & FUBLKIX & FUEL & FMATL & REP \\
\hline 236 & CASO1 & 0.9979 & 0.0022 & 24.9978 & н & 93.20 & 0 & & s & SP & НОМ & NITRATE & U235 & 12 \\
\hline 237 & CASO2 & 0.9960 & 0.0023 & 24.9338 & $\mathbf{H}$ & 93.20 & v & & $\mathbf{s}$ & SP & ном & NITRATE & U235 & 12 \\
\hline 238 & $\mathrm{CASO}_{3}$ & 0.9920 & 0.0027 & 24.8696 & H & 93.20 & U & & $\mathbf{s}$ & SP & HOK & NITRATE & U235 & 12 \\
\hline 239 & CASO4 & 1.0020 & 0.0024 & 24.8423 & H & 93.20 & v & & s & SP & ном & NITRATE & U235 & 12 \\
\hline 240 & CASO5 & 1.0086 & 0.0022 & 24.6298 & H & 97.70 & u & & $\mathbf{s}$ & SP & HOM & NITRATE & 0233 & 12 \\
\hline 241 & CASO6 & 1.0079 & 0.0025 & 24.6005 & H & 97.70 & u & & $\mathbf{s}$ & SP & ном & NITRATE & บ233 & 12 \\
\hline 242 & CASO7 & 1.0045 & 0.0023 & 24.5747 & H & 97.70 & v & & s & SP & ном & NITRATE & U233 & 12 \\
\hline 243 & CASOB & 1.0065 & 0.0023 & 24.5493 & H & 97.70 & u & & $\mathbf{s}$ & SP & HOM & NITRATE & U233 & 12 \\
\hline 244 & CAS09 & 1.0084 & 0.0023 & 24.5177 & H & 97.70 & u & & $\mathbf{s}$ & SP & HOM & NITRATE & U233 & 12 \\
\hline 245 & CAS 10 & 0.9979 & 0.0017 & 25.0633 & H & 93.20 & $\mathbf{v}$ & & $\mathbf{s}$ & SP & Hом & NITRATE & U235 & 12 \\
\hline 246 & CAS11 & 0.9959 & 0.0017 & 24.7279 & H & 97.70 & $\mathbf{u}$ & & $\mathbf{s}$ & SP & HOM & NITRATE & U233 & 12 \\
\hline 247 & CAS 12 & 0.9989 & 0.0023 & 25.0338 & H & 93.20 & v & & $\mathbf{s}$ & CY & Hом & NITRATE & U235 & 12 \\
\hline 248 & CAS 13 & 0.9983 & 0.0022 & 25.0378 & $\mathbf{H}$ & 93.20 & $\mathbf{u}$ & & $\mathbf{s}$ & CY & ном & NITRATE & U235 & 12 \\
\hline 249 & CAS 14 & 0.9992 & 0.0017 & 25.0618 & H & 93.20 & $\mathbf{u}$ & & $\mathbf{s}$ & $C Y$ & HOM & NITRATE & U235 & 12 \\
\hline 250 & CAS 15 & 0.9925 & 0.0019 & 25.0692 & H & 93.20 & u & & $s$ & CY & HOM & NITRATE & U235 & 12 \\
\hline 251 & CAS 16 & 0.9976 & 0.0016 & 25.0784 & H & 93.20 & $\mathbf{u}$ & & $\mathbf{s}$ & $\mathbf{C Y}$ & HOM & NITRATE & U235 & 12 \\
\hline 252 & CAS 17 & 0.9952 & 0.0020 & 24.6961 & H & 97.70 & U & & $\mathbf{s}$ & $\mathbf{C Y}$ & HOM & NITRATE & U233 & 12 \\
\hline 253 & CAS18 & 0.9973 & 0.0018 & 24.7122 & H & 97.70 & v & & $\mathbf{s}$ & CY & ном & NITRATE & 0233 & 12 \\
\hline 254 & CAS 19 & 0.9916 & 0.0015 & 24.7286 & H & 97.70 & $\mathbf{u}$ & & s & CY & ном & NITRATE & U233 & 12 \\
\hline 255 & CAS20 & 0.9927 & 0.0015 & 24.7480 & H & 97.70 & u & & $\mathbf{s}$ & CY & ном & NITRATE & U233 & 12 \\
\hline 256 & CAS21 & 0.9923 & 0.0015 & 25.0740 & H & 93.20 & $\mathbf{u}$ & & $\mathbf{s}$ & CY & Hом & NITRATE & U235 & 12 \\
\hline 257 & CAS 22 & 0.9915 & 0.0014 & 25.0786 & H & 93.20 & $\mathbf{u}$ & & $\mathbf{s}$ & CY & HOM & NITRATE & U235 & 12 \\
\hline 258 & CAS 23 & 0.9991 & 0.0014 & 25.0846 & H & 93.20 & u & & $\mathbf{s}$ & CY & HOM & NITRATE & U235 & 12 \\
\hline
\end{tabular}


ORNL/CSD/TM-238

\section{INTERNAL DISTRIBUTION}

1. B. L. Broadhead

2. J. A. Bucholz

3. R. L. Childs

4. M. G. Culbertson

5. H. L. Dodds

6. H. R. Dyer

7. W. E. Ford

8. V. M. Forsberg

9. O. W. Hermann

10. J. C. Ingram

11-15. W. C. Jordan

16-20. N. F. Landers

21. R. P. Leinius/G. E. Whitesides/ Central Research Library

22-26.

27-31.
32. J. P. Renier

33. J. S. Tang

34. R. G. Taylor

35. J. T. Thomas

36. J. C. Turner

37. C. C. Webster

38. R. M. Westfall

39. R. Q. Wright

40. Central Research Library

41-43. Enrichment Technology Library

44-45. Laboratory Records Department

46. Laboratory Records, RC

47-61. Nuclear Criticality Safety Analytical Methods Resource Ctr.

62. ORNL Patent Office

63. ORNL-Y-12 Technical Library, Document Reference Section

\section{EXTERNAL DISTRIBUTION}

64. J. B. Briggs, EG\&G Idaho, Inc., Reactor Physics Branch, P.O. Box 1625, Idaho Falls, ID 83415

65. L. C. Dolan, Manager, Regulatory Compliance, Westinghouse Materials Company of Ohio, P.O. Box 398704, Cincinnati, OH 45239

66. A. S. Garcia, Criticality Safety Representative, Argonne National Laboratory, Reactor Experiments \& Examination Div., ANL-W 765-A, P.O. Box 2528, Idaho Falls, ID 83403-2528

67. S. T. Huang, Manager, Nuclear Technology, The Ralph M. Parsons Company, 100 W. Walnut St., Pasadena, CA 91124

68. R. E. Miles, Nuclear Criticality Specialist, Rockwell International, Critical Mass Laboratory, P.O. Box 464, Golden, CO 80401

69. J. N. Rogers, Div. 8324, Sandia Laboratories, Livermore,CA 94550

70. Division of Engineering, Mathematics and Geosciences, U. S. Department of Energy, Washington, DC 20545

71. Office of Assistant Manager for Energy Research and Development, U. S. Department of Energy, Oak Ridge Operations, P.O. Box E, Oak Ridge, TN 37831

72-101. Office of Scientific and Technical Information, U. S. Department of Energy, P.O. Box 62, Oak Ridge, TN 37831 